\title{
$\therefore \therefore$
}

R E S E A R C H

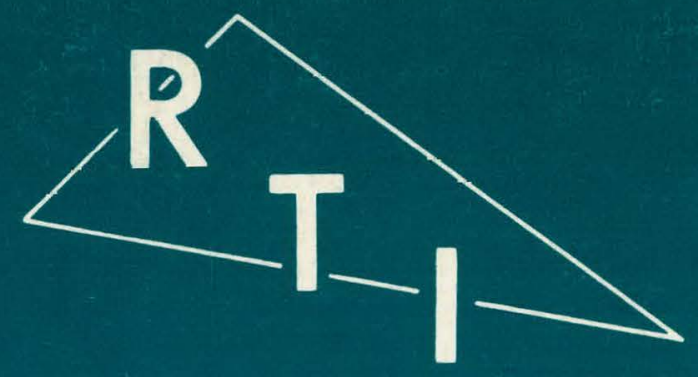

Final Report

Walter D. Bach, Jr. and

Fred M. Vukovich

Research Triangle Institute

Research Triangle Park, North Carolina 27709

Apri1 1980

Prepared for the
United States Department of Energy
Division of Solar Energy

DOE Contract No. EG-77-02-4470 


\section{DISCLAIMER}

This report was prepared as an account of work sponsored by an agency of the United States Government. Neither the United States Government nor any agency Thereof, nor any of their employees, makes any warranty, express or implied, or assumes any legal liability or responsibility for the accuracy, completeness, or usefulness of any information, apparatus, product, or process disclosed, or represents that its use would not infringe privately owned rights. Reference herein to any specific commercial product, process, or service by trade name, trademark, manufacturer, or otherwise does not necessarily constitute or imply its endorsement, recommendation, or favoring by the United States Government or any agency thereof. The views and opinions of authors expressed herein do not necessarily state or reflect those of the United States Government or any agency thereof. 


\section{DISCLAIMER}

Portions of this document may be illegible in electronic image products. Images are produced from the best available original document. 


\title{
COASTAL-INLAND \\ SOLAR RADIATION DIFFERENCE STUDY
}

\author{
Final Report
}

\author{
Walter D. Bach, Jr. \\ and \\ Fred M. Vukovich \\ Research Triangle Institute \\ Research Triangle Park, North Carolina 27709
}

Apri广 1980

Prepared for the
United States Department of Energy
Division of Solar Energy

DOE Contract No. EG-77-02-4470

This book was prepared as an account of work sponsored by an agency of the United States Government. Neithes tha United States Government nor any agency thereot, nor any of their employees. makes any

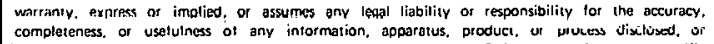

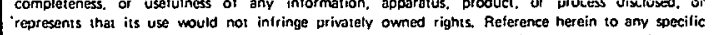
represents that its use would not iniringe privitely owmed rights, Reference herein

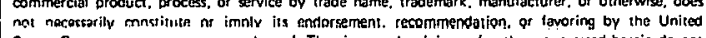


This study on coastal-inland solar radiation differences and the potential affect of the sea breeze circulation on those differences was performed under contract to the U.S. Department of Energy under contract number EG-77-C-02-4470. In order to perform this study, a field program was initiated in the period 1978 . Measurements were made of global, direct, and ultraviolet radiation from the sun and various meteorological parameters. The basic analysis of the field data was performed by the staff of the Geosciences Department of the Research Triangle Institute (RTI). Dr. Walter D. Bach, Jr. of RTI was project leader. Another portion of the analysis was performed under a subcontract to Duke University by Mr. Paul J. Gunthorpe to satisfy his thesis requirements for a Masters Degree in the School of Forestry and Environmental. Studies. His thesis is presented in Appendix 3.

Portions of the results of his thesis are also summarized in the body of the report.

RTI acknowledges the cooperation and support of Mr. Ed Foss and students at the Cape Fear Technical Institute; Mr. B. N. Ayscue, Superintendent of the Horticultural Crop Research Station at $\mathrm{Cl}$ inton, N.C. ; the personnel at the United States Marine Corps at Camp LeJuene; Mr. C. H. Eden; Mr. Wayne Rich, Manager of the Henderson Airport at Wallace; and Mr. John Lossie, Manager of the Ellis Airport. The National Weather Service at the Raleigh-Durham Airport provided GOES imagery and plots of coastal weather reports to the project. 
The purpose of this study was to quantify the characteristics of solar insolation in the coastal zone and to determine the effect of the sea breeze circulation on the global insolation. In order to satsify these objectives, the Research Triangle Institute (RTI) established a six station sampling network in the coastal plain of southeastern North Carolina (Figure 1), where previous evidence has indicated that the sea breeze circulation is almost a daily occurrence from late May through October. Three sites [Sloop Point, Onslow Beach, and Cape Fear Technical Institute (CFTI)] were located near the coast (coastal sites) to assess the insolation at the coast. A site (Clinton) was located in an area seldom affected by the sea breeze (about $100 \mathrm{~km}$ from the coast). Two additional sites, wallace and Ellis Airport, located between the coastal sites and the control site, were to be used to assess the transient impact of the sea breeze upon the insolation. Pyranometers were located at each site to measure the global insolation. Direct normal insolation measured by a pyrheliometer and ultraviolet radiation measured by UV radiometers were observed at the Sloop Point and Clinton sites only. Data were collected during the calendar year 1978.

The results of the study indicated that the global insolation had greater variability over the network during the summer season (June, July, and August). During the summer, there was a systematic diurnal variation of the difference in global insolation between the inland and the coastal sites. These differences were statistically significant at $0.5 \%$ level according to the Students-t test in the hours 1100 to 1500 EST. The data indicated that there was a depletion of global insolation at the inland sites. The average depletion over the day was $4.9 \%$, and the most significant depletion occurred in the period 1000 to 1600 EST and was $7.0 \%$.

The deficit of global insolation at the inland sites also occurred in spring (March, April, and May) and fall (September, October, and November); but the differences in those seasons were not 


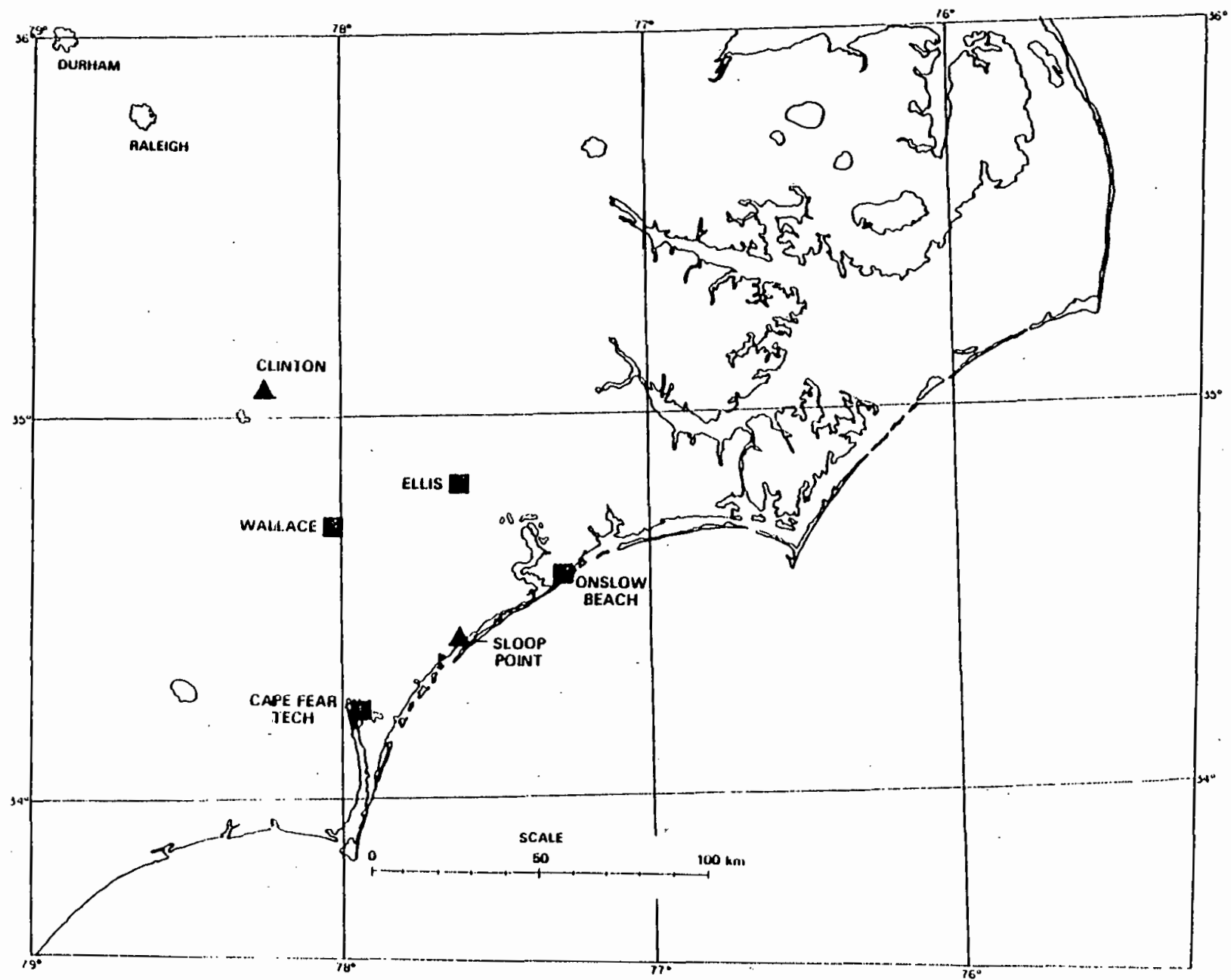

Figure 1. Sampling network for coastal-inland solar radiation study. All sites had pyranometers; the triangles refer to sites which also had pyrheliometers. 
statistically significant at the $1 \%$ level. In the spring, there was an average $3.7 \%$ depletion between 1000 and 1600 EST. In the fall, the diurnal minimum occurred in the.period 1100 to 1500 EST with an average depletion of $3.3 \%$. Though the differences in the spring and fall were not statistically significant, the diurnal trends were similar to those in the summer season suggesting that these seasons may also be affected by the same phenomena influencing the summer. No diurnal depletion of global insolation was noted at the inland site or at any site in the wintertime (December, January, February).

GOES satellite data were used to identify 32 days in the period May through September 1978, when the study area was affected by the sea breeze. In the GOES imagery, the sea breeze was identified as a line separating a zone of cloudiness on the inland side from a zone of relatively clear skies on the coastal side. Sites behind the front consistently received $5.0 \%$ more global insolation than those ahead of the front irrespective of the cloud amount. The depletion of global insolation by cloudiness associated with sea breeze fronts, which had greater vertical extent and horizontal dimensions than air mass cumulus, was greater than that produced by air mass cumulus by 5 percentage points.

Twenty-four of the 32 days were in the summer. Global insolation data on those summer days showed a similar systematic diurnal trend as found in the summer season; i.e., there was a depletion of global insolation at the inland sites relative to both the coastal and Clinton sites. However, in the average of the 24 days, the depletion was noted in the period 1100 to 1700 EST, whereas in the summer season and in a data set containing all the remaining summer days in which sea breeze characteristics could not be identified, the depletion occurred in the period 1000 to 1600 EST. In the respective time periods, there was $7.8 \%$ depletion of global insolation on definite sea breeze days, $7.2 \%$ for the data set noted as all other summer days, and $7.0 \%$ for the summer season. The set of 24 sea breeze days also showed a depletion of global insolation at the Clinton and inland sites after 1600 EST. This effect was not noted in any other data set. 
The 24 days identified as definite sea breeze days were probably days when the sea breeze circulation was very strong. This is supported by the persistence of the sea breeze until late in the day and by evidence that it may have affected the clinton site. It is expected that only very intense sea breeze circulations would penetrate as far as $100 \mathrm{~km}$ inland.

Over the 24 days in the summer in which definite sea breeze characteristics were identified, the patterns and trends were similar to those in the summer season. This would suggest that the summer season was governed by the sea breeze circulation. Undoubtedly, the sea breeze circulation existed on many of the summer days in which definite sea breeze characteristics could not be identified. On those days, the maximum intensity of the sea breeze circulation may have been reached earlier in the day. This would explain the rather high percentage differences found in the period 1000 to 1200 in the data set noted as all other summer days compared to the data set in which definite sea breeze characteristics were identified.

The inland sites did not always fall within the realm of maximum cloud activity associated with the sea breeze front. The analysis of the days identified as definite sea breeze days indicated that most fronts passed through at $10 \mathrm{~km}$ band about $20 \mathrm{~km}$ inland from the coast. Furthermore, frontal passage at the inland sites occurred around 1500 to 1600 hours, a time when the sea breeze normally begins to dissipate. This would suggest that the depletion of global insolation experienced at the inland sites may not be the maximum amount of depletion associated with the sea breeze cloudiness.

There was a depletion of direct normal insolation at the Sloop Point site relative to the Clinton site in the spring and fall. The depletion amounted to $20.5 \%$ in the spring, and $23: 2 \%$ in the fall. The depletion of direct normal insolation at the Sloop Point site was most pronounced in March and April and in October and November. There was no statistically significant differences in direct normal insolation between the coastal and inland site in the summer and winter. 
An examination of the synoptic meteorological data during this period revealed no mechanism which could be responsible for the marked depletion in the spring and fall. Furthermore, no significant statistical differences were noted in the global insolation data between sloop Point and Clinton in the spring and fall. In essence, the diffuse radiation made up for the loss in the direct normal insolation. This suggests that aerosols, water vapor, or even thin cirrus may be possible causes for the loss. To date, no adequate explanation for the cause of the marked depletion of direct normal insolation in the spring and fall have been determined. 
Foreword. . . . . . . . . . . . . . . . . . $i$

Summary . . . . . . . . . . . . . . . . . . . . $i i$

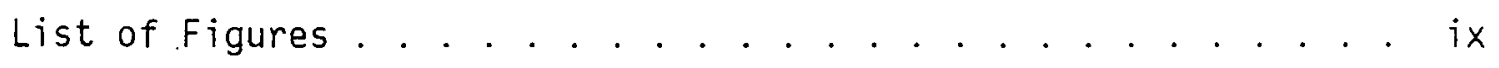

List of Tables. . . . . . . . . . . . . . . . . . . $x i$

1.0 Introduction. ............... 1

2.0 Research Plan............... 3

2.1 Network Design : . . . . . . . . . . . . 3

2.2 Instrumentation. . . . . . . . . . . . . . 7

2.3 Quality Assurance. . . . . . . . . . . . 9

2.4 Data Processing. . . . . . . . . . . 12

3.0 Global Insolation In The
Coastal-Inland Zone.................. 17

3.1 Seasonal Analysis. . . . . . . . . . . . 17

3.2 The Influence of The Sea Breeze Circulation
On The Global Insolation . . . . . . 2 27

3.2.1 Sea Breeze Characteristics. . . . . . . . . 31

3.2.2 Sea Breeze Cloudiness Versus
Global Insolation........ . . . 35

3.2.3 Global Insolation and Sea Breeze
Frontal Passages......... . . 37

3.2.4 Diurnal Variations of Global Insolation
on Sea Breeze Days. . . . . . . . . 38

4.0 Direct Normal Insolation In The Coastal-Inland. . . . 45 Zone

5.0 Summary and Conclusions ........... 51 
TABLE OF CONTENTS (continued)

Page

6.0 Topics For Further Research . . . . . . . . . . . . . 55

References. . . . . . .. . . . . . . . . . . . 57

Appendix 1: Field Operator Manual. . . . . . . . . . . . 59

Appendix 2: Monthly Data Tabulation. . . . . . . . . . 69

Appendix 3: Effects of the Sea Breeze Frontal Passage and

Associated Sea Breeze Induced Cloud Cover On

The Global Insolation of the Onslow Bay Region

of North Carolina. . . . . . . . . . . . 191 
1 Sampling network for coastal-inland solar radiation study. . . . . . . . . . . . . . . 4

2 Instrumentation at the Clinton Site. . . . . . . . . 5

3 Example of daily log at each station......... . 13

4 Mean hourly difference in global insolation between the Coastal and $C l$ inton classes (solid line) and the Inland and Clinton classes (dashed line) for each season. . . . . . . . . 21

5 The mean diurnal variation of the trend lines for global insolation for the COASTAL INLAND, and CLINTON classes for each. season..................... . . 25

6 GOES visible image for 17 June 1978 showing the line of demarcation (the sea breeze front) between the inland cloudiness and relatively clear skies at the coast............... . . 30

7 The spatial distribution over the network of the mean hour of sea breeze frontal passage based on the 32 days identified as sea breeze days using GOES data for the period May through September. . . . . . . . . . . . . . . . 32

8 Frequency of occurrence in days of sea breeze frontal passage over the network based on the 32 days identified as seabreeze days using GOES satelilite data in the period May through September. . . . . . . . . . 34

9 The value of $\bar{Q}^{\star}$ versus cloud amount for air mass cumulus (solid line) and sea breeze cumulus (dashed line)............... . 36

10 The value of $\bar{Q}^{*}$ versus hours in the day for locations ahead of (solid line) and behind (dashed line) the sea breeze front. . . . . . . 36 


\section{LIST OF FIGURES (continued)}

Figure Number

$\underline{\text { Page }}$

11 The mean diurnal variation of the trend lines for global insolation for the COASTAL, INLAND, and CLINTON classes passed on 24 days with definite sea breeze characteristics in the summer and on all other summer days... . . . . . . . . . . . . . 43

12 Mean hourly values of direct normal insolation at Clinton (solid line) and at Sloop Point (dashed line)

for each season. ... . . . . . . . . . . . . . 46 
1 Instrumentation sensitivity $(S)$ derived by experimentation and given by the manufacturer for each instrument at each site. . . . . . . . . . . . 10

2 Calibration values for the reference instruments for the two major calibrations and the percent difference between the values... . . . . . . . . . . . 11

3 The amount of data available in percent for each site and for each instrument by month. . . . . . . . 15

4 Correlation coefficients calculated using the hourly global insolation data for each station pair for each season. . . . . . . . . . . . . . . . 18

5 The ranked distribution of the normalized variance O for each season. . . . . . . . . . . . . . 20

6 Mean hourly global insolation differences between the Coastal and Inland classes for each season and the results for each hour of the students-t test. . . . . 23

7 Mean hourly percent difference in global insolation between the Coastal and Inland classes for each season............... . . 24

8 Sea breeze frontal passage times and number of occurrences for the six study location sites ordered in increasing distance from the

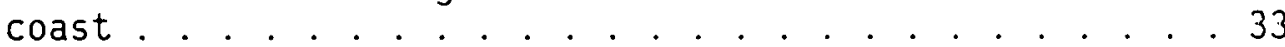

9 Trend classifications of the global insolation across the sea breeze front and the frequency of occurrence of each trend classification based on the 14 days the sea breeze was identified. . . . . . . 39

10 Mean hourly differences in global insolation (watts $/ \mathrm{m}^{2}$ ) and percent differences for the 24 days in th summer in which the sea breeze was identified. 


\section{LIST OF TABLES (continued)}

Table Number

Page

11 Mean hourly percent difference in global

insolation between the Coastal and Inland

classes for the 24 days in the summer when

the sea breeze was identified and for all

remaining summer days . . . . . . . . . . . . . . . . . 42

12 Mean hourly difference and percent difference

in direct normal insolation between Sloop Point

and $\mathrm{Cl}$ inton for each season. . . . . . . . . . . . . 47

13 Mean monthly percent difference in direct normal

insolation between Sloop Point and Clinton. . . . . . . . 49

14 Mean seasonal ratios of direct normal insolation

to the total horizontal insolation (global

insolation) calculated from observed data at

Sloop Point and.Clinton and estimated at other

Coastal and Inland sites from Boes et al's (1978) data. 50 


\section{THIS PAGE WAS INTENTIONALLY \\ LEFT BLANK}




\subsection{Introduction}

The United States Department of Energy is conducting a comprehensive resource assessment of the availability of solar insolation (as an energy source) across the United States. This report treats aspects of the resource availability in the coastal-inland regions of the United States. Very little information on the characteristics of solar insolation along the coastal zone of the United States is available. Information on the insolation along the coastal zone is important because $60 \%$ of the nations population lives within $100 \mathrm{~km}$ of the Atlantic, Gulf, Great Lakes, and Pacific coasts. Consequently, greater energy demands exist within these highly populated areas.

It is important to understand those factors which might affect the solar insolation received at the surface in the coastal zone. One of the most significant factors affecting the solar insolation is the cloud cover associated with the sea breeze circulation. The sea breeze is a convective circulation system which under certain moisture conditions can produce extensive cloud cover which will inhibit the amount of solar radiation received at the surface in the coastal zone. In order to quantify the characteristics of solar insolation in the coastal zone and to determine the effect of the sea breeze circulation on the solar insolation, Research Triangle Institute (RTI) conducted a research program with the following specific objectives:

- Measure hourly average amounts of global, direct and ultraviolet solar insolation on the scale of the sea breeze circulation for twelve months.

- Analyze those data for the effects of sea breeze circulation on the measured differences.

- Develop methodologies to translate the results to other coastal locations.

Insolation measurements were made at six sitcs in the coastal plain of southeastern North Carolina during the calendar year 1978. In this region, the sea breeze circulation is most prevalent in the late spring and early sunmer months when a maximum temperature difference exists between the land and water surfaces, though weaker 
sea breeze circulations may exist in the fall. Frequency of the sea breeze occurrences decreases toward the end of the warm season with the reduction of the land-sea temperature difference. The sea breeze front in this region is characterized by tall and dense cumulus clouds when sufficient water vapor exists. It is these cloud systems that can have significant effect on the solar radiation received at the surface. 
2.0 Research Plan

2.1 Network Design

A six station sampling network (Figure 1) was established in the coastal plain of southeastern North Carolina, where Hinn (1978) has indicated that the sea breeze circulation is a nearly daily occurrence from late May through October. The area is well suited to study the effects of sea breeze circulation on insolation because there are few other factors that affect the insolation. The terrain is flat. The land is used for growing timber or farming which generally have no affect on a large local circulation such as the sea breeze. Inland marsh lands are largely uninhabited and have an abundant moisture supply. Heavy industry and population centers are distant so local pollution is not a factor in the insolation. The coast line, oriented northeast to southwest is reasonably uniform, broken only by occasional bays and inlets.

Three sites, Sloop Point, Onslow Beach, and Cape Fear Technical Institute (CFTI), were located near the coast to assess the insolation at the coast. A site was located at Clinton, an area seldom affected by the sea breeze. Two additional sites, Wallace and Ellis Airport, were located between the coastal sites and the Clinton site, and were to be used to assess the transient impact of the sea breeze upon the insolation.

Sites were chosen on the basis of field of view, the availability of electrical power, and the availability of a secure but accessible location. A specific description of each station follows.

CLINTON - This station was located on the Horticultural Crops Research Station of the North Carolina Department of Agriculture about $6 \mathrm{~km}$ northeast of Clinton, North Carolina and was used as the primary control site. The site is approximately $100 \mathrm{~km}$ from the coast and is approximately $400 \mathrm{~m}$ south-southwest of the main buildings of the researeh station, about $5 \mathrm{~m}$ from an access road, and adjacent to a $1.2 \mathrm{~m}$ high row of myrtle bushes. The instruments were located on top of the shelter about $2 \mathrm{~m}$ above the ground. A pyranometer was mounted to the south, a pyrheliometer towards the north, with a UV radiometer between (see Figure 2). The site has excellent exposure to all sides. The tallest trees are approximately $15 \mathrm{~m}$ high and $150 \mathrm{~m}$ to the south-southeast of the site. 


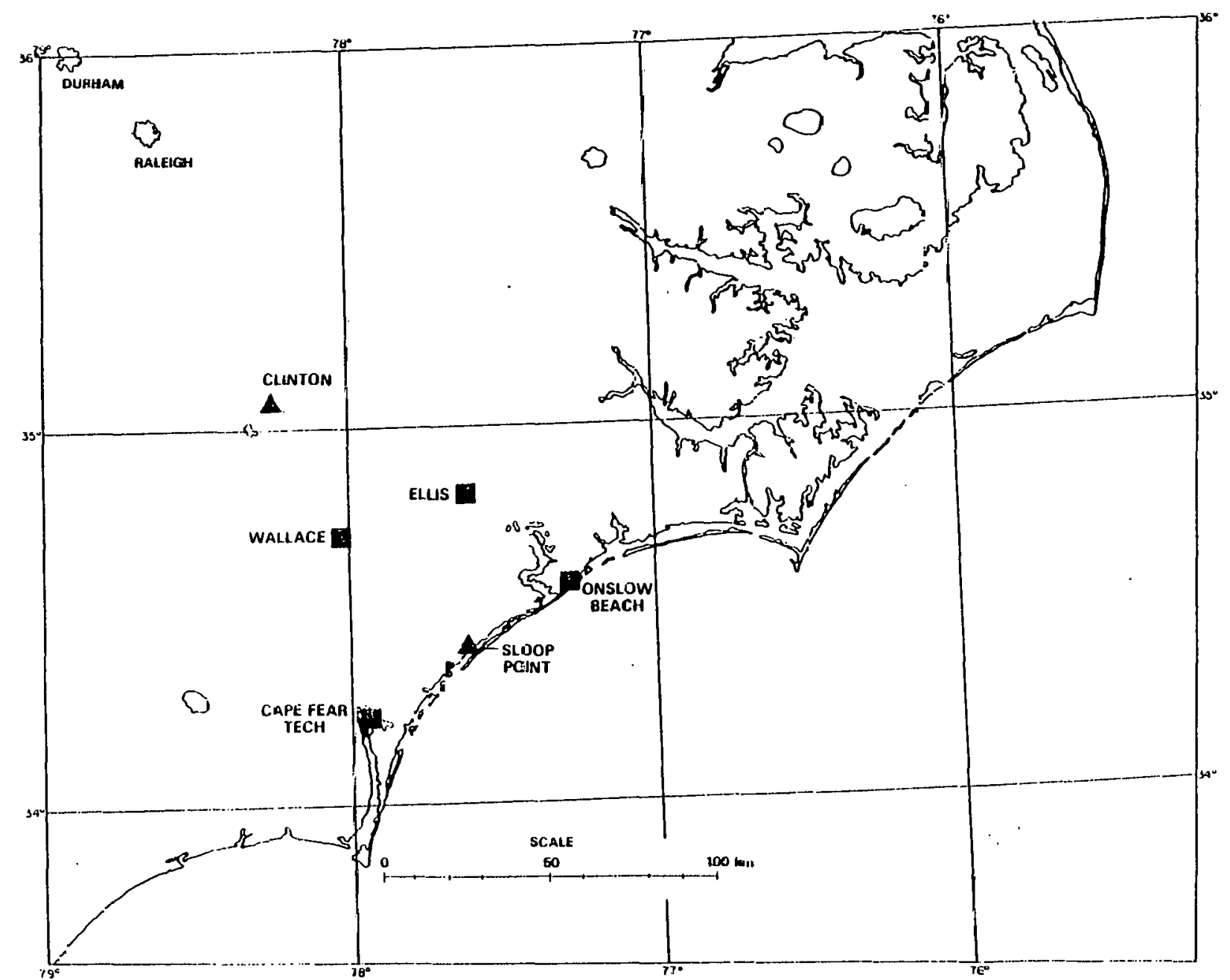

Figure 1. Sampling network for coastal-inland solar radiation study. All sites had pyranometers; the triangles refer to sites which alsc had purheliometers. 


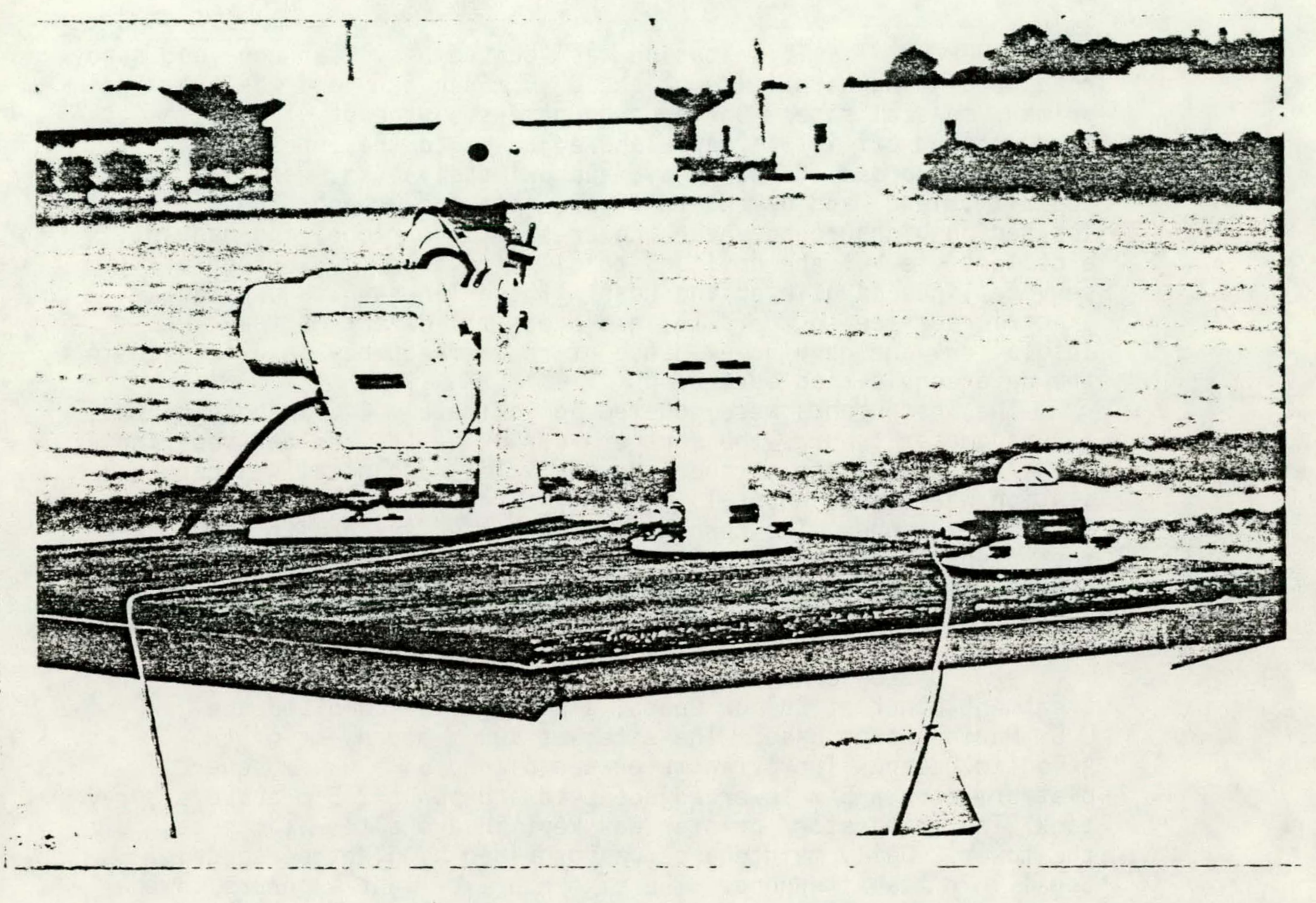

Figure 2. Instrumentation at the Clinton site. To the left is the pyrheliometer; center, the UV radiometer; and right, the pyranometer. 
A mechanical weather station was set up $20 \mathrm{~m}$ north-northwest of the shelter with excellent exposure for air flow from all

directions. The anemometer was approximately $3 \mathrm{~m}$ above ground. The station was exceptionally well maintained because of the active interest of the station attendant and that of the research station superintendent.

SLOOP POINT - This station was located beside an unpaved, sandy road separating cornfields on the $\mathrm{C}$. H. Eden farm and was the primary coastal site. One hundred meters southeast of the site, the land dropped off into a marshland adjacent to the Intercoastal Waterway. Across the waterway, approximately $4 \mathrm{~km}$, was Tupsail Island, and beyond that, the Atlantic Ocean. The site was located on high ground, near the crest of the ridge, and had an excellent view of the horizons to all directions. nverhead utility service lines paralleled the rudd. These lines which provided electric service to the site, had a minimal affect on the insolation, and gave no evidence of radio frequency interference in the data acquisition systems.

The instruments were mounted approximately $2.5 \mathrm{~m}$ above ground, a pyranometer towards the southern corner of the shelter roof and a pyrheliometer to the northern corner. The mechanical weather station was placed about $15 \mathrm{~m}$ down the road towards the water and $3 \mathrm{~m}$ above ground. The corn crop never interferred with the instrument exposure. Lacking rainfall in June and July, the plant. growth was stunted, never growing more than $1 \mathrm{~m}$ high.

ONSLOW BEACH - This site was located at the sewage treatment plant at Onslow Beach, a part of the Camp LeJeune, II. S. Marine Corps Base. The site was $100 \mathrm{~m}$ northwest of the Atlantic 0cean. The pyranometer was placed on a $0.4 \mathrm{~m}$ square platform atop a $5 \mathrm{~m}$ tower adjacent to and about $2.5 \mathrm{~m}$ above a spray tank. The integrator/ printer was kept in a shelter at the base of the tower. Daily maintenance was provided by plant personnel, usually in the aftemuon. The site had cxcellent exposure ton the horizon in all directions. The ground albedo at this sile is probably the greatest of any site in the study because of the white sand and lie lighly reflective metal roofs to the snuth of the site. However, the effect of reflections from roofs were minimized since lie pyranometer was mounted at a high pnint. along the beach above the rnof tops. The effects of a water tower about $800 \mathrm{~m}$ northeast of the site upon the installation are not known.

CAPE FEAR TECHNICAL INSTITUTE - A pyranometer was located on a small platform $15 \mathrm{~m}$ above the water level on the southeast corner of the upper deck of the Albert Lennon Marine Laboratory at the Cape Fear Technical Institute (CFTI). The laboratory is a moored barge in the Cape Fear River, adjacent to downtown Wilmington, North Carolina, about $15 \mathrm{~km}$ inland (west) from the Atlantic Ocean. To the east and southeast two buildings extend more than $10^{\circ}$ above the 
horizon, but there were no obstructions to the south and west. The integrator/printer was located in a secure office one floor below the sensor. A CFTI student tended the station daily. The station is approximately $7 \mathrm{~km}$ south of the National Weather Service Office, New Hanover County Airport, Wilmington, North Carolina.

Since the barge is subject to oscillations because of occasional river traffic, a brief study of the effects of those oscillations upon an hourly integrated value was conducted.

Regardless of the season, as long as the period of oscillation is less than 6 min., the integrated hourly error is less than one percent. The plane of the oscillation makes very little difference.

WALLACE - A pyranometer was located atop the one story terminal building of the Henderson Airport, Wallace, North Carolina, a site located about $55 \mathrm{~km}$ from the coast. The site has an unobstructed view of the sky from northeast to northwest. The local OMNI radio antenna and a utility pole with an attached mercury vapor lamp were located in the northern quadrant. The pyranometer cable lay across the roof of the building, entering a secure office through a conduit opening to the integrator printer. The site was tended daily by a local resident, charged with airport operations. The initial installation was completely destroyed on July 27 during an electrical storm. Damage apparently resulted from a line surge through the integrater printer, and the cable to the thermopile of the pyranometer. Spare units were installed promptly and remained in service throughout the rest of the field program.

ELLIS AIRPORT - A pyranometer was mounted atop an instrument shelter, approximately $20 \mathrm{~m}$ southeast of the middle marker on the approach to runway 05 at the Albert S. Ellis Airport near Richlands, North Carolina. This station was located about $45 \mathrm{~km}$ from the coast. Electric service was obtained through cooperation of the Federal Aviation Administration. The instrument was tended daily by airport security personnel making their routine checks, under the direction of the Airport Manager. In this location, the horizon was clearly visible in all directions with no obstructions to $3^{\circ}$.

\subsection{Instrumentation}

Eppley Precision Spectral Pyranometers (PSP) were used to measure the global insolation at all sites. Direct insolation was measured using an Eppley Normal Incident Pyrheliometer (NIP). Ultraviolet radiation was measured with an Eppley UV radiumeter. Only two NIPS and ultraviolet radiometers were used in the field program, and these were located at the Clinton and Sloop Point sites. Each NIP was mounted on an electrically driven, polar axis tracking mount made by Eppley. The data were recorded using Eppley integrator printer units 
$(I / P)$. These units accumulated the input voltage in counts (1000 counts per hour is equivalent to a $10 \mathrm{mv}$ output) for the hours from midnight to midnight. At predetermined intervals, the time and accumulated counts since midnight were printed on paper tape. The amount of energy incident upon the sensor at a given time period is proportional to the difference in the counts over that period. In normal operations, the data were printed hourly.

At Clinton, Sloop Point, Onslow Beach, and Ellis Airport, waterproof shelters were constructed to house the I/P units recording the insolation. In the winter, these shelters were painted dark green and were heated hy a thermostatically controlled 100-wat.t lightbulb. In the summer, the sides were painted white and air was drawn through the shelters by a small fan to help maintain proper temperature conditions. At C.FTI, the I/P units were housed in facilities provided for by CFTI; and at Wallace, in facilities at the Henderson Airport.

The tracking units for the pyrhelinmeters were aligned on the local meridian. At the minute of true solar noon, the shadow of a plumb line was permanently marked across the top of the instrument shelters at $\mathrm{Clinton}$ and Sloop Point. The base of the tracker unit was aligned along that line and was fastened to the top of the shelter. The pyrheliometer was mounted on the tracker and aligned in accordance with the manufacturer's instructions. The alignment was checked each morning and afternoon using the pin-hole and target spot that are a part of the instrument. Routine maintenance, described in Appendix 1, was scheduled as a daily responsibility of the station attendant. Adjustments to the declination angle were done on days with enough direct insolation to permit alignment using the pin-hole and target spot. The azimuth was readjusted by the field operator after power failures.

Mechanical weather stations manufactured by Meteorology Research, Incorporated were placed in the field at the Clinton and Sloop Point sites. This system measures wind run (miles of wind), wind direction, temperature, humidity, and rainfall. Slope of the wind run trace is proportional to wind speed. The temperature stylus is driven by a bimetal coil and the humidity stylus, by expansion and contraction of 
beads of Zerex. Precipitation is measured in 0.01 inch increments by a tripping bucket gauge.

\subsection{Quality Assurance}

A two-phase data quality assurance program was carried out to insure the integrity of the field data. Before the field program, an intercomparison of all instruments and data acquisition systems was performed on the campus of RTI. One instrument of each type was calibrated at the National Oceanic and Atmospheric Administrations Solar Radiation Facility before field deployment. Those instruments were retained at RTI for periodic checks of in-field instruments and as spares in case of field instrument malfunction or failure. Upon completion of the field program, an intercomparison of all systems (instruments and integrator/printers) was conducted for three weeks at RTI. The reference instruments were returned to NOAA for recalibration.

In the intercomparison tests before the field program, the sensitivity of each non-calibrated instrument was determined by comparison with the calibrated instrument. Results of the intercomparison are presented in Table 1 . The pyranometer sensitivities are consistently $3 \%$ lower than the manufacturer's value. The relative dispersion of the readings is about $1.3 \%$. This dispersion includes the total system--pyranometer, integrator, and printer. Some of the variance arises from the sensor. A constant 10 mv was applied to each integrator/printer unit. In each hour, the I/P units responded with 992 to 1002 counts for a maximum of $0.8 \%$ departure from the expected count. Most I/P units showed less than $0.5 \%$ error in an hour.

When the instruments were returned from the field, a similar analysis was conducted for a three week period. The calibration of the reference instruments changed only slightly. Table 2 shows the calibration values for each of the major calibrations. The changes in the pyranometers and pyrheliometers is less than $1 \%$ which is considered within anticipated error. The $2 \%$ change in the UV calibration is also within the expected range. Based on these results, the derived calibrations for the sensors did not change. 
TABLE 1. INSTRUMENT SENSITIVITY (S) DERIVED AND GIVEN BY THE MANIITAGTURER FOR EACH INETRUMENT AT EACH SITE

\begin{tabular}{lccc}
\hline \hline Instrument Type & Location & $\begin{array}{c}S^{*} \\
\text { (derived) }\end{array}$ & $\begin{array}{c}S^{*} \\
\text { (manufacturers) }\end{array}$ \\
\hline Pyranometer & Onslow Beach & 10.17 & 10.46 \\
& Sioop Point & 9.44 & 9.62 \\
& CFTI & 11.27 & 11.63 \\
& Ellis Airport & 10.99 & 11.09 \\
& Wallace & 11.00 & 11.23 \\
& Clinton & 11.00 & 11.24 \\
RTI & $10.37^{\dagger}$ & 10.61 \\
Pyrholiomoter & Sloop Puint & 8.85 & 8.85 \\
& Clinton & 8.29 & 8.29 \\
RTI & $8.64^{\dagger}$ & 8.70 \\
UV Radlometer & Sloop Point & 156 & 129 \\
& Clinton & 313 & 223 \\
& RTI & $153^{\dagger}$ & 133 \\
\hline \hline
\end{tabular}

* Number of millivolts required for nne watt/moter ${ }^{2}$

†NOAA-ERL calibration 
TABLE 2. CALIBRATION VALUES FOR THE REFERENCE INSTRUMENTS FOR THE TWO MAJOR CALIBRATIONS AND THE PERCENT DIFFERENCE BETWEEN THE VALUES

\begin{tabular}{lccc}
\hline & Pyranometer & Pyrheliometer & UV Radiometer \\
\hline Serial \# & $16142 \mathrm{F3}$ & $16220 \mathrm{E} 6$ & 16374 \\
Fall 1977 & $10.37^{*}$ & $8.64^{*}$ & $153.00^{*}$ \\
Spring 1979 & 10.46 & 8.60 & 156.10 \\
Percent Difference & 0.86 & 0.47 & 2.03 \\
\hline \hline
\end{tabular}

*Units are millivolts/watt meter ${ }^{-2}$ 
In-field calibrations were conducted during the first part of the field program. The reference instruments were removed from RTI and taken sequentially to each site. Sensor output was recorded on a dual pen strip chart recorder, and were integrated over the sample period, usually two hours. The instantaneous insolation recorded by each instrument was compared at quarter hourly intervals.

Each field station operator was thoroughly briefed upon proper station maintenance, i.e., daily cleaning of the optical opening of the instrument, daily check of the wiring, the dessicant, and the level of the unit. After performing these tasks, the time of day was recorded on the printer tape of each insolation instrument. The wind vane on the mechanical weather station was rotated through a full circle and the date and time were written on the strip chart. A daily log was maintained at each station (Figure 3 ). Also, a field stalion manual of operation was developed and kept at each location for reference (Appendix 1). Operators notified RTT whenever problems developed so that corrective maintenance could be performed.

\subsection{Data Processing}

Field insolation data and station logs were mailed to RTI by the station attendants twice each month. Each spt. of data was closcly rhecked for consistency in the cuunt values and in the time of the observation, especially when clocks are reset after power outages. After checking the data, the hourly count data were entered into a computer, stored on magnetic tape, and bulk processer The processing essentially converted counts to energy, $E\left(\mathrm{~kJ} / \mathrm{m}^{2}\right)$, through the follnwing formula:

$$
E=36 \wedge \Gamma / S
$$

where $\Delta C$ is the increment of counts in the hour, and $S$ is the instrument sensitivity determined by calibration.

Some of the I/P units were particularly sensitivity to intermittent signals which appeared on the power line. This typically caused increasing counts during the nighttime hours. In most cases, the counting was systematic and gave an approximate constant increment in counts during the nighttime hours. Lacking any further knowledge 
RESEARCH TRIANGLE INSTITUTE

COASTAL.INLAND SOLAR RAOIATION DIFFERENCE STUDY

DAILY LOG

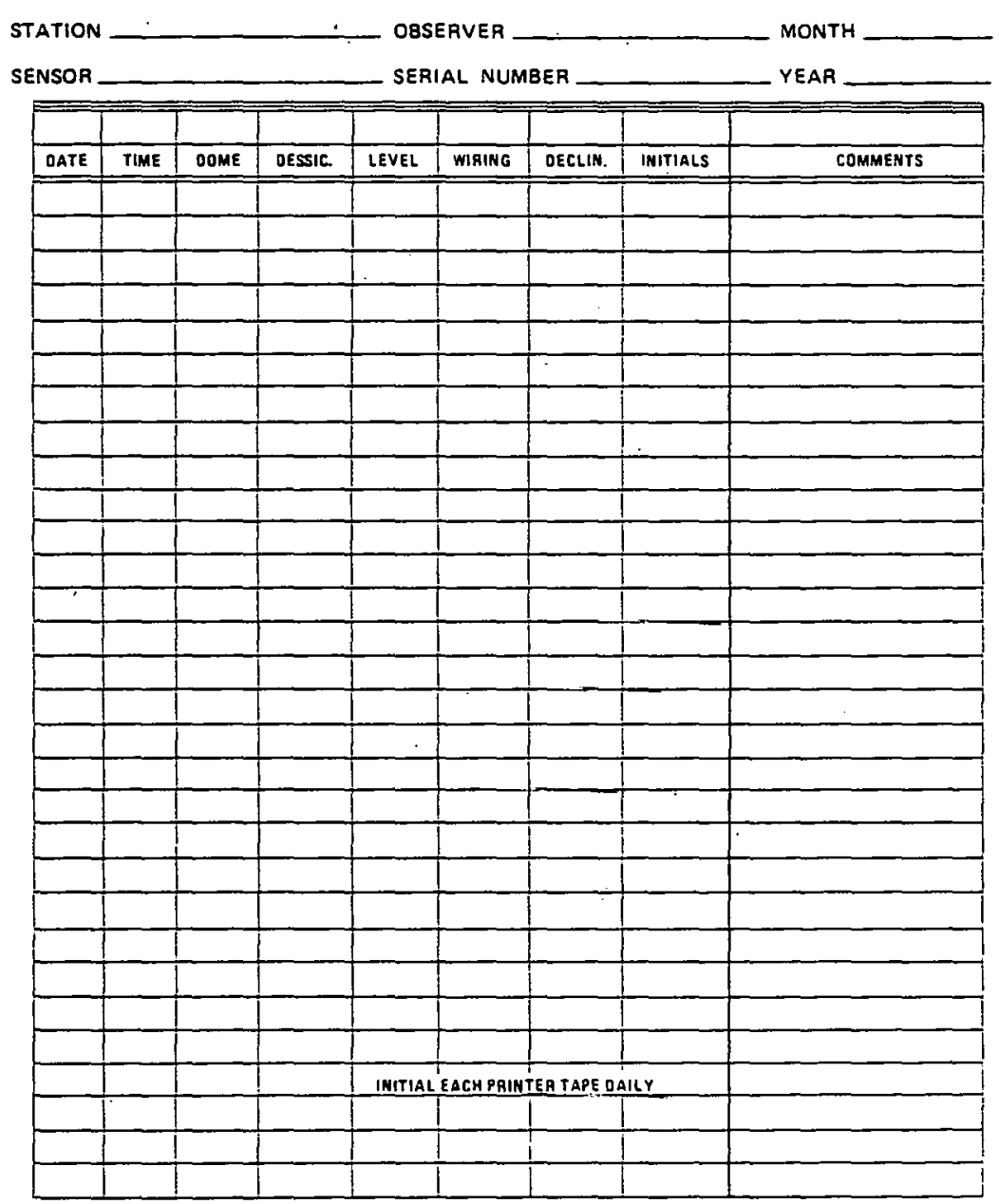

Figure 3. Exdmile of daily log at each station. 
of the data, the same trends were presumed to persist during the daytime hours. To remove the trends, the mean hourly increment of energy was computed using the nighttime values and subtracted from both the nighttime and daytime values. Infrequently, but significantly, transient signals caused a spurious, large number of counts. To remove the effect of these transients, only the energy increment less than $100 \mathrm{~kJ} / \mathrm{m}^{2}$ for the PSP or the NIP were used to determine the mean trend. At least two more than half of the nocturnal hourly observations including otherwise missing data were required to compute the mean trend. When there were insufficient data to compute the trend, all hourly data values for that day were considered erroneous and were indicated as missing.

After the nocturnal trends were removed, all nocturnal values were set to zero and all daytime values less than $90 / \mathrm{s} \mathrm{kJ} / \mathrm{m}^{2} \mathrm{hr}$ were also set to zero, i.e., for $\Delta C$ values generally less than 3 . The remaining values were compared with an idealized maximum value for that month and hour. The value was determined as the insolation received through an atmosphere of optical depth 0.92 on the longest day of the month. Low solar altitude was handled by requiring the sine of the solar elevation angle to exceed 0.15 during daylight hours. The maximum UV irradiance was assumed to be 8 percent of the maximum global radiation. Data that exceeded these maximum values were flagged and examined for possible errors. This procedure provided a filter for spurious data resulting from transient signals. It also indicated timing errors that occurred when station operators recorded the wrong time. It was also helpful in identifying count values that may have been ontered improperly into the computer. Unusual or extreme values were checked against original records and station logs, and were corrected when possible or designated as missing if there were no rationale for making a correction. Hourly values of incident radiation were plotted for each instrument for each month. Data and time of unusual patterns of insolation were identified and checked against the original records. Discrepancies or defficiencies in data were corrected. The availability of data in percent for each instrument at each station are given in Table 3 . 
- TABLE 3. THE AMOUNT OF DATA AVAILABLE IN PERCENT OF TOTAL AT EACH SITE AND FOR EACH INSTRUMENT BY MONTH. EXPLANATIONS ARE GIVEN FOR MAJOR DATA LOSSES.

\begin{tabular}{|c|c|c|c|c|c|c|c|c|c|c|c|c|c|c|}
\hline Instrument & Location & JAN & FEB & MAR & APR & MAY & JUNE & JULY & AUG & SEPT & $\mathrm{OCT}$ & NOV & DEC & Average \\
\hline \multirow{6}{*}{$\begin{array}{l}\text { Pyranometers } \\
\text { (global } \\
\text { insolation) }\end{array}$} & Onslow Beach & 81 & $36^{1}$ & 70 & 78 & 74 & 87 & 78 & $39^{2}$ & $80^{3}$ & 95 & 98 & 80 & 75 \\
\hline & Sloop Point & 76 & 76 & 98 & 95 & 99 & 95 & 76 & 96 & $81^{3}$ & 99 & $43^{5}$ & 79 & 81 \\
\hline & CFTI & 89 & $29^{1}$ & 89 & 94 & 97 & 90 & 86 & 96 & $63^{3}$ & 96 & 92 & 99 & 86 \\
\hline & Ellis Airport & 85 & 85 & 83 & 79 & $67^{1}$ & $77^{1}$ & 90 & 92 & 92 & $52^{1}$ & 66 & 95 & 80 \\
\hline & Wallace & 89 & 86 & 86 & 94 & 92 & 91 & $75^{6}$ & $88^{2}$ & 98 & 98 & 99 & 94 & 91 \\
\hline & Clinton & 97 & 96 & 87 & 97 & 99 & 90 & 90 & 98 & 77 & 98 & 94 & 97 & 93 \\
\hline \multirow{2}{*}{$\begin{array}{l}\text { Pyrheliometer } \\
\text { (direct normal } \\
\text { insolation) }\end{array}$} & Sloop Point & 53 & 83 & 99 & $81^{7}$ & 94 & 82 & $61^{4}$ & $34^{5}$ & $25^{5,3}$ & $40^{5}$ & 80 & 63 & 66 \\
\hline & Clinton & $26^{4}$ & 88 & 88 & 98 & 98 & 94 & 91 & 98 & 80 & 90 & 97 & 99 & 87 \\
\hline \multirow[t]{2}{*}{ UV Radiometer } & Sloop Point & 70 & 69 & 99 & 99 & -8 & -8 & 97 & 95 & $66^{3}$ & 95 & $44^{5}$ & 92 & 69 \\
\hline & Clinton & 97 & 99 & 75 & 97 & -8 & -8 & 99 & 97 & 96 & 98 & 97 & 99 & 80 \\
\hline
\end{tabular}

\footnotetext{
IIntegrator malfunctioned

2 Printer malfunctioned

3 Instrument dowr time due to severe weather (hurricane)

${ }^{4}$ Tracking unit malfunctioned

${ }^{5}$ Human factor

${ }^{6}$ Lightening damaje to instrumentation

${ }^{7}$ Moisture problem inside lens

${ }^{8}$ Calibration prob:em
} 
The data were also transferred to magnetic tape in the research-cooperative format of the National Climatic Center (NCC). The following WBAN numbers were assigned to the monitoring stations by NCC.

$\begin{array}{ll}\text { Clinton } & 93748 \\ \text { Wallace } & 93749 \\ \text { Wilmington (CFTI) } & 93750 \\ \text { Sloop Point } & 93751 \\ \text { Camp Lejeune (Enslow Beach) } & 93752 \\ \text { Jacksunville (Ellis Airport) } & 93753\end{array}$

These data will be available at NCC in that format at the close of this research project. Monthly summaries are presented in Appendix 2. 


\subsection{Global Insolation In The Coastal-Inland Zone}

\subsection{Seasonal Analysis}

Correlation coefficients were computed using the hourly insolation values across the network for each season of the year (Table 4). The summer season included the months June, July, and August; the fall, September, October, and November; the winter, December, January, and February; and the spring, March, Apri1, and May. Stations in the table are listed in order of their distance from the coast. The correlation coefficients are rather large for all seasons. In the fall, winter, and spring, correlation coefficients are greater than or equal to 0.86 . The average correlation coefficient among all station pairs for the fall, winter, or spring was approximately 0.93 for each season. However, in the summer, the correlation coefficient seldom exceeded 0.86 . The average correlation coefficient for the summer for all station pairs was approximately 0.84. The relatively high correlation coefficients in the fall, winter, and spring suggests that the insolation over the network had similar trends. The lower coefficients in the summer indicated that the summer data was more independent.

The data were stratified into three classes in order to simplify analysis: the COASTAL class (those stations within $15 \mathrm{~km}$ of the coast); the INLAND class (those stations from 16 to $70 \mathrm{~km}$ from the coast); and the CLINTON class (stations more than $70 \mathrm{~km}$ from the coast). The global insolation for the COASTAL class was defined as the mean of the hourly global insolation at Onslow Beach, Sloop Point, and CFTI sites. For the INLAND class, it was defined as the mean of the hourly global insolation at Wallace and Ellis Airport sites. The CLINTON class, in this case, refers to the measurements made at Clinton, North Carolina. This station was initially chosen as a reference location because it was expected that the site would be independent of coastal influences.

Seasonal mean differences between any two of the three coastal sites were computed for each hour. Statistical analysis indicated that the mean differences were not significantly different from zero. A similar analysis for the Wallace and Ellis Airport data also showed 
TABLE 4. CORRELATION COEFFICIENTS CALCULATED USING THE HOURLY GLOBAL INSOLATION DATA FOR EACH STATION PAIR FOR EACH SEASON

\begin{tabular}{|c|c|c|c|c|c|c|c|}
\hline Site & $\begin{array}{l}\text { Onslow } \\
\text { Beach }\end{array}$ & $\begin{array}{l}\text { Sloop } \\
\text { Point }\end{array}$ & CFTI & Ellis & Wallace & Clinton & \\
\hline $\begin{array}{l}\text { Onslow } \\
\text { Beach }\end{array}$ & & 0.86 & 0.82 & 0.84 & 0.81 & $\cap 7.9$ & \\
\hline $\begin{array}{l}\text { Sloop } \\
\text { Point }\end{array}$ & 0.95 & & 0.88 & 0.83 & 0.84 & ก 8? & \\
\hline CFTI & 0.92 & 0.95 & & 0.86 & 0.85 & 0.83 & $\sum_{\Sigma}^{W}$ \\
\hline Ellis & 0.92 & 0.94 & 0.93 & & 0.90 & 0.85 & $\vec{c}$ \\
\hline Wallace & 0.89 & 0.92 & 0.92 & 0.96 & & 0.88 & \\
\hline Clinton & 0.89 & 0.92 & 0.91 & 0.95 & 0.94 & & \\
\hline & \multicolumn{7}{|c|}{ SPRING } \\
\hline
\end{tabular}

\begin{tabular}{|c|c|c|c|c|c|c|c|}
\hline Site & $\begin{array}{l}\text { Onslow } \\
\text { Beach }\end{array}$ & $\begin{array}{l}\text { Sloop } \\
\text { Point }\end{array}$ & CFTI & Ellis & Wallace & Clinton & . \\
\hline $\begin{array}{l}\text { Onslow } \\
\text { Beach }\end{array}$ & & 0.90 & 0.90 & 0.86 & 0.91 & 0.88 & \\
\hline $\begin{array}{l}\text { Sloop } \\
\text { Point }\end{array}$ & 0.95 & & 0.98 & 0.92 & 0.96 & 0.95 & \\
\hline CFTI & 0.94 & 0.96 & & 0.93 & 0.97 & 0.96 & 岁 \\
\hline Fllis & 0.81 & 0.92 & 0.87 & & 0.93 & 0.93 & $\xi$ \\
\hline Wallace & 0.95 & 0.84 & 0.33 & 0.92 & & 0.97 & \\
\hline Clinton & 0.91 , & 0.91 & 0.91 & 0.90 & 0.95 & & \\
\hline \multicolumn{7}{|c|}{ FALL } & \\
\hline
\end{tabular}


that the mean differences were not significant. Results of the analysis indicated that the development of the COASTAL and INLAND classes using combined data were justified.

The normalized variance, $D$, was computed to quantify the variability of insolation across the network using data in the three defined classes for each season of the year. The parameter $D$ was defined:

$$
D=\frac{1}{12} \sum_{h r=7}^{18}\left\{(\text { INLAND-CLINTON })_{h r}^{2}+(\text { COASTAL-CLINTON })_{h r}^{2}\right\} / C L I N T O N
$$

When the insolation across the network is uniform for a given day, $D$ is zero. However, when there is a large variability, 0 will be large. The values of $D$ in Table 5 are ranked from low values to the high values for each season. The summer has the most variability compared to the other seasons across $99 \%$ of the rank. The values of $D$ in the spring are not significantly different from that in the fall or winter, which show the least variability, across the first $75 \%$ of the rank. In the remaining $25 \%$ of the rank, the D-values in the spring are more like those in the summer season, suggesting the possibility that those days in the spring may be influenced by the same phenomena that influences the summer season.

The mean hourly differences of global insolation between the COASTAL and CLINTON classes and between the INLAND and CLINTON classes are shown for each season of the year in Figure 4. In each season, the COASTAL and CLINTON class differences are small and generally positive, and there is no apparent trend from season to season.

On the other hand, large variations are noted in the hourly differences between the INLAND and CLINTON classes. In the summer, the differences are negative (indicating a depletion of radiation at the inland sites) and are greater than those found in the other seasons. The spring differences are also negative and are larger than those found in the fall or winter. In the fall, the trends in both curves are nearly identical indicating that all sites were influenced by the same phenomena. Winter differences are small throughout the day. These data indicated that the principal differences occurring in the network are consistently between the COASTAL and INLAND classes. 
TABLE 5. THE RANK DISTRIBUTION OF THE NORMALIZED VARIANCE D FOR EACH SEASON. THE LOWEST VALUE OF D HAS A RANK OF 1; AND THE HIGHEST, A RANK OF 81.

\begin{tabular}{rrrrr}
\hline \hline Rank & Winter & Spring & Summer & \multicolumn{1}{c}{ Fall } \\
\hline 1 & 2.9 & 1.5 & 4.9 & 5.7 \\
11 & 8.5 & 9.1 & 34.3 & 26.4 \\
21 & 33.4 & 23.5 & 63.7 & 48.2 \\
31 & 63.9 & 72.0 & 136.5 & 74.0 \\
41 & 109.3 & 109.5 & 189.0 & 109.1 \\
51 & 151.3 & 178.6 & 294.1 & 224.0 \\
61 & 211.3 & 242.2 & 389.8 & 270.1 \\
71 & 310.7 & 529.3 & 615.9 & 415.4 \\
81 & 782.3 & $1,010.7$ & $1,265.5$ & 746.9 \\
\hline \hline
\end{tabular}



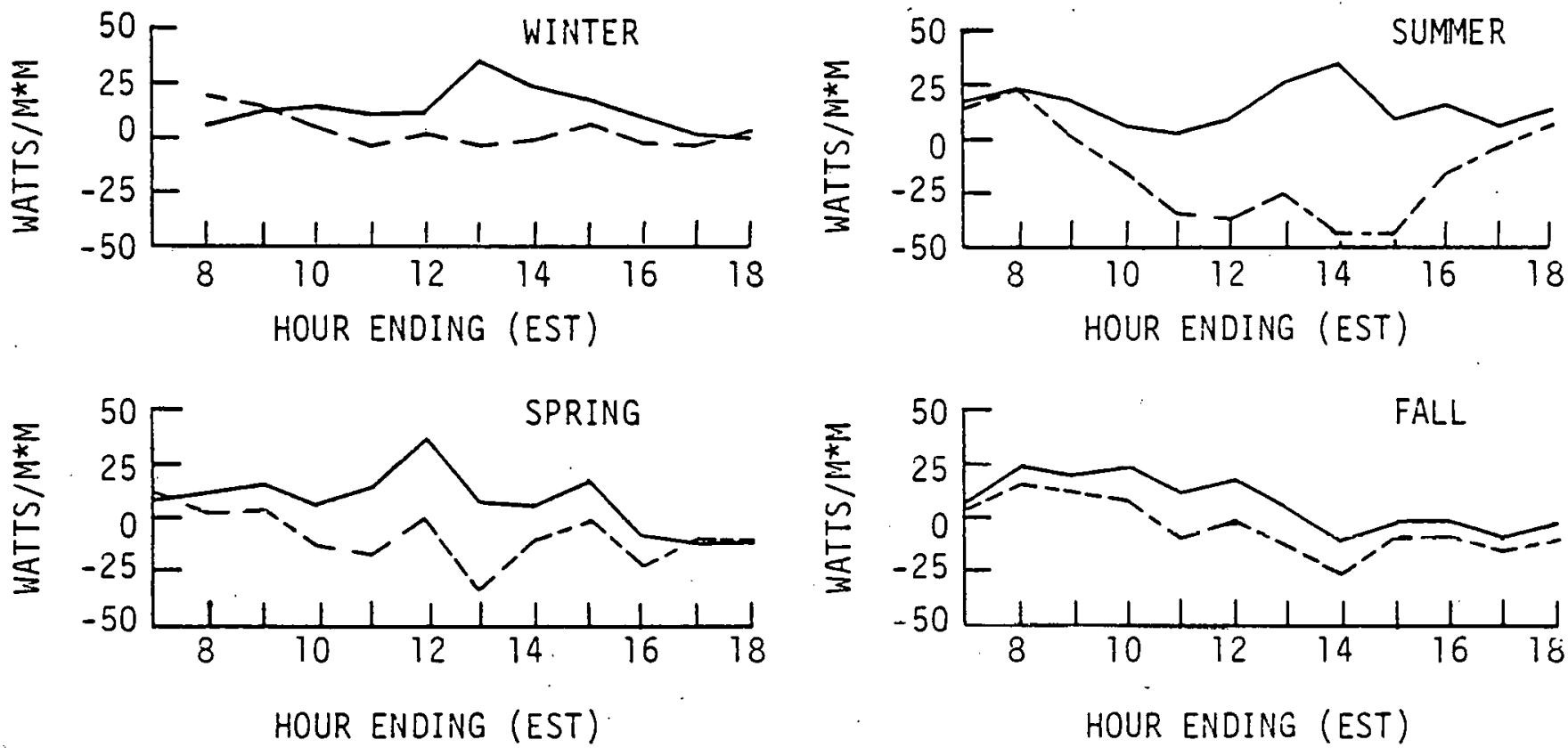

Figure 4. Mean hourly difference in global insolation between the Coastal' and Clinton classes (solid line) and the Inland and Clinton classes (dashed line) for each season. 
The mean hourly global insolation differences between the COASTAL and INLAND classes for each season are given in Table 6 . In this case positive differences indicate a depletion of solar radiation at the inland sites. Systematic diurnal trends are noted in spring, summer and fall, but not in the winter. The winter diurnal pattern is reminescent of random noise. The maximum difference in the summer occurs at around 1400 EST and is the largest compared to all seasons. Spring maximum occurs at 1300 EST and the fall maximum at 1200 EST. The fact that the maximum occurs around mid-day suggests that the differences may be related to the available solar insolation. The Students-t test rejected at the $0.5 \%$ level the hypothesis that the mean hourly differences were zero in the summer between 1100 and 1500 EST, and at the 5.0\% level, between 1000 and 1600 EST. In other seasons and particularly in the spring and fall, statistical significance at a low percentage level was not found in any time interval, but the hypothesis was rejected at the $1.0 \%$ level at 1300 EST in the spring and winter.

The hourly percentage difference between COASTAL and INLAND classes of global insolation relative to the COASTAL class is given in Table 7. Neglecting the values at low sun angle, the largest percentage depletion of global insolation at the inland sites (10.6\%) occurs in the summer. The maximum percentage depletion at the inland sites in the winter is $8.2 \%$. Though the winter period has the second largest percentage depletion of global insolation, it is associated with a diurnal difference pattern which does not have a systematic diurnal trend, suggesting that the value, though the difference is significant at the $1.0 \%$ level, may have a lower probability of recurrence. Of the seasons with diurnal patterns which were systematic, the spring season had the second lorgest (5.5\%) rullowed by the fall (4.3\%). The average depletion of global insolation in the summer over all hours was approximately $4.9 \%$, that in the spring was $1.2 \%$, and in the fall $3.4 \%$.

Figure 5 shows the hourly trend of global insolation across the 
TABLE 6. MEAN HOURLY GLOBAL INSOLATION DIFFERENCES BETWEEN THE COASTAL AND INLAND CLASSES FOR EACH SEASON AND THE RESULTS FOR EACH HOUR OF THE STUDENTS-T TEST

\begin{tabular}{|c|c|c|c|c|c|c|c|c|}
\hline \multirow{2}{*}{$\begin{array}{l}\text { Hour } \\
\text { (EST) }\end{array}$} & \multicolumn{2}{|c|}{ Spring } & \multicolumn{2}{|c|}{ Summer } & \multicolumn{2}{|c|}{ Fall } & \multicolumn{2}{|c|}{ Winter } \\
\hline & $\Delta^{*}$ & $p^{\dagger}$ & $\Delta^{*}$ & $\mathrm{pt}^{t}$ & $\Delta^{*}$ & $\mathrm{pt}^{\dagger}$ & $\Delta^{*}$ & $\mathbf{p t}^{\dagger}$ \\
\hline 0700 & -5.7 & .138 & 1.1 & .754 & 0.3 & .768 & & \\
\hline .0800 & 9.4 & .116 & 2.9 & .670 & 6.3 & .104 & -13.7 & .0001 \\
\hline 0900 & 10.6 & .184 & 17.3 & .093 & 4.8 & .517 & 3.7 & .474 \\
\hline 1000 & 16.9 & .149 & 31.2 & .030 & 13.0 & .214 & 7.4 & .266 \\
\hline 1100 & 27.4 & .059 & 44.6 & .0022 & 21.8 & .083 & 10.6 & .257 \\
\hline 1200 & 33.3 & .034 & 51.5 & .0017 & 24.3 & .027 & 3.0 & .751 \\
\hline 1300 & 38.0 & .006 & 54.8 & .0013 & 16.9 & .125 & 37.4 & .0004 \\
\hline 1400 & 13.4 & .292 & 78.4 & .0001 & 14.0 & .153 & 22.6 & .013 \\
\hline 1500 & 13.7 & .203 & 48.6 & .0031 & 7.5 & .431 & 8.8 & .221 \\
\hline 1600 & 12.9 & .22 & 30.8 & .041 & 2.1 & .762 & 11.2 & .030 \\
\hline 1700 & -1.3 & .86 & 9.1 & .363 & 2.9 & .340 & 2.6 & .360 \\
\hline 1800 & -1.7 & .67 & 3.9 & .433 & 3.6 & .050 & & \\
\hline
\end{tabular}

${ }^{*} \Delta$-mean difference, watts/meter ${ }^{2}$

$t_{\text {P-probability of exceeding } t}$ 
TABLE 7. MEAN HOURLY PERC.ENT DIFFERENCE IN GLODAL INSOLATION BETWEEN THE COASTAL AND INLAND CLASSES FOR EACH SEASON

\begin{tabular}{crrrr}
\hline $\begin{array}{c}\text { Hour } \\
\text { (EST) }\end{array}$ & Spring & Summer & Fall & Winter \\
\hline 0700 & -7.3 & 0.8 & 1.0 & - \\
0800 & 4.4 & 1.0 & 5.0 & -5.5 \\
0900 & 2.8 & 3.7 & 1.8 & 2.7 \\
1000 & 3.2 & 5.1 & 3.2 & 2.8 \\
1100 & 4.3 & 6.1 & 4.3 & 2.9 \\
1200 & 4.7 & 6.5 & 4.3 & 0.6 \\
1300 & 5.5 & 6.9 & 3.0 & 8.2 \\
1400 & 2.1 & 10.6 & 2.9 & 5.4 \\
1500 & 2.6 & 7.6 & 1.9 & 2.6 \\
1600 & 3.2 & 6.1 & 0.8 & 5.2 \\
1700 & -0.5 & 2.7 & 2.5 & 3.1 \\
1800 & -1.6 & 2.2 & 10.1 & 10.1 \\
\hline \hline
\end{tabular}



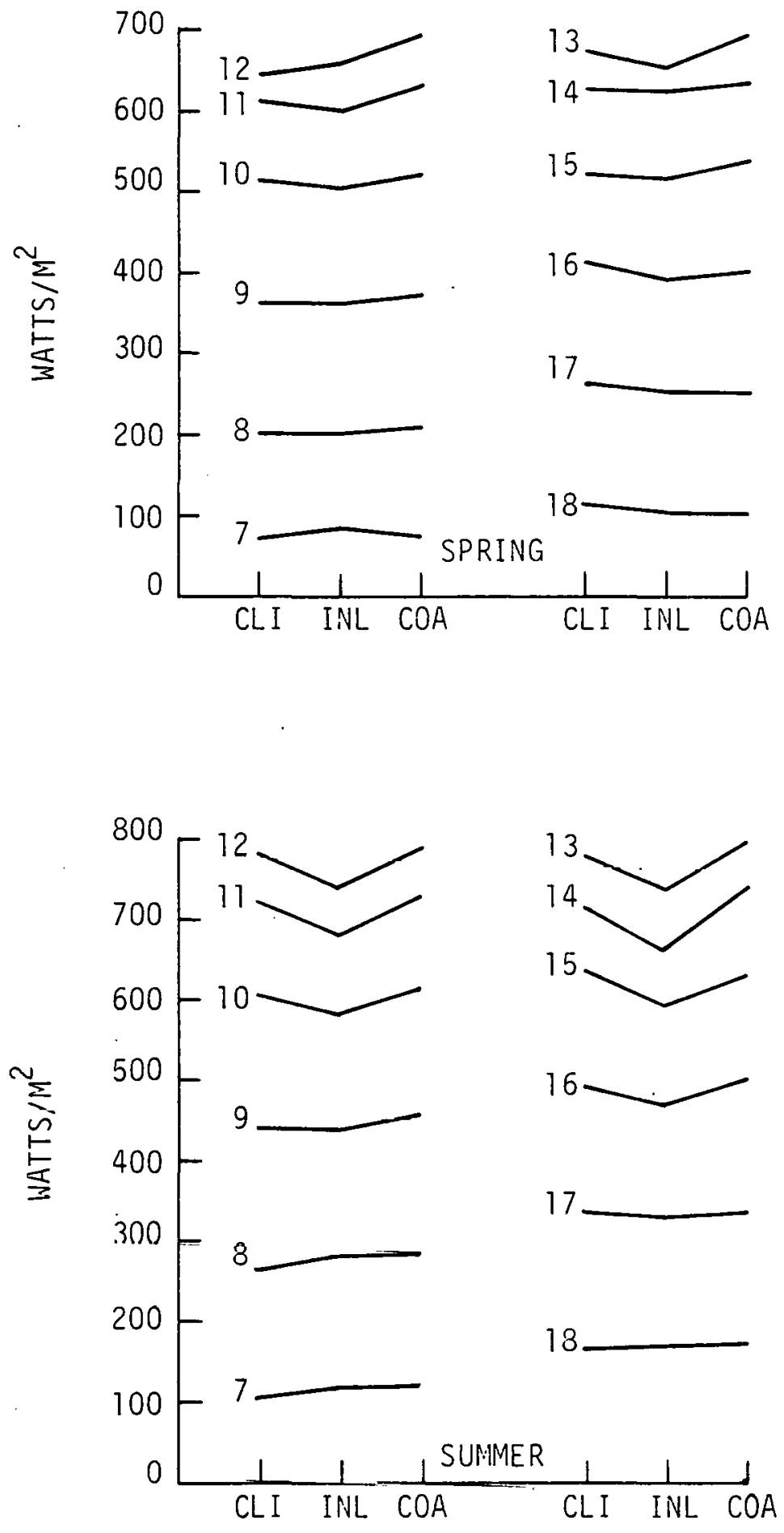

Figure 5. The mean diurnal variation of the trend lines for global insolation for the COASTAL, INLAND, and CLINTON classes for each season. 

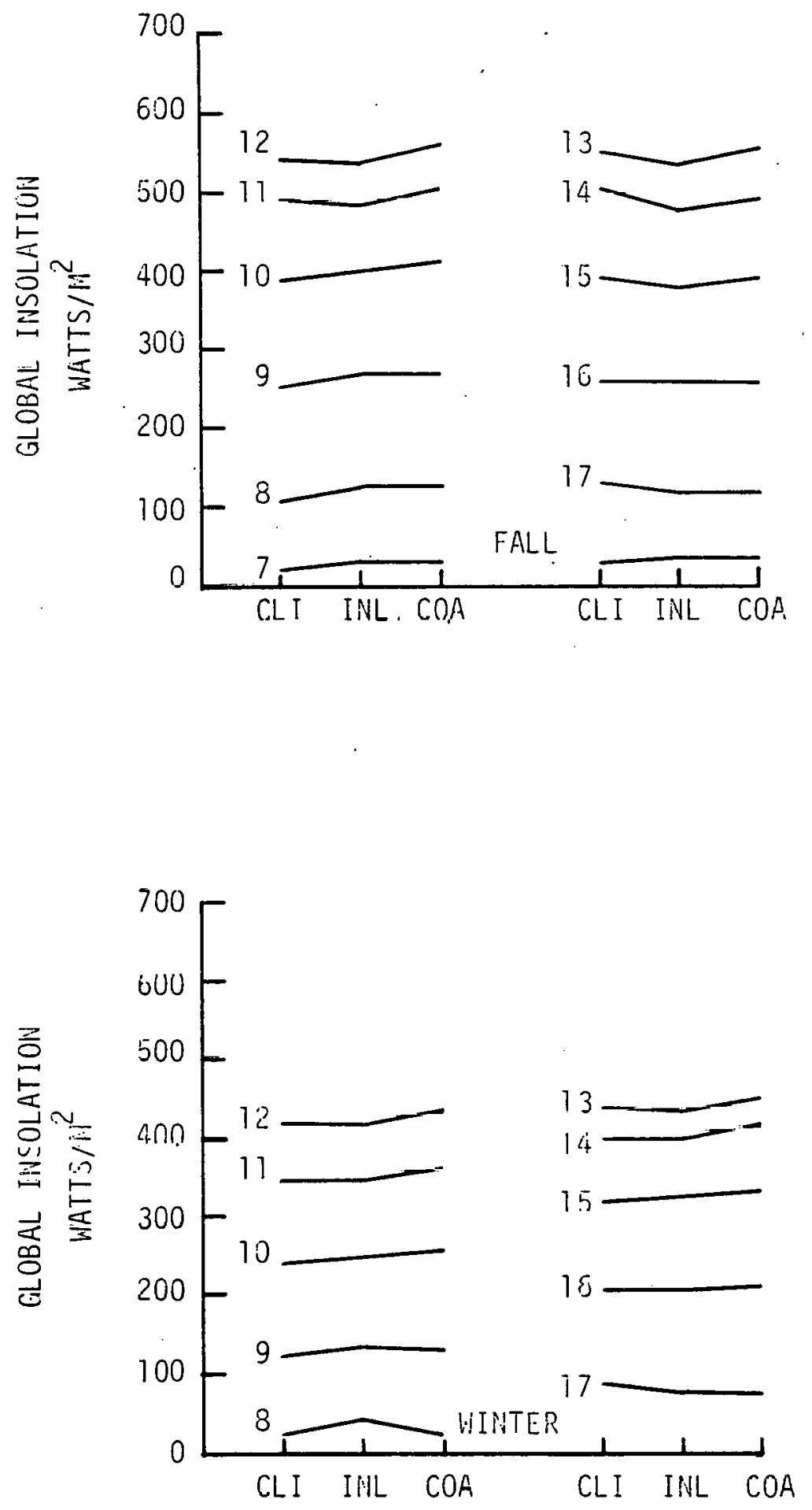

Figure 5. (continued) 
three classes in each season. The figure shows that in the summer after 1000 EST, a local minimum develops at the INLAND class. The difference between the INLAND class and the other two classes reaches a maximum at 1400 EST. After 1400 EST, the differences diminish rapidly becoming insignificant after 1600 EST. In the period 1000 to 1600 EST, the average depletion of global insolation at the INLAND class relative to the COASTAL class is $7.0 \%$.

A similar pattern is noted in the spring in the same time period. During the period 1000 to 1600 EST, there was an average $3.7 \%$ depletion of global insolation at the INLAND class. In the fall, the pattern was noted during the period 1100 to 1500 EST with an average depletion of global insolation during that period of $3.3 \%$. In the winter, no such pattern was noted. Though the differences in the fall and spring are not statistically significant, the similarity in trends with the summer trend suggests a real effect with a mechanism similar to that found in the summer but having less intensity.

\subsection{The Influence of The Sea Breeze Circulation On The Global Insolation \\ The data presented in the previous section indicate a systematic} diurnal trend characterized by a localized minimum in the global insolation at the inland sites relative to the coastal sites and to the Clinton site in the summer, and to a lesser extent in the spring and the fall. As indicated earlier, the sea breeze circulation is a nearly daily occurrence from late spring through the summer and through part of early fall in the North Carolina coastal region. This suggests the potential that cloud systems associated with the sea breeze may be influencing the global insolation received at the inland sites. This section describes the results of a study specifically designed to determine the influence of the sea breeze circulation on the global insolation.

The sea breeze circulation is a circulation induced by the land-sea temperature difference. During the day, the land is heated more than the water due to differences in the specific heat of soil and water. The air over the land is heated through conduction and convection. Continuity demands that the rising air is repiaced, 
setting up a low level transport of air from the ocean to the land. The warm rising air over the land expands and sets up a localized zone of high pressure in the upper air. This produces accelerations in the the air aloft from the land to the water. The air over the water subsides. If the rising air over the land is moist, the potential exists to develop clouds.

As the circulation develops, it generally moves further inland. The extent of the penetration of the low level air from the ocean, which is generally cooler and more moist, is controlled by the air-sea temperature difference and strength of the prevailing flow. When the boundary between the oceanic cool, moist air and the warm, drier air over the laild (the sed ureeze front) passes a location, the wind direction changes abruptly to a direction consistent with air coming from the sea, the humidity increases, and the temperature decreases. In the afternoon, terrestrial cooling decreases the temperature difference between the air and the sea and the driving mechanism no longer exists. At this time, the circulation and the cloudiness associated with the sea breeze dissipates.

In order to identify the sea breeze, meteorological data from the National Weather Service Station in Wilmington, from the Ell is Airport, and from the Sloop Point and Clinton sites were examined for abrupt changes in the wind direction, for humidity increases, and for temperature decreases, that would signify the passage of the sea breeze front. Unfortunately, no systematic occurrence of the specific features associated with the passage of the sea breeze front could be identified in these meteorological data. The network needed to identify the sea breeze circulation requires significantly more observing stations then were used by or available to this study. A mesoscale wind, temperature, and humidity sampling network must be established to identify the sea breeze circulation. Because these kind of data were not available, the results of using the available meteorological data were fruitless.

The Wilmington National Weather Service Station has a WSR-57 weather radar which keeps a constant surveillance of $250 \mathrm{~km}$ radius about the city of Wilmington. Every five minutes, a $35 \mathrm{~mm}$ pictlire of 
the PDI scope is taken. Those pictures were examined to identify the occurrence of sea breeze fronts by locating a line or group of convective cells generally thought to be indicative of that front. Examination of nine months of data did not reveal even a suggestion of the occurrence of the sea breeze.

However, definite sea breeze characteristics were identified on 32 days using GOES satellite data. The GOES satellite is a geosynchronous satellite positioned some 22,000 miles away from the earth. A geosynchronous satellite has an orbital speed identical to the rotation of the earth at the equator. Under these circumstances, the subsatellite point, the position of the satellite relative to a point on the earth, remains constant in time and space. Therefore, the satellite is able to collect synoptic scale data in the same region at essentially the same satellite look angle. The GOES satellite collects visible images from radiation in the 0.55 to 0.70 $\mu \mathrm{m}$ wavelength band and infrared images in the 10.5 to $12.6 \mu \mathrm{m}$ band. The resolution of the visible data is $0.8 \mathrm{~km}$, and that for the infrared data is $10 \mathrm{~km}$. This satellite provides infrared and visible images for a given region on a half-hourly basis. The visible imagery were used to determine the sea breeze front.

In the imagery, air mass and sea breeze cumulus clouds were classified on the basis of the vertical extent as determined by their relative brightness and by the size of the convective cells. In most cases, cumulus convection over land appears as a pattern consisting of cumulus humulus and the more vertically developed cumulus congestus. In the satellite imagery, sea breeze front was defined as the line between the inland cloudiness and this typically cloud-free area on the seaward side (Figure 6). Generally, the sea breeze front was found inland from the coast. Clouds along the sea breeze front had greater relative brightness in the visible images which generally means they have either greater vertical depth or greater horizontal dimensions than the air mass cumulii found further inland from the sea breeze front.

Diurnal variation of the sea breeze cloudiness was also depicted in the GOES imagery. The cloudiness associated with the sea breeze 


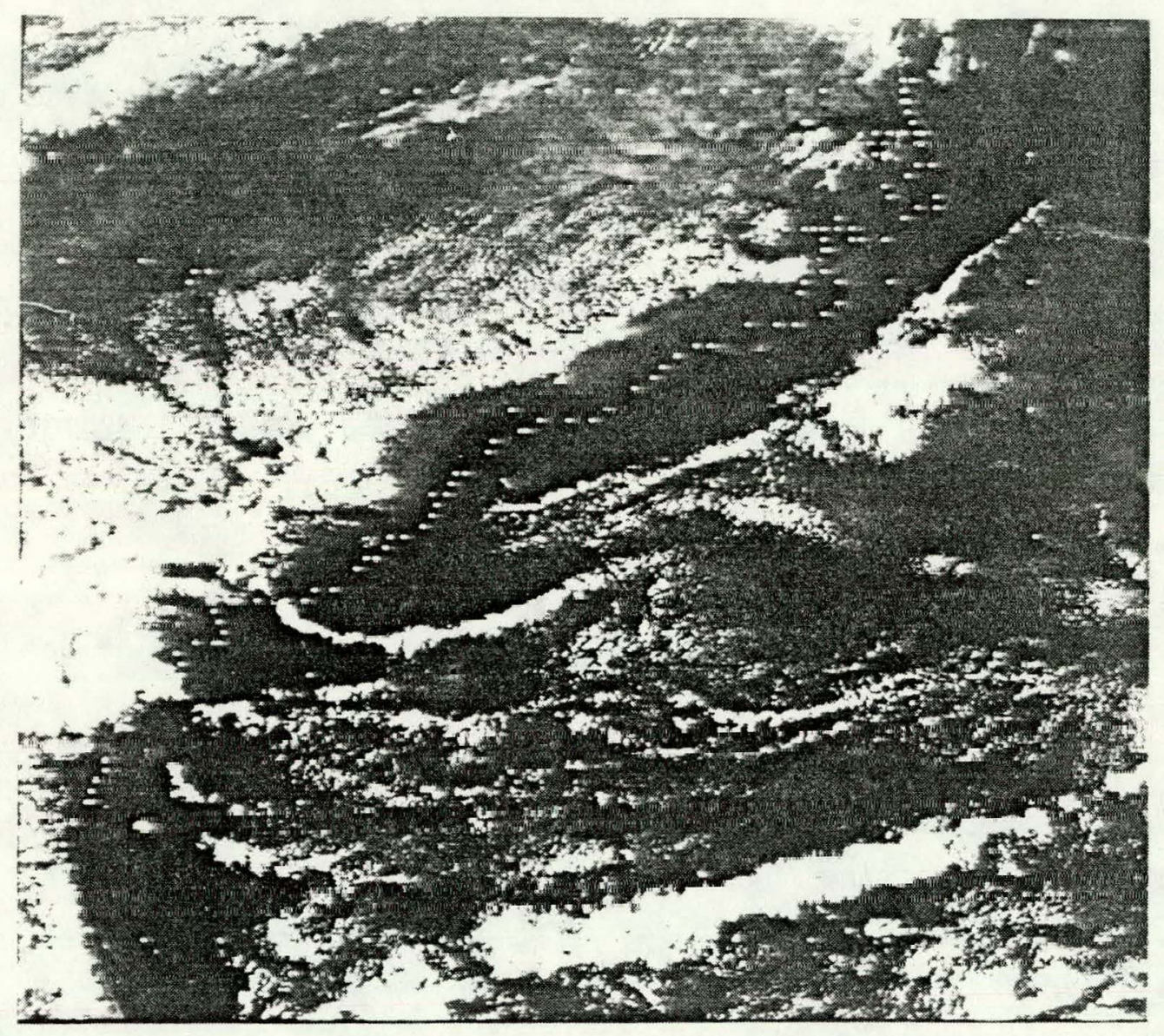

Figure 6. GOES visible image for 17 June 1978 showing the 1 ine of demarcation (the sea-breeze front) between the inland cloudiness and relatively clear skies at the coast. 
front formed over the 1 and somewhere between 0930 and 1000 EST near the coast. As the day progressed, the cloud cover seemed to increase in depth and horizontal extent, and the front moved further inland. Depending upon the circumstances, the line generally moved inland until about 1500 to 1600 EST in the afternoon. Thereafter, the line dissipated.

The satellite imagery was the sole basis on which the sea breeze front and the cloudiness associated with the sea breeze front could be determined. Only 32 days clearly showed the sea breeze in the period May through September 1978. The following describes the analysis of the satellite data and the global insolation data collected during that period.

\subsubsection{Sea Breeze Characteristics}

Characteristics of the sea breeze frontal passages were derived from the 32 sea breeze days. Sea breeze frontal passages were defined to occur when the seaward edge of the sea breeze cumulus cloud line passed over a given location and a clearing trend developed. The mean hour of sea breeze frontal passages relative to different locations in the area of the network is shown in Figure 7; and a summary of the time of sea breeze frontal passages and the number of occurrences for all six study sites is given in Table 8 . These data indicate that in only about $42 \%$ of the cases did a sea breeze frontal passage occur at the inland sites (Wallace and Ellis Airport). Frontal passages generally occurred between 1500 and 1600 hours at the inland sites which is about the time the sea breeze begins to dissipate.

Based on the 32 cases available, the maximum number of sea breeze frontal passages occurred within a $10 \mathrm{~km}$ wide area, approximately 20 $\mathrm{km}$ inland from the coast (Figure 8 ). Fewer frontal passages occurred closer to the coast due to frontal developmenl a few kilometers inland from the coast. These data indicate that the inland sites were located inland from the region of maximum sea breeze frontal activity and cloudiness associated with that frontal activity. Furthermore, 


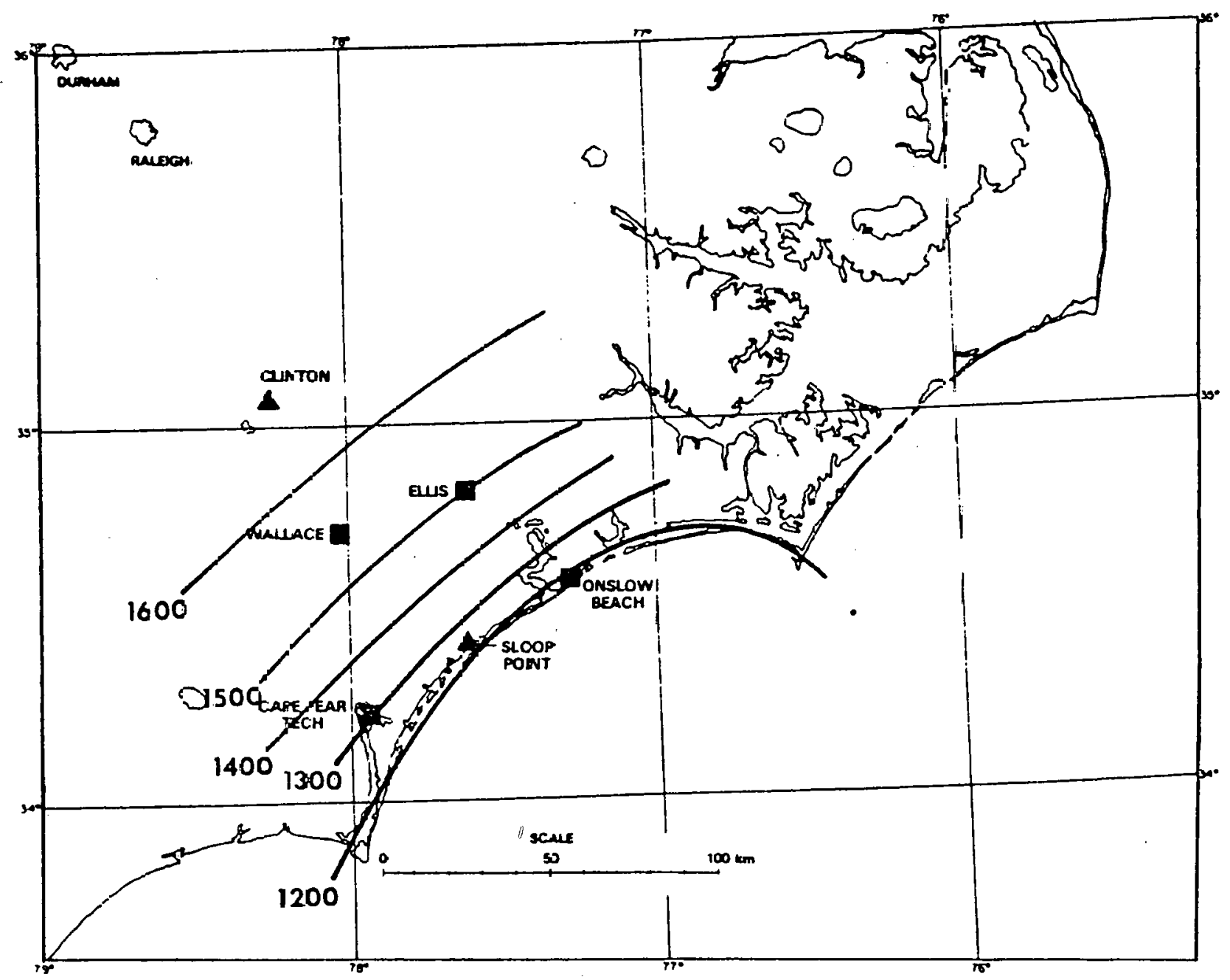

Figure 7. The spatial distribution over the network of the mean hour of sea breeze frontal passage based on the 32 days identified as sea breeze days using GOES data for the period May through September. 
TABLE 8. SEA BREEZE FRONTAL PASSAGE TIMES AND NUMBER OF OCCURRENCES FOR SIX STUDY LOCATION SITES ORDERED IN INCREASING DISTANCE FROM THE COAST

\begin{tabular}{lcccccc}
\hline \multirow{2}{*}{$\begin{array}{c}\text { Passage } \\
\text { time (EST) }\end{array}$} & Onslow. Beach & Sloop Point & Cape Fear & Ellis & Wallace & Clinton \\
\cline { 2 - 7 } $0600-1100$ & 7 & 7 & 3 & 0 & 0 & 0 \\
$1100-1400$ & 11 & 13 & 16 & 6 & 3 & 0 \\
$1400-1800$ & 2 & 1 & 5 & 15 & 12 & 0 \\
\hline \hline
\end{tabular}




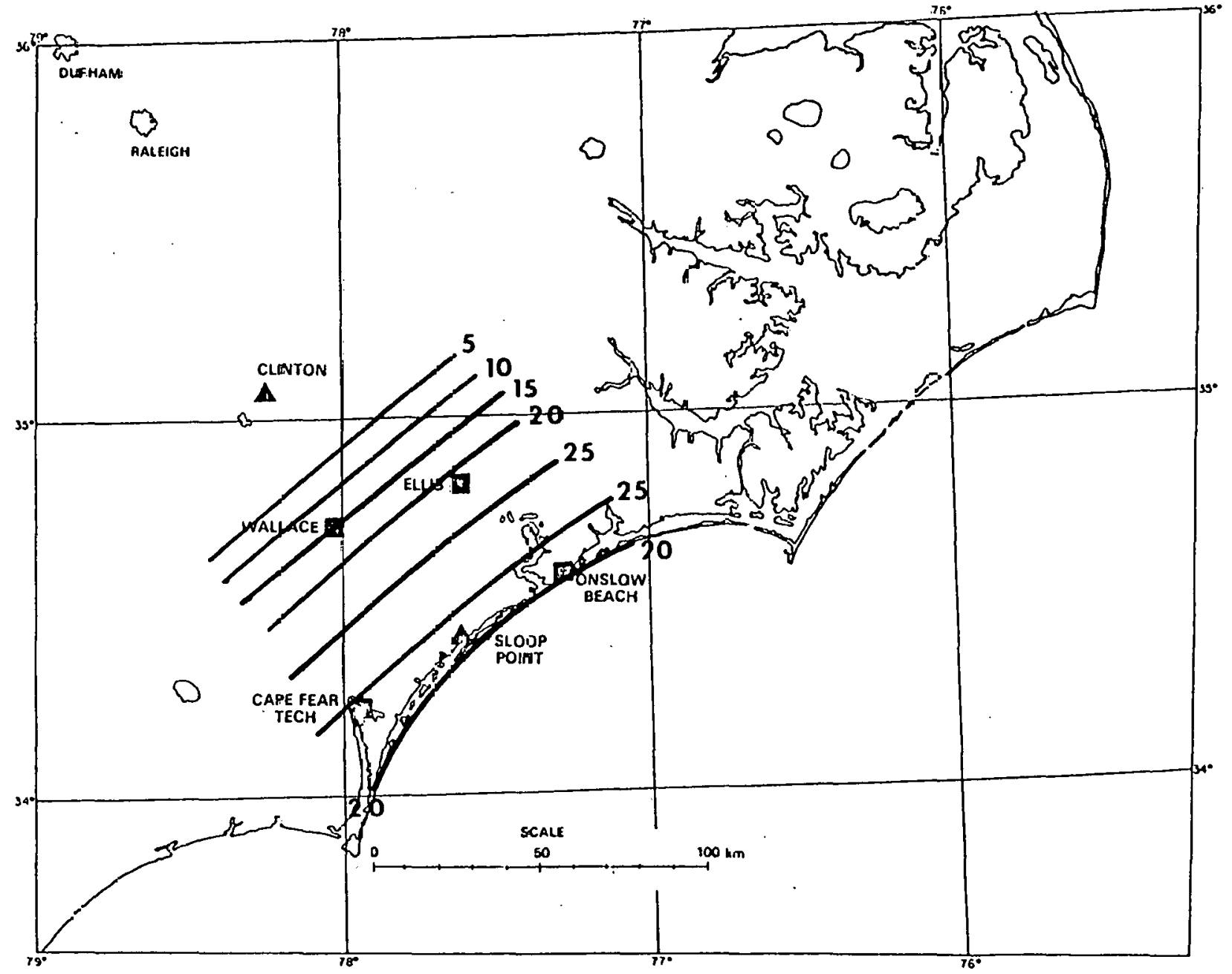

Figure 5 . Frequency of occurrence in days of sea breeze frontal fassage over the network based on the 32 days identified as sea bretze days using GOE'S satellite data in the period May through Sepzember. 
the data suggests that the inland sites experienced sea breeze frontal passage around the time when the sea breeze was in its dissipation stage.

\subsubsection{Sea Breeze Cloudiness Versus Global Insolation}

Maximum monthly values of global insolation for each daylight hour were determined for clear sky conditions from the available data base on sea breeze days as defined by the GOES imagery. These values were used to produce a dimensionless quantity, $Q^{*}$, the ratio of the measured global insolation under a specific cloud amount to the clear sky global insolation for a given month and hour. The GOES imagery were used to determine cloud amounts. Cloud fields centered within a $20 \mathrm{~km}$ radius of each study site were used to determine the cloud amounts, a technique formulated by Avaste (1964). Estimates of the percentage of cloud cover over each of the study sites were made using a transparency placed over the imagery. This transparency included reference points for positioning, observation points marking locations of each of the study sites, and a $20 \mathrm{~km}$ radi $i$ circle centered on each of the observation sites. Visual estimates were made of the cloud amount in tenths of sky cover within these circles. This was done for both air mass and sea breeze cumulus clouds. Hourly cloud cover data were calculated by averaging cloud amounts measured from two half-hourly images taken during a particular hour.

A large number of cloud observations obtained during the late afternoon period made possible the comparison of the quantity $Q^{*}$ for sea breeze and air mass cumulus. There was a depletion of qlobal insolation by as much as 5 percent at locations under sea breeze cumulus relative to locations under air mass cumulus for cloud amounts ranging from 0.1 to 0.7 (Figure 9). Insufficient data prevented comparisons for cloud amounts greater than 0.7 . The difference in $Q^{*}$ for the two types of cumulus clouds are attributed to the greater vertical extent and horizontal dimensions of the sea breeze cumulus.

The value of $Q^{*}$ associated with a constant amount of sea breeze cumulus cloud cover decreases with time of day. Increasing thickness of individual clouds in a sea breeze convergence zone with time of day resulted in the reduction of $Q^{*}$ from 0.858 in the late morning and 


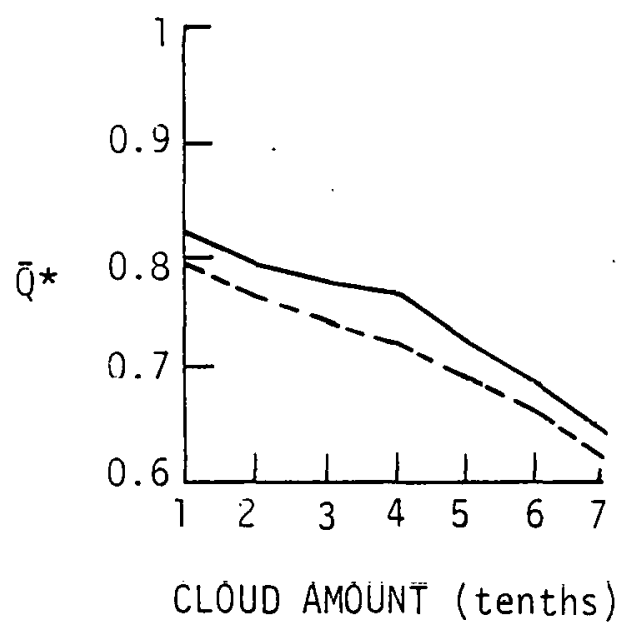

Figure 9. The value of $\bar{Q}^{*}$ versus cloud amount for air mass cumulus (solid line) and sea breeze cumulus (dashed line).

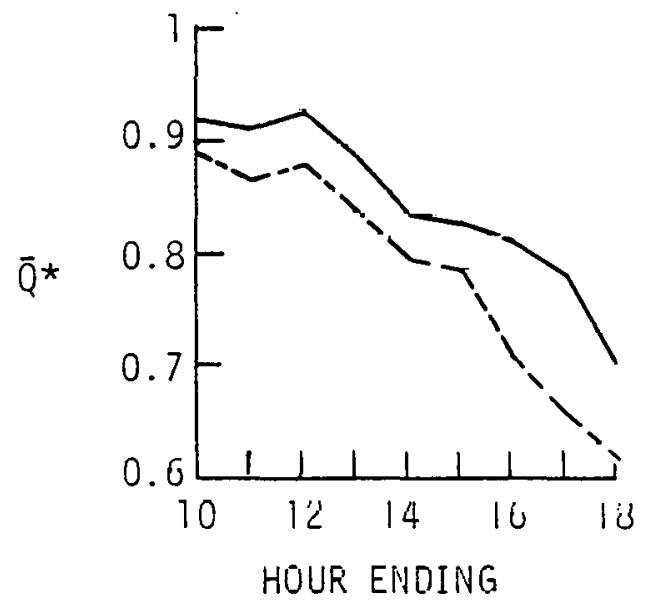

Figure 10. The value of $\bar{Q}^{*}$ versus hours in the day for locations ahead of (dashed line) and behind (solid line) the sea breeze front. 
midday hours to 0.763 during the late afternoon hours for a less than 0.2 sky cover. Air mass cumulus clouds exhibit a smaller diurnal increase in vertical extent for small cloud amounts. The diurnal change in solar elevation angle along with small increases in cloud thickness for air mass cumulus clouds with less than 0.2 sky cover produces a corresponding diurnal change in $Q^{*}$ from 0.897 in the late morning and midday hours to 0.807 in the late afternoon hours.

\subsubsection{Global Insolation and Sea Breeze Frontal Passages}

Sea breeze frontal passage was usually characterized by a marked decrease in cloud cover and an increase in global insolation at the sites behind the front. Locations behind the sea breeze fronts consistently exhibit larger $Q^{*}$ values than those sites ahead of the front during the same hour of the day (Figure 10). The values of $Q^{*}$ observed ahead of the front range from 0.886 to 0.622 , and that behind the front, from 0.923 to 0.699 . The difference between $Q^{\star}$ values ahead of and behind the sea breeze front increased with time of day after the midday period. This spatial difference in $Q^{\star}$ increases from 0.043 at 1400 EST to 0.105 at 1700 EST as a result of temporal increases in cumulus cloud amounts and in vertical extent of the cloudiness ahead of the front.

Global insolation values from the six site were distributed into three catgories. The coastal category were those values obtained when the sea breeze front was inland of the measuring site. The frontal category were those values obtained when the measuring site was directly influenced by the sea breeze frontal zone. The inland category included those values obtained from measuring sites which had not yet experienced sea breeze frontal passages. A subset of 14 of the 32 days were chosen which included days in which all three catgories were present. For each of these selected days, the mean global insolation was computed for each of the three categories and for three different time periods (0600 to 1100 EST, 1100 to 1400 EST, and 1400 to 1800 EST). Values of the global insolation for the three categories were then summarized in the form of trend lines for each of the three time periods. Mean global insolation gradients among the 
three categories were determined using a $5 \%$ threshold value. Nine classifications of trend lines are possible. The trend analysis results are summarized in Table 9.

The major trend occurring during the period 0600 to 1100 EST was classification I, occurring in approximately $60 \%$ of the cases. Classification I indicates less than $5 \%$ difference in the mean global insolation among the three categories. Between 1100 and 1400 EST, classification VI was most prevalent, occurring $43 \%$ of the time; and classification I also occurred in $29 \%$ of the cases. Classification VI is characterized by having lower levels of global insolation at the frontal sites. Classification VI occurs as a result of increased cloud cover with greater vertical extent along the sea breeze convergence zone. Classifications II and VI occurred most often (29\% of the time) during the period 1400 to 1800 EST, with trend classification IV occurring $21 \%$ of the time. Trend classifications II and VI represent cases when the sea breeze front passes frontal sites relatively late in the day. In those cases when classification IV developed in the period 1400 to 1800 EST, the sea breeze front had passed a majority of the sites in the frontal category, and the frontal cloudiness remained close enough to those locations to reduce the global insolation under lower solar elevations.

\subsubsection{Diurnal Variations of Global Insolation on Sea Breeze Days}

The diurnal variation of the global insolation was examined on days when the sea breeze was definitely identified in the summer season (June, July, and August). This was done in order to compare diurnal trends established in this study with tlie summer seasonal trends discussed in the previous section. of the 32 sea breeze days identified using the GOES satcllite data, 24 occurred during the summer season. The mean hourly differences between the three previously defined classes-COASTAL, INLAND, and CLINTON-are given in Table 10. Also included is the percentage difference relative to the COASTAL class in the case of the COASTAL and INLAND class differences and the COASTAL and CLINTON class differences, and relative to the CLINTON class in the case of the CLINTON and INLAND class differences. 
TABLE 9. TREND CLASSIFICATIONS OF GLOBAL INSOLATION ACROSS A SEA BREEZE FRONT AND THE FREQUENCY OF OCCURRENCE OF EACH TREND CLASSIFICATION BASED ON THE 14 DAYS THE SEA BREEZE WAS IDENTIFIED
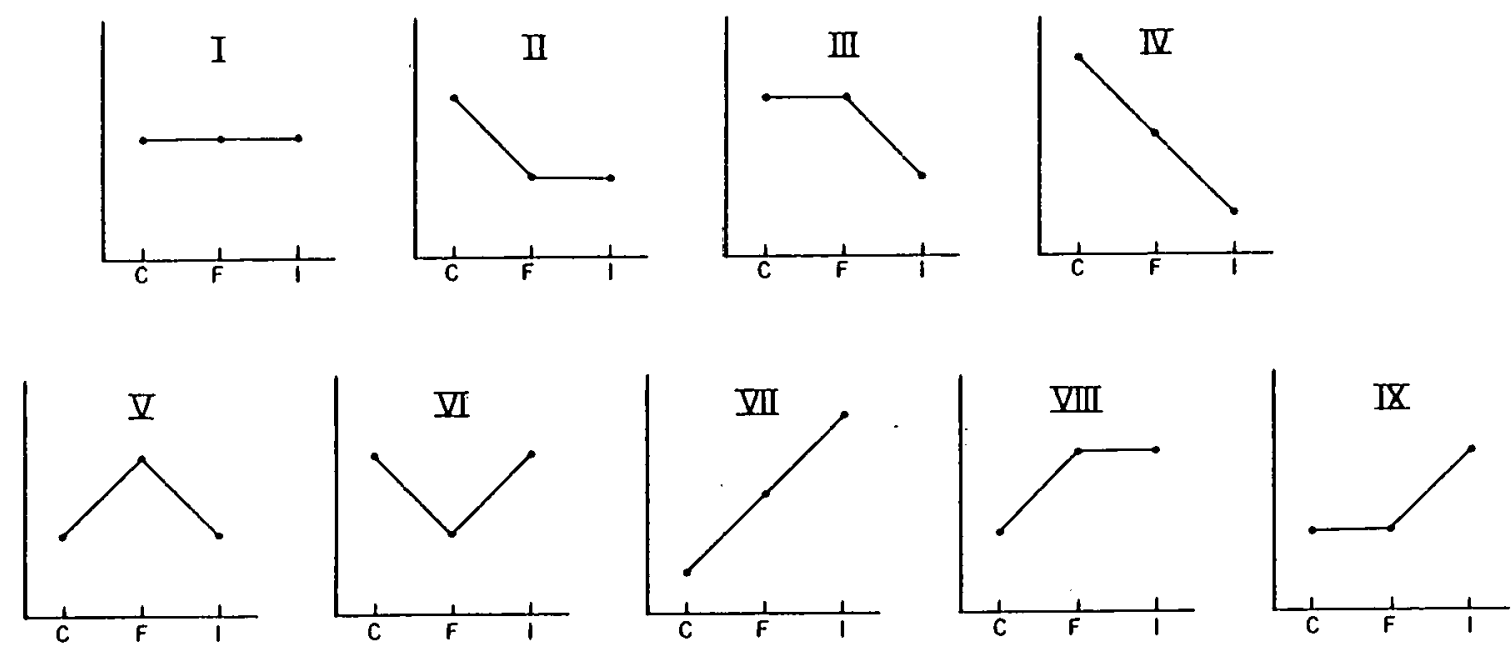

\begin{tabular}{lcccccccccc} 
& \multicolumn{10}{c}{ Tread Represeatations } \\
\cline { 2 - 7 } JOURS (EST) & I & II & III & IV & V & VI & VII & VIII & IX & Total \\
\hline $1600-1100$ & 8 & 2 & 0 & 1 & 0 & 3 & 0 & 0 & 0 & 14 \\
$1100-1400$ & 4 & 1 & 1 & 1 & 0 & 6 & 0 & 0 & 0 & 13 \\
$1400-1800$ & 1 & 4 & 1 & 3 & 0 & 4 & 0 & 1 & 0 & 14 \\
\hline
\end{tabular}


TABLE 10. MEAN HOURLY DIFFERENCES $(\Delta)$ IN GLOBAL INSOLATION (WATTS/METER 2 ) AND PERCENT DIFFERENCES $(\% \Delta)$ FOR THE 24 DAYS IN THE SUMMER WHEN THE SEA BREEZE WAS IDENITIFIED

\begin{tabular}{|c|c|c|c|c|c|c|}
\hline \multirow{2}{*}{$\begin{array}{l}\text { Hour } \\
\text { (EST) }\end{array}$} & \multicolumn{2}{|c|}{$\begin{array}{l}\text { COASTAL minus } \\
\text { CLINTON classes } \\
\end{array}$} & \multicolumn{2}{|c|}{$\begin{array}{l}\text { COASTAL minus } \\
\text { INLAND classes }\end{array}$} & \multicolumn{2}{|c|}{$\begin{array}{l}\text { CLINTON minus } \\
\text { INLAND classes }\end{array}$} \\
\hline & $\Delta$ & $\% \Delta$ & $\Delta$ & $\% \Delta$ & $\Delta$ & $\% \Delta$ \\
\hline 0700 & 16.8 & 11.9 & 3.6 & 2.6 & -9.4 & -7.3 \\
\hline 0800 & 21.7 & 6.6 & 5.3 & 1.6 & -16.1 & -5.3 \\
\hline 0900 & 20.3 & 3.9 & 2.8 & 3.9 & -17.4 & -3.5 \\
\hline 1000 & 14.3 & 2.0 & 9.1 & 2.0 & -5.3 & -0.7 \\
\hline 1100 & -19.2 & -2.4 & 22.5 & 2.8 & 41.6 & 3.1 \\
\hline 1200 & -15.9 & -1.7 & 40.1 & 4.5 & 57.8 & 6.4 \\
\hline 1300 & 6.5 & 0.7 & 60.4 & 6.8 & 42.8 & 4.9 \\
\hline 1400 & 7.0 & 0.8 & 85.0 & 10.2 & 75.0 & 9.2 \\
\hline 1500 & 15.8 & 2.1 & 49.1 & 6.8 & 24.6 & 4.2 \\
\hline 16n & 59.2 & 10.4 & 65.3 & 11.4 & 6.0 & 1.2 \\
\hline 1700 & 32.4 & 8.2 & 46.9 & 11.9 & 14.5 & 4.0 \\
\hline 1800 & 16.1 & 7.7 & 16.6 & 7.8 & 0.4 & 0.3 \\
\hline
\end{tabular}


The data indicate that there is a systematic diurnal trend in the COASTAL and INLAND class differences similar to that found in the summer season discussed earlier in this 'section. The maximum difference occurred at 1400 EST; and the maximum percentage difference was at 1700 EST. The data indicated that the period of 1600 EST through 1800 EST have relatively high percentage differences. These values, to some respect, are influenced by the low sun angle. Overal1, there appeared to be approximately a $6.0 \%$ depletion of global insolation at the inland sites.

A somewhat similar pattern was noted in the CLINTON and INLAND class differences in global insolation except that the high percentage differences noted late in the day were not apparent in these data. Although the COASTAL and CLINTON class differences do not show a systematic diurnal pattern, they do indicate large percentage differences in the period 1600 through 1800 EST. The data indicate that the phenomena causing large percentage differences in the period 1600 through 1800 EST was affecting the inland sites and Clinton producing a relatively large depletion of global insolation relative to the coastal sites.

The percent differences between the COASTAL and INLAND classes for the 24 summer sea breeze days and the percent difference between the COASTAL and INLAND classes for the remaining summer days are given in Table 11. Both data sets indicate a similar diurnal pattern. The COASTAL and INLAND class differences in global insolation (not the percent difference) were a maximum in both data sets at 1400 .EST. However, the data set in which definite sea breeze characteristics were identified has the persistence of high percentage differences between 1600 and 1800 EST which does not occur in the remaining data. The percentage difference in the period 1000 to 1200 EST is about twice as large in the data set containing all the other summer days than that for the definite sea breeze days.

Figure 11 shows the diurnal variation of the trend lines for the COASTAL, INLAND, and CLINTON classes for those days when definite sea breeze characteristics were identified and for all other summer days. In the case where a definite sea breeze characteristics were 
TABLE 11. MEAN HOURLY PERCENT DIFFERENCE IN GLOBAL INSOLATION BETWEEN THE COASTAL AND INLAND CLASSES FOR THE 24 DAYS IN THE SUMMER WHEN THE SEABREEZE WAS IDENTIFIED $(\% \Delta)$ AND FOR ALL THE REMAINING SUMMER DAYS $(\% \Delta R)$

\begin{tabular}{ccc}
\hline $\begin{array}{c}\text { Hour } \\
\text { (EST) }\end{array}$ & $\% \Delta$ & $\% \Delta R$ \\
\hline 0700 & 2.6 & 0.0 \\
0800 & 1.6 & 0.0 \\
0900 & 3.9 & 0.5 \\
1000 & 2.0 & 6.7 \\
1100 & 2.8 & 7.6 \\
1200 & 4.5 & 7.4 \\
1300 & 6.8 & 6.8 \\
1400 & 10.2 & 10.6 \\
1500 & 6.8 & 8.0 \\
1800 & 11.4 & 3.0 \\
1700 & 11.9 & -1.7 \\
1800 & 7.8 & -0.6 \\
\hline
\end{tabular}



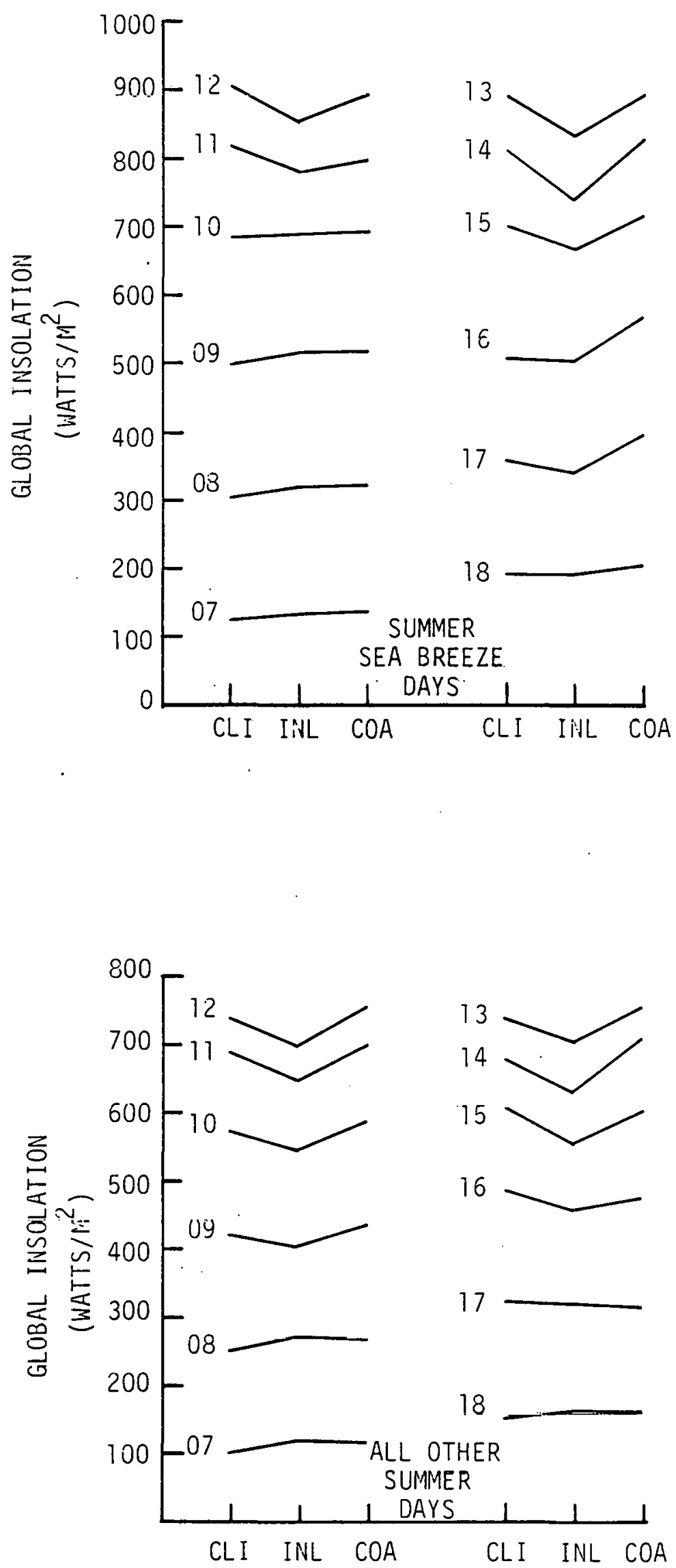

Figure 11. The mean diurnal variation of the trend lines for global insolation for the COASTAL, INLAND, and CLINTON classes based on 24 days with definite sea breeze characteristics in the summer and on all other summer days. 
noted, a specific trend of depletion of global insolation at the inland sites begins at 1100 EST and persists to 1700 EST. During that period the average depletion of global insolation for the INLAND class relative to the COASTAL class was approximately $7.8 \%$. On the other hand, the data set containing all other summer days indicate a depletion of global insolation for the INLAND class in the period 1000 through 1600 EST, a circumstance that is similar to that observed in the mean summer season data. The average depletion of global insolation for the INLAND class relative to the COASTAL class in the period 1000 through 1600 EST was $7.2 \%$ for the data set containing all other summer days.

The frequency distribution for the global insolation for the 24 definite sea breeze days were compared with that for the remaining summer days across the COASTAL, INLAND, and CLINTON classes. For each class, the Chi-squared test showed that two distributions have statistically different distributions. There is a higher hourly mean for the global insolation throughout the day for the definite sea breeze days; and 22 out of the 24 days are included in the first half of the rank of the D-values in Table 5 , those values having the least variability across the network. These three factors indicate that the definite sea breeze days did not characterize the summer season. This may be due, in part, to the fact that the sea breeze was better defined which may be a result of the absence of high clouds, which, in turn, permitted the identification of the sea breeze using GOES data. 


\subsection{Direct Insolation Measurements in the Coastal-Inland Zone}

Direct insolation data were obtained at the Clinton and Sloop Point sites onily. The mean hourly direct normal insolation for each season is shown in Figure 12 . The differences in the direct normal insolation between the Sloop point and Clinton site and the percentage difference relative to the $\mathrm{Clinton}$ site are given in Table 12 . There is a marked depletion of direct normal insolation at the Sloop Point site relative to the $\mathrm{Clinton}$ site in the fall and spring. The average percent depletion over the entire daylight hours is $20.5 \%$ in the spring (if only the period 1000 to 1600 EST is considered when the solar elevation angle is high, the average depletion is $25.1 \%$ in the spring), and is $23.2 \%$ in the fall (the average depletion in the hours 1000 to 1600 EST is $28.3 \%$ in the fall).

In the winter and summer, there was, on the average, a small depletion of direct normal insolation at the Clinton site relative to the sloop Point site. In the winter, a systematic diurnal trend in the difference in the direct normal insolation data was not found. large percentage differences were noted at sunrise and at sunset. In the period with high sun angle (1000 hrs to 1600 EST), the average depletion of direct normal insolation amounted to $3.4 \%$. In the summer, a systematic diurnal trend was noted in the hours 0900 to 1600 EST. Large percent differences were once again noted at the hours around sunrise and sunset. The average depletion of direct normal insolation at the Clinton site in the summer and in the period 1000 to 1600 EST was $7.9 \%$. The Students-t test indicated that in the winter and summer the differences in direct normal insolation were not significant at the $1 \%$ level. In the summer, the percent difference found in direct normal insolation is comparable to the percent difference in the global insolation, but the percent difference in direct normal insolation is not statistically significant, whereas it is significant for the global insolation.

The monthly mean percent difference in direct normal insolation between $\mathrm{Clinton}$ and Sloop Point relative to the Clinton site is 

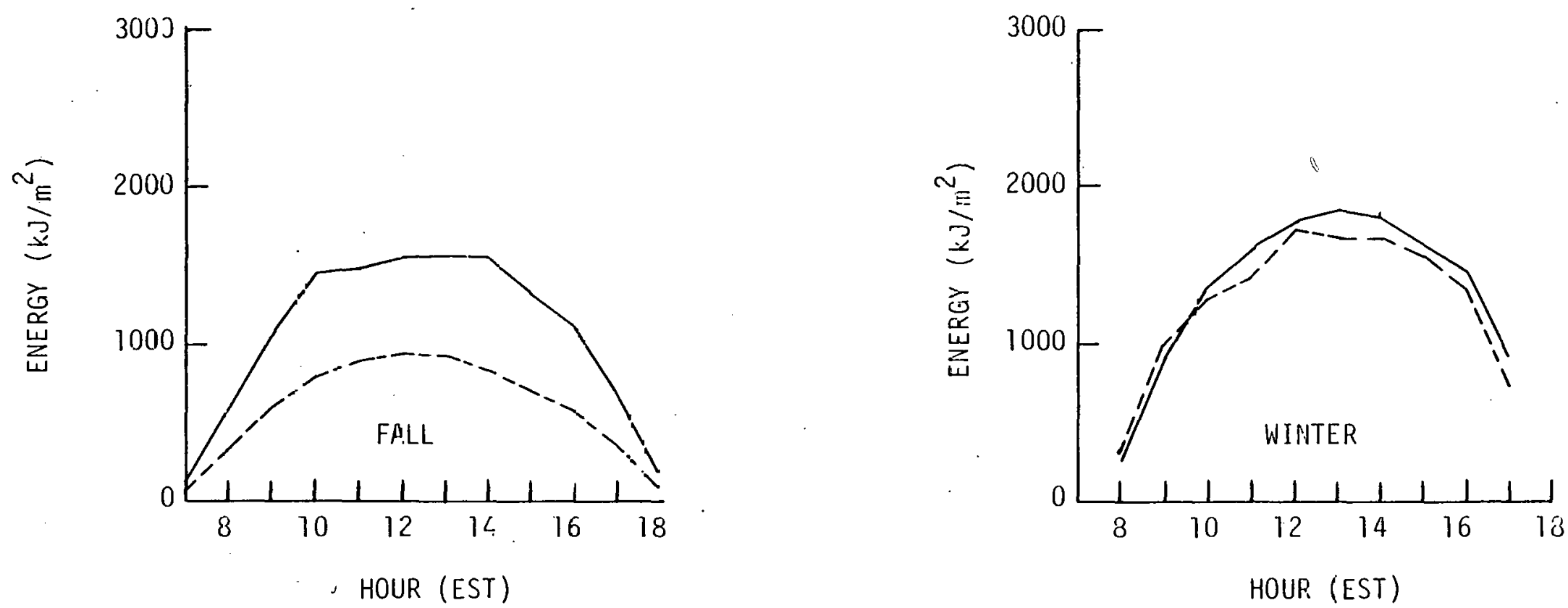

뭉
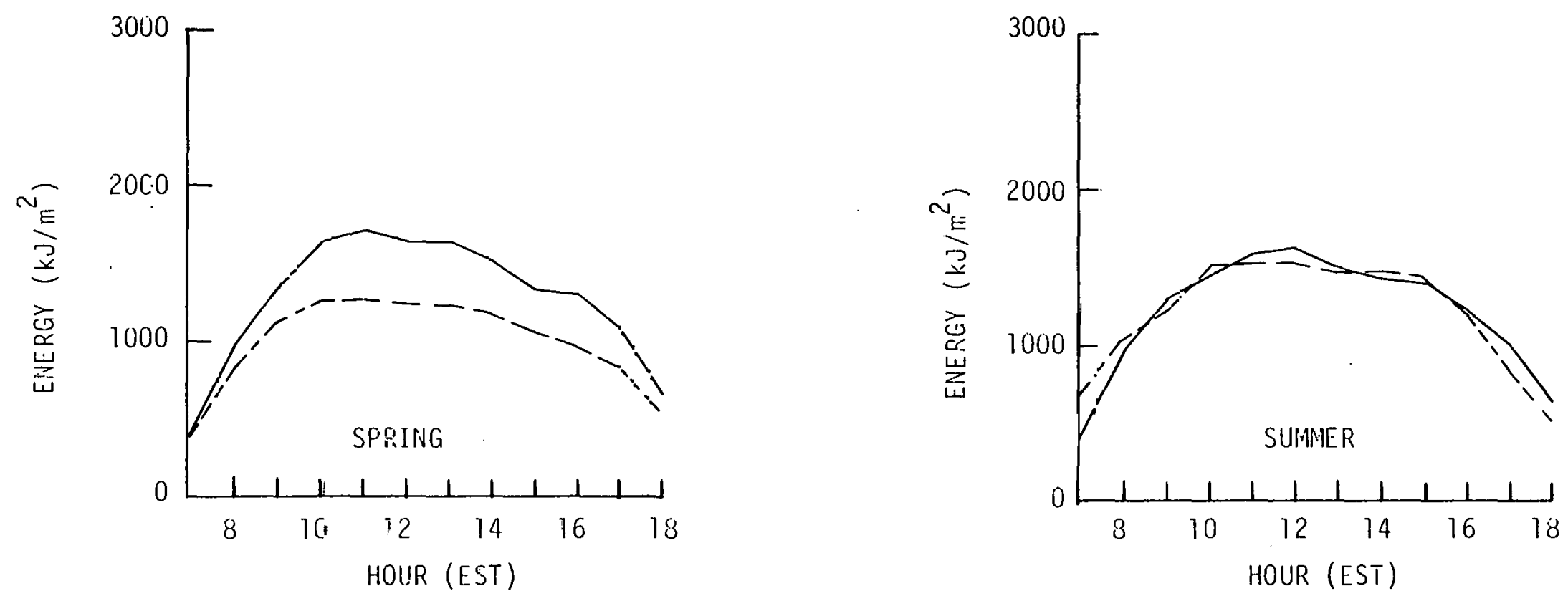

Figure 1\%. Maan hourly yalues of diract normal insolation at Clinton (solid line) and at Sloop Point Figure 12. Mean hourly yalues of direct nor 
TABLE 12. MEAN HOURLY DIFFERENCE $(\Delta)$ AND PERCENT DIFFERENCE $(\% \Delta)$ IN DIRECT NORMAL INSOLATION BETWEEN SLOOP POINT AND CLINTON FOR EACH SEASON. DIFFERENCES ARE IN WATTS/METER2

\begin{tabular}{|c|c|c|c|c|c|c|c|c|}
\hline \multirow{2}{*}{$\begin{array}{l}\text { Hour } \\
\text { (EST) }\end{array}$} & \multicolumn{2}{|c|}{ Spring } & \multicolumn{2}{|c|}{ Summer } & \multicolumn{2}{|c|}{ Fall } & \multicolumn{2}{|c|}{ Winter } \\
\hline & $\Delta$ & $\% \Delta$ & $\Delta$ & $\% \Delta$ & $\Delta$ & $\% \Delta$ & $\Delta$ & $\% \Delta$ \\
\hline 0700 & 6.8 & 6.3 & 86.1 & 77.7 & -1.9 & -5.9 & 31.0 & 18.9 \\
\hline 0800 & -31.8 & -11.9 & 28.3 & 10.7 & -10.6 & -6.6 & 33.3 & 47.8 \\
\hline 0900 & -63.1 & -17.0 & 5.6 & 1.6 & -57.5 & -19.5 & 66.9 & 27.2 \\
\hline 1000 & -106.2 & -23.3 & 42.2 & 10.6 & -119.4 & -30.0 & 10.0 & 2.7 \\
\hline 1100 & -120.8 & -25.3 & 29.1 & 6.6 & -112.7 & -27.5 & -15.8 & -3.6 \\
\hline 1200 & -116.6 & -25.6 & 26.4 & 5.8 & -144.4 & -33.5 & -17.6 & -3.6 \\
\hline 1300 & -124.3 & -27.1 & 37.7 & 8.9 & -111.1 & -25.7 & 20.0 & 3.9 \\
\hline 1400 & -106.6 & -25.2 & 40.0 & 10.0 & -137.2 & -32.0 & 31.7 & 6.4 \\
\hline 1500 & -84.5 & -22.6 & 51.4 & 13.1 & -89.4 & -24.4 & 49.7 & 11.0 \\
\hline 1600 & -97.6 & -26.7 & 1.4 & 0.4 & -78.1 & -25.2 & 27.9 & 6.9 \\
\hline 1700 & -78.8 & -25.7 & -33.6 & -12.0 & -35.0 & -17.9 & -11.5 & -4.6 \\
\hline 1800 & -40.2 & -21.7 & -20.3 & -11.1 & -15.0 & -30.0 & 3.3 & 11.0 \\
\hline
\end{tabular}


given in Table 13. These data indicate that the large average depletion in the spring and fall seasons are brought about by affects occurring in two of the three months representing each season. In the spring, the months are March and Apri1; and in the fall, they are october and November. Large negative percent difference in August and the large positive percent difference in September are biased by the fact that data were available for only $34 \%$ and $25 \%$ of the days in the month, respectively, due to instrument difficulties (see Table 3). In October, data were only available for $40 \%$ of the days which may also bias the results in that month.

The seasonaliy averaged ratio between the direct normal insolation and the global insolation were computed and comparsil with seasonal estimates of the ratio for locations in other coastal zones from data prepared by Boes et al (1978). The data in Table 14 are grouped according to sites which are near the coast and those which are approximately $40 \mathrm{~km}$ or greater from the coast. It is noted that both in the spring and in the fall, the sloop polnt site has a lower ratio than any of the other coastal sites. The average difference in the spring is approximately 0.17 , and in the fall, 0.47 . The Clinton site has a ratio which compares reasonable well with the ratios determined for other inland sites.

In the summer, the sloop Point site has a ratio that is lower than all the other coastal sites with the exception of Miami. The ratio is, 0.04 less than that computed for Charleston, South Carolina and 0.16 less than that at Cape Hatteras, North Carolina both of which are less than two hundred miles from sloop polnt. The ratio at the Clinton site is onnsiderably lower than that in either Brownsville or Lake Charles in the summer. The average difference is 0.17 .

In the winter, the Sloop point site has a value which, in the average, compares well with the other sites. lhe Clinluil site, however, has a value that is 0.21 greater than that at the other sites, but is comparable to the coastal sites. 
TABLE 13. MEAN MONTHLY PERCENT DIFFERENCE $(\% \Delta)$ IN DIRECT . NORMAL INSOLATION BETWEEN SLOOP POINT AND CLINTON

\begin{tabular}{lrlr}
\hline \hline Month & $\% \Delta$ & Month & $\% \Delta$ \\
\hline Jan & 7.2 & July & 1.0 \\
Feb & -3.1 & Aug & -19.0 \\
Mar & -30.4 & Sept & 24.0 \\
Apr & -24.4 & Oct & -51.9 \\
May & -1.0 & Nov & -47.4 \\
June & 4.2 & Dec & 9.6 \\
\hline
\end{tabular}


TABI_E 14. MEAN SEASONAL RATIOS OF THE DIRECT NORMAL IINSULATION TO THE TOTAL HORIZONTAL INEOLATION (GLOBAL INSOLATION) CALCULATED FROM OBSERVED DATA AT SLOOP POINT AND CLINTON AND ESTIMATED AT OTHER COASTAL AND INLAND SITES FROM BOES ET AL'S (1978) DATA

\begin{tabular}{lcccc}
\hline \multicolumn{1}{c}{ Location } & Spring & Summer & Fall & Winter \\
\hline Sloop Point & 0.68 & 0.67 & 0.55 & 1.26 \\
Appalachacola & 0.88 & 0.78 & 1.07 & 1.14 \\
Miami & 0.76 & 0.64 & 0.85 & 1.08 \\
Boston & 0.86 & 0.79 & 1.11 & 1.29 \\
Cape Hatteras & 0.91 & 0.83 & 1.08 & 1.29 \\
Charleston & 0.82 & 0.71 & 1.00 & 1.19 \\
Clinton & 0.76 & 0.64 & 1.06 & 1.24 \\
Brownsville & 0.78 & 0.87 & 1.00 & 1.03 \\
Lake Charles & 0.76 & 0.75 & 0.97 & 1.03 \\
\hline \hline
\end{tabular}




\subsection{Summary and Conclusions}

The global insolation data indicated greater variability over the network during the summer season. The summer variability was characterized by systematic diurnal differences in global insolation between the inland sites and the coastal sites. The differences were found to be statistically significant at the $0.5 \%$ leve 1 in the summer in the principal hours of the day, i.e., 1100 to 1500 EST. 'The average depletion at the inland sites over the day amounted to $4.9 \%$ of the global insolation relative to the coastal sites. However, the most significant depletion occurred between 1000 and 1600 EST and amounted to an average of $7.0 \%$. The maximum depletion (10.6\%) occurred at 1400 EST.

A systematic diurnal depletion of global insolation at the inland site was also noted in the spring and fall. However, the differences in those seasons were not.statistically significant at the $5 \%$ level for most hours in the day. A minimum in the global insolation was noted at the inland site relative to the coastal sites and the Clinton site during the period 1000 to 1600 EST in the spring with an average depletion over that period of approximately 3.7\%. The maximum depletion occurred at 1300 EST and was $5.5 \%$. In the fall, the minimum occurred in the period 1100 to 1500 EST with an average depletion over that period of $3.3 \%$. The maximum depletion was $4.3 \%$ at 1200 EST. Though the differences in the spring and fall were not statistically significant, the fact that the diurnal trends were similar to that in the summer suggest that these seasons may also be affected by the same pheriumena influencing the summer. No systematic diurnal trends of depletion of global insolation was noted at the inland site or at any site in the winter.

GOES satellite data were used to identify 32 days in the period May through September 1978 in which there was a definite occurrence of the sea breeze in the North Carolina coastal regions. The sea breeze front was identified in the GOES imagery as a line separating a zone 
of cloudiness on the inland side from a zone of relatively clear skies on the coastal side. Analysis of the global insolation data on those days indicated that the sites behind the front consistently receive more global insolation than those ahead of the front by 5 percent irrespective of the cloud amount. Furthermore, the depletion of global insolation by the cloudiness associated with the sea breeze fronts was more significant by 5 percent than the depletion by air mass cumulus clouds.

of the 32 days identified as having definite sea breeze characteristics, 24 were in the summertime. Global insolation data on the 24 summer days showed a similar diurnal pattern as found in the summer season. There were, however, some characteristic differences. A definite trend of depletion of global insolation at the inland sites was noted in the period 1100 to 1700 EST in the average of the 24 days. A similar pattern was noted in the summer season and in the data set containing all the remaining summer days which sea breeze characteristics could not be identified, in the period 1000 to 1600 EST. In the respective time periods, $7.8 \%$ depletion of global insolation was noted on the definite sea breeze days, $7.2 \%$ for the data set noted as all other summer days, and $7.0 \%$ for the summer season. Furthermore, there was a depletion of global insolation at the Clinton site after 1600 EST in the average of the 24 definite sea breeze days. This effect was not noted in the data set containing all the other summer days or the summer season data. The sea breeze circulation most probably exists every day during the summer. The 24 days identified in the satellite data which had definite sea breeze characteristics were probably days when the sea breezc circulation was vory intense, which is supported by the persistance of the sea breeze late in lie day and by evidence that the sea hreeze may have even affected the clinton site. It is expected that only very intense sea breeze circulations would penetrate as far as $100 \mathrm{~km}$ inland. The intense sea hreeze may be due to development of a large land-sea 
temperature difference because there was no middle or high cloud cover. Under those conditions the satellite could detect the sea breeze and the solar insolation would reach the ground with little or no scattering.

Over the 24 days in the summer in which definite sea breeze characteristics were identified, the patterns and trends were qualitatively similar to those in the summer season. This would suggest that the summer seasonal pattern was governed by the sea breeze circulation. The sea breeze circulation most probably existed on many of the summer days in which definite sea breeze characteristics could not be identified because the sea breeze effects were subtle compared to the other localized effects. Sea breeze circulations of little intensity are most often due to small land-sea temperature differences. Middle and high cloudiness (e:g., cirrus overcast) can reduce the land-sea temperature difference by impeding the solar insolation from reaching the ground. Furthermore, on those days when the sea breeze could not be identified, the maximum intensity of the sea breeze circulation on a diurnal basis may, in many circumstances, have been reached earlier in the day. This would explain the rather high percentage differences found in the period 1000 to 1200 EST in the data set noted as all other summer days compared to the data set in which definite sea breeze characteristics were identified.

The inland sites used in this study did not always fall within the realm of the maximum cloud activity associated with the sea breeze front. The analysis of the definile sea breeze days indicated that the maximum frontal activity occurred in approximately a $10 \mathrm{~km}$ band centered about $20 \mathrm{~km}$ from the coast. Furthermore, frontal passage at the inland sites occurred around 1500 to 1600 EST, a time when the sea breeze normally begins to dissipate. This would suggest that the depletion of global insolation experienced at the inland sites may not be the maximum amount of depletion associated with the sea breeze effect. 
The direct normal insolation data indicated a depletion of direct normal insolation at the coast (the Sloop Point site) relative to a far inland site (the Clinton site) in the spring and fall. The depletion amounted to $20.5 \%$ in the spring and $23.2 \%$ in the fall. The depletion of direct normal insolation was most pronounced in March and April, and in the fall, in October and November. No statistically significant differences in the direct normal insolation between the coastal and inland sites specified above were noted in the summer and winter.

Comparisons of the ratio of the direct normal insolation to the global insolation calculated using the Sloop point data and data prepared by Boes et al (1978) at other coastal biles indicated that the ratios at sloop Point were smaller than those estimated for other coastal sites in the spring and fall seasons. The ratio calculated using the Clinton data compared reasonably well with the ratio estimated for other inland sites in the spring and fall; and was smaller in the summer season and larger in the winter.

Examination of meteorological and satellite data revealed no mechanism which could be responsible for the marked depletion in the spring and fall. Furthermore, no significant statistical differences were noted in the global insolation data between sloop point and clinton in the spring and fali; i.e., the diffuse radiation made up for the loss in the direct normal insolation. This suggests that aerosols, water vapor, or thin cirrus may be cause for the loss. To date, no adequate explanation for the cause of the marked depletion of direct norilal insolation in the spring and fall have been determined. 


\subsection{Topics For Further Research}

The results of this study indicate that there is a direct effect of the sea breeze circulation on the depletion of global insolation inland from the coast. Though, a magnitude effect was determined from this study, the magnitude effect was most probably not the maximum effect of the sea breeze circulation, because the inland sites were not located in the region of maximum sea breeze frontal activity. Limited data analysis indicated that the sea breeze activity off the North Carolina coast occurred approximately $20 \mathrm{~km}$ inland. The inland sites in this study were located approximately 40 to $50 \mathrm{~km}$ inland. Because of the potential application of solar energy in coastal regions, it is necessary to determine the maximum effect of the sea breeze circulation on the depletion of global insolation. In order to accomplish this, a high resolution study should be performed along the line from the coast approximately $100 \mathrm{~km}$ inland. Stations measuring global insolations should be located approximately 5 to $10 \mathrm{~km}$ apart. Since this study indicated that the most significant (i.e., the statistically significant) depletions of global insolations would take place in the summer time in the North Carolina coastal region, high resolution study should only be performed during that season.

Satellite data were used to identify the regions of sea breeze frontal activity. Given the analysis of the estimated frequency of occurrence of sea breeze circulation from satelitite data, the results of this study can be used to estimate the depletion of global insolation as a result of sea breeze cloud effects. Of course, better estimates could be accomplished if high resolution estimates of the depletion of global insolation were available. However, before such a technique should be placed in an applied mode, it should be tested. This test could be accomplished by determining a specific region, performing the methodology described above, making estimates of depletion associated with sea breeze cloud effects, and setting up a field program in the region to determine the accuracy of the estimates. 
A more than $20 \%$ depletion of direct normal insolation at the coastal site was noted in the spring and fall. No physical explanation could be made on the basis of available data. Because of the potential effect this depletion can have on concentrated solar systems, it is necessary to find the reason for this depletion. Studies should be performed in which a very detailed assessment of the meteorology at the coastal sites and inland sites should be made while direct normal insolation measurements are taken during the fall and spring seasons. This should include high resolution measurements of wind, temperature, pressure, turbidity and dew point in the region of interest; temporal assessment of the cloud cover using all sky cameras; measurements of the land and sea temperature difference on a diurnal basis; and the use of applicable satellite data. It is only through a high temporal and spatial resolution study that the mechanism which produces the depletion of direct normal insulation in the spring and fall at the coast can be determined. 


\section{REFERENCES}

Avaste, 0. A., 1964: "Transfer of Solar Radiation in the Atmosphere". NASA Technical Translation F-323, National Aeronautics and Space Administration, Washington, D.C. 1154-66.

Hinn, A., 1978: Personal communication (Mr. Hinn is Meteorologist-in-Charge, National Weather Service Office, Wilmington, N.C.).

Boes, E. C., H. E. Anderson, I. J. Hall, R. E. Prairie, and R. T. Stromberg, 1977: "Availability of Direct, Total, and Diffuse Solar Radiation to Fixed and Tracking Collecters in the U.S.A. (Addendum)". SAND77-0885, Sandia Laboratories, Albuquerque, N. M. 
THIS PAGE

\section{WAS INTENTIONALLY LEFT BLANK}


APPENDIX 1

FIELD OPERATOR MANUAL 
PESEAFCH TRIANGLE INSTITUTE

$=O S T O F F I C E$ BO X 12194

RESEARCHTTAI ANGLE PARK. NORTHCAROLINAMITOO

COASTAL - INLAND

SOLAR. RAOIATION DIFFERENCE STUDY

\section{OBJECTIVES}

- Collect a year sample of hourly solar radiation and meteorological data in southeastern North Carolina.

- Analyze those data for differences of direct, diffuse, and UV radiation between coastal and inland areas at the sea breeze scale.

- Develop methodologies to translate the results to other coastal locations. 


\section{INSTRUMENTS}

To get high quality data, we have purchased the highest quality instruments available. However, these instruments are no better than the care they are given. Although you have limited experience with these types of instruments, I know you will give them good care.

\section{PRINCIPLES OF OPERATION}

The sunshine, directly from the sun, the sunlight scattered by the air, and the clouds and the trees, produce a very small electric current in the instrument. That current is transmitted to the counter which amplifies the current and counts the energy. Every hour, the printer, the time and accumulated counts are printed on the folding tape.

The process is rather simple, but, things can, and do, go wrong. 
PRECISION SFECTRAL PYRANOMETER (PSP)

YOUR RESPONSIBILITIES

1. CLEAN THE GLASS DOME DAILY

Wash the dome with the alcohol and water mixture.

Wipe the dome with the tissues provided.

2. CHECK tHE DESSICANT (BLUE CRYSTALS)

The crystals should be a bright-dark blue.

They absorb moisture to prevent water condensing on the inside of the dome. As they turn pink, call kil.

3. CHECK THE BUBBLE LEVEL

The bubble should be inside the small circle.

slowly adjust the three thumb screws if leveling is needed.

4. RECORD THE TIME YOU DID THESE CHORES

Press the PRINT button on the printer.

The current time (hours and minutes since midnight) and counts

should be printed. THIS IS IMPORTANT, IT IS OUR RECORD OF YOUR WORK

Initial the printer tape and date it. If there has been even a very brief power failure, the clock in the printer will indicate 99:99. It must be reset to the present time. Follow the instructions provided.

5. RECORD YOUR WORK IN THE DAILY LOG

Add any comments you may wish to make.

Use more than one line if you wịsh.

6. RETURN THE DATA

On the first and fifteenth of each month, return your DAILY LOG and the printed tapes to RTI in the envelope provided.

7. PROBLEMS (?)

If you have a problem or suspect a problem with the equipmerit, call

Dr. Walter D. Bach, Jr. Research Triangle Institute

(919) 541-5855---COLLECT or (919) 489-8781 (Weekends)

Research Triangle Park, N. C. 27709 
EPPLTY PRECISIOI PYRANOHTITSR

$$
\text { ModeI PSP }
$$
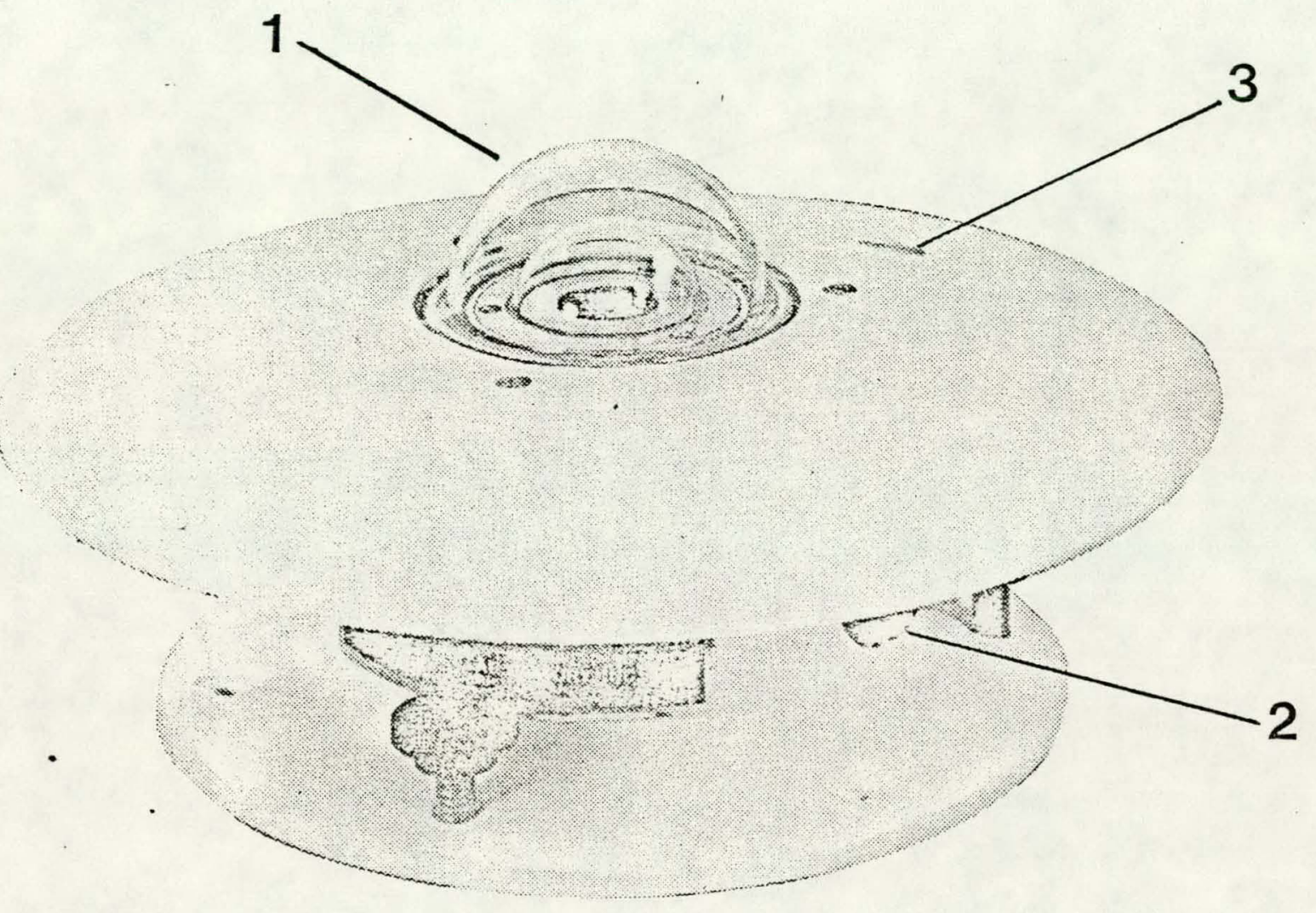
ULTRAVIOLET RADIOMETER

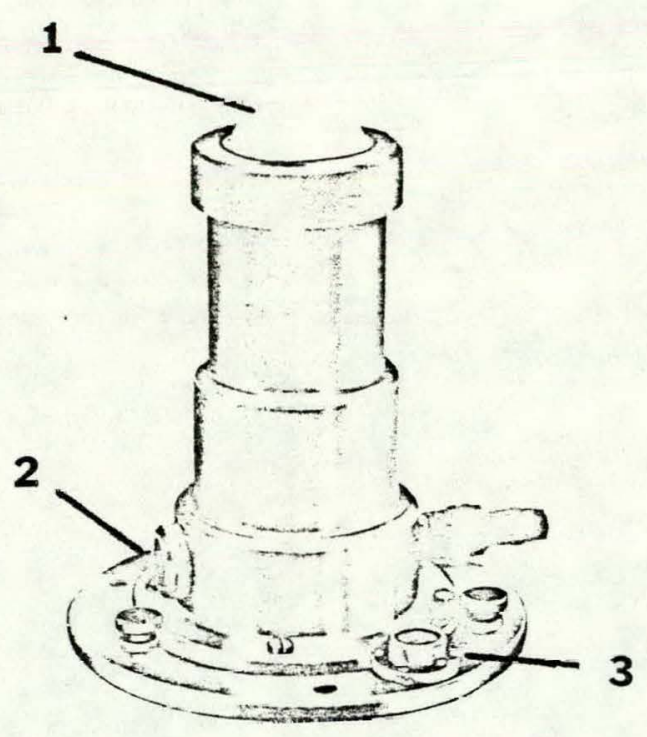

YOUR RESPONSTRTT TTIES

\author{
(Same as with the PSP)
}


The clock may be set to any desired time by using the following procedure:

1. Insert the finger into the top part of the front pane! bezel hole and pull the door open.

2. Locate the clock switches in the upper right hand corner. (See Figure 2)

3. Turn the power off then turn on again.

4. Depress the front panel PRINT button. The printout will indicate 99:99.

5. Move the TENS/UNITS switch to the UNITS position. (See Figure 6)

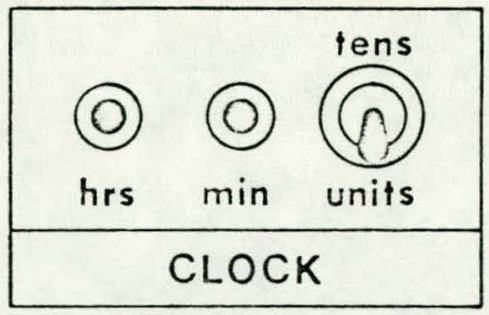

Figure 6

Clock Switches

6. Depress the MIN button once to reset the clock to 00:00. Depress the MIN button one time for each digit desired in the minutes column.

NOTE: If the MIN button is depressed when the minutes column is printing the number nine (9). the number will change to zero $(0)$ and the tensof-minutes column will advance by one (1).

7. Depress the front panel PRINT button to check printout.

8. Muve the TENS/UNITS switch to the TENS position.

9. Depress the MIN button one time for each digit desired in the tens-of-minutes column.

NOTE: If the MIN button is depressed when the tens-of-minutes column is printing the number six (6). the number will change to zero (0) and the hours column will advance by one (1).

10. Depress the front panel PRINT button to check printout.

11. Move the TENS/UNITS switch to UNITS position.

12. Depress the HRS button one time for each digit desired in the hours column.

NOTE: If the HRS button is depressed when the hours column is printing the number nine (9), the number will change to zero $(0)$ and the tens-ofhours column will advance by one (1) EXCEPT when the tens-of-hours column contains the number iwo (2) and the hours column contains the number three (3), depressing the HRS will Ulien reset both the 2 and the 3 to zero.

13. Depress the front panel PRINT button to check printout.

14. Move the TENS/UNITS switch to the TENS position.

15. Depress the HRS button one time for each digit desired in the tens-of-hours column.

16. Depress the front psinel PRINT button to check time setting on print. 


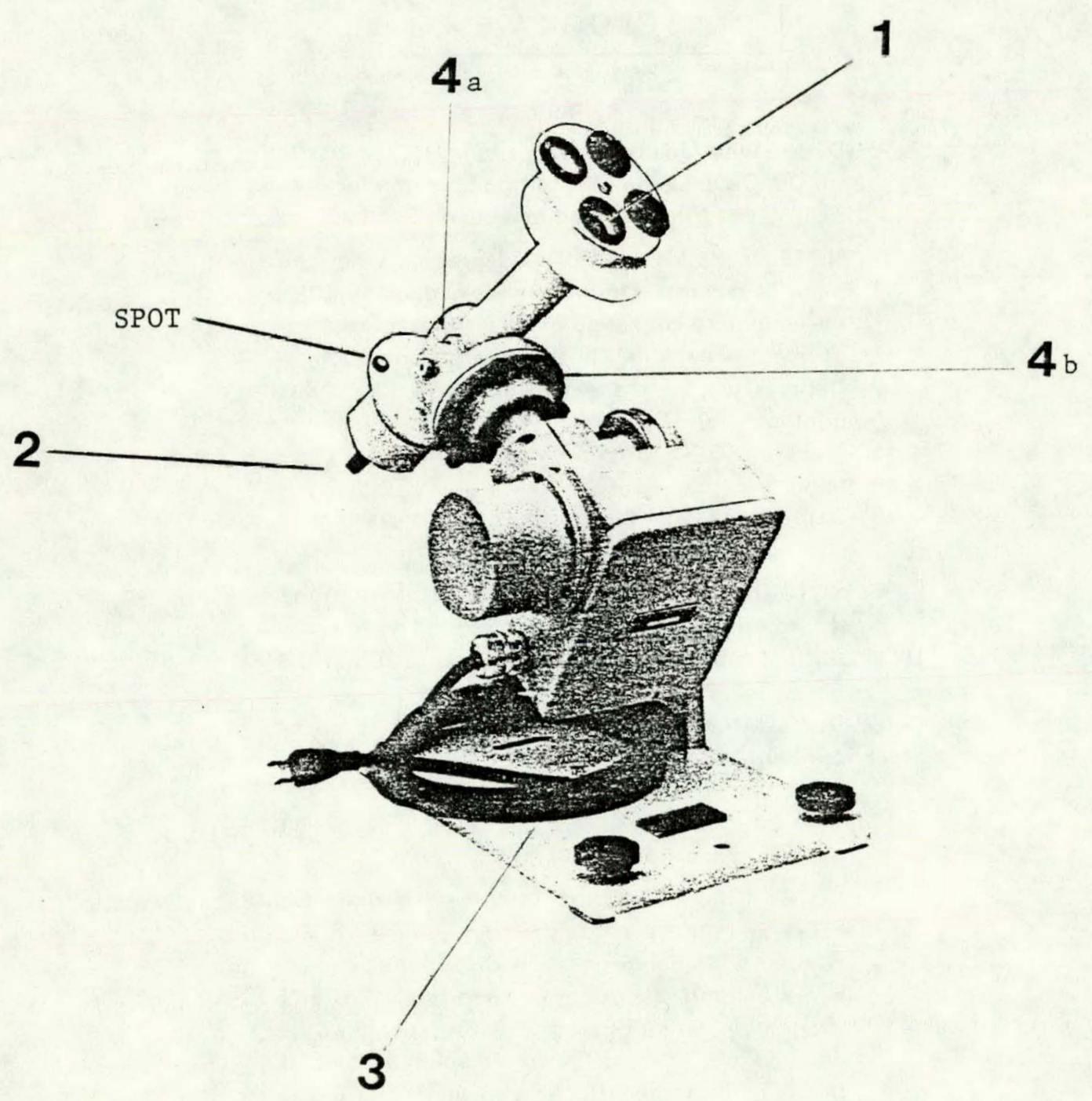




\section{NORMAL INCIDENCE PYRHELIOMETER (NIP) AND \\ SOLAR TRACER UNIT \\ YOUR RESPONSIBILITIES}

1. CLEAN THE OPENING DAILY

Wash the opening with the alcohol and water mixture.

Wipe with the tissues provided.

2. UNWIND THE LEAD WIRE DAILY

The wire leading from the NIP to the integrator unit will wrap around the tracker. Unplug the wire from the NIP, pushing the connector in slightly then turning. Unwrap the wire from the tracker. Replace the plug in the socket.

3. CHECK THE BUBBLE LEVEL

The bubble should be in the center of the small circle. Adjust the leading screws as necessary.

4. CHECK THE ALIGNMENT (requires direct sunlight)

The sunlight should be on the white disk with the black center dot at the rear of the NIP.

(a) The elevation angle, or declination, is adjusted by loosening the clamping hand screw in the " $U$ " block, adjusting the angle (between $+23^{\circ}$ to $-23^{\circ}$ ) for the right date, and retightening the hand screw.

(b) If there has been a power failure, loosen the three thumb screws under the instrument support plate, rotate the instrument until the sun's image lines up on the black center dot, tighten the thumb screws.

5. RE..ORD THE TIME AND YOUR WORK

A Table of Solar Declination is on the next page. 
All data are for $O^{*}$ Greenwich Civil Time in the year 1950. Variations of these data from year to year are negligible for most meteorological purposes, the largest variation occurs through the 4-year leap-year cycle. The year 1950 was sclected to rcpresent a mean condition in this cycle.

The declination oi the sun is its angular distance. north $(+)$ or south $(-)$ of the celestial equator.

The longitude of the sun is the angular distance of the meridian of sun from the vernal equinox (mean equinox of 1950.0) measured eastward along the ecliptic.

The equation of time (apparent - mean) is the correction to be applied to mean solar time in order to obtain apparent (true) solar time.

The radius vector of the earth is the distance from the center of the earth to the center of the sun expressed in terms of the length of the semimajor axis of the earth's orbit.

IU. S. Naval Observatory. The American ephemeris and nautical almanac for tbe year 1950, Wasbington. 1948 .

\section{EPHEMERIS OF THE SUN}

\begin{tabular}{|c|c|c|c|c|c|c|c|}
\hline Date & & & $\begin{array}{l}\text { Lon } \\
\text { tud }\end{array}$ & & $\begin{array}{c}\text { Equ } \\
\text { of }\end{array}$ & & $\begin{array}{c}\text { Radiug } \\
\text { vertinr }\end{array}$ \\
\hline & $\bullet$ & 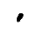 & & ${ }^{\circ}$ & m. & s. & \\
\hline an. $\begin{array}{r}1 \\
5 \\
9 \\
13 \\
17 \\
21 \\
25 \\
29\end{array}$ & $\begin{array}{r}-23 \\
23 \\
22 \\
21 \\
20 \\
20 \\
19 \\
18\end{array}$ & $\begin{array}{r}4 \\
47 \\
13 \\
37 \\
54 \\
5 \\
9\end{array}$ & $\begin{array}{l}280 \\
284 \\
288 \\
292 \\
296 \\
300 \\
304 \\
308\end{array}$ & $\begin{array}{l}1 \\
5 \\
10 \\
14 \\
19 \\
23 \\
27\end{array}$ & $\begin{array}{r}-3 \\
5 \\
6 \\
8 \\
9 \\
11 \\
12 \\
13\end{array}$ & $\begin{array}{r}14 \\
6 \\
50 \\
27 \\
54 \\
10 \\
14 \\
5\end{array}$ & $\begin{array}{r}0.98 .324 \\
.98324 \\
.98333 \\
.98352 \\
.98378 \\
.98410 \\
.98448 \\
.98493\end{array}$ \\
\hline
\end{tabular}

Mar. 1 - $753 \quad 33951-12 \quad 380.99084^{\circ}$ $\begin{array}{rrrrrrrr}5 & 6 & 21 & 343 & 51 & 11 & 43 & .99182 \\ 9 & 4 & 48 & 347 & 51 & 10 & 51 & .99287 \\ 13 & 3 & 14 & 351 & 51 & 9 & 49 & .99396 \\ 17 & 1 & 39 & 355 & 50 & 8 & 42 & .99508 \\ 21 & -0 & 5 & 359 & 49 & 7 & 32 & .99619 \\ 25 & +1 & 30 & 3 & 47 & 6 & 20 & .99731 \\ 29 & 3 & 4 & 7 & 44 & 5 & 7 & .99943\end{array}$

May $1+1450404+2501.00759$

$\begin{array}{rrrrrrrr}1 & +14 & 50 & 40 & 4 & +2 & 50 & 1.00759 \\ 5 & 16 & 2 & 43 & 56 & 3 & 17 & 1.00859 \\ 9 & 17 & 9 & 47 & 48 & 3 & 35 & 1.00057 \\ 13 & 18 & 11 & 51 & 40 & 3 & 44 & 1.01051 \\ 17 & 19 & 9 & 55 & 32 & 3 & 44 & 1.01138 \\ 17 & 20 & 2 & 59 & 23 & 3 & 34 & 1.01218 \\ 21 & 20 & 49 & 63 & 14 & 3 & 16 & 1.01291 \\ 25 & 20 & 3 & 2 \\ 29 & 21 & 30 & 67 & 4 & 2 & 51 & 1.01358\end{array}$

July $1+23 \quad 10 \quad 98 \quad 36-331 \quad 1.01667$

$\begin{array}{rrrrrrrrr}5 & 22 & 52 & 102 & 24 & 4 & 16 & 1.01671 \\ 9 & 22 & 28 & 106 & 13 & 4 & 56 & 1.01669 \\ 13 & 21 & 57 & 110 & 2 & 5 & 30 & 1.01659 \\ 17 & 21 & 21 & 113 & 51 & 5 & 57 & 1.01639 \\ 21 & 20 & 38 & 117 & 40 & 6 & 15 & 1.01610 \\ 25 & 19 & 50 & 121 & 29 & 6 & 24 & 1.01573 \\ 29 & 18 & 57 & 125 & 19 & 6 & 23 & 1.01530\end{array}$

Sept. $1+83515759-0151.00917$

$$
\begin{array}{rrrrrrrr}
5 & 7 & 7 & 161 & 52 & +1 & 2 & 1.141422 \\
9 & 5 & 37 & 165 & 45 & 2 & 22 & 1.00723 \\
13 & 4 & 6 & 169 & 38 & 3 & 45 & 1.00519 \\
17 & 2 & 34 & 173 & 32 & 5 & 10 & 1.00510 \\
21 & +1 & 1 & 177 & 26 & 6 & 35 & 1.00397 \\
25 & -0 & 32 & 181 & 21 & 8 & 0 & 1.00283 \\
29 & 2 & 6 & 185 & 16 & 9 & 22 & 1.00170
\end{array}
$$

Nov. $1-14 \quad 1121759+16 \quad 210.99249$

$\begin{array}{rrrrrrrr}5 & 15 & 27 & 222 & 0 & 16 & 23 & .99150 \\ 9 & 16 & 38 & 226 & 1 & 16 & 12 & .99054 \\ 13 & 17 & 45 & 230 & 2 & 15 & 47 & .98960 \\ 17 & 18 & 18 & 234 & 4 & 15 & 10 & .98369 \\ 21 & 19 & 45 & 238 & 6 & 14 & 18 & .98784 \\ 25 & 20 & 36 & 242 & 8 & 13 & 15 & .98706 \\ 29 & 21 & 21 & 246 & 11 & 11 & 59 & .98636\end{array}$
Date Decli- Longi- Equation Radius Dativil lude oftime vector -, , m. s.

Feb: $\quad \begin{array}{lllllllll}1 & \cdots 17 & 13 & 311 & 34 & -1.3 & 34 & 0.98533\end{array}$ $\begin{array}{rrrrrrrr}5 & 16 & 10 & 315 & 37 & 14 & 2 & .98593 \\ 9 & 14 & 55 & 319 & 40 & 14 & 17 & .98662 \\ 13 & 13 & 37 & 323 & 43 & 14 & 20 & .98738 \\ 17 & 12 & 15 & 327 & 46 & 14 & 10 & .98819 \\ 21 & 10 & 50 & 331 & 48 & 13 & 50 & .98903 \\ 25 & 9 & 23 & 335 & 49 & 13 & 19 & .98991\end{array}$

Apr. $1+4141042-4120.99928$ $5+546 \quad 1439 \quad 3 \quad 1 \quad 1.00043$ $\begin{array}{llllllll}9 & 7 & 17 & 18 & 35 & 1 & 52 & 1.00160\end{array}$ $\begin{array}{lllllllll}13 & 8 & 46 & 22 & 30 & -0 & 47 & 1.00276\end{array}$ $17 \quad 10122625+0131.00390$ $\begin{array}{llllllllllll}21 & 11 & 35 & 30 & 20 & 1 & 6 & 1.00500\end{array}$ $\begin{array}{llllllll}25 & 12 & 56 & 34 & 14 & 1 & 53 & 1.00606\end{array}$

June $1+21576956+2271.01405$ $\begin{array}{llllllll}5 & 22 & 28 & 73 & 46 & 1 & 49 & 1.01465\end{array}$

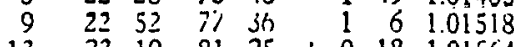
$13 \quad 2310 \quad 8125+0181.01564$ $\begin{array}{llllllll}17 & 23 & 22 & 85 & 15 & -0 & 33 & 1.0160 ?\end{array}$ $\begin{array}{llllllll}21 & 23 & 27 & 89 & 4 & 1 & 2.5 & 1.01630\end{array}$ $\begin{array}{llllllll}25 & 23 & 25 & 92 & 53 & 2 & 17 & 1.01649\end{array}$

Aug. $1+18 \quad 14 \quad 128 \quad 11 \quad-6 \quad 17 \quad 1.01494$ $\begin{array}{rrrrrrrr}5 & 17 & 12 & 132 & 0 & -5 & 59 & 1.01442\end{array}$ $9 \quad 16 \begin{array}{lllllll}9 & 6 & 135 & 50 & 5 & 33 & 1.01384\end{array}$ $\begin{array}{lllllllll}13 & 14 & 55 & 139 & 41 & \cdot & 4 & 57 & 1.01318\end{array}$ $\begin{array}{lllllllll}17 & 13 & 41 & 143 & 31 & 4 & 12 & 1.01244\end{array}$ $\begin{array}{llllllll}21 & 12 & 23 & 147 & 22 & 3 & 19 & 1.01163\end{array}$ $\begin{array}{lllllllll}25 & 11 & 2 & 151 & 14 & 2 & 18 & 1.01076\end{array}$ $\begin{array}{llllllll}29 & 9 & 39 & 155 & 5 & 1 & 10 & 1.00986\end{array}$

Oct. $1-253 \quad 187 \quad 14+10 \quad 1 \quad 1: 0011$ $\begin{array}{rrrrrrrr}5 & 4 & 26 & 191 & 11 & 11 & 17 & 1.00001 \\ 9 & 5 & 58 & 195 & 7 & 12 & 27 & 0.99883 \\ 13 & 7 & 29 & 199 & 5 & 13 & 30 & .99771 \\ 17 & 8 & 58 & 203 & 3 & 14 & 25 & .99659 \\ 21 & .10 & 25 & 207 & 1 & 15 & 10 & .99544 \\ 25 & 11 & 50 & 211 & 0 & 15 & 46 & .99433 \\ 29 & 13 & 12 & 214 & 59 & 16 & 10 & .99326\end{array}$

Dec. $1-214124813+11 \quad 160.98604$ $\begin{array}{rrrrrrrr}5 & 22 & 16 & 252 & 16 & 9 & 43 & .985+6 \\ 9 & 22 & 45 & 256 & 20 & 8 & 1 & .98494 \\ 13 & 23 & 6 & 266 & 24 & 6 & 17 & .98446 \\ 17 & 23 & 20 & 264 & 28 & 4 & 17 & .98405 \\ 21 & 23 & 26 & 268 & 32 & 2 & 19 & .98372 \\ 25 & 23 & 25 & 27 & 37 & +0 & 20 & .98348 \\ 29 & 23 & 17 & 276 & 41 & -1 & 39 & .98334\end{array}$ 
APPENDIX 2

MONTHLY DATA TABULATION 
KEY TO ERROR CODES

$\begin{array}{ll}-99 & \text { Missing Data } \\ -999 & \text { Missing Data } \\ -555 & \text { Insufficient Data To Establish A Trend } \\ -666 & \text { Computed Incident Energy Too Large } \\ -888 & >4000 \mathrm{~kJ} / \mathrm{m}^{2}\end{array}$


CIJASTAL - IIILAIID SUI.AR RADIATIIIN DIFFERENCF. STUUY IISTRIIPENT : PSP H 2 MUNTHLY DATA SUMHARY

SEIISITIVITY 10. MOE-6 V/W/SO.M TREIND REMOUVED

EINERGY KILOJIUULES PER SQUARE METER

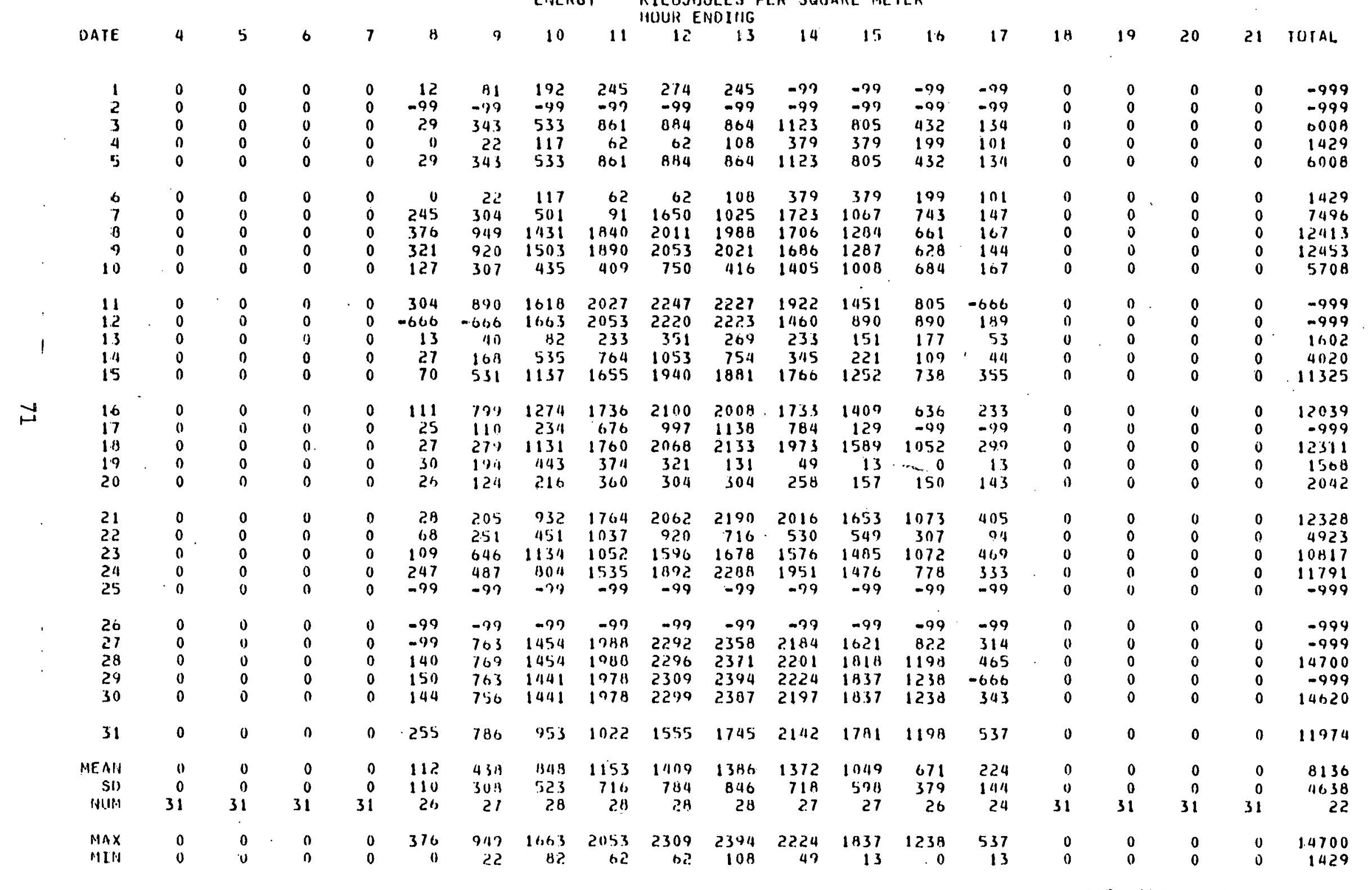


CUASTAL. - IHLANII) SULLAR RADIATIOH DIFFEREHCE STUOY MUNTIHLY DATA SUMAAAHY

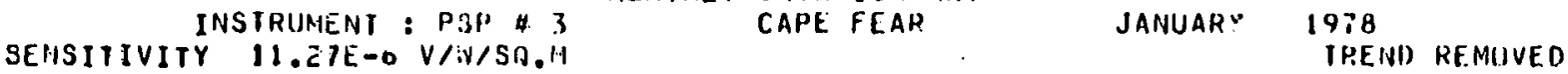

F.NERG KILUJDULES PF.R SIJUARE METER

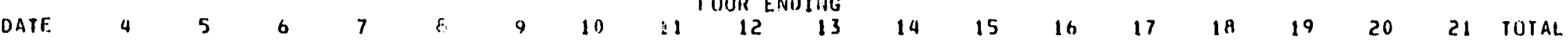

$\begin{array}{ll}1 & 0 \\ 2 & 0 \\ 3 & 0 \\ 1 & 0 \\ 5 & 0 \\ & \\ 6 & 0 \\ 7 & 0 \\ 6 & 0 \\ 9 & 0 \\ 10 & 0 \\ 11 & 0 \\ 12 & 0 \\ 13 & 0 \\ 14 & 0 \\ 15 & 0\end{array}$

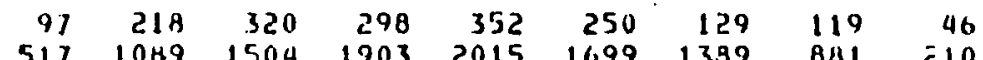

$\begin{array}{rrrrr}0 & 0 & 0 & 0 & 1846 \\ 0 & 0 & 0 & 0 & 11309 \\ 0 & 0 & 0 & 0 & 12631 \\ 0 & 0 & 0 & 0 & 12305 \\ 0 & 0 & 0 & 0 & 9684 \\ 0 & 0 & 0 & 0 & -999 \\ 0 & 0 & 0 & 0 & -999 \\ 0 & 0 & 0 & 0 & -999 \\ 0 & 0 & 0 & 0 & 11579 \\ 0 & 0 & 0 & 0 & 13216 \\ 0 & 0 & 0 & 0 & 13159 \\ 0 & 0 & 0 & 0 & 10593 \\ 0 & 0 & 0 & 0 & 1856 \\ 0 & 0 & 0 & 0 & 4330 \\ 0 & 0 & 0 & 0 & -999 \\ 0 & 0 & 0 & 0 & 11913 \\ 0 & 0 & 0 & 0 & 3961 \\ 0 & 0 & 0 & 0 & 12239 \\ 0 & 0 & 0 & 0 & 1626 \\ 0 & 0 & 0 & 0 & 2413 \\ 0 & 0 & 0 & 0 & 10798 \\ 0 & 0 & 0 & 0 & 7581 \\ 0 & 0 & 0 & 0 & 13638 \\ 0 & 0 & 0 & 0 & 11967 \\ 0 & 0 & 0 & 0 & 2124 \\ 0 & 0 & 0 & 0 & -999 \\ 0 & 0 & 0 & 0 & -999 \\ 0 & 0 & 0 & 0 & -999 \\ 0 & 0 & 0 & 0 & -999 \\ 0 & 0 & 0 & 0 & 14701 \\ 0 & 0 & 0 & 0 & 11605 \\ 0 & 0 & 0 & 0 & 9024 \\ 0 & 0 & 0 & 0 & 4453 \\ 0 & 31 & 31 & 31 & 23 \\ 0 & 0 & 0 & 0 & 14701 \\ 0 & 0 & 0 & 1626\end{array}$


COASIAL - INI.ANO SOLAR RADIATION DIFTEREIICE STIJOY MONTHILY DATA SUMMARY

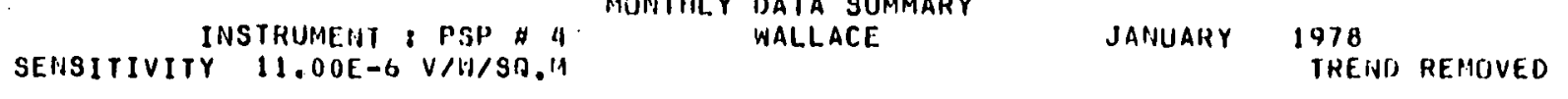

ENERGY KILOJOULES PER SOUARE METTR

\begin{tabular}{|c|c|c|c|c|c|c|c|c|c|c|c|c|c|c|c|c|c|c|c|}
\hline \multicolumn{20}{|c|}{ HOUR ENDING } \\
\hline DATE: & 4 & 5 & 6 & 7 & B & 7 & 10 & 11 & 12 & 13 & .14 & 15 & 16 & 17 & 18 & 19 & 20 & 21 & IOIAL \\
\hline $\begin{array}{l}1 \\
2 \\
3 \\
4 \\
5\end{array}$ & $\begin{array}{l}0 \\
0 \\
0 \\
0 \\
0\end{array}$ & $\begin{array}{l}0 \\
0 \\
0 \\
0 \\
0\end{array}$ & $\begin{array}{l}0 \\
0 \\
0 \\
0 \\
0\end{array}$ & $\begin{array}{l}0 \\
0 \\
0 \\
0 \\
0\end{array}$ & $\begin{array}{r}318 \\
61 \\
81 \\
-666 \\
54\end{array}$ & $\begin{array}{l}116 \\
516 \\
680 \\
769 \\
447\end{array}$ & $\begin{array}{r}165 \\
1105 \\
1233 \\
1292 \\
918\end{array}$ & $\begin{array}{r}253 \\
1596 \\
1714 \\
1711 \\
1337\end{array}$ & $\begin{array}{r}250 \\
1880 \\
1999 \\
1993 \\
1674\end{array}$ & $\begin{array}{r}230 \\
2014 \\
2055 \\
2055 \\
1393\end{array}$ & $\begin{array}{l}181 \\
1831 \\
1901 \\
1806 \\
1494\end{array}$ & $\begin{array}{l}122 \\
1455 \\
1505 \\
1449 \\
1353\end{array}$ & $\begin{array}{r}89 \\
830 \\
926 \\
903 \\
584\end{array}$ & $\begin{array}{l}299 \\
159 \\
301 \\
278 \\
185\end{array}$ & $\begin{array}{l}0 \\
0 \\
0 \\
0 \\
0\end{array}$ & $\begin{array}{l}0 \\
0 \\
0 \\
0 \\
0\end{array}$ & $\begin{array}{l}0 \\
0 \\
0 \\
0 \\
0\end{array}$ & $\begin{array}{l}0 \\
0 \\
0 \\
0 \\
0\end{array}$ & $\begin{array}{r}2023 \\
11447 \\
12395 \\
-999 \\
9439\end{array}$ \\
\hline $\begin{array}{r}6 \\
7 \\
4 \\
9 \\
10\end{array}$ & $\begin{array}{l}0 \\
0 \\
0 \\
0 \\
0\end{array}$ & $\begin{array}{l}0 \\
0 \\
0 \\
0 \\
0\end{array}$ & $\begin{array}{l}0 \\
0 \\
0 \\
0 \\
0\end{array}$ & $\begin{array}{l}0 \\
0 \\
0 \\
0 \\
0\end{array}$ & $\begin{array}{r}420 \\
-666 \\
29 \\
-666 \\
-666\end{array}$ & $\begin{array}{r}178 \\
99 \\
252 \\
64 \\
654\end{array}$ & $\begin{array}{r}413 \\
165 \\
716 \\
539 \\
1299\end{array}$ & $\begin{array}{r}198 \\
227 \\
1306 \\
1072 \\
1803\end{array}$ & $\begin{array}{r}744 \\
387 \\
808 \\
2185 \\
2091\end{array}$ & $\begin{array}{r}220 \\
636 \\
913 \\
2260 \\
2153\end{array}$ & $\begin{array}{r}191 \\
413 \\
510 \\
2106 \\
1983\end{array}$ & $\begin{array}{r}73 \\
472 \\
589 \\
1710 \\
1603\end{array}$ & $\begin{array}{r}73 \\
338 \\
461 \\
1098 \\
1024\end{array}$ & $\begin{array}{l}292 \\
178 \\
101 \\
418 \\
373\end{array}$ & $\begin{array}{l}0 \\
0 \\
0 \\
0 \\
0\end{array}$ & $\begin{array}{l}0 \\
0 \\
0 \\
0 \\
0\end{array}$ & $\begin{array}{l}0 \\
0 \\
0 \\
0 \\
0\end{array}$ & $\begin{array}{l}0 \\
0 \\
0 \\
0 \\
0\end{array}$ & $\begin{array}{l}3102 \\
-999 \\
5685 \\
-999 \\
-999\end{array}$ \\
\hline $\begin{array}{l}11 \\
12 \\
13 \\
14 \\
15\end{array}$ & $\begin{array}{l}0 . \\
0 \\
0 \\
0 \\
0\end{array}$ & $\begin{array}{l}0 \\
0 \\
0 \\
0 \\
0\end{array}$ & $\begin{array}{l}0 \\
0 \\
0 \\
0 \\
0\end{array}$ & $\begin{array}{l}0 \\
0 \\
0 \\
0 \\
0\end{array}$ & $\begin{array}{r}98 \\
-666 \\
0 \\
275 \\
-666\end{array}$ & $\begin{array}{r}651 \\
662 \\
-808 \\
206 \\
524\end{array}$ & $\begin{array}{r}1272 \\
1208 \\
71 \\
523 \\
1081\end{array}$ & $\begin{array}{r}1786 \\
1657 \\
198 \\
1142 \\
1588\end{array}$ & $\begin{array}{r}2074 \\
1955 \\
254 \\
1060 \\
1919\end{array}$ & $\begin{array}{r}2127 \\
1981 \\
274 \\
841 \\
1536\end{array}$ & $\begin{array}{r}1947 \\
1804 \\
190 \\
422 \\
1212\end{array}$ & $\begin{array}{r}1561 \\
1392 \\
110 \\
157 \\
1480\end{array}$ & $\begin{array}{r}905 \\
534 \\
133 \\
59 \\
960\end{array}$ & $\begin{array}{r}350 \\
171 \\
61 \\
206 \\
295\end{array}$ & $\begin{array}{l}0 \\
0 \\
0 \\
0 \\
0\end{array}$ & $\begin{array}{l}0 \\
0 \\
0 \\
0 \\
0\end{array}$ & $\begin{array}{l}0 \\
0 \\
0 \\
0 \\
0\end{array}$ & $\begin{array}{l}0 \\
0 \\
0 \\
0 \\
0\end{array}$ & $\begin{array}{r}12871 \\
-999 \\
-999 \\
4891 \\
-999\end{array}$ \\
\hline $\begin{array}{l}16 \\
17 \\
18 \\
19 \\
20\end{array}$ & $\begin{array}{l}0 \\
0 \\
0 \\
0 \\
0\end{array}$ & $\begin{array}{l}0 \\
0 \\
0 \\
0 \\
0\end{array}$ & $\begin{array}{l}0 \\
0 \\
0 \\
0 \\
0\end{array}$ & $\begin{array}{l}0 \\
0 \\
0 \\
0 \\
0\end{array}$ & $\begin{array}{r}-666 \\
-606 \\
328 \\
28 \\
16\end{array}$ & $\begin{array}{r}777 \\
72 \\
570 \\
143 \\
98\end{array}$ & $\begin{array}{r}1215 \\
536 \\
1140 \\
385 \\
272\end{array}$ & $\begin{array}{r}1707 \\
782 \\
1699 \\
323 \\
399\end{array}$ & $\begin{array}{r}2044 \\
896 \\
2023 \\
251 \\
268\end{array}$ & $\begin{array}{r}1704 \\
851 \\
2102 \\
90 \\
291\end{array}$ & $\begin{array}{r}1828 \\
481 \\
1977 \\
31 \\
265\end{array}$ & $\begin{array}{r}1118 \\
46 \\
1627 \\
12 \\
187\end{array}$ & $\begin{array}{r}561 \\
134 \\
986 \\
22 \\
173\end{array}$ & $\begin{array}{r}224 \\
229 \\
318 \\
31 \\
111\end{array}$ & $\begin{array}{l}0 \\
0 \\
0 \\
0 \\
0\end{array}$ & $\begin{array}{l}0 \\
0 \\
0 \\
0 \\
0\end{array}$ & $\begin{array}{l}0 \\
0 \\
0 \\
0 \\
0\end{array}$ & $\begin{array}{l}0 \\
0 \\
0 \\
0 \\
0\end{array}$ & $\begin{array}{r}-999 \\
-999 \\
12770 \\
1316 \\
2080\end{array}$ \\
\hline $\begin{array}{l}21 \\
23 \\
23 \\
24 \\
25\end{array}$ & $\begin{array}{l}0 \\
0 \\
0 \\
0 \\
0\end{array}$ & $\begin{array}{l}0 \\
0 \\
0 \\
0 \\
0\end{array}$ & $\begin{array}{l}0 \\
0 \\
0 \\
0 \\
0\end{array}$ & $\begin{array}{l}0 \\
0 \\
0 \\
0 \\
0\end{array}$ & $\begin{array}{r}-666 \\
38 \\
534 \\
188 \\
10\end{array}$ & $\begin{array}{r}259 \\
257 \\
634 \\
365 \\
33\end{array}$ & $\begin{array}{r}959 \\
450 \\
1083 \\
897 \\
72\end{array}$ & $\begin{array}{r}1626 \\
787 \\
985 \\
1435 \\
120\end{array}$ & $\begin{array}{r}2062 \\
790 \\
1649 \\
2041 \\
1.77\end{array}$ & $\begin{array}{r}2166 \\
516 \\
1528 \\
2266 \\
281\end{array}$ & $\begin{array}{r}2032 \\
466 \\
1685 \\
2018 \\
226\end{array}$ & $\begin{array}{r}1662 \\
745 \\
1358 \\
1438 \\
157\end{array}$ & $\begin{array}{r}1099 \\
342 \\
1093 \\
030 \\
419\end{array}$ & $\begin{array}{l}419 \\
139 \\
399 \\
280 \\
167\end{array}$ & $\begin{array}{l}0 \\
0 \\
0 \\
0 \\
0\end{array}$ & $\begin{array}{l}0 \\
0 \\
0 \\
0 \\
0\end{array}$ & $\begin{array}{l}0 \\
0 \\
0 \\
0 \\
0\end{array}$ & $\begin{array}{l}0 \\
0 \\
0 \\
0 \\
0\end{array}$ & $\begin{array}{r}-999 \\
4530 \\
10953 \\
11668 \\
1670\end{array}$ \\
\hline $\begin{array}{l}26 \\
27 \\
28 \\
29 \\
30\end{array}$ & $\begin{array}{l}0 \\
0 \\
0 \\
0 \\
0\end{array}$ & $\begin{array}{l}0 \\
0 \\
0 \\
0 \\
0\end{array}$ & $\begin{array}{l}0 \\
0 \\
0 \\
0 \\
0\end{array}$ & $\begin{array}{l}0 \\
0 \\
0 \\
0 \\
0\end{array}$ & $\begin{array}{r}-99 \\
-666 \\
104 \\
-666 \\
-606\end{array}$ & $\begin{array}{l}-97 \\
671 \\
680 \\
755 \\
680\end{array}$ & $\begin{array}{l}-99 \\
1358 \\
1354 \\
1361 \\
1358\end{array}$ & $\begin{array}{r}-99 \\
1901 \\
1911 \\
1904 \\
1901\end{array}$ & $\begin{array}{r}-79 \\
2248 \\
2258 \\
2261 \\
2258\end{array}$ & $\begin{array}{r}-99 \\
2362 \\
2379 \\
2392 \\
2415\end{array}$ & $\begin{array}{r}-99 \\
2150 \\
2235 \\
2267 \\
2267\end{array}$ & $\begin{array}{l}-99 \\
1770 \\
1871 \\
1907 \\
1894\end{array}$ & $\begin{array}{r}-99 \\
899 \\
1305 \\
1341 \\
1286\end{array}$ & $\begin{array}{r}-99 \\
402 \\
-666 \\
-666 \\
487\end{array}$ & $\begin{array}{l}0 \\
0 \\
0 \\
0 \\
0\end{array}$ & $\begin{array}{l}0 \\
0 \\
0 \\
0 \\
0\end{array}$ & $\begin{array}{l}0 \\
0 \\
0 \\
0 \\
0\end{array}$ & $\begin{array}{l}0 \\
0 \\
0 \\
0 \\
0\end{array}$ & $\begin{array}{l}-999 \\
-999 \\
-999 \\
-999 \\
-999\end{array}$ \\
\hline 31 & 0 & 0 & 0 & 0 & 1167 & BOS & Aก1 & 913 & 1282 & 1832 & 2081 & 1888 & 1315 & $-6,66$ & 0 & 0 & 0 & 0 & -999 \\
\hline $\begin{array}{c}\text { HEAH } \\
\text { SD } \\
\text { HUM }\end{array}$ & $\begin{array}{r}0 \\
31\end{array}$ & $\begin{array}{r}0 \\
0 \\
31\end{array}$ & $\begin{array}{r}0 \\
0 \\
31\end{array}$ & $\begin{array}{r}0 \\
31\end{array}$ & $\begin{array}{r}169 \\
170 \\
18\end{array}$ & $\begin{array}{r}4.34 \\
2622 \\
29\end{array}$ & $\begin{array}{r}840 \\
435 \\
30\end{array}$ & $\begin{array}{r}1209 \\
602 \\
30\end{array}$ & $\begin{array}{r}1459 \\
752 \\
30\end{array}$ & $\begin{array}{r}1462 \\
811 \\
30\end{array}$ & $\begin{array}{r}1333 \\
812 \\
30\end{array}$ & $\begin{array}{r}1090 \\
681 \\
30\end{array}$ & $\begin{array}{r}693 \\
427 \\
30\end{array}$ & $\begin{array}{r}254 \\
116 \\
27\end{array}$ & $\begin{array}{r}0 \\
0 \\
31\end{array}$ & $\begin{array}{r}0 \\
0 \\
31\end{array}$ & $\begin{array}{r}0 \\
0 \\
31\end{array}$ & $\begin{array}{r}0 \\
0 \\
31\end{array}$ & $\begin{array}{r}7122 \\
4449 \\
15\end{array}$ \\
\hline $\begin{array}{l}\text { MAX } \\
\text { MIH }\end{array}$ & $\begin{array}{l}0 \\
0\end{array}$ & $\begin{array}{l}0 \\
0\end{array}$ & $\begin{array}{l}0 \\
0\end{array}$ & $\begin{array}{l}0 \\
0\end{array}$ & $\begin{array}{r}539 \\
0\end{array}$ & $\begin{array}{r}105 \\
33\end{array}$ & $\begin{array}{r}1361 \\
72\end{array}$ & $\begin{array}{r}1911 \\
128\end{array}$ & $\begin{array}{r}2261 \\
177\end{array}$ & $\begin{array}{r}2415 \\
90\end{array}$ & $\begin{array}{r}2267 \\
31\end{array}$ & $\begin{array}{r}1907 \\
12\end{array}$ & $\begin{array}{r}1341 \\
22\end{array}$ & $\begin{array}{r}487 \\
31\end{array}$ & $\begin{array}{l}0 \\
0\end{array}$ & $\begin{array}{l}0 \\
0\end{array}$ & $\begin{array}{l}0 \\
0\end{array}$ & $\begin{array}{l}0 \\
0\end{array}$ & $\begin{array}{r}12871 \\
1316\end{array}$ \\
\hline
\end{tabular}


COASTAL - INLAITO SOLAR RADIATIUN DIFFEREHCE STIUDY MIONTHLY DATA SUMMARY

IMSTRUMENT: PSP 5
SENSITIVITY $9.44 E-6$ VIW/SO.M

ENERGY KILUJOULES PER SQUARE METER HOUR ENDING

\begin{tabular}{|c|c|c|c|c|c|c|c|c|c|c|c|c|c|c|c|c|c|c|c|}
\hline \multicolumn{20}{|c|}{ HOUR ENDING } \\
\hline DATE & 4 & 5 & 6 & 7 & 8 & 9 & 11) & 11 & 12 & 13 & 14 & 15 & 16 & 17 & 18 & 19 & 20 & 21 & TOTAL \\
\hline $\begin{array}{l}1 \\
2 \\
3 \\
4 \\
5\end{array}$ & $\begin{array}{l}0 \\
0 \\
0 \\
0 \\
0\end{array}$ & $\begin{array}{l}0 \\
0 \\
0 \\
0 \\
0\end{array}$ & $\begin{array}{l}0 \\
0 \\
0 \\
0 \\
0\end{array}$ & $\begin{array}{l}0 \\
0 \\
0 \\
0 \\
0\end{array}$ & $\begin{array}{l}17 \\
94 \\
26 \\
95 \\
45\end{array}$ & $\begin{array}{l}131 \\
525 \\
575 \\
613 \\
335\end{array}$ & $\begin{array}{r}268 \\
986 \\
1216 \\
1227 \\
919\end{array}$ & $\begin{array}{r}348 \\
1.558 \\
1738 \\
1.750 \\
1262\end{array}$ & $\begin{array}{l}337 \\
1707 \\
2036 \\
2040 \\
1205\end{array}$ & $\begin{array}{r}268 \\
1978 \\
2097 \\
2112 \\
1372\end{array}$ & $\begin{array}{r}242 \\
1856 \\
1941 \\
1910 \\
1014\end{array}$ & $\begin{array}{r}146 \\
1326 \\
1544 \\
1487 \\
911\end{array}$ & $\begin{array}{l}104 \\
837 \\
961 \\
926 \\
877\end{array}$ & $\begin{array}{l}28 \\
223 \\
320 \\
305 \\
183\end{array}$ & $\begin{array}{l}0 \\
0 \\
0 \\
0 \\
0\end{array}$ & $\begin{array}{l}0 \\
0 \\
0 \\
0 \\
0\end{array}$ & $\begin{array}{l}0 \\
0 \\
0 \\
0 \\
0\end{array}$ & $\begin{array}{l}0 \\
0 \\
0 \\
0 \\
0\end{array}$ & $\begin{array}{r}1889 \\
11090 \\
12454 \\
12473 \\
8123\end{array}$ \\
\hline $\begin{array}{r}6 \\
7 \\
0 \\
9 \\
10\end{array}$ & $\begin{array}{l}0 \\
0 \\
0 \\
0 \\
0\end{array}$ & $\begin{array}{l}1) \\
0 \\
0 \\
0 \\
0\end{array}$ & $\begin{array}{l}0 \\
0 \\
0 \\
0 \\
0\end{array}$ & $\begin{array}{l}0 \\
0 \\
0 \\
0 \\
0\end{array}$ & $\begin{array}{r}0 \\
20 \\
61 \\
-99 \\
-99\end{array}$ & $\begin{array}{r}45 \\
100 \\
450 \\
-99 \\
-97\end{array}$ & $\begin{array}{l}228 \\
230 \\
626 \\
-99 \\
-99\end{array}$ & $\begin{array}{r}777 \\
322 \\
1194 \\
-79 \\
-99\end{array}$ & $\begin{array}{r}797 \\
539 \\
1850 \\
-99 \\
-99\end{array}$ & $\begin{array}{r}1727 \\
493 \\
1861 \\
-99 \\
-99\end{array}$ & $\begin{array}{r}907 \\
440 \\
1388 \\
-99 \\
-99\end{array}$ & $\begin{array}{r}209 \\
192 \\
1003 \\
-99 \\
-99\end{array}$ & $\begin{array}{r}87 \\
154 \\
519 \\
-99 \\
-99\end{array}$ & $\begin{array}{r}102 \\
66 \\
133 \\
-97 \\
-99\end{array}$ & $\begin{array}{l}0 \\
0 \\
0 \\
0 \\
0\end{array}$ & $\begin{array}{l}11 \\
0 \\
0 \\
0 \\
0\end{array}$ & $\begin{array}{l}0 \\
0 \\
0 \\
0 \\
0\end{array}$ & $\begin{array}{l}0 \\
0 \\
0 \\
0 \\
0\end{array}$ & $\begin{array}{l}4879 \\
2556 \\
9090 \\
-999 \\
-999\end{array}$ \\
\hline $\begin{array}{l}11 \\
12 \\
13 \\
14 \\
15\end{array}$ & $\begin{array}{l}0 \\
0 \\
0 \\
0 \\
0\end{array}$ & $\begin{array}{l}0 \\
0 \\
0 \\
0 \\
0\end{array}$ & $\begin{array}{l}0 \\
0 \\
0 \\
0 \\
0\end{array}$ & $\begin{array}{l}0 \\
0 \\
0 \\
0 \\
0\end{array}$ & $\begin{array}{r}-99 \\
118 \\
11 \\
53 \\
53\end{array}$ & $\begin{array}{r}-99 \\
728 \\
22 \\
381 \\
514\end{array}$ & $\begin{array}{r}-97 \\
1201 \\
64 \\
957 \\
968\end{array}$ & $\begin{array}{r}-97 \\
1671 \\
144 \\
1399 \\
1544\end{array}$ & $\begin{array}{l}-99 \\
-99 \\
-99 \\
-99 \\
-99\end{array}$ & $\begin{array}{r}-99 \\
1841 \\
442 \\
705 \\
1792\end{array}$ & $\begin{array}{r}-99 \\
1750 \\
381 \\
514 \\
1929\end{array}$ & $\begin{array}{r}930 \\
865 \\
373 \\
236 \\
1460\end{array}$ & $\begin{array}{r}1022 \\
591 \\
202 \\
40 \\
961\end{array}$ & $\begin{array}{r}377 \\
194 \\
57 \\
3.7 \\
354\end{array}$ & $\begin{array}{l}0 \\
0 \\
0 \\
0 \\
0\end{array}$ & $\begin{array}{l}0 \\
0 \\
0 \\
0 \\
0\end{array}$ & $\begin{array}{l}0 \\
0 \\
0 \\
0 \\
0\end{array}$ & $\begin{array}{l}0 \\
0 \\
0 \\
0 \\
0\end{array}$ & $\begin{array}{l}-999 \\
-999 \\
-949 \\
-999 \\
-999\end{array}$ \\
\hline $\begin{array}{l}16 \\
17 \\
18 \\
19 \\
20\end{array}$ & $\begin{array}{l}0 \\
0 \\
0 \\
0 \\
0\end{array}$ & $\begin{array}{l}0 \\
0 \\
0 \\
0 \\
0\end{array}$ & $\begin{array}{l}0 \\
0 \\
0 \\
0 \\
0\end{array}$ & $\begin{array}{l}0 \\
0 \\
0 \\
0 \\
0\end{array}$ & $\begin{array}{r}179 \\
15 \\
90 \\
44 \\
49\end{array}$ & $\begin{array}{l}592 \\
163 \\
518 \\
1115 \\
360\end{array}$ & $\begin{array}{r}1267 \\
508 \\
1047 \\
521 \\
401\end{array}$ & $\begin{array}{r}1727 \\
682 \\
1467 \\
494 \\
765\end{array}$ & $\begin{array}{l}-79 \\
-99 \\
-99 \\
-99 \\
-99\end{array}$ & $\begin{array}{r}2124 \\
960 \\
2035 \\
216 \\
423\end{array}$ & $\begin{array}{r}1742 \\
380 \\
1993 \\
29 \\
343\end{array}$ & $\begin{array}{r}1144 \\
91 \\
1623 \\
0 \\
305\end{array}$ & $\begin{array}{r}606 \\
179 \\
1043 \\
0 \\
163\end{array}$ & $\begin{array}{r}247 \\
19.7 \\
407 \\
14 \\
150\end{array}$ & $\begin{array}{l}0 \\
0 \\
0 \\
0 \\
0\end{array}$ & $\begin{array}{l}0 \\
0 \\
0 \\
0 \\
0\end{array}$ & $\begin{array}{l}0 \\
0 \\
0 \\
0 \\
0\end{array}$ & $\begin{array}{l}0 \\
0 \\
0 \\
0 \\
0\end{array}$ & $\begin{array}{l}-999 \\
-999 \\
-199 \\
-999 \\
-999\end{array}$ \\
\hline $\begin{array}{l}21 \\
22 \\
23 \\
24 \\
25\end{array}$ & $\begin{array}{l}0 \\
0 \\
0 \\
0 \\
0\end{array}$ & $\begin{array}{l}0 \\
0 \\
0 \\
0 \\
0\end{array}$ & $\begin{array}{l}0 \\
0 \\
0 \\
0 \\
0\end{array}$ & $\begin{array}{l}0 \\
0 \\
0 \\
0 \\
0\end{array}$ & $\begin{array}{r}19 \\
95 \\
133 \\
171 \\
11\end{array}$ & $\begin{array}{r}171 \\
404 \\
682 \\
492 \\
68\end{array}$ & $\begin{array}{r}503 \\
873 \\
1365 \\
854 \\
106\end{array}$ & $\begin{array}{r}1224 \\
785 \\
1953 \\
1540 \\
102\end{array}$ & $\begin{array}{l}-99 \\
-99 \\
-99 \\
-99 \\
-99\end{array}$ & $\begin{array}{l}21113 \\
1731 \\
2006 \\
2166 \\
163\end{array}$ & $\begin{array}{r}2013 \\
781 \\
2055 \\
2005 \\
297\end{array}$ & $\begin{array}{r}1666 \\
606 \\
1578 \\
1697 \\
385\end{array}$ & $\begin{array}{r}1117 \\
247 \\
1136 \\
682 \\
518\end{array}$ & $\begin{array}{l}145 \\
171 \\
385 \\
315 \\
133\end{array}$ & $\begin{array}{l}0 \\
0 \\
0 \\
0 \\
0\end{array}$ & $\begin{array}{l}0 \\
0 \\
0 \\
0 \\
0\end{array}$ & $\begin{array}{l}0 \\
0 \\
0 \\
0 \\
0\end{array}$ & $\begin{array}{l}0 \\
0 \\
0 \\
0 \\
0\end{array}$ & $\begin{array}{l}-999 \\
-999 \\
-999 \\
-999 \\
-999\end{array}$ \\
\hline $\begin{array}{l}26 \\
27 \\
28 \\
27 \\
30\end{array}$ & $\begin{array}{l}0 \\
0 \\
0 \\
0 \\
0\end{array}$ & $\begin{array}{l}0 \\
0 \\
0 \\
0 \\
0\end{array}$ & $\begin{array}{l}0 \\
0 \\
0 \\
0 \\
0\end{array}$ & $\begin{array}{l}0 \\
0 \\
0 \\
0 \\
0\end{array}$ & $\begin{array}{r}-99 \\
-99 \\
0 \\
0 \\
141\end{array}$ & $\begin{array}{l}-99 \\
-99 \\
347 \\
339 \\
766\end{array}$ & $\begin{array}{r}-79 \\
-97 \\
1033 \\
1033 \\
1437\end{array}$ & $\begin{array}{r}-97 \\
-97 \\
1674 \\
1677 \\
198 .\end{array}$ & $\begin{array}{r}-99 \\
-99 \\
-99 \\
-99 \\
2314\end{array}$ & $\begin{array}{r}-99 \\
-99 \\
2372 \\
-666 \\
2417\end{array}$ & $\begin{array}{r}-99 \\
-99 \\
2375 \\
2272 \\
2269\end{array}$ & $\begin{array}{r}-99 \\
-99 \\
-666 \\
1891 \\
1883\end{array}$ & $\begin{array}{r}-99 \\
392 \\
-666 \\
1300 \\
1296\end{array}$ & $\begin{array}{r}-97 \\
499 \\
-360 \\
-360 \\
3813\end{array}$ & $\begin{array}{l}0 \\
0 \\
0 \\
0 \\
0\end{array}$ & $\begin{array}{l}0 \\
0 \\
0 \\
0 \\
0\end{array}$ & $\begin{array}{l}0 \\
0 \\
0 \\
0 \\
0\end{array}$ & $\begin{array}{l}0 \\
0 \\
0 \\
0 \\
0\end{array}$ & $\begin{array}{r}-999 \\
-999 \\
-999 \\
-999 \\
14897\end{array}$ \\
\hline 31 & 0 & 0 & 0 & 0 & 148 & 713 & 1022 & 737 & 1121 & 1525 & 2047 & $178 B$ & 1197 & 545 & 0 & ? & 0 & 0 & 10875 \\
\hline $\begin{array}{r}\text { HEAN } \\
\text { SD } \\
\text { HIUIM }\end{array}$ & $\begin{array}{r}0 \\
0 \\
.31\end{array}$ & $\begin{array}{r}0 \\
0 \\
31\end{array}$ & $\begin{array}{r}0 \\
0 \\
31\end{array}$ & $\begin{array}{r}0 \\
0 \\
31\end{array}$ & $\begin{array}{l}64 \\
54 \\
26\end{array}$ & $\begin{array}{r}406 \\
242 \\
26\end{array}$ & $\begin{array}{r}804 \\
390 \\
26\end{array}$ & $\begin{array}{r}1174 \\
572 \\
25\end{array}$ & $\begin{array}{r}1394 \\
655 \\
10\end{array}$ & $\begin{array}{r}1481 \\
748 \\
25\end{array}$ & $\begin{array}{r}1341 \\
777 \\
20\end{array}$ & $\begin{array}{r}974 \\
637 \\
23\end{array}$ & $\begin{array}{r}636 \\
420 \\
27\end{array}$ & $\begin{array}{r}241 \\
149 \\
20\end{array}$ & $\begin{array}{r}0 \\
0 \\
31\end{array}$ & $\begin{array}{r}0 \\
0 \\
31\end{array}$ & $\begin{array}{r}0 \\
0 \\
31\end{array}$ & $\begin{array}{r}0 \\
0 \\
31\end{array}$ & $\begin{array}{r}8832 \\
4197 \\
10\end{array}$ \\
\hline $\begin{array}{l}\text { MAX } \\
\text { MIPI }\end{array}$ & $\begin{array}{l}0 \\
0\end{array}$ & $\begin{array}{l}0 \\
0\end{array}$ & $\begin{array}{l}0 \\
0\end{array}$ & $\begin{array}{l}0 \\
0\end{array}$ & $\begin{array}{r}179 \\
0\end{array}$ & $\begin{array}{r}892 \\
2 ?\end{array}$ & $\begin{array}{r}1437 \\
64\end{array}$ & $\begin{array}{r}198 \pi \\
10 ?\end{array}$ & $\begin{array}{r}2314 \\
337\end{array}$ & $\begin{array}{r}2417 \\
163\end{array}$ & $\begin{array}{r}2375 \\
29\end{array}$ & $\begin{array}{r}1891 \\
0\end{array}$ & $\begin{array}{r}1300 \\
.0\end{array}$ & $\begin{array}{r}545 \\
14\end{array}$ & $\begin{array}{l}0 \\
0\end{array}$ & $\begin{array}{l}0 \\
0\end{array}$ & $\begin{array}{l}0 \\
0\end{array}$ & $\begin{array}{l}0 \\
0\end{array}$ & $\begin{array}{r}14897 \\
1889\end{array}$ \\
\hline
\end{tabular}


COASTAL - INLAND SOLAR RADIATION DIFFERENCE STUOY

INSTRUMENT: PSP 6 MONTILY DATA SUMMARY
CLINTON
$11.00 E-6$ VIW/90. IN

INSTRUMENT: PSP 6
SENSITIVITY $11.00 E-6$ VIW/90. IA
FiNERGY KII.OJUULES PER SOUARE HETER

FiNERGY KII.UJUULES PER SOUARE HETER
HUUR ENDING

\begin{tabular}{|c|c|c|c|c|c|c|c|c|c|c|c|c|c|c|c|c|c|c|c|}
\hline \multicolumn{20}{|c|}{ HUUR ENDING } \\
\hline DATE & 4 & 5 & 6 & 7 & 8 & 9 & 10 & 11 & 12 & 13 & 14 & 15 & 16 & 17 & 18 & 19 & 20 & 21 & TOTAL \\
\hline $\begin{array}{l}1 \\
2 \\
3 \\
4 \\
5\end{array}$ & $\begin{array}{l}0 \\
0 \\
0 \\
0 \\
0\end{array}$ & $\begin{array}{l}0 \\
0 \\
0 \\
0 \\
0\end{array}$ & $\begin{array}{l}0 \\
0 \\
0 \\
0 \\
0\end{array}$ & $\begin{array}{l}0 \\
0 \\
0 \\
0 \\
0\end{array}$ & $\begin{array}{l}13 \\
56 \\
78 \\
88 \\
36\end{array}$ & $\begin{array}{r}42 \\
175 \\
595 \\
697 \\
523\end{array}$ & $\begin{array}{r}131 \\
1140 \\
1201 \\
1191 \\
946\end{array}$ & $\begin{array}{l}177 \\
1568 \\
1691 \\
1678 \\
1276\end{array}$ & $\begin{array}{l}233 \\
1938 \\
1986 \\
1957 \\
1440\end{array}$ & $\begin{array}{r}229 \\
1994 \\
2068 \\
2015 \\
11433\end{array}$ & $\begin{array}{l}177 \\
1824 \\
1898 \\
1793 \\
1718\end{array}$ & $\begin{array}{l}167 \\
1392 \\
1528 \\
1361 \\
1073\end{array}$ & $\begin{array}{l}102 \\
678 \\
952 \\
811 \\
854\end{array}$ & $\begin{array}{r}33 \\
184 \\
320 \\
297 \\
291\end{array}$ & $\begin{array}{l}0 \\
0 \\
0 \\
0 \\
0\end{array}$ & $\begin{array}{l}0 \\
0 \\
0 \\
0 \\
0\end{array}$ & $\begin{array}{l}0 \\
0 \\
0 \\
0 \\
0\end{array}$ & $\begin{array}{l}0 \\
0 \\
0 \\
0 \\
0\end{array}$ & $\begin{array}{r}1354 \\
11249 \\
12317 \\
11891 \\
9590\end{array}$ \\
\hline $\begin{array}{r}6 \\
7 \\
8 \\
9 \\
10\end{array}$ & $\begin{array}{l}0 \\
0 \\
0 \\
0 \\
0\end{array}$ & $\begin{array}{l}0 \\
0 \\
0 \\
0 \\
0\end{array}$ & $\begin{array}{l}0 \\
0 \\
0 \\
0 \\
0\end{array}$ & $\begin{array}{l}0 \\
0 \\
0 \\
0 \\
0\end{array}$ & $\begin{array}{r}37 \\
10 \\
40 \\
9 \\
70\end{array}$ & $\begin{array}{l}175 \\
102 \\
184 \\
112 \\
601\end{array}$ & $\begin{array}{r}279 \\
249 \\
452 \\
1141 \\
1232\end{array}$ & $\begin{array}{r}165 \\
314 \\
1103 \\
1698 \\
1730\end{array}$ & $\begin{array}{r}171 \\
527 \\
071 \\
2005 \\
2034\end{array}$ & $\begin{array}{r}175 \\
661 \\
1201 \\
2100 \\
2109\end{array}$ & $\begin{array}{r}109 \\
609 \\
530 \\
1930 \\
1949\end{array}$ & $\begin{array}{r}113 \\
566 \\
337 \\
1570 \\
1579\end{array}$ & $\begin{array}{r}83 \\
305 \\
223 \\
1000 \\
1016\end{array}$ & $\begin{array}{r}31 \\
128 \\
62 \\
372 \\
3 \text { A1 }\end{array}$ & $\begin{array}{l}0 \\
0 \\
0 \\
0 \\
0\end{array}$ & $\begin{array}{l}0 \\
0 \\
0 \\
0 \\
0\end{array}$ & $\begin{array}{l}0 \\
0 \\
0 \\
0 \\
0\end{array}$ & $\begin{array}{l}0 \\
0 \\
0 \\
0 \\
0\end{array}$ & $\begin{array}{r}1338 \\
3471 \\
5003 \\
11997 \\
12701\end{array}$ \\
\hline $\begin{array}{l}11 \\
12 \\
13 \\
14 \\
15\end{array}$ & $\begin{array}{l}0 \\
0 \\
0 \\
0 \\
0\end{array}$ & $\begin{array}{l}0 \\
0 \\
0 \\
0 \\
0\end{array}$ & $\begin{array}{l}0 \\
0 \\
0 \\
0 \\
0\end{array}$ & $\begin{array}{l}0 \\
0 \\
0 \\
0 \\
0\end{array}$ & $\begin{array}{r}71 \\
82 \\
0 \\
27 \\
65\end{array}$ & $\begin{array}{r}6132 \\
572 \\
21 \\
214 \\
474\end{array}$ & $\begin{array}{r}1243 \\
1158 \\
96 \\
453 \\
1070\end{array}$ & $\begin{array}{r}1718 \\
1616 \\
217 \\
603 \\
1590\end{array}$ & $\begin{array}{r}2029 \\
1944 \\
152 \\
659 \\
1898\end{array}$ & $\begin{array}{r}2114 \\
1954 \\
132 \\
1330 \\
1963\end{array}$ & $\begin{array}{r}1940 \\
1747 \\
207 \\
741 \\
1613\end{array}$ & $\begin{array}{r}1590 \\
1384 \\
93 \\
479 \\
1522\end{array}$ & $\begin{array}{r}1037 \\
674 \\
96 \\
129 \\
1021\end{array}$ & $\begin{array}{r}301 \\
183 \\
63 \\
24 \\
392\end{array}$ & $\begin{array}{l}0 \\
0 \\
0 \\
0 \\
0\end{array}$ & $\begin{array}{l}0 \\
0 \\
0 \\
0 \\
0\end{array}$ & $\begin{array}{l}0 \\
0 \\
0 \\
0 \\
0\end{array}$ & $\begin{array}{l}0 \\
0 \\
0 \\
0 \\
0\end{array}$ & $\begin{array}{r}12645 \\
11314 \\
1077 \\
4679 \\
11848\end{array}$ \\
\hline $\begin{array}{l}16 \\
17 \\
18 \\
19 \\
20\end{array}$ & $\begin{array}{l}0 \\
0 \\
0 \\
0 \\
0\end{array}$ & $\begin{array}{l}0 \\
0 \\
0 \\
0 \\
0\end{array}$ & $\begin{array}{l}0 \\
0 \\
0 \\
0 \\
0\end{array}$ & $\begin{array}{l}0 \\
0 \\
0 \\
0 \\
0\end{array}$ & $\begin{array}{l}85 \\
19 \\
54 \\
13 \\
10\end{array}$ & $\begin{array}{r}559 \\
107 \\
408 \\
154 \\
67\end{array}$ & $\begin{array}{r}1168 \\
140 \\
1105 \\
245 \\
236\end{array}$ & $\begin{array}{r}1633 \\
768 \\
1678 \\
367 \\
308\end{array}$ & $\begin{array}{r}1371 \\
1079 \\
2011 \\
226 \\
242\end{array}$ & $\begin{array}{r}2083 \\
8 A 9 \\
2100 \\
75 \\
262\end{array}$ & $\begin{array}{r}1584 \\
464 \\
1831 \\
33 \\
285\end{array}$ & $\begin{array}{r}1148 \\
61 \\
1550 \\
13 \\
341\end{array}$ & $\begin{array}{r}533 \\
297 \\
1013 \\
52 \\
269\end{array}$ & $\begin{array}{r}193 \\
146 \\
437 \\
29 \\
148\end{array}$ & $\begin{array}{l}0 \\
0 \\
0 \\
0 \\
0\end{array}$ & $\begin{array}{l}0 \\
0 \\
0 \\
0 \\
0\end{array}$ & $\begin{array}{l}0 \\
0 \\
0 \\
0 \\
0\end{array}$ & $\begin{array}{l}0 \\
0 \\
0 \\
0 \\
0\end{array}$ & $\begin{array}{r}10362 \\
4010 \\
12187 \\
1207 \\
2170\end{array}$ \\
\hline $\begin{array}{l}21 \\
22 \\
33 \\
21 \\
25\end{array}$ & $\begin{array}{l}0 \\
0 \\
0 \\
0 \\
0\end{array}$ & $\begin{array}{l}0 \\
0 \\
0 \\
0 \\
0\end{array}$ & $\begin{array}{l}0 \\
0 \\
0 \\
0 \\
0\end{array}$ & $\begin{array}{l}0 \\
0 \\
0 \\
0 \\
0\end{array}$ & $\begin{array}{r}37 \\
24 \\
49 \\
107 \\
0\end{array}$ & $\begin{array}{r}32 ? \\
135 \\
42.8 \\
3.42 \\
76\end{array}$ & $\begin{array}{r}1209 \\
181 \\
1132 \\
715 \\
151\end{array}$ & $\begin{array}{r}1729 \\
305 \\
1734 \\
1269 \\
200\end{array}$ & $\begin{array}{r}2053 \\
384 \\
1814 \\
1920 \\
236\end{array}$ & $\begin{array}{r}2111 \\
449 \\
1151 \\
2008 \\
429\end{array}$ & $\begin{array}{r}1974 \\
655 \\
1757 \\
2159 \\
223\end{array}$ & $\begin{array}{r}580 \\
325 \\
1158 \\
1278 \\
145\end{array}$ & $\begin{array}{r}1101 \\
340 \\
975 \\
640 \\
59\end{array}$ & $\begin{array}{r}430 \\
217 \\
428 \\
342 \\
50\end{array}$ & $\begin{array}{l}0 \\
0 \\
0 \\
0 \\
0\end{array}$ & $\begin{array}{l}0 \\
0 \\
0 \\
0 \\
0\end{array}$ & $\begin{array}{l}0 \\
0 \\
0 \\
0 \\
0\end{array}$ & $\begin{array}{r}0 \\
0 \\
0 \\
0 \\
0\end{array}$ & $\begin{array}{r}11596 \\
3055 \\
10654 \\
10780 \\
1509\end{array}$ \\
\hline $\begin{array}{l}26 \\
27 \\
28 \\
29 \\
30\end{array}$ & $\begin{array}{l}0 \\
0 \\
0 \\
0 \\
0\end{array}$ & $\begin{array}{l}0 \\
0 \\
0 \\
0 \\
0\end{array}$ & $\begin{array}{l}0 \\
0 \\
0 \\
0 \\
0\end{array}$ & $\begin{array}{l}0 \\
0 \\
0 \\
0 \\
0\end{array}$ & $\begin{array}{r}100 \\
100 \\
-6166 \\
111 \\
117\end{array}$ & $\begin{array}{l}578 \\
692 \\
703 \\
697 \\
703\end{array}$ & $\begin{array}{l}1357 \\
1357 \\
1387 \\
1390 \\
1387\end{array}$ & $\begin{array}{l}1707 \\
1887 \\
1917 \\
2225 \\
1914\end{array}$ & $\begin{array}{l}2289 \\
2217 \\
2241 \\
2261 \\
2254\end{array}$ & $\begin{array}{l}2260 \\
2312 \\
2317 \\
2375 \\
2218\end{array}$ & $\begin{array}{l}2149 \\
2083 \\
2150 \\
2231 \\
2209\end{array}$ & $\begin{array}{l}1779 \\
1556 \\
1754 \\
1858 \\
1832\end{array}$ & $\begin{array}{l}1173 \\
1065 \\
1207 \\
1295 \\
1227\end{array}$ & $\begin{array}{r}-666 \\
486 \\
-666 \\
-666 \\
-666\end{array}$ & $\begin{array}{l}0 \\
0 \\
0 \\
0 \\
0\end{array}$ & $\begin{array}{l}0 \\
0 \\
0 \\
0 \\
0\end{array}$ & $\begin{array}{l}0 \\
0 \\
0 \\
0 \\
0\end{array}$ & $\begin{array}{l}0 \\
0 \\
0 \\
0 \\
0\end{array}$ & $\begin{array}{r}-999 \\
13761 \\
-999 \\
-999 \\
-999\end{array}$ \\
\hline 31 & 0 & 0 & 0 & 0 & 136 & 476 & 761 & 1196 & 1746 & 2100 & 2109 & $180 B$ & 1216 & -666 & 0 & $\mathbf{0}$ & 0 & 0 & -999 \\
\hline $\begin{array}{r}\text { MEAII } \\
\text { SD } \\
\text { iIUM }\end{array}$ & $\begin{array}{r}0 \\
0 \\
31\end{array}$ & $\begin{array}{r}0 \\
0 \\
31\end{array}$ & $\begin{array}{r}0 \\
0 \\
31\end{array}$ & $\begin{array}{r}0 \\
0 \\
31\end{array}$ & $\begin{array}{l}55 \\
38 \\
30\end{array}$ & $\begin{array}{r}396 \\
229 \\
31\end{array}$ & $\begin{array}{r}836 \\
472 \\
31\end{array}$ & $\begin{array}{r}1225 \\
652 \\
31\end{array}$ & $\begin{array}{r}1426 \\
780 \\
31\end{array}$ & $\begin{array}{r}1505 \\
796 \\
31\end{array}$ & $\begin{array}{r}1383 \\
780 \\
31\end{array}$ & $\begin{array}{r}10.30 \\
642 \\
31\end{array}$ & $\begin{array}{r}693 \\
420 \\
31\end{array}$ & $\begin{array}{r}231 \\
147 \\
26\end{array}$ & $\begin{array}{r}0 \\
0 \\
31\end{array}$ & $\begin{array}{r}0 \\
0 \\
31\end{array}$ & $\begin{array}{r}0 \\
0 \\
31\end{array}$ & $\begin{array}{r}0 \\
0 \\
31\end{array}$ & $\begin{array}{r}7839 \\
4618 \\
26\end{array}$ \\
\hline $\begin{array}{l}\text { MAX } \\
\text { HIN }\end{array}$ & $\begin{array}{l}0 \\
0\end{array}$ & $\begin{array}{l}0 \\
0\end{array}$ & $\begin{array}{l}0 \\
0\end{array}$ & $\begin{array}{l}0 \\
0\end{array}$ & $\begin{array}{r}136 \\
0\end{array}$ & $\begin{array}{r}703 \\
21\end{array}$ & $\begin{array}{r}1390 \\
76\end{array}$ & $\begin{array}{r}2.25 \\
165\end{array}$ & $\begin{array}{r}2289 \\
152\end{array}$ & $\begin{array}{r}2375 \\
75\end{array}$ & $\begin{array}{r}2231 \\
33\end{array}$ & $\begin{array}{r}185 B \\
13\end{array}$ & $\begin{array}{r}1295 \\
.52\end{array}$ & $\begin{array}{r}4116 \\
24\end{array}$ & $\begin{array}{l}11 \\
0\end{array}$ & $\begin{array}{l}0 \\
0\end{array}$ & $\begin{array}{l}0 \\
0\end{array}$ & $\begin{array}{l}0 \\
0\end{array}$ & $\begin{array}{r}13761 \\
1077\end{array}$ \\
\hline
\end{tabular}


COASTAL - IIILAND SOLAR RADIATIUN IJIFFERENCE. STUDT

INSTRUMENT: PSP A 7 MUNTILY DATA SUMMARY
SENS.ITIVITY IO.17E-6 V/W/SO. ONSLUH GEACI

ENERGY KILOJOHLES PER SUUARE METER

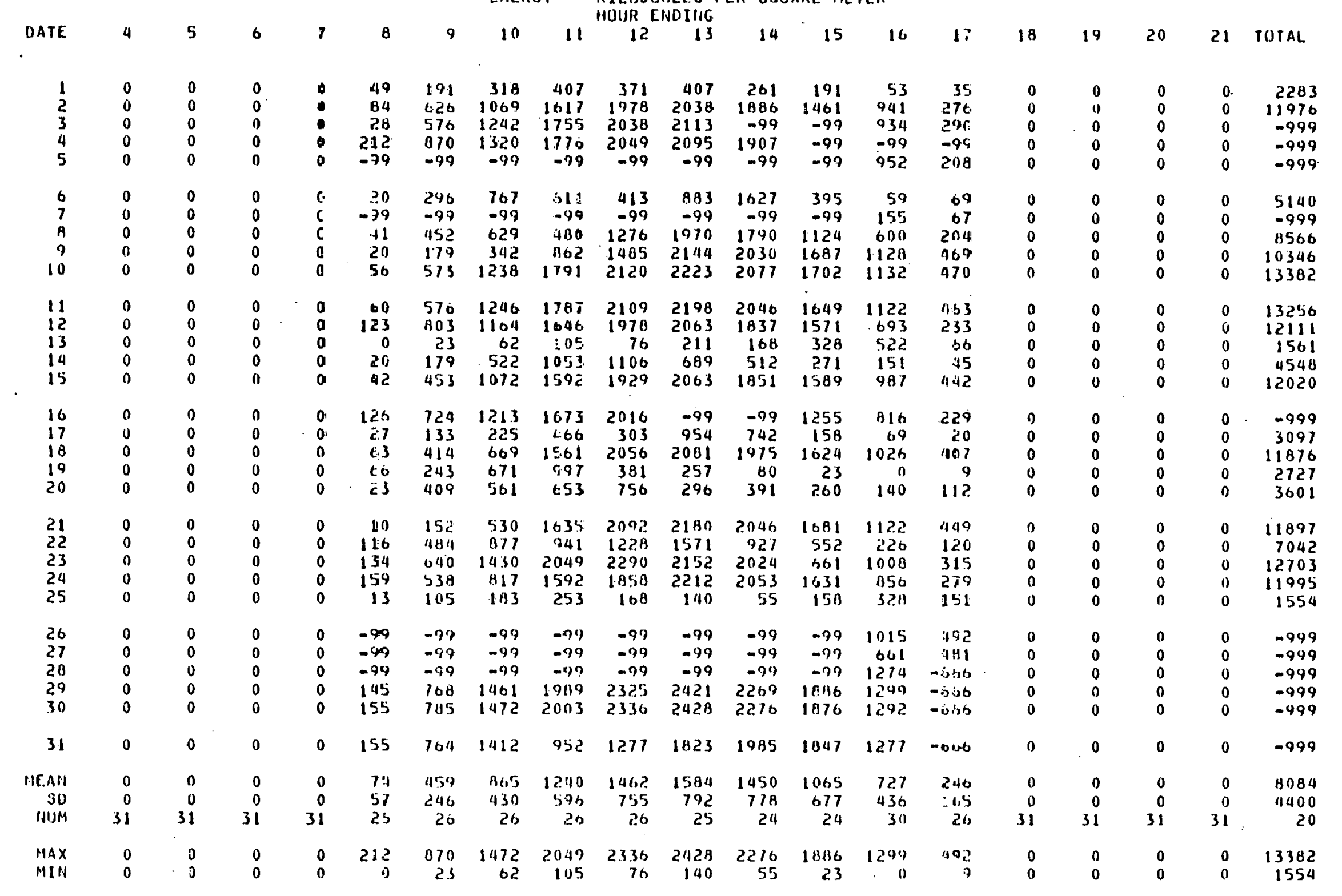


COASTAL - INLAND SOLAH RADIATIUN DIFFERENCE STUDY

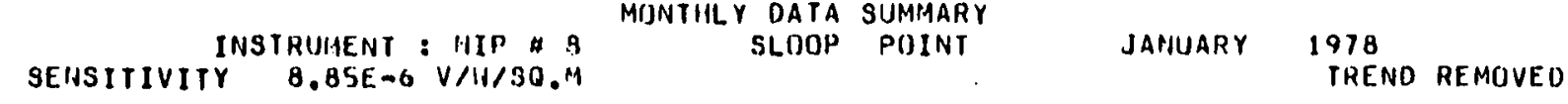

ENERGY KILUJOULES PER SOUARE METER HOUR ENOING

$10 \quad 112^{12} 13$

$14 \quad 15$

$16 \quad 17$

171

$19 \quad 20$

21 TUTAL

$\begin{array}{lll}1 & 0 & 0 \\ 2 & 0 & 0 \\ 3 & 0 & 0 \\ 4 & 0 & 0 \\ 5 & 0 & 0\end{array}$

$\begin{array}{rrrrrrrrrr}0 & 0 & 0 & 0 & 0 & 0 & 0 & 0 & 0 & 0 \\ 485 & 1490 & 1538 & 2210 & 2022 & 3109 & 3161 & 2291 & 2132 & 355 \\ 69 & 2213 & 29135 & 3323 & 3490 & 3526 & 3490 & 3335 & 2957 & 1867 \\ 790 & 2470 & 3080 & 3373 & 3491 & 3532 & 3296 & 2698 & 2421 & 163 \\ 0 & 200 & 1278 & 900 & 501 & 542 & 326 & 1066 & 1453 & 82\end{array}$

$\begin{array}{rrrrrrrrrr}12 & 32 & 223 & 85 & 0 & 415 & 105 & 0 & 0 & 11 \\ 0 & 0 & 0 & 0 & 0 & 0 & 0 & 0 & 0 & 0 \\ 0 & 0 & 0 & 10 & 1332 & 1767 & 994 & 315 & 51 & 0 \\ -99 & -99 & -99 & -99 & -99 & -99 & -79 & -99 & -99 & -99 \\ -99 & -99 & -99 & -97 & -99 & -99 & -99 & -79 & -99 & -99\end{array}$

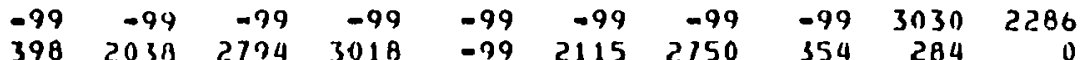

$\begin{array}{llll}0 & 0 & 0 & 0\end{array}$

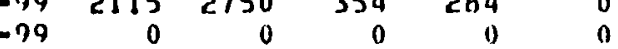

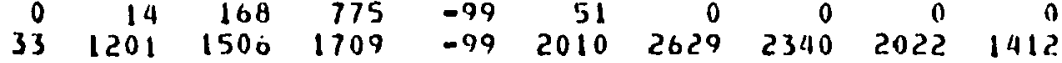

$\begin{array}{rrrrrrrrrr}516 & 2058 & 2811 & 2957 & -99 & 3132 & 2233 & 1090 & 557 & 53 \\ 0 & 0 & 66 & 17 & -99 & 25 & 0 & 0 & 0 & 0 \\ 151 & 1624 & 733 & 1274 & -99 & 2771 & 3190 & 3035 & 2604 & 1538 \\ 0 & 0 & 0 & 0 & -99 & 0 & 0 & 0 & 0 & 0 \\ 0 & 2211 & 17 & 269 & -99 & 0 & 0 & 0 & 0 & 0\end{array}$

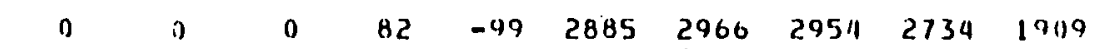

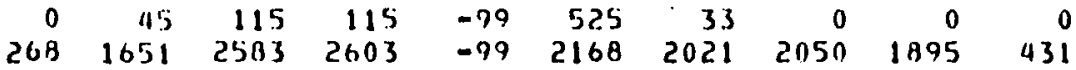

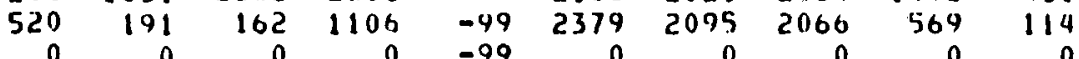

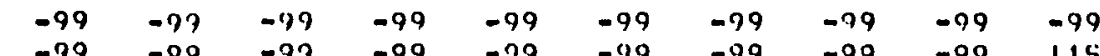

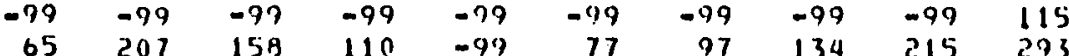

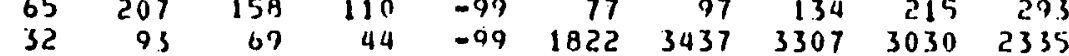

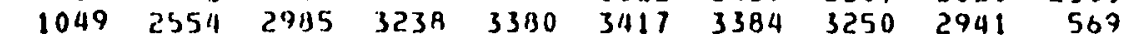

310000

$\begin{array}{llllllllll}909 & 2364 & 106 ? & 0 & 13 & 493 & 2405 & 2828 & 2543 & 1795\end{array}$

MEAN

NIJ)

$\begin{array}{llllllllll}206 & 794 & 935 & 1046 & 1422 & 1413 & 1485 & 1273 & 1164 & 599 \\ 317 & 906 & 1160 & 1259 & 1471 & 1335 & 1424 & 1321 & 1229 & 918\end{array}$

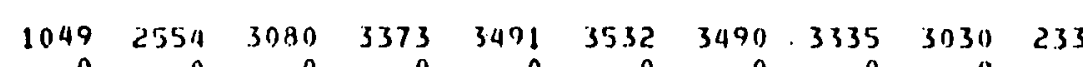

\begin{tabular}{|c|c|c|c|c|}
\hline 0 & 0 & 0 & 0 & 0 \\
\hline 0 & 0 & 0 & 0 & 18793 \\
\hline 0 & 0 & 0 & 0 & 27255 \\
\hline 0 & 0 & 0 & 0 & 26783 \\
\hline 0 & 0 & 0 & 0 & 6348 \\
\hline 0 & 0 & 0 & 0 & 872 \\
\hline 0 & 0 & 0 & 0 & 0 \\
\hline 0 & 0 & 0 & 0 & 4469 \\
\hline 0 & 0 & 0 & 0 & -999 \\
\hline 0 & 0 & 0 & 0 & -999 \\
\hline 0 & 0 & 0 & 0 & -499 \\
\hline 0 & 0 & 0 & 0 & -999 \\
\hline 0 & 0 & 0 & 0 & -499 \\
\hline 0 & 0 & 0 & 0 & -999 \\
\hline 0 & 0 & 0 & 0 & -999 \\
\hline 0 & 0 & 0 & 0 & -999 \\
\hline 0 & 0 & 0 & 0 & -999 \\
\hline 0 & 0 & 0 & 0 & -994 \\
\hline 0 & 0 & 0 & 0 & -499 \\
\hline 0 & 0 & 0 & 0 & -999 \\
\hline n & 0 & 0 & 0 & -999 \\
\hline 0 & 0 & 0 & 0 & -999 \\
\hline 0 & 0 & 0 & 0 & -999 \\
\hline 0 & 0 & 0 & 0 & -999 \\
\hline 0 & 0 & 0 & 0 & -999 \\
\hline 0 & $n$ & 0 & 0 & -999 \\
\hline 0 & 0 & 0 & 0 & -999 \\
\hline 0 & 0 & 0 & 0 & -999 \\
\hline 0 & 0 & 0 & 0 & .999 \\
\hline 0 & 0 & 0 & 0 & 26767 \\
\hline 0 & 0 & 0 & 0 & 14492 \\
\hline 0 & 0 & 0 & 0 & 12577 \\
\hline 0 & 0 & 0 & 0 & 11040 \\
\hline 31 & 31 & 31 & 31 & 10 \\
\hline 0 & 0 & 0 & 0 & 27255 \\
\hline 0 & 0 & 0 & 0 & 0 \\
\hline
\end{tabular}


COASTAL - INLAND SULAR RADIATION DIFFERENCE STUDY

MIJUTHLY DATA SUMMARY

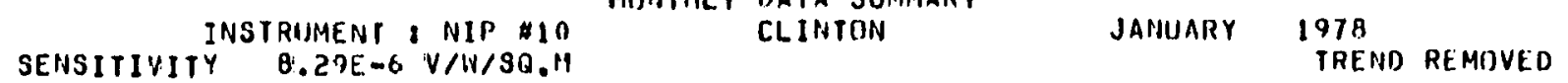

ENERGY KILOJOLLES PER SUUARE METER

H:JUR F.O.IHG

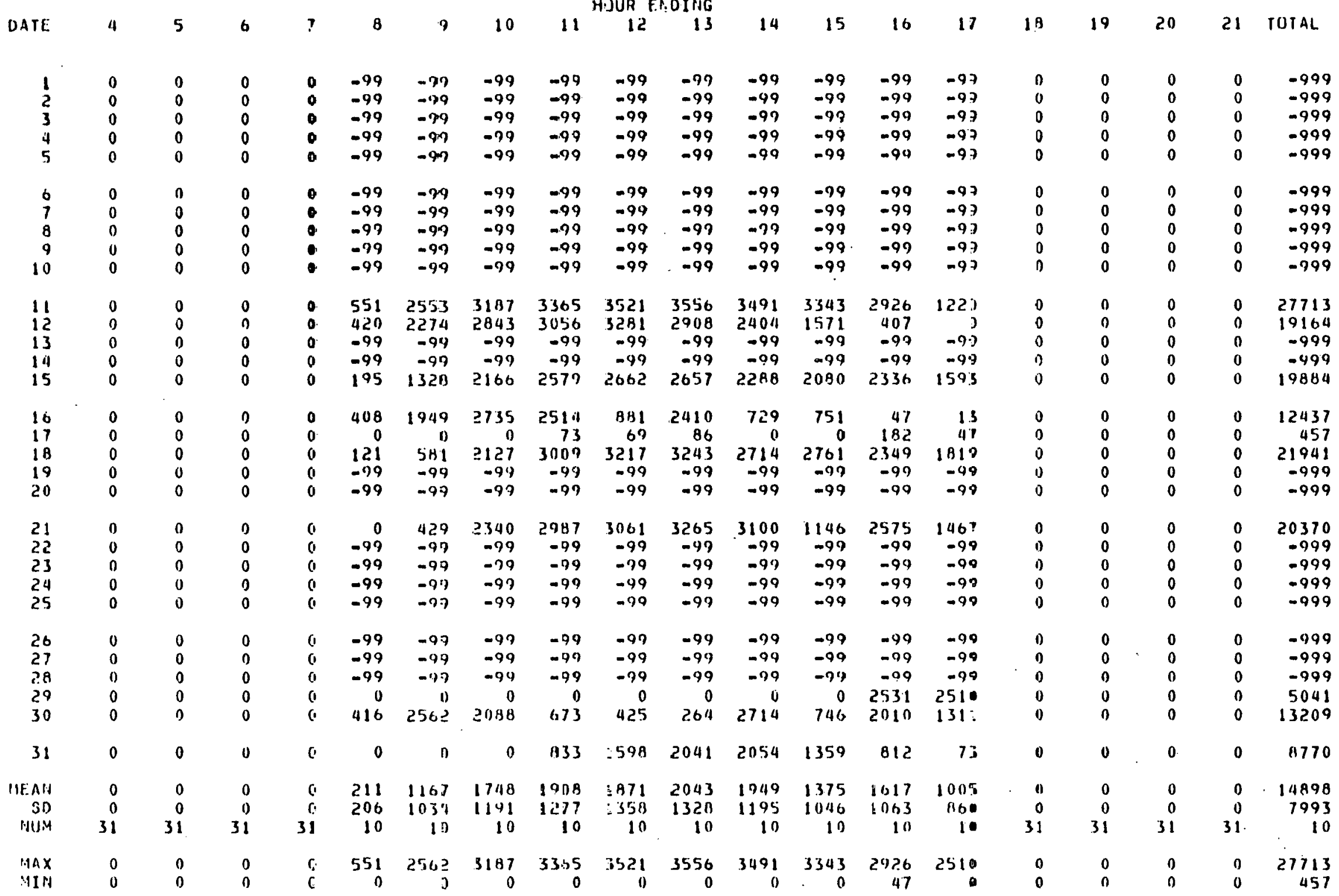


COASTAL - INLAND STLAR RADIATION DIFFERENCE STUDY IIISTRUMENT UV MONTHLY DATA SUMPARY

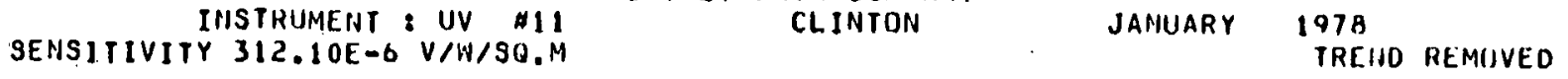

ENERGY KILOJOULES PER SOUARE IAETER

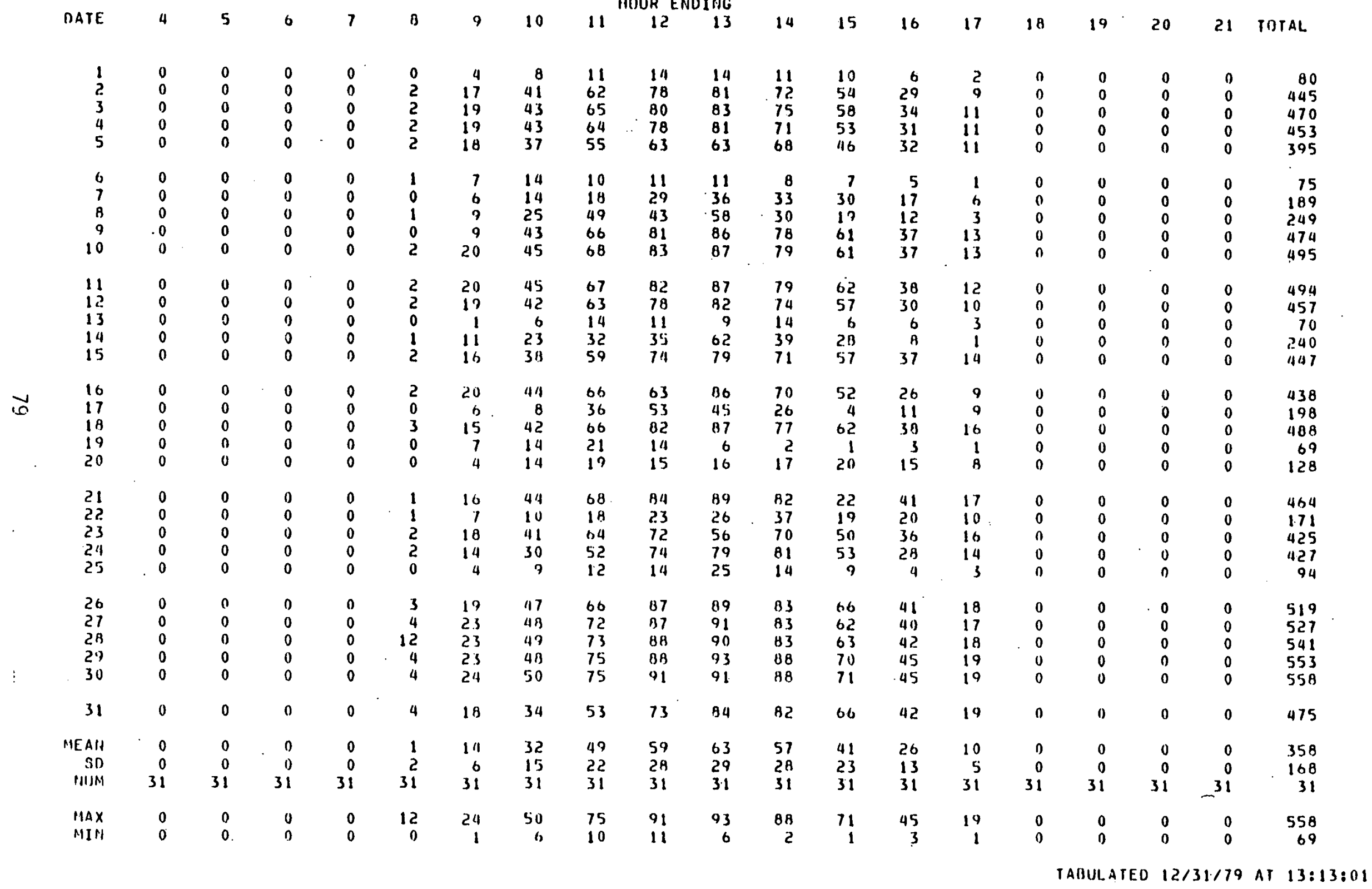


COASTAL - IILAHD SOLAR RADIATION DIFFERENICE STIJOY

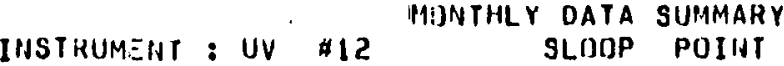

INSTRUMENT: UV H12
SENSITIVITY $154.40 E-6$ V/W/SO.M

JANUARY 1978

ENERGY KILOJOULES PER SQUARE METER

\section{HOUR ENDING}

1
2
3
4
5

$\begin{array}{llll}0 & 0 & 0 & 0 \\ 0 & 0 & 0 & 0 \\ 0 & 0 & 0 & 0 \\ 0 & 0 & 0 & 0 \\ 0 & 0 & 0 & 0\end{array}$

$\begin{array}{ll}1 & \\ 3 & 2 \\ 3 & 2 \\ 3 & 2 \\ 3 & 1\end{array}$

$\begin{array}{ll}9 & 16 \\ 21 & 4 \\ 22 & 4 \\ 23 & 48 \\ 18 & 42\end{array}$

162121

$11 \quad 6.4 \quad 75$

$48 \quad 6.9$

6983

$\begin{array}{rrrr}3 & 14 & 38 & 42 \\ 1 & 7 & 15 & 20 \\ 2 & 19 & 32 & 55\end{array}$

$\begin{array}{lll}-99 & -97 & -99\end{array}$

$-99-9$

-99
-99

$\begin{array}{rr}-99 & -7 \\ 0 & 1 \\ 0 & \\ 0 & \end{array}$

$\begin{array}{rr}-97 & -99 \\ 34 & 59 \\ 3 & \\ 29 & 49\end{array}$

$\stackrel{\infty}{\complement}$

$\begin{array}{ll}16 & 0 \\ 17 & 0 \\ 18 & 0 \\ 19 & 0 \\ 20 & 0\end{array}$

0
0
0
0
0

$\begin{array}{rr}11 & 36 \\ 3 & 18 \\ 11 & 31 \\ 5 & 17 \\ 8 & 14\end{array}$

21

22
23
24

25

26
27

27

29

MEAN

$\begin{array}{rrr}\text { SD } & 0 & 0 \\ \text { HUM } & 31 & 31\end{array}$

$3{ }^{9}$

$\begin{array}{rrrr}0 & 4 & 17 & \\ 0 & 10 & 27 & \\ 0 & 12 & 37 & \\ -99 & -17 & -97 & -97 \\ -99 & -99 & -99 & -97 \\ & & & \\ -99 & -49 & -97 & -97 \\ -99 & -19 & -99 & -97 \\ 0 & 12 & 39 & \\ 0 & 12 & 33 & \\ 6 & 29 & 50 & \end{array}$

$\begin{array}{rr}59 & -99 \\ 8 & -99 \\ 49 & -99\end{array}$

99
-99
-99

13

$14 \quad 15$

16

$\begin{array}{ll}18 & 16 \\ 83 & 76 \\ 86 & 79 \\ 87 & 79 \\ 63 & 49\end{array}$

$\begin{array}{lll}10 & 7 & 2 \\ 56 & 34 & 11 \\ 61 & 37 & 13 \\ 60 & 36 & 13 \\ 39 & 6 & 10\end{array}$

2
11
13
13
10

TREHD REMUVED 
CCIASTAL - INLAHD SOLAR RADIATIUN DIFFERENCE STUDY

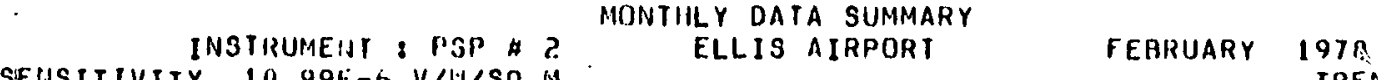

SEIISITIVITY $10.99 E-6$ V/W/SB.M .

ENERGY KILUJUULES PER SIJUARE HETER

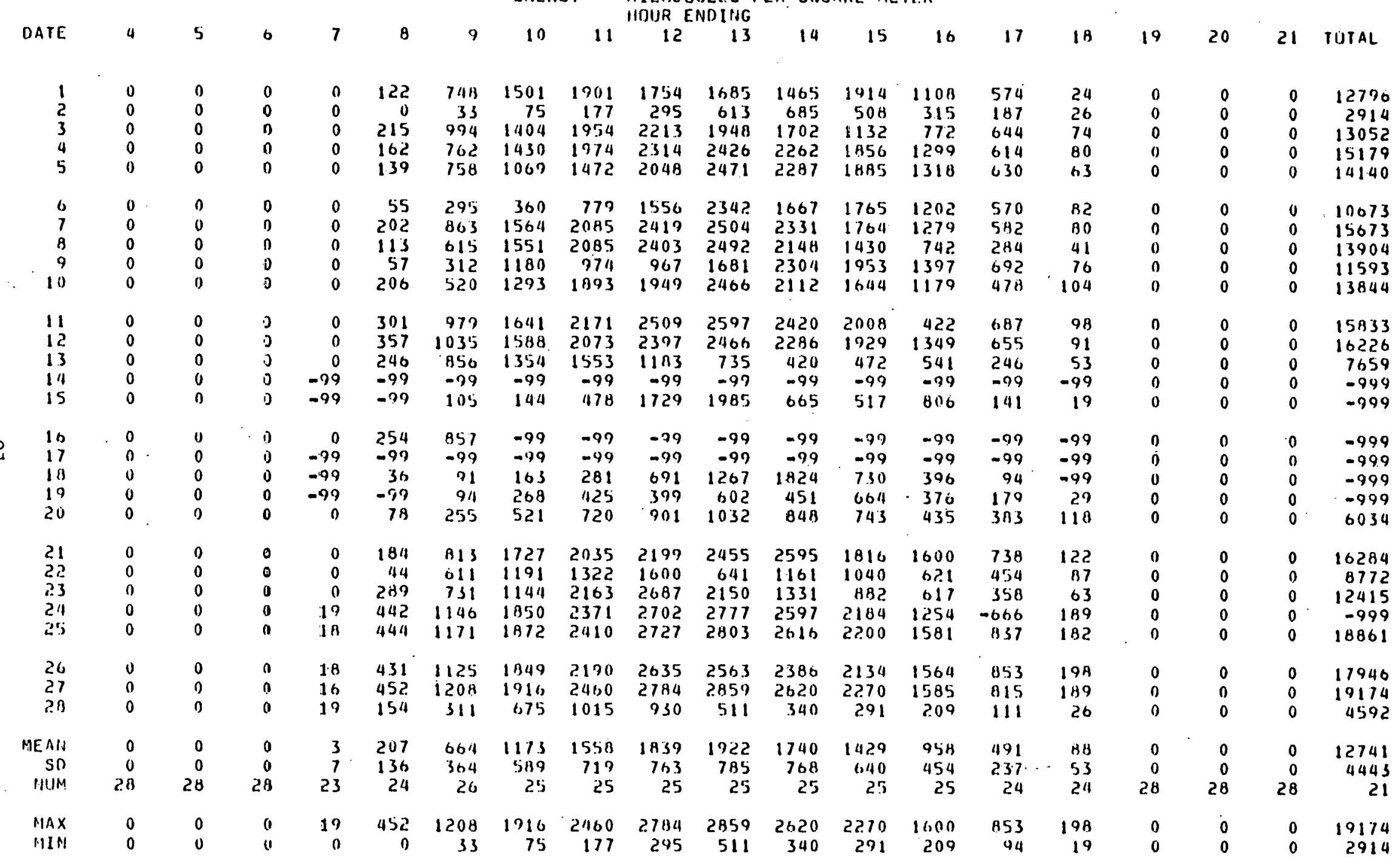


CUASTAL - INLAIJD SOL.AR RADIATION DIFFERENCF. STUIJY STHU.IENT: P3P \#3 MOHTHLY DATA SUMMARY

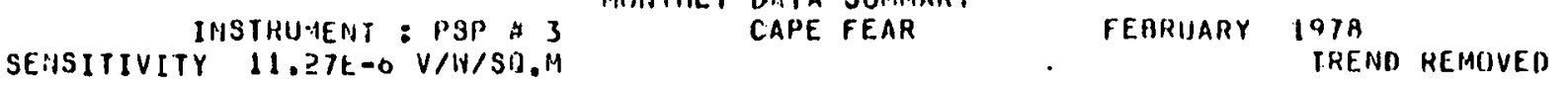

ENERGY KILUJUULES PER SQUARE METER

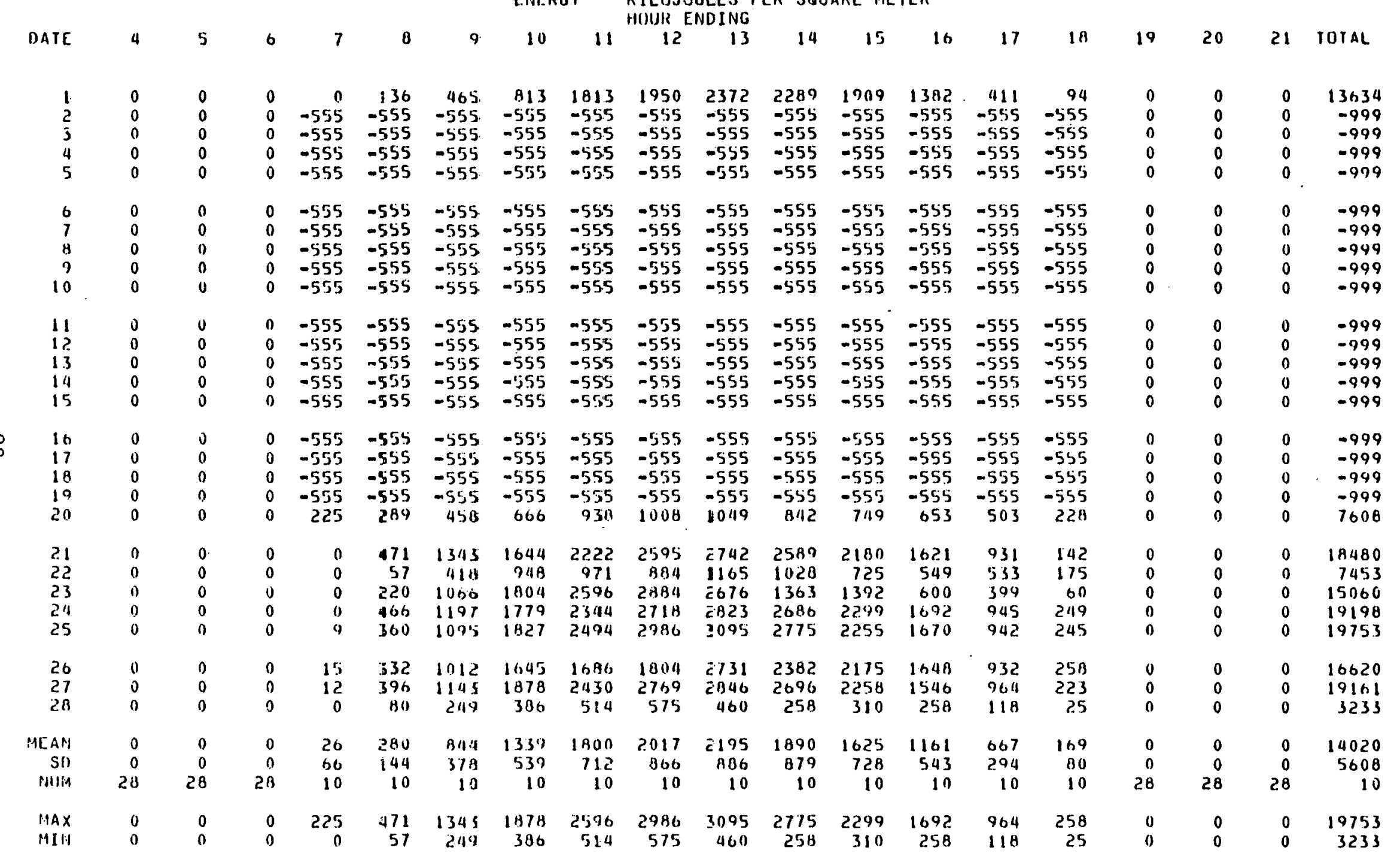

TABULATED $12 / 31 / 79$ AT $13: 12: 34$ 
COASTAL - IHLAHD SILAR RADIATION DIFFERENCE. STUDY MUNTHLY DATA SUMMARY

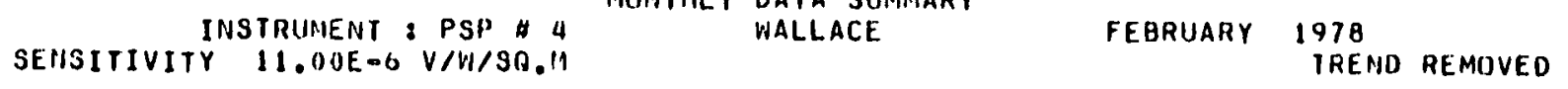

EHERGY KILUJDULES PER SQUARE METER

III)UR ENDING

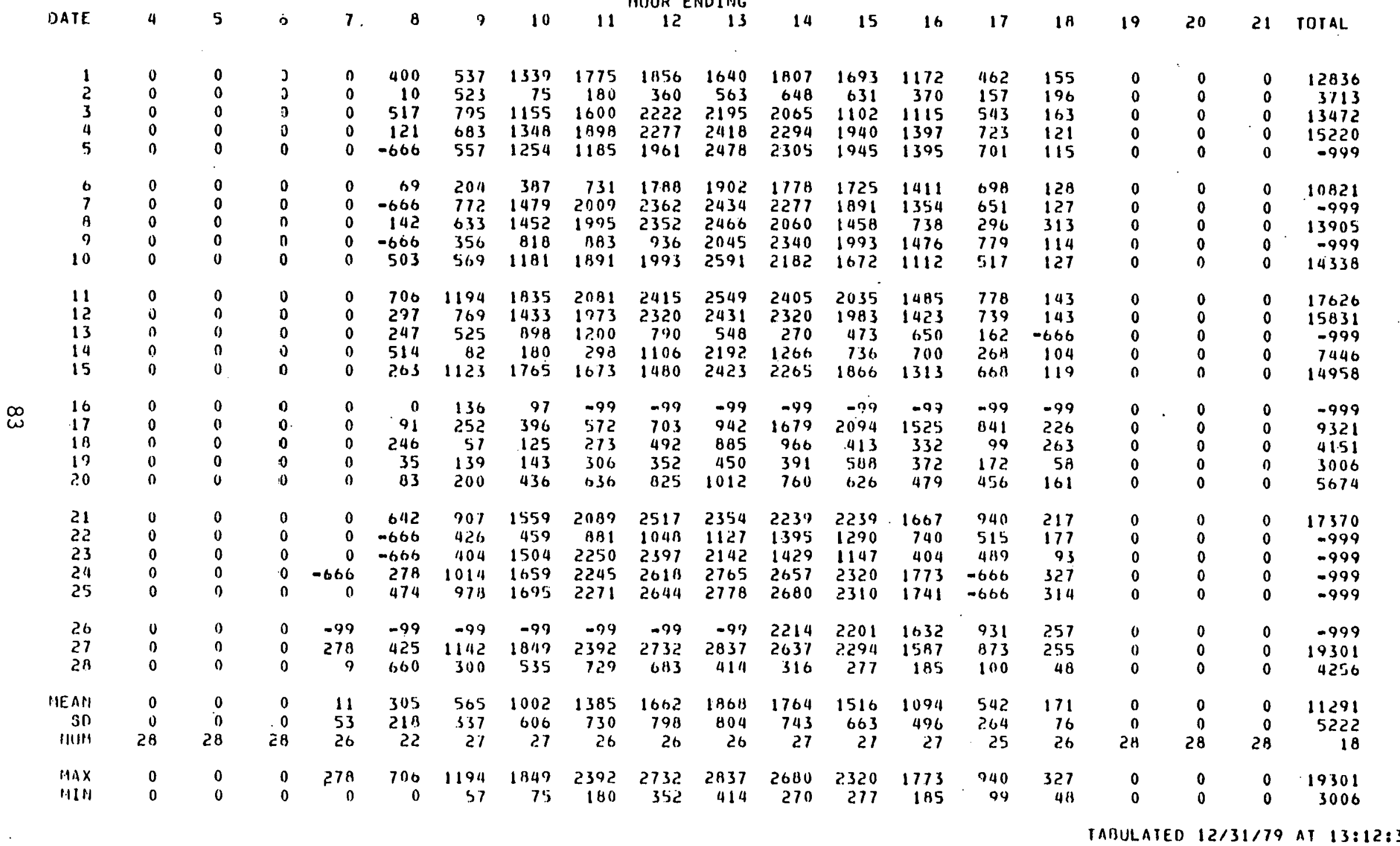


CIASTAL - ITL.AND SILLAR RAOIATIUN DIFFERCMCE STIUY MONTHLY DATA SUMMARY

IHSTRUMENT: PSP $\$ 5$
SENSITIVITY $9.4 \mathrm{HE}-6 \mathrm{V/W/SH.M}$ SI.OUP POINT

FEGRUARY 19 TO

ENERGY KILUJOULES PER SQUARE METER

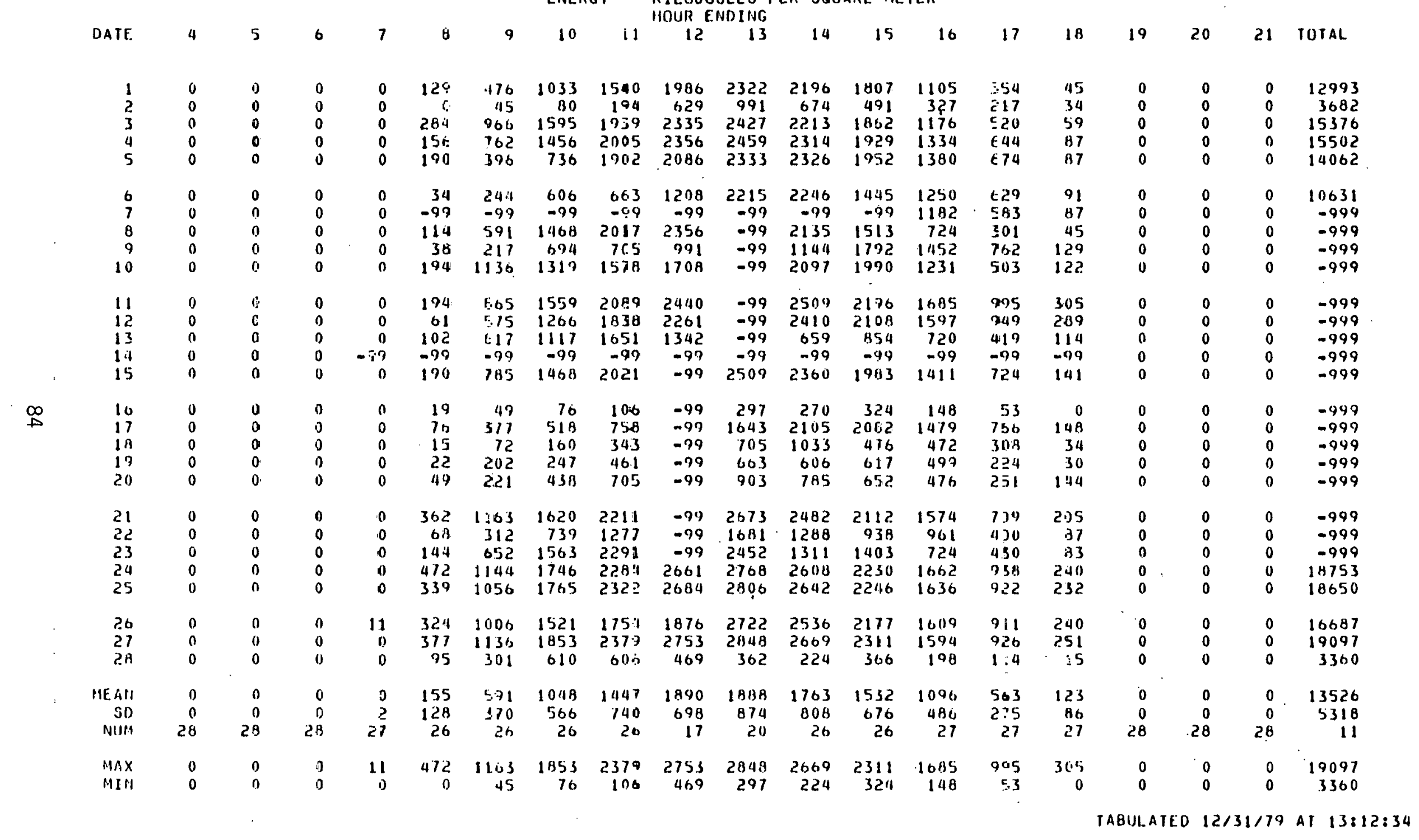


COASTAL - INLAND SOLAR RADIATIUN DIFFERENICE STIJNY HUHTHLY DATA SUMMIARY

IMSTRUMEIIT : PSP \# 6
SENSITIVITY $11.00 E-6$ VIVISO.M

EHERGY KILOJUULF.S PER STUARE METER

\begin{tabular}{|c|c|c|c|c|c|c|c|c|c|c|c|c|c|c|c|c|c|c|c|}
\hline \multirow[b]{2}{*}{ DATE } & \multirow[b]{2}{*}{4} & \multirow[b]{2}{*}{5} & \multirow[b]{2}{*}{6} & \multirow[b]{2}{*}{7} & \multirow[b]{2}{*}{ A } & \multirow[b]{2}{*}{9} & \multirow[b]{2}{*}{10} & \multicolumn{3}{|c|}{ HOUR ENDIHG } & \multicolumn{3}{|c|}{ 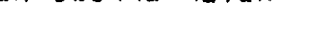 } & \multirow[b]{2}{*}{17} & \multirow[b]{2}{*}{10} & \multirow[b]{2}{*}{19} & \multirow[b]{2}{*}{20} & \multirow[b]{2}{*}{21} & \multirow[b]{2}{*}{ TOTAL. } \\
\hline & & & & & & & & 11 & 12 & 13 & 14 & 15 & 16 & & & & & & \\
\hline $\begin{array}{l}1 \\
? \\
3 \\
4 \\
5\end{array}$ & $\begin{array}{l}0 \\
0 \\
0 \\
0 \\
0\end{array}$ & $\begin{array}{l}n \\
0 \\
0 \\
0 \\
0\end{array}$ & $\begin{array}{l}0 \\
0 \\
0 \\
0 \\
0\end{array}$ & $\begin{array}{l}0 \\
0 \\
0 \\
0 \\
0\end{array}$ & $\begin{array}{r}147 \\
11 \\
147 \\
150 \\
98\end{array}$ & $\begin{array}{r}65 B \\
50 \\
602 \\
700 \\
507\end{array}$ & $\begin{array}{r}1420 \\
132 \\
1040 \\
1364 \\
736\end{array}$ & $\begin{array}{r}1409 \\
240 \\
1616 \\
1911 \\
1532\end{array}$ & $\begin{array}{r}1407 \\
597 \\
1983 \\
2271 \\
1836\end{array}$ & $\begin{array}{r}1463 \\
940 \\
2313 \\
2392 \\
2268\end{array}$ & $\begin{array}{r}1567 \\
574 \\
1655 \\
2271 \\
1973\end{array}$ & $\begin{array}{r}1656 \\
407 \\
1276 \\
1904 \\
1921\end{array}$ & $\begin{array}{r}1096 \\
420 \\
1305 \\
1354 \\
1381\end{array}$ & $\begin{array}{l}599 \\
384 \\
638 \\
674 \\
609\end{array}$ & $\begin{array}{r}72 \\
66 \\
91 \\
104 \\
92\end{array}$ & $\begin{array}{l}0 \\
0 \\
0 \\
0 \\
0\end{array}$ & $\begin{array}{l}0 \\
0 \\
0 \\
0 \\
0\end{array}$ & $\begin{array}{l}0 \\
0 \\
0 \\
0 \\
0\end{array}$ & $\begin{array}{r}11489 \\
3821 \\
12606 \\
15075 \\
12953\end{array}$ \\
\hline $\begin{array}{r}6 \\
7 \\
8 \\
9 \\
10\end{array}$ & $\begin{array}{l}0 \\
0 \\
0 \\
0 \\
0\end{array}$ & $\begin{array}{l}0 \\
0 \\
0 \\
0 \\
0\end{array}$ & $\begin{array}{l}0 \\
0 \\
0 \\
0 \\
0\end{array}$ & $\begin{array}{l}0 \\
0 \\
0 \\
0 \\
0\end{array}$ & $\begin{array}{r}39 \\
159 \\
157 \\
89 \\
121\end{array}$ & $\begin{array}{l}186 \\
800 \\
759 \\
445 \\
445\end{array}$ & $\begin{array}{r}448 \\
1491 \\
1.453 \\
744 \\
1090\end{array}$ & $\begin{array}{r}1076 \\
2018 \\
2019 \\
937 \\
1456\end{array}$ & $\begin{array}{l}2026 \\
2342 \\
2359 \\
2046 \\
1466\end{array}$ & $\begin{array}{l}2261 \\
2430 \\
2444 \\
2495 \\
1619\end{array}$ & $\begin{array}{l}1963 \\
2322 \\
2235 \\
2295 \\
1006\end{array}$ & $\begin{array}{l}1728 \\
1972 \\
1332 \\
1942 \\
1921\end{array}$ & $\begin{array}{r}1479 \\
1380 \\
756 \\
1405 \\
1018\end{array}$ & $\begin{array}{l}710 \\
653 \\
317 \\
695 \\
517\end{array}$ & $\begin{array}{r}121 \\
10 n \\
52 \\
50 \\
144\end{array}$ & $\begin{array}{l}0 \\
0 \\
0 \\
0 \\
0\end{array}$ & $\begin{array}{l}0 \\
0 \\
0 \\
0 \\
0\end{array}$ & $\begin{array}{l}0 \\
0 \\
0 \\
0 \\
0\end{array}$ & $\begin{array}{l}12037 \\
15673 \\
13883 \\
13183 \\
11803\end{array}$ \\
\hline $\begin{array}{l}11 \\
12 \\
13 \\
14 \\
15\end{array}$ & $\begin{array}{l}0 \\
0 \\
0 \\
0 \\
0\end{array}$ & $\begin{array}{l}0 \\
0 \\
0 \\
0 \\
0\end{array}$ & $\begin{array}{l}0 \\
0 \\
0 \\
0 \\
0\end{array}$ & $\begin{array}{l}0 \\
0 \\
0 \\
0 \\
9\end{array}$ & $\begin{array}{r}294 \\
224 \\
189 \\
16 \\
133\end{array}$ & $\begin{array}{r}886 \\
794 \\
614 \\
72 \\
427\end{array}$ & $\begin{array}{r}1492 \\
1452 \\
912 \\
121 \\
1331\end{array}$ & $\begin{array}{r}2035 \\
1988 \\
804 \\
713 \\
1960\end{array}$ & $\begin{array}{l}2411 \\
2322 \\
666 \\
1865 \\
2316\end{array}$ & $\begin{array}{r}2510 \\
2440 \\
447 \\
2248 \\
2427\end{array}$ & $\begin{array}{r}2356 \\
2319 \\
300 \\
1011 \\
2293\end{array}$ & $\begin{array}{r}2000 \\
1949 \\
859 \\
596 \\
1923\end{array}$ & $\begin{array}{r}1420 \\
1373 \\
280 \\
475 \\
1360\end{array}$ & $\begin{array}{r}749 \\
692 \\
45 \\
200 \\
6136\end{array}$ & $\begin{array}{r}127 \\
123 \\
22 \\
39 \\
133\end{array}$ & $\begin{array}{l}0 \\
0 \\
0 \\
0 \\
0\end{array}$ & $\begin{array}{l}0 \\
0 \\
0 \\
0 \\
0\end{array}$ & $\begin{array}{l}0 \\
0 \\
0 \\
0 \\
0\end{array}$ & $\begin{array}{r}16286 \\
15676 \\
5130 \\
7356 \\
15004\end{array}$ \\
\hline $\begin{array}{l}16 \\
17 \\
10 \\
19 \\
20\end{array}$ & $\begin{array}{l}0 \\
0 \\
0 \\
0 \\
0\end{array}$ & $\begin{array}{l}0 \\
0 \\
0 \\
0 \\
0\end{array}$ & $\begin{array}{l}0 \\
0 \\
0 \\
0 \\
0\end{array}$ & $\begin{array}{l}0 \\
0 \\
0 \\
0 \\
0\end{array}$ & $\begin{array}{l}21 \\
84 \\
21 \\
51 \\
78\end{array}$ & $\begin{array}{l}60 \\
212 \\
103 \\
169 \\
294\end{array}$ & $\begin{array}{l}109 \\
3199 \\
159 \\
264 \\
589\end{array}$ & $\begin{array}{l}221 \\
540 \\
281 \\
195 \\
769\end{array}$ & $\begin{array}{l}558 \\
827 \\
414 \\
532 \\
990\end{array}$ & $\begin{array}{r}381 \\
1059 \\
440 \\
431 \\
1175\end{array}$ & $\begin{array}{r}417 \\
1249 \\
506 \\
451 \\
1142\end{array}$ & $\begin{array}{r}240 \\
1897 \\
316 \\
316 \\
968\end{array}$ & $\begin{array}{r}90 \\
1357 \\
142 \\
303 \\
658\end{array}$ & $\begin{array}{r}34 \\
693 \\
64 \\
97 \\
487\end{array}$ & $\begin{array}{r}14 \\
153 \\
31 \\
25 \\
80\end{array}$ & $\begin{array}{l}0 \\
0 \\
0 \\
0 \\
0\end{array}$ & $\begin{array}{l}0 \\
0 \\
0 \\
0 \\
0\end{array}$ & $\begin{array}{l}0 \\
0 \\
0 \\
0 \\
0\end{array}$ & $\begin{array}{l}2145 \\
8426 \\
2482 \\
2834 \\
7246\end{array}$ \\
\hline $\begin{array}{l}21 \\
22 \\
23 \\
211 \\
25\end{array}$ & $\begin{array}{l}0 \\
0 \\
0 \\
0 \\
0\end{array}$ & $\begin{array}{l}0 \\
0 \\
0 \\
0 \\
0\end{array}$ & $\begin{array}{l}0 \\
0 \\
0 \\
0 \\
0\end{array}$ & $\begin{array}{l}0 \\
0 \\
0 \\
0 \\
0\end{array}$ & $\begin{array}{l}246 \\
119 \\
183 \\
368 \\
344\end{array}$ & $\begin{array}{r}1042 \\
623 \\
553 \\
1062 \\
1074\end{array}$ & $\begin{array}{r}1588 \\
6913 \\
1436 \\
1367 \\
1768\end{array}$ & $\begin{array}{r}2141 \\
999 \\
2300 \\
2316 \\
2311\end{array}$ & $\begin{array}{l}2449 \\
1055 \\
2549 \\
2649 \\
2661\end{array}$ & $\begin{array}{l}2403 \\
1611 \\
2500 \\
2774 \\
2773\end{array}$ & $\begin{array}{l}2498 \\
1353 \\
2225 \\
2640 \\
2629\end{array}$ & $\begin{array}{r}2154 \\
963 \\
1024 \\
2250 \\
2233\end{array}$ & $\begin{array}{l}1441 \\
1032 \\
733 \\
16613 \\
16117\end{array}$ & $\begin{array}{l}813 \\
525 \\
726 \\
944 \\
914\end{array}$ & $\begin{array}{l}132 \\
105 \\
104 \\
241 \\
240\end{array}$ & $\begin{array}{l}0 \\
0 \\
0 \\
0 \\
0\end{array}$ & $\begin{array}{l}0 \\
0 \\
0 \\
0 \\
0\end{array}$ & $\begin{array}{l}0 \\
0 \\
0 \\
0 \\
0\end{array}$ & $\begin{array}{r}16907 \\
9143 \\
14333 \\
18779 \\
18594\end{array}$ \\
\hline $\begin{array}{l}26 \\
27 \\
211\end{array}$ & $\begin{array}{l}0 \\
0 \\
0\end{array}$ & $\begin{array}{l}0 \\
0 \\
0\end{array}$ & $\begin{array}{l}n \\
0 \\
0\end{array}$ & $\begin{array}{l}0 \\
B \\
9\end{array}$ & $\begin{array}{r}349 \\
305 \\
84\end{array}$ & $\begin{array}{r}1046 \\
1121 \\
359\end{array}$ & $\begin{array}{r}2001 \\
1828 \\
591\end{array}$ & $\begin{array}{r}2334 \\
2391 \\
76 ?\end{array}$ & $\begin{array}{r}2224 \\
2734 \\
729\end{array}$ & $\begin{array}{r}1880 \\
2836 \\
395\end{array}$ & $\begin{array}{r}2417 \\
2640 \\
444\end{array}$ & $\begin{array}{r}2217 \\
2257 \\
290\end{array}$ & $\begin{array}{r}165 B \\
1628 \\
186\end{array}$ & $\begin{array}{l}967 \\
912 \\
110\end{array}$ & $\begin{array}{r}273 \\
224 \\
32\end{array}$ & $\begin{array}{l}n \\
0 \\
0\end{array}$ & $\begin{array}{l}0 \\
0 \\
0\end{array}$ & $\begin{array}{l}0 \\
0 \\
0\end{array}$ & $\begin{array}{r}17370 \\
18944 \\
3991\end{array}$ \\
\hline $\begin{array}{r}\text { MEAN } \\
30 \\
\text { PHJ" }\end{array}$ & $\begin{array}{r}0 \\
0 \\
20\end{array}$ & $\begin{array}{r}0 \\
0 \\
2 B\end{array}$ & $\begin{array}{r}0 \\
0 \\
28\end{array}$ & $\begin{array}{r}0 \\
2 \\
28\end{array}$ & $\begin{array}{r}152 \\
107 \\
20\end{array}$ & $\begin{array}{r}560 \\
333 \\
28\end{array}$ & $\begin{array}{r}016 \\
589 \\
28\end{array}$ & $\begin{array}{r}1103 \\
739 \\
28\end{array}$ & $\begin{array}{r}1724 \\
769 \\
28\end{array}$ & $\begin{array}{r}1841 \\
823 \\
23\end{array}$ & $\begin{array}{r}1698 \\
785 \\
2.8\end{array}$ & $\begin{array}{r}1447 \\
694 \\
2 B\end{array}$ & $\begin{array}{r}1037 \\
514 \\
28\end{array}$ & $\begin{array}{r}551 \\
283 \\
28\end{array}$ & $\begin{array}{r}109 \\
69 \\
28\end{array}$ & $\begin{array}{r}0 \\
0 \\
2 H\end{array}$ & $\begin{array}{r}0 \\
0 \\
28\end{array}$ & $\begin{array}{r}0 \\
0 \\
28\end{array}$ & $\begin{array}{r}11544 \\
5263 \\
28\end{array}$ \\
\hline $\begin{array}{l}\ln x \\
\operatorname{sil} 11\end{array}$ & $\begin{array}{l}0 \\
0\end{array}$ & $\begin{array}{l}0 \\
0\end{array}$ & $\begin{array}{l}0 \\
0\end{array}$ & $\begin{array}{l}? \\
0\end{array}$ & $\begin{array}{r}368 \\
11\end{array}$ & $\begin{array}{r}1121 \\
50\end{array}$ & $\begin{array}{r}2001 \\
109\end{array}$ & $\begin{array}{r}2391 \\
195\end{array}$ & $\begin{array}{r}2734 \\
414\end{array}$ & $\begin{array}{r}2836 \\
381\end{array}$ & $\begin{array}{r}2640 \\
300\end{array}$ & $\begin{array}{r}2257 \\
240\end{array}$ & $\begin{array}{r}1668 \\
90\end{array}$ & $\begin{array}{r}967 \\
34\end{array}$ & $\begin{array}{r}273 \\
14\end{array}$ & $\begin{array}{l}0 \\
0\end{array}$ & $\begin{array}{l}0 \\
0\end{array}$ & $\begin{array}{l}0 \\
0\end{array}$ & $\begin{array}{r}18944 \\
2145\end{array}$ \\
\hline
\end{tabular}


CINSTAL - INLAJD SOLAR RADIATIUN DIFFEREHCE STHDY WISTK JMENT: PSP \# 7 . WTHLY DATA SUMMARY
ONSLOW BEACH

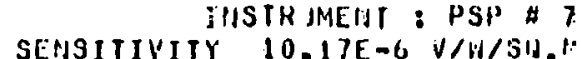

FF.BRUARY 1978

ENERGY KILOJUULES PER SOUARE METER

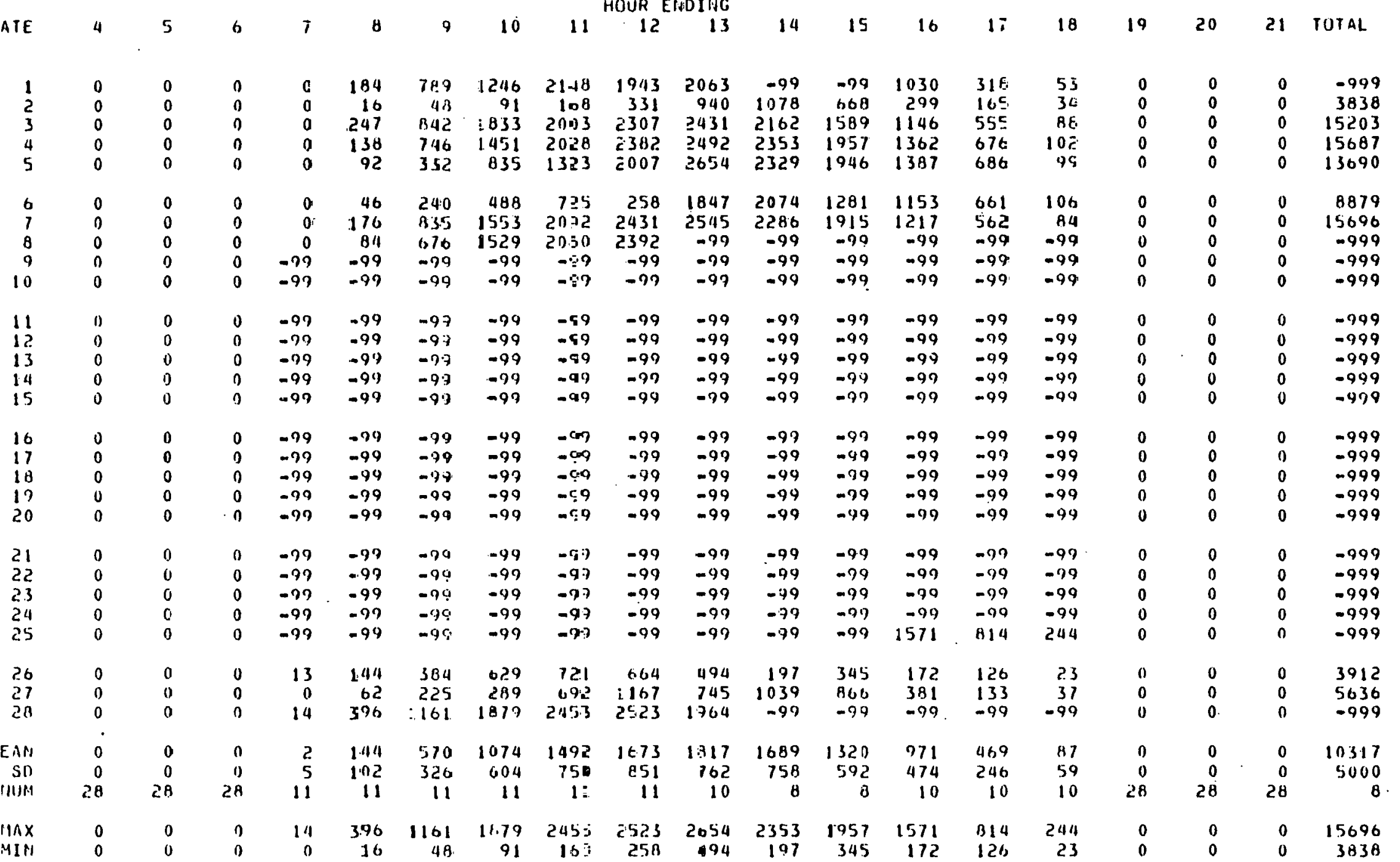




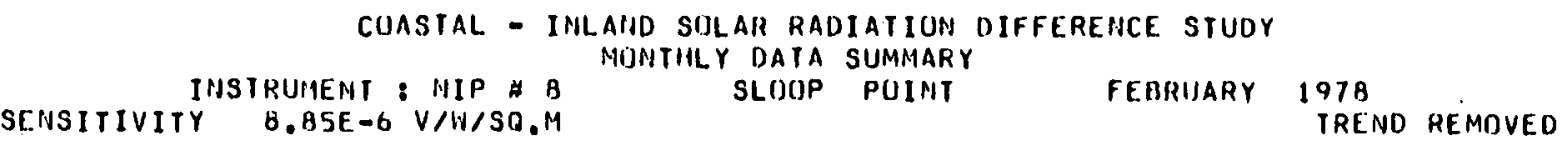

ENF.RGY KILOJOULES PER SOUARE. METER HOUUH ENDING

DATE

$\begin{array}{lll}10 & 11 & 12 \\ & & 13\end{array}$
$14 \quad 15$

16

17

18

$17 \quad 20$

21 TOTAL

1
2
3
4
5
6
7
4
9
10
11
12
13
14
15 
CUASTAL - INLAND SULAR RAOIATION DIFFERENICE STHOY MONTHLY DATA SUMRIARY

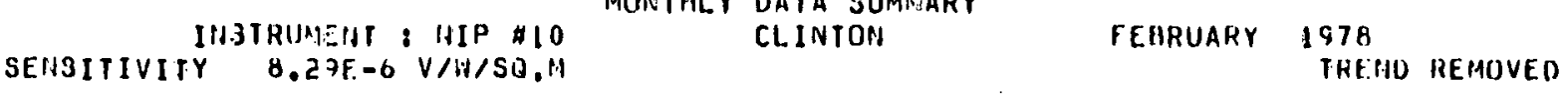

ENERGY KILUJOULES PER ȘMUARE METER

HOUR ENDING

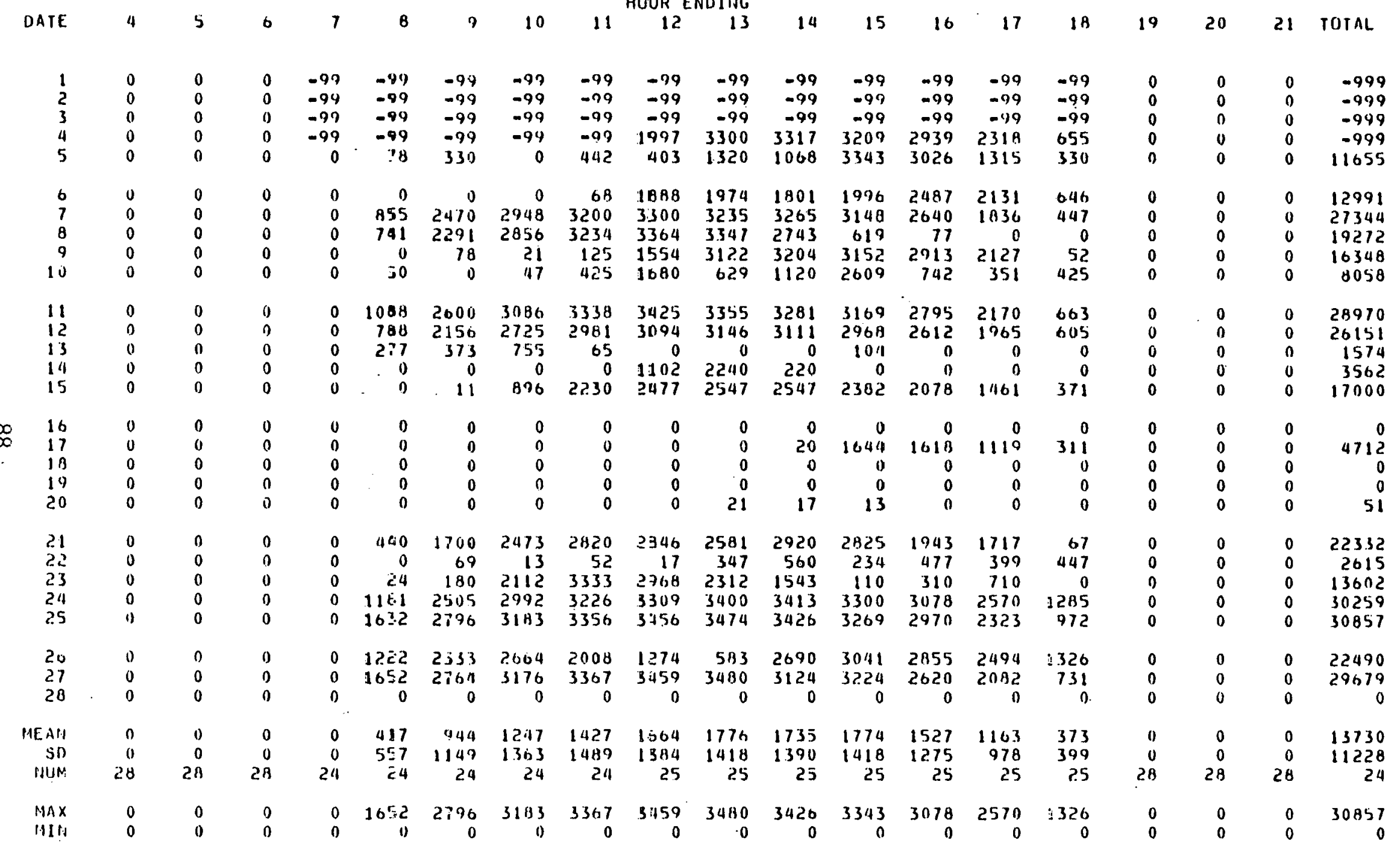

FABULATED $12 / 31 / 79$ AT 13:12:34. 
COASTAL - IHLAPID SIJLAR RADIATIOH DIFFEPEIICE STUDY MUNTHLY DATA SUIMMARY

INSTRUMENI: UV NII
SEHSITIVITY $312,10 E-6$ V/W/SG,

CLINTON

FEBRUARY 1978

TREND REMOVED

EIIERGY KILOJOULFS PER SOUJARE METER

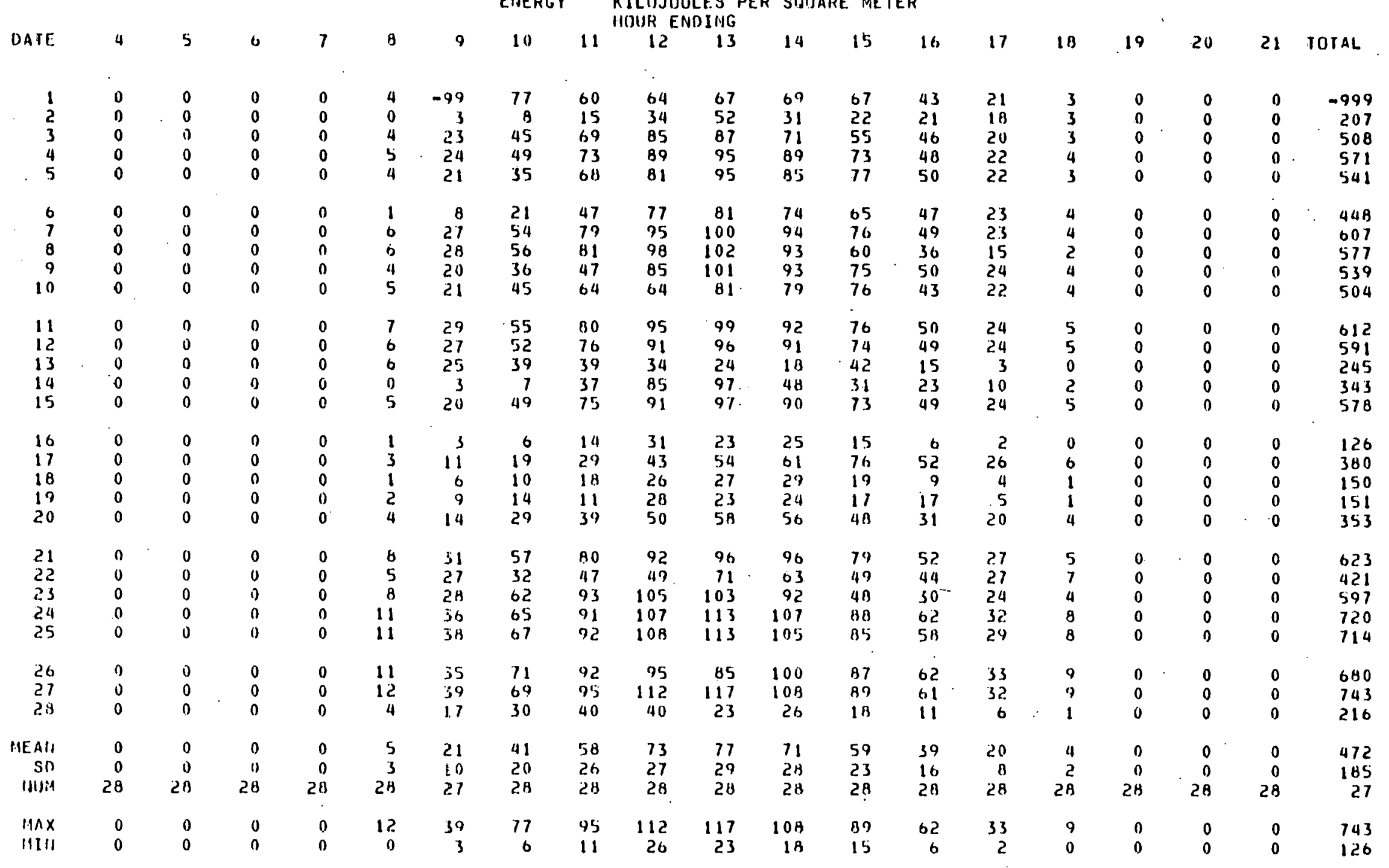


CGASTAL. - (NLAIHI) SILLR RADIATION DIFFERENCE 9TUDY MONTHLY DAIA SLMMAKY

IHSTRIJMET: UV HE TLEOP POIHI FERRUARY 1978

SEINSITIVIT'Y $154.40 E-6$ V/H/SO.

ENERGY KILOJOULES PEH SQUART. HETER

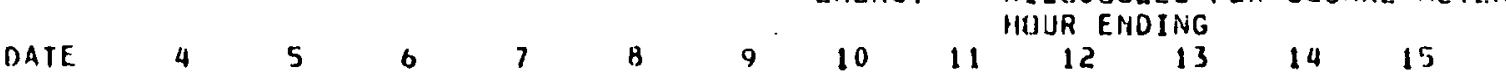

$\begin{array}{lll}1 & 0 & 0 \\ 2 & 0 & 0 \\ 3 & 0 & 0 \\ 4 & 0 & 0 \\ 5 & 0 & 0 \\ 0 & 0 & \\ 6 & 0 & 0 \\ 7 & 0 & 0 \\ 0 & 0 & 0 \\ 7 & 0 & 0 \\ 0 & 0 & 0 \\ 1 & 0 & \\ 1 & 0 & 0 \\ 2 & 0 & 0 \\ 3 & 0 & 0 \\ 4 & 0 & 0 \\ 5 & 0 & 0\end{array}$

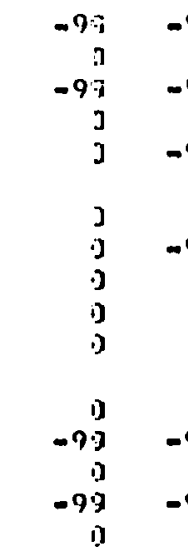

$-99-59-99$

90 
COASTAL - INLAIID SOLAR RADIATION DIFFEREHICE STUDY MUHTHLY DATA SUMHARY

INSTHUMENT \& PSP 2
SENSITIVITY $10.99 E-6$ V/W/SD.

EHERGY KILUJOULES PER SUIUARE METER

\begin{tabular}{|c|c|c|c|c|c|c|c|c|c|c|c|c|c|c|c|c|c|c|c|c|}
\hline & \multicolumn{9}{|c|}{ EHERGY } & \multicolumn{11}{|c|}{$\begin{array}{l}\text { KILUJOULES PER SWUARE METER } \\
\text { HOUR ENDING }\end{array}$} \\
\hline & DATE & 4 & 5 & 0 & 7 & 8 & $?$ & 10 & 11 & 12 & 13 & 14 & 15 & 16 & 17 & 18 & 19 & 20 & 21 & TOTAL \\
\hline & $\begin{array}{l}1 \\
2 \\
3 \\
4 \\
5\end{array}$ & $\begin{array}{l}0 \\
0 \\
0 \\
0 \\
0\end{array}$ & $\begin{array}{l}0 \\
0 \\
0 \\
0 \\
0\end{array}$ & $\begin{array}{l}0 \\
0 \\
0 \\
0 \\
0\end{array}$ & $\begin{array}{r}0 \\
-99 \\
-99 \\
-99 \\
112\end{array}$ & $\begin{array}{r}45 \\
-99 \\
30 \\
-99 \\
592\end{array}$ & $\begin{array}{r}183 \\
1231 \\
66 \\
1077 \\
11005\end{array}$ & $\begin{array}{r}274 \\
1948 \\
79 \\
940 \\
2125\end{array}$ & $\begin{array}{r}386 \\
2466 \\
70 \\
1811 \\
2606\end{array}$ & $\begin{array}{r}-99 \\
2413 \\
151 \\
1343 \\
-99\end{array}$ & $\begin{array}{r}-49 \\
1411 \\
381 \\
1700 \\
-99\end{array}$ & $\begin{array}{r}-99 \\
1378 \\
509 \\
1768 \\
2862\end{array}$ & $\begin{array}{r}-99 \\
1041 \\
332 \\
1667 \\
2424\end{array}$ & $\begin{array}{r}-99 \\
942 \\
502 \\
1329 \\
1814\end{array}$ & $\begin{array}{r}-99 \\
382 \\
361 \\
835 \\
1031\end{array}$ & $\begin{array}{r}-99 \\
61 \\
115 \\
216 \\
278\end{array}$ & $\begin{array}{l}0 \\
0 \\
0 \\
0 \\
0\end{array}$ & $\begin{array}{l}0 \\
0 \\
0 \\
0 \\
0\end{array}$ & $\begin{array}{l}0 \\
0 \\
0 \\
0 \\
0\end{array}$ & $\begin{array}{l}-999 \\
-999 \\
-999 \\
-999 \\
-999\end{array}$ \\
\hline & $\begin{array}{r}6 \\
7 \\
8 \\
9 \\
10\end{array}$ & $\begin{array}{l}0 \\
0 \\
0 \\
0 \\
0\end{array}$ & $\begin{array}{l}0 \\
0 \\
0 \\
0 \\
0\end{array}$ & $\begin{array}{l}0 \\
0 \\
0 \\
0 \\
0\end{array}$ & $\begin{array}{r}-99 \\
33 \\
13 \\
44 \\
52\end{array}$ & $\begin{array}{r}-99 \\
39 ? \\
72 \\
71 \\
98\end{array}$ & $\begin{array}{r}1269 \\
1214 \\
173 \\
152 \\
203\end{array}$ & $\begin{array}{r}1168 \\
1980 \\
196 \\
437 \\
521\end{array}$ & $\begin{array}{r}2210 \\
2455 \\
-99 \\
893 \\
419\end{array}$ & $\begin{array}{r}2806 \\
2776 \\
-99 \\
755 \\
347\end{array}$ & $\begin{array}{r}2891 \\
2891 \\
-99 \\
840 \\
327\end{array}$ & $\begin{array}{r}2698 \\
2698 \\
-99 \\
794 \\
311\end{array}$ & $\begin{array}{r}2275 \\
22116 \\
-99 \\
428 \\
284\end{array}$ & $\begin{array}{r}1682 \\
1509 \\
-99 \\
287 \\
124\end{array}$ & $\begin{array}{r}942 \\
555 \\
-90 \\
110 \\
55\end{array}$ & $\begin{array}{r}244 \\
126 \\
-99 \\
44 \\
22\end{array}$ & $\begin{array}{l}0 \\
0 \\
0 \\
0 \\
0\end{array}$ & $\begin{array}{l}0 \\
0 \\
0 \\
0 \\
0\end{array}$ & $\begin{array}{l}0 \\
0 \\
0 \\
0 \\
0\end{array}$ & $\begin{array}{r}-999 \\
18880 \\
-999 \\
4855 \\
2766\end{array}$ \\
\hline & $\begin{array}{l}11 \\
12 \\
13 \\
14 \\
15\end{array}$ & $\begin{array}{l}0 \\
0 \\
0 \\
0 \\
0\end{array}$ & $\begin{array}{l}0 \\
0 \\
0 \\
0 \\
0\end{array}$ & $\begin{array}{l}0 \\
0 \\
0 \\
0 \\
0\end{array}$ & $\begin{array}{r}75 \\
82 \\
-99 \\
-99 \\
-99\end{array}$ & $\begin{array}{r}556 \\
289 \\
530 \\
386 \\
1297\end{array}$ & $\begin{array}{r}1297 \\
1275 \\
871 \\
671 \\
1408\end{array}$ & $\begin{array}{l}2099 \\
1171 \\
1729 \\
1533 \\
2171\end{array}$ & $\begin{array}{l}2686 \\
2126 \\
2502 \\
1637 \\
2640\end{array}$ & $\begin{array}{r}2954 \\
888 \\
2653 \\
812 \\
2997\end{array}$ & $\begin{array}{r}2993 \\
803 \\
2640 \\
507 \\
3059\end{array}$ & $\begin{array}{r}-79 \\
986 \\
2424 \\
979 \\
2709\end{array}$ & $\begin{array}{r}-99 \\
1684 \\
1729 \\
265 \\
2306\end{array}$ & $\begin{array}{r}-99 \\
1193 \\
1198 \\
701 \\
1775\end{array}$ & $\begin{array}{l}-99 \\
868 \\
861 \\
642 \\
809\end{array}$ & $\begin{array}{l}-79 \\
177 \\
252 \\
239 \\
242\end{array}$ & $\begin{array}{l}0 \\
0 \\
0 \\
0 \\
0\end{array}$ & $\begin{array}{l}0 \\
0 \\
0 \\
0 \\
0\end{array}$ & $\begin{array}{l}0 \\
0 \\
0 \\
0 \\
0\end{array}$ & $\begin{array}{r}-999 \\
11845 \\
-999 \\
-999 \\
-999\end{array}$ \\
\hline $\mathscr{\omega}$ & $\begin{array}{l}16 \\
17 \\
18 \\
19 \\
20\end{array}$ & $\begin{array}{l}0 \\
0 \\
0 \\
0 \\
0\end{array}$ & $\begin{array}{l}0 \\
n \\
n \\
0 \\
0\end{array}$ & $\begin{array}{l}0 \\
0 \\
0 \\
0 \\
0\end{array}$ & $\begin{array}{r}74 \\
104 \\
134 \\
-99 \\
-99\end{array}$ & $\begin{array}{l}146 \\
490 \\
799 \\
-99 \\
-99\end{array}$ & $\begin{array}{l}765 \\
1221 \\
1625 \\
1591 \\
1531\end{array}$ & $\begin{array}{l}1185 \\
2095 \\
2335 \\
2247 \\
2135\end{array}$ & $\begin{array}{l}1943 \\
2659 \\
2892 \\
2323 \\
2600\end{array}$ & $\begin{array}{l}2216 \\
3219 \\
3217 \\
3108 \\
21341\end{array}$ & $\begin{array}{r}2377 \\
3222 \\
3275 \\
-94 \\
3016\end{array}$ & $\begin{array}{r}2226 \\
2112 \\
3043 \\
-99 \\
2804\end{array}$ & $\begin{array}{r}1627 \\
1840 \\
2581 \\
-99 \\
2436\end{array}$ & $\begin{array}{r}1152 \\
634 \\
1023 \\
-99 \\
1758\end{array}$ & $\begin{array}{l}762 \\
651 \\
-99 \\
-99 \\
991\end{array}$ & $\begin{array}{l}195 \\
215 \\
-99 \\
-99 \\
297\end{array}$ & $\begin{array}{l}0 \\
0 \\
0 \\
0 \\
0\end{array}$ & $\begin{array}{l}0 \\
0 \\
0 \\
0 \\
0\end{array}$ & $\begin{array}{l}0 \\
0 \\
0 \\
0 \\
0\end{array}$ & $\begin{array}{r}14568 \\
18462 \\
-999 \\
-999 \\
-999\end{array}$ \\
\hline & $\begin{array}{l}21 \\
22 \\
23 \\
24 \\
25\end{array}$ & $\begin{array}{l}0 \\
0 \\
0 \\
0 \\
0\end{array}$ & $\begin{array}{l}0 \\
0 \\
0 \\
0 \\
0\end{array}$ & $\begin{array}{l}0 \\
0 \\
0 \\
0 \\
0\end{array}$ & $\begin{array}{r}100 \\
-555 \\
134 \\
23 \\
57\end{array}$ & $\begin{array}{r}588 \\
-555 \\
B 12 \\
59 \\
306\end{array}$ & $\begin{array}{r}1351 \\
-555 \\
1555 \\
1055 \\
506\end{array}$ & $\begin{array}{r}2029 \\
-555 \\
2253 \\
1949 \\
932\end{array}$ & $\begin{array}{l}1682 \\
-5.55 \\
2784 \\
26136 \\
1009\end{array}$ & $\begin{array}{l}2049 \\
-555 \\
3066 \\
2994 \\
1558\end{array}$ & $\begin{array}{r}1895 \\
-555 \\
3131 \\
3024 \\
962\end{array}$ & $\begin{array}{r}1839 \\
-555 \\
2918 \\
2395 \\
1335\end{array}$ & $\begin{array}{l}1564 \\
-555 \\
2532 \\
1536 \\
1109\end{array}$ & $\begin{array}{r}1436 \\
-555 \\
1903 \\
619 \\
510\end{array}$ & $\begin{array}{r}873 \\
-555 \\
1189 \\
789 \\
359\end{array}$ & $\begin{array}{r}362 \\
-555 \\
425 \\
246 \\
136\end{array}$ & $\begin{array}{l}0 \\
0 \\
0 \\
0 \\
0\end{array}$ & $\begin{array}{l}0 \\
0 \\
0 \\
0 \\
0\end{array}$ & $\begin{array}{l}0 \\
0 \\
0 \\
0 \\
0\end{array}$ & $\begin{array}{r}15768 \\
-999 \\
22702 \\
17375 \\
8859\end{array}$ \\
\hline & $\begin{array}{l}26 \\
27 \\
28 \\
29 \\
30\end{array}$ & $\begin{array}{l}0 \\
0 \\
0 \\
0 \\
0\end{array}$ & $\begin{array}{l}0 \\
0 \\
0 \\
0 \\
0\end{array}$ & $\begin{array}{l}0 \\
0 \\
0 \\
0 \\
0\end{array}$ & $\begin{array}{l}-99 \\
-99 \\
206 \\
190 \\
206\end{array}$ & $\begin{array}{l}-99 \\
175 \\
835 \\
977 \\
884\end{array}$ & $\begin{array}{r}328 \\
352 \\
1600 \\
-99 \\
1759\end{array}$ & $\begin{array}{r}0 \\
598 \\
2450 \\
2356 \\
2427\end{array}$ & $\begin{array}{r}66 \\
1122 \\
2912 \\
2897 \\
2961\end{array}$ & $\begin{array}{r}165 \\
2059 \\
3233 \\
3208 \\
3265\end{array}$ & $\begin{array}{r}515 \\
2059 \\
3292 \\
3231 \\
3318\end{array}$ & $\begin{array}{r}646 \\
1889 \\
3098 \\
3038 \\
3092\end{array}$ & $\begin{array}{l}2.2711 \\
1230 \\
2676 \\
2586 \\
2663\end{array}$ & $\begin{array}{r}1737 \\
644 \\
2044 \\
1976 \\
2142\end{array}$ & $\begin{array}{r}797 \\
339 \\
1264 \\
1216 \\
1195\end{array}$ & $\begin{array}{r}66 \\
218 \\
514 \\
453 \\
494\end{array}$ & $\begin{array}{l}0 \\
0 \\
0 \\
0 \\
0\end{array}$ & $\begin{array}{l}0 \\
0 \\
0 \\
0 \\
0\end{array}$ & $\begin{array}{l}0 \\
0 \\
0 \\
0 \\
0\end{array}$ & $\begin{array}{r}-999 \\
-999 \\
24204 \\
-999 \\
24406\end{array}$ \\
\hline & 31 & 0 & 0 & 0 & 2110 & 1176 & 16117 & 2308 & 2376 & 3194 & 3223 & 3001 & 2568 & 1949 & 1219 & 1185 & 0 & 0 & 0 & 23972 \\
\hline & $\begin{array}{r}\text { MENN } \\
\text { SD } \\
\text { HIINM }\end{array}$ & $\begin{array}{r}0 \\
0 \\
3 !\end{array}$ & $\begin{array}{r}0 \\
0 \\
31\end{array}$ & $\begin{array}{r}0 \\
0 \\
31\end{array}$ & $\begin{array}{l}76 \\
70 \\
19\end{array}$ & $\begin{array}{r}483 \\
369 \\
24\end{array}$ & $\begin{array}{r}1017 \\
5113 \\
29\end{array}$ & $\begin{array}{r}1535 \\
798 \\
30\end{array}$ & $\begin{array}{r}2029 \\
917 \\
29\end{array}$ & $\begin{array}{r}22.34 \\
1050 \\
27\end{array}$ & $\begin{array}{r}2191 \\
1080 \\
26\end{array}$ & $\begin{array}{r}2059 \\
897 \\
26\end{array}$ & $\begin{array}{r}1765 \\
779 \\
26\end{array}$ & $\begin{array}{r}1287 \\
605 \\
26\end{array}$ & $\begin{array}{r}763 \\
339 \\
25\end{array}$ & $\begin{array}{r}244 \\
1110 \\
25\end{array}$ & $\begin{array}{r}0 \\
0 \\
31\end{array}$ & $\begin{array}{r}0 \\
0 \\
31\end{array}$ & $\begin{array}{r}0 \\
0 \\
31\end{array}$ & $\begin{array}{r}16052 \\
6976 \\
13\end{array}$ \\
\hline & $\begin{array}{l}\text { MAX } \\
\text { IIIH }\end{array}$ & $\begin{array}{l}\mathbf{0} \\
0\end{array}$ & $\begin{array}{l}0 \\
0\end{array}$ & $\begin{array}{l}0 \\
0\end{array}$ & $\begin{array}{r}2116 \\
0\end{array}$ & $\begin{array}{r}1297 \\
30\end{array}$ & $\begin{array}{r}1759 \\
60\end{array}$ & $\begin{array}{r}2450 \\
0\end{array}$ & $\begin{array}{r}2961 \\
66\end{array}$ & $\begin{array}{r}3265 \\
151\end{array}$ & $\begin{array}{r}3318 \\
32.7\end{array}$ & $\begin{array}{r}3078 \\
311\end{array}$ & $\begin{array}{r}26,76 \\
265\end{array}$ & $\begin{array}{r}2142 \\
1.24\end{array}$ & $\begin{array}{r}1264 \\
55\end{array}$ & $\begin{array}{r}514 \\
22\end{array}$ & $\begin{array}{l}0 \\
0\end{array}$ & $\begin{array}{l}n \\
0\end{array}$ & $\begin{array}{l}0 \\
0\end{array}$ & $\begin{array}{r}241106 \\
2766\end{array}$ \\
\hline
\end{tabular}


CIASTAL - INLAHO SILLAR RADIATIUN DIFFEREMCE STUDY HOHTHLY DATA SUMAARY

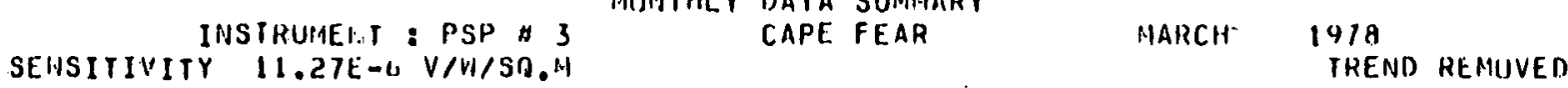

EHERGY KILOJOULES PEH SIUUARE IIETER

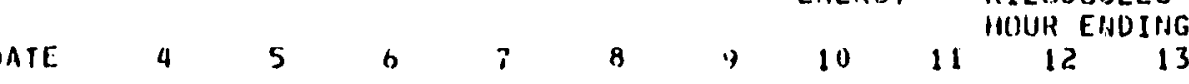

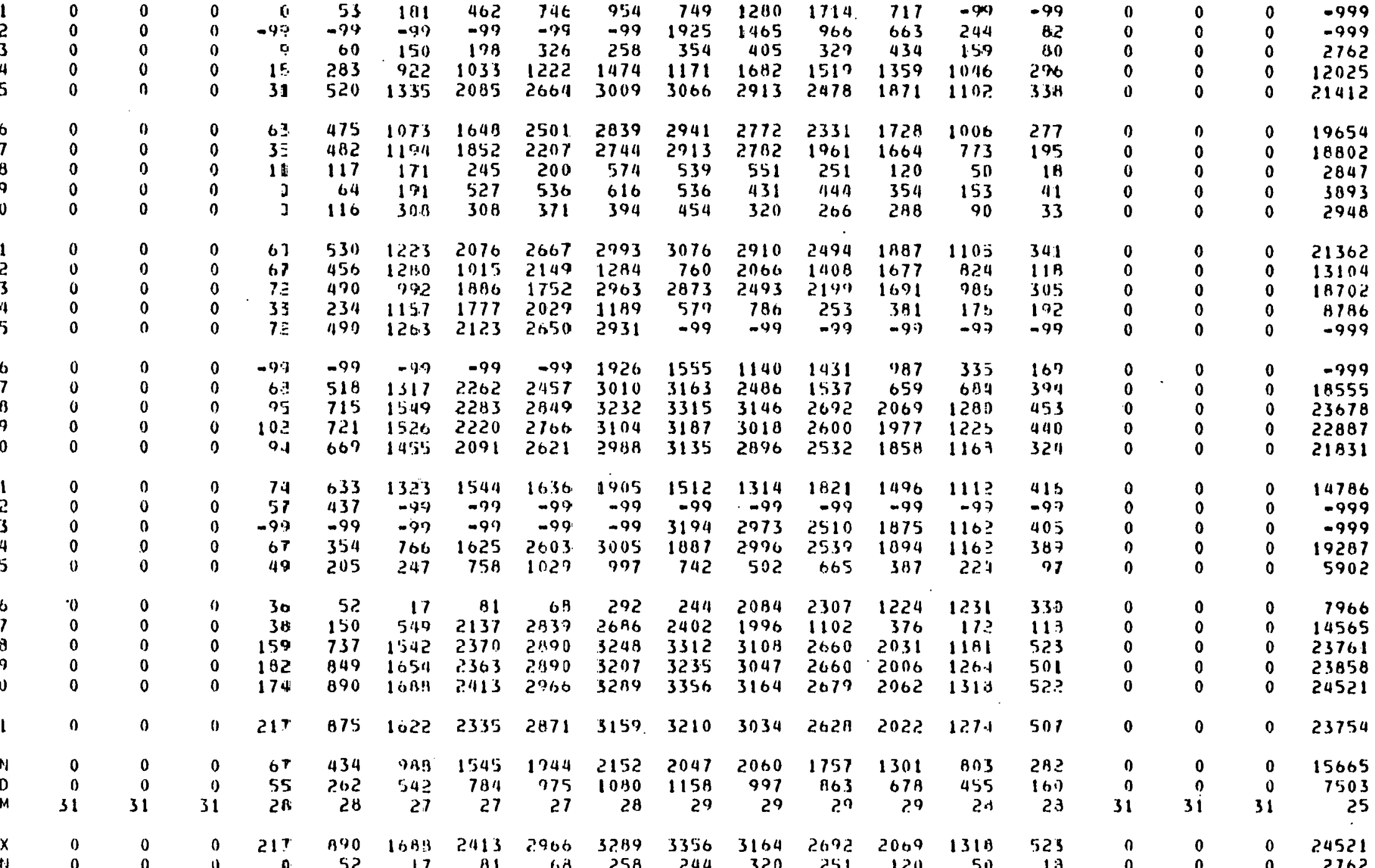

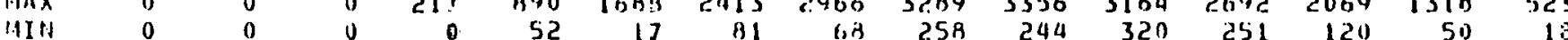


COASTAL - INLAIID SILLAR RADIATIUH WIFFERFMCE STUOY

MUNTHLY DATA SUMHAKY

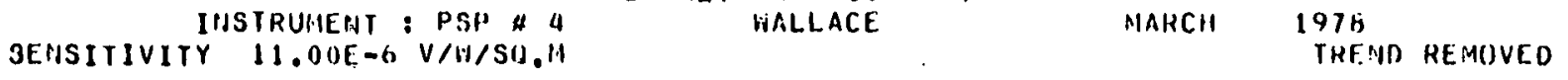

ENERGY KILOJOULES PER SOUARE METER

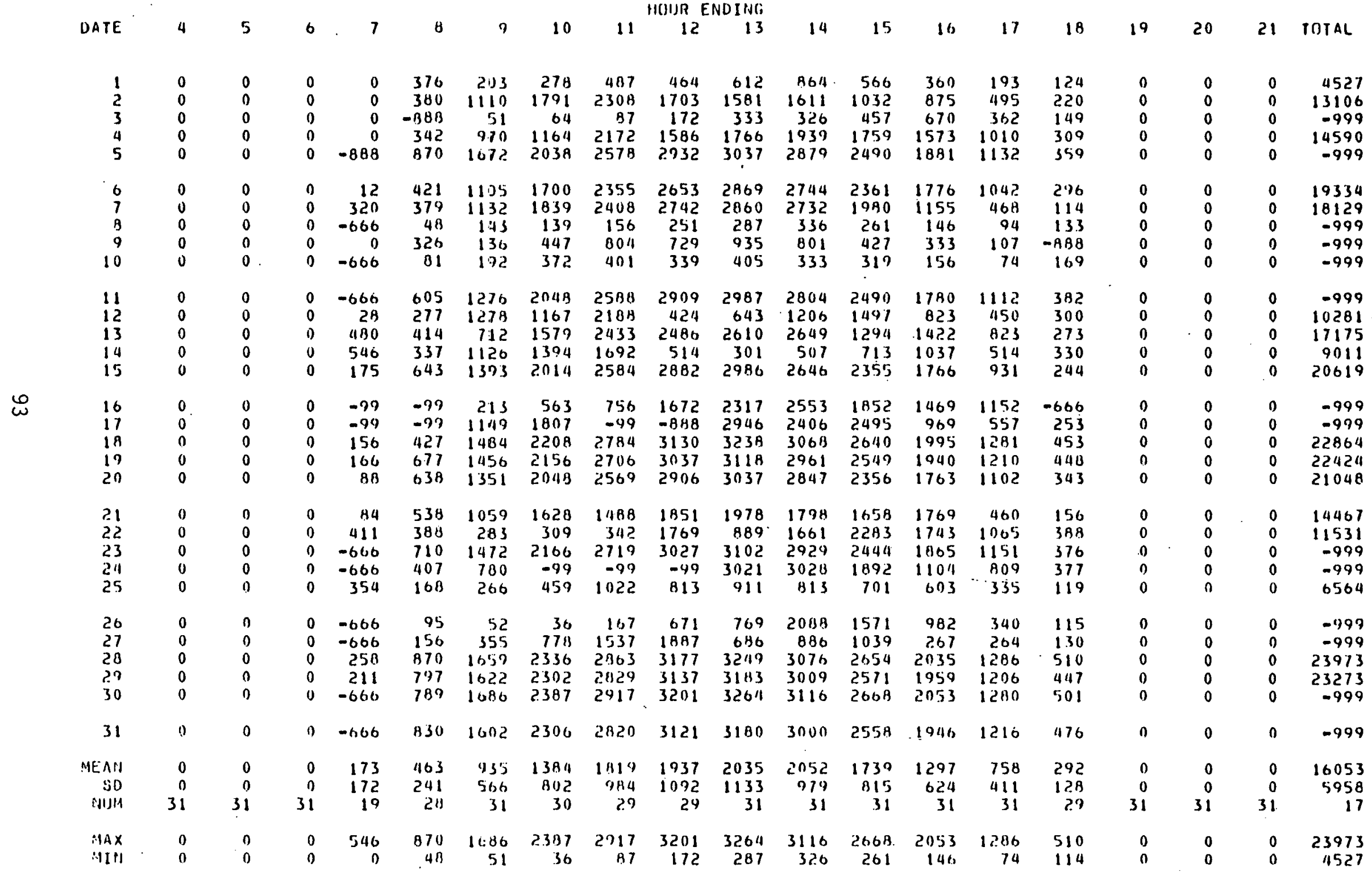


CUASIAL - INLAFID SIJLAR RADIATION DIFFERENCE STIJOY INSTRUMENI PSP \# 5 MONTHLY DATA SUMMARY

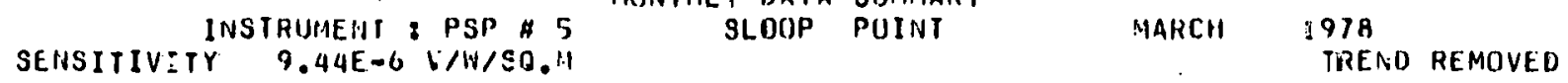

ENEFG KILOJOULES PER SIJUARE METER

HOIIR ENDIING

0
0
0
0
0
0
0
0
0
0
0
0
0
0
0

0
0
0
0
0
0
0
0
0
0
0
0
0
0
0

$\begin{array}{rr}0 & \\ 11 & 38 \\ 0 & \\ 19 & 38 \\ 2.2 & 484 \\ 19 & 373 \\ 19 & 375 \\ 0 & 152 \\ 0 & \\ 0 & 171 \\ 45 & \\ 110 & 588 \\ 106 & 587 \\ 57 & 524 \\ 144 & 559\end{array}$

$\begin{array}{rrr}49 & 186 & \\ 388 & 1121 & 1 \\ 13 & 150 & \\ 388 & 919 & 109\end{array}$

10

11

184121

312
857 2261

29311110

$12 \quad 13$

14

$\begin{array}{ll}098 & 1 \\ 021 & 257\end{array}$

375

$\begin{array}{lrllll}40 & 934 & 1083 & 1357 & 564 & 228\end{array}$

$\begin{array}{lll}373 & 1174 & 187\end{array}$

$\begin{array}{ll}872 & 2407 \\ 070 & 2265\end{array}$

$742963 \quad 30692905 \quad 2501 \quad 1887 \quad 1032$

132.

68

97

54

$\begin{array}{llllllll}2650 & 2867 & 2757 & 1929 & 1262 & 741 & 152\end{array}$

$177 \quad 244$

$\begin{array}{lll}788 & 1079 & 202 \\ 579 & 1308 & 11976\end{array}$

$\begin{array}{lllllll}743 & 621 & 678 & 549 & 461 & 392 & 175\end{array}$

4.1

$\begin{array}{lllllllll}1178 & 1887 & 2044 & 1151 & 915 & 583 & 270 & 179 & 198\end{array}$

$\begin{array}{llllllllllll}129 & 518 & 831 & 1140 & 1719 & 1773 & 1914 & 1178 & 1243 & 983 & 343\end{array}$

$518 \quad 831 \quad 1140$

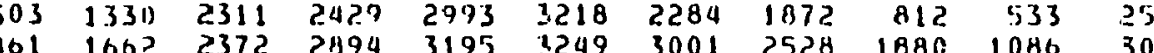

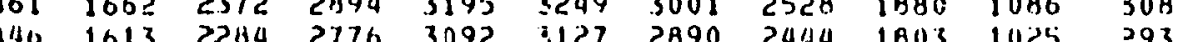

$160 \quad 189 \quad 1521 \quad 2227 \quad 2715 \quad 3073 \quad 1119$ 2822 $2127 \quad 1735 \quad 911 \quad 232$

$\begin{array}{lllllllllll}720 & 1365 & 1765 & 1853 & 2269 & 1395 & 1510 & 1544 & 1563 & 1132 & 385\end{array}$

$\begin{array}{lllllllllll}324 & 1090 & 2162 & 2692 & 3024 & 7157 & 3.001 & 2375 & 1395 & 1144 & 704 \\ 560 & 1372 & 2208 & 2761 & 3096 & 1203 & 2993 & 2558 & 1944 & 1106 & 354\end{array}$

$\begin{array}{lllllllllll}575 & 1242 & 2124 & 20116 & 2982 & 3085 & 2917 & 2509 & 1999 & 1128 & 391\end{array}$

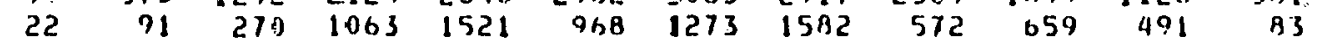

$\begin{array}{ll}26 & 0 \\ 27 & 0 \\ 28 & 0 \\ 29 & 0 \\ 30 & 0\end{array}$

17

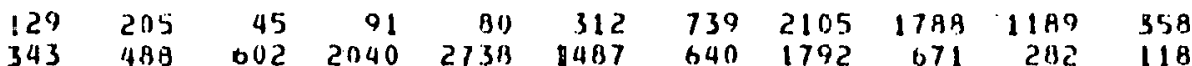

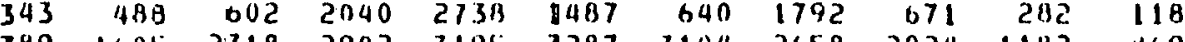

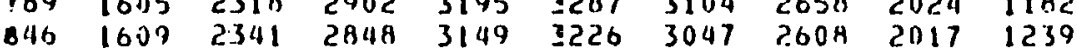

8H8 160i) $2374 \quad 2913 \quad 3233 \quad 3302 \quad 3100 \quad 2661 \quad 2032 \quad 1259$

465

3100

221

THS 1611233

$\begin{array}{rr}\text { HEAH } & 0 \\ \text { SO } & 0\end{array}$

$\operatorname{lig}$

$\because[\mathrm{N}$

$\begin{array}{llllllllllll}83 \quad 42 \quad 103 n & 1595 & 2012 & 2180 & 2113 & 2028 & 1739 & 1306 & 758 & 243\end{array}$

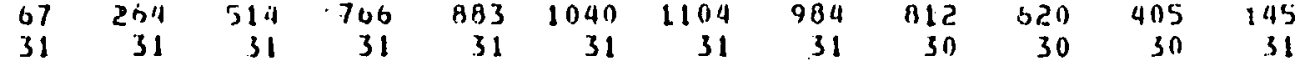

488

TABULATED $12 / 31 / 79$ AT $13: 19848$ 
CIAASTAL - INLAND SILLAR RAOIATION DIFFERENCE. STUDY HONTHLY DATA SIJMMARY

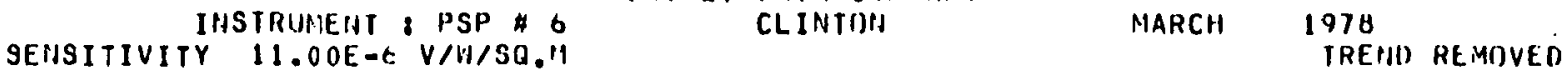

EHERGY KILOJOULES PER SHUARE METER HOUR ENUING

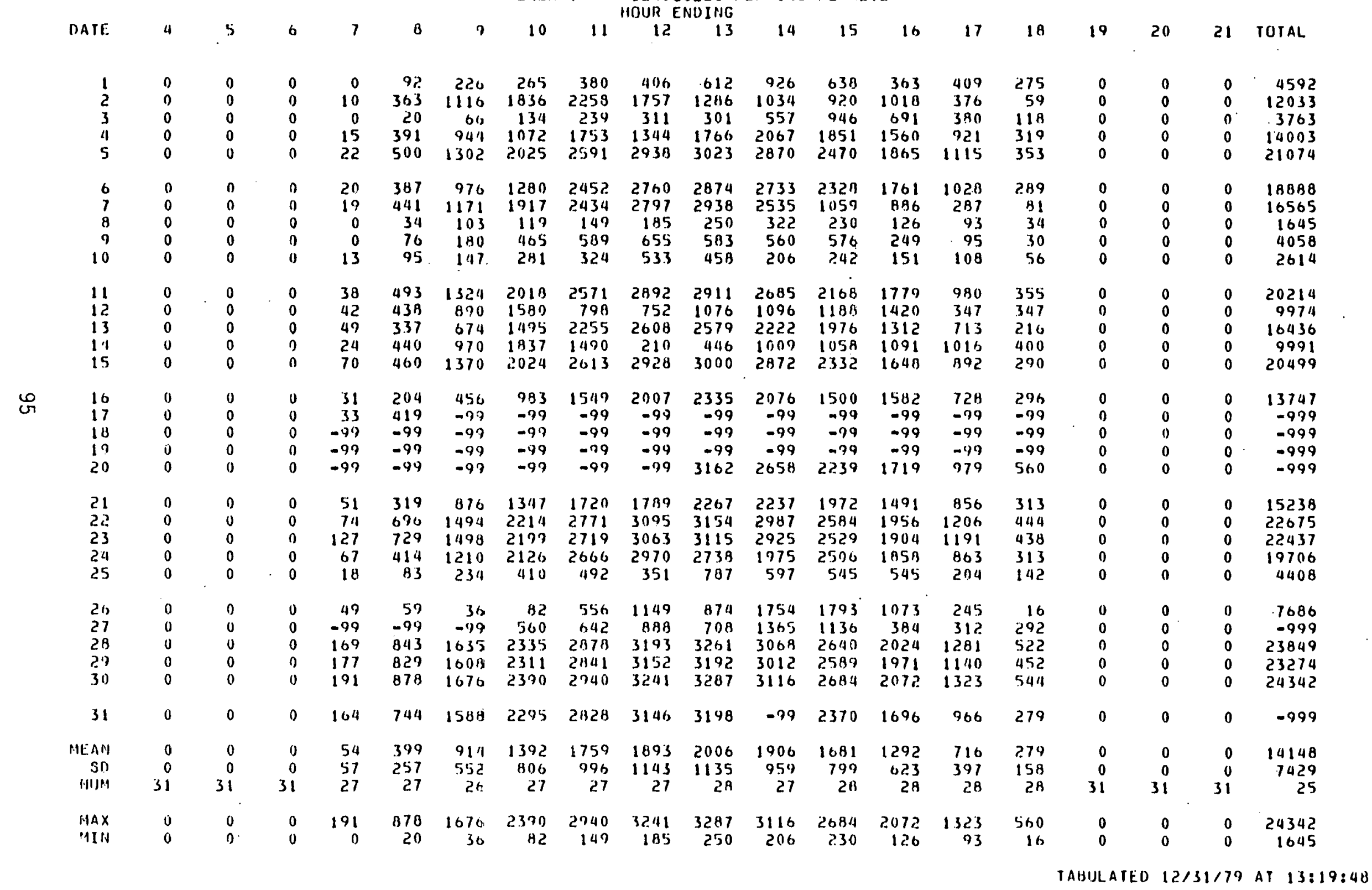


CUASTAL - INLAND SILLAR RADIATIUN DIFFERENCE STUDY

INBTRUMENT: PSP A 7 ONSLOW BEACH MARCH 197A

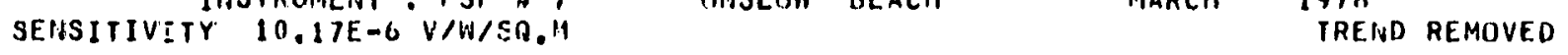

ENERGY KILOJOLLES PER SHUARE METER

\begin{tabular}{|c|c|c|c|c|c|c|c|c|c|c|c|c|c|c|c|c|c|c|c|}
\hline \multirow[b]{2}{*}{ DATE } & \multirow[b]{2}{*}{4} & \multirow[b]{2}{*}{5} & \multirow[b]{2}{*}{6} & & & & & & HOUR E & VBITG & & & & \multirow[b]{2}{*}{17} & \multirow[b]{2}{*}{18} & \multirow[b]{2}{*}{19} & \multirow[b]{2}{*}{20} & \multirow[b]{2}{*}{21} & \multirow[b]{2}{*}{ TOIAL } \\
\hline & & & & 7 & 8 & 9 & 10 & 11 & 12 & 13 & 14 & 15 & 16 & & & & & & \\
\hline $\begin{array}{l}1 \\
2 \\
3 \\
1 \\
5\end{array}$ & $\begin{array}{l}0 \\
0 \\
0 \\
0 \\
0\end{array}$ & $\begin{array}{l}0 \\
0 \\
0 \\
0 \\
0\end{array}$ & $\begin{array}{l}0 \\
0 \\
0 \\
0 \\
0\end{array}$ & $\begin{array}{r}-99 \\
-99 \\
0 \\
38 \\
38\end{array}$ & $\begin{array}{r}-99 \\
-99 \\
26 \\
382 \\
555\end{array}$ & $\begin{array}{r}-99 \\
-99 \\
171 \\
966 \\
1380\end{array}$ & $\begin{array}{r}-99 \\
-99 \\
234 \\
1228 \\
2109\end{array}$ & $\begin{array}{r}-99 \\
-99 \\
394 \\
1192 \\
2672\end{array}$ & $\begin{array}{r}-99 \\
-94 \\
571 \\
1224 \\
2998\end{array}$ & $\begin{array}{r}-99 \\
-99 \\
295 \\
1550 \\
2072\end{array}$ & $\begin{array}{r}-99 \\
-99 \\
164 \\
1571 \\
2899\end{array}$ & $\begin{array}{r}-99 \\
-99 \\
245 \\
1079 \\
2474\end{array}$ & $\begin{array}{r}-99 \\
346 \\
539 \\
1423 \\
1847\end{array}$ & $\begin{array}{r}-99 \\
431 \\
334 \\
860 \\
1083\end{array}$ & $\begin{array}{r}-99 \\
81 \\
97 \\
251 \\
315\end{array}$ & $\begin{array}{l}0 \\
0 \\
0 \\
0 \\
0\end{array}$ & $\begin{array}{l}0 \\
0 \\
0 \\
0 \\
0\end{array}$ & $\begin{array}{l}0 \\
0 \\
0 \\
0 \\
0\end{array}$ & $\begin{array}{r}-799 \\
-999 \\
1074 \\
11764 \\
21442\end{array}$ \\
\hline $\begin{array}{r}6 \\
7 \\
0 \\
9 \\
10\end{array}$ & $\begin{array}{l}0 \\
0 \\
0 \\
0 \\
0\end{array}$ & $\begin{array}{l}0 \\
0 \\
0 \\
0 \\
0\end{array}$ & $\begin{array}{l}0 \\
0 \\
0 \\
0 \\
0\end{array}$ & $\begin{array}{r}28 \\
31 \\
9 \\
0 \\
15\end{array}$ & $\begin{array}{r}375 \\
831 \\
200 \\
47 \\
153\end{array}$ & $\begin{array}{r}1139 \\
1281 \\
313 \\
196 \\
302\end{array}$ & $\begin{array}{r}1576 \\
1932 \\
338 \\
412 \\
118\end{array}$ & $\begin{array}{r}1932 \\
2399 \\
235 \\
982 \\
557\end{array}$ & $\begin{array}{r}2598 \\
2697 \\
320 \\
746 \\
454\end{array}$ & $\begin{array}{r}2803 \\
2877 \\
441 \\
914 \\
571\end{array}$ & $\begin{array}{r}2711 \\
2725 \\
430 \\
713 \\
525\end{array}$ & $\begin{array}{r}2293 \\
1975 \\
175 \\
482 \\
107\end{array}$ & $\begin{array}{r}1699 \\
1083 \\
129 \\
373 \\
245\end{array}$ & $\begin{array}{l}962 \\
644 \\
193 \\
171 \\
7 ?\end{array}$ & $\begin{array}{r}261 \\
100 \\
9 \\
36 \\
22\end{array}$ & $\begin{array}{l}0 \\
0 \\
0 \\
0 \\
0\end{array}$ & $\begin{array}{l}0 \\
0 \\
0 \\
0 \\
0\end{array}$ & $\begin{array}{l}0 \\
0 \\
0 \\
0 \\
0\end{array}$ & $\begin{array}{r}18447 \\
18255 \\
2792 \\
5272 \\
3151\end{array}$ \\
\hline $\begin{array}{l}11 \\
12 \\
13 \\
14 \\
15\end{array}$ & $\begin{array}{l}0 \\
0 \\
0 \\
0 \\
0\end{array}$ & $\begin{array}{l}0 \\
0 \\
0 \\
0 \\
0\end{array}$ & $\begin{array}{l}0 \\
0 \\
0 \\
0 \\
1)\end{array}$ & $\begin{array}{l}74 \\
53 \\
8 B \\
37 \\
92\end{array}$ & $\begin{array}{l}658 \\
633 \\
584 \\
240 \\
612\end{array}$ & $\begin{array}{r}142.3 \\
934 \\
1030 \\
802 \\
.253\end{array}$ & $\begin{array}{l}2120 \\
2063 \\
1115 \\
172.9 \\
2077\end{array}$ & $\begin{array}{l}2686 \\
1671 \\
2584 \\
2317 \\
2598\end{array}$ & $\begin{array}{l}3023 \\
2736 \\
2846 \\
1567 \\
2959\end{array}$ & $\begin{array}{r}3030 \\
1631 \\
2707 \\
689 \\
2784\end{array}$ & $\begin{array}{r}2920 \\
1603 \\
2456 \\
554 \\
2746\end{array}$ & $\begin{array}{r}2492 \\
1851 \\
1964 \\
618 \\
2198\end{array}$ & $\begin{array}{r}1791 \\
1136 \\
1277 \\
122 \\
1776\end{array}$ & $\begin{array}{r}1061 \\
69.3 \\
342 \\
115 \\
785\end{array}$ & $\begin{array}{r}3116 \\
138 \\
272 \\
91 \\
258\end{array}$ & $\begin{array}{l}0 \\
0 \\
0 \\
0 \\
0\end{array}$ & $\begin{array}{l}0 \\
0 \\
0 \\
0 \\
0\end{array}$ & $\begin{array}{l}0 \\
0 \\
0 \\
0 \\
0\end{array}$ & $\begin{array}{r}21624 \\
15145 \\
18465 \\
8487 \\
20338\end{array}$ \\
\hline $\begin{array}{l}17 \\
17 \\
18 \\
19 \\
20\end{array}$ & $\begin{array}{l}0 \\
0 \\
0 \\
0 \\
0\end{array}$ & $\begin{array}{l}0 \\
0 \\
0 \\
0 \\
0\end{array}$ & $\begin{array}{l}0 \\
0 \\
0 \\
0 \\
0\end{array}$ & $\begin{array}{r}87 \\
47 \\
106 \\
109 \\
-97\end{array}$ & $\begin{array}{l}409 \\
291 \\
761 \\
750 \\
-99\end{array}$ & $\begin{array}{r}760 \\
711 \\
1576 \\
1543 \\
-99\end{array}$ & $\begin{array}{r}1308 \\
1435 \\
2315 \\
2233 \\
-99\end{array}$ & $\begin{array}{r}2232 \\
2550 \\
2877 \\
2764 \\
-99\end{array}$ & $\begin{array}{r}1754 \\
2925 \\
3214 \\
-99 \\
-49\end{array}$ & $\begin{array}{r}1783 \\
3137 \\
3295 \\
-99 \\
-99\end{array}$ & $\begin{array}{r}1383 \\
3105 \\
3086 \\
-99 \\
-99\end{array}$ & $\begin{array}{r}940 \\
1530 \\
2637 \\
-79 \\
-99\end{array}$ & $\begin{array}{r}760 \\
1845 \\
1985 \\
-99 \\
-99\end{array}$ & $\begin{array}{r}416 \\
868 \\
1210 \\
-99 \\
-99\end{array}$ & $\begin{array}{l}197 \\
426 \\
414 \\
-99 \\
-99\end{array}$ & $\begin{array}{l}0 \\
0 \\
0 \\
0 \\
0\end{array}$ & $\begin{array}{l}0 \\
0 \\
0 \\
0 \\
0\end{array}$ & $\begin{array}{l}0 \\
0 \\
0 \\
0 \\
0\end{array}$ & $\begin{array}{r}12149 \\
18900 \\
23496 \\
-999 \\
-999\end{array}$ \\
\hline $\begin{array}{l}21 \\
22 \\
23 \\
24 \\
25\end{array}$ & $\begin{array}{l}0 \\
0 \\
0 \\
0 \\
0\end{array}$ & $\begin{array}{l}0 \\
0 \\
0 \\
0 \\
0\end{array}$ & $\begin{array}{l}0 \\
0 \\
0 \\
0 \\
0\end{array}$ & $\begin{array}{r}-99 \\
-99 \\
158 \\
95 \\
37\end{array}$ & $\begin{array}{l}-49 \\
-79 \\
674 \\
638 \\
172\end{array}$ & $\begin{array}{r}-99 \\
-911 \\
1457 \\
1447 \\
833\end{array}$ & $\begin{array}{r}-79 \\
-97 \\
2261 \\
2127 \\
629\end{array}$ & $\begin{array}{r}-99 \\
-99 \\
2316 \\
2670 \\
979\end{array}$ & $\begin{array}{r}-99 \\
-99 \\
3131 \\
3001 \\
1528\end{array}$ & $\begin{array}{r}-99 \\
-99 \\
3209 \\
3079 \\
1.79\end{array}$ & $\begin{array}{r}-99 \\
-99 \\
3025 \\
2488 \\
1397\end{array}$ & $\begin{array}{r}-99 \\
-99 \\
2575 \\
2489 \\
1659\end{array}$ & $\begin{array}{r}-99 \\
-99 \\
1945 \\
1854 \\
1050\end{array}$ & $\begin{array}{r}-99 \\
-99 \\
1195 \\
1115 \\
006\end{array}$ & $\begin{array}{l}-99 \\
399 \\
391 \\
364 \\
232\end{array}$ & $\begin{array}{l}0 \\
0 \\
0 \\
0 \\
0\end{array}$ & $\begin{array}{l}0 \\
0 \\
0 \\
0 \\
0\end{array}$ & $\begin{array}{l}0 \\
0 \\
0 \\
0 \\
0\end{array}$ & $\begin{array}{r}-999 \\
-999 \\
22766 \\
21106 \\
11151\end{array}$ \\
\hline $\begin{array}{l}26 \\
27 \\
27 \\
27 \\
30\end{array}$ & $\begin{array}{l}0 \\
0 \\
0 \\
0 \\
0\end{array}$ & $\begin{array}{l}0 \\
0 \\
0 \\
0 \\
0\end{array}$ & $\begin{array}{l}0 \\
0 \\
0 \\
0 \\
0\end{array}$ & $\begin{array}{r}115 \\
87 \\
-99 \\
-99 \\
169\end{array}$ & $\begin{array}{l}1 \geq 9 \\
735 \\
-99 \\
-79 \\
916\end{array}$ & $\begin{array}{r}126 \\
977 \\
-99 \\
-97 \\
1716\end{array}$ & $\begin{array}{r}66 \\
1346 \\
-99 \\
-99 \\
2244\end{array}$ & $\begin{array}{r}137 \\
2006 \\
-99 \\
-99 \\
3125\end{array}$ & $\begin{array}{r}37 \\
2802 \\
-99 \\
-99 \\
3242\end{array}$ & $\begin{array}{r}52 \\
3 \div 59 \\
-79 \\
-99 \\
-99\end{array}$ & $\begin{array}{l}207 \\
-99 \\
-97 \\
-99 \\
-99\end{array}$ & $\begin{array}{r}1634 \\
-79 \\
-99 \\
-99 \\
-99\end{array}$ & $\begin{array}{r}1899 \\
-99 \\
-99 \\
-99 \\
-99\end{array}$ & $\begin{array}{r}1135 \\
-99 \\
-99 \\
-99 \\
-99\end{array}$ & $\begin{array}{l}360 \\
-99 \\
-99 \\
-99 \\
-99\end{array}$ & $\begin{array}{l}0 \\
0 \\
0 \\
0 \\
0\end{array}$ & $\begin{array}{l}0 \\
0 \\
0 \\
0 \\
0\end{array}$ & $\begin{array}{l}0 \\
0 \\
0 \\
0 \\
0\end{array}$ & $\begin{array}{l}5897 \\
-999 \\
-999 \\
-999 \\
-999\end{array}$ \\
\hline 31 & 0 & 0 & 0 & -99 & -99 & -9.7 & -99 & -99 & -99 & -99 & -99 & -79 & -99 & -97 & .99 & 0 & 0 & 0 & -999 \\
\hline $\begin{array}{r}\text { HEAN } \\
\text { SD } \\
\text { HUW" }\end{array}$ & $\begin{array}{r}0 \\
0 \\
31\end{array}$ & $\begin{array}{r}0 \\
0 \\
31\end{array}$ & $\begin{array}{r}0 \\
31\end{array}$ & $\begin{array}{l}65 \\
46 \\
23\end{array}$ & $\begin{array}{r}451 \\
249 \\
23\end{array}$ & $\begin{array}{r}9131 \\
476 \\
23\end{array}$ & $\begin{array}{r}1491 \\
767 \\
2.3\end{array}$ & $\begin{array}{r}1932 \\
932 \\
23\end{array}$ & $\begin{array}{r}2116 \\
1062 \\
22\end{array}$ & $\begin{array}{r}2052 \\
11.31 \\
21\end{array}$ & $\begin{array}{r}1835 \\
1064 \\
20\end{array}$ & $\begin{array}{r}1570 \\
853 \\
20\end{array}$ & $\begin{array}{r}1220 \\
639 \\
21\end{array}$ & $\begin{array}{r}713 \\
360 \\
21\end{array}$ & $\begin{array}{r}233 \\
132 \\
22\end{array}$ & $\begin{array}{r}0 \\
0 \\
31\end{array}$ & $\begin{array}{r}0 \\
0 \\
31\end{array}$ & $\begin{array}{r}0 \\
0 \\
31\end{array}$ & $\begin{array}{r}14221 \\
7088 \\
20\end{array}$ \\
\hline $\begin{array}{l}\ln x \\
M I H\end{array}$ & $\begin{array}{l}0 \\
0\end{array}$ & $\begin{array}{l}0 \\
0\end{array}$ & $\begin{array}{l}0 \\
0\end{array}$ & $\begin{array}{r}169 \\
0\end{array}$ & $\begin{array}{r}916 \\
76 b\end{array}$ & $\begin{array}{r}1710 \\
120\end{array}$ & $\begin{array}{r}2515 \\
06\end{array}$ & $\begin{array}{r}3125 \\
137\end{array}$ & $\begin{array}{r}32112 \\
37\end{array}$ & $\begin{array}{r}32.95 \\
52\end{array}$ & $\begin{array}{r}3105 \\
164\end{array}$ & $\begin{array}{r}2637 \\
107\end{array}$ & $\begin{array}{r}1985 \\
122\end{array}$ & $\begin{array}{r}1210 \\
12\end{array}$ & $\begin{array}{r}126 \\
9\end{array}$ & $\begin{array}{l}0 \\
0\end{array}$ & $\begin{array}{l}0 \\
0\end{array}$ & $\begin{array}{l}n \\
0\end{array}$ & $\begin{array}{r}23496 \\
2792\end{array}$ \\
\hline
\end{tabular}


COABTAL - INLAND SOLAR RADIATIUH DIFFERENCE STUDY MONTHLY DATA SUMMARY

INSTRUMEHT : HIP H 8
3EHSITIVITY $8.85 E-6$ VIH/SA.M

riJERGY KILOJUULES PER SIJUAHE METER IIDUR ENDING

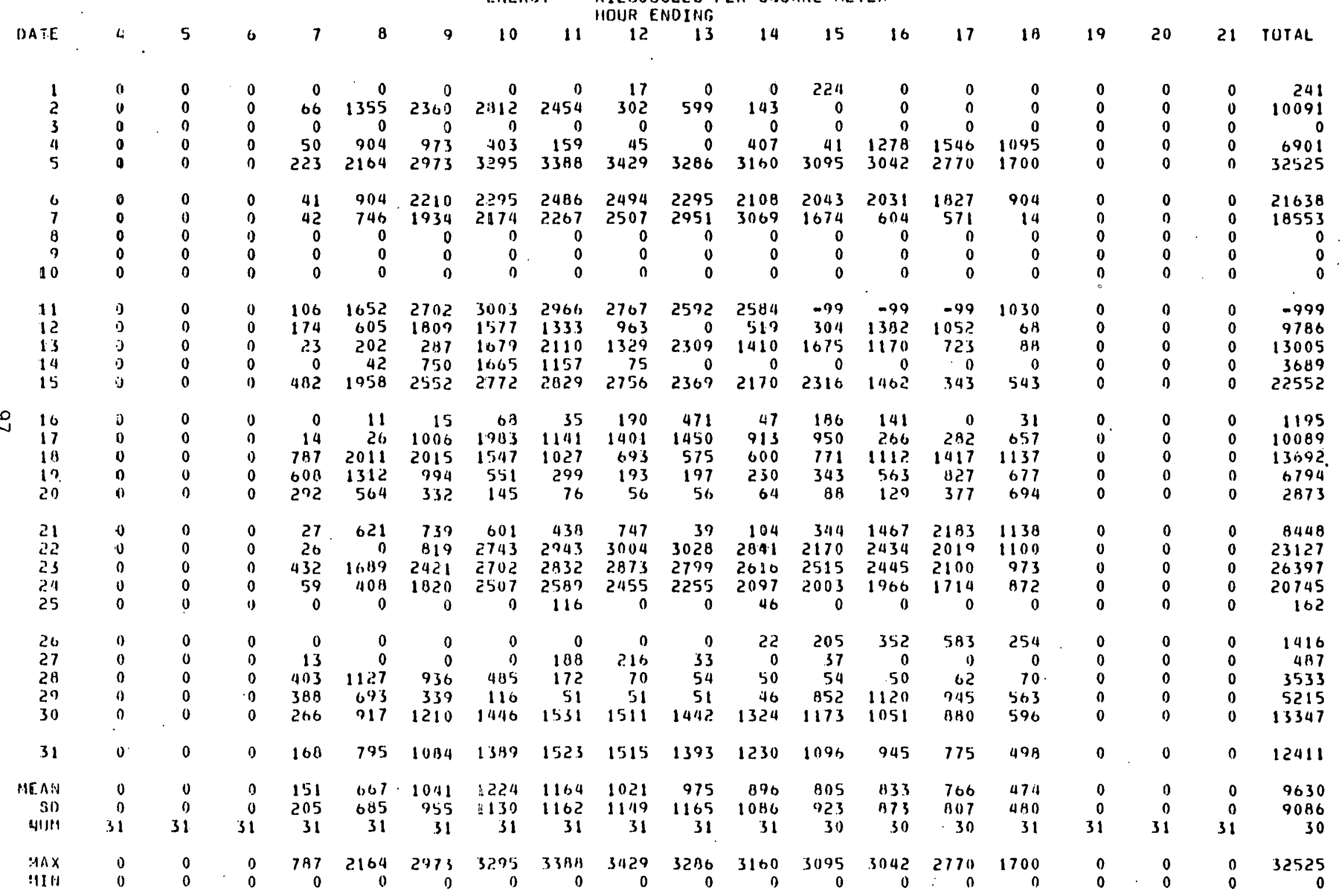


CUASTAL - INLAND SIILAR RAOIATIONN DIFFERENCE STIJO* MIBHTHLY Y DATA SUMMARY

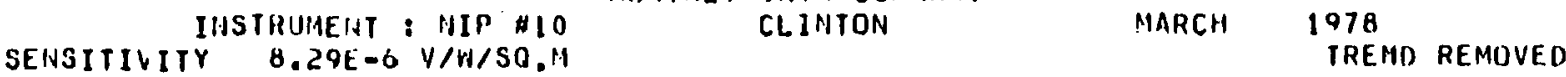

ENERGY KILOJOULES PER SIJUARE. METER IIDUR ENDING

\begin{tabular}{|c|c|c|c|c|c|c|c|c|c|c|c|c|c|c|c|c|c|c|c|}
\hline DATE & 4 & 5 & 6 & 7 & 8 & 9 & 10 & 11 & 12 & 13 & 14 & 15 & 16 & 17 & 16 & 19 & 20 & 21 & TUTAL \\
\hline $\begin{array}{l}1 \\
2 \\
3 \\
4 \\
5\end{array}$ & $\begin{array}{l}0 \\
0 \\
0 \\
0 \\
0\end{array}$ & $\begin{array}{l}0 \\
0 \\
0 \\
0 \\
0\end{array}$ & $\begin{array}{l}0 \\
0 \\
0 \\
0 \\
0\end{array}$ & $\begin{array}{r}0 \\
17 \\
0 \\
25 \\
130\end{array}$ & $\begin{array}{r}52 \\
1150 \\
0 \\
1041 \\
2240\end{array}$ & $\begin{array}{r}0 \\
2356 \\
0 \\
715 \\
3152\end{array}$ & $\begin{array}{r}0 \\
2835 \\
0 \\
94 \\
3474\end{array}$ & $\begin{array}{r}0 \\
2258 \\
0 \\
524 \\
3643\end{array}$ & $\begin{array}{r}0 \\
121 \\
0 \\
0 \\
3717\end{array}$ & $\begin{array}{r}-19 \\
0 \\
0 \\
289 \\
3717\end{array}$ & $\begin{array}{r}0 \\
0 \\
0 \\
1002 \\
3665\end{array}$ & $\begin{array}{r}13 \\
0 \\
0 \\
785 \\
3569\end{array}$ & $\begin{array}{r}0 \\
143 \\
0 \\
1254 \\
3374\end{array}$ & $\begin{array}{r}160 \\
0 \\
1701 \\
2930\end{array}$ & $\begin{array}{r}455 \\
0 \\
0 \\
1275 \\
186 \bar{c}\end{array}$ & $\begin{array}{l}0 \\
0 \\
0 \\
0 \\
0\end{array}$ & $\begin{array}{l}0 \\
0 \\
0 \\
0 \\
0\end{array}$ & $\begin{array}{l}0 \\
0 \\
0 \\
0 \\
0\end{array}$ & $\begin{array}{r}-999 \\
8860 \\
0 \\
8705 \\
35482\end{array}$ \\
\hline $\begin{array}{r}0 \\
7 \\
8 \\
7 \\
10\end{array}$ & $\begin{array}{l}0 \\
0 \\
0 \\
0 \\
0\end{array}$ & $\begin{array}{l}0 \\
0 \\
0 \\
0 \\
0\end{array}$ & $\begin{array}{l}0 \\
0 \\
0 \\
0 \\
0\end{array}$ & $\begin{array}{c}10 \\
0 \\
0 \\
0 \\
0\end{array}$ & $\begin{array}{r}628 \\
1132 \\
0 \\
0 \\
0\end{array}$ & $\begin{array}{r}1423 \\
2343 \\
0 \\
0 \\
0\end{array}$ & $\begin{array}{r}637 \\
2778 \\
0 \\
0 \\
0\end{array}$ & $\begin{array}{r}3021 \\
2969 \\
0 \\
0 \\
0\end{array}$ & $\begin{array}{r}1038 \\
7225 \\
0 \\
0 \\
0\end{array}$ & $\begin{array}{r}3260 \\
3234 \\
0 \\
0 \\
0\end{array}$ & $\begin{array}{r}3247 \\
2209 \\
0 \\
0 \\
0\end{array}$ & $\begin{array}{r}3116 \\
55 \\
0 \\
0 \\
0\end{array}$ & $\begin{array}{r}2964 \\
94 \\
0 \\
0 \\
0\end{array}$ & $\begin{array}{r}240 c \\
6 \\
6 \\
c \\
c\end{array}$ & $\begin{array}{r}1084 \\
C \\
C \\
C \\
1 \bar{c}\end{array}$ & $\begin{array}{l}0 \\
0 \\
0 \\
0 \\
0\end{array}$ & $\begin{array}{l}0 \\
0 \\
0 \\
0 \\
0\end{array}$ & $\begin{array}{l}0 \\
0 \\
0 \\
0 \\
0\end{array}$ & $\begin{array}{r}24834 \\
18039 \\
0 \\
0 \\
12\end{array}$ \\
\hline $\begin{array}{ll}1 & 1 \\
1 & 2 \\
1 & 5 \\
1 & 11 \\
15\end{array}$ & $\begin{array}{l}0 \\
0 \\
0 \\
0 \\
0\end{array}$ & $\begin{array}{l}0 \\
0 \\
0 \\
0 \\
0\end{array}$ & $\begin{array}{l}0 \\
0 \\
0 \\
0 \\
0\end{array}$ & $\begin{array}{r}79 \\
27 \\
0 \\
0 \\
86\end{array}$ & $\begin{array}{r}917 \\
335 \\
42 \\
146 \\
451\end{array}$ & $\begin{array}{r}2411 \\
218 \\
29 \\
757 \\
1853\end{array}$ & $\begin{array}{r}3128 \\
826 \\
638 \\
1302 \\
2462\end{array}$ & $\begin{array}{r}3262 \\
23 \\
1579 \\
112 \\
3144\end{array}$ & $\begin{array}{r}1149 \\
0 \\
1666 \\
0 \\
3230\end{array}$ & $\begin{array}{r}2789 \\
14 \\
1310 \\
0 \\
3204\end{array}$ & $\begin{array}{r}2054 \\
0 \\
1006 \\
0 \\
3139\end{array}$ & $\begin{array}{r}2233 \\
70 \\
1206 \\
52 \\
2718\end{array}$ & $\begin{array}{r}2276 \\
849 \\
641 \\
629 \\
1984\end{array}$ & $\begin{array}{r}118 E \\
4 \\
177 \\
1276 \\
1351\end{array}$ & $\begin{array}{r}657 \\
678 \\
20 \\
1276 \\
361\end{array}$ & $\begin{array}{l}0 \\
0 \\
0 \\
n \\
0\end{array}$ & $\begin{array}{l}0 \\
0 \\
0 \\
0 \\
0\end{array}$ & $\begin{array}{l}0 \\
0 \\
0 \\
0 \\
0\end{array}$ & $\begin{array}{r}25037 \\
3039 \\
8374 \\
6192 \\
23999\end{array}$ \\
\hline $\begin{array}{l}16 \\
17 \\
18 \\
19 \\
20\end{array}$ & $\begin{array}{l}c \\
0 \\
0 \\
0 \\
0\end{array}$ & $\begin{array}{l}0 \\
0 \\
0 \\
0 \\
0\end{array}$ & $\begin{array}{l}0 \\
0 \\
0 \\
0 \\
0\end{array}$ & $\begin{array}{r}0 \\
0 \\
-97 \\
-94 \\
-97\end{array}$ & $\begin{array}{r}0 \\
724 \\
-94 \\
-99 \\
-99\end{array}$ & $\begin{array}{r}0 \\
-97 \\
-97 \\
-99 \\
-99\end{array}$ & $\begin{array}{r}0 \\
-99 \\
-199 \\
-99 \\
-99\end{array}$ & $\begin{array}{l}191 \\
-99 \\
-99 \\
-99 \\
-99\end{array}$ & $\begin{array}{l}868 \\
-99 \\
-99 \\
-99 \\
-99\end{array}$ & $\begin{array}{r}1315 \\
-99 \\
-99 \\
-99 \\
3035\end{array}$ & $\begin{array}{r}864 \\
-99 \\
-97 \\
-97 \\
2314\end{array}$ & $\begin{array}{r}642 \\
-99 \\
-99 \\
-99 \\
1971\end{array}$ & $\begin{array}{r}1367 \\
-99 \\
-99 \\
-99 \\
1962\end{array}$ & $\begin{array}{r}343 \\
-99 \\
-99 \\
-99 \\
1446\end{array}$ & $\begin{array}{r}30 \\
-99 \\
-99 \\
-97 \\
317\end{array}$ & $\begin{array}{l}0 \\
0 \\
0 \\
0 \\
0\end{array}$ & $\begin{array}{l}0 \\
0 \\
0 \\
0 \\
0\end{array}$ & $\begin{array}{l}0 \\
0 \\
0 \\
0 \\
0\end{array}$ & $\begin{array}{l}5620 \\
-909 \\
-999 \\
-999 \\
-999\end{array}$ \\
\hline $\begin{array}{l}21 \\
23 \\
23 \\
21 \\
25\end{array}$ & $\begin{array}{l}0 \\
0 \\
0 \\
0 \\
0\end{array}$ & $\begin{array}{l}0 \\
0 \\
0 \\
0 \\
0\end{array}$ & $\begin{array}{l}0 \\
0 \\
0 \\
0 \\
0\end{array}$ & $\begin{array}{r}0 \\
176 \\
166 \\
0 \\
0\end{array}$ & $\begin{array}{r}0 \\
1.943 \\
1.564 \\
0 \\
0\end{array}$ & $\begin{array}{r}124 \\
2609 \\
2580 \\
981 \\
0\end{array}$ & $\begin{array}{r}329 \\
3050 \\
2967 \\
2657 \\
0\end{array}$ & $\begin{array}{r}572 \\
3250 \\
3119 \\
2848 \\
0\end{array}$ & $\begin{array}{r}415 \\
3346 \\
3240 \\
2913 \\
0\end{array}$ & $\begin{array}{r}923 \\
3311 \\
3236 \\
2479 \\
0\end{array}$ & $\begin{array}{r}1375 \\
3280 \\
3154 \\
1241\end{array}$ & $\begin{array}{r}1536 \\
3176 \\
3032 \\
2275 \\
0\end{array}$ & $\begin{array}{r}1405 \\
2907 \\
2732 \\
1758 \\
0\end{array}$ & $\begin{array}{r}1106 \\
2486 \\
2281 \\
612 \\
0\end{array}$ & $\begin{array}{r}481 \\
1609 \\
1430 \\
360 \\
0\end{array}$ & $\begin{array}{l}0 \\
0 \\
0 \\
0 \\
0\end{array}$ & $\begin{array}{l}0 \\
0 \\
0 \\
0 \\
0\end{array}$ & $\begin{array}{l}0 \\
0 \\
0 \\
0 \\
0\end{array}$ & $\begin{array}{r}8266 \\
31102 \\
29601 \\
18124 \\
0\end{array}$ \\
\hline $\begin{array}{l}26 \\
27 \\
213 \\
29 \\
30\end{array}$ & $\begin{array}{l}0 \\
0 \\
0 \\
0 \\
0\end{array}$ & $\begin{array}{l}0 \\
0 \\
0 \\
0 \\
0\end{array}$ & $\begin{array}{l}0 \\
0 \\
0 \\
0 \\
0\end{array}$ & $\begin{array}{r}21 \\
-99 \\
609 \\
721 \\
7119\end{array}$ & $\begin{array}{r}13 \\
-99 \\
2342 \\
2097 \\
2130\end{array}$ & $\begin{array}{r}0 \\
-99 \\
2784 \\
2727 \\
2864\end{array}$ & $\begin{array}{r}0 \\
-99 \\
3154 \\
3016 \\
3172\end{array}$ & $\begin{array}{r}0 \\
0 \\
3275 \\
3239 \\
3337\end{array}$ & $\begin{array}{r}91 \\
0 \\
3340 \\
3296 \\
3359\end{array}$ & $\begin{array}{r}26 \\
0 \\
3345 \\
3265 \\
3307\end{array}$ & $\begin{array}{r}425 \\
0 \\
3314 \\
3213 \\
3285\end{array}$ & $\begin{array}{r}807 \\
0 \\
3136 \\
3031 \\
3176\end{array}$ & $\begin{array}{r}364 \\
0 \\
3010 \\
3762 \\
3016\end{array}$ & $\begin{array}{r}0 \\
157 \\
2593 \\
1989 \\
2646\end{array}$ & $\begin{array}{r}0 \\
127 \\
1803 \\
1164 \\
1860\end{array}$ & $\begin{array}{l}0 \\
0 \\
0 \\
0 \\
0\end{array}$ & $\begin{array}{l}0 \\
0 \\
0 \\
0 \\
0\end{array}$ & $\begin{array}{l}0 \\
0 \\
0 \\
0 \\
0\end{array}$ & $\begin{array}{r}1747 \\
-999 \\
32405 \\
30570 \\
32901\end{array}$ \\
\hline 31 & 0 & 0 & 0 & 82 & 740 & 2271 & 2766 & 2979 & $3 \cap 83$ & 5070 & 2952 & 2770 & 2457 & 1993 & 908 & 0 & o & 0 & 26137 \\
\hline $\begin{array}{c}\text { MEAN } \\
\text { SOD } \\
\text { MIJM }\end{array}$ & $\begin{array}{r}0 \\
0 \\
31\end{array}$ & $\begin{array}{r}0 \\
0 \\
31\end{array}$ & $\begin{array}{r}0 \\
0 \\
31\end{array}$ & $\begin{array}{r}107 \\
214 \\
27\end{array}$ & $\begin{array}{r}731 \\
779 \\
27\end{array}$ & $\begin{array}{r}1240 \\
11 \mathrm{AO} \\
3 \%\end{array}$ & $\begin{array}{r}1515 \\
1381 \\
26\end{array}$ & $\begin{array}{r}1616 \\
1480 \\
27\end{array}$ & $\begin{array}{r}1559 \\
1550 \\
27\end{array}$ & $\begin{array}{r}1671 \\
1501 \\
27\end{array}$ & $\begin{array}{r}1512 \\
1401 \\
2 B\end{array}$ & $\begin{array}{r}1406 \\
1337 \\
28\end{array}$ & $\begin{array}{r}1355 \\
1189 \\
28\end{array}$ & $\begin{array}{r}1041 \\
1026 \\
28\end{array}$ & $\begin{array}{r}636 \\
651 \\
28\end{array}$ & $\begin{array}{r}0 \\
0 \\
31\end{array}$ & $\begin{array}{r}0 \\
31\end{array}$ & $\begin{array}{l}0 \\
0 \\
31\end{array}$ & $\begin{array}{r}15161 \\
12576 \\
25\end{array}$ \\
\hline $\begin{array}{l}\text { MAX } \\
\text { ?IIN }\end{array}$ & $\begin{array}{l}0 \\
0\end{array}$ & $\begin{array}{l}0 \\
0\end{array}$ & $\begin{array}{l}0 \\
0\end{array}$ & $\begin{array}{r}749 \\
0\end{array}$ & $\begin{array}{r}2240 \\
0\end{array}$ & 3152 & $\begin{array}{r}3474 \\
0\end{array}$ & $\begin{array}{r}3643 \\
0\end{array}$ & $\begin{array}{r}3717 \\
0\end{array}$ & $\begin{array}{r}1717 \\
0\end{array}$ & $\begin{array}{r}3665 \\
0\end{array}$ & $\begin{array}{r}356 ? \\
0\end{array}$ & $\begin{array}{r}3374 \\
0\end{array}$ & $\begin{array}{r}2939 \\
0\end{array}$ & $\begin{array}{r}18 b 2 \\
0\end{array}$ & $\begin{array}{l}0 \\
0\end{array}$ & $\begin{array}{l}0 \\
0\end{array}$ & $\begin{array}{l}0 \\
0\end{array}$ & $\begin{array}{r}35482 \\
0\end{array}$ \\
\hline
\end{tabular}


CHA3TAL - IHLANO SOLAK RADIATION DIFFERENCE STUDY IHSTRIJHENT : UV \#II MONTHLY DATA SUMMARY
CLINTIOH SEHSITIVITY $312,10 E-6$ V/H/SO.M

TARCH

EHERGY KILOJOULES PER SMUARE METER

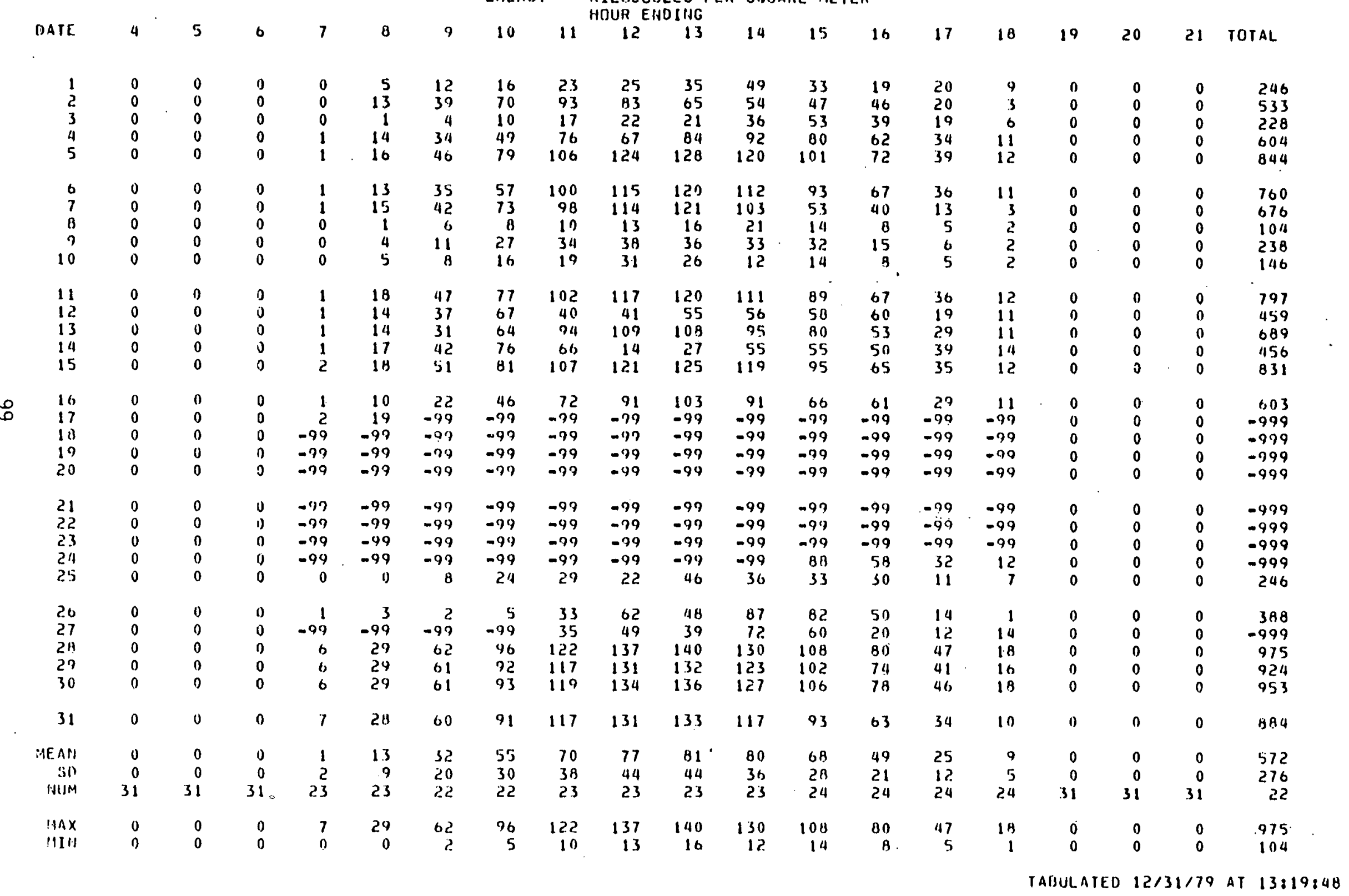


COISTAL - IIILAIND SOLAR RADIATIOH DIFFEREHCE STUOY INSTRUIAE IT UV $\$ 12$
SEHSITIVIIY $154.40-6$ V/W/SO.N

ENERG KILUJUULES PEA SIJUARE HETER HOUR ENDING

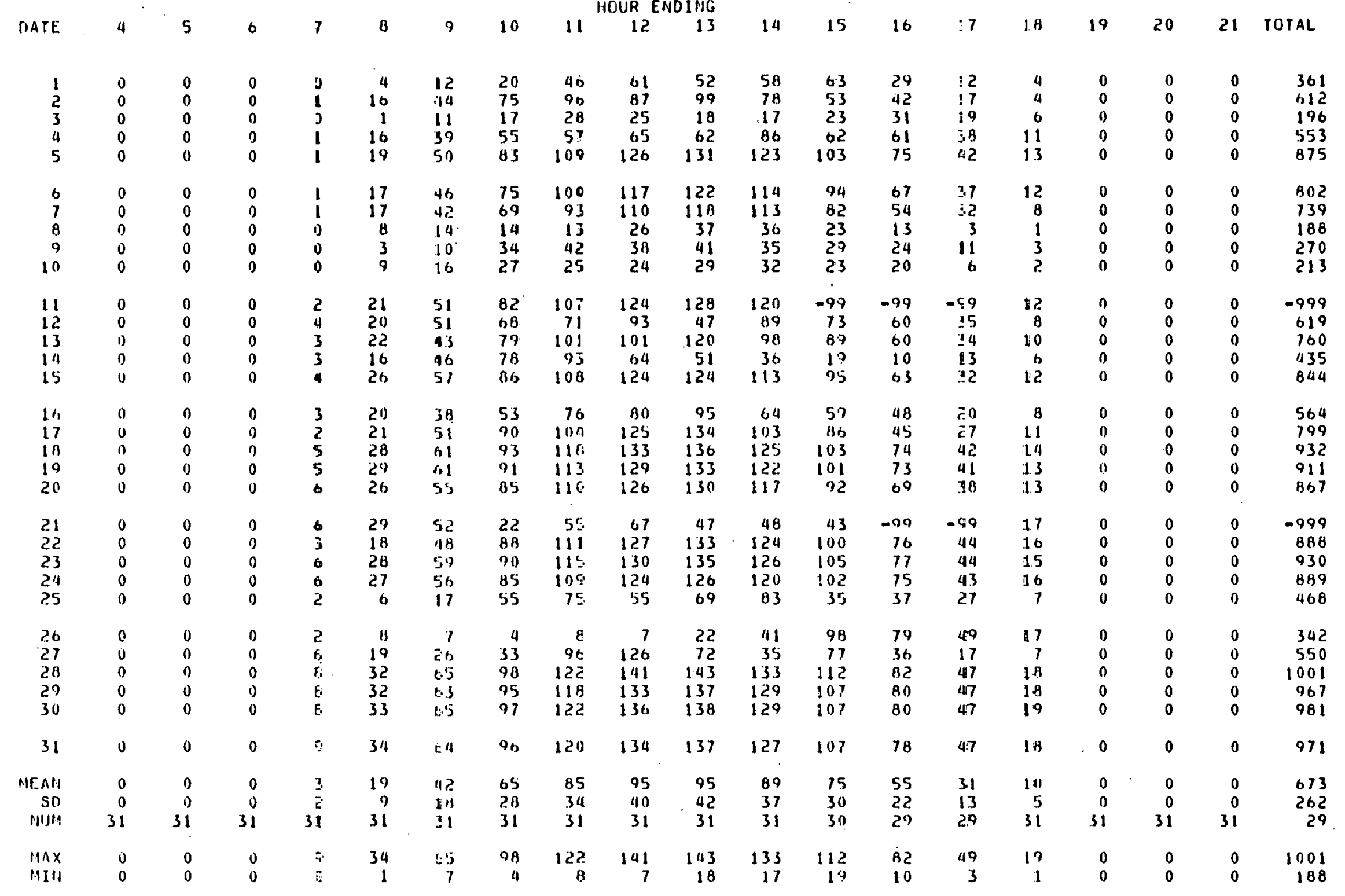


CIIASTAL - IALAHD SIILAR RADIATIUN DIFFERENCE STUDY mojivthly dota summary

IHSTRUIIEUI : PSP 42
SEINSITIVITY $10.99 E-6$ VIVISIJ.

EHERGY KILUJOULES PER SOUARE HETER HOUR ENDING

\begin{tabular}{|c|c|c|c|c|c|c|c|c|c|c|c|c|c|c|c|c|c|c|c|}
\hline & & & & & & & & & & & & & & & & & & & \\
\hline DATE & 4 & 5 & 6 & 7 & B & ) & 10 & 11 & 12 & 13 & 14 & 15 & 16 & 17 & 19 & 19 & 20 & 21 & TOTAL. \\
\hline $\begin{array}{l}1 \\
2 \\
3 \\
11 \\
5\end{array}$ & $\begin{array}{l}0 \\
0 \\
0 \\
0 \\
0\end{array}$ & $\begin{array}{l}0 \\
0 \\
0 \\
0 \\
0\end{array}$ & $\begin{array}{r}-99 \\
0 \\
0 \\
-555 \\
-99\end{array}$ & $\begin{array}{r}-99 \\
392 \\
73 \\
-595 \\
-99\end{array}$ & $\begin{array}{r}-99 \\
788 \\
400 \\
-555 \\
949\end{array}$ & $\begin{array}{r}1530 \\
1680 \\
587 \\
-555 \\
1727\end{array}$ & $\begin{array}{l}2257 \\
2406 \\
1046 \\
-555 \\
2397\end{array}$ & $\begin{array}{r}2780 \\
2914 \\
803 \\
-555 \\
2915\end{array}$ & $\begin{array}{l}3079 \\
3212 \\
1540 \\
-555 \\
3164\end{array}$ & $\begin{array}{r}3145 \\
32145 \\
947 \\
-555 \\
3039\end{array}$ & $\begin{array}{l}2958 \\
3042 \\
1553 \\
-555 \\
2970\end{array}$ & $\begin{array}{r}2565 \\
2590 \\
905 \\
-555 \\
2646\end{array}$ & $\begin{array}{r}1933 \\
1849 \\
341 \\
-555 \\
1994\end{array}$ & $\begin{array}{r}1193 \\
1093 \\
587 \\
-555 \\
1290\end{array}$ & $\begin{array}{r}426 \\
457 \\
-99 \\
-555 \\
546\end{array}$ & $\begin{array}{r}33 \\
35 \\
-666 \\
-555 \\
35\end{array}$ & $\begin{array}{l}0 \\
0 \\
0 \\
0 \\
0\end{array}$ & $\begin{array}{l}0 \\
0 \\
0 \\
0 \\
0\end{array}$ & $\begin{array}{r}-999 \\
23709 \\
-999 \\
-999 \\
-999\end{array}$ \\
\hline $\begin{array}{r}6 \\
7 \\
8 \\
9 \\
10\end{array}$ & $\begin{array}{l}0 \\
0 \\
0 \\
0 \\
0\end{array}$ & $\begin{array}{l}0 \\
0 \\
0 \\
0 \\
0\end{array}$ & $\begin{array}{r}-555 \\
8 \\
-97 \\
-97 \\
0\end{array}$ & $\begin{array}{r}-555 \\
274 \\
-79 \\
-79 \\
261\end{array}$ & $\begin{array}{r}-555 \\
991 \\
-49 \\
-99 \\
7112\end{array}$ & $\begin{array}{r}-555 \\
1813 \\
1917 \\
-39 \\
1614\end{array}$ & $\begin{array}{r}-555 \\
2488 \\
2608 \\
-77 \\
1156\end{array}$ & $\begin{array}{r}-555 \\
3006 \\
3106 \\
-99 \\
2276\end{array}$ & $\begin{array}{l}-555 \\
3287 \\
3371 \\
3224 \\
3124\end{array}$ & $\begin{array}{l}-555 \\
3323 \\
3411 \\
3273 \\
2665\end{array}$ & $\begin{array}{l}-555 \\
3114 \\
3217 \\
30133 \\
2050\end{array}$ & $\begin{array}{l}-555 \\
2695 \\
2729 \\
2667 \\
1562\end{array}$ & $\begin{array}{r}-555 \\
2069 \\
2094 \\
2048 \\
-99\end{array}$ & $\begin{array}{r}-555 \\
1329 \\
1331 \\
1324 \\
-99\end{array}$ & $\begin{array}{r}-555 \\
539 \\
538 \\
555 \\
-199\end{array}$ & $\begin{array}{r}-555 \\
41 \\
53 \\
60 \\
-99\end{array}$ & $\begin{array}{l}0 \\
0 \\
0 \\
0 \\
0\end{array}$ & $\begin{array}{l}0 \\
0 \\
0 \\
0 \\
0\end{array}$ & $\begin{array}{r}-999 \\
249611 \\
-999 \\
-999 \\
-999\end{array}$ \\
\hline $\begin{array}{l}11 \\
12 \\
13 \\
14 \\
15\end{array}$ & $\begin{array}{l}0 \\
0 \\
0 \\
0 \\
0\end{array}$ & $\begin{array}{l}0 \\
0 \\
0 \\
0 \\
0\end{array}$ & $\begin{array}{r}-99 \\
0 \\
-99 \\
21 \\
16\end{array}$ & $\begin{array}{r}-99 \\
85 \\
-97 \\
44 ! \\
370\end{array}$ & $\begin{array}{r}-99 \\
451 \\
55 \\
1236 \\
1051\end{array}$ & $\begin{array}{r}7611 \\
1315 \\
915 \\
2039 \\
1919\end{array}$ & $\begin{array}{r}1641 \\
-99 \\
161 \\
2723 \\
2604\end{array}$ & $\begin{array}{r}-99 \\
-79 \\
272 \\
3218 \\
3079\end{array}$ & $\begin{array}{r}-99 \\
-99 \\
213 \\
31137 \\
3387\end{array}$ & $\begin{array}{r}-99 \\
-79 \\
429 \\
3510 \\
3429\end{array}$ & $\begin{array}{r}-49 \\
-94 \\
360 \\
3280 \\
3233\end{array}$ & $\begin{array}{r}2293 \\
-99 \\
635 \\
2412 \\
2460\end{array}$ & $\begin{array}{r}1962 \\
-97 \\
367 \\
2163 \\
1919\end{array}$ & $\begin{array}{r}1186 \\
-79 \\
331 \\
1384 \\
1343\end{array}$ & $\begin{array}{l}283 \\
-99 \\
137 \\
542 \\
576\end{array}$ & $\begin{array}{r}42 \\
-99 \\
19 \\
44 \\
55\end{array}$ & $\begin{array}{l}0 \\
0 \\
0 \\
0 \\
0\end{array}$ & $\begin{array}{l}0 \\
0 \\
0 \\
0 \\
0\end{array}$ & $\begin{array}{r}-999 \\
-999 \\
-999 \\
26903 \\
25148\end{array}$ \\
\hline $\begin{array}{l}16 \\
17 \\
16 \\
19 \\
20\end{array}$ & $\begin{array}{l}0 \\
11 \\
0 \\
0 \\
0\end{array}$ & $\begin{array}{l}0 \\
0 \\
0 \\
0 \\
n\end{array}$ & $\begin{array}{r}-99 \\
-99 \\
-90 \\
43 \\
-99\end{array}$ & $\begin{array}{l}-99 \\
-89 \\
-99 \\
212 \\
-99\end{array}$ & $\begin{array}{r}18.11 \\
-99 \\
-99 \\
23.3 \\
-99\end{array}$ & $\begin{array}{r}2011 \\
839 \\
120 \\
102 \\
2075\end{array}$ & $\begin{array}{r}2695 \\
123 \\
138 \\
1148 \\
27110\end{array}$ & $\begin{array}{r}3157 \\
1216 \\
397 \\
858 \\
3228\end{array}$ & $\begin{array}{r}3593 \\
1249 \\
801 \\
1130 \\
3510\end{array}$ & $\begin{array}{r}3553 \\
3511 \\
1561 \\
1517 \\
35116\end{array}$ & $\begin{array}{r}3334 \\
414 \\
1840 \\
2293 \\
3051\end{array}$ & $\begin{array}{r}2908 \\
342 \\
1774 \\
17110 \\
2580\end{array}$ & $\begin{array}{r}2214 \\
534 \\
696 \\
12109 \\
-49\end{array}$ & $\begin{array}{l}-99 \\
116 \\
415 \\
-99 \\
-99\end{array}$ & $\begin{array}{r}-99 \\
53 \\
97 \\
-99 \\
549\end{array}$ & $\begin{array}{r}-99 \\
0 \\
15 \\
-99 \\
64\end{array}$ & $\begin{array}{l}0 \\
0 \\
0 \\
0 \\
0\end{array}$ & $\begin{array}{l}0 \\
0 \\
0 \\
0 \\
0\end{array}$ & $\begin{array}{l}-999 \\
-999 \\
-999 \\
-999 \\
-999\end{array}$ \\
\hline $\begin{array}{l}21 \\
22 \\
23 \\
24 \\
25\end{array}$ & $\begin{array}{l}0 \\
0 \\
0 \\
0 \\
0\end{array}$ & $\begin{array}{l}0 \\
0 \\
0 \\
0 \\
0\end{array}$ & $\begin{array}{r}-99 \\
0 \\
36 \\
14 \\
211\end{array}$ & $\begin{array}{l}-99 \\
461 \\
478 \\
269 \\
499\end{array}$ & $\begin{array}{r}1041 \\
1212 \\
1803 \\
911 \\
1177\end{array}$ & $\begin{array}{l}1950 \\
2073 \\
2155 \\
1365 \\
1501\end{array}$ & $\begin{array}{l}2.6201 \\
2777 \\
2761 \\
2.608 \\
1904\end{array}$ & $\begin{array}{r}3036 \\
3337 \\
3200 \\
3047 \\
797\end{array}$ & $\begin{array}{r}3167 \\
3577 \\
31168 \\
2677 \\
1111\end{array}$ & $\begin{array}{r}3136 \\
3613 \\
3557 \\
2910 \\
594\end{array}$ & $\begin{array}{r}3042 \\
3396 \\
3320 \\
2752 \\
345\end{array}$ & $\begin{array}{r}2201 \\
2954 \\
2751 \\
2615 \\
338\end{array}$ & $\begin{array}{r}524 \\
2286 \\
2106 \\
2019 \\
217\end{array}$ & $\begin{array}{r}425 \\
1575 \\
1343 \\
1252 \\
47\end{array}$ & $\begin{array}{r}370 \\
717 \\
4134 \\
567 \\
86\end{array}$ & $\begin{array}{r}26 \\
117 \\
75 \\
129 \\
37\end{array}$ & $\begin{array}{l}0 \\
0 \\
0 \\
0 \\
0\end{array}$ & $\begin{array}{l}0 \\
0 \\
0 \\
0 \\
0\end{array}$ & $\begin{array}{r}-949 \\
281095 \\
27605 \\
23635 \\
8042\end{array}$ \\
\hline $\begin{array}{l}26 \\
27 \\
213 \\
29 \\
30\end{array}$ & $\begin{array}{l}11 \\
0 \\
0 \\
0 \\
0\end{array}$ & $\begin{array}{l}0 \\
0 \\
0 \\
0 \\
0\end{array}$ & $\begin{array}{r}0 \\
-51.5 \\
65 \\
0 \\
-90\end{array}$ & $\begin{array}{r}109 \\
-555 \\
512 \\
-562 \\
-99\end{array}$ & $\begin{array}{r}384 \\
-555 \\
1657 \\
1369 \\
697\end{array}$ & $\begin{array}{r}154 \\
-5513 \\
2355 \\
2155 \\
13615\end{array}$ & $\begin{array}{l}1469 \\
-555 \\
2110 \\
21162 \\
1745\end{array}$ & $\begin{array}{r}2799 \\
-555 \\
3324 \\
3321 \\
3039\end{array}$ & $\begin{array}{r}3316 \\
-5.55 \\
3586 \\
3596 \\
2941\end{array}$ & $\begin{array}{r}3120 \\
-555 \\
3613 \\
3622 \\
2414\end{array}$ & $\begin{array}{l}3005 \\
-555 \\
3403 \\
3410 \\
2640\end{array}$ & $\begin{array}{l}2035 \\
-555 \\
29118 \\
2981 \\
1654\end{array}$ & $\begin{array}{l}2114 \\
-555 \\
2319 \\
2197 \\
1247\end{array}$ & $\begin{array}{r}646 \\
-555 \\
15112 \\
1529 \\
43 ?\end{array}$ & $\begin{array}{r}286 \\
-555 \\
773 \\
789 \\
127\end{array}$ & $\begin{array}{r}-99 \\
-555 \\
124 \\
134 \\
19\end{array}$ & $\begin{array}{l}0 \\
0 \\
0 \\
0 \\
0\end{array}$ & $\begin{array}{l}0 \\
0 \\
0 \\
0 \\
0\end{array}$ & $\begin{array}{r}-999 \\
-999 \\
29171 \\
-999 \\
-999\end{array}$ \\
\hline $\begin{array}{c}\text { MEAU } \\
\text { SO } \\
\text { IJUM }\end{array}$ & $\begin{array}{r}0 \\
0 \\
30\end{array}$ & $\begin{array}{r}0 \\
0 \\
30\end{array}$ & $\begin{array}{l}1.2 \\
1.7 \\
1.5\end{array}$ & $\begin{array}{r}326 \\
160 \\
14\end{array}$ & $\begin{array}{r}923 \\
152 \\
20\end{array}$ & $\begin{array}{r}1461 \\
687 \\
212\end{array}$ & $\begin{array}{r}31.37 \\
1127 \\
0.5\end{array}$ & $\begin{array}{r}3 ! 124 \\
1034 \\
211\end{array}$ & $\begin{array}{r}2725 \\
1076 \\
25\end{array}$ & $\begin{array}{r}2737 \\
1038 \\
25\end{array}$ & $\begin{array}{r}2606 \\
954 \\
25\end{array}$ & $\begin{array}{r}2167 \\
801 \\
21\end{array}$ & $\begin{array}{r}1602 \\
716 \\
211\end{array}$ & $\begin{array}{r}1008 \\
476 \\
2 ?\end{array}$ & $\begin{array}{r}431 \\
2.17 \\
22\end{array}$ & $\begin{array}{l}35 \\
38 \\
21\end{array}$ & $\begin{array}{r}0 \\
0 \\
30\end{array}$ & $\begin{array}{r}0 \\
0 \\
30\end{array}$ & $\begin{array}{r}24176 \\
5486 \\
9\end{array}$ \\
\hline $\begin{array}{l}M 4 X \\
H 1 N\end{array}$ & $\begin{array}{l}0 \\
0\end{array}$ & $\begin{array}{l}0 \\
0\end{array}$ & $\begin{array}{r}1.5 \\
0\end{array}$ & $\begin{array}{r}317 \\
73\end{array}$ & $\begin{array}{r}136.3 \\
3,5\end{array}$ & $\begin{array}{r}2345 \\
75\end{array}$ & $\begin{array}{r}7.962 \\
161\end{array}$ & $\begin{array}{r}3337 \\
272\end{array}$ & $\begin{array}{r}3496 \\
213\end{array}$ & $\begin{array}{r}3622 \\
1129\end{array}$ & $\begin{array}{r}3410 \\
345\end{array}$ & $\begin{array}{r}2944 \\
3.38\end{array}$ & $\begin{array}{r}2319 \\
217\end{array}$ & $\begin{array}{r}1532 \\
47\end{array}$ & $\begin{array}{r}7119 \\
53\end{array}$ & $\begin{array}{r}134 \\
0\end{array}$ & $\begin{array}{l}0 \\
0\end{array}$ & $\begin{array}{l}0 \\
0\end{array}$ & $\begin{array}{r}29171 \\
5000\end{array}$ \\
\hline
\end{tabular}


CJKSTAL - IHLARO SOLAK RADIATIUH DIFFERENCE STUIYY MiCITIILY DATA SIJMMAKY

IHSTIVUIEHT: PSP \#3 CAPE FEAK APRIL 197H

SENSITIVITY 11.2.7E-ذ V/H/SO."H

EWERGY KILUJUULES PER SUUARE METER

\begin{tabular}{|c|c|c|c|c|c|c|c|c|c|c|c|c|c|c|c|c|c|c|c|}
\hline & & & & & & & & & HOUR E & ADIAT & & & & & & & & & \\
\hline DATE & 4 & 5 & 6 & $i$ & 8 & 9 & 10 & 11 & 12 & 13 & 14 & 15 & 16 & 1.7 & 10 & 19 & 20 & 21 & TOTAL \\
\hline $\begin{array}{l}1 \\
2 \\
3 \\
4 \\
5\end{array}$ & $\begin{array}{l}0 \\
0 \\
0 \\
0 \\
0\end{array}$ & $\begin{array}{l}0 \\
0 \\
0 \\
0 \\
0\end{array}$ & $\begin{array}{l}0 \\
0 \\
0 \\
0 \\
0\end{array}$ & $\begin{array}{l}207 \\
189 \\
172 \\
153 \\
22^{13}\end{array}$ & $\begin{array}{l}831 \\
828 \\
393 \\
428 \\
891\end{array}$ & $\begin{array}{r}1 \text { Sish } \\
1620 \\
897 \\
1401 \\
1696\end{array}$ & $\begin{array}{l}2278 \\
2323 \\
1127 \\
2201 \\
2370\end{array}$ & $\begin{array}{r}2818 \\
2872 \\
-95 \\
2526 \\
2911\end{array}$ & $\begin{array}{l}3131 \\
3205 \\
1479 \\
2958 \\
3325\end{array}$ & $\begin{array}{r}3214 \\
3310 \\
955 \\
3063 \\
3168\end{array}$ & $\begin{array}{r}3045 \\
3007 \\
645 \\
2105 \\
2456\end{array}$ & $\begin{array}{r}21330 \\
25138 \\
594 \\
11172 \\
2066\end{array}$ & $\begin{array}{r}2051 \\
1940 \\
396 \\
1003 \\
2034\end{array}$ & $\begin{array}{r}1294 \\
1151 \\
163 \\
1154 \\
1124\end{array}$ & $\begin{array}{r}525 \\
423 \\
96 \\
469 \\
405\end{array}$ & $\begin{array}{l}49 \\
42 \\
16 \\
57 \\
38\end{array}$ & $\begin{array}{l}0 \\
0 \\
0 \\
0 \\
0\end{array}$ & $\begin{array}{l}0 \\
0 \\
0 \\
0 \\
0\end{array}$ & $\begin{array}{r}23605 \\
23628 \\
-999 \\
19057 \\
22715\end{array}$ \\
\hline $\begin{array}{r}6 \\
7 \\
1 \\
7 \\
11\end{array}$ & $\begin{array}{l}0 \\
0 \\
0 \\
0 \\
0\end{array}$ & $\begin{array}{l}0 \\
0 \\
0 \\
0 \\
0\end{array}$ & $\begin{array}{l}0 \\
0 \\
0 \\
0 \\
0\end{array}$ & $\begin{array}{l}273 \\
215 \\
321 \\
220 \\
6110\end{array}$ & $\begin{array}{r}960 \\
907 \\
1009 \\
977 \\
1157\end{array}$ & $\begin{array}{l}1755 \\
1676 \\
18513 \\
1710 \\
1738\end{array}$ & $\begin{array}{l}2305 \\
2424 \\
2509 \\
2453 \\
2035\end{array}$ & $\begin{array}{l}2922 \\
2973 \\
3030 \\
2922 \\
2412\end{array}$ & $\begin{array}{l}3046 \\
3322 \\
3343 \\
3274 \\
2911\end{array}$ & $\begin{array}{l}3276 \\
3334 \\
3384 \\
3360 \\
3000\end{array}$ & $\begin{array}{l}2905 \\
3092 \\
3193 \\
3095 \\
2620\end{array}$ & $\begin{array}{l}1953 \\
2016 \\
2720 \\
2667 \\
2310\end{array}$ & $\begin{array}{l}1315 \\
2053 \\
2116 \\
2111 \\
1122\end{array}$ & $\begin{array}{l}605 \\
1255 \\
13155 \\
1277 \\
1055\end{array}$ & $\begin{array}{l}222 \\
543 \\
526 \\
597 \\
442\end{array}$ & $\begin{array}{l}24 \\
57 \\
59 \\
73 \\
74\end{array}$ & $\begin{array}{l}0 \\
0 \\
0 \\
0 \\
0\end{array}$ & $\begin{array}{l}0 \\
0 \\
0 \\
0 \\
0\end{array}$ & $\begin{array}{l}21467 \\
24517 \\
25433 \\
24766 \\
21506\end{array}$ \\
\hline $\begin{array}{l}11 \\
12 \\
13 \\
14 \\
15\end{array}$ & $\begin{array}{l}0 \\
0 \\
0 \\
0 \\
0\end{array}$ & $\begin{array}{l}0 \\
0 \\
0 \\
0 \\
0\end{array}$ & $\begin{array}{r}11 \\
-79 \\
0 \\
11 \\
10\end{array}$ & $\begin{array}{r}267 \\
-90 \\
30 \\
401 \\
365 .\end{array}$ & $\begin{array}{r}369 \\
-99 \\
113 \\
1209 \\
1314\end{array}$ & $\begin{array}{r}957 \\
-79 \\
2199 \\
2040 \\
1940\end{array}$ & $\begin{array}{r}2382 \\
1598 \\
177 \\
2723 \\
25132\end{array}$ & $\begin{array}{r}2921 \\
1218 \\
90 \\
3250 \\
3096\end{array}$ & $\begin{array}{r}2608 \\
2352 \\
403 \\
3531 \\
334 ?\end{array}$ & $\begin{array}{r}3088 \\
3432 \\
662 \\
3567 \\
3396\end{array}$ & $\begin{array}{r}2663 \\
3211 \\
611 \\
3327 \\
3240\end{array}$ & $\begin{array}{r}2439 \\
2793 \\
347 \\
28365 \\
2786\end{array}$ & $\begin{array}{r}1736 \\
1941 \\
429 \\
22611 \\
2179\end{array}$ & $\begin{array}{r}118101 \\
1416 \\
327 \\
11993 \\
140.3\end{array}$ & $\begin{array}{l}32.8 \\
621 \\
196 \\
513 \\
633\end{array}$ & $\begin{array}{l}50 \\
62 \\
10 \\
72 \\
84\end{array}$ & $\begin{array}{l}0 \\
0 \\
0 \\
0 \\
0\end{array}$ & $\begin{array}{l}0 \\
0 \\
0 \\
0 \\
0\end{array}$ & $\begin{array}{r}21000 \\
-999 \\
3724 \\
27283 \\
26373\end{array}$ \\
\hline $\begin{array}{l}16 \\
17 \\
17 \\
19 \\
20\end{array}$ & $\begin{array}{l}0 \\
0 \\
0 \\
0 \\
0\end{array}$ & $\begin{array}{l}0 \\
0 \\
0 \\
0 \\
0\end{array}$ & $\begin{array}{r}13 \\
0 \\
11 \\
10 \\
25\end{array}$ & $\begin{array}{r}365 \\
111 \\
52 \\
190 \\
450\end{array}$ & $\begin{array}{r}1165 \\
530 \\
135 \\
282 \\
1242\end{array}$ & $\begin{array}{r}1555 \\
635 \\
420 \\
36 \\
2034\end{array}$ & $\begin{array}{r}2435 \\
693 \\
1691 \\
1511 \\
27110\end{array}$ & $\begin{array}{r}31911 \\
1325 \\
1635 \\
3116 \\
3255\end{array}$ & $\begin{array}{r}3511 \\
725 \\
1272 \\
978 \\
3545\end{array}$ & $\begin{array}{r}3546 \\
434 \\
1962 \\
1326 \\
3596\end{array}$ & $\begin{array}{r}3348 \\
217 \\
1771 \\
1751 \\
3318\end{array}$ & $\begin{array}{r}2863 \\
306 \\
1950 \\
1496 \\
2925\end{array}$ & $\begin{array}{r}2269 \\
303 \\
1563 \\
732 \\
22413\end{array}$ & $\begin{array}{r}14 \times 6 \\
93 \\
742 \\
3 \text { fil } \\
7122\end{array}$ & $\begin{array}{r}6817 \\
41 \\
212 \\
12.5 \\
41.8\end{array}$ & $\begin{array}{l}93 \\
16 \\
17 \\
39 \\
99\end{array}$ & $\begin{array}{l}0 \\
0 \\
0 \\
0 \\
0\end{array}$ & $\begin{array}{l}0 \\
0 \\
0 \\
0 \\
0\end{array}$ & $\begin{array}{r}26300 \\
5428 \\
13420 \\
7455 \\
26597\end{array}$ \\
\hline $\begin{array}{l}21 \\
22 \\
23 \\
24 \\
25\end{array}$ & $\begin{array}{l}0 \\
0 \\
0 \\
0 \\
0\end{array}$ & $\begin{array}{l}0 \\
0 \\
0 \\
0 \\
0\end{array}$ & $\begin{array}{r}22 \\
31 \\
-99 \\
21 \\
35\end{array}$ & $\begin{array}{l}351 \\
475 \\
-90 \\
345 \\
460\end{array}$ & $\begin{array}{r}841 \\
1245 \\
-95 \\
1046 \\
1220\end{array}$ & $\begin{array}{r}17112 \\
2079 \\
-139 \\
113313 \\
1058\end{array}$ & $\begin{array}{r}2660 \\
2.709 \\
-97 \\
1079 \\
1324\end{array}$ & $\begin{array}{r}3127 \\
3267 \\
-99 \\
1813 \\
2578\end{array}$ & $\begin{array}{r}3009 \\
-30 \\
-79 \\
2212 \\
3664\end{array}$ & $\begin{array}{r}3580 \\
-99 \\
3657 \\
2675 \\
1635\end{array}$ & $\begin{array}{l}3306 \\
3300 \\
2506 \\
1110\end{array}$ & $\begin{array}{r}2519 \\
-97 \\
2823 \\
2174 \\
1173\end{array}$ & $\begin{array}{r}-99 \\
-99 \\
1977 \\
1493 \\
140 ?\end{array}$ & $\begin{array}{r}360 \\
-90 \\
185 \\
1059 \\
256\end{array}$ & $\begin{array}{l}3.35 \\
-99 \\
425 \\
67.6 \\
124\end{array}$ & $\begin{array}{r}99 \\
-99 \\
60 \\
101 \\
16\end{array}$ & $\begin{array}{l}0 \\
0 \\
0 \\
0 \\
0\end{array}$ & $\begin{array}{l}0 \\
0 \\
0 . \\
0 \\
0\end{array}$ & $\begin{array}{r}-999 \\
-999 \\
-999 \\
19716 \\
16129\end{array}$ \\
\hline $\begin{array}{l}20 \\
27 \\
28 \\
29 \\
30\end{array}$ & $\begin{array}{l}0 \\
0 \\
0 \\
0 \\
0\end{array}$ & $\begin{array}{l}0 \\
0 \\
0 \\
0 \\
0\end{array}$ & $\begin{array}{r}1 \\
0 \\
54 \\
53 \\
20\end{array}$ & $\begin{array}{r}167 \\
615 \\
555 \\
555 \\
384\end{array}$ & $\begin{array}{r}282 \\
107 \\
13411 \\
1353 \\
1011\end{array}$ & $\begin{array}{r}1003 \\
215 \\
2127 \\
2145 \\
1015\end{array}$ & $\begin{array}{r}2931 \\
487 \\
2314 \\
2851 \\
2272\end{array}$ & $\begin{array}{l}25117 \\
1001 \\
3373 \\
3314 \\
3035\end{array}$ & $\begin{array}{r}2211 \\
621 \\
3638 \\
3577 \\
2914\end{array}$ & $\begin{array}{r}2790 \\
409 \\
36116 \\
3589 \\
1952\end{array}$ & $\begin{array}{r}1950 \\
532 \\
-99 \\
3173 \\
2741\end{array}$ & $\begin{array}{r}1384 \\
253 \\
-94 \\
2921 \\
2.584\end{array}$ & $\begin{array}{r}114 B \\
138 \\
-99 \\
20411 \\
2093\end{array}$ & $\begin{array}{r}12 \text { (ats } \\
112 \\
1610 \\
17 \mathrm{~m} \\
12 \mathrm{gn}\end{array}$ & $\begin{array}{r}44.8 \\
97 \\
798 \\
7048 \\
547\end{array}$ & $\begin{array}{r}14 \\
42 \\
110 \\
59 \\
106\end{array}$ & $\begin{array}{l}0 \\
0 \\
0 \\
0 \\
0\end{array}$ & $\begin{array}{l}0 \\
0 \\
0 \\
0 \\
0\end{array}$ & $\begin{array}{r}19012 \\
4657 \\
-999 \\
28115 \\
22570\end{array}$ \\
\hline $\begin{array}{r}\text { HEAII } \\
\text { SII } \\
\text { HIISA }\end{array}$ & $\begin{array}{r}0 \\
0 \\
30\end{array}$ & $\begin{array}{r}0 \\
0 \\
30\end{array}$ & $\begin{array}{l}11 \\
16 \\
2 B\end{array}$ & $\begin{array}{r}27 \% \\
150 \\
2 \pi\end{array}$ & $\begin{array}{r}8.33 \\
1100 \\
23\end{array}$ & $\begin{array}{r}1471 \\
617 \\
213\end{array}$ & $\begin{array}{r}2051 \\
7131 \\
20\end{array}$ & $\begin{array}{r}2113 B \\
906 \\
2[1\end{array}$ & $\begin{array}{r}2068 \\
1013 \\
28\end{array}$ & $\begin{array}{r}2739 \\
1010 \\
29\end{array}$ & $\begin{array}{r}2447 \\
971 \\
28\end{array}$ & $\begin{array}{r}207 ? \\
8117 \\
20\end{array}$ & $\begin{array}{r}15213 \\
691 \\
27\end{array}$ & $\begin{array}{r}973 \\
4(121) \\
207\end{array}$ & $\begin{array}{r}422 \\
205 \\
29\end{array}$ & $\begin{array}{l}57 \\
32 \\
29\end{array}$ & $\begin{array}{r}0 \\
0 \\
30\end{array}$ & $\begin{array}{r}0 \\
0 \\
30\end{array}$ & $\begin{array}{r}14788 \\
1304 \\
24\end{array}$ \\
\hline $\begin{array}{l}\text { MAX } \\
\text { MIII }\end{array}$ & $\begin{array}{l}0 \\
0\end{array}$ & $\begin{array}{l}0 \\
0\end{array}$ & $\begin{array}{r}54 \\
0\end{array}$ & $\begin{array}{c}6.110 \\
3 r\end{array}$ & $\begin{array}{r}1353 \\
113\end{array}$ & $\begin{array}{r}21: 15 \\
30\end{array}$ & $\begin{array}{l}2.361 \\
-154\end{array}$ & $\begin{array}{r}337.5 \\
90\end{array}$ & $\begin{array}{r}36 b l \\
4(1) .3\end{array}$ & $\begin{array}{r}36866 \\
434\end{array}$ & $\begin{array}{r}3360 \\
217\end{array}$ & $\begin{array}{r}2925 \\
253\end{array}$ & $\begin{array}{r}2261) \\
130\end{array}$ & $\begin{array}{r}17407 \\
m ?\end{array}$ & $\begin{array}{r}7931 \\
111\end{array}$ & $\begin{array}{r}140 \\
10\end{array}$ & $\begin{array}{l}0 \\
0\end{array}$ & $\begin{array}{l}0 \\
0\end{array}$ & $\begin{array}{r}28115 \\
3724\end{array}$ \\
\hline
\end{tabular}

TABIHA.ATEO O1/02/80 AT 13:08:53 
CIJATTAL - INLAHO GULAR RAOIATIUN UIFFERENCE STUOY M(I)ITHLY DATA SUMMARY

INSTHUMENT: I'SP $\# 4 \quad$ WALLACE
SEHSITIVITY $11.00 F-6$ V/HISO.N

FHERGY KILUJUULES PEH SIJUARE METER HONR ENDING

\begin{tabular}{|c|c|c|c|c|c|c|c|c|c|c|c|c|c|c|c|c|c|c|c|}
\hline \multicolumn{20}{|c|}{ ל } \\
\hline DATE & 1 & 5 & 6 & 7 & B & 9 & 10 & 11 & 12 & 13 & 14 & 15 & 16 & 17 & 18 & 19 & 20 & 21 & TUIAL \\
\hline $\begin{array}{l}1 \\
2 \\
3 \\
4 \\
5\end{array}$ & $\begin{array}{l}0 \\
0 \\
0 \\
0 \\
0\end{array}$ & $\begin{array}{l}0 \\
0 \\
0 \\
0 \\
0\end{array}$ & $\begin{array}{r}0 \\
11 \\
0 \\
0 \\
-606\end{array}$ & $\begin{array}{l}696 \\
303 \\
35 \\
233 \\
250\end{array}$ & $\begin{array}{l}833 \\
843 \\
257 \\
3711 \\
929\end{array}$ & $\begin{array}{r}1569 \\
16.38 \\
470 \\
155 \\
1605\end{array}$ & $\begin{array}{r}2234 \\
2345 \\
6.34 \\
1964 \\
2359\end{array}$ & $\begin{array}{l}2761 \\
21369 \\
1236 \\
2671 \\
2876\end{array}$ & $\begin{array}{l}3065 \\
3160 \\
1161 \\
3008 \\
3148\end{array}$ & $\begin{array}{r}3117 \\
3225 \\
886 \\
2848 \\
2935\end{array}$ & $\begin{array}{l}2937 \\
3027 \\
1845 \\
2216 \\
2621\end{array}$ & $\begin{array}{r}2378 \\
2534 \\
794 \\
1264 \\
2647\end{array}$ & $\begin{array}{l}1897 \\
1959 \\
342 \\
1644 \\
1960\end{array}$ & $\begin{array}{l}1220 \\
1229 \\
179 \\
1091 \\
1204\end{array}$ & $\begin{array}{l}447^{3} \\
463 \\
103 \\
534 \\
595\end{array}$ & $\begin{array}{r}64 \\
110 \\
54 \\
122 \\
117\end{array}$ & $\begin{array}{l}0 \\
0 \\
0 \\
0 \\
0\end{array}$ & $\begin{array}{l}0 \\
0 \\
0 \\
0 \\
0\end{array}$ & $\begin{array}{r}23224 \\
23806 \\
7999 \\
18828 \\
-999\end{array}$ \\
\hline $\begin{array}{r}6 \\
7 \\
6 \\
9 \\
10\end{array}$ & $\begin{array}{l}0 \\
0 \\
0 \\
0 \\
0\end{array}$ & $\begin{array}{l}0 \\
0 \\
0 \\
0 \\
0\end{array}$ & $\begin{array}{r}13 \\
130 \\
17 \\
-600 \\
-606\end{array}$ & $\begin{array}{l}286 \\
255 \\
330 \\
315 \\
191\end{array}$ & $\begin{array}{r}961 \\
958 \\
1004 \\
1019 \\
714\end{array}$ & $\begin{array}{l}1707 \\
1691 \\
1855 \\
1781 \\
1333\end{array}$ & $\begin{array}{l}2352 \\
2125 \\
2516 \\
2110 \\
2131\end{array}$ & $\begin{array}{l}2594 \\
2722 \\
3020 \\
2920 \\
2678\end{array}$ & $\begin{array}{l}2914 \\
3213 \\
3315 \\
3192 \\
2959\end{array}$ & $\begin{array}{l}2977 \\
3269 \\
3361 \\
3287 \\
2593\end{array}$ & $\begin{array}{l}2777 \\
3009 \\
3171 \\
3035 \\
2684\end{array}$ & $\begin{array}{l}1635 \\
2690 \\
2719 \\
2229 \\
2537\end{array}$ & $\begin{array}{l}1416 \\
2078 \\
2091 \\
1719 \\
367\end{array}$ & $\begin{array}{r}525 \\
1354 \\
1322 \\
1248 \\
672\end{array}$ & $\begin{array}{l}257 \\
56 ? \\
559 \\
479 \\
299\end{array}$ & $\begin{array}{l}113 \\
130 \\
107 \\
164 \\
63\end{array}$ & $\begin{array}{l}0 \\
0 \\
0 \\
0 \\
0\end{array}$ & $\begin{array}{l}0 \\
0 \\
0 \\
0 \\
0\end{array}$ & $\begin{array}{r}20522 \\
24812 \\
25389 \\
-999 \\
-999\end{array}$ \\
\hline $\begin{array}{l}11 \\
12 \\
13 \\
14 \\
15\end{array}$ & $\begin{array}{l}0 \\
0 \\
0 \\
0 \\
0\end{array}$ & $\begin{array}{l}0 \\
0 \\
0 \\
0 \\
0\end{array}$ & $\begin{array}{r}-606 \\
8 \\
11 \\
-666 \\
1116\end{array}$ & $\begin{array}{r}148 \\
93 \\
30 \\
330 \\
320\end{array}$ & $\begin{array}{l}233 \\
100 \\
135 \\
1063 \\
1078\end{array}$ & $\begin{array}{r}911 \\
973 \\
57 \\
1973 \\
1867\end{array}$ & $\begin{array}{r}1057 \\
2295 \\
132 \\
2660 \\
2535\end{array}$ & $\begin{array}{r}2629 \\
2341 \\
204 \\
3154 \\
3003\end{array}$ & $\begin{array}{r}2573 \\
3342 \\
227 \\
3433 \\
3337\end{array}$ & $\begin{array}{r}2587 \\
3405 \\
489 \\
3459 \\
3386\end{array}$ & $\begin{array}{r}2478 \\
3189 \\
263 \\
3256 \\
3278\end{array}$ & $\begin{array}{r}2426 \\
2766 \\
500 \\
2827 \\
2672\end{array}$ & $\begin{array}{r}1912 \\
2138 \\
744 \\
2116 \\
1590\end{array}$ & $\begin{array}{r}1117 \\
1398 \\
397 \\
1377 \\
1393\end{array}$ & $\begin{array}{r}305 \\
593 \\
86 \\
461 \\
61.0\end{array}$ & $\begin{array}{l}115 \\
129 \\
266 \\
157 \\
224\end{array}$ & $\begin{array}{l}0 \\
0 \\
0 \\
0 \\
0\end{array}$ & $\begin{array}{l}0 \\
0 \\
0 \\
0 \\
0\end{array}$ & $\begin{array}{r}-999 \\
23090 \\
3538 \\
-999 \\
25454\end{array}$ \\
\hline $\begin{array}{l}16 \\
17 \\
18 \\
17 \\
20\end{array}$ & $\begin{array}{l}0 \\
0 \\
0 \\
0 \\
0\end{array}$ & $\begin{array}{l}0 \\
0 \\
0 \\
0 \\
0\end{array}$ & $\begin{array}{r}24 \\
0 \\
-99 \\
-49 \\
-99\end{array}$ & $\begin{array}{l}380 \\
250 \\
-29 \\
-99 \\
-99\end{array}$ & $\begin{array}{r}1140 \\
734 \\
-99 \\
-97 \\
1073\end{array}$ & $\begin{array}{r}19413 \\
541 \\
667 \\
2314 \\
1849\end{array}$ & $\begin{array}{r}3652 \\
1117 \\
323 \\
719 \\
2539\end{array}$ & $\begin{array}{r}3165 \\
449 \\
385 \\
880 \\
3076\end{array}$ & $\begin{array}{l}3476 \\
2397 \\
1220 \\
1698 \\
3351\end{array}$ & $\begin{array}{r}3441 \\
869 \\
2470 \\
2140 \\
3167\end{array}$ & $\begin{array}{r}3296 \\
289 \\
2303 \\
2536 \\
3056\end{array}$ & $\begin{array}{r}2750 \\
800 \\
948 \\
2195 \\
27112\end{array}$ & $\begin{array}{r}27.79 \\
3197 \\
673 \\
1891 \\
1737\end{array}$ & $\begin{array}{r}1493 \\
93 \\
372 \\
1351 \\
1489\end{array}$ & $\begin{array}{r}6131 \\
37 \\
-99 \\
166 \\
768\end{array}$ & $\begin{array}{l}148 \\
119 \\
-79 \\
-99 \\
130\end{array}$ & $\begin{array}{l}0 \\
0 \\
0 \\
0 \\
0\end{array}$ & $\begin{array}{l}0 \\
0 \\
0 \\
0 \\
0\end{array}$ & $\begin{array}{r}26876 \\
8106 \\
-999 \\
-999 \\
-999\end{array}$ \\
\hline $\begin{array}{l}21 \\
22 \\
23 \\
24 \\
25\end{array}$ & $\begin{array}{l}0 \\
0 \\
0 \\
0 \\
0\end{array}$ & $\begin{array}{l}0 \\
0 \\
0 \\
0 \\
0\end{array}$ & $\begin{array}{r}9 \\
-8138 \\
20 \\
0 \\
-006\end{array}$ & $\begin{array}{l}2513 \\
327 \\
654 \\
197 \\
359\end{array}$ & $\begin{array}{r}922 \\
971 \\
1246 \\
907 \\
502\end{array}$ & $\begin{array}{l}1517 \\
1970 \\
1913 \\
1724 \\
1763\end{array}$ & $\begin{array}{l}2101 \\
2603 \\
26114 \\
2462 \\
3.104\end{array}$ & $\begin{array}{l}21321 \\
3187 \\
3131 \\
2432 \\
1282\end{array}$ & $\begin{array}{r}27119 \\
35115 \\
31106 \\
2419 \\
8150\end{array}$ & $\begin{array}{r}3187 \\
3577 \\
3478 \\
2390 \\
437\end{array}$ & $\begin{array}{r}2663 \\
3413 \\
3226 \\
3159 \\
323\end{array}$ & $\begin{array}{r}2320 \\
2994 \\
2811 \\
2249 \\
201\end{array}$ & $\begin{array}{l}11137 \\
2336 \\
2133 \\
21108 \\
2311\end{array}$ & $\begin{array}{r}549 \\
10146 \\
1309 \\
1434 \\
84\end{array}$ & $\begin{array}{l}147 \\
82.1 \\
435 \\
8116 \\
110\end{array}$ & $\begin{array}{l}1111 \\
134 \\
127 \\
125 \\
182\end{array}$ & $\begin{array}{l}0 \\
0 \\
0 \\
0 \\
0\end{array}$ & $\begin{array}{l}0 \\
0 \\
0 \\
0 \\
0\end{array}$ & $\begin{array}{r}20604 \\
-999 \\
26576 \\
22417 \\
-999\end{array}$ \\
\hline $\begin{array}{l}20 \\
27 \\
27 \\
29 \\
30\end{array}$ & $\begin{array}{l}0 \\
0 \\
0 \\
0 \\
0\end{array}$ & $\begin{array}{l}0 \\
1 \\
0 \\
0 \\
0\end{array}$ & $\begin{array}{r}0 \\
10 \\
113 \\
70 \\
-666\end{array}$ & $\begin{array}{l}225 \\
484 \\
499 \\
493 \\
275\end{array}$ & $\begin{array}{r}300 \\
1013 \\
1324 \\
1344 \\
553\end{array}$ & $\begin{array}{r}932 \\
291 \\
21193 \\
2122 \\
1100\end{array}$ & $\begin{array}{r}1681 \\
2209 \\
2764 \\
26115 \\
1253\end{array}$ & $\begin{array}{r}2771 \\
199 \\
3261 \\
3251 \\
2372\end{array}$ & $\begin{array}{r}1750 \\
334 \\
3543 \\
3513 \\
2706\end{array}$ & $\begin{array}{l}2555 \\
1497 \\
3585 \\
3507 \\
3171\end{array}$ & $\begin{array}{l}1737 \\
1993 \\
3396 \\
3320 \\
3001\end{array}$ & $\begin{array}{l}1243 \\
1044 \\
2950 \\
2878 \\
1911\end{array}$ & $\begin{array}{r}-99 \\
601 \\
2348 \\
2227 \\
107 ?\end{array}$ & $\begin{array}{r}-97 \\
334 \\
1599 \\
1461 \\
1076\end{array}$ & $\begin{array}{l}-94 \\
242 \\
790 \\
715 \\
337\end{array}$ & $\begin{array}{r}-99 \\
-848 \\
120 \\
133 \\
62\end{array}$ & $\begin{array}{l}0 \\
0 \\
0 \\
0 \\
0\end{array}$ & $\begin{array}{l}0 \\
0 \\
0 \\
0 \\
0\end{array}$ & $\begin{array}{r}-999 \\
-999 \\
28385 \\
27726 \\
-999\end{array}$ \\
\hline $\begin{array}{r}\text { HEAN } \\
\text { SD } \\
\text { HUM. }\end{array}$ & $\begin{array}{r}0 \\
0 \\
30\end{array}$ & $\begin{array}{r}0 \\
0 \\
30\end{array}$ & $\begin{array}{l}33 \\
55 \\
19\end{array}$ & $\begin{array}{l}3011 \\
155 \\
27\end{array}$ & $\begin{array}{r}736 \\
357 \\
2 H\end{array}$ & $\begin{array}{r}1383 \\
604 \\
311\end{array}$ & $\begin{array}{r}1153 \\
1115 \\
311\end{array}$ & $\begin{array}{r}2365 \\
960 \\
30\end{array}$ & $\begin{array}{r}2633 ? \\
369 \\
30\end{array}$ & $\begin{array}{r}2710 \\
929 \\
30\end{array}$ & $\begin{array}{r}2505 \\
886 \\
30\end{array}$ & $\begin{array}{r}2030 \\
819 \\
30\end{array}$ & $\begin{array}{r}1571 \\
\text { ofs. } \\
29\end{array}$ & $\begin{array}{r}1035 \\
1105 \\
29\end{array}$ & $\begin{array}{r}1143 \\
236 \\
26\end{array}$ & $\begin{array}{r}126 \\
45 \\
26\end{array}$ & $\begin{array}{r}0 \\
0 \\
30\end{array}$ & $\begin{array}{r}0 \\
0 \\
30\end{array}$ & $\begin{array}{r}21020 \\
7209 \\
17\end{array}$ \\
\hline $\begin{array}{l}\ln x \\
M I \| 1\end{array}$ & $\begin{array}{l}0 \\
y\end{array}$ & $\begin{array}{l}0 \\
0\end{array}$ & $\begin{array}{r}190 \\
0\end{array}$ & $\begin{array}{r}696 \\
311\end{array}$ & $\begin{array}{r}13114 \\
108\end{array}$ & $\begin{array}{r}2122 \\
51\end{array}$ & $\begin{array}{r}27611 \\
132\end{array}$ & $\begin{array}{r}3261 \\
100\end{array}$ & $\begin{array}{r}3543 \\
227\end{array}$ & $\begin{array}{r}3585 \\
137\end{array}$ & $\begin{array}{r}3413 \\
263\end{array}$ & $\begin{array}{r}29911 \\
2.118\end{array}$ & $\begin{array}{r}25113 \\
234\end{array}$ & $\begin{array}{r}1646 \\
114\end{array}$ & $\begin{array}{r}821 \\
37\end{array}$ & $\begin{array}{r}266 \\
54\end{array}$ & $\begin{array}{l}0 \\
0\end{array}$ & $\begin{array}{l}0 \\
0\end{array}$ & $\begin{array}{r}28385 \\
3538\end{array}$ \\
\hline
\end{tabular}


COASTAL - ENLAHA SOLAR RADIATIOR. WIFFERENCE STUIOY MUNTHLY DATA SUMMAAFY

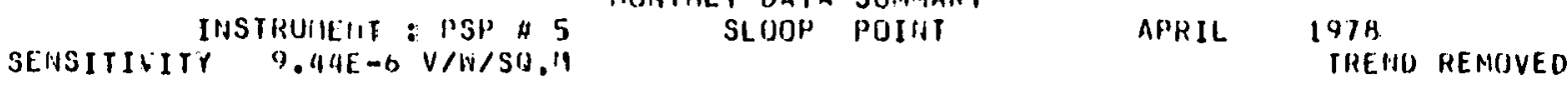

REERGY KILIJJOULES PEH SUUARE HETER

DATE

\section{IIIJUR ENDING}

$\begin{array}{ll}1 & 0 \\ 2 & 0 \\ 3 & 0 \\ 4 & 0 \\ 5 & 0 \\ 0 & 0 \\ 7 & 0 \\ 0 & 0 \\ 9 & 0 \\ 0 & 0 \\ 1 & 0 \\ 2 & 0 \\ 3 & 0 \\ 4 & 0 \\ 5 & 0\end{array}$

1

$\begin{array}{rrrrrrrrrrr}19) & 713 & 1571 & 2253 & 2757 & 3054 & 3161 & 2997 & 2581 & 1979 & 1254 \\ 197 & 819 & 1604 & 2333 & 285 ? & 3157 & 3237 & 3035 & 2597 & 1967 & 1203 \\ 91 & 360 & 713 & 701 & 663 & 1147 & 934 & 537 & 500 & 390 & 16 J \\ 148 & 667 & 300 & 1853 & 2616 & 2886 & 2644 & 2455 & 1292 & 1193 & 1027\end{array}$

$148067 \quad 1853 \quad 2616 \quad 2886 \quad 2644 \quad 2455 \quad 1292 \quad 1193 \quad 1027$

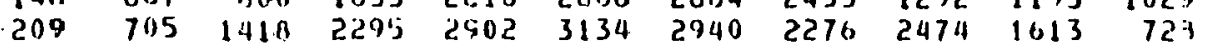

274 968 1693 $2452 \quad 2903 \quad 30693153 \quad 2776 \quad 2009$ 1266 685

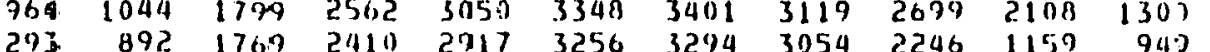

24

305. 486

92. 176.9211

636964

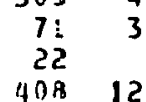

$888 \quad 7742307$

072560

8i) 2067

344

$205 \quad 1998$

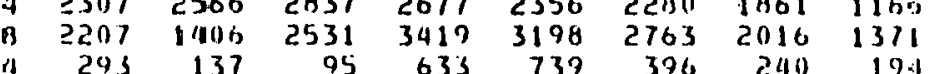

$134 \quad 1750$

07 253

161 64A 1165 1067 1141

$25.5 \quad 347$

727247

2457

45

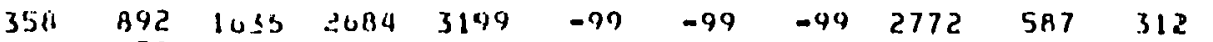

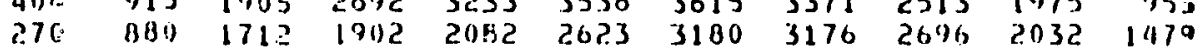

$23 \overline{6} \quad 655 \quad 10172 \quad 2169 \quad 2715 \quad 3211 \quad 3550 \quad 2375 \quad 2375 \quad 1826 \quad 1250$

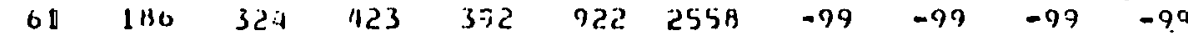

$\begin{array}{lllllllll}-97 & -97 & -79 & -97 & 3615 & 3409 & 2966 & 2337 & 1565\end{array}$

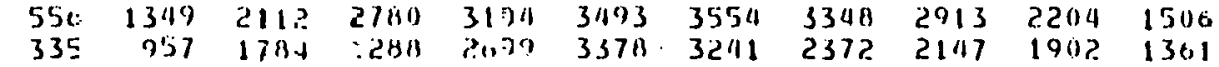

MEAH 0

I. SIIM 30 s

$\ln x$

MIN

20

763139

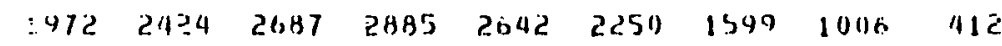

4

3

26113

197 21227

$9613 \quad 018$

243

1)

41 H7 137

$\begin{array}{rrrrrr}3569 & 3617 & 3109 & 29126 & 2337 & 1563 \\ 99 & 633 & 434 & 335 & 240 & 168\end{array}$

781
45

TAHILATED 01/02/AO AT 13:08:53

23028

23480

18141

21120

21572

24089

32676

22676
19575

20104

20738

3163

26735

26790

8902

12874

26782

$-999$

28179

25566

$\begin{array}{r}-999 \\ \hline\end{array}$

18774

23023

$-999$

27593

22123

20430

$8 B 28$

28179
3163 
CUASTAL - IHLAIII SULAR RADIATIUN DIFFERENCE SIUIOY IHSTRUHEMT : BGH MIJNTHLY DATA SUMHARY

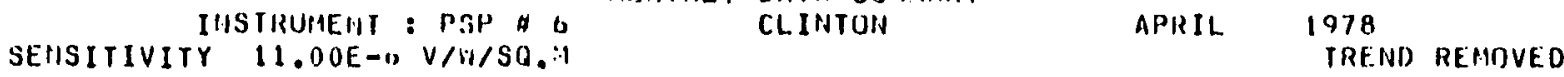

ENERGY KILUJOIJLES PER SIJUARE METFR

\begin{tabular}{|c|c|c|c|c|c|c|c|c|c|c|c|c|c|c|c|c|c|c|c|}
\hline \multirow[b]{2}{*}{ DATE } & & & & & & & & & 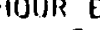 & 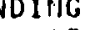 & & & \multirow[b]{2}{*}{16} & \multirow[b]{2}{*}{17} & \multirow[b]{2}{*}{18} & \multirow[b]{2}{*}{19} & \multirow[b]{2}{*}{20} & \multirow[b]{2}{*}{21} & \multirow[b]{2}{*}{ IOTAL } \\
\hline & 4 & 5 & i) & 7 & $B$ & 9 & 10 & 11 & 12 & 13 & 14 & 15 & & & & & & & \\
\hline $\begin{array}{l}1 \\
2 \\
3 \\
4 \\
5\end{array}$ & $\begin{array}{l}0 \\
0 \\
0 \\
0 \\
0\end{array}$ & $\begin{array}{l}0 \\
0 \\
0 \\
0 \\
0\end{array}$ & $\begin{array}{r}19 \\
0 \\
0 \\
0 \\
0\end{array}$ & $\begin{array}{r}370 \\
197 \\
80 \\
121 \\
247\end{array}$ & $\begin{array}{r}1093 \\
100 \\
247 \\
442 \\
905\end{array}$ & $\begin{array}{r}1076 \\
1656 \\
714 \\
962 \\
1061\end{array}$ & $\begin{array}{r}2204 \\
2350 \\
809 \\
2095 \\
2345\end{array}$ & $\begin{array}{l}2791 \\
2863 \\
1071 \\
2782 \\
2892\end{array}$ & $\begin{array}{l}3112 \\
3174 \\
11117 \\
21387 \\
3232\end{array}$ & $\begin{array}{l}3178 \\
3227 \\
1605 \\
2772 \\
3288\end{array}$ & $\begin{array}{l}3047 \\
3043 \\
1467 \\
1807 \\
3130\end{array}$ & $\begin{array}{l}2602 \\
2618 \\
1035 \\
1937 \\
2708\end{array}$ & $\begin{array}{r}1954 \\
1983 \\
574 \\
1757 \\
2067\end{array}$ & $\begin{array}{r}1168 \\
1224 \\
541 \\
1325 \\
1357\end{array}$ & $\begin{array}{l}392 \\
4911 \\
253 \\
514 \\
529\end{array}$ & $\begin{array}{l}42 \\
39 \\
18 \\
46 \\
36\end{array}$ & $\begin{array}{l}0 \\
0 \\
0 \\
0 \\
0\end{array}$ & $\begin{array}{l}0 \\
0 \\
0 \\
0 \\
0\end{array}$ & $\begin{array}{r}23108 \\
23730 \\
9721 \\
19447 \\
24399\end{array}$ \\
\hline $\begin{array}{r}6 \\
7 \\
8 \\
9 \\
10\end{array}$ & $\begin{array}{l}0 \\
0 \\
0 \\
0 \\
0\end{array}$ & $\begin{array}{l}0 \\
0 \\
0 \\
0 \\
0\end{array}$ & $\begin{array}{r}0 \\
0 \\
0 \\
10 \\
0\end{array}$ & $\begin{array}{l}282 \\
236 \\
356 \\
268 \\
206\end{array}$ & $\begin{array}{r}789 \\
920 \\
1060 \\
972 \\
461\end{array}$ & $\begin{array}{l}1509 \\
1725 \\
1395 \\
1715 \\
1037\end{array}$ & $\begin{array}{l}2324 \\
2116 \\
2539 \\
2386 \\
1914\end{array}$ & $\begin{array}{l}2783 \\
2927 \\
3966 \\
2932 \\
2757\end{array}$ & $\begin{array}{l}3035 \\
3257 \\
3361 \\
3207 \\
2876\end{array}$ & $\begin{array}{l}3080 \\
3342 \\
3400 \\
3312 \\
2389\end{array}$ & $\begin{array}{l}2553 \\
3165 \\
3200 \\
2873 \\
2572\end{array}$ & $\begin{array}{l}1968 \\
2760 \\
2745 \\
2651 \\
1145\end{array}$ & $\begin{array}{l}1415 \\
2148 \\
2133 \\
2071 \\
1780\end{array}$ & $\begin{array}{l}613 \\
1394 \\
1384 \\
1345 \\
1211\end{array}$ & $\begin{array}{l}205 \\
609 \\
602 \\
563 \\
432\end{array}$ & $\begin{array}{l}50 \\
60 \\
62 \\
62 \\
65\end{array}$ & $\begin{array}{l}0 \\
0 \\
0 \\
0 \\
0\end{array}$ & $\begin{array}{l}0 \\
0 \\
0 \\
0 \\
0\end{array}$ & $\begin{array}{l}20686 \\
24963 \\
25759 \\
24367 \\
18547\end{array}$ \\
\hline $\begin{array}{l}11 \\
12 \\
13 \\
14 \\
15\end{array}$ & $\begin{array}{l}0 \\
0 \\
0 \\
0 \\
0\end{array}$ & $\begin{array}{l}0 \\
0 \\
0 \\
0 \\
0\end{array}$ & $\begin{array}{r}10 \\
0 \\
0 \\
12 \\
13\end{array}$ & $\begin{array}{r}72 \\
72 \\
35 \\
401 \\
343\end{array}$ & $\begin{array}{r}255 \\
377 \\
71 \\
1196 \\
11813\end{array}$ & $\begin{array}{r}668 \\
1516 \\
140 \\
1938 \\
1855\end{array}$ & $\begin{array}{r}1803 \\
2546 \\
349 \\
26160 \\
25117\end{array}$ & $\begin{array}{r}2396 \\
3096 \\
339 \\
3160 \\
3050\end{array}$ & $\begin{array}{r}3034 \\
3391 \\
336 \\
3435 \\
3377\end{array}$ & $\begin{array}{r}2782 \\
3450 \\
519 \\
3461 \\
3220\end{array}$ & $\begin{array}{r}2533 \\
3148 \\
549 \\
3261 \\
3155\end{array}$ & $\begin{array}{r}2350 \\
2838 \\
729 \\
2823 \\
1774\end{array}$ & $\begin{array}{l}1545 \\
2186 \\
1118 \\
2115 \\
1957\end{array}$ & $\begin{array}{r}979 \\
1434 \\
889 \\
1373 \\
1371\end{array}$ & $\begin{array}{l}380 \\
661 \\
251 \\
512 \\
5,95\end{array}$ & $\begin{array}{l}52 \\
56 \\
58 \\
74 \\
75\end{array}$ & $\begin{array}{l}0 \\
0 \\
0 \\
0 \\
0\end{array}$ & $\begin{array}{l}0 \\
0 \\
0 \\
0 \\
0\end{array}$ & $\begin{array}{r}18849 \\
24861 \\
5383 \\
26507 \\
24480\end{array}$ \\
\hline $\begin{array}{l}16 \\
17 \\
18 \\
19 \\
20\end{array}$ & $\begin{array}{l}0 \\
0 \\
0 \\
0 \\
0\end{array}$ & $\begin{array}{l}0 \\
0 \\
0 \\
0 \\
0\end{array}$ & $\begin{array}{r}16 \\
0 \\
-99 \\
10 \\
21\end{array}$ & $\begin{array}{r}386 \\
179 \\
-99 \\
3.3 \\
453\end{array}$ & $\begin{array}{r}1142 \\
431 \\
-99 \\
113 \\
1222\end{array}$ & $\begin{array}{r}1956 \\
555 \\
75 \\
412 \\
1917\end{array}$ & $\begin{array}{r}26,17 \\
703 \\
163 \\
12 / 11 \\
26 / 5\end{array}$ & $\begin{array}{r}3158 \\
978 \\
536 \\
1441 \\
3172\end{array}$ & $\begin{array}{r}3420 \\
16112 \\
857 \\
1826 \\
-79\end{array}$ & $\begin{array}{r}3524 \\
595 \\
1531 \\
2906 \\
-99\end{array}$ & $\begin{array}{r}3338 \\
477 \\
2310 \\
2203 \\
-99\end{array}$ & $\begin{array}{r}21557 \\
650 \\
1007 \\
2026 \\
2135\end{array}$ & $\begin{array}{r}2310 \\
467 \\
120 \\
1830 \\
1961\end{array}$ & $\begin{array}{r}1531 \\
159 \\
-99 \\
1106 \\
973\end{array}$ & $\begin{array}{r}730 \\
61 \\
55 \\
102 \\
381\end{array}$ & $\begin{array}{r}98 \\
0 \\
12 \\
125 \\
73\end{array}$ & $\begin{array}{l}0 \\
0 \\
0 \\
0 \\
0\end{array}$ & $\begin{array}{l}0 \\
0 \\
0 \\
0 \\
0\end{array}$ & $\begin{array}{r}27107 \\
6897 \\
-999 \\
15389 \\
-994\end{array}$ \\
\hline $\begin{array}{l}21 \\
22 \\
23 \\
211 \\
25\end{array}$ & $\begin{array}{l}0 \\
0 \\
0 \\
0 \\
0\end{array}$ & $\begin{array}{l}0 \\
0 \\
0 \\
0 \\
0\end{array}$ & $\begin{array}{r}25 \\
29 \\
37 \\
-555 \\
73\end{array}$ & $\begin{array}{r}345 \\
530 \\
433 \\
-555 \\
246\end{array}$ & $\begin{array}{r}1.121 \\
1214 \\
11127 \\
-5155 \\
754\end{array}$ & $\begin{array}{l}1671 \\
2055 \\
22811 \\
-555 \\
13.51\end{array}$ & $\begin{array}{l}2318 \\
2745 \\
2704 \\
-555 \\
1598\end{array}$ & $\begin{array}{l}3216 \\
3260 \\
3198 \\
-555 \\
1281\end{array}$ & $\begin{array}{r}2967 \\
3554 \\
3424 \\
-555 \\
1320\end{array}$ & $\begin{array}{l}3380 \\
3609 \\
3522 \\
-555 \\
1667\end{array}$ & $\begin{array}{r}3160 \\
3387 \\
3200 \\
-555 \\
314\end{array}$ & $\begin{array}{r}1785 \\
2978 \\
2861 \\
-555 \\
233\end{array}$ & $\begin{array}{r}1124 \\
2323 \\
2004 \\
-555 \\
269\end{array}$ & $\begin{array}{r}-97 \\
1570 \\
1251 \\
-555 \\
246\end{array}$ & $\begin{array}{r}162 \\
769 \\
531 \\
-555 \\
83\end{array}$ & $\begin{array}{r}165 \\
114 \\
92 \\
-555 \\
14\end{array}$ & $\begin{array}{l}0 \\
0 \\
0 \\
0 \\
0\end{array}$ & $\begin{array}{l}0 \\
0 \\
0 \\
0 \\
0\end{array}$ & $\begin{array}{r}-999 \\
28143 \\
20732 \\
-999 \\
9432\end{array}$ \\
\hline $\begin{array}{l}26 \\
27 \\
20 \\
29 \\
30\end{array}$ & $\begin{array}{l}0 \\
0 \\
0 \\
0 \\
0\end{array}$ & $\begin{array}{l}0 \\
0 \\
0 \\
0 \\
0\end{array}$ & $\begin{array}{l}0 \\
14 \\
51 \\
55 \\
24\end{array}$ & $\begin{array}{r}48 \\
60 \\
532 \\
448 \\
155\end{array}$ & $\begin{array}{r}173 \\
154 \\
1376 \\
1247 \\
541\end{array}$ & $\begin{array}{r}705 \\
229 \\
2090 \\
2186 \\
10184\end{array}$ & $\begin{array}{r}1501 \\
174 \\
2918 \\
2814 \\
2125\end{array}$ & $\begin{array}{r}2372 \\
253 \\
3241 \\
3305 \\
2314\end{array}$ & $\begin{array}{r}-99 \\
341 \\
3576 \\
3570 \\
2806\end{array}$ & $\begin{array}{r}-79 \\
482 \\
3621 \\
31777 \\
2914\end{array}$ & $\begin{array}{l}3154 \\
1029 \\
3428 \\
3354 \\
2462\end{array}$ & $\begin{array}{l}1148 \\
1434 \\
3003 \\
2912 \\
1222\end{array}$ & $\begin{array}{l}1256 \\
1025 \\
2361 \\
2291 \\
1814\end{array}$ & $\begin{array}{r}-79 \\
548 \\
1612 \\
1518 \\
1146\end{array}$ & $\begin{array}{r}112 \\
678 \\
797 \\
667 \\
335\end{array}$ & $\begin{array}{r}55 \\
53 \\
149 \\
127 \\
53\end{array}$ & $\begin{array}{l}0 \\
0 \\
0 \\
0 \\
0\end{array}$ & $\begin{array}{l}0 \\
0 \\
0 \\
0 \\
0\end{array}$ & $\begin{array}{r}-999 \\
6493 \\
28804 \\
28071 \\
19029\end{array}$ \\
\hline
\end{tabular}

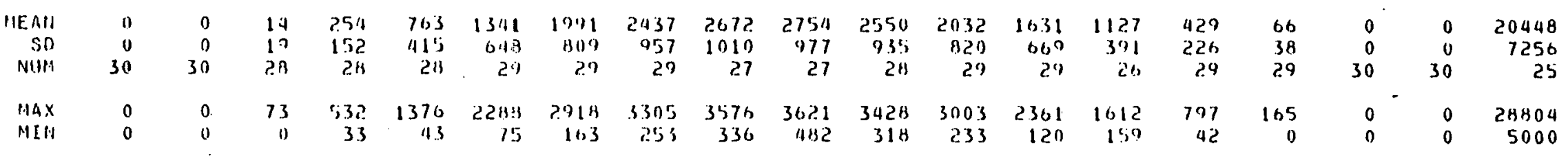


CIASTAL. - IN_AND SILLAR RADIATIGI DIFFEREMICE STIJIOY HOHTHLY DATA SUMMATY

\begin{tabular}{|c|c|c|c|}
\hline $\begin{array}{l}\text { IHSTISUNEHT : PSP E } 7 \\
\text { SENSITIVITY } 10,17 \mathrm{~F}-6 \mathrm{~V} / \mathrm{H} / \mathrm{SO}, \mathrm{H}\end{array}$ & ONGLOW BEACH & APRIL & $\begin{array}{l}1978 \\
\text { TREND HEMOVEU }\end{array}$ \\
\hline
\end{tabular}

ENERGY KILOJOULES PER SU'JARE HETER

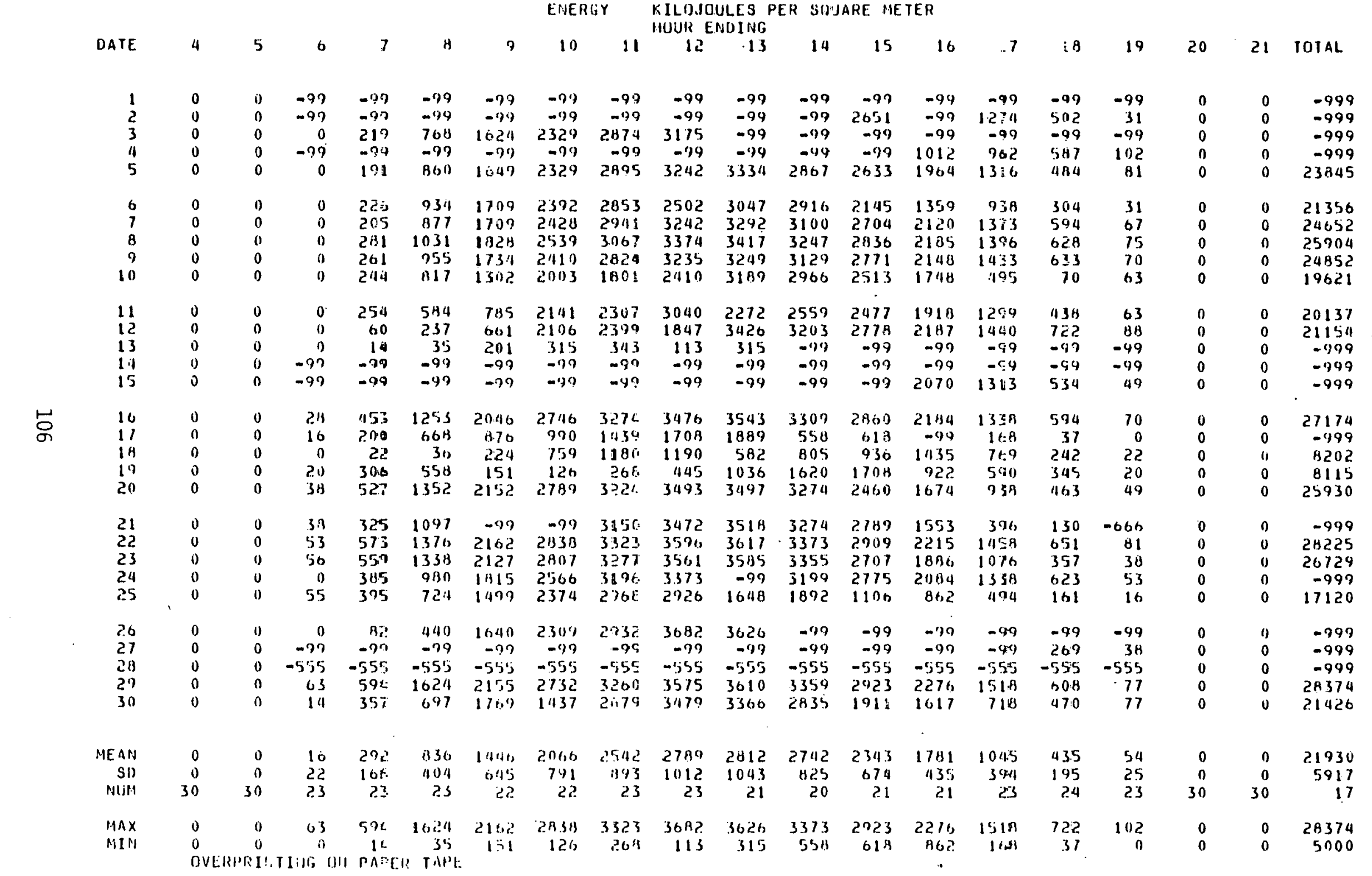


CIJASTAL - INLAIID SOLAR RADIATIUN DIFFERFNCE STUOY

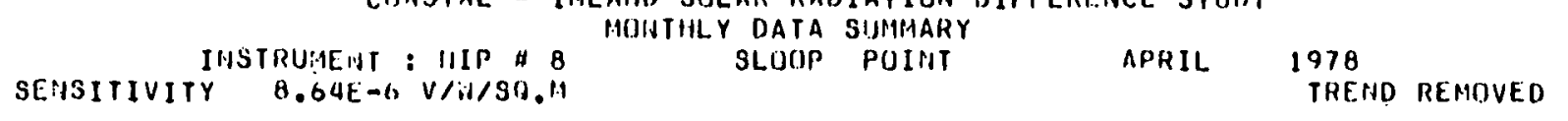

EHERG KILOJOULES PER SOUARF. METER

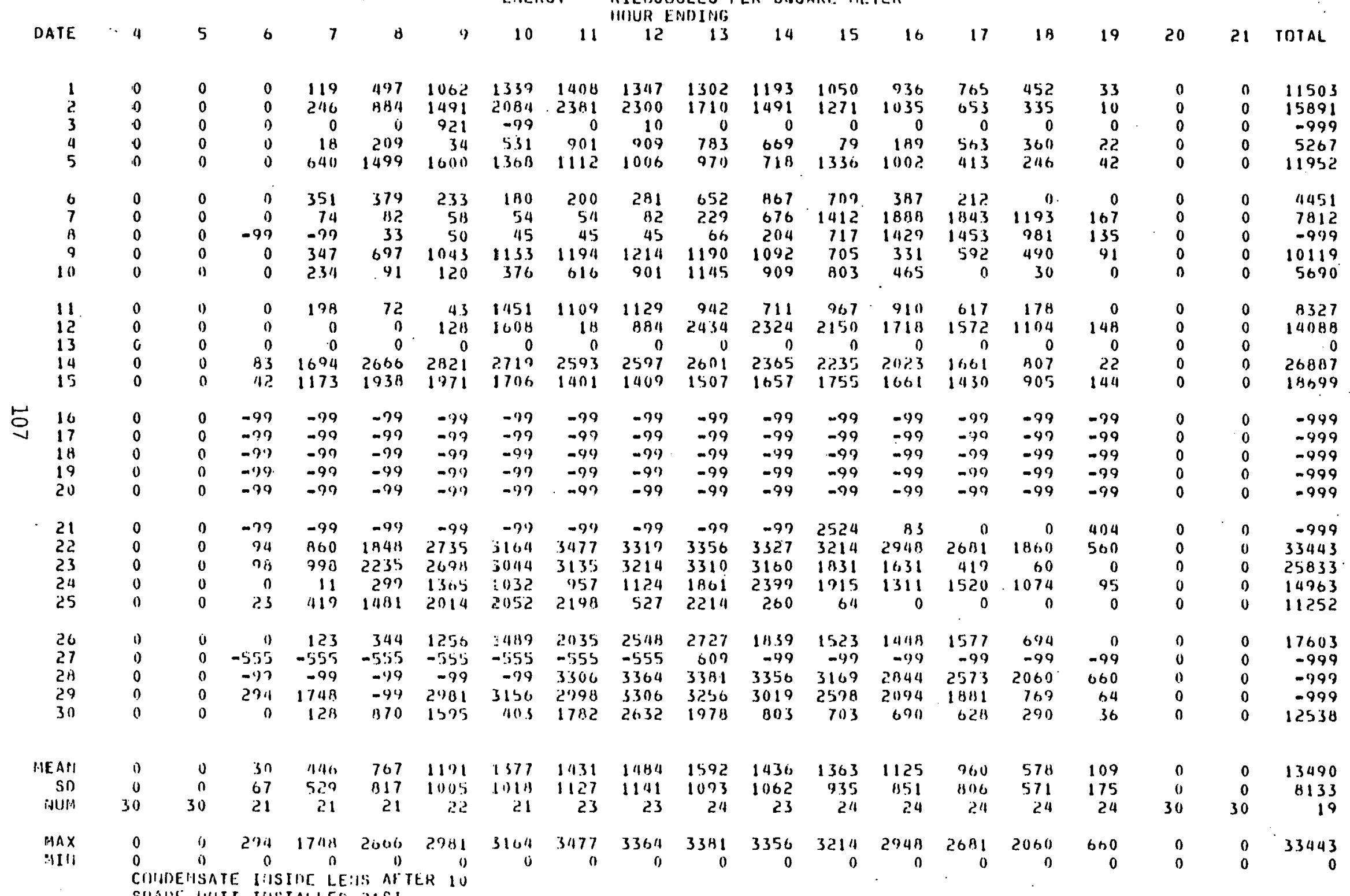




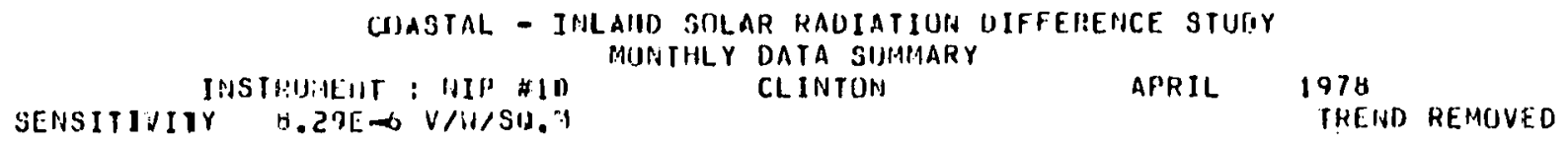

CIJERGY TILOJJULLES PER SOIJARE METER

DATE HUIJR ENDING

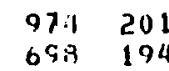


COF.STAL - IILLAHO SOLAR RADIAYIUH DIFFERENCE STUDY MONTHLY DATA SUMAARY

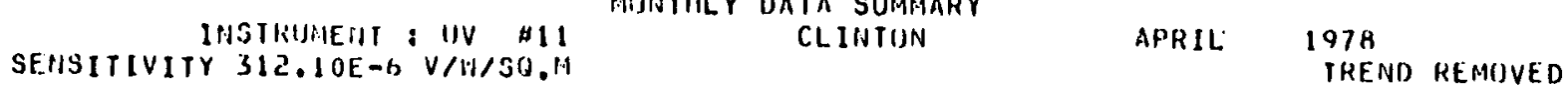

EHERGY KILOJOULES PER SIUARE METER HIOUR ENDINI;

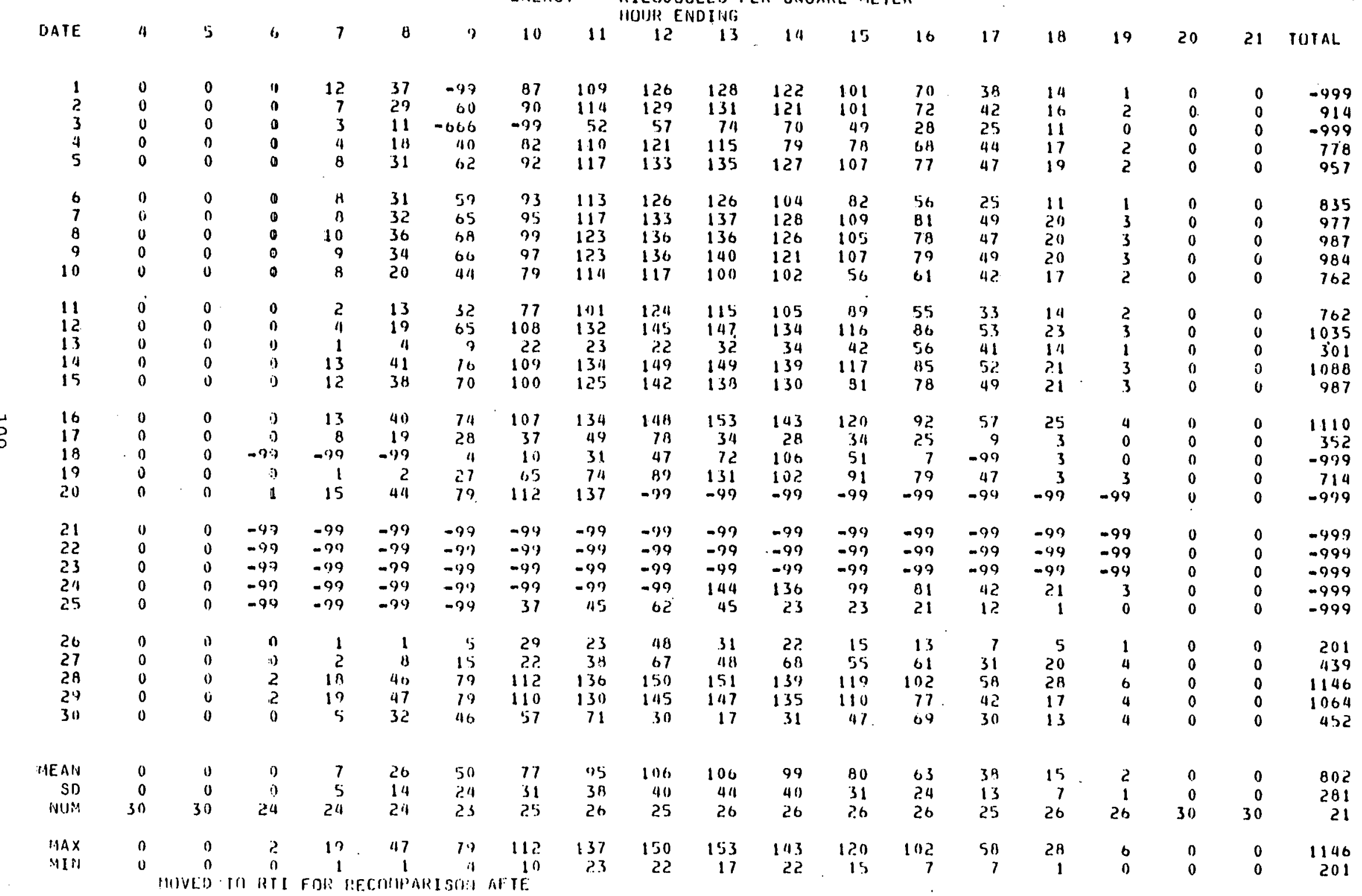


CIJASTAL - IHLAHI SOLAR RADIATION DIFFEREINCE STUMY MIJITHLY DATA SUMMARY

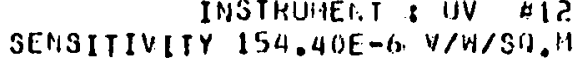
S.OOP POINI

APRIL. $\quad 1978$

EWRAGY KILOJOUIES PER SUUARE METER

$\begin{array}{ll}0 & 0 \\ 0 & 0 \\ 0 & 0 \\ 0 & 0 \\ 0 & 0\end{array}$

$\begin{array}{ll}8 \\ 0 & 8 \\ 0 & 5 \\ 0 & 8 \\ 0 & 10\end{array}$

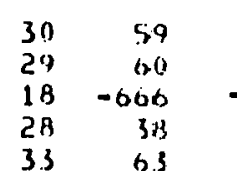

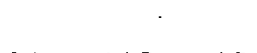

$12 \quad 13$

$\begin{array}{rr}0 & 10 \\ 0 & 9 \\ 0 & -666 \\ 0 & 11 \\ 1 & 11\end{array}$

$\begin{array}{lll}11 & 0 & 0 \\ 12 & 0 & 0 \\ 13 & 0 & 0 \\ 14 & 0 & 0 \\ 15 & 0 & 0\end{array}$

11
11
2
15
15

1
0
1
1

$\begin{array}{lllllll}35 & 65 & 94 & 116 & 124 & 127 & 114\end{array}$

31
37
35
213

$\begin{array}{llll}61 & 99 & 111 & 122 \\ 67 & 96 & 116 & 131 \\ 67 & 95 & 121 & 138\end{array}$

21
10
65
42

38

$\begin{array}{ll}3 \\ 79 & 110 \\ 4 & 1\end{array}$

90
90
20
10
102

$\begin{array}{rl}107 & 118 \\ 711 & 117 \\ 10 & \\ 133 & \end{array}$

$\begin{array}{rr}57 & 42 \\ 7 & 31 \\ 3 & 5 \\ 12 & 17\end{array}$

72
48

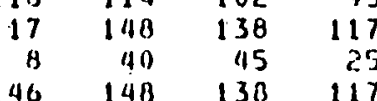

$\begin{array}{rrrrr}10 & 8 & 40 & 15 & 23 \\ 5 & 146 & 148 & 138 & 117 \\ 50 & 145 & 133 & 112\end{array}$

32

$\begin{array}{lll}131 & 145 & 147 \\ 63 & 112\end{array}$

$47 \quad 137$

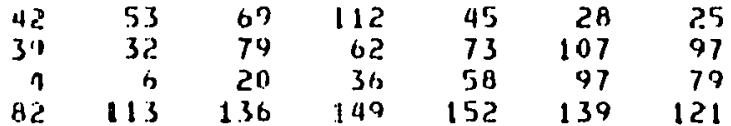

$20 \quad 0$

2

21
22
23
24
25

$\begin{array}{ll}20 & 0 \\ 27 & 0 \\ 28 & 0 \\ 29 & 0 \\ 30 & 0\end{array}$

$\begin{array}{rrrrr}\text { IIEAH } & 0 & 11 & 0 & 10 \\ \text { SII } & 0 & 0 & 0 & 10 \\ \text { HUM } & 30 & 30 & 27 & 26\end{array}$

iI I

MII

1)

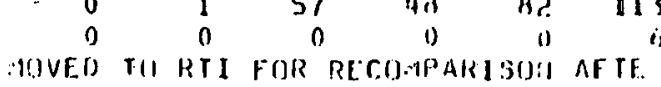

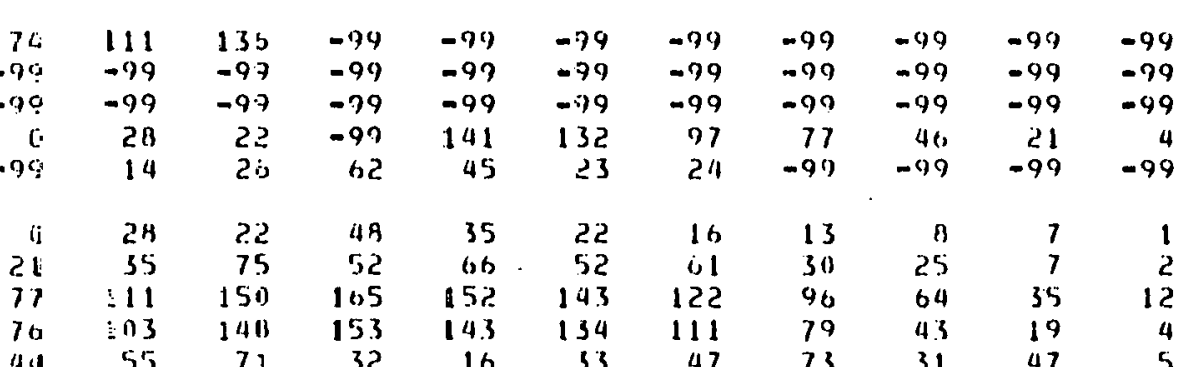

TRE HO REMUVEO 
CIASTAL - IWLANO SOLAR RADIATIUN DIFFEREIICE STUUY

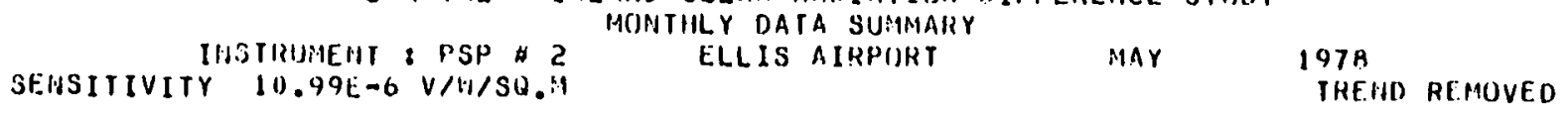

EHERGY KILUJDULES PER SUUARF METER

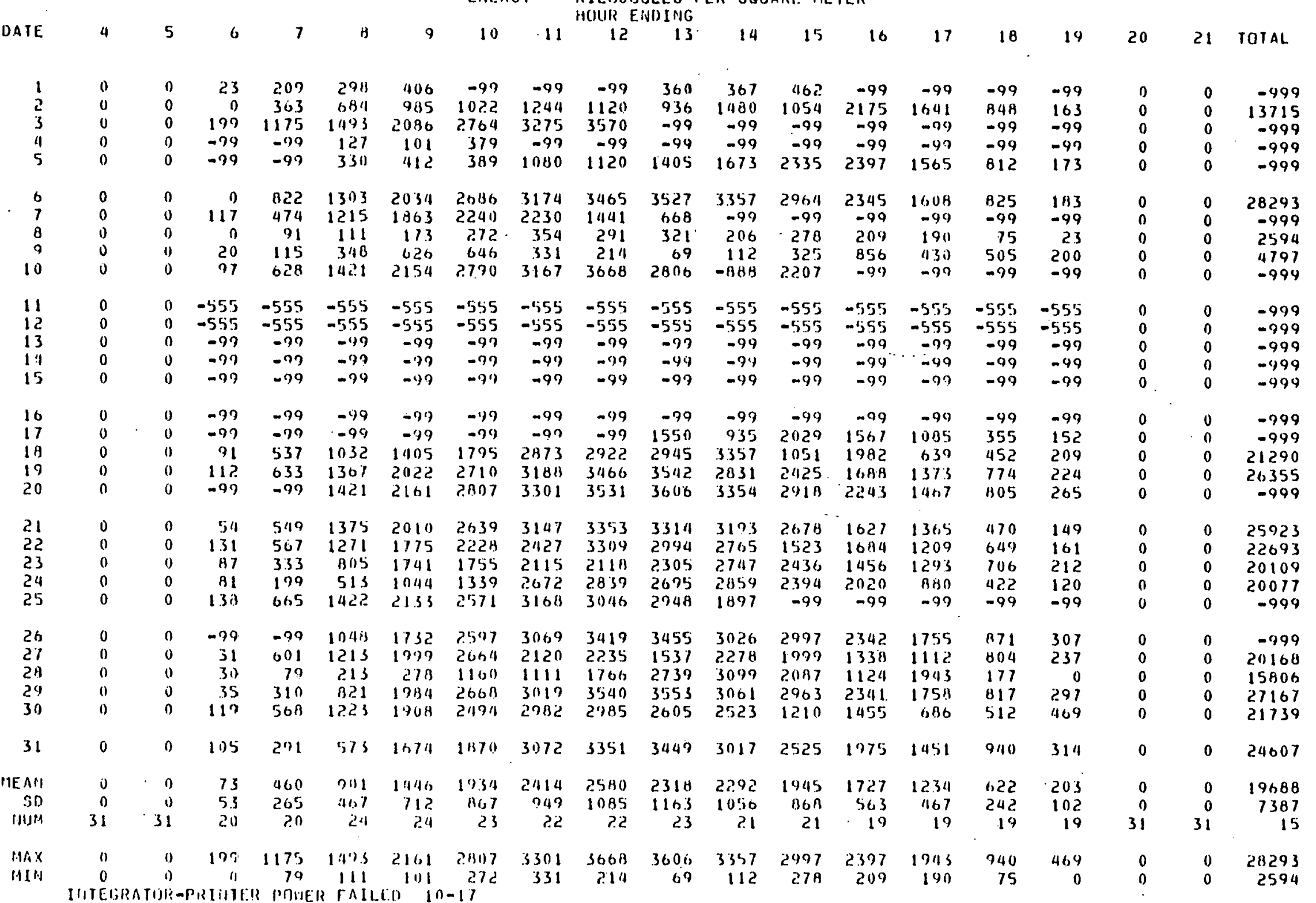


COASTAL - INLAIID SIJLAR RADIATIUN OIFFERENICE STUDY

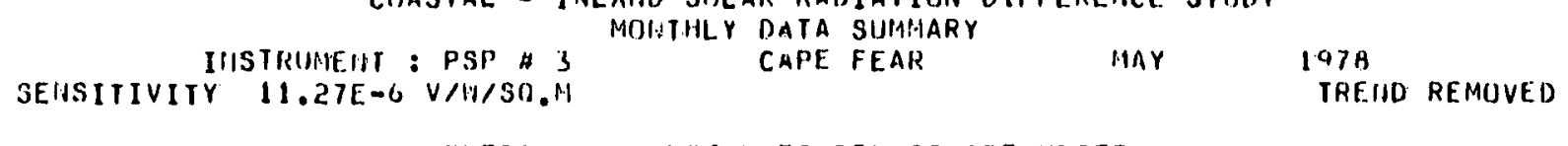

EHERG KILOJUULF.S PER SQUARE METER

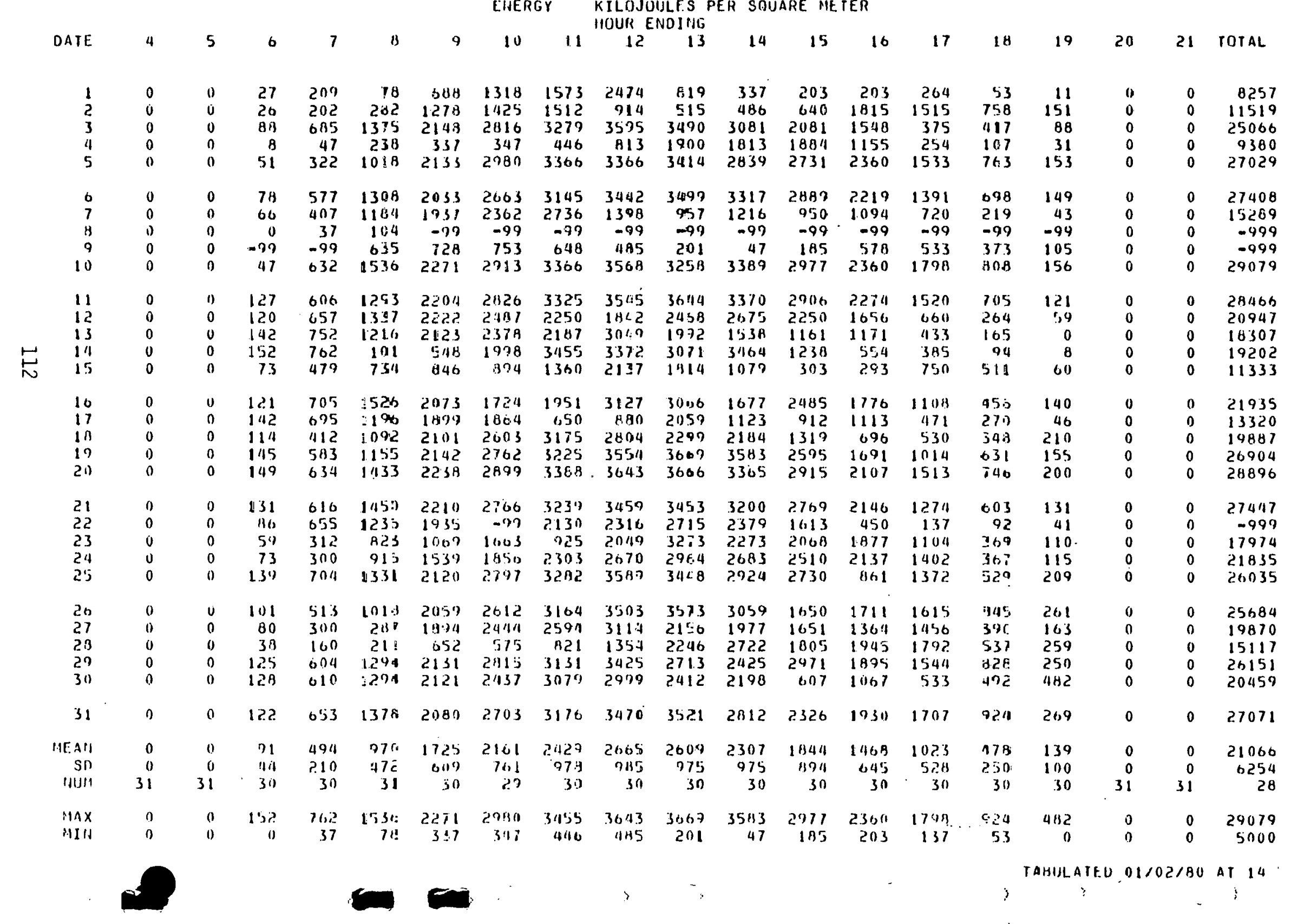


CUASTAL - IHLAHD SHLAR RADIATIUN DIFFERENCE STIJDY IIISIRUIAENT : PGP $\#$ A MONTHLY DATA SUMMARY WALLACE MAY 1978

SENSITIVITY $11.00 E-6$ V/W/SP.M

EHERGY KILIJUIILES PER SHUARE METEH IIIUR ENDING

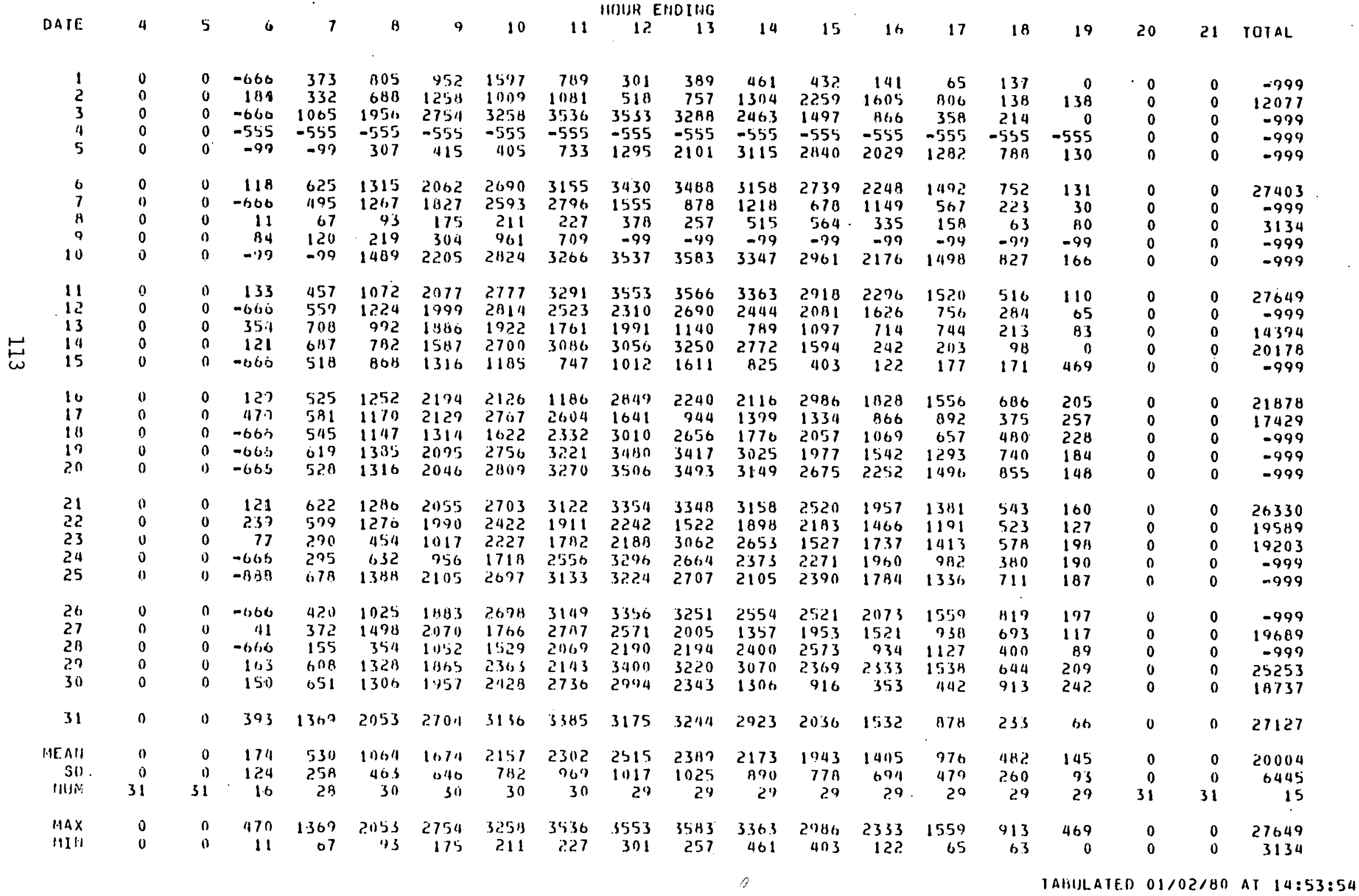


CIJASTAL - INLAIID SILARR RADIATION DIFFFREIICE STUDY

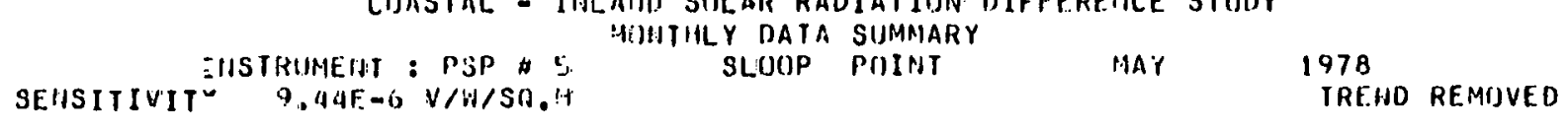

EHERGY KILOJIJULES PER SQUARE METER HOUR ENDING

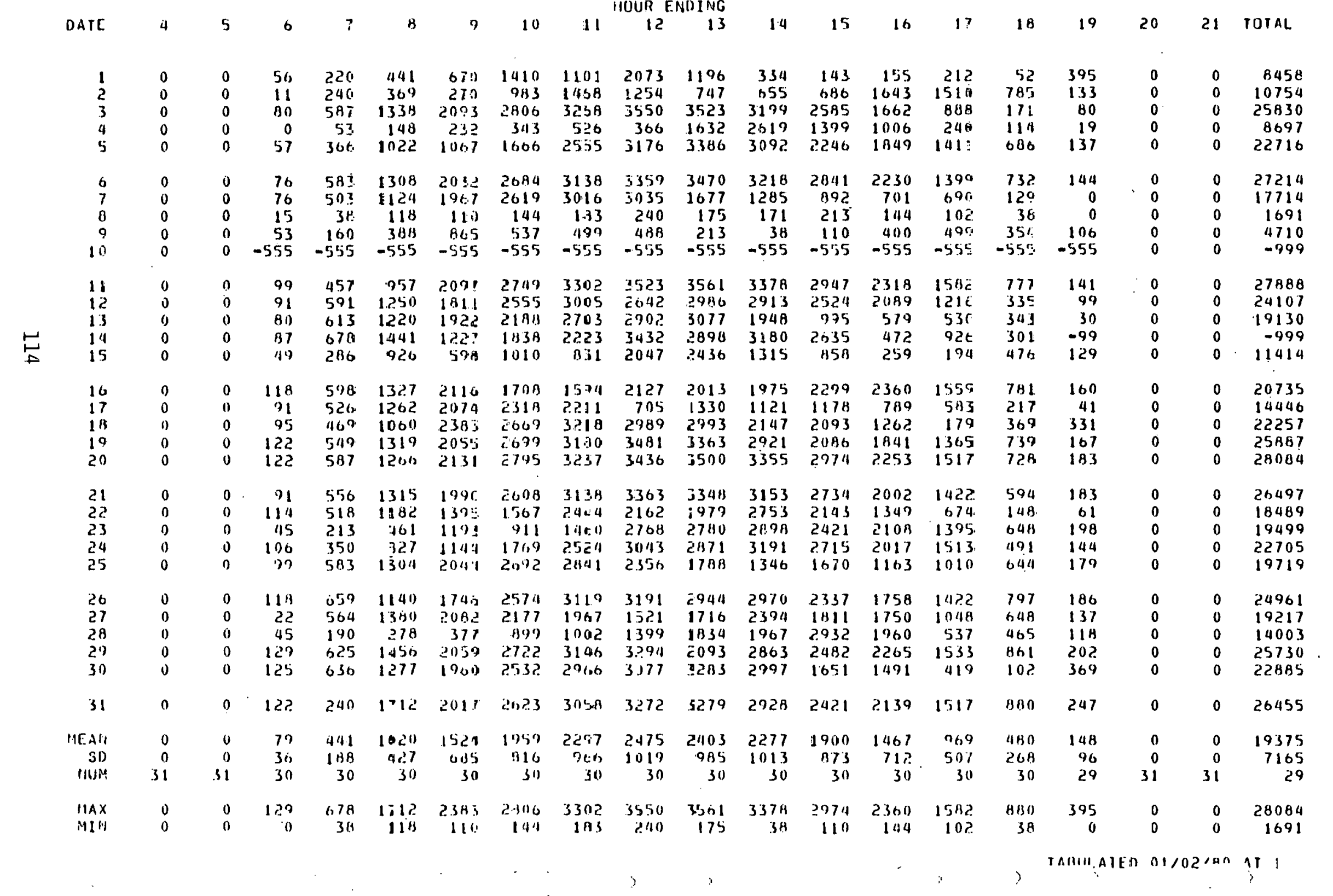


CUASTAL - INLAHO SIULAR RADIATIINN DIFFEREHCE STUUY

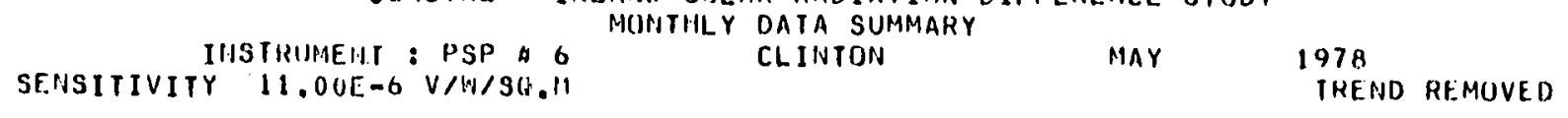

EMERGY KILUJOULES PER SOUARE METER

DNTE HOUR EHDING

\begin{tabular}{|c|c|c|c|c|c|c|c|c|c|c|c|c|c|c|c|c|c|c|c|c|}
\hline & DATE & 4 & 5 & 6 & 7 & B & 9 & 10 & 11 & 12 & 13 & 14 & 15 & 16 & 17 & 18 & 19 & 20 & 21 & TUTAL \\
\hline & $\begin{array}{l}1 \\
2 \\
3 \\
4 \\
5\end{array}$ & $\begin{array}{l}1) \\
0 \\
0 \\
0 \\
0\end{array}$ & $\begin{array}{l}0 \\
0 \\
0 \\
0 \\
0\end{array}$ & $\begin{array}{l}19 \\
25 \\
79 \\
10 \\
19\end{array}$ & $\begin{array}{r}2013 \\
218 \\
655 \\
49 \\
120\end{array}$ & $\begin{array}{r}3410 \\
382 \\
1375 \\
115 \\
382\end{array}$ & $\begin{array}{r}487 \\
1151 \\
2370 \\
157 \\
660\end{array}$ & $\begin{array}{r}421 \\
879 \\
2799 \\
252 \\
1248\end{array}$ & $\begin{array}{r}519 \\
1030 \\
3280 \\
462 \\
1727\end{array}$ & $\begin{array}{r}601 \\
591 \\
3499 \\
570 \\
1972\end{array}$ & $\begin{array}{r}467 \\
627 \\
3607 \\
1440 \\
3419\end{array}$ & $\begin{array}{r}696 \\
1468 \\
3344 \\
799 \\
2990\end{array}$ & $\begin{array}{r}791 \\
2725 \\
2540 \\
616 \\
2015\end{array}$ & $\begin{array}{r}397 \\
2205 \\
1670 \\
1106 \\
2241\end{array}$ & $\begin{array}{r}202 \\
1553 \\
1045 \\
298 \\
1439\end{array}$ & $\begin{array}{r}58 \\
794 \\
439 \\
121 \\
719\end{array}$ & $\begin{array}{r}15 \\
162 \\
96 \\
10 \\
123\end{array}$ & $\begin{array}{l}0 \\
0 \\
0 \\
0 \\
0\end{array}$ & $\begin{array}{l}0 \\
0 \\
0 \\
0 \\
0\end{array}$ & $\begin{array}{r}5422 \\
13810 \\
26803 \\
6005 \\
19134\end{array}$ \\
\hline & $\begin{array}{r}6 \\
7 \\
8 \\
9 \\
10\end{array}$ & $\begin{array}{l}0 \\
0 \\
0 \\
0 \\
0\end{array}$ & $\begin{array}{l}0 \\
0 \\
0 \\
0 \\
0\end{array}$ & $\begin{array}{r}73 \\
83 \\
12 \\
20 \\
101\end{array}$ & $\begin{array}{l}567 \\
525 \\
107 \\
128 \\
654\end{array}$ & $\begin{array}{r}1303 \\
1163 \\
101 \\
347 \\
1423\end{array}$ & $\begin{array}{r}2053 \\
1900 \\
219 \\
475 \\
2170\end{array}$ & $\begin{array}{r}2704 \\
2511 \\
385 \\
1103 \\
2811\end{array}$ & $\begin{array}{r}3198 \\
3061 \\
615 \\
471 \\
3001\end{array}$ & $\begin{array}{r}3496 \\
3005 \\
313 \\
508 \\
397.7\end{array}$ & $\begin{array}{r}3558 \\
2056 \\
526 \\
292 \\
3619\end{array}$ & $\begin{array}{r}3323 \\
413 \\
474 \\
1129 \\
3367\end{array}$ & $\begin{array}{r}2933 \\
246 \\
510 \\
1303 \\
26611\end{array}$ & $\begin{array}{r}2305 \\
338 \\
235 \\
622 \\
2281\end{array}$ & $\begin{array}{r}1575 \\
201 \\
251 \\
616 \\
1682\end{array}$ & $\begin{array}{r}809 \\
40 \\
88 \\
645 \\
890\end{array}$ & $\begin{array}{r}171 \\
17 \\
9 \\
2116 \\
203\end{array}$ & $\begin{array}{l}0 \\
0 \\
0 \\
0 \\
0\end{array}$ & $\begin{array}{l}0 \\
0 \\
0 \\
0 \\
0\end{array}$ & $\begin{array}{r}28068 \\
15565 \\
3845 \\
5305 \\
28793\end{array}$ \\
\hline$\stackrel{\boxminus}{\Xi}$ & $\begin{array}{l}11 \\
12 \\
13 \\
14 \\
15\end{array}$ & $\begin{array}{l}0 \\
0 \\
0 \\
0 \\
0\end{array}$ & $\begin{array}{l}0 \\
0 \\
0 \\
0 \\
0\end{array}$ & $\begin{array}{r}107 \\
192 \\
124 \\
110 \\
-555\end{array}$ & $\begin{array}{r}1228 \\
660 \\
504 \\
666 \\
-555\end{array}$ & $\begin{array}{l}1097 \\
1285 \\
1008 \\
1210 \\
-555\end{array}$ & $\begin{array}{r}2072 \\
2162 \\
.1777 \\
2162 \\
-555\end{array}$ & $\begin{array}{l}2796 \\
2923 \\
1646 \\
2594 \\
-555\end{array}$ & $\begin{array}{r}3251 \\
3062 \\
982 \\
2561 \\
-555\end{array}$ & $\begin{array}{r}3575 \\
2709 \\
1132 \\
2853 \\
-555\end{array}$ & $\begin{array}{l}3581 \\
3180 \\
1859 \\
2938 \\
-555\end{array}$ & $\begin{array}{l}3411 \\
2961 \\
1181 \\
1809 \\
-555\end{array}$ & $\begin{array}{r}2976 \\
26,30 \\
779 \\
1020 \\
-555\end{array}$ & $\begin{array}{r}2300 \\
2.054 \\
1364 \\
244 \\
-555\end{array}$ & $\begin{array}{r}1637 \\
171 \\
1158 \\
241 \\
-555\end{array}$ & $\begin{array}{r}829 \\
359 \\
396 \\
189 \\
-555\end{array}$ & $\begin{array}{r}171 \\
68 \\
104 \\
51 \\
-555\end{array}$ & $\begin{array}{l}0 \\
0 \\
0 \\
0 \\
0\end{array}$ & $\begin{array}{l}0 \\
0 \\
0 \\
0 \\
0\end{array}$ & $\begin{array}{r}29129 \\
24916 \\
14014 \\
18648 \\
-999\end{array}$ \\
\hline & $\begin{array}{l}16 \\
17 \\
18 \\
19 \\
20\end{array}$ & $\begin{array}{l}0 \\
0 \\
0 \\
0 \\
0\end{array}$ & $\begin{array}{l}0 \\
0 \\
0 \\
0 \\
0\end{array}$ & $\begin{array}{r}116 \\
64 \\
88 \\
119 \\
99\end{array}$ & $\begin{array}{l}620 \\
509 \\
477 \\
623 \\
465\end{array}$ & $\begin{array}{r}1602 \\
984 \\
1063 \\
1320 \\
1290\end{array}$ & $\begin{array}{l}202.4 \\
2106 \\
140.0 \\
21102 \\
2114.8\end{array}$ & $\begin{array}{l}2626 \\
2715 \\
2179 \\
2763 \\
2766\end{array}$ & $\begin{array}{l}1521 \\
2602 \\
2742 \\
3741 \\
3231\end{array}$ & $\begin{array}{l}1975 \\
1933 \\
2034 \\
3509 \\
3516\end{array}$ & $\begin{array}{l}1927 \\
1930 \\
2945 \\
3447 \\
3503\end{array}$ & $\begin{array}{l}2554 \\
1485 \\
1656 \\
2766 \\
3339\end{array}$ & $\begin{array}{l}1903 \\
1655 \\
1306 \\
1876 \\
3015\end{array}$ & $\begin{array}{r}1841 \\
434 \\
1152 \\
1964 \\
2387\end{array}$ & $\begin{array}{l}1065 \\
198 \\
1063 \\
1516 \\
1631\end{array}$ & $\begin{array}{l}711 \\
179 \\
723 \\
806 \\
865\end{array}$ & $\begin{array}{l}228 \\
221 \\
258 \\
236 \\
141\end{array}$ & $\begin{array}{l}0 \\
0 \\
0 \\
0 \\
0\end{array}$ & $\begin{array}{l}0 \\
0 \\
0 \\
0 \\
0\end{array}$ & $\begin{array}{l}20751 \\
17095 \\
19706 \\
26294 \\
28396\end{array}$ \\
\hline & $\begin{array}{l}21 \\
22 \\
23 \\
24 \\
25\end{array}$ & $\begin{array}{l}0 \\
0 \\
0 \\
0 \\
0\end{array}$ & $\begin{array}{l}0 \\
0 \\
n \\
0 \\
0\end{array}$ & $\begin{array}{r}122 \\
117 \\
66 \\
80 \\
131\end{array}$ & $\begin{array}{l}596 \\
568 \\
263 \\
33 ? \\
623\end{array}$ & $\begin{array}{r}1240 \\
127 \mathrm{i3} \\
557 \\
741 \\
1320\end{array}$ & $\begin{array}{r}2013 \\
2002 \\
993 \\
01253 \\
18.50\end{array}$ & $\begin{array}{l}2634 \\
22411 \\
2148 \\
1873 \\
20155\end{array}$ & $\begin{array}{l}3087 \\
2522 \\
2953 \\
2861 \\
31184\end{array}$ & $\begin{array}{l}3316 \\
2859 \\
2672 \\
3097 \\
2845\end{array}$ & $\begin{array}{l}3365 \\
3417 \\
2884 \\
2560 \\
2691\end{array}$ & $\begin{array}{l}3198 \\
2884 \\
2953 \\
2760 \\
2138\end{array}$ & $\begin{array}{l}2501 \\
2133 \\
2056 \\
2217 \\
2167\end{array}$ & $\begin{array}{r}1820 \\
1584 \\
930 \\
803 \\
2177\end{array}$ & $\begin{array}{r}1068 \\
1255 \\
1336 \\
534 \\
1261\end{array}$ & $\begin{array}{l}626 \\
529 \\
629 \\
469 \\
750\end{array}$ & $\begin{array}{l}223 \\
107 \\
129 \\
296 \\
217\end{array}$ & $\begin{array}{l}0 \\
0 \\
0 \\
0 \\
0\end{array}$ & $\begin{array}{l}0 \\
0 \\
0 \\
0 \\
0\end{array}$ & $\begin{array}{l}25854 \\
23806 \\
20569 \\
19488 \\
23932\end{array}$ \\
\hline & $\begin{array}{l}26 \\
27 \\
28 \\
29 \\
30\end{array}$ & $\begin{array}{l}0 \\
0 \\
0 \\
0 \\
0\end{array}$ & $\begin{array}{l}0 \\
0 \\
0 \\
0 \\
0\end{array}$ & $\begin{array}{r}77 \\
41 . \\
55 \\
131 \\
123\end{array}$ & $\begin{array}{l}1127 \\
516 \\
225 \\
677 \\
581\end{array}$ & $\begin{array}{r}1285 \\
1318 \\
407 \\
1430 \\
1216\end{array}$ & $\begin{array}{r}2021 \\
2191 \\
076 \\
2081 \\
1864\end{array}$ & $\begin{array}{l}2613 \\
2734 \\
1213 \\
2731 \\
2.179\end{array}$ & $\begin{array}{l}3118 \\
3271 \\
1249 \\
3375 \\
2977\end{array}$ & $\begin{array}{r}3386 \\
2479 \\
988 \\
2631 \\
2947\end{array}$ & $\begin{array}{l}3407 \\
2777 \\
1701 \\
3803 \\
2584\end{array}$ & $\begin{array}{l}3304 \\
2479 \\
3043 \\
2850 \\
1674\end{array}$ & $\begin{array}{l}2675 \\
1077 \\
2336 \\
2267 \\
1707\end{array}$ & $\begin{array}{l}2106 \\
1635 \\
1878 \\
2378 \\
1975\end{array}$ & $\begin{array}{r}1612 \\
1125 \\
660 \\
1602 \\
16114\end{array}$ & $\begin{array}{l}312 \\
4111 \\
183 \\
864 \\
8114\end{array}$ & $\begin{array}{r}264 \\
126 \\
78 \\
229 \\
254\end{array}$ & $\begin{array}{l}0 \\
0 \\
0 \\
0 \\
0\end{array}$ & $\begin{array}{l}0 \\
0 \\
0 \\
0 \\
0\end{array}$ & $\begin{array}{l}27282 \\
22984 \\
14952 \\
27062 \\
22899\end{array}$ \\
\hline & 31 & 0 & 0 & คI & 147 & $106 ?$ & 1723 & 2.673 & 3134 & 3383 & 3311 & 2915 & 2911 & 2014 & $160 ?$ & 945 & 267 & 0 & 0 & 26875 \\
\hline & $\begin{array}{r}\text { HENAS } \\
\text { SD } \\
\text { IIUSA }\end{array}$ & $\begin{array}{r}0 \\
31\end{array}$ & $\begin{array}{r}0 \\
0 \\
31\end{array}$ & $\begin{array}{l}83 \\
43 \\
30\end{array}$ & $\begin{array}{r}475 \\
237 \\
30\end{array}$ & $\begin{array}{r}992 \\
430 \\
30\end{array}$ & $\begin{array}{r}1603 \\
608 \\
30\end{array}$ & $\begin{array}{r}21313 \\
111 \\
310\end{array}$ & $\begin{array}{r}2420 \\
970 \\
30\end{array}$ & $\begin{array}{r}2431 \\
10185 \\
30\end{array}$ & $\begin{array}{r}2580 \\
104.3 \\
30\end{array}$ & $\begin{array}{r}2312 \\
459 \\
30\end{array}$ & $\begin{array}{r}1952 \\
773 \\
30\end{array}$ & $\begin{array}{r}1573 \\
734 \\
30\end{array}$ & $\begin{array}{r}100.4 \\
52.4 \\
3.0\end{array}$ & $\begin{array}{r}560 \\
2 B S \\
30\end{array}$ & $\begin{array}{r}157 \\
86 \\
30\end{array}$ & $\begin{array}{r}0 \\
0 \\
31\end{array}$ & $\begin{array}{r}0 \\
0 \\
31\end{array}$ & $\begin{array}{r}20346 \\
7238 \\
30\end{array}$ \\
\hline & $\begin{array}{l}M A x \\
M I \| I\end{array}$ & $\begin{array}{l}0 \\
0\end{array}$ & $\begin{array}{l}0 \\
0\end{array}$ & $\begin{array}{r}122 \\
10\end{array}$ & $\begin{array}{r}1230 \\
119\end{array}$ & $\begin{array}{r}16,02 \\
101\end{array}$ & $\begin{array}{r}2370 \\
151\end{array}$ & $\begin{array}{r}21123 \\
7.52\end{array}$ & $\begin{array}{r}3325 \\
142\end{array}$ & $\begin{array}{r}3927 \\
313\end{array}$ & $\begin{array}{r}3003 \\
24 \%\end{array}$ & $\begin{array}{r}3117 \\
415\end{array}$ & $\begin{array}{r}31115 \\
246\end{array}$ & $\begin{array}{r}2414 \\
235\end{array}$ & $\begin{array}{r}1694 \\
198\end{array}$ & $\begin{array}{r}945 \\
40\end{array}$ & $\begin{array}{r}296 \\
9\end{array}$ & $\begin{array}{l}0 \\
0\end{array}$ & $\begin{array}{l}\dot{0} \\
0\end{array}$ & $\begin{array}{r}29129 \\
3445\end{array}$ \\
\hline
\end{tabular}


CUASTAL - IRLAHO SOLAR RADIATION DIFFERENCE STUDS MU"THLY DATA SUMMARY

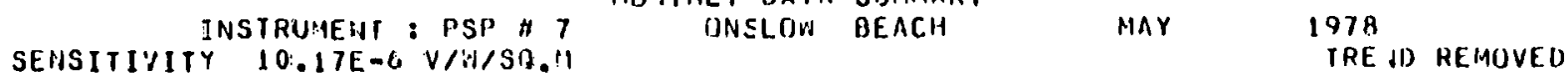

SENSITIVITY 10:17E-6 V/AISO."1 TRE ID REMOVED

KILUJOULES PER SMUARE HETER

ENERGY KILUJOULES

\begin{tabular}{|c|c|c|c|c|c|c|c|c|c|c|c|c|c|c|c|c|c|c|c|}
\hline \multicolumn{20}{|c|}{ HDIJR E.MDING } \\
\hline DATE & 1 & 5 & 6 & 7 & 8 & 9 & 10 & 11 & 12 & 13 & 14 & 15 & $1 \mathrm{~h}$ & 17 & 10 & 19 & 20 & 21 & TOTAL \\
\hline $\begin{array}{l}1 \\
2 \\
3 \\
4 \\
5\end{array}$ & $\begin{array}{l}0 \\
0 \\
0 \\
0 \\
0\end{array}$ & $\begin{array}{l}0 \\
0 \\
0 \\
0 \\
0\end{array}$ & $\begin{array}{r}30 \\
17 \\
-99 \\
13 \\
56\end{array}$ & $\begin{array}{r}35= \\
19 ! \\
-9 ! \\
45 \\
520\end{array}$ & $\begin{array}{r}770 \\
346 \\
-99 \\
112 \\
1369\end{array}$ & $\begin{array}{r}5: 8 \\
256 \\
-57 \\
15.5 \\
2205\end{array}$ & $\begin{array}{r}1571 \\
6.37 \\
-99 \\
314 \\
1897\end{array}$ & $\begin{array}{r}1669 \\
725 \\
-99 \\
1173 \\
1417\end{array}$ & $\begin{array}{r}2604 \\
739 \\
-99 \\
597 \\
21110\end{array}$ & $\begin{array}{r}1655 \\
761 \\
-99 \\
1202 \\
2567\end{array}$ & $\begin{array}{r}1772 \\
-99 \\
-99 \\
2629 \\
3274\end{array}$ & $\begin{array}{r}222 \\
-99 \\
2718 \\
2007 \\
2799\end{array}$ & $\begin{array}{r}140 \\
-99 \\
1706 \\
1375 \\
2254\end{array}$ & $\begin{array}{r}126 \\
-99 \\
955 \\
568 \\
1401\end{array}$ & $\begin{array}{r}83 \\
-93 \\
311 \\
260 \\
683\end{array}$ & $\begin{array}{r}9 \\
-99 \\
53 \\
48 \\
113\end{array}$ & $\begin{array}{l}0 \\
0 \\
0 \\
0 \\
0\end{array}$ & $\begin{array}{l}0 \\
0 \\
0 \\
0 \\
0\end{array}$ & $\begin{array}{r}11525 \\
-999 \\
-999 \\
9810 \\
22987\end{array}$ \\
\hline $\begin{array}{r}6 \\
7 \\
8 \\
7 \\
10\end{array}$ & $\begin{array}{l}0 \\
0 \\
0 \\
0 \\
0\end{array}$ & $\begin{array}{l}0 \\
0 \\
0 \\
0 \\
0\end{array}$ & $\begin{array}{r}88 \\
123 \\
9 \\
77 \\
120\end{array}$ & $\begin{array}{r}640 \\
50 n \\
45 \\
346 \\
690\end{array}$ & $\begin{array}{r}1376 \\
1189 \\
913 \\
559 \\
1472\end{array}$ & $\begin{array}{r}2071) \\
1493 \\
100 \\
1171 \\
2201\end{array}$ & $\begin{array}{r}2764 \\
2028 \\
133 \\
2031 \\
21531\end{array}$ & $\begin{array}{r}319 \bar{c} \\
2083 \\
363 \\
927 \\
3323\end{array}$ & $\begin{array}{r}3454 \\
1886 \\
352 \\
145 \\
3347\end{array}$ & $\begin{array}{r}3511 \\
1394 \\
303 \\
176 \\
3599\end{array}$ & $\begin{array}{r}3295 \\
952 \\
236 \\
60 \\
1649\end{array}$ & $\begin{array}{r}2792 \\
453 \\
207 \\
67 \\
2265\end{array}$ & $\begin{array}{r}2215 \\
371 \\
115 \\
304 \\
1752\end{array}$ & $\begin{array}{r}1518 \\
403 \\
105 \\
481 \\
1505\end{array}$ & $\begin{array}{r}743 \\
113 \\
66 \\
392 \\
838\end{array}$ & $\begin{array}{r}148 \\
0 \\
16 \\
106 \\
100\end{array}$ & $\begin{array}{l}0 \\
0 \\
0 \\
0 \\
0\end{array}$ & $\begin{array}{l}0 \\
0 \\
0 \\
0 \\
0\end{array}$ & $\begin{array}{r}27806 \\
12999 \\
2188 \\
6442 \\
25895\end{array}$ \\
\hline $\begin{array}{l}11 \\
13 \\
13 \\
14 \\
15\end{array}$ & $\begin{array}{r}0 \\
.0 \\
0 \\
0 \\
0\end{array}$ & $\begin{array}{l}0 \\
0 \\
0 \\
0 \\
0\end{array}$ & $\begin{array}{r}173 \\
99 \\
74 \\
106 \\
42\end{array}$ & $\begin{array}{l}615 \\
59 ! \\
520 \\
69 ? \\
300\end{array}$ & $\begin{array}{r}1118 \\
1327 \\
1313 \\
1529 \\
669\end{array}$ & $\begin{array}{c}1971 \\
2205 \\
1907 \\
1553 \\
1363\end{array}$ & $\begin{array}{r}2849 \\
2538 \\
2729 \\
1727 \\
934\end{array}$ & $\begin{array}{l}3305 \\
2491 \\
3214 \\
2134 \\
1263\end{array}$ & $\begin{array}{l}3553 \\
2322 \\
3256 \\
2899 \\
216 ?\end{array}$ & $\begin{array}{l}3571 \\
2623 \\
3076 \\
3518 \\
2670\end{array}$ & $\begin{array}{l}3362 \\
1982 \\
2672 \\
2630 \\
2180\end{array}$ & $\begin{array}{l}29311 \\
2775 \\
1050 \\
2980 \\
1607\end{array}$ & $\begin{array}{r}2300 \\
1801 \\
574 \\
1451 \\
1131\end{array}$ & $\begin{array}{r}1570 \\
1397 \\
263 \\
495 \\
257\end{array}$ & $\begin{array}{l}778 \\
442 \\
3011 \\
31.8 \\
47.4\end{array}$ & $\begin{array}{r}180 \\
67 \\
42 \\
53 \\
169\end{array}$ & $\begin{array}{l}0 \\
0 \\
0 \\
0 \\
0\end{array}$ & $\begin{array}{l}0 \\
0 \\
0 \\
0 \\
0\end{array}$ & $\begin{array}{l}28291 \\
22640 \\
21332 \\
22090 \\
14151\end{array}$ \\
\hline $\begin{array}{l}16 \\
17 \\
18 \\
19 \\
20\end{array}$ & $\begin{array}{l}0 \\
0 \\
0 \\
0 \\
0\end{array}$ & $\begin{array}{l}0 \\
0 \\
0 \\
0 \\
n\end{array}$ & $\begin{array}{r}127 \\
-29 \\
-97 \\
-97 \\
0\end{array}$ & $\begin{array}{l}715 \\
-99 \\
-99 \\
-99 \\
160\end{array}$ & $\begin{array}{r}1486 \\
-99 \\
-99 \\
-99 \\
708\end{array}$ & $\begin{array}{r}2102 \\
-97 \\
-97 \\
-79 \\
1401\end{array}$ & $\begin{array}{r}2003 \\
-99 \\
-99 \\
-919 \\
21130\end{array}$ & $\begin{array}{r}1702 \\
-99 \\
-99 \\
-99 \\
21119\end{array}$ & $\begin{array}{r}2672 \\
-99 \\
-99 \\
-99 \\
3309\end{array}$ & $\begin{array}{r}3270 \\
-99 \\
-97 \\
-99 \\
3553\end{array}$ & $\begin{array}{r}1876 \\
-99 \\
-99 \\
-99 \\
3568\end{array}$ & $\begin{array}{r}1019 \\
-99 \\
-99 \\
-99 \\
3338\end{array}$ & $\begin{array}{r}-79 \\
-94 \\
-99 \\
21492 \\
2867\end{array}$ & $\begin{array}{r}-59 \\
-59 \\
-59 \\
1964 \\
2157\end{array}$ & $\begin{array}{r}-79 \\
-59 \\
-79 \\
1359 \\
5 \leftarrow 2\end{array}$ & $\begin{array}{r}-99 \\
-94 \\
-99 \\
-666 \\
70\end{array}$ & $\begin{array}{l}0 \\
0 \\
0 \\
0 \\
0\end{array}$ & $\begin{array}{l}0 \\
0 \\
0 \\
0 \\
0\end{array}$ & $\begin{array}{r}-999 \\
-999 \\
-999 \\
-999 \\
26806\end{array}$ \\
\hline $\begin{array}{l}21 \\
22 \\
23 \\
24 \\
25\end{array}$ & $\begin{array}{l}0 \\
0 \\
0 \\
0 \\
0\end{array}$ & $\begin{array}{l}0 \\
0 \\
0 \\
0 \\
0\end{array}$ & $\begin{array}{r}11 \\
-97 \\
-97 \\
-97 \\
-97\end{array}$ & $\begin{array}{l}110 \\
-59 \\
-52 \\
-5.7 \\
-51\end{array}$ & $\begin{array}{l}681 \\
-99 \\
-99 \\
-99 \\
-99\end{array}$ & $\begin{array}{r}14105 \\
-99 \\
1409 \\
-79 \\
-97\end{array}$ & $\begin{array}{r}2014 \\
-99 \\
-99 \\
-97 \\
-99\end{array}$ & $\begin{array}{r}265: \\
-99 \\
-818 \\
-99 \\
-79\end{array}$ & $\begin{array}{r}1189 \\
-99 \\
2309 \\
-99 \\
-90\end{array}$ & $\begin{array}{r}3440 \\
-99 \\
3553 \\
-99 \\
-97\end{array}$ & $\begin{array}{r}3316 \\
-99 \\
3561 \\
-99 \\
-99\end{array}$ & $\begin{array}{r}3111 \\
-97 \\
3338 \\
-99 \\
1826\end{array}$ & $\begin{array}{r}2654 \\
-99 \\
2867 \\
-99 \\
11108\end{array}$ & $\begin{array}{r}205.8 \\
-0.9 \\
215.7 \\
-0.9 \\
1304\end{array}$ & $\begin{array}{r}116.4 \\
-6.9 \\
-6.9 \\
-99 \\
623\end{array}$ & $\begin{array}{r}-666 \\
-99 \\
-99 \\
-99 \\
180\end{array}$ & $\begin{array}{l}0 \\
0 \\
0 \\
0 \\
0\end{array}$ & $\begin{array}{l}0 \\
0 \\
0 \\
0 \\
0\end{array}$ & $\begin{array}{l}-999 \\
-999 \\
-999 \\
-999 \\
-999\end{array}$ \\
\hline $\begin{array}{l}26 \\
27 \\
28 \\
29 \\
30\end{array}$ & $\begin{array}{l}0 \\
0 \\
0 \\
0 \\
0\end{array}$ & $\begin{array}{l}0 \\
0 \\
0 \\
0 \\
0\end{array}$ & $\begin{array}{r}B 4 \\
159 \\
27 \\
113 \\
148\end{array}$ & $\begin{array}{l}53 ! 1 \\
507 \\
131 \\
659 \\
654\end{array}$ & $\begin{array}{r}944 \\
1210 \\
304 \\
1404 \\
1345\end{array}$ & $\begin{array}{r}1+38 \\
1775 \\
431 \\
2815 \\
2028\end{array}$ & $\begin{array}{r}2615 \\
1692 \\
753 \\
2814 \\
2630\end{array}$ & $\begin{array}{l}3192 \\
1617 \\
1189 \\
3263 \\
3054\end{array}$ & $\begin{array}{l}3500 \\
1861 \\
2067 \\
2477 \\
2863\end{array}$ & $\begin{array}{l}3044 \\
1985 \\
1784 \\
2987 \\
1670\end{array}$ & $\begin{array}{l}2527 \\
2155 \\
1281 \\
31119 \\
1886\end{array}$ & $\begin{array}{l}2339 \\
1801 \\
2463 \\
2392 \\
2807\end{array}$ & $\begin{array}{l}2104 \\
1161 \\
2155 \\
2343 \\
1316\end{array}$ & $\begin{array}{r}1360 \\
962 \\
7 \% 6 \\
15: 1 \\
707\end{array}$ & $\begin{array}{l}743 \\
325 \\
650 \\
851 \\
180\end{array}$ & $\begin{array}{l}346 \\
169 \\
2013 \\
223 \\
162\end{array}$ & $\begin{array}{l}0 \\
0 \\
0 \\
0 \\
0\end{array}$ & $\begin{array}{l}0 \\
0 \\
0 \\
0 \\
0\end{array}$ & $\begin{array}{l}25034 \\
17439 \\
14273 \\
26775 \\
21454\end{array}$ \\
\hline 31 & 0 & 0 & 133 & 573 & 1476 & 5030 & 2587 & 3065 & 3323 & 3192 & 3238 & 2651 & 2141 & $14: 1$ & 711 & 237 & 0 & 0 & $2671 \mathrm{~B}$ \\
\hline $\begin{array}{r}\text { HEAH } \\
\text { SD } \\
\text { HIJM }\end{array}$ & $\begin{array}{r}0 \\
0 \\
31\end{array}$ & $\begin{array}{r}0 \\
u \\
31\end{array}$ & $\begin{array}{l}19 \\
52 \\
23\end{array}$ & $\begin{array}{r}1122 \\
227 \\
23\end{array}$ & $\begin{array}{r}1001 \\
457 \\
23\end{array}$ & $\begin{array}{r}19.75 \\
635 \\
24\end{array}$ & $\begin{array}{r}1225 \\
828 \\
23\end{array}$ & $\begin{array}{r}21.37 \\
983 \\
23\end{array}$ & $\begin{array}{r}2430 \\
1021 \\
24\end{array}$ & $\begin{array}{r}2471 \\
1078 \\
24\end{array}$ & $\begin{array}{r}2327 \\
1003 \\
23\end{array}$ & $\begin{array}{r}2073 \\
1001 \\
25\end{array}$ & $\begin{array}{r}16,24 \\
841 \\
25\end{array}$ & $\begin{array}{r}1105 \\
646 \\
25\end{array}$ & $\begin{array}{r}5.36 \\
323 \\
24\end{array}$ & $\begin{array}{r}126 \\
46 \\
2 ?\end{array}$ & $\begin{array}{r}0 \\
0 \\
31\end{array}$ & $\begin{array}{r}0 \\
0 \\
31\end{array}$ & $\begin{array}{r}19356 \\
7525 \\
20\end{array}$ \\
\hline $\begin{array}{l}M A X \\
M I \|\end{array}$ & $\begin{array}{l}0 \\
0\end{array}$ & $\begin{array}{l}0 \\
0\end{array}$ & $\begin{array}{r}173 \\
10\end{array}$ & $\begin{array}{r}715 \\
115\end{array}$ & $\begin{array}{r}1529 \\
48\end{array}$ & $\begin{array}{r}2215 \\
14 \pi\end{array}$ & $\begin{array}{r}2849 \\
133\end{array}$ & $\begin{array}{r}3323 \\
363\end{array}$ & $\begin{array}{r}3553 \\
145\end{array}$ & $\begin{array}{r}3597 \\
176\end{array}$ & $\begin{array}{r}3560 \\
60\end{array}$ & $\begin{array}{r}3338 \\
67\end{array}$ & $\begin{array}{r}2367 \\
115\end{array}$ & $\begin{array}{r}2117 \\
105\end{array}$ & $\begin{array}{r}1359 \\
66\end{array}$ & $\begin{array}{r}346 \\
0\end{array}$ & $\begin{array}{l}0 \\
0\end{array}$ & $\begin{array}{l}0 \\
0\end{array}$ & $\begin{array}{r}28291 \\
2188\end{array}$ \\
\hline
\end{tabular}


COMSTAL - IIILAND SOLAR RAOIATIUN DIFFEREHCE STUIYY IHSTRUHEMT : HIP $\$ 8$
SEHSITIVITY MUHTHLY DATA SUMMARY

EHERGY KILIJOULES PER. SUUART METER HOUR ENDING

ATE

$10111^{10} 12^{13}$

$14 \quad 15 \quad 16$

17

18

19

20

21 TOTAL

1
2
3
4
5

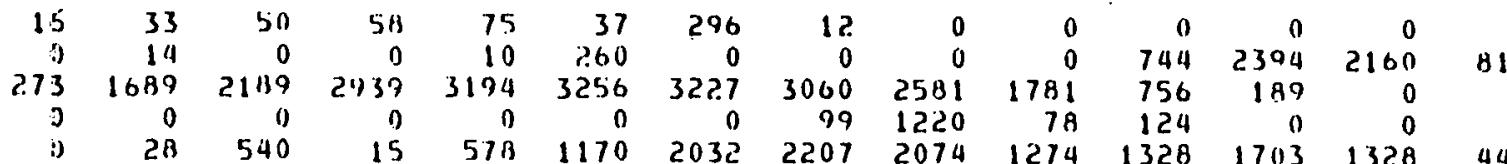

$\begin{array}{llllllllllllllll}332 & 1536 & 2095 & 2361 & 25190 & 2645 & 2703 & 2842 & 2807 & 2761 & 2549 & 1953 & 1565 & 478\end{array}$

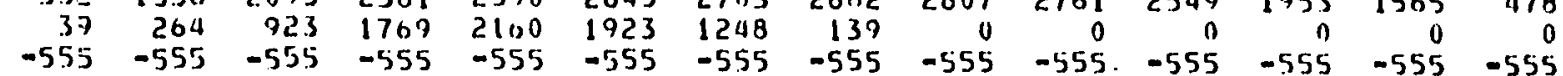

$\begin{array}{llllllllllllll}-555 & -555 & -555 & -555 & -555 & -555 & -555 & -555 & -555 & -555 & -555 & -555 & -555 & -555 \\ -555 & -555 & -555 & -555 & -555 & -555 & -555 & -555 & -555 & -555 & -555 & -555 & -555 & -555\end{array}$

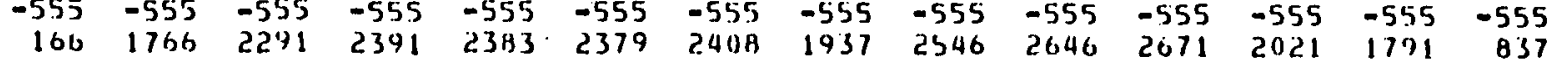

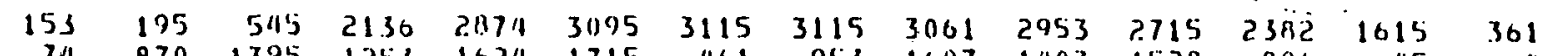

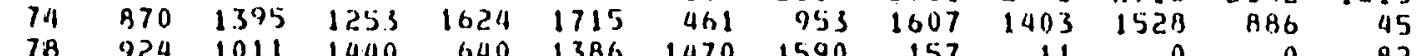

$78 \quad 924 \quad 1011 \quad 1440$

1470

$\begin{array}{lr}1590 & 157\end{array}$

11

11

$\begin{array}{rrr}0 & 0 \\ 70 & 315 & 0\end{array}$

0
5

$\begin{array}{rrr}403 & 2020 & 2049 \\ 0 & 0 & 519\end{array}$

0

$\begin{array}{rrrr}0 & 324 & 2324 & 1270 \\ 0 & 0 & 214 & 794\end{array}$

14

227

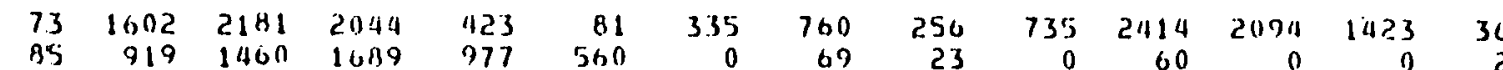

$\begin{array}{llllllllllllll}0 & 0 & 503 & 1403 & 1186 & 1474 & 865 & 807 & 240 & 332 & 220 & 0 & 132 & 161\end{array}$

$\begin{array}{lllllllllllllllll}101 & 761 & 1074 & 1207 & 1220 & 12140 & 1299 & 1503 & 1853 & 1107 & 1253 & 1220 & 1090 & 255\end{array}$

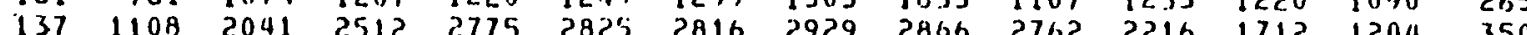

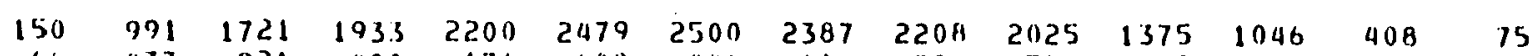

66

433921

$\begin{array}{rrrrrrrrrrr}33 & 921 & 400 & 171 & 608 & 446 & 116 & 701 & 501 & 162 & 29 \\ 0 & 0 & 58 & 0 & 41 & 537 & 512 & 756 & 891 & 1204 & 1333 \\ 0 & 71 & 116 & 223 & 725 & 1021 & 1404 & 2016 & 1608 & 1312 & 1300\end{array}$

0
12

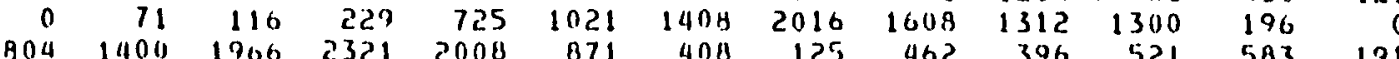

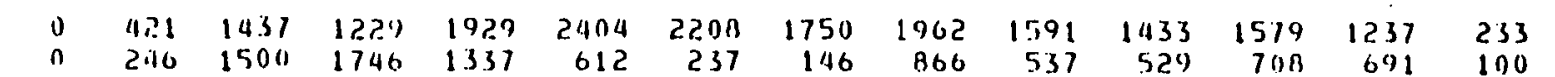

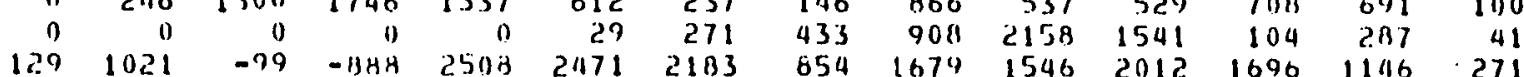

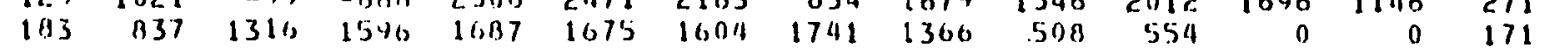

$223950 \quad 1581 \quad 1913021173 \quad 2277 \quad 2235 \quad 2052 \quad 1764 \quad 1443 \quad 1535 \quad 1452 \quad 1243 \quad 556$

3

$\begin{array}{rr}\text { MEAN } & 0 \\ \text { SI) } & 11 \\ \text { (HII! } & 31\end{array}$

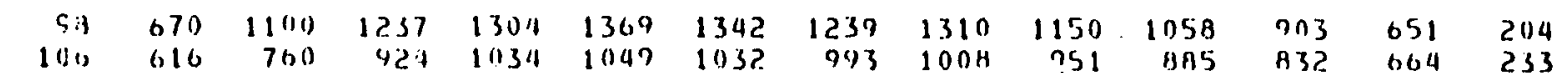

$\operatorname{MAX}$

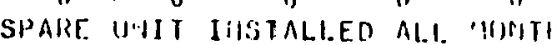

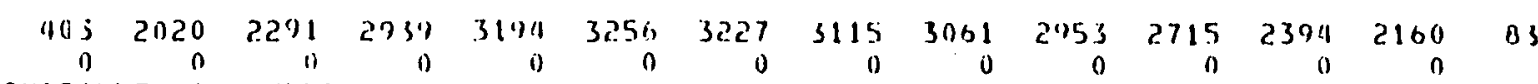


CGASTAL - IHLAHL SIJLAR RADIATEON OIFFERENCE STUDY MOMTHLY DATA SUMMARY

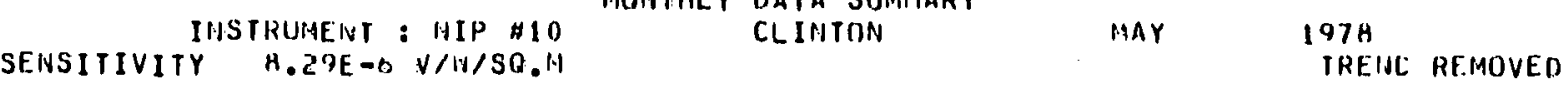

EHERGY RILOJJOULES PER SIJUARE HETER.

HOLIR ENDIHL

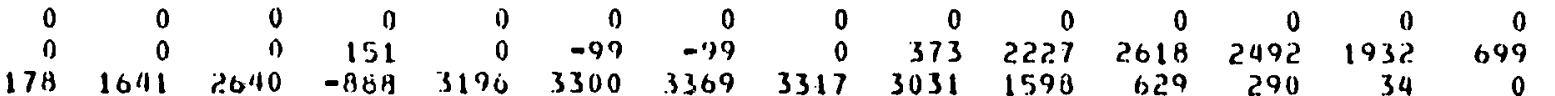

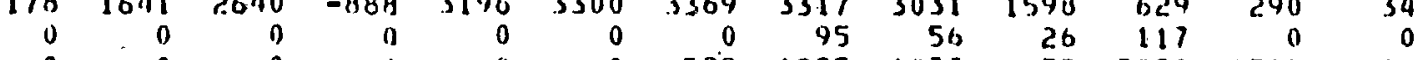

$269 \quad 1389$

403

011

052

$\begin{array}{rr}0 & 0 \\ 234 & 1936\end{array}$

1233
0
0

$\div 728$

$2683 \quad 2831$

29 197

$5 \quad 1433$

$2670 \quad 29443074 \quad 3239$

$1889 \quad 42$

212831

36732080

$56 \quad 269$

30

$65 \quad 160$

712
5110

24713009

$\begin{array}{rrrr}0 & 0 & 0 & 0 \\ 0 & 91 & 165 & 0 \\ 96 & 3100 & 1080 & 2119\end{array}$

$170,2 \quad 1111$

$\begin{array}{ll}-555 & -555\end{array}$

$738 \quad 39$

3009310

0

$52592 \quad 2305 \quad 1763$ i)

0
0

000

$1)$
0
186

655
0

0

26

390

0
0

160

73

130

$196 \quad 3204$

$5350 \% 52570 \quad 1724$

$\begin{array}{rrrr}56 & 1932 & 1645 & 1337\end{array}$

2570
21

6314

78

51 6B1

833

$275 \quad 165$

$\begin{array}{rr}0 & 0 \\ -555 & -555\end{array}$

$-555$

$421 \quad 334$

143
746

$\begin{array}{llrr}1844 & 69 & -99\end{array}$

555

$\begin{array}{rrrrr}191 & 112 & 338 & 573 & 525 \\ 1728 & 2297 & 2031 & 2757 & 2.779\end{array}$

234

$1016 \quad 1528$

2481 2761

595
568

946

$1103 \quad 1399$

$\begin{array}{rrrrrr}2449 & 1341 & 34 & 39 & 82 & 125\end{array}$

20

22

24

147

195

0

$\begin{array}{llllll}528 & 1958 & 2171 & 2253 & 2206 & 2227\end{array}$

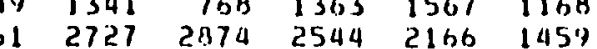

738
99

60

616
251

82

$998 \quad 151$

$01003 \quad 920$

$\begin{array}{lllllll}2206 & 2227 & 2106 & 1298 & 742 & 451 & 138\end{array}$

$\begin{array}{rrrrrrrrrr}1 & 551 & 1202 & 812 & 1116 & 12119 & 586 & 104 & 816 & 382 \\ 13 & 177 & 1189 & 1111 & 725 & 1068 & 920 & 57 & 0 & 212\end{array}$

0
39

39
156

$23 \mathrm{H}$

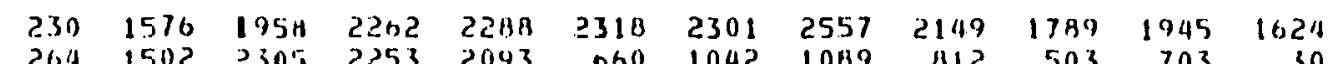

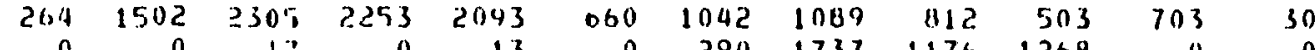

664

$\begin{array}{rrrrrrrrrrrrr}86 & 977 & 1376 & 2045 & 2336 & 2311 & 1406 & 2635 & 1606 & 1506 & 2236 & 1489 & -8188 \\ 112 & 590 & 933 & 1003 & 1430 & 1521 & 1312 & 846 & 334 & 347 & 994 & 1237 & 641\end{array}$

$4311369 \quad 1116 \quad 2171 \quad 2314 \quad 2566 \quad 2331 \quad 2093 \quad 2423 \quad 2292 \quad 1910 \quad 1602$

502

MEAH SH

SI)

B4

502

$75 \quad 12001491 \quad 1.192 \quad 131$

$13811251 \quad 1068$ 1095 921 636

0
0
-09

238

560

220

279
29

MAX

$72^{17}$

$936 \quad 3743 \quad 2910$

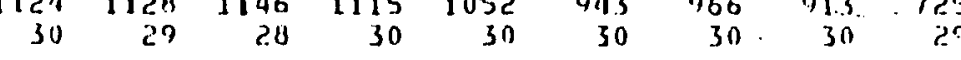

HIII

0

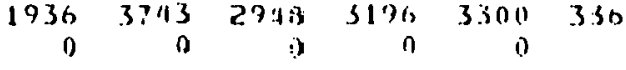

1) 3.31

320

$\begin{array}{rrrr}3122 & 2952 & 2570 & 2023 \\ 0 & 0 & 0 & 0\end{array}$ 
COASTAL - IIILAND SIILAR RADIATIUN DIFFERENCE. STUDY

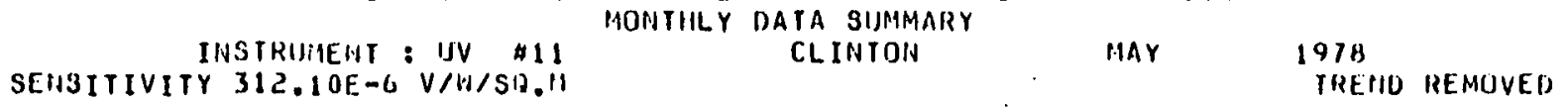

EHERGY KILOJUULES PER SIJUARE. METER HDUR EHDING

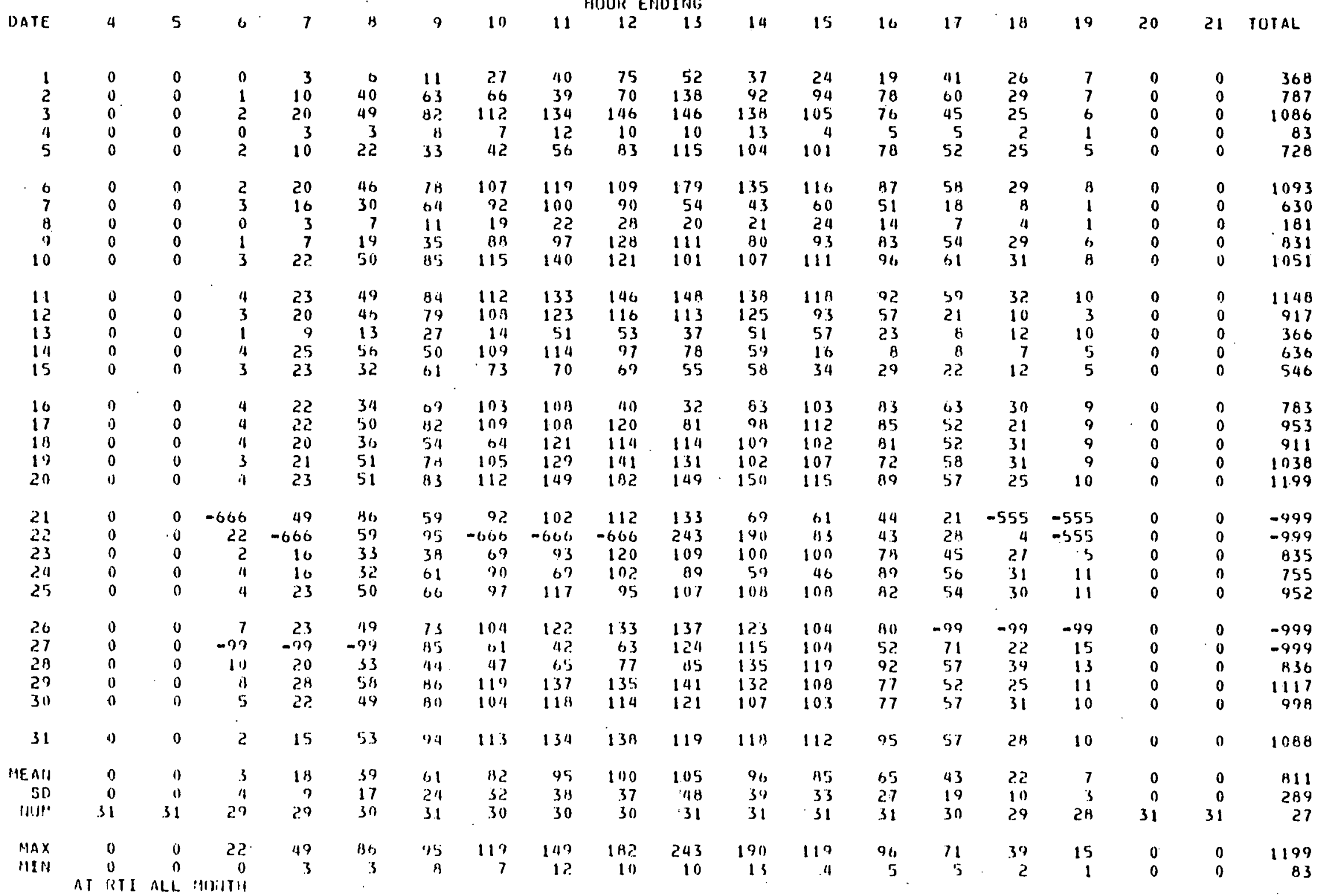


CLIASTAL - IALAHD SOLAR RADIATIUN DIFFERENCE 3TIMY HONTHL DATA SUMAIRY

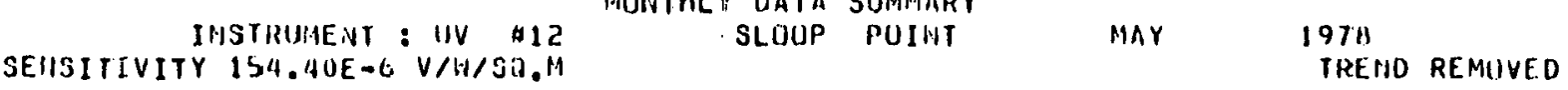

EINERGY KILOJDULES PER SIJUARE HETER IOUR ENDIHG

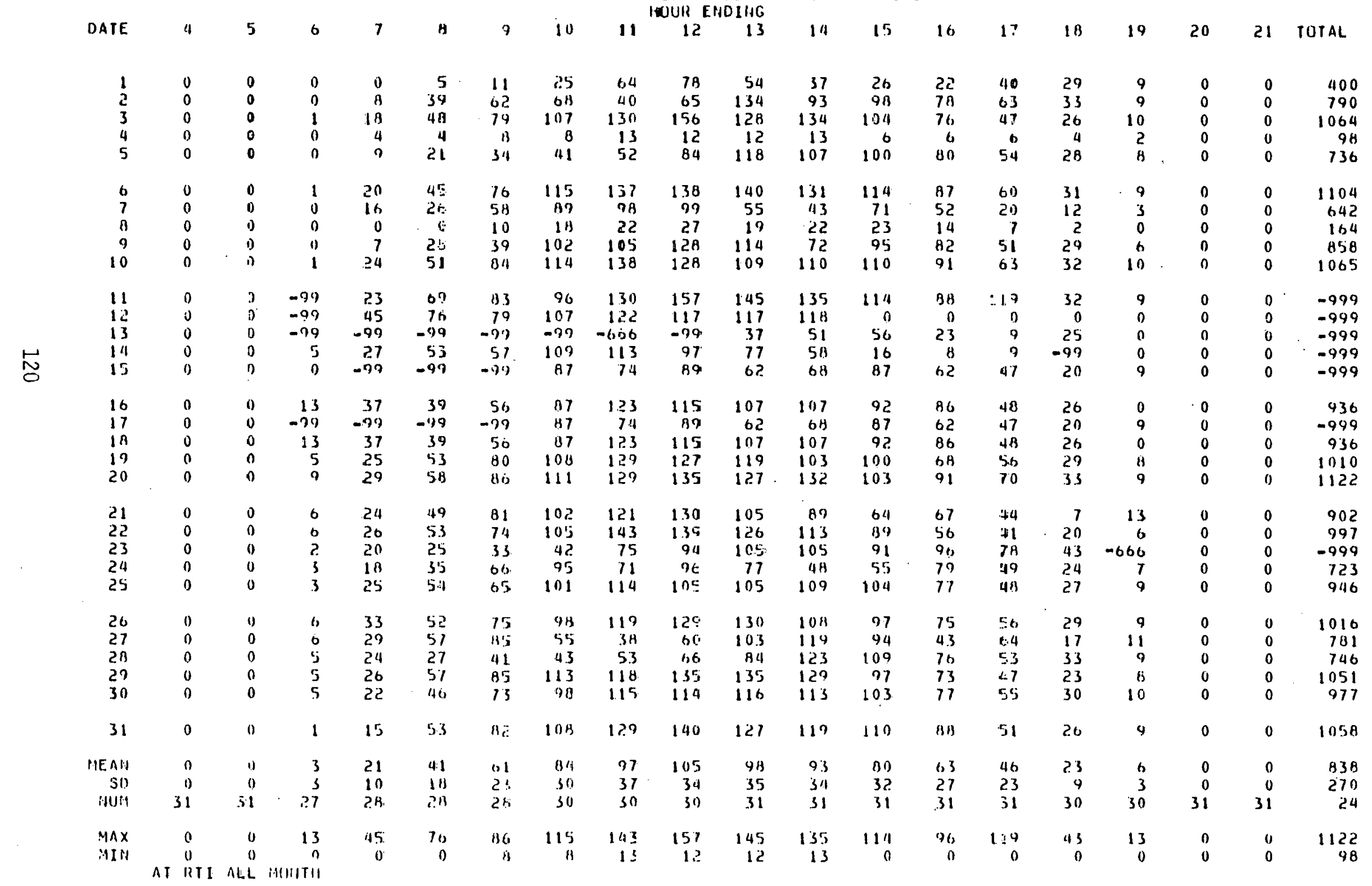


CUASTAL - IIJLAHD GMLAR RADIATIIJN DIFFEREIICE BTUDY MONTHLY DATA SUMHAHY

IHSTRUIAENT: PSP 2
SEHITIVITY $10.99 E-6$ VIN/SI.H.

EIJERLYY KILIJJOULES PER SIJUARE MEIER IIOUR ENOINC:

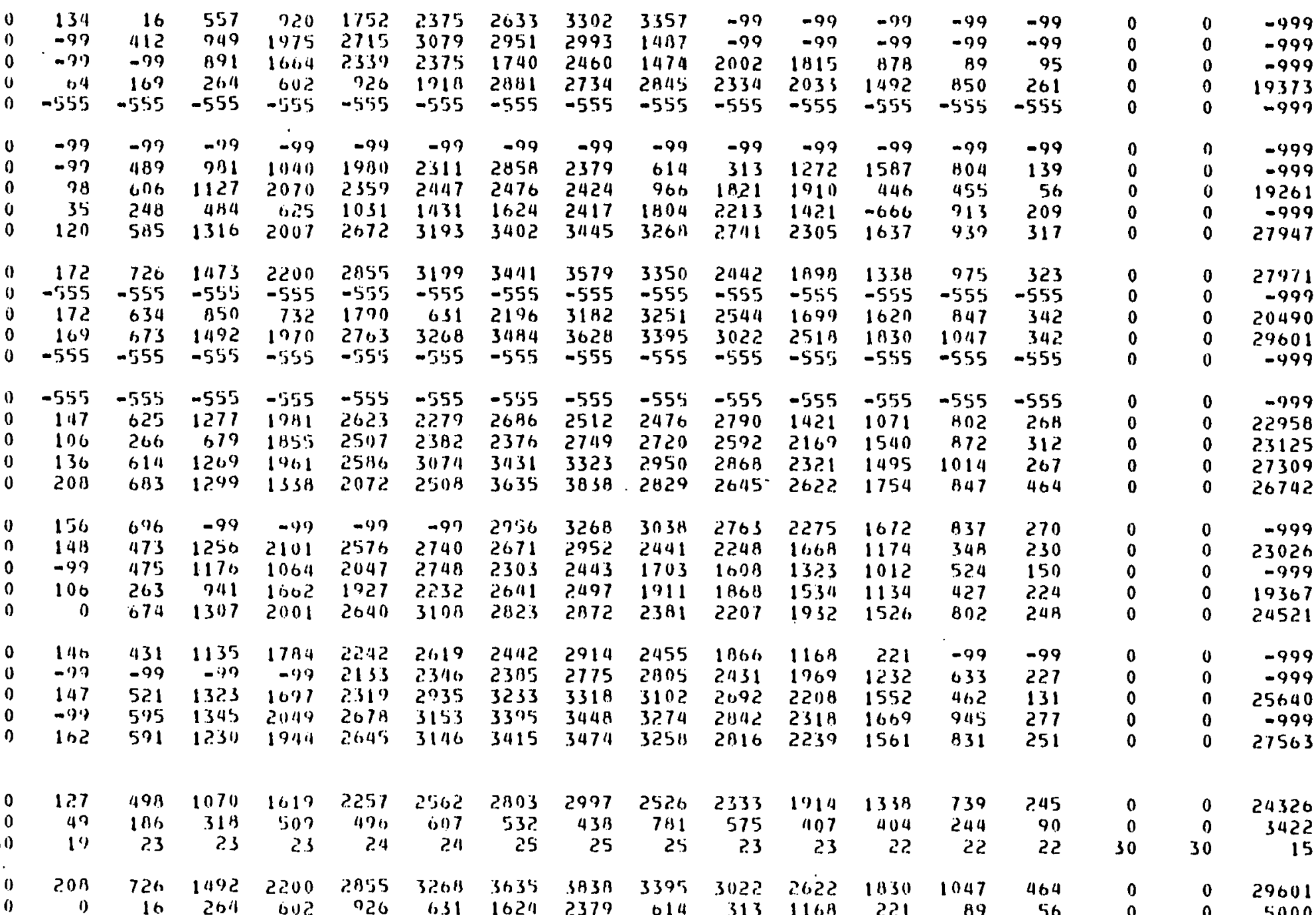


CIIASTAL - INLAIII) SULAR RAOIATIUN DIFFERENCE. ST.JDY H(INTHILY DATA SUMMAIRY

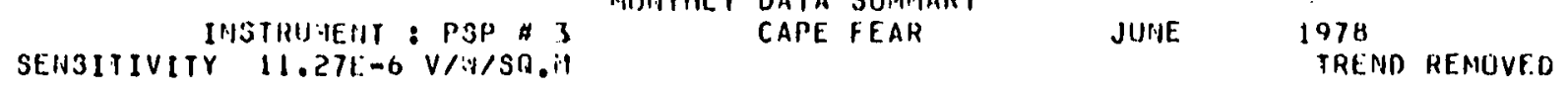

EHERGY KILUJOULES PER SWUARE METER HiI) F. F.IIIIHG

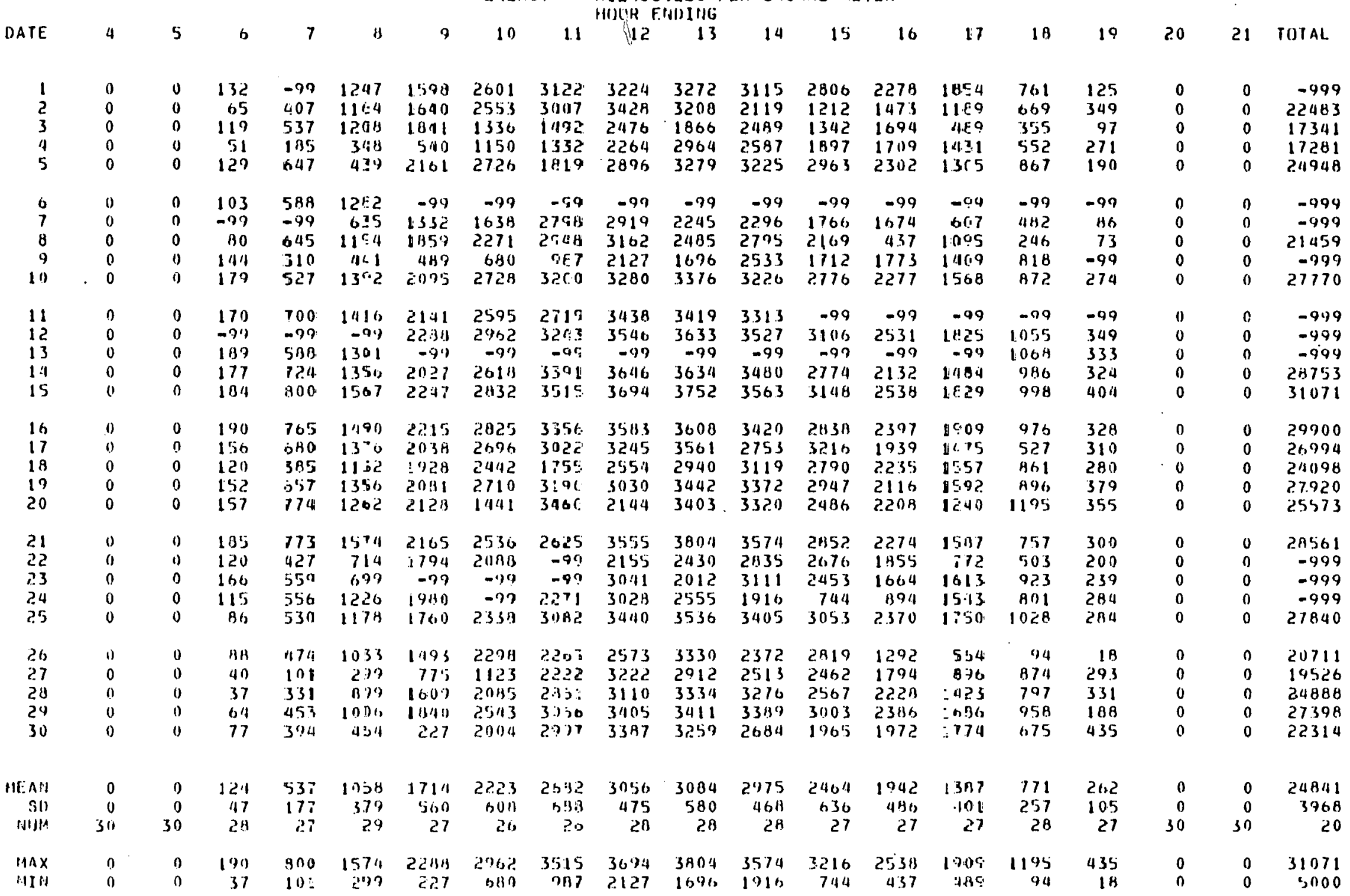


COASTAL - INLAIIN SOLAR RADIATIUN DIFFERENCE STUDY

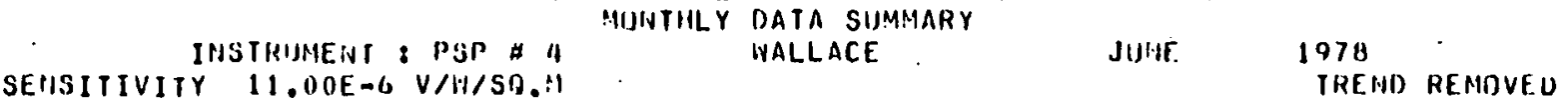

E.MEHIY KILOJOULES PER SUUARE METER

\begin{tabular}{|c|c|c|c|c|c|c|c|c|c|c|c|c|c|c|c|c|c|c|c|}
\hline \multirow[b]{2}{*}{ DATE } & \multirow[b]{2}{*}{4} & \multirow[b]{2}{*}{5} & \multirow[b]{2}{*}{ D } & \multirow[b]{2}{*}{7} & & & & & C & 20181, & \multirow{2}{*}{\multicolumn{2}{|c|}{15}} & \multirow[b]{2}{*}{16} & \multirow[b]{2}{*}{17} & \multirow[b]{2}{*}{18} & \multirow[b]{2}{*}{19} & \multirow[b]{2}{*}{20} & \multirow[b]{2}{*}{21} & \multirow[b]{2}{*}{ TOTAL } \\
\hline & & & & & 8 & 9 & 10 & 11 & 12 & 13 & & & & & & & & & \\
\hline $\begin{array}{l}1 \\
2 \\
3 \\
4 \\
5\end{array}$ & $\begin{array}{l}n \\
0 \\
11 \\
0 \\
0\end{array}$ & $\begin{array}{l}0 \\
0 \\
0 \\
0 \\
0\end{array}$ & $\begin{array}{r}129 \\
475 \\
178 \\
-8 B A \\
218\end{array}$ & $\begin{array}{l}571 \\
298 \\
620 \\
181 \\
030\end{array}$ & $\begin{array}{r}8913 \\
6213 \\
1271 \\
305 \\
1625\end{array}$ & $\begin{array}{r}1756 \\
1699 \\
1969 \\
650 \\
2129\end{array}$ & $\begin{array}{l}2698 \\
2641 \\
2246 \\
1254 \\
2718\end{array}$ & $\begin{array}{l}3117 \\
2743 \\
1264 \\
2236 \\
2653\end{array}$ & $\begin{array}{l}3373 \\
3240 \\
1902 \\
3375 \\
2334\end{array}$ & $\begin{array}{l}3013 \\
1967 \\
1317 \\
3257 \\
1838\end{array}$ & $\begin{array}{r}2741 \\
203 \\
1182 \\
2776 \\
2970\end{array}$ & $\begin{array}{r}1504 \\
605 \\
2485 \\
2416 \\
1416\end{array}$ & $\begin{array}{l}1314 \\
1113 \\
1506 \\
1418 \\
1713\end{array}$ & $\begin{array}{r}1494 \\
893 \\
426 \\
1454 \\
1298\end{array}$ & $\begin{array}{r}725 \\
586 \\
19 \\
796 \\
601\end{array}$ & $\begin{array}{l}221 \\
200 \\
-99 \\
224 \\
139\end{array}$ & $\begin{array}{l}0 \\
0 \\
0 \\
0 \\
0\end{array}$ & $\begin{array}{l}0 \\
0 \\
0 \\
0 \\
0\end{array}$ & $\begin{array}{r}23784 \\
17294 \\
-999 \\
-999 \\
27532\end{array}$ \\
\hline $\begin{array}{r}0 \\
7 \\
0 \\
9 \\
10\end{array}$ & $\begin{array}{l}0 \\
0 \\
0 \\
0 \\
0\end{array}$ & $\begin{array}{l}0 \\
0 \\
0 \\
0 \\
0\end{array}$ & $\begin{array}{r}-660 \\
-666 \\
-848 \\
-99 \\
330\end{array}$ & $\begin{array}{l}653 \\
585 \\
628 \\
288 \\
520\end{array}$ & $\begin{array}{r}1422 \\
928 \\
1499 \\
497 \\
1279\end{array}$ & $\begin{array}{r}1903 \\
1219 \\
2117 \\
432 \\
1913\end{array}$ & $\begin{array}{l}2162 \\
2502 \\
2297 \\
1099 \\
2521\end{array}$ & $\begin{array}{r}2214 \\
247.1 \\
-99 \\
1754 \\
2367\end{array}$ & $\begin{array}{r}3082 \\
1537 \\
-99 \\
1456 \\
3403\end{array}$ & $\begin{array}{r}2558 \\
1419 \\
-99 \\
2327 \\
31113\end{array}$ & $\begin{array}{r}1393 \\
755 \\
-99 \\
1682 \\
3260\end{array}$ & $\begin{array}{r}1147 \\
997 \\
-99 \\
1990 \\
21370\end{array}$ & $\begin{array}{r}981 \\
1083 \\
-99 \\
2330 \\
2297\end{array}$ & $\begin{array}{r}1213 \\
990 \\
-99 \\
1777 \\
11561\end{array}$ & $\begin{array}{r}297 \\
853 \\
-99 \\
1047 \\
697\end{array}$ & $\begin{array}{r}74 \\
143 \\
-99 \\
262 \\
330\end{array}$ & $\begin{array}{l}0 \\
0 \\
0 \\
0 \\
0\end{array}$ & $\begin{array}{l}0 \\
0 \\
0 \\
0 \\
0\end{array}$ & $\begin{array}{r}-999 \\
-999 \\
-999 \\
-999 \\
271467\end{array}$ \\
\hline $\begin{array}{l}11 \\
12 \\
13 \\
14 \\
15\end{array}$ & $\begin{array}{l}0 \\
0 \\
0 \\
0 \\
0\end{array}$ & $\begin{array}{l}0 \\
0 \\
0 \\
0 \\
0\end{array}$ & $\begin{array}{r}-606 \\
185 \\
136 \\
349 \\
-606\end{array}$ & $\begin{array}{l}6,66 \\
748 \\
573 \\
457 \\
076\end{array}$ & $\begin{array}{r}13139 \\
1471 \\
714 \\
1402 \\
1543\end{array}$ & $\begin{array}{l}2117 \\
2201 \\
1061 \\
2073 \\
2260\end{array}$ & $\begin{array}{l}2744 \\
2810 \\
1333 \\
2764 \\
2906\end{array}$ & $\begin{array}{l}2810 \\
3317 \\
1523 \\
3245 \\
33110\end{array}$ & $\begin{array}{l}3441 \\
3509 \\
2436 \\
3559 \\
3441\end{array}$ & $\begin{array}{l}3431 \\
3648 \\
3427 \\
3661 \\
3386\end{array}$ & $\begin{array}{l}3098 \\
3481 \\
3486 \\
3487 \\
3415\end{array}$ & $\begin{array}{l}2067 \\
3022 \\
3054 \\
3101 \\
3036\end{array}$ & $\begin{array}{l}1789 \\
2496 \\
2331 \\
2522 \\
2446\end{array}$ & $\begin{array}{l}12113 \\
1 B 51 \\
1814 \\
1821 \\
1769\end{array}$ & $\begin{array}{r}823 \\
1075 \\
1058 \\
1095 \\
797\end{array}$ & $\begin{array}{l}336 \\
378 \\
357 \\
345 \\
352\end{array}$ & $\begin{array}{l}0 \\
0 \\
0 \\
0 \\
0\end{array}$ & $\begin{array}{l}0 \\
0 \\
0 \\
0 \\
0\end{array}$ & $\begin{array}{r}-999 \\
30255 \\
23623 \\
29941 \\
-999\end{array}$ \\
\hline $\begin{array}{l}16 \\
17 \\
18 \\
17 \\
20\end{array}$ & $\begin{array}{l}0 \\
0 \\
0 \\
0 \\
0\end{array}$ & $\begin{array}{l}0 \\
0 \\
0 \\
0 \\
0\end{array}$ & $\begin{array}{r}-666 \\
129 \\
162 \\
-606 \\
172\end{array}$ & $\begin{array}{l}709 \\
565 \\
554 \\
591 \\
460\end{array}$ & $\begin{array}{l}1119 \\
1236 \\
1153 \\
1236 \\
1347\end{array}$ & $\begin{array}{r}2129 \\
1946 \\
1814 \\
1939 \\
-99\end{array}$ & $\begin{array}{r}2751 \\
2004 \\
1943 \\
2508 \\
-09\end{array}$ & $\begin{array}{r}3227 \\
2944 \\
1014 \\
3047 \\
-99\end{array}$ & $\begin{array}{l}3471 \\
2466 \\
3165 \\
3013 \\
3320\end{array}$ & $\begin{array}{l}3612 \\
2397 \\
3264 \\
2551 \\
2653\end{array}$ & $\begin{array}{l}2954 \\
2024 \\
2241 \\
2564 \\
31132\end{array}$ & $\begin{array}{l}3013 \\
2132 \\
2210 \\
1978 \\
2191\end{array}$ & $\begin{array}{l}1648 \\
1596 \\
1709 \\
1749 \\
1815\end{array}$ & $\begin{array}{l}1389 \\
1208 \\
1339 \\
1226 \\
1046\end{array}$ & $\begin{array}{l}908 \\
787 \\
717 \\
656 \\
375\end{array}$ & $\begin{array}{l}329 \\
277 \\
292 \\
303 \\
-99\end{array}$ & $\begin{array}{l}0 \\
0 \\
0 \\
0 \\
0\end{array}$ & $\begin{array}{l}0 \\
0 \\
0 \\
0 \\
0\end{array}$ & $\begin{array}{r}-999 \\
22405 \\
22729 \\
-999 \\
-999\end{array}$ \\
\hline $\begin{array}{l}21 \\
22 \\
23 \\
21 \\
25\end{array}$ & $\begin{array}{l}0 \\
0 \\
0 \\
0 \\
0\end{array}$ & $\begin{array}{l}0 \\
0 \\
0 \\
0 \\
0\end{array}$ & $\begin{array}{r}472 \\
-066 \\
-99 \\
110 \\
119\end{array}$ & $\begin{array}{l}773 \\
698 \\
547 \\
3115 \\
551\end{array}$ & $\begin{array}{r}1464 \\
1304 \\
511 \\
241 \\
1123\end{array}$ & $\begin{array}{r}2138 \\
2046 \\
639 \\
1612 \\
1955\end{array}$ & $\begin{array}{l}2173 \\
2685 \\
1045 \\
2119 \\
2616\end{array}$ & $\begin{array}{l}2989 \\
2073 \\
2249 \\
2538 \\
3074\end{array}$ & $\begin{array}{l}34114 \\
3071 \\
1935 \\
2637 \\
3355\end{array}$ & $\begin{array}{r}3787 \\
2730 \\
-99 \\
2414 \\
3447\end{array}$ & $\begin{array}{l}3457 \\
2292 \\
2373 \\
2581 \\
2635\end{array}$ & $\begin{array}{l}2900 \\
3172 \\
2763 \\
2273 \\
1722\end{array}$ & $\begin{array}{l}1922 \\
2133 \\
2193 \\
1939 \\
1336\end{array}$ & $\begin{array}{l}1303 \\
1169 \\
1732 \\
990 \\
1496\end{array}$ & $\begin{array}{l}952 \\
724 \\
681 \\
693 \\
757\end{array}$ & $\begin{array}{l}194 \\
233 \\
308 \\
277 \\
285\end{array}$ & $\begin{array}{l}0 \\
0 \\
0 \\
0 \\
0\end{array}$ & $\begin{array}{l}0 \\
0 \\
0 \\
0 \\
0\end{array}$ & $\begin{array}{r}28428 \\
-999 \\
-999 \\
21509 \\
214471\end{array}$ \\
\hline $\begin{array}{l}26 \\
27 \\
20 \\
29 \\
30\end{array}$ & $\begin{array}{l}0 \\
0 \\
0 \\
0 \\
0\end{array}$ & $\begin{array}{l}0 \\
0 \\
0 \\
0 \\
0\end{array}$ & $\begin{array}{r}2130 \\
0 \\
-99 \\
-666 \\
3211\end{array}$ & $\begin{array}{r}539 \\
1393 \\
-99 \\
5115 \\
464\end{array}$ & $\begin{array}{r}941 \\
354 \\
1132 \\
1203 \\
1110\end{array}$ & $\begin{array}{l}1305 \\
902 \\
1916 \\
2001 \\
11027\end{array}$ & $\begin{array}{l}25013 \\
2057 \\
20148 \\
2026 \\
2560\end{array}$ & $\begin{array}{l}23013 \\
20117 \\
29112 \\
3121 \\
30441\end{array}$ & $\begin{array}{l}2974 \\
2178 \\
3145 \\
3428 \\
33119\end{array}$ & $\begin{array}{l}3042 \\
2666 \\
3331 \\
31135 \\
3323\end{array}$ & $\begin{array}{l}1933 \\
2872 \\
3083 \\
3304 \\
2960\end{array}$ & $\begin{array}{l}1487 \\
2630 \\
2670 \\
2385 \\
2406\end{array}$ & $\begin{array}{r}568 \\
1998 \\
2274 \\
2394 \\
7305\end{array}$ & $\begin{array}{r}51 \\
1416 \\
1597 \\
1694 \\
1611\end{array}$ & $\begin{array}{r}38 \\
621 \\
759 \\
921 \\
907\end{array}$ & $\begin{array}{r}0 \\
133 \\
265 \\
277 \\
321\end{array}$ & $\begin{array}{l}0 \\
0 \\
0 \\
0 \\
0\end{array}$ & $\begin{array}{l}0 \\
0 \\
0 \\
0 \\
0\end{array}$ & $\begin{array}{r}19024 \\
21272 \\
-499 \\
-994 \\
26529\end{array}$ \\
\hline $\begin{array}{r}\text { HEAN } \\
30 \\
\text { IIIII! }\end{array}$ & $\begin{array}{r}0 \\
0 \\
30\end{array}$ & $\begin{array}{r}0 \\
\text { 1) } \\
30\end{array}$ & $\begin{array}{r}257 \\
142 \\
17\end{array}$ & $\begin{array}{r}509 \\
208 \\
29\end{array}$ & $\begin{array}{r}1111 \\
31,0 \\
310\end{array}$ & $\begin{array}{r}1737 \\
505 \\
27\end{array}$ & $\begin{array}{r}2350 \\
523 \\
29\end{array}$ & $\begin{array}{r}2,08 \\
564 \\
28\end{array}$ & $\begin{array}{r}2735 \\
616 \\
21\end{array}$ & $\begin{array}{r}2905 \\
672 \\
28\end{array}$ & $\begin{array}{r}2567 \\
136 \\
24\end{array}$ & $\begin{array}{r}2291 \\
674 \\
27\end{array}$ & $\begin{array}{r}1822 \\
409 \\
29\end{array}$ & $\begin{array}{r}1342 \\
404 \\
39\end{array}$ & $\begin{array}{r}729 \\
257 \\
29\end{array}$ & $\begin{array}{r}255 \\
92 \\
27\end{array}$ & $\begin{array}{r}0 \\
0 \\
30\end{array}$ & $\begin{array}{r}0 \\
0 \\
30\end{array}$ & $\begin{array}{r}24084 \\
3673 \\
15\end{array}$ \\
\hline $\begin{array}{l}\ln x \\
\ln 11\end{array}$ & $\begin{array}{l}0 \\
0\end{array}$ & $\begin{array}{l}0 \\
0\end{array}$ & $\begin{array}{r}475 \\
0\end{array}$ & $\begin{array}{r}1393 \\
131\end{array}$ & $\begin{array}{r}1625 \\
3015\end{array}$ & 2.619 & $\begin{array}{l}21013 \\
11) 155\end{array}$ & $\begin{array}{l}3340 \\
13611\end{array}$ & $\begin{array}{l}3569 \\
1456\end{array}$ & $\begin{array}{l}3787 \\
1317\end{array}$ & $\begin{array}{r}3497 \\
203\end{array}$ & $\begin{array}{r}317 ? \\
605\end{array}$ & $\begin{array}{r}2527 \\
560\end{array}$ & 1851 & $\begin{array}{r}1075 \\
38\end{array}$ & $\begin{array}{r}385 \\
0\end{array}$ & $\begin{array}{l}0 \\
0\end{array}$ & $\begin{array}{l}0 \\
0\end{array}$ & $\begin{array}{r}30255 \\
5000\end{array}$ \\
\hline
\end{tabular}




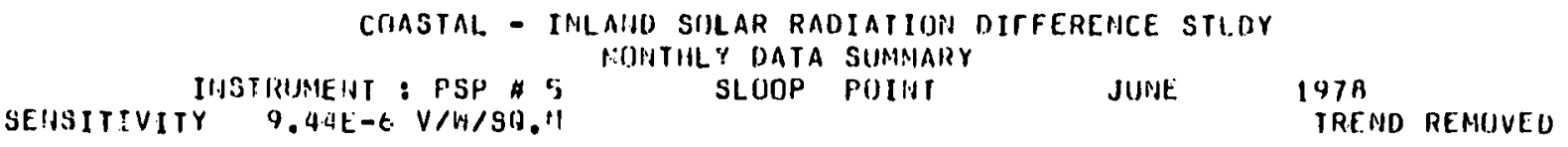

ENEPG KILUJUULES PER SUUAPE METER

$10 \quad 11$ MIDUR ENDING

DATE

113
87
133
63
152
137
57
64
137
129

$$
\begin{array}{rr}
533 & 11 \\
396 & 7 \\
526 & 11 \\
194 & 3 \\
760 & 14
\end{array}
$$

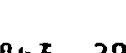

793 1:357

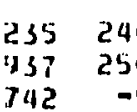

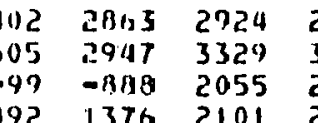

$2471 \quad 2272 \quad 094$

516

17

18

19

20

21 TOIAI.

$\begin{array}{lllllllllll}659 & 1284 & 1790 & 2661 & 2833 & 3413 & 3157 & 2281 & 1037 & 2215 & 1098\end{array}$

$\begin{array}{lllllllllll}11 & 1033 & 1725 & 2680 & 2998 & 3390 & 1872 & 1983 & 1540 & 1929 & 1300\end{array}$

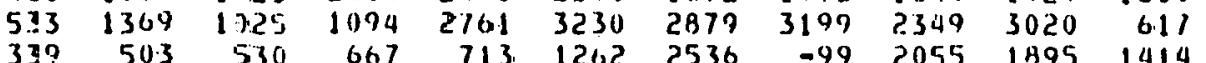

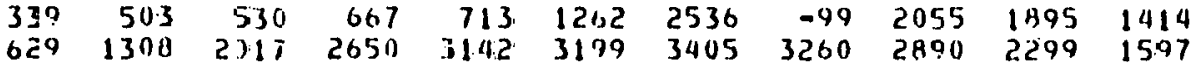

$\begin{array}{llllllllllll}667 & 11122 & 2177 & 2318 & 3291 & 3531 & 3554 & 3416 & 3031 & 2448 & 17011 & 1029\end{array}$

$713 \quad 1491$ 2234 2898 337A. $3626 \quad 3619 \quad 3477 \quad 3092 \quad 2505$ 1803 1037

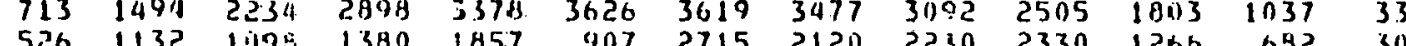

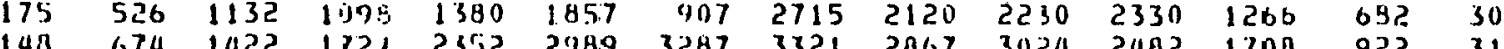

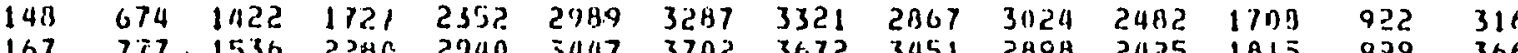

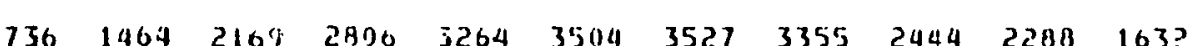

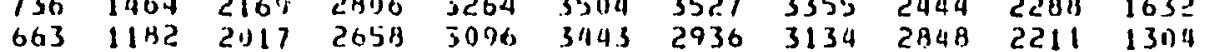

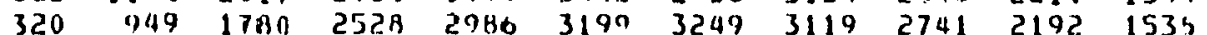

B73 297

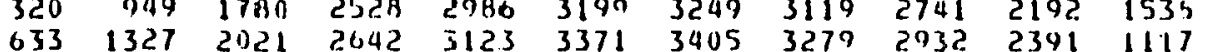

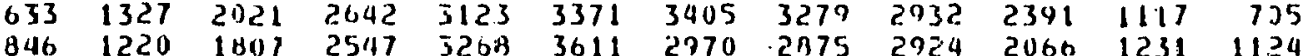

$\begin{array}{llllllllllll}743 & 1513 & 2522 & 2841 & 3333 & 3470 & 3424 & 3340 & 2947 & 2375 & 1715 & 438\end{array}$

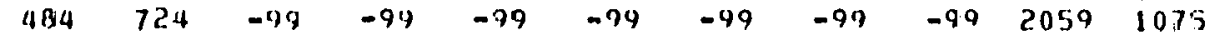

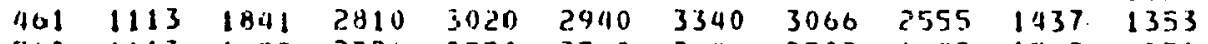

71

$5601113 \quad 1073 \quad 23265030$

572
617

$335 \% \quad 3394 \quad 3249 \quad 2825 \quad 2204 \quad 1473$

$\begin{array}{ll}1134 & 240 \\ A 5 B & 217\end{array}$

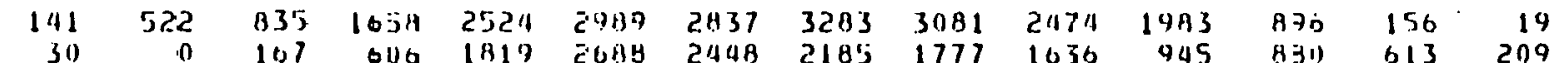

$\begin{array}{llllllllllllll}-79 & -99 & -179 & -.17 & 2490 & 2302 & 3066 & 3149 & 3176 & 2539 & 2166 & 771 & 607 & 175\end{array}$

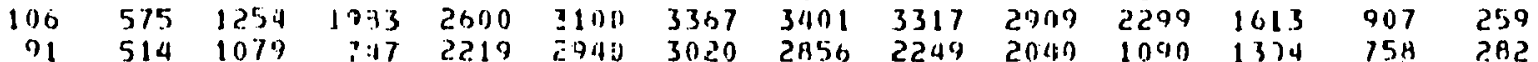

$552 \quad 1127$

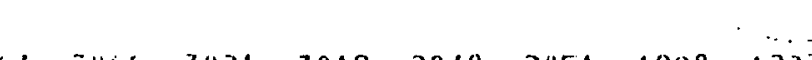

12

248

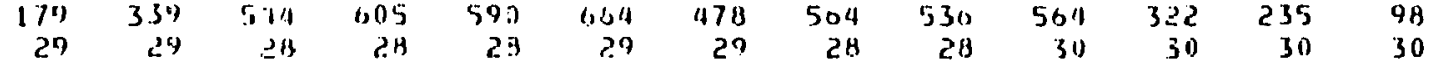

$\operatorname{lnx}$

30) 30

175

1346 1530 232? 2940 3417 370? $367 ? \quad 3477 \quad 3097 \quad 3020 \quad 1815 \quad 1124$

511
19 
CIJASTAL - IIJLAIJO SOLAR RADIATION DIFFERENCE STUDY

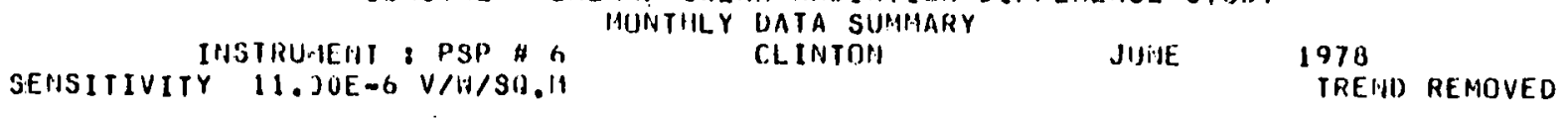

EHERGY KILOJUULES PER SUUARE IIETER

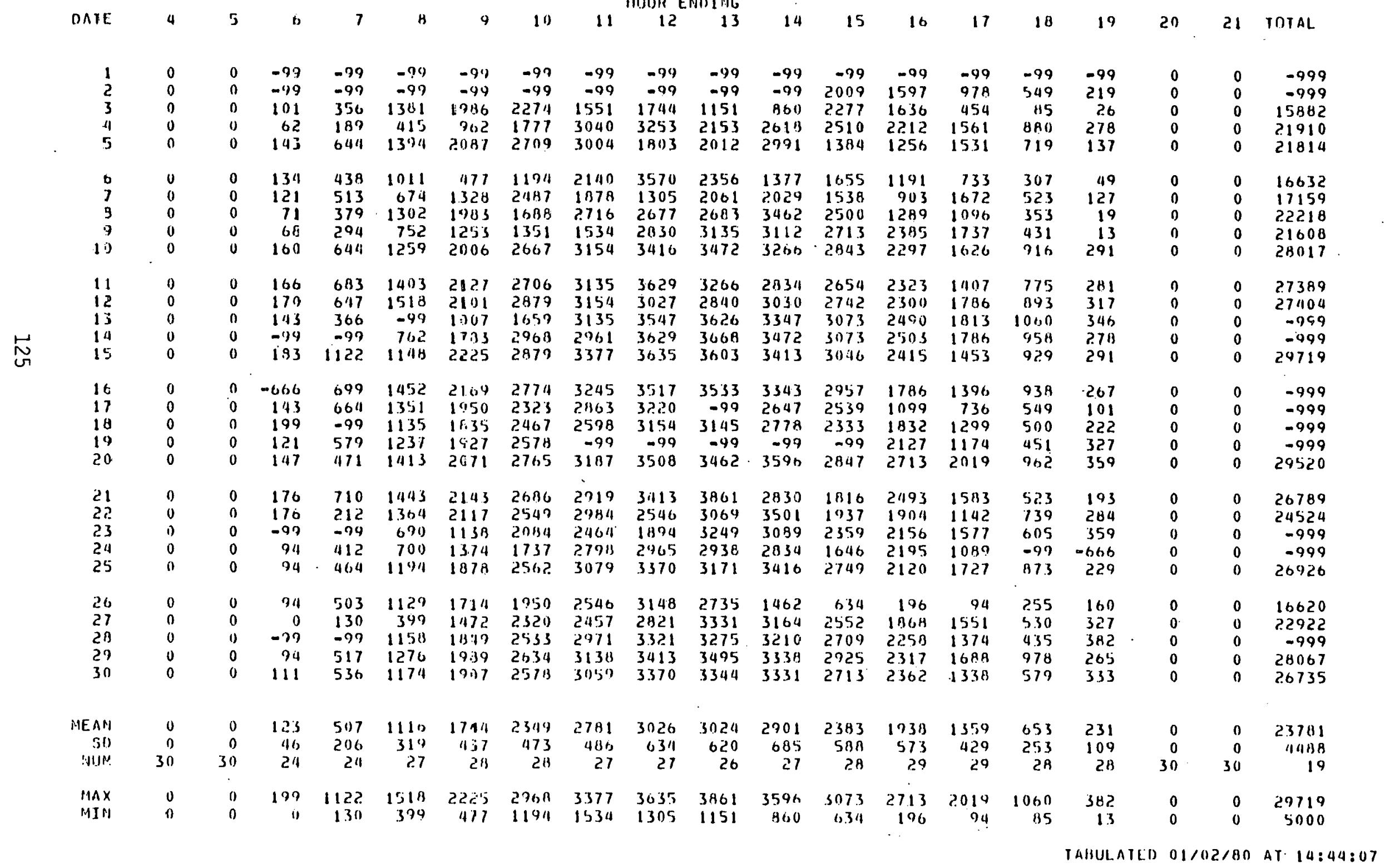


COASTAL - IHLRIID SILLAR RAOIATLOUN DIFFERFHCE STUDY

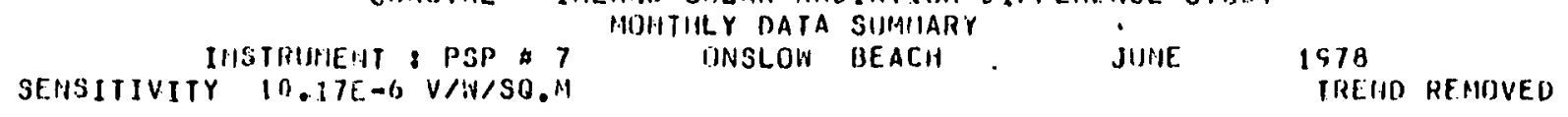

EHERGY KILOJOULES PER SHUARE METER

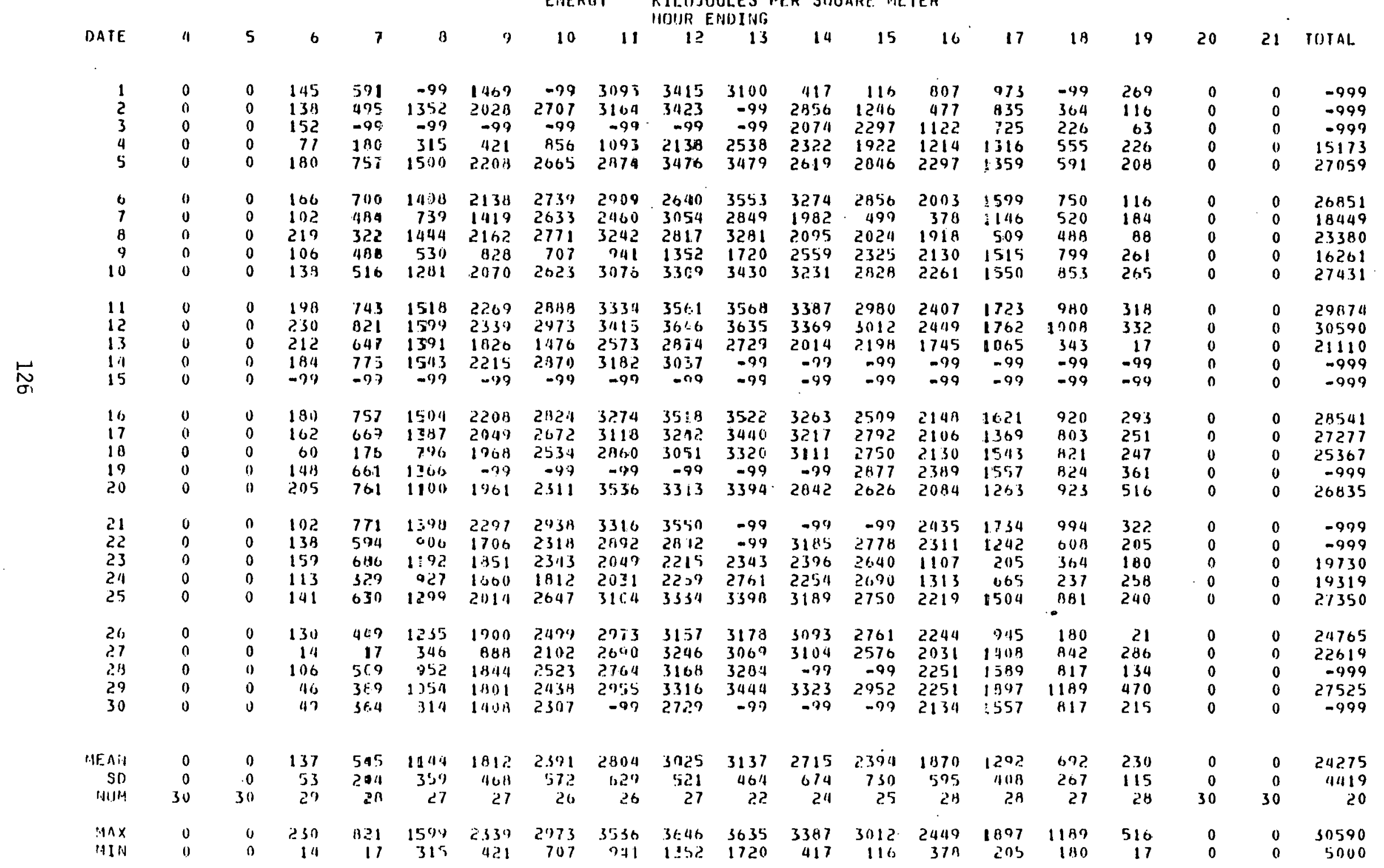


CIJASTAL. - INLAHD SIILAR RADIATIUN DIFFERENCE STUIOY

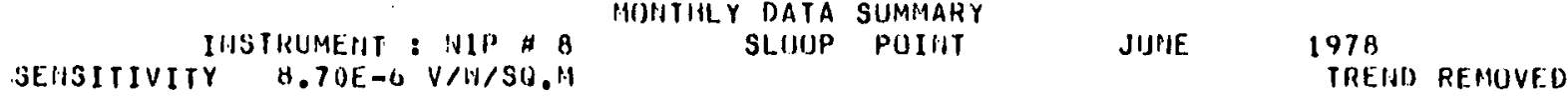

EIIERGY KILIJJOULES PER SPUNRE: METER HOUR ENDING,

\begin{tabular}{|c|c|c|c|c|c|c|c|c|c|c|c|c|c|c|c|c|c|c|c|c|}
\hline & & & & & & & & & & IOUR & IDING & & & & & & & & & \\
\hline DATE & 4 & & 5 & 6 & 7 & 8 & 4 & 10 & 11 & 12 & 13 & 14 & 15 & 16 & 17 & 18 & 19 & 20 & 21 & TOTAL \\
\hline $\begin{array}{l}1 \\
? \\
3 \\
4 \\
5\end{array}$ & $\begin{array}{l}0 \\
0 \\
0 \\
0 \\
0\end{array}$ & & $\begin{array}{l}0 \\
0 \\
0 \\
0 \\
0 .\end{array}$ & $\begin{array}{r}-77 \\
0 \\
174 \\
0 \\
383\end{array}$ & $\begin{array}{r}-79 \\
10 \\
302 \\
0 \\
1523\end{array}$ & $\begin{array}{r}-49 \\
60 \\
752 \\
0 \\
1793\end{array}$ & $\begin{array}{r}327 \\
1043 \\
931 \\
0 \\
1948\end{array}$ & $\begin{array}{r}1352 \\
1.377 \\
643 \\
0 \\
2314\end{array}$ & $\begin{array}{r}1560 \\
1793 \\
218 \\
23 \\
2410\end{array}$ & $\begin{array}{r}1931 \\
2198 \\
2114 \\
127 \\
1377\end{array}$ & $\begin{array}{r}1352 \\
2260 \\
168 \\
785 \\
506\end{array}$ & $\begin{array}{r}964 \\
2243 \\
143 \\
381 \\
1156\end{array}$ & $\begin{array}{r}-99 \\
1123 \\
518 \\
602 \\
2206\end{array}$ & $\begin{array}{r}0 \\
1289 \\
252 \\
360 \\
1689\end{array}$ & $\begin{array}{l}377 \\
668 \\
277 \\
335 \\
593\end{array}$ & $\begin{array}{r}456 \\
102 \\
0 \\
39 \\
148\end{array}$ & $\begin{array}{r}127 \\
0 \\
0 \\
206 \\
27\end{array}$ & $\begin{array}{l}0 \\
0 \\
0 \\
0 \\
0\end{array}$ & $\begin{array}{l}0 \\
0 \\
0 \\
0 \\
0\end{array}$ & $\begin{array}{r}-999 \\
14674 \\
4616 \\
2858 \\
18271\end{array}$ \\
\hline $\begin{array}{r}6 \\
7 \\
8 \\
9 \\
10\end{array}$ & $\begin{array}{l}0 \\
0 \\
0 \\
0 \\
0\end{array}$ & & $\begin{array}{l}0 \\
0 \\
0 \\
0 \\
0\end{array}$ & $\begin{array}{r}206 \\
0 \\
0 \\
61 \\
i 13\end{array}$ & $\begin{array}{r}827 \\
56 \\
539 \\
11 \\
46 ?\end{array}$ & $\begin{array}{r}1314 \\
410 \\
1852 \\
0 \\
1286\end{array}$ & $\begin{array}{r}1627 \\
1323 \\
2356 \\
0 \\
1625\end{array}$ & $\begin{array}{r}1981 \\
1973 \\
2027 \\
0 \\
1904\end{array}$ & $\begin{array}{r}1514 \\
1110 \\
2706 \\
0 \\
2113\end{array}$ & $\begin{array}{r}2048 \\
1943 \\
1377 \\
0 \\
1890\end{array}$ & $\begin{array}{r}1752 \\
73 \\
2127 \\
724 \\
2204\end{array}$ & $\begin{array}{r}923 \\
202 \\
1198 \\
-99 \\
2209\end{array}$ & $\begin{array}{r}52 \\
223 \\
2727 \\
694 \\
2110\end{array}$ & $\begin{array}{r}1760 \\
964 \\
102 \\
803 \\
1836\end{array}$ & $\begin{array}{r}510 \\
893 \\
308 \\
1203 \\
1460\end{array}$ & $\begin{array}{l}698 \\
168 \\
235 \\
957 \\
930\end{array}$ & $\begin{array}{r}18 \\
18 \\
0 \\
390 \\
359\end{array}$ & $\begin{array}{l}0 \\
0 \\
0 \\
0 \\
0\end{array}$ & $\begin{array}{l}0 \\
0 \\
0 \\
0 \\
0\end{array}$ & $\begin{array}{r}15234 \\
9676 \\
14344 \\
-999 \\
20492\end{array}$ \\
\hline $\begin{array}{l}11 \\
12 \\
13 \\
11 \\
15\end{array}$ & $\begin{array}{l}0 \\
0 \\
0 \\
0 \\
0\end{array}$ & & $\begin{array}{l}0 \\
0 \\
11 \\
0 \\
0\end{array}$ & $\begin{array}{l}314 \\
613 \\
232 \\
339 \\
785\end{array}$ & $\begin{array}{r}1200 \\
1755 \\
497 \\
1394 \\
2051\end{array}$ & $\begin{array}{r}1732 \\
2430 \\
676 \\
1767 \\
2613\end{array}$ & $\begin{array}{r}2354 \\
2827 \\
191 \\
604 \\
-1389\end{array}$ & $\begin{array}{r}2607 \\
3063 \\
96 \\
600 \\
3567\end{array}$ & $\begin{array}{r}1494 \\
3187 \\
423 \\
1191 \\
3409\end{array}$ & $\begin{array}{r}3385 \\
3237 \\
0 \\
1523 \\
-99\end{array}$ & $\begin{array}{r}2793 \\
3162 \\
671 \\
1709 \\
-99\end{array}$ & $\begin{array}{r}2838 \\
3150 \\
679 \\
1460 \\
-99\end{array}$ & $\begin{array}{r}2793 \\
3075 \\
1399 \\
2516 \\
-97\end{array}$ & $\begin{array}{r}2590 \\
2901 \\
2132 \\
2437 \\
-99\end{array}$ & $\begin{array}{r}2300 \\
2637 \\
12.48 \\
2.089 \\
-99\end{array}$ & $\begin{array}{r}1825 \\
2161 \\
969 \\
1502 \\
-99\end{array}$ & $\begin{array}{r}931 \\
1201 \\
895 \\
740 \\
-99\end{array}$ & $\begin{array}{l}0 \\
0 \\
0 \\
0 \\
0\end{array}$ & $\begin{array}{l}0 \\
0 \\
0 \\
0 \\
0\end{array}$ & $\begin{array}{r}29964 \\
35407 \\
10160 \\
19871 \\
-999\end{array}$ \\
\hline $\begin{array}{l}16 \\
17 \\
19 \\
19 \\
20\end{array}$ & $\begin{array}{l}0 \\
0 \\
0 \\
0 \\
0\end{array}$ & 9 & $\begin{array}{l}0 \\
0 \\
0 \\
0 \\
0\end{array}$ & $\begin{array}{r}-9 n \\
323 \\
n \\
17 n \\
253\end{array}$ & $\begin{array}{r}-99 \\
1139 \\
0 \\
874 \\
15130\end{array}$ & $\begin{array}{r}-99 \\
1362 \\
341 \\
-94 \\
1357\end{array}$ & $\begin{array}{r}-99 \\
1979 \\
1045 \\
-818 \\
1627\end{array}$ & $\begin{array}{r}-99 \\
2223 \\
1442 \\
2024 \\
1471\end{array}$ & $\begin{array}{r}-94 \\
2.77 \\
11113 \\
2194 \\
2.421\end{array}$ & $\begin{array}{r}-99 \\
2260 \\
1467 \\
2245 \\
2427\end{array}$ & $\begin{array}{l}-99 \\
1532 \\
1727 \\
2339 \\
1110\end{array}$ & $\begin{array}{r}-99 \\
2045 \\
1789 \\
2392 \\
1751\end{array}$ & $\begin{array}{r}-99 \\
2103 \\
1760 \\
2459 \\
2508\end{array}$ & $\begin{array}{l}-99 \\
1710 \\
1573 \\
2335 \\
1072\end{array}$ & $\begin{array}{r}-92 \\
733 \\
1322 \\
812 \\
336\end{array}$ & $\begin{array}{r}1382 \\
352 \\
425 \\
-848 \\
1346\end{array}$ & $\begin{array}{r}716 \\
295 \\
254 \\
-99 \\
1209\end{array}$ & $\begin{array}{l}0 \\
0 \\
0 \\
0 \\
0\end{array}$ & $\begin{array}{l}0 \\
0 \\
0 \\
0 \\
0\end{array}$ & $\begin{array}{r}-999 \\
20388 \\
14963 \\
-999 \\
21254\end{array}$ \\
\hline $\begin{array}{l}21 \\
22 \\
23 \\
211 \\
25\end{array}$ & $\begin{array}{l}0 \\
0 \\
0 \\
0 \\
0\end{array}$ & & $\begin{array}{l}0 \\
0 \\
0 \\
0 \\
0\end{array}$ & $\begin{array}{r}381 \\
69 \\
27 . \\
39 \\
122\end{array}$ & $\begin{array}{r}1739 \\
156 \\
180 \\
494 \\
929\end{array}$ & $\begin{array}{r}2120 \\
114 \\
3117 \\
718 \\
1591\end{array}$ & $\begin{array}{r}2181 \\
-999 \\
9182 \\
342 \\
1934\end{array}$ & $\begin{array}{r}2521 \\
-99 \\
1657 \\
1169 \\
2021\end{array}$ & $\begin{array}{r}2885 \\
-99 \\
1744 \\
1222 \\
2050\end{array}$ & $\begin{array}{l}2239 \\
1099 \\
1491 \\
1086 \\
2216\end{array}$ & $\begin{array}{r}2479 \\
1116 \\
1934 \\
920 \\
2257\end{array}$ & $\begin{array}{r}2802 \\
1215 \\
1806 \\
966 \\
2170\end{array}$ & $\begin{array}{r}2410 \\
2088 \\
1723 \\
643 \\
1796\end{array}$ & $\begin{array}{r}2604 \\
1463 \\
701 \\
672 \\
1591\end{array}$ & $\begin{array}{r}2223 \\
226 \\
734 \\
792 \\
1185\end{array}$ & $\begin{array}{r}1238 \\
40 \\
291 \\
89 \\
949\end{array}$ & $\begin{array}{r}646 \\
11 \\
0 \\
27 \\
151\end{array}$ & $\begin{array}{l}0 \\
0 \\
0 \\
0 \\
0\end{array}$ & $\begin{array}{l}0 \\
0 \\
0 \\
0 \\
0\end{array}$ & $\begin{array}{r}29224 \\
-999 \\
13913 \\
9679 \\
21162\end{array}$ \\
\hline $\begin{array}{l}26 \\
27 \\
29 \\
29 \\
30\end{array}$ & $\begin{array}{l}0 \\
0 \\
0 \\
0 \\
0\end{array}$ & & $\begin{array}{l}0 \\
0 \\
0 \\
0 \\
0\end{array}$ & $\begin{array}{r}60 \\
0 \\
-94 \\
-99 \\
-70\end{array}$ & $\begin{array}{r}330 \\
0 \\
-79 \\
-97 \\
-97\end{array}$ & $\begin{array}{r}37 ? \\
0 \\
-99 \\
-99 \\
-79\end{array}$ & $\begin{array}{r}152 \\
11 \\
-99 \\
-99 \\
-97\end{array}$ & $\begin{array}{r}1360 \\
520 \\
-99 \\
-99 \\
-97\end{array}$ & $\begin{array}{r}1724 \\
1779 \\
-79 \\
-79 \\
-99\end{array}$ & $\begin{array}{r}1120 \\
768 \\
-49 \\
-99 \\
-199\end{array}$ & $\begin{array}{r}1931 \\
392 \\
-99 \\
-99 \\
-97\end{array}$ & $\begin{array}{r}1794 \\
263 \\
-90 \\
-99 \\
-97\end{array}$ & $\begin{array}{r}1364 \\
346 \\
-99 \\
-99 \\
-99\end{array}$ & $\begin{array}{r}1037 \\
110 \\
-40 \\
-94 \\
-99\end{array}$ & $\begin{array}{l}330 \\
259 \\
-49 \\
-99 \\
-97\end{array}$ & $\begin{array}{r}0 \\
272 \\
-99 \\
-99 \\
-99\end{array}$ & $\begin{array}{r}0 \\
90 \\
-99 \\
-99 \\
-99\end{array}$ & $\begin{array}{l}0 \\
0 \\
0 \\
0 \\
0\end{array}$ & $\begin{array}{l}0 \\
0 \\
0 \\
0 \\
0\end{array}$ & $\begin{array}{r}12119 \\
4099 \\
-999 \\
-999 \\
-999\end{array}$ \\
\hline $\begin{array}{r}\text { HENH } \\
\text { SD } \\
\text { HITN }\end{array}$ & $\begin{array}{r}0 \\
0 \\
30\end{array}$ & & $\begin{array}{r}0 \\
0 \\
30\end{array}$ & $\begin{array}{r}194 \\
198 \\
25\end{array}$ & $\begin{array}{r}720 \\
612 \\
25\end{array}$ & $\begin{array}{r}1071 \\
340 \\
24\end{array}$ & $\begin{array}{r}1231 \\
023 \\
23\end{array}$ & $\begin{array}{r}16,10 \\
9010 \\
25\end{array}$ & $\begin{array}{r}1694 \\
197 \\
25\end{array}$ & $\begin{array}{r}1620 \\
963 \\
25\end{array}$ & $\begin{array}{r}1521 \\
82.11 \\
25\end{array}$ & $\begin{array}{r}1522 \\
848 \\
24\end{array}$ & $\begin{array}{r}1572 \\
\sin 8 \\
24\end{array}$ & $\begin{array}{r}1350 \\
840 \\
25\end{array}$ & $\begin{array}{r}961 \\
691 \\
25\end{array}$ & $\begin{array}{r}678 \\
612 \\
25\end{array}$ & $\begin{array}{r}3311 \\
392 \\
25\end{array}$ & $\begin{array}{r}0 \\
0 \\
30\end{array}$ & $\begin{array}{r}0 \\
0 \\
30\end{array}$ & $\begin{array}{r}16494 \\
3284 \\
21\end{array}$ \\
\hline $\begin{array}{l}\text { MAX } \\
\text { MI!U }\end{array}$ & $\begin{array}{l}0 \\
0\end{array}$ & & $\begin{array}{l}0 \\
0\end{array}$ & $\begin{array}{r}745 \\
1)\end{array}$ & 2051 & $\begin{array}{r}26113 \\
0\end{array}$ & $\begin{array}{r}283.7 \\
0\end{array}$ & $\begin{array}{r}3367 \\
0\end{array}$ & $\begin{array}{r}34013 \\
0\end{array}$ & $\begin{array}{r}3785 \\
0\end{array}$ & $\begin{array}{r}3162 \\
73\end{array}$ & $\begin{array}{r}3150 \\
143\end{array}$ & $\begin{array}{r}3075 \\
52\end{array}$ & $\begin{array}{r}2901 \\
0\end{array}$ & $\begin{array}{r}2637 \\
226\end{array}$ & $\begin{array}{r}2161 \\
0\end{array}$ & $\begin{array}{r}1209 \\
0\end{array}$ & $\begin{array}{l}0 \\
0\end{array}$ & $\begin{array}{l}0 \\
0\end{array}$ & $\begin{array}{r}35407 \\
2858\end{array}$ \\
\hline
\end{tabular}


CUASTAL - I\%IAIID SOLAH RADIATIUH DIFFEHENCE STUOY

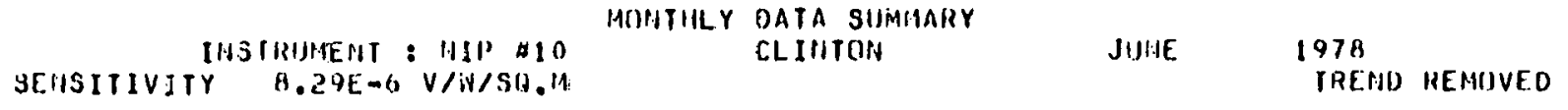

EHERGY KILOJIULES PER SOJUAPE METER

\begin{tabular}{|c|c|c|c|c|c|c|c|c|c|c|c|c|c|c|c|c|c|c|c|}
\hline \multicolumn{20}{|c|}{ moure Eisosing } \\
\hline DATE & 4 & 5 & 0 & 7 & B & $?$ & 10 & 11 & 12 & 13 & 14 & 15 & 16 & 17 & 1 ค & 19 & 20 & 21 & TOIAL \\
\hline $\begin{array}{l}1 \\
? \\
3 \\
4 \\
5\end{array}$ & $\begin{array}{l}0 \\
0 \\
0 \\
0 \\
0\end{array}$ & $\begin{array}{l}0 \\
0 \\
0 \\
0 \\
0\end{array}$ & $\begin{array}{r}131 \\
0 \\
1) \\
0 \\
125\end{array}$ & $\begin{array}{r}803 \\
0 \\
82 \\
0 \\
364\end{array}$ & $\begin{array}{r}1767 \\
0 \\
1050 \\
175\end{array}$ & $\begin{array}{r}2071 \\
43 \\
8554 \\
74 \\
1745\end{array}$ & $\begin{array}{r}1971 \\
1406 \\
894 \\
408 \\
2054\end{array}$ & $\begin{array}{r}19700 \\
2006 \\
02 \\
18>1 \\
1796\end{array}$ & $\begin{array}{r}2392 \\
22116 \\
113 \\
1862 \\
303\end{array}$ & $\begin{array}{r}1763 \\
1871 \\
0 \\
486 \\
225\end{array}$ & $\begin{array}{r}1380 \\
438 \\
21 \\
1155 \\
1324\end{array}$ & $\begin{array}{r}2275 \\
812 \\
381 \\
1502 \\
321\end{array}$ & $\begin{array}{r}1741 \\
468 \\
720 \\
1763 \\
429\end{array}$ & $\begin{array}{r}1094 \\
412 \\
0 \\
1710 \\
855\end{array}$ & $\begin{array}{r}699 \\
21 ? \\
0 \\
1246 \\
321\end{array}$ & $\begin{array}{r}351 \\
95 \\
0 \\
686 \\
0\end{array}$ & $\begin{array}{l}0 \\
0 \\
0 \\
0 \\
0\end{array}$ & $\begin{array}{l}0 \\
0 \\
0 \\
0 \\
0\end{array}$ & $\begin{array}{r}20417 \\
10369 \\
5127 \\
12767 \\
12057\end{array}$ \\
\hline $\begin{array}{r}0 \\
7 \\
8 \\
9 \\
10\end{array}$ & $\begin{array}{l}0 \\
0 \\
0 \\
0 \\
0\end{array}$ & $\begin{array}{l}0 \\
0 \\
0 \\
0 \\
0\end{array}$ & $\begin{array}{r}0 \\
0 \\
0 \\
121\end{array}$ & $\begin{array}{r}21 \\
17 \\
6 \\
17 \\
499\end{array}$ & $\begin{array}{r}100 \\
0 \\
1007 \\
1+3 \\
803\end{array}$ & $\begin{array}{r}10 \\
273 \\
1645 \\
608 \\
1.37\end{array}$ & $\begin{array}{r}13 \\
1116 \\
304 \\
91 \\
1767\end{array}$ & $\begin{array}{r}392 \\
156 \\
1515 \\
136 \\
2114\end{array}$ & $\begin{array}{r}1767 \\
0 \\
10311 \\
1398 \\
2140\end{array}$ & $\begin{array}{r}625 \\
60 \\
1215 \\
1889 \\
2219\end{array}$ & $\begin{array}{r}455 \\
256 \\
2466 \\
1324 \\
2059\end{array}$ & $\begin{array}{r}716 \\
117 \\
1741 \\
1810 \\
1911\end{array}$ & $\begin{array}{r}399 \\
321 \\
690 \\
2111 \\
1837\end{array}$ & $\begin{array}{r}26 \\
2106 \\
386 \\
2097 \\
2019\end{array}$ & $\begin{array}{r}0 \\
594 \\
26 \\
434 \\
1211\end{array}$ & $\begin{array}{r}0 \\
0 \\
0 \\
0 \\
529\end{array}$ & $\begin{array}{l}0 \\
0 \\
0 \\
0 \\
0\end{array}$ & $\begin{array}{l}0 \\
0 \\
0 \\
0 \\
0\end{array}$ & $\begin{array}{r}4564 \\
5016 \\
12783 \\
11711 \\
20090\end{array}$ \\
\hline $\begin{array}{l}11 \\
12 \\
13 \\
14 \\
15\end{array}$ & $\begin{array}{l}0 \\
0 \\
0 \\
0 \\
0\end{array}$ & $\begin{array}{l}0 \\
0 \\
0 \\
0 \\
0\end{array}$ & $\begin{array}{r}225 \\
1.30 \\
30 \\
0 \\
-555\end{array}$ & $\begin{array}{r}916 \\
58 ! \\
52 \\
0 \\
-555\end{array}$ & $\begin{array}{r}1534 \\
1867 \\
-94 \\
0 \\
-555\end{array}$ & $\begin{array}{r}1028 \\
2253 \\
-79 \\
13 \\
-555\end{array}$ & $\begin{array}{r}2080 \\
2466 \\
225 \\
17 \\
-545\end{array}$ & $\begin{array}{r}1097 \\
2177 \\
2201 \\
17 \\
-555\end{array}$ & $\begin{array}{r}24110 \\
1906 \\
2914 \\
0 \\
-555\end{array}$ & $\begin{array}{r}1871 \\
1567 \\
2961 \\
13 \\
-555\end{array}$ & $\begin{array}{r}1641 \\
1089 \\
2005 \\
0 \\
-555\end{array}$ & $\begin{array}{r}1984 \\
2900 \\
3009 \\
13 \\
-555\end{array}$ & $\begin{array}{r}1938 \\
2027 \\
2000 \\
13 \\
-555\end{array}$ & $\begin{array}{r}12114 \\
2275 \\
2492 \\
0 \\
-555\end{array}$ & $\begin{array}{r}972 \\
1576 \\
2027 \\
13 \\
-555\end{array}$ & $\begin{array}{r}509 \\
1055 \\
1237 \\
0 \\
-555\end{array}$ & $\begin{array}{l}0 \\
0 \\
0 \\
0 \\
0\end{array}$ & $\begin{array}{l}0 \\
0 \\
0 \\
0 \\
0\end{array}$ & $\begin{array}{r}21293 \\
23471 \\
-999 \\
99 \\
-999\end{array}$ \\
\hline $\begin{array}{l}16 \\
17 \\
18 \\
19 \\
20\end{array}$ & $\begin{array}{l}0 \\
0 \\
0 \\
1) \\
0\end{array}$ & $\begin{array}{l}0 \\
0 \\
0 \\
0 \\
0\end{array}$ & $\begin{array}{r}1022 \\
186 \\
-99 \\
125 \\
113\end{array}$ & $\begin{array}{r}1605 \\
885 \\
-79 \\
716 \\
208\end{array}$ & $\begin{array}{r}2325 \\
1530 \\
503 \\
1155 \\
1572\end{array}$ & $\begin{array}{l}2077 \\
1+59 \\
1129 \\
1345 \\
1771\end{array}$ & $\begin{array}{l}2855 \\
1291 \\
1391 \\
1875 \\
2910\end{array}$ & $\begin{array}{r}2528 \\
1+511 \\
r .59 \\
\bar{c} 0100 \\
2557\end{array}$ & $\begin{array}{r}2933 \\
18117 \\
1493 \\
-99 \\
2483\end{array}$ & $\begin{array}{r}2742 \\
-99 \\
1376 \\
-99 \\
2301\end{array}$ & $\begin{array}{r}2559 \\
-99 \\
1020 \\
-99 \\
2935\end{array}$ & $\begin{array}{r}2442 \\
-99 \\
820 \\
-99 \\
2496\end{array}$ & $\begin{array}{r}401 \\
-99 \\
868 \\
-99 \\
2974\end{array}$ & $\begin{array}{r}935 \\
-09 \\
690 \\
-99 \\
2648\end{array}$ & $\begin{array}{r}1517 \\
-99 \\
56 \\
-99 \\
1506\end{array}$ & $\begin{array}{r}540 \\
-79 \\
95 \\
-99 \\
1120\end{array}$ & $\begin{array}{l}0 \\
0 \\
0 \\
0 \\
0\end{array}$ & $\begin{array}{l}0 \\
0 \\
0 \\
0 \\
0\end{array}$ & $\begin{array}{r}27541 \\
-999 \\
-999 \\
-999 \\
27224\end{array}$ \\
\hline $\begin{array}{l}21 \\
23 \\
23 \\
23 \\
21 \\
25\end{array}$ & $\begin{array}{l}0 \\
0 \\
0 \\
0 \\
0\end{array}$ & $\begin{array}{l}0 \\
0 \\
0 \\
0 \\
n\end{array}$ & $\begin{array}{r}351 \\
251 \\
-99 \\
21 \\
56\end{array}$ & $\begin{array}{r}1732 \\
369 \\
-79 \\
47 \\
334\end{array}$ & $\begin{array}{r}2766 \\
1 \geq 32 \\
0 \\
47 \\
338\end{array}$ & $\begin{array}{r}2637 \\
2062 \\
0 \\
273 \\
1437\end{array}$ & $\begin{array}{r}2579 \\
1975 \\
547 \\
395 \\
1893\end{array}$ & $\begin{array}{r}2340 \\
: 323 \\
310 \\
1307 \\
3301\end{array}$ & $\begin{array}{r}2427 \\
1042 \\
277 \\
-8138 \\
2153\end{array}$ & $\begin{array}{r}2770 \\
1572 \\
1459 \\
-99 \\
1697\end{array}$ & $\begin{array}{l}1823 \\
2318 \\
1611 \\
1146 \\
2036\end{array}$ & $\begin{array}{r}916 \\
990 \\
1159 \\
277 \\
1376\end{array}$ & $\begin{array}{r}2158 \\
1089 \\
1259 \\
998 \\
1276\end{array}$ & $\begin{array}{r}1402 \\
385 \\
1168 \\
179 \\
1454\end{array}$ & $\begin{array}{r}60 \\
312 \\
156 \\
104 \\
529\end{array}$ & $\begin{array}{r}0 \\
125 \\
138 \\
377 \\
60\end{array}$ & $\begin{array}{l}0 \\
0 \\
0 \\
0 \\
0\end{array}$ & $\begin{array}{l}0 \\
0 \\
0 \\
0 \\
0\end{array}$ & $\begin{array}{r}23551 \\
16545 \\
-999 \\
-999 \\
17340\end{array}$ \\
\hline $\begin{array}{l}26 \\
27 \\
28 \\
217 \\
3 n\end{array}$ & $\begin{array}{l}0 \\
0 \\
0 \\
0 \\
0\end{array}$ & $\begin{array}{l}0 \\
0 \\
0 \\
0 \\
0\end{array}$ & $\begin{array}{r}17 \\
0 \\
-99 \\
131 \\
117\end{array}$ & $\begin{array}{r}478 \\
0 \\
-19 \\
612 \\
777\end{array}$ & $\begin{array}{r}768 \\
0 \\
1092 \\
1715 \\
1302\end{array}$ & $\begin{array}{r}929 \\
317 \\
1515 \\
6097 \\
1915\end{array}$ & $\begin{array}{r}1110 \\
860 \\
1936 \\
21153 \\
2210\end{array}$ & $\begin{array}{r}1116 \\
3107 \\
2101 \\
21048 \\
2375\end{array}$ & $\begin{array}{l}15100 \\
1159 \\
232.3 \\
2692 \\
2 \$ 114\end{array}$ & $\begin{array}{r}801 \\
1732 \\
2132 \\
2805 \\
2236\end{array}$ & $\begin{array}{r}21 \\
1697 \\
2067 \\
2735 \\
2275\end{array}$ & $\begin{array}{r}0 \\
1302 \\
1637 \\
2493 \\
1706\end{array}$ & $\begin{array}{r}0 \\
781 \\
1367 \\
2123 \\
1737\end{array}$ & $\begin{array}{r}01 \\
781 \\
786 \\
1962 \\
807\end{array}$ & $\begin{array}{r}0 \\
117 \\
0 \\
1359 \\
169\end{array}$ & $\begin{array}{r}0 \\
78 \\
264 \\
375 \\
343\end{array}$ & $\begin{array}{l}0 \\
0 \\
0 \\
0 \\
0\end{array}$ & $\begin{array}{l}0 \\
0 \\
0 \\
0 \\
0\end{array}$ & $\begin{array}{r}6336 \\
9699 \\
-999 \\
26117 \\
20313\end{array}$ \\
\hline $\begin{array}{r}\text { HEAH } \\
\text { SDD } \\
\text { PHIM }\end{array}$ & $\begin{array}{r}0 \\
0 \\
30\end{array}$ & $\begin{array}{r}0 \\
n \\
30\end{array}$ & $\begin{array}{r}113 \\
202 \\
26\end{array}$ & $\begin{array}{l}146 \\
436 \\
26\end{array}$ & $\begin{array}{r}1006 \\
767 \\
23\end{array}$ & $\begin{array}{r}.252 \\
653 \\
28\end{array}$ & $\begin{array}{r}1377 \\
974 \\
29\end{array}$ & $\begin{array}{r}1536 \\
1125 \\
29\end{array}$ & $\begin{array}{r}1678 \\
1596 \\
.27\end{array}$ & $\begin{array}{r}1556 \\
878 \\
26\end{array}$ & $\begin{array}{r}1520 \\
863 \\
27\end{array}$ & $\begin{array}{r}1382 \\
863 \\
27\end{array}$ & $\begin{array}{r}1271 \\
809 \\
27\end{array}$ & $\begin{array}{r}1110 \\
778 \\
27\end{array}$ & $\begin{array}{r}563 \\
609 \\
27\end{array}$ & $\begin{array}{r}296 \\
360 \\
27\end{array}$ & $\begin{array}{r}0 \\
0 \\
30\end{array}$ & $\begin{array}{r}0 \\
0 \\
30\end{array}$ & $\begin{array}{r}15219 \\
7960 \\
22\end{array}$ \\
\hline $\begin{array}{l}\text { HAX } \\
\text { HIHI }\end{array}$ & $\begin{array}{l}0 \\
0\end{array}$ & $\begin{array}{l}0 \\
0\end{array}$ & $\begin{array}{r}1022 \\
n\end{array}$ & $\begin{array}{r}1732 \\
0\end{array}$ & $\begin{array}{r}236 \% \\
0\end{array}$ & $\begin{array}{r}2017 \\
0\end{array}$ & $\begin{array}{r}24515 \\
13\end{array}$ & $\begin{array}{r}-928 \\
17\end{array}$ & $\begin{array}{r}29114 \\
0\end{array}$ & $\begin{array}{r}2961 \\
0\end{array}$ & $\begin{array}{r}2935 \\
11\end{array}$ & $\begin{array}{r}3009 \\
0\end{array}$ & $\begin{array}{r}2974 \\
0\end{array}$ & $\begin{array}{r}2648 \\
0\end{array}$ & $\begin{array}{r}2027 \\
0\end{array}$ & $\begin{array}{r}1237 \\
0\end{array}$ & $\begin{array}{l}0 \\
0\end{array}$ & $\begin{array}{l}0 \\
0\end{array}$ & $\begin{array}{r}27541 \\
99\end{array}$ \\
\hline
\end{tabular}


COASTAL - IIILAND SILLAR RADIATIUN DIFFERENICE STURY MIONTIILY DATA SUMMARY

IIISTRUHENT: UV H11
SEIISITIVITY $312.10 E-6$, V/H/SO.M

ENERG KILIJJOULES PER SQUARE METER

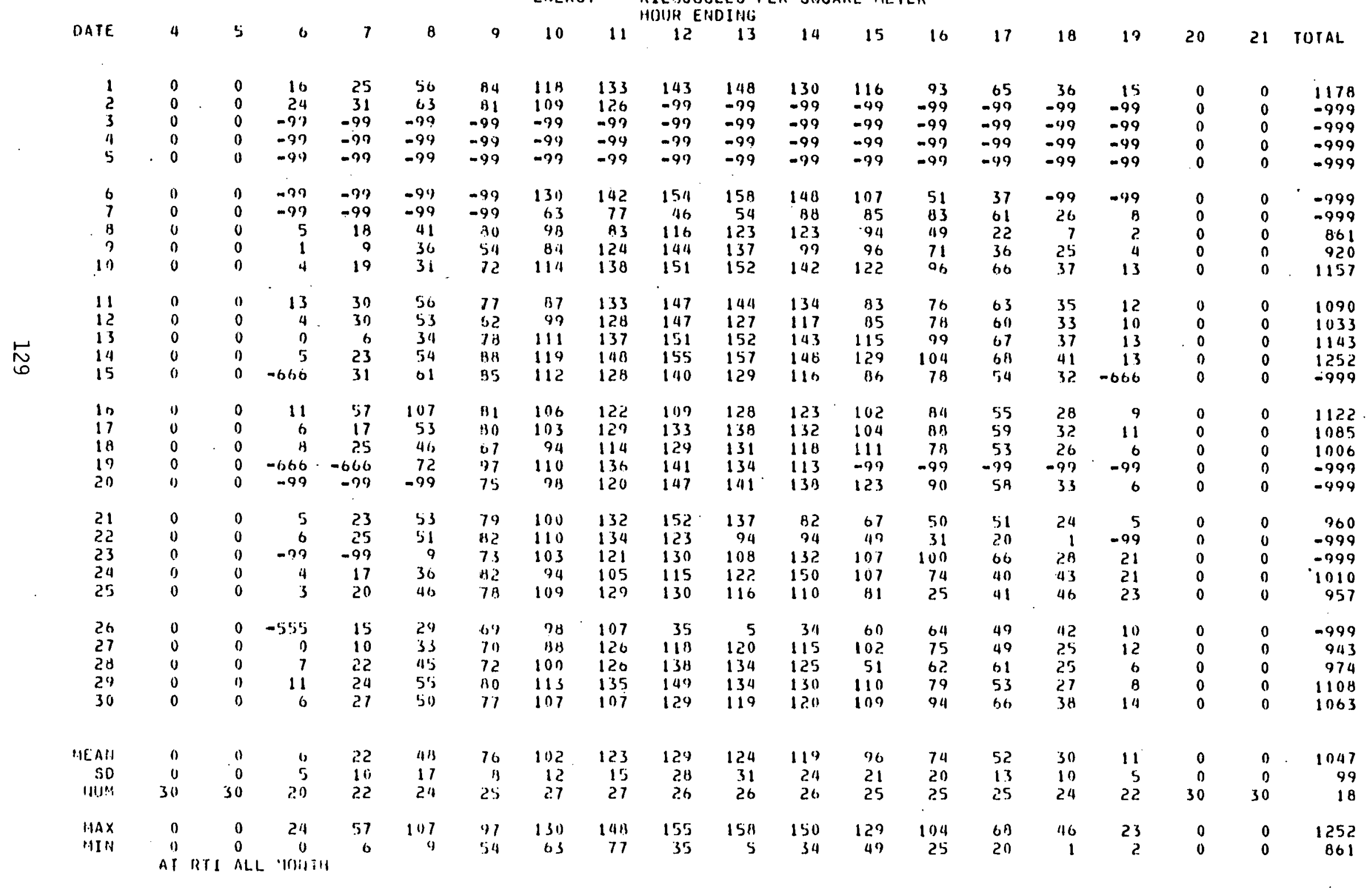


COASTAL - IMLAHD GOLAR RADIATIUN DIFFEHENCE STUDY

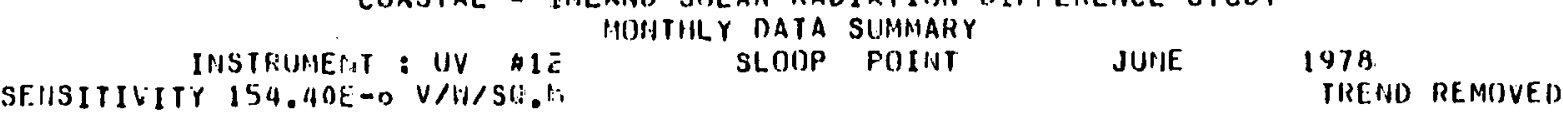

ENERGY KILINJULES PER SAUARE METER HOUR EMDING

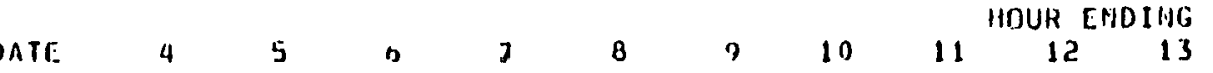

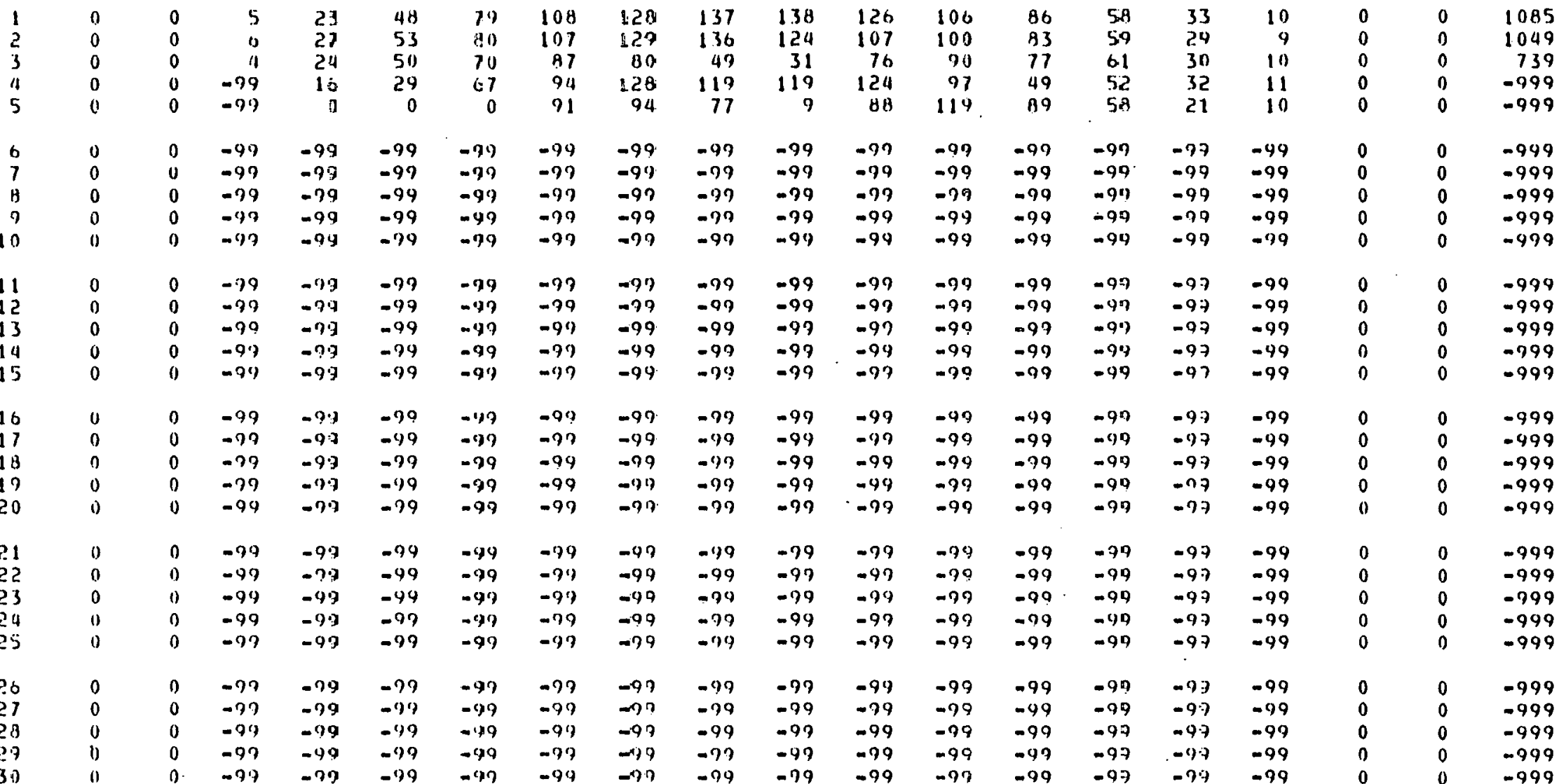

\begin{tabular}{|c|c|c|c|c|c|c|c|c|c|c|c|c|c|c|c|c|c|c|c|}
\hline MEAII & 0 & ) & 5 & 18 & 30 & $5 ?$ & 97 & 111 & 113 & $8 / 1$ & 104 & $10 ?$ & 76 & 5.7 & 27 & 10 & 0 & 0 & 957 \\
\hline SD & 0 & 0 & 1) & 9 & 19 & 30 & $n$ & .20 & 34 & 53 & 19 & 9 & 14 & 3 & 4 & 0 & 0 & 0 & 155 \\
\hline NIIM & 30 & 30 & 3 & 5 & 5 & 5 & 5 & 5 & ; & 5 & 5 & 5 & 5 & 5 & 5 & 5 & 30 & 30 & 3 \\
\hline$H \cap x$ & 0 & 1) & 0 & 27 & 53 & 810 & $101]$ & 1.27 & 137 & 134 & 126 & 119 & no & 61 & 33 & 11 & 0 & 0 & 1085 \\
\hline [11i] & 0 & 0 & 4 & $n$ & i) & 0 & 117 & $3 n$ & 149 & 9 & 76 & 90 & 49 & $5 ?$ & 21 & 9 & 0. & 0 & 739 \\
\hline
\end{tabular}


COASTAL - IHLAND SILAR RADIATIUN DIFFERENCE STUISY

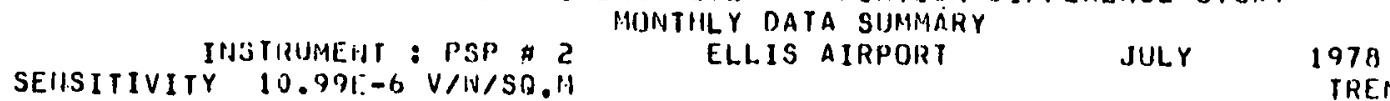

TRENII REMOIED

EHERGY KILOJOUI.ES PER SGUARE METER

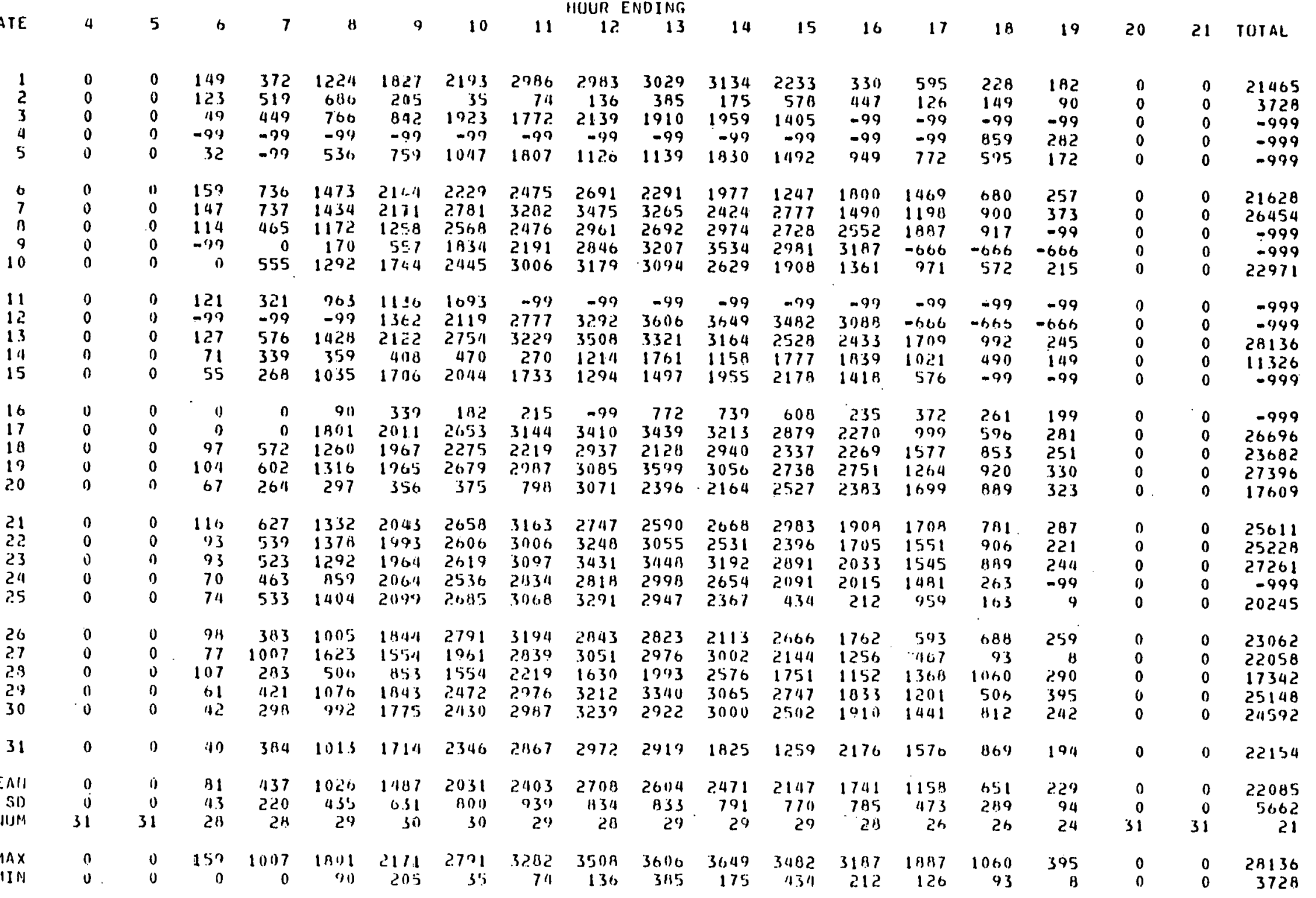


CIIASTAL - IMLANID SULAK RADIATION DIFFFREIICE STUDY IHSTHIJME.HT: PSP H 3
SENSITIVITY II.2TE-6 V/H/SO.H

EHERGY KILOJUIJES PER SOIJARE IIETER IIOUR ENDING

DATE

$10 \quad 11 \quad 12 \quad 13$

14

16

17

IA

19

20

21 TOTAL

1
2
3
4
5

-999
-999
-999
-999
12901
26438
-999
22472
25819
25273
-999
-999
29485
10192
14226
-999
-999
27758
26881
-999
-999
25745
25226
21625
-999
-999
25936
18388
24796
22163
26628
22886
5318
18
29485
5000


COASTAL - IMLAHD SULAR RADIATION DIFFERENCE STIJDY

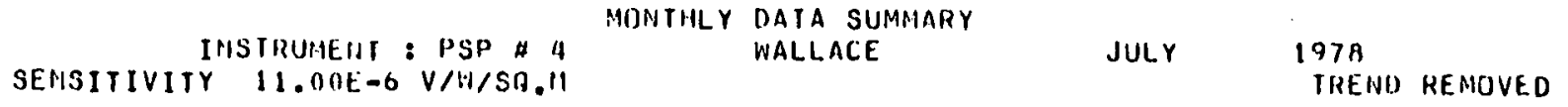

EHERTY KILUJOULES PER SOUARE METER

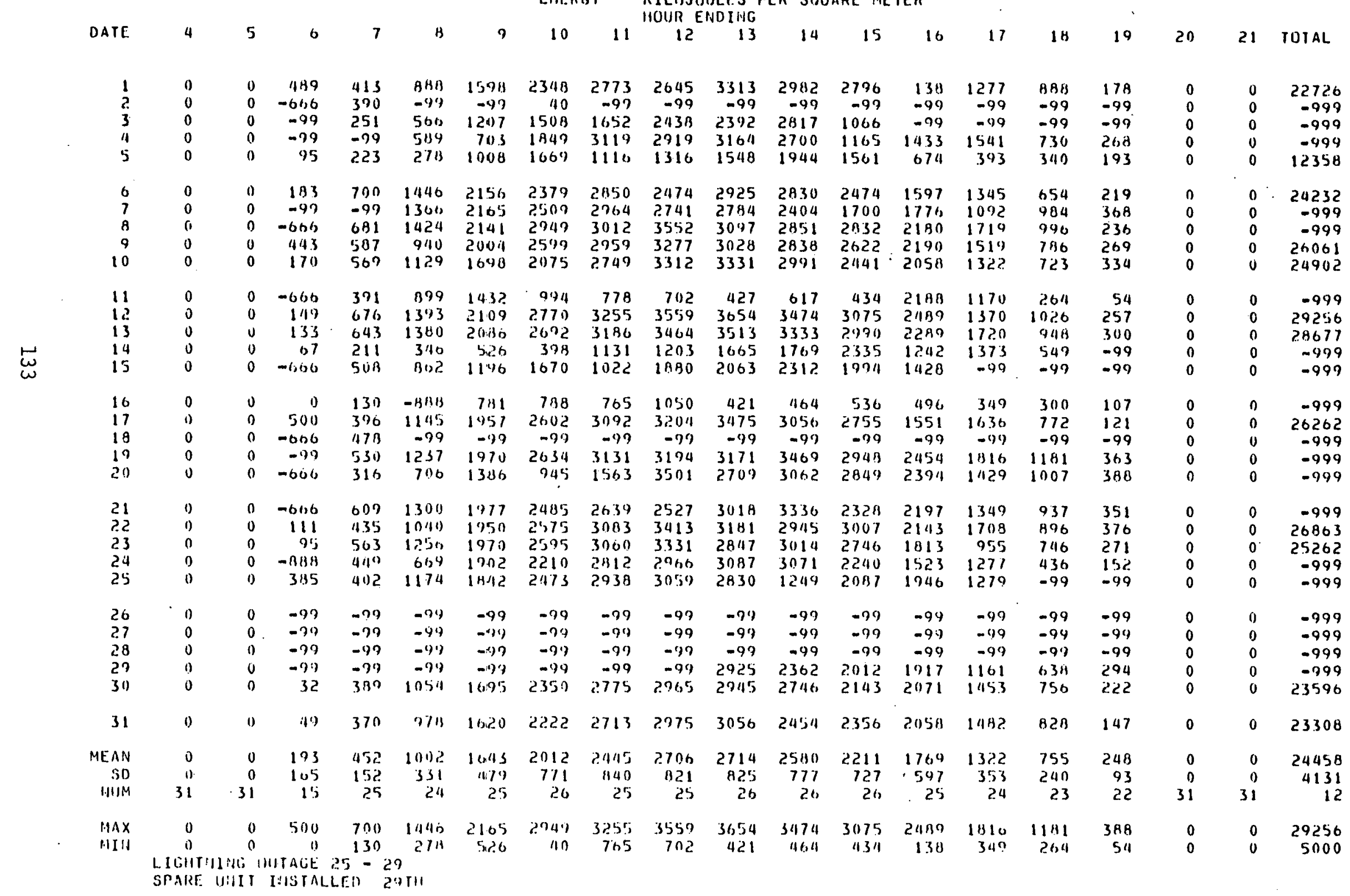


CIIASTAL. - IHLALI SOLAR RADIATIIIN DIFFEREHCE STIJOY

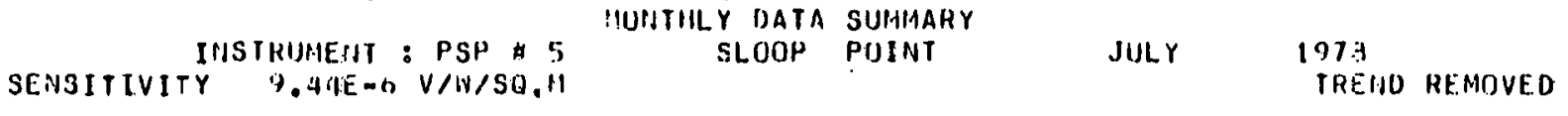

ENERGY RILOJCULES PER SUUARE METER

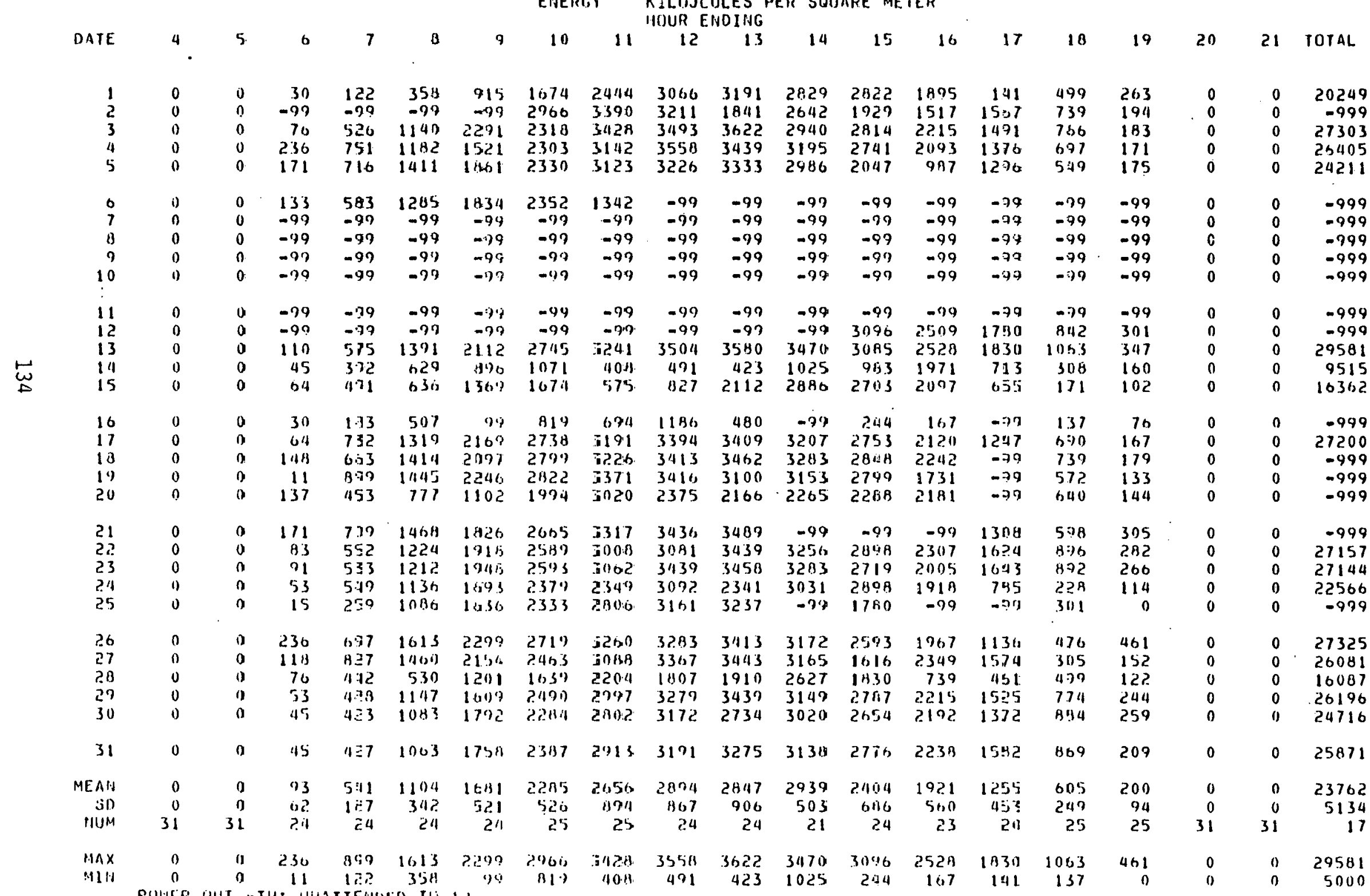

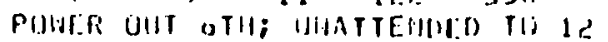




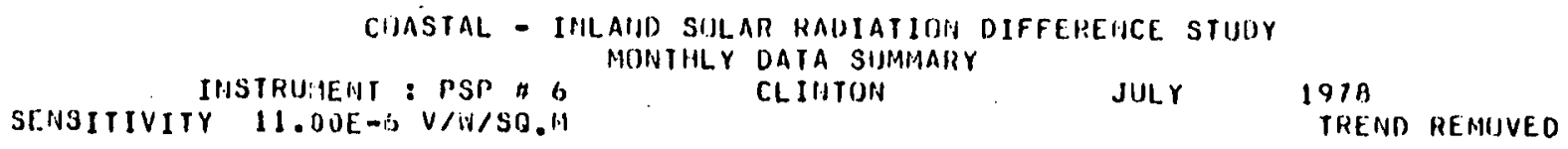

ENERGY KILUJOULES PER SUUARE METER IIOUIR ENDING

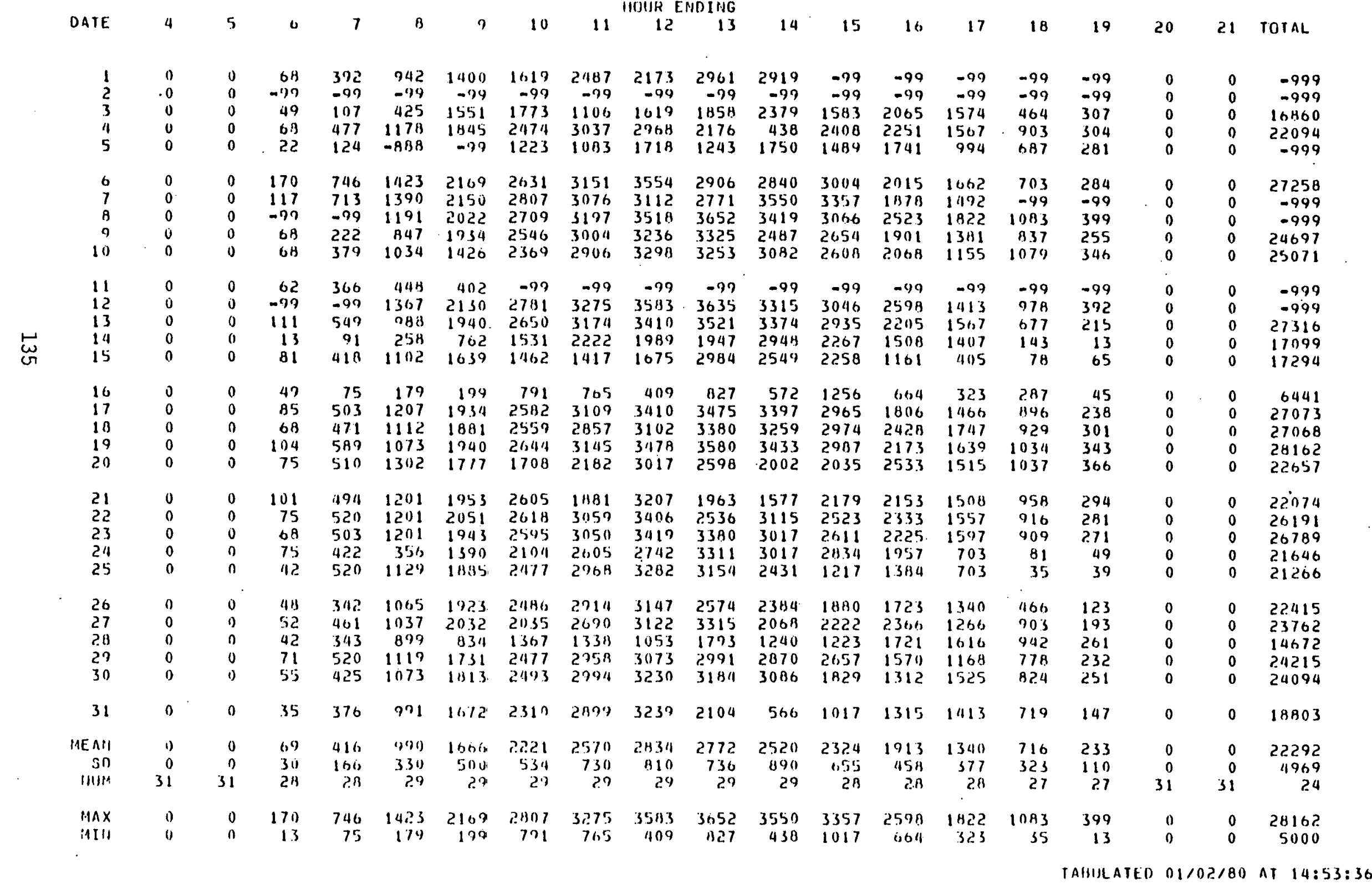


CIIASTAL - INLANO SULAR RADIATIOH DIFFEREHCE STIORY

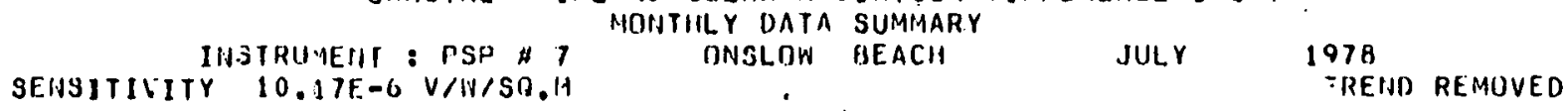

FIHFRGY KILIJJOULES PER SNUARE METER

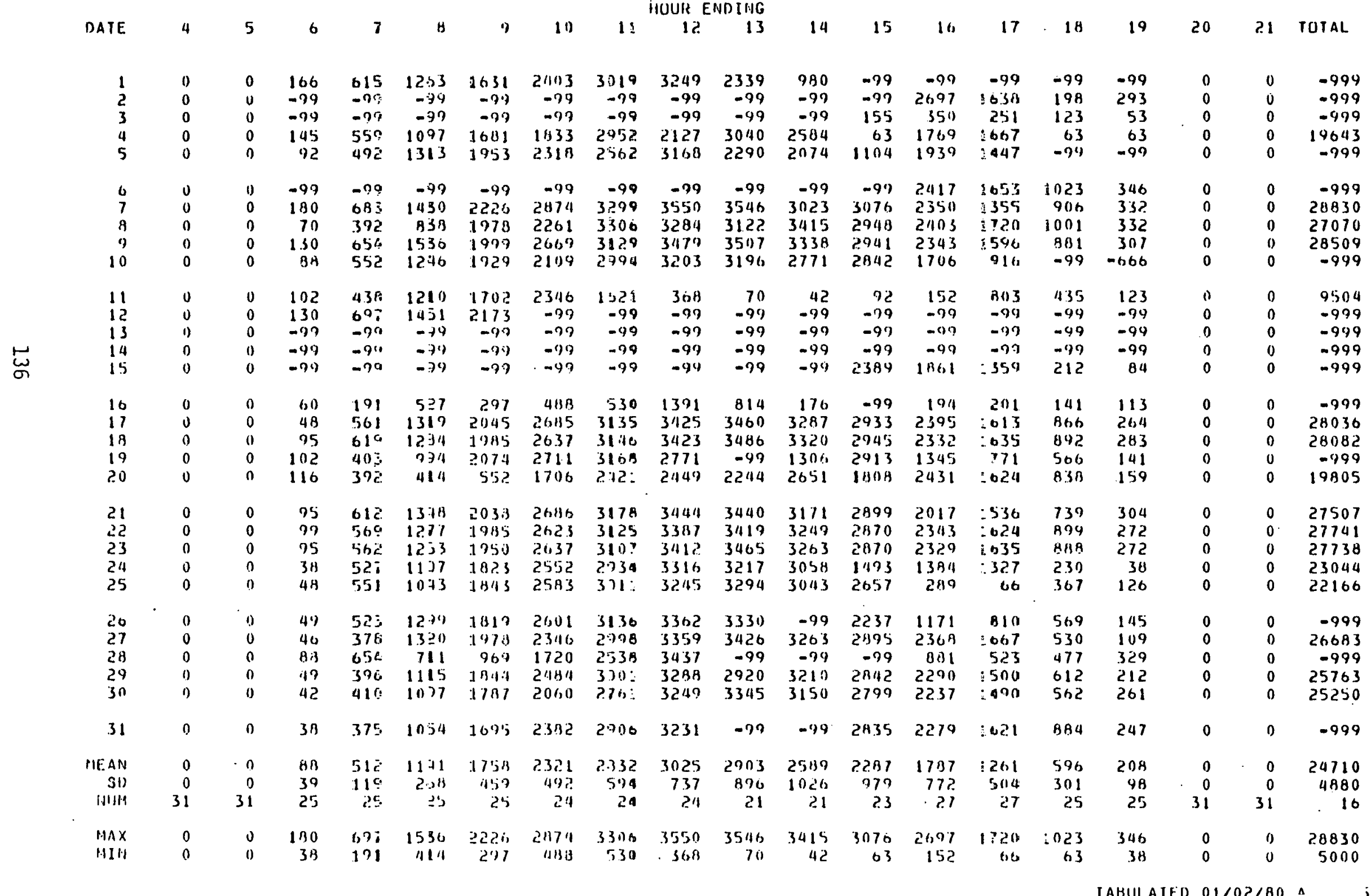


COASTAL - INLANO SULAR RADIATIUN DIFFEREHCE STUDY

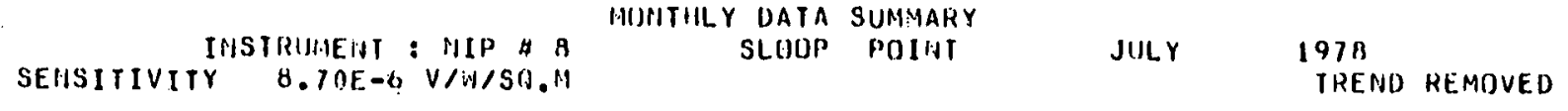

EHERG KILUJOULES PER SIJUARE METER

$10 \quad 11 \frac{12}{1} 13 \quad 14 \quad 15$

$$
-2
$$

$\begin{array}{rrrrr}0 & 0 & 0 & 0 & 17 \\ -99 & -99 & -99 & -99 & -77 \\ 0 & 0 & 0 & 0 & 0 \\ 0 & -719 & -888 & 0 & 0 \\ -99 & -99 & -97 & -97 & -99\end{array}$

$\begin{array}{rrr}12 & 1 \\ 0 & 19 & 2 \\ 9 & 479 & 9\end{array}$

12
0
0
479

12
0
0
312

-99
0
0
364

-99
0
0
357
-99

$\begin{array}{lllll}-99 & -99 & -99 & -99 & -99 \\ -79 & -99 & -99 & -99 & -97 \\ -99 & -99 & -99 & -99 & -97 \\ -99 & -90 & -99 & -99 & -99 \\ -97 & -99 & -94 & -99 & -99\end{array}$

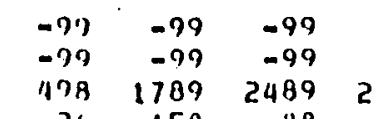

$\begin{array}{lllll}178 & 1789 & 2489 & 2778 & 2842 \\ 2052\end{array}$

$-99 \quad-99$

$-99$

$-99 \quad-99$

-99
-99

$-99 \quad-99$

-99
-99

$-99-99 \quad-99$

$\begin{array}{ll}-99 & -99 \\ -99 & -99\end{array}$

$-99$

$-99$

$-99$

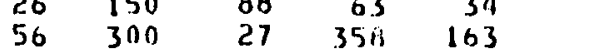

$\begin{array}{rrr}0 & 73 & \\ -555 & -555 & -5 \\ -99 & -79 & - \\ -19 & -179 & - \\ -79 & -99 & -99\end{array}$

$\begin{array}{rr}-99 & -79 \\ 111 & 9 \\ 11 & 82 \\ 58 & 11\end{array}$

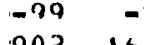

16139 2

1659 2205 गद5.

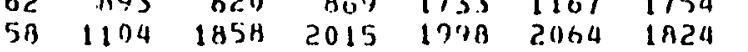

$\begin{array}{rll}144 & 1152 & 158 \\ 44 & 1186 & 172\end{array}$

2.19 732

$72 \quad 795 \quad 1288 \quad 101$ 101

$\begin{array}{lllll}1424 & 2119 & 2348 & 2285 & \end{array}$

0

$39 \quad 57$

MEAi
SD
MIIPH

$\ln x$

MIN

93.691

31

(1)

1

$17 \quad 469$

110211001620

$586 \quad 1934$

906

$\begin{array}{rrrrr}-99 & -99 & -99 & -99 & -99 \\ -99 & -97 & -99 & 2803 & 2253\end{array}$

060

3118

$\begin{array}{lllll}4803 & 2253 & \text { BA3 } & 238\end{array}$

$2207 \quad 1198$ 
CUA3IAL. - IHLATH SIILAR RADIATIOH DIFFERTHCE STHIYY

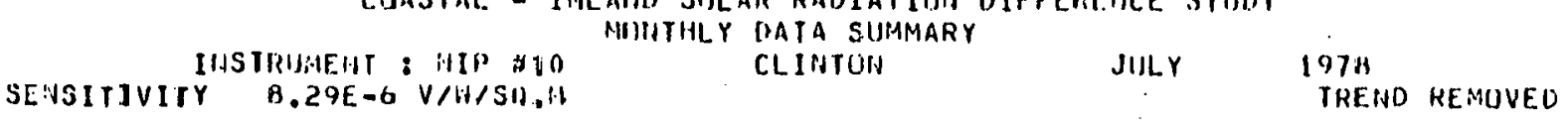

ENERT'i RILOJOULES PER SIJUARF. METER

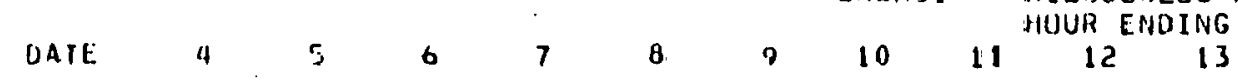

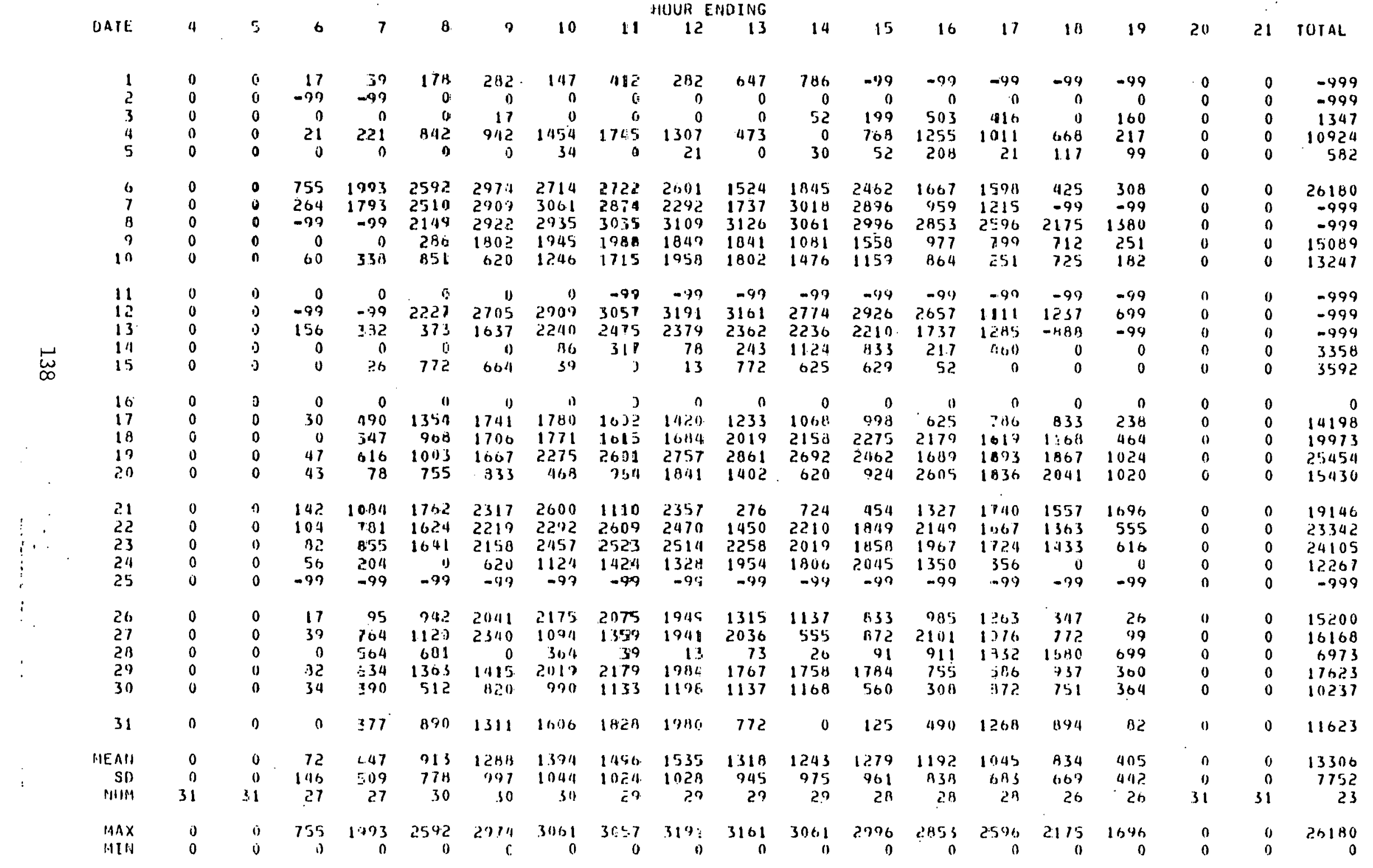




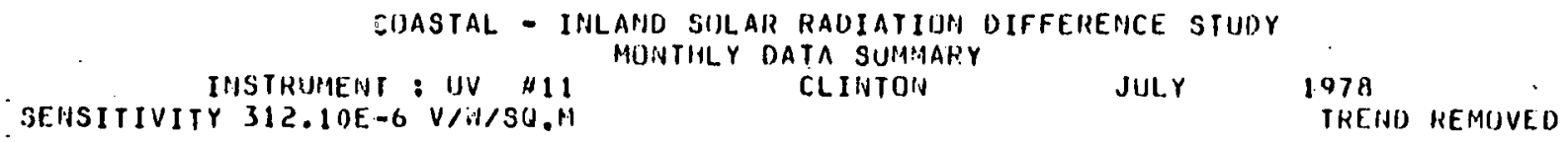

EIIFRGY KILOJUULES PER SIUURE METFE HOUR ENDING

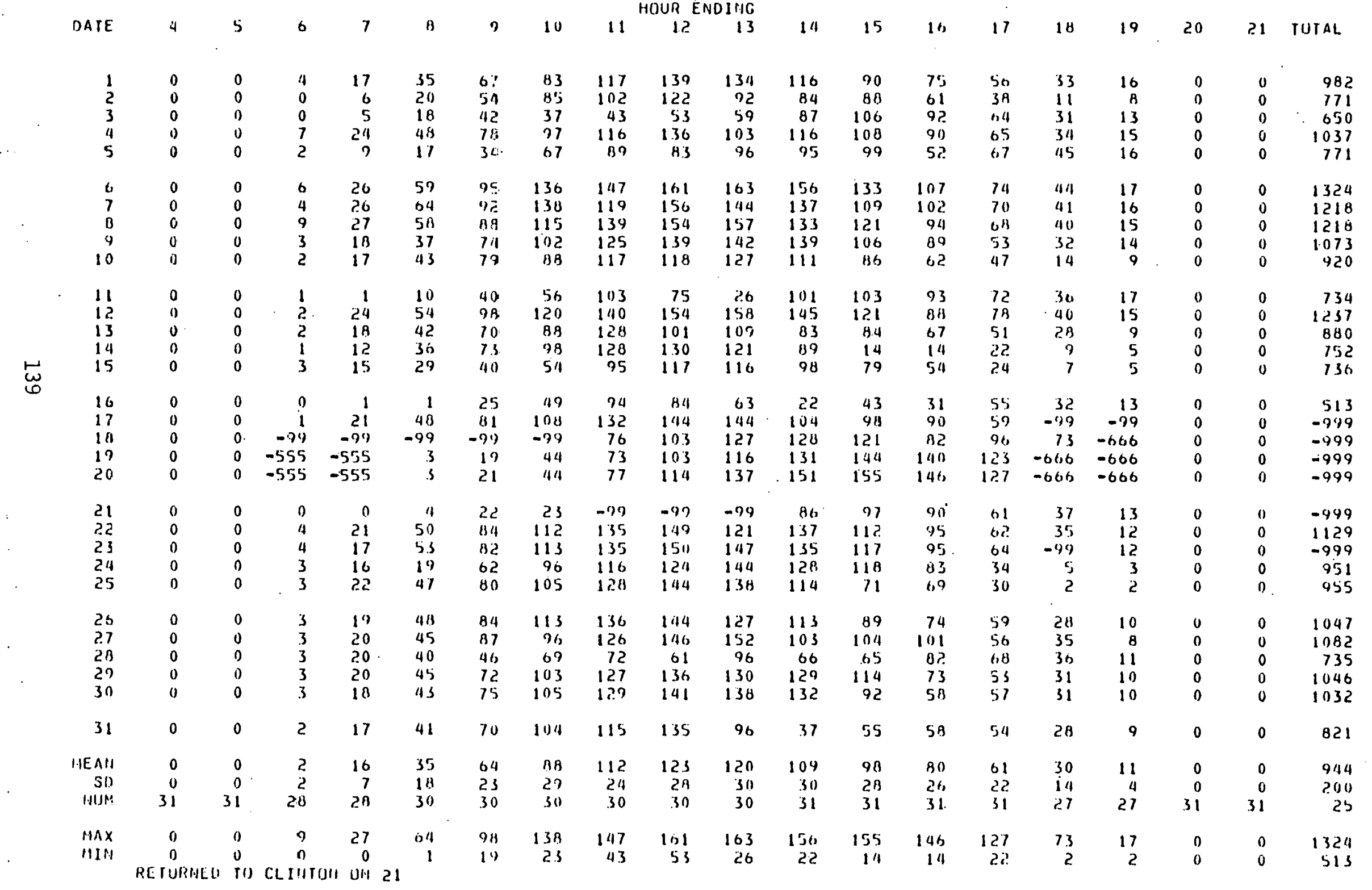



COASTAL - IML.MHD SULAR RADIATION DIFFERENCE STHDY
MUNTHLY DATA SUMMARY
SENSITIVITY 154.40 -6 V/W/SA.M

ENERGY KILUJDULES PER SUUARE METER HOUR ENDING

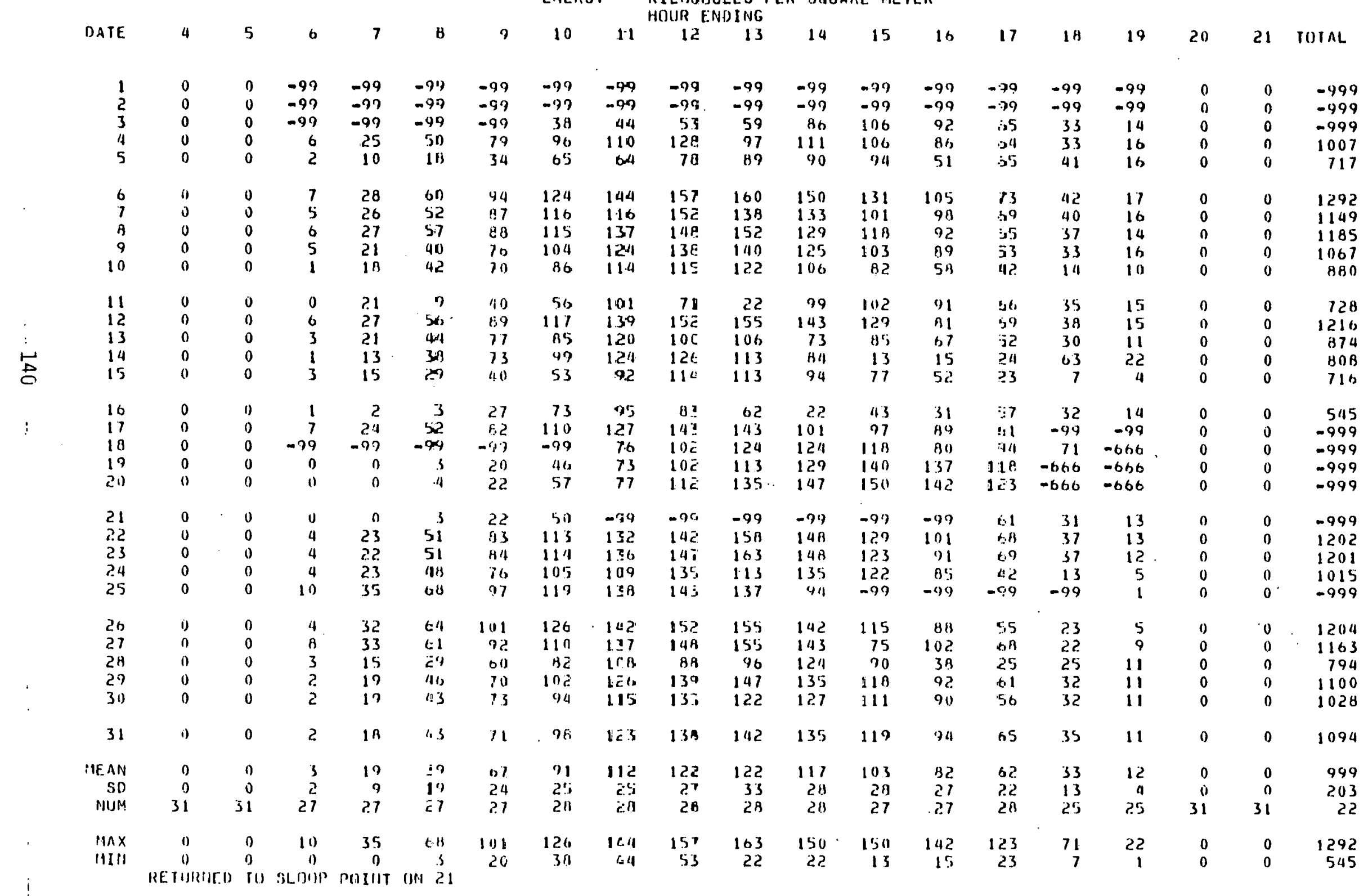


COASTAL - IHLAND 3IJLAR RADIATION DIFFEREIICE STUDY

IHSTRUMENT: PSP \# 2
SENSITIVITY $10.99 E-6$ VIW/SO.M

EHERGY KILUJUULES PER SPUAAE METER

\begin{tabular}{|c|c|c|c|c|c|c|c|c|c|c|c|c|c|c|c|c|c|c|c|}
\hline \multirow[b]{2}{*}{ ATE. } & \multirow[b]{2}{*}{4} & \multirow[b]{2}{*}{5} & \multirow[b]{2}{*}{6} & \multirow[b]{2}{*}{7} & & & & & H(N)RR E & VUING & & & \multirow[b]{2}{*}{16} & \multirow[b]{2}{*}{17} & & \multirow[b]{2}{*}{20} & \multirow[b]{2}{*}{21} & \multirow[b]{2}{*}{ TOTAL } \\
\hline & & & & & B & 7 & 10 & 11 & 12 & 13 & 14 & 15 & & & 18 & 19 & & & \\
\hline $\begin{array}{l}1 \\
2 \\
3 \\
1 \\
5\end{array}$ & $\begin{array}{l}0 \\
0 \\
0 \\
0 \\
0\end{array}$ & $\begin{array}{l}0 \\
0 \\
0 \\
0 \\
0\end{array}$ & $\begin{array}{l}18 \\
37 \\
76 \\
54 \\
55\end{array}$ & $\begin{array}{l}444 \\
374 \\
5118 \\
510 \\
422\end{array}$ & $\begin{array}{r}1083 \\
1321 \\
866 \\
1207 \\
11 B 9\end{array}$ & $\begin{array}{r}1614 \\
831 \\
1707 \\
132.5 \\
1723\end{array}$ & $\begin{array}{r}1166 \\
253 \\
1865 \\
2390 \\
175\end{array}$ & $\begin{array}{r}2564 \\
689 \\
2792 \\
2.337 \\
762\end{array}$ & $\begin{array}{r}1208 \\
2232 \\
2166 \\
3037 \\
167\end{array}$ & $\begin{array}{r}1234 \\
1838 \\
2605 \\
3389 \\
750\end{array}$ & $\begin{array}{r}136 \\
754 \\
3165 \\
3445 \\
1153\end{array}$ & $\begin{array}{r}133 \\
9114 \\
1911 \\
2727 \\
12.118\end{array}$ & $\begin{array}{l}303 \\
1583 \\
1282 \\
1571 \\
1091\end{array}$ & $\begin{array}{r}389 \\
1149 \\
564 \\
1159 \\
907\end{array}$ & $\begin{array}{r}330 \\
11111 \\
391 \\
572 \\
566\end{array}$ & $\begin{array}{r}143 \\
289 \\
125 \\
80 \\
157\end{array}$ & $\begin{array}{l}0 \\
0 \\
0 \\
0 \\
0\end{array}$ & $\begin{array}{l}0 \\
0 \\
0 \\
0 \\
0\end{array}$ & $\begin{array}{l}11065 \\
137.37 \\
20050 \\
24165 \\
10165\end{array}$ \\
\hline $\begin{array}{r}6 \\
7 \\
6 \\
9 \\
10\end{array}$ & $\begin{array}{l}0 \\
0 \\
0 \\
0 \\
0\end{array}$ & $\begin{array}{l}0 \\
0 \\
0 \\
0 \\
0\end{array}$ & $\begin{array}{r}97 \\
31 \\
-97 \\
-99 \\
31\end{array}$ & $\begin{array}{l}762 \\
529 \\
-99 \\
560 \\
529\end{array}$ & $\begin{array}{l}1568 \\
1306 \\
1205 \\
1244 \\
1099\end{array}$ & $\begin{array}{l}1457 \\
1325 \\
1742 \\
1781 \\
1567\end{array}$ & $\begin{array}{l}2357 \\
1892 \\
2142 \\
2686 \\
1699\end{array}$ & $\begin{array}{l}2350 \\
2616 \\
3370 \\
3030 \\
2098\end{array}$ & $\begin{array}{l}2806 \\
3130 \\
2833 \\
2617 \\
2419\end{array}$ & $\begin{array}{l}2906 \\
3117 \\
3033 \\
3360 \\
2157\end{array}$ & $\begin{array}{r}2288 \\
3058 \\
2037 \\
2296 \\
-99\end{array}$ & $\begin{array}{r}1292 \\
2855 \\
1922 \\
2571 \\
.99\end{array}$ & $\begin{array}{r}916 \\
2423 \\
1998 \\
1732 \\
-99\end{array}$ & $\begin{array}{r}749 \\
1630 \\
1611 \\
1080 \\
-99\end{array}$ & $\begin{array}{l}500 \\
909 \\
8611 \\
671 \\
-99\end{array}$ & $\begin{array}{l}130 \\
185 \\
180 \\
167 \\
-99\end{array}$ & $\begin{array}{l}0 \\
0 \\
0 \\
0 \\
n\end{array}$ & $\begin{array}{l}0 \\
0 \\
0 \\
0 \\
0\end{array}$ & $\begin{array}{r}20253 \\
25006 \\
-999 \\
-999 \\
-999\end{array}$ \\
\hline $\begin{array}{l}11 \\
12 \\
13 \\
14 \\
15\end{array}$ & $\begin{array}{l}0 \\
0 \\
0 \\
0 \\
0\end{array}$ & $\begin{array}{l}0 \\
0 \\
0 \\
0 \\
0\end{array}$ & $\begin{array}{r}-99 \\
30 \\
41 \\
-99 \\
31\end{array}$ & $\begin{array}{l}303 \\
371 \\
395 \\
294 \\
2.97\end{array}$ & $\begin{array}{r}1116 \\
3615 \\
827 \\
1071 \\
10611\end{array}$ & $\begin{array}{r}1667 \\
895 \\
1457 \\
1735 \\
1797\end{array}$ & $\begin{array}{l}1656 \\
1458 \\
1997 \\
2338 \\
2413\end{array}$ & $\begin{array}{l}2042 \\
2490 \\
3140 \\
21353 \\
2488\end{array}$ & $\begin{array}{l}1030 \\
2523 \\
3327 \\
3138 \\
3048\end{array}$ & $\begin{array}{r}424 \\
1337 \\
2249 \\
3038 \\
2537\end{array}$ & $\begin{array}{r}244 \\
469 \\
912 \\
2663 \\
1708\end{array}$ & $\begin{array}{r}591 \\
2343 \\
-99 \\
704 \\
2573\end{array}$ & $\begin{array}{r}958 \\
1655 \\
-91 \\
176 \\
2121\end{array}$ & $\begin{array}{r}3114 \\
17114 \\
-99 \\
242 \\
1442\end{array}$ & $\begin{array}{l}323 \\
748 \\
-99 \\
386 \\
680\end{array}$ & $\begin{array}{r}77 \\
181 \\
-99 \\
85 \\
31\end{array}$ & $\begin{array}{l}0 \\
0 \\
0 \\
0 \\
0\end{array}$ & $\begin{array}{l}0 \\
0 \\
0 \\
0 \\
0\end{array}$ & $\begin{array}{r}-999 \\
14572 \\
-997 \\
-999 \\
22246\end{array}$ \\
\hline $\begin{array}{l}16 \\
17 \\
18 \\
17 \\
20\end{array}$ & $\begin{array}{l}0 \\
0 \\
0 \\
0 \\
0\end{array}$ & $\begin{array}{l}0 \\
0 \\
0 \\
0 \\
0\end{array}$ & $\begin{array}{r}-99 \\
31 \\
20 \\
27 \\
28\end{array}$ & $\begin{array}{l}321 \\
375 \\
325 \\
322 \\
323\end{array}$ & $\begin{array}{r}936 \\
1037 \\
892 \\
905 \\
908\end{array}$ & $\begin{array}{r}1667 \\
1777 \\
-79 \\
1612 \\
1633\end{array}$ & $\begin{array}{r}2339 \\
2406 \\
-79 \\
2150 \\
2446\end{array}$ & $\begin{array}{r}2777 \\
28611 \\
-99 \\
2690 \\
2521\end{array}$ & $\begin{array}{r}3203 \\
2780 \\
-99 \\
3096 \\
3029\end{array}$ & $\begin{array}{r}2168 \\
2852 \\
-99 \\
2713 \\
2950\end{array}$ & $\begin{array}{r}3822 \\
2475 \\
-99 \\
3008 \\
3209\end{array}$ & $\begin{array}{r}2544 \\
2311 \\
-99 \\
2644 \\
21172\end{array}$ & $\begin{array}{r}1985 \\
1823 \\
-99 \\
2078 \\
1879\end{array}$ & $\begin{array}{r}1428 \\
624 \\
-99 \\
1305 \\
1111\end{array}$ & $\begin{array}{r}641 \\
67 \\
-99 \\
509 \\
1987\end{array}$ & $\begin{array}{r}121 \\
36 \\
-99 \\
20 \\
58\end{array}$ & $\begin{array}{l}0 \\
0 \\
0 \\
0 \\
0\end{array}$ & $\begin{array}{l}0 \\
0 \\
0 \\
0 \\
0\end{array}$ & $\begin{array}{r}-999 \\
21464 \\
-997 \\
23079 \\
22844\end{array}$ \\
\hline $\begin{array}{l}22 \\
23 \\
24 \\
25\end{array}$ & $\begin{array}{l}0 \\
0 \\
0 \\
0 \\
0\end{array}$ & $\begin{array}{l}0 \\
0 \\
0 \\
0 \\
0\end{array}$ & $\begin{array}{l}26 \\
25 \\
21 \\
22 \\
25\end{array}$ & $\begin{array}{l}403 \\
411 \\
392 \\
347 \\
264\end{array}$ & $\begin{array}{l}1104 \\
1001 \\
1101 \\
1058 \\
935\end{array}$ & $\begin{array}{l}1857 \\
1921 \\
1912 \\
1805 \\
1020\end{array}$ & $\begin{array}{l}2525 \\
2527 \\
2440 \\
21196 \\
2226\end{array}$ & $\begin{array}{l}3049 \\
31119 \\
2288 \\
3115 \\
26713\end{array}$ & $\begin{array}{l}3341 \\
3291 \\
3258 \\
3308 \\
2976\end{array}$ & $\begin{array}{l}3436 \\
3327 \\
3399 \\
3311 \\
3045\end{array}$ & $\begin{array}{l}3253 \\
2956 \\
3102 \\
3108 \\
21119\end{array}$ & $\begin{array}{l}2820 \\
2671 \\
2724 \\
2609 \\
2233\end{array}$ & $\begin{array}{l}1844 \\
2105 \\
2115 \\
2099 \\
11443\end{array}$ & $\begin{array}{l}1438 \\
1123 \\
1414 \\
1320 \\
1040\end{array}$ & $\begin{array}{l}609 \\
021 \\
483 \\
563 \\
424\end{array}$ & $\begin{array}{l}85 \\
84 \\
61 \\
75 \\
41\end{array}$ & $\begin{array}{l}0 \\
0 \\
0 \\
0 \\
0\end{array}$ & $\begin{array}{l}0 \\
0 \\
0 \\
0 \\
0\end{array}$ & $\begin{array}{l}25790 \\
25378 \\
24459 \\
25319 \\
21369\end{array}$ \\
\hline $\begin{array}{l}? .6 \\
27 \\
20 \\
29 \\
30\end{array}$ & $\begin{array}{l}0 \\
0 \\
0 \\
0 \\
0\end{array}$ & $\begin{array}{l}0 \\
0 \\
0 \\
0 \\
0\end{array}$ & $\begin{array}{r}14 \\
17 \\
-99 \\
0 \\
12\end{array}$ & $\begin{array}{l}240 \\
197 \\
206 \\
245 \\
179\end{array}$ & $\begin{array}{r}620 \\
866 \\
1035 \\
887 \\
536\end{array}$ & $\begin{array}{r}148.1 \\
879 \\
671 \\
1972 \\
130.3\end{array}$ & $\begin{array}{r}1724 \\
1170 \\
625 \\
2234 \\
1709\end{array}$ & $\begin{array}{r}21480 \\
2022 \\
490 \\
2731 \\
2397\end{array}$ & $\begin{array}{l}2346 \\
2127 \\
2227 \\
3046 \\
2927\end{array}$ & $\begin{array}{r}2772 \\
980 \\
2640 \\
3079 \\
3216\end{array}$ & $\begin{array}{r}2612 \\
106 \\
2656 \\
27187 \\
2803\end{array}$ & $\begin{array}{r}2241 \\
224 \\
1248 \\
2476 \\
2085\end{array}$ & $\begin{array}{r}1347 \\
718 \\
-99 \\
1922 \\
1705\end{array}$ & $\begin{array}{r}666 \\
1082 \\
-09 \\
1169 \\
1165\end{array}$ & $\begin{array}{l}129 \\
577 \\
-97 \\
965 \\
480\end{array}$ & $\begin{array}{r}0 \\
40 \\
-99 \\
36 \\
32\end{array}$ & $\begin{array}{l}0 \\
0 \\
0 \\
0 \\
0\end{array}$ & $\begin{array}{l}0 \\
0 \\
0 \\
0 \\
0\end{array}$ & $\begin{array}{r}18672 \\
11005 \\
-999 \\
22649 \\
20549\end{array}$ \\
\hline 31 & 0 & 0 & 17 & 335 & 714 & 1501 & 2395 & 2500 & 2009 & 1291 & 430 & 2107 & 2035 & 1334 & 522 & 56 & 0 & 0 & 17276 \\
\hline SI & $\begin{array}{r}0 \\
0 \\
31\end{array}$ & $\begin{array}{r}0 \\
0 \\
31\end{array}$ & $\begin{array}{l}31 \\
20 \\
25\end{array}$ & $\begin{array}{r}374 \\
125 \\
30\end{array}$ & $\begin{array}{r}1006 \\
235 \\
31\end{array}$ & $\begin{array}{r}15.30 \\
320 \\
30\end{array}$ & $\begin{array}{r}1949 \\
623 \\
30\end{array}$ & $\begin{array}{r}21151 \\
090 \\
30\end{array}$ & $\begin{array}{r}2623 \\
73.7 \\
30\end{array}$ & $\begin{array}{r}2509 \\
861 \\
30\end{array}$ & $\begin{array}{r}2177 \\
1116 \\
29\end{array}$ & $\begin{array}{r}1971 \\
914 \\
29\end{array}$ & $\begin{array}{r}1589 \\
563 \\
.27\end{array}$ & $\begin{array}{r}1122 \\
341 \\
27\end{array}$ & $\begin{array}{r}514 \\
217 \\
27\end{array}$ & $\begin{array}{l}95 \\
65 \\
27\end{array}$ & $\begin{array}{r}0 \\
0 \\
31\end{array}$ & $\begin{array}{r}0 \\
0 \\
31\end{array}$ & $\begin{array}{r}20159 \\
4804 \\
22\end{array}$ \\
\hline & $\begin{array}{l}0 \\
0\end{array}$ & $\begin{array}{l}0 \\
0\end{array}$ & $\begin{array}{r}97 \\
0\end{array}$ & $\begin{array}{l}762 \\
179\end{array}$ & $\begin{array}{r}15613 \\
3013\end{array}$ & $\begin{array}{r}1981 \\
6.71\end{array}$ & $\begin{array}{r}2686 \\
253\end{array}$ & $\begin{array}{r}3370 \\
26 ?\end{array}$ & $\begin{array}{r}3341 \\
167\end{array}$ & $\begin{array}{r}3436 \\
424\end{array}$ & $\begin{array}{r}3822 \\
106\end{array}$ & $\begin{array}{r}2155 \\
133\end{array}$ & $\begin{array}{r}2423 \\
176\end{array}$ & $\begin{array}{r}1704 \\
242\end{array}$ & $\begin{array}{r}1140 \\
67\end{array}$ & $\begin{array}{r}289 \\
0\end{array}$ & $\begin{array}{l}0 \\
0\end{array}$ & $\begin{array}{l}0 \\
0\end{array}$ & $\begin{array}{r}25790 \\
5000\end{array}$ \\
\hline
\end{tabular}


CIIASTAL - INLAIID SIILAR RADIATION UIFFEREHCE STUUV

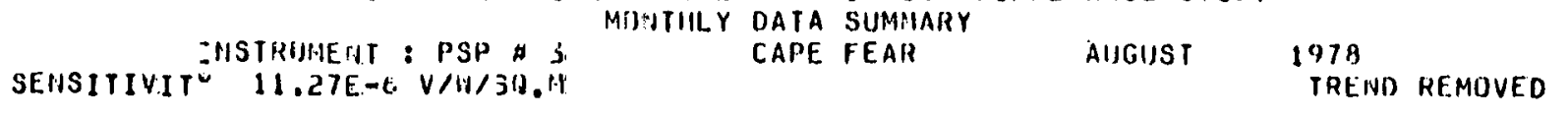

EHERGY KLLUJUULES PER SIJUARE HETER

\begin{tabular}{|c|c|c|c|c|c|c|c|c|c|c|c|c|c|c|c|c|c|c|c|}
\hline & & & & & & & & & -11JUR E & IND INSF & & & & & & & & & \\
\hline DATE & 4 & 5 & 6 & 7 & 8 & 7 & 10 & 11 & 12 & 13 & 14 & 15 & 10 & 17 & 18 & 19 & 20 & 21 & TOTAL \\
\hline $\begin{array}{l}1 \\
2 \\
3 \\
4 \\
5\end{array}$ & $\begin{array}{l}0 \\
0 \\
0 \\
0 \\
0\end{array}$ & $\begin{array}{l}0 \\
0 \\
0 \\
0 \\
0\end{array}$ & $\begin{array}{l}53 \\
52 \\
71 \\
62 \\
48\end{array}$ & $\begin{array}{l}516 \\
471 \\
541 \\
506 \\
530\end{array}$ & $\begin{array}{r}1037 \\
1371 \\
1240 \\
1247 \\
770\end{array}$ & $\begin{array}{l}2037 \\
18119 \\
1453 \\
1563 \\
175\end{array}$ & $\begin{array}{r}2398 \\
1074 \\
2176 \\
2649 \\
478\end{array}$ & $\begin{array}{r}2925 \\
1477 \\
2579 \\
2630 \\
476\end{array}$ & $\begin{array}{r}3037 \\
2515 \\
2697 \\
3150 \\
287\end{array}$ & $\begin{array}{l}2724 \\
2758 \\
3141 \\
3224 \\
1897\end{array}$ & $\begin{array}{r}1321 \\
2428 \\
2464 \\
3339 \\
059\end{array}$ & $\begin{array}{l}1401 \\
2432 \\
1857 \\
2790 \\
2124\end{array}$ & $\begin{array}{r}999 \\
1605 \\
1400 \\
2138 \\
1163\end{array}$ & $\begin{array}{r}587 \\
1752 \\
1413 \\
1509 \\
176\end{array}$ & $\begin{array}{r}449 \\
032 \\
854 \\
538 \\
96\end{array}$ & $\begin{array}{r}130 \\
103 \\
2.34 \\
70 \\
32\end{array}$ & $\begin{array}{l}0 \\
0 \\
0 \\
0 \\
0\end{array}$ & $\begin{array}{l}0 \\
0 \\
0 \\
0 \\
0\end{array}$ & $\begin{array}{r}19617 \\
21083 \\
22125 \\
25483 \\
9512\end{array}$ \\
\hline $\begin{array}{r}6 \\
7 \\
8 \\
9 \\
10\end{array}$ & $\begin{array}{l}0 \\
0 \\
0 \\
0 \\
0\end{array}$ & $\begin{array}{l}0 \\
0 \\
0 \\
0 \\
0\end{array}$ & $\begin{array}{l}75 \\
52 \\
55 \\
33 \\
23\end{array}$ & $\begin{array}{l}535 \\
477 \\
509 \\
346 \\
336\end{array}$ & $\begin{array}{r}1312 \\
1179 \\
-94 \\
953 \\
1135\end{array}$ & $\begin{array}{r}1762 \\
943 \\
-99 \\
1917 \\
1266\end{array}$ & $\begin{array}{r}2145 \\
1355 \\
-49 \\
2748 \\
1997\end{array}$ & $\begin{array}{r}2372 \\
1668 \\
-99 \\
3189 \\
2531\end{array}$ & $\begin{array}{r}2081 \\
2637 \\
-99 \\
3476 \\
3224\end{array}$ & $\begin{array}{r}3362 \\
2042 \\
-99 \\
3333 \\
3537\end{array}$ & $\begin{array}{l}2775 \\
3495 \\
2933 \\
3480 \\
2515\end{array}$ & $\begin{array}{l}2190 \\
3205 \\
2135 \\
2611 \\
1282\end{array}$ & $\begin{array}{r}1593 \\
2451 \\
2150 \\
2419 \\
477\end{array}$ & $\begin{array}{l}919 \\
1619 \\
1630 \\
1560 \\
106\end{array}$ & $\begin{array}{r}383 \\
876 \\
763 \\
742 \\
23\end{array}$ & $\begin{array}{r}185 \\
180 \\
205 \\
253 \\
17\end{array}$ & $\begin{array}{l}0 \\
0 \\
0 \\
0 \\
0\end{array}$ & $\begin{array}{l}0 \\
0 \\
0 \\
0 \\
0\end{array}$ & $\begin{array}{r}21594 \\
22170 \\
-999 \\
27060 \\
18469\end{array}$ \\
\hline $\begin{array}{l}11 \\
12 \\
13 \\
14 \\
15\end{array}$ & $\begin{array}{l}0 \\
0 \\
0 \\
0 \\
0\end{array}$ & $\begin{array}{l}0 \\
0 \\
0 \\
0 \\
0\end{array}$ & $\begin{array}{r}314 \\
0 \\
17 \\
2.0 \\
20\end{array}$ & $\begin{array}{r}31 \pi \\
2 \pi \\
237 \\
403 \\
387\end{array}$ & $\begin{array}{r}954 \\
104 \\
1215 \\
799 \\
1071\end{array}$ & $\begin{array}{r}1465 \\
68 ? \\
1787 \\
740 \\
1769\end{array}$ & $\begin{array}{l}1091 \\
1401 \\
2355 \\
2099 \\
21213\end{array}$ & $\begin{array}{r}317 \\
2420 \\
2972 \\
2338 \\
2539\end{array}$ & $\begin{array}{r}69 \\
360 \\
3301 \\
23137 \\
3016\end{array}$ & $\begin{array}{r}210 \\
788 \\
2451 \\
908 \\
2911\end{array}$ & $\begin{array}{r}503 \\
791 \\
1758 \\
496 \\
1681\end{array}$ & $\begin{array}{r}708 \\
1589 \\
909 \\
2363 \\
1451\end{array}$ & $\begin{array}{r}1436 \\
1634 \\
1767 \\
553 \\
2262\end{array}$ & $\begin{array}{r}470 \\
1700 \\
290 \\
460 \\
1112\end{array}$ & $\begin{array}{l}226 \\
740 \\
250 \\
7115 \\
496\end{array}$ & $\begin{array}{r}63 \\
187 \\
87 \\
49 \\
189\end{array}$ & $\begin{array}{l}0 \\
0 \\
0 \\
0 \\
0\end{array}$ & $\begin{array}{l}0 \\
0 \\
0 \\
0 \\
0\end{array}$ & $\begin{array}{r}7947 \\
12428 \\
19470 \\
14448 \\
21347\end{array}$ \\
\hline $\begin{array}{l}16 \\
17 \\
18 \\
19 \\
20\end{array}$ & $\begin{array}{l}0 \\
0 \\
0 \\
0 \\
0\end{array}$ & $\begin{array}{l}0 \\
0 \\
0 \\
0 \\
0\end{array}$ & $\begin{array}{l}18 \\
23 \\
17 \\
17 \\
25\end{array}$ & $\begin{array}{l}302 \\
330 \\
282 \\
272 \\
212\end{array}$ & $\begin{array}{r}986 \\
1039 \\
847 \\
995 \\
910\end{array}$ & $\begin{array}{r}1703 \\
1737 \\
883 \\
1653 \\
1511\end{array}$ & $\begin{array}{l}2398 \\
2273 \\
12013 \\
2323 \\
2236\end{array}$ & $\begin{array}{l}2752 \\
2919 \\
26 ! 7 \\
2 n \div 3 \\
2805\end{array}$ & $\begin{array}{l}2765 \\
2817 \\
2572 \\
3198 \\
2897\end{array}$ & $\begin{array}{l}3199 \\
21138 \\
2432 \\
3029 \\
3098\end{array}$ & $\begin{array}{l}2 B 13 \\
2 B 85 \\
2984 \\
3032 \\
2575\end{array}$ & $\begin{array}{l}1986 \\
2643 \\
2473 \\
2647 \\
2380\end{array}$ & $\begin{array}{r}2142 \\
1882 \\
1087 \\
1972 \\
942\end{array}$ & $\begin{array}{r}1458 \\
950 \\
1160 \\
1192 \\
255\end{array}$ & $\begin{array}{l}829 \\
329 \\
585 \\
585 \\
182\end{array}$ & $\begin{array}{r}167 \\
49 \\
103 \\
129 \\
102\end{array}$ & $\begin{array}{l}0 \\
0 \\
0 \\
0 \\
0\end{array}$ & $\begin{array}{l}0 \\
0 \\
0 \\
0 \\
0\end{array}$ & $\begin{array}{l}23518 \\
27311 \\
19327 \\
23840 \\
20132\end{array}$ \\
\hline $\begin{array}{l}21 \\
22 \\
23 \\
24 \\
25\end{array}$ & $\begin{array}{l}0 \\
0 \\
.0 \\
0 \\
0\end{array}$ & $\begin{array}{l}n \\
0 \\
0 \\
0 \\
0\end{array}$ & $\begin{array}{l}2.3 \\
17 \\
20 \\
13 \\
12\end{array}$ & $\begin{array}{l}314 \\
373 \\
38 \% \\
350 \\
330\end{array}$ & $\begin{array}{r}975 \\
1092 \\
1119 \\
1004 \\
9110\end{array}$ & $\begin{array}{l}1763 \\
1875 \\
1903 \\
1865 \\
1654\end{array}$ & $\begin{array}{l}2377 \\
2546 \\
2566 \\
2460 \\
2328\end{array}$ & $\begin{array}{l}29155 \\
30917 \\
3122 \\
3019 \\
21108\end{array}$ & $\begin{array}{l}3278 \\
3344 \\
3332 \\
3310 \\
3057\end{array}$ & $\begin{array}{l}3323 \\
3342 . \\
3355 \\
3371 \\
3042\end{array}$ & $\begin{array}{l}3176 \\
3 \geq 04 \\
3058 \\
3163 \\
2.994\end{array}$ & $\begin{array}{l}2847 \\
2327 \\
2470 \\
27118 \\
3443\end{array}$ & $\begin{array}{l}2711 \\
2284 \\
2189 \\
2141 \\
1753\end{array}$ & $\begin{array}{r}1524 \\
472 \\
1486 \\
1445 \\
1035\end{array}$ & $\begin{array}{l}793 \\
283 \\
723 \\
681 \\
498\end{array}$ & $\begin{array}{r}148 \\
73 \\
116 \\
112 \\
44\end{array}$ & $\begin{array}{l}0 \\
0 \\
0 \\
0 \\
0\end{array}$ & $\begin{array}{l}0 \\
0 \\
0 \\
0 \\
0\end{array}$ & $\begin{array}{l}25737 \\
24835 \\
25828 \\
25718 \\
22850\end{array}$ \\
\hline $\begin{array}{l}26 \\
27 \\
28 \\
29 \\
30\end{array}$ & $\begin{array}{l}0 \\
0 \\
0 \\
0 \\
0\end{array}$ & $\begin{array}{l}0 \\
0 \\
0 \\
0 \\
0\end{array}$ & $\begin{array}{r}3 \\
-97 \\
12 \\
8 \\
24\end{array}$ & $\begin{array}{l}245 \\
-96 \\
179 \\
276 \\
356\end{array}$ & $\begin{array}{r}832 \\
-99 \\
859 \\
931 \\
1155\end{array}$ & $\begin{array}{r}1407 \\
-7.7 \\
1552 \\
1604 \\
1561\end{array}$ & $\begin{array}{r}2049 \\
-97 \\
11333 \\
23110 \\
1925\end{array}$ & $\begin{array}{l}21111 \\
1090 \\
2555 \\
21138 \\
21137\end{array}$ & $\begin{array}{r}2733 \\
972 \\
3121 \\
3167 \\
2973\end{array}$ & $\begin{array}{r}2845 \\
758 \\
2565 \\
3081 \\
3123\end{array}$ & $\begin{array}{l}2129 \\
2304 \\
2360 \\
2136 \\
21399\end{array}$ & $\begin{array}{l}-97 \\
1049 \\
2517 \\
2236 \\
2577\end{array}$ & $\begin{array}{r}-99 \\
333 \\
1801 \\
1928 \\
1752\end{array}$ & $\begin{array}{r}-99 \\
45 \vdots \\
50 ! \\
12110 \\
37 ?\end{array}$ & $\begin{array}{l}-90 \\
430 \\
175 \\
481 \\
436\end{array}$ & $\begin{array}{r}-99 \\
30 \\
32 \\
37 \\
53\end{array}$ & $\begin{array}{l}0 \\
0 \\
0 \\
0 \\
0 .\end{array}$ & $\begin{array}{l}0 \\
0 \\
0 \\
0 \\
0\end{array}$ & $\begin{array}{r}-999 \\
-999 \\
20062 \\
22398 \\
22201\end{array}$ \\
\hline 31 & 0 & 0 & 21 & 376. & 1034 & 1577 & 1755 & 2614 & 31107 & 3426 & 2560 & 2385 & 2091 & $128 \%$ & 523 & 53 & 0 & 0 & 23335 \\
\hline $\begin{array}{r}\text { MEAH } \\
30 \\
\text { IIUP }\end{array}$ & $\begin{array}{r}n \\
0 \\
31\end{array}$ & $\begin{array}{r}0 \\
0 \\
31\end{array}$ & $\begin{array}{l}27 \\
19 \\
310\end{array}$ & $\begin{array}{r}35 ! \\
120 \\
30\end{array}$ & $\begin{array}{r}1000 \\
2213 \\
29\end{array}$ & $\begin{array}{r}1512 \\
922 \\
27\end{array}$ & $\begin{array}{r}2052 \\
505 \\
24\end{array}$ & $\begin{array}{r}2146 \\
714 \\
30\end{array}$ & $\begin{array}{r}2040 \\
934 \\
30\end{array}$ & $\begin{array}{r}2600 \\
803 \\
30\end{array}$ & $\begin{array}{r}2438 \\
941 \\
31\end{array}$ & $\begin{array}{r}2181 \\
614 \\
311\end{array}$ & $\begin{array}{r}1685 \\
575 \\
30\end{array}$ & $\begin{array}{r}102: \\
510^{\circ} \\
30\end{array}$ & $\begin{array}{r}518 \\
240 \\
30\end{array}$ & $\begin{array}{r}104 \\
63 \\
30\end{array}$ & $\begin{array}{r}0 \\
0 \\
31\end{array}$ & $\begin{array}{r}0 \\
0 \\
31\end{array}$ & $\begin{array}{r}20848 \\
4647 \\
28\end{array}$ \\
\hline $\begin{array}{l}\text { MAX } \\
\text { MIN }\end{array}$ & $\begin{array}{l}0 \\
0\end{array}$ & $\begin{array}{l}0 \\
0\end{array}$ & $\begin{array}{r}75 \\
0\end{array}$ & $\begin{array}{r}560 \\
24\end{array}$ & $\begin{array}{r}1371 \\
104\end{array}$ & $\begin{array}{r}2037 \\
173\end{array}$ & $\begin{array}{r}27114 \\
11711\end{array}$ & $\begin{array}{r}31.79 \\
51 ?\end{array}$ & $\begin{array}{r}3476 \\
69\end{array}$ & $\begin{array}{r}3537 \\
210\end{array}$ & $\begin{array}{r}3495 \\
196\end{array}$ & $\begin{array}{r}3205 \\
704\end{array}$ & $\begin{array}{r}2451 \\
3.33\end{array}$ & $\begin{array}{r}1752 \\
106\end{array}$ & $\begin{array}{r}876 \\
2: 5\end{array}$ & $\begin{array}{r}253 \\
17\end{array}$ & $\begin{array}{l}0 \\
0\end{array}$ & $\begin{array}{l}0 \\
0\end{array}$ & $\begin{array}{r}27060 \\
5000\end{array}$ \\
\hline
\end{tabular}


CUASTAL - IMLAND SOLAR RADIATIUN DIFFEREHCE STUDY MOMTILY DATA SUMMARY

\begin{tabular}{|c|c|c|c|}
\hline $\begin{array}{l}\text { IMSTRUMEIIT = PSP } \# \| 1 \\
\text { SEHSITIVITY } 10.37 E-6 \text { V/M/SO, }\end{array}$ & WALLACE & AlJGiJS T & $\begin{array}{l}1978 \\
\text { IREND REMOVEO }\end{array}$ \\
\hline
\end{tabular}

ENERGY KILOJUULES PER SUUARE METER

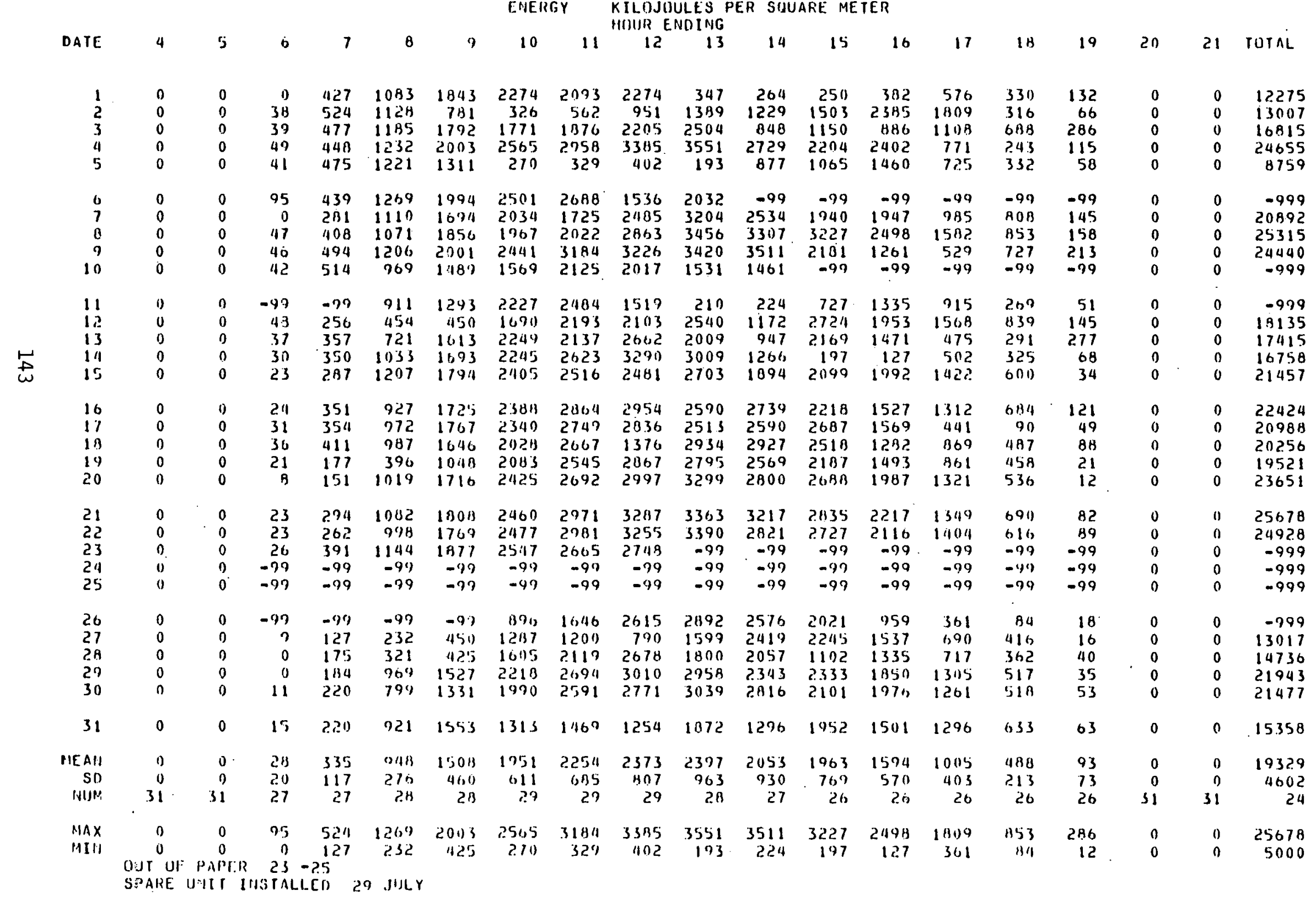




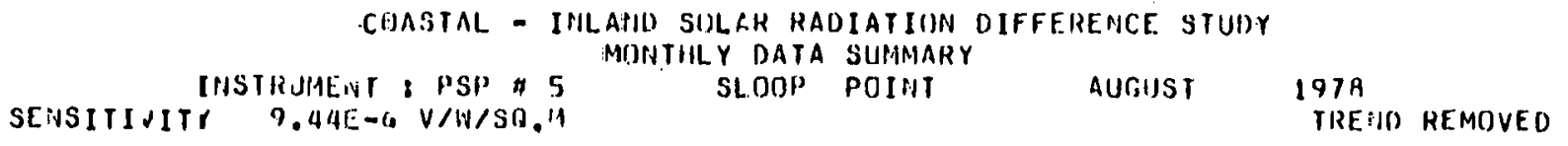

EIJERGY KILUJOULES PER SQUARE METER

DATE 400 H 500 HIIUR ENDIING

\begin{tabular}{|c|c|c|c|c|c|c|c|c|c|c|c|c|c|c|c|c|c|c|c|}
\hline DATE & 4 & 5 & 6 & 7 & B & $?$ & 10 & 11 & 12 & 13 & 14 & 15 & 16 & 17 & 19 & 19 & 20 & 21 & TOTAL \\
\hline $\begin{array}{l}1 \\
2 \\
3 \\
4 \\
5\end{array}$ & $\begin{array}{l}0 \\
0 \\
0 \\
0 . \\
0\end{array}$ & $\begin{array}{l}0 \\
0 \\
0 \\
0 \\
0\end{array}$ & $\begin{array}{l}87 \\
15 \\
45 \\
38 \\
45\end{array}$ & $\begin{array}{l}457 \\
453 \\
408 \\
472 \\
381\end{array}$ & $\begin{array}{r}1210 \\
499 \\
: 452 \\
: 292 \\
: 025\end{array}$ & $\begin{array}{r}1979 \\
545 \\
1967 \\
20197 \\
446\end{array}$ & $\begin{array}{r}2600 \\
938 \\
2609 \\
2673 \\
419\end{array}$ & $\begin{array}{r}3127 \\
1086 \\
3047 \\
3146 \\
312\end{array}$ & $\begin{array}{r}3401 \\
2246 \\
3352 \\
3374 \\
732\end{array}$ & $\begin{array}{l}3176 \\
2764 \\
2909 \\
31181 \\
1216\end{array}$ & $\begin{array}{l}3558 \\
1967 \\
1759 \\
3291 \\
1845\end{array}$ & $\begin{array}{l}1773 \\
2471 \\
2566 \\
2913 \\
978\end{array}$ & $\begin{array}{l}941 \\
1658 \\
2265 \\
2318 \\
1609\end{array}$ & $\begin{array}{r}561 \\
1753 \\
1273 \\
152.5 \\
691\end{array}$ & $\begin{array}{r}407 \\
1002 \\
774 \\
600 \\
114\end{array}$ & $\begin{array}{r}106 \\
297 \\
122 \\
68 \\
15\end{array}$ & $\begin{array}{l}0 \\
0 \\
0 \\
0 \\
0\end{array}$ & $\begin{array}{l}0 \\
0 \\
0 \\
0 \\
0\end{array}$ & $\begin{array}{r}23393 \\
17699 \\
24542 \\
27210 \\
9730\end{array}$ \\
\hline $\begin{array}{r}0 \\
7 \\
8 \\
9 \\
10\end{array}$ & $\begin{array}{l}0 \\
0 \\
0 \\
0 \\
0\end{array}$ & $\begin{array}{l}0 \\
0 \\
0 \\
0 \\
0\end{array}$ & $\begin{array}{l}49 \\
311 \\
41 \\
38 \\
26\end{array}$ & $\begin{array}{l}499 \\
537 \\
488 \\
488 \\
278\end{array}$ & $\begin{array}{l}0121 \\
0086 \\
1136 \\
1147 \\
896\end{array}$ & $\begin{array}{l}2002 \\
1705 \\
1986 \\
1602 \\
1540\end{array}$ & $\begin{array}{r}2612 \\
2593 \\
2669 \\
2284 \\
-94\end{array}$ & $\begin{array}{r}2909 \\
3069 \\
3092 \\
2856 \\
-99\end{array}$ & $\begin{array}{l}3317 \\
3336 \\
3359 \\
3382 \\
2276\end{array}$ & $\begin{array}{l}3451 \\
3397 \\
3455 \\
3348 \\
3718\end{array}$ & $\begin{array}{l}3130 \\
3226 \\
2879 \\
3184 \\
2822\end{array}$ & $\begin{array}{l}2707 \\
2841 \\
3073 \\
2863 \\
2047\end{array}$ & $\begin{array}{r}17083 \\
2271 \\
1544 \\
2242 \\
305\end{array}$ & $\begin{array}{r}1525 \\
1493 \\
1624 \\
1610 \\
33\end{array}$ & $\begin{array}{r}591 \\
785 \\
827 \\
655 \\
95\end{array}$ & $\begin{array}{r}87 \\
232 \\
163 \\
205 \\
61\end{array}$ & $\begin{array}{l}0 \\
0 \\
0 \\
0 \\
0\end{array}$ & $\begin{array}{l}0 \\
0 \\
0 \\
0 \\
0\end{array}$ & $\begin{array}{r}25788 \\
26693 \\
26386 \\
25970 \\
-999\end{array}$ \\
\hline $\begin{array}{l}11 \\
12 \\
13 \\
14 \\
15\end{array}$ & $\begin{array}{l}0 \\
0 \\
0 \\
0 \\
0\end{array}$ & $\begin{array}{l}0 \\
0 \\
0 \\
0 \\
0\end{array}$ & $\begin{array}{l}0 \\
0 \\
0 \\
0 \\
0\end{array}$ & $\begin{array}{r}183 \\
53 \\
186 \\
255 \\
29 ?\end{array}$ & $\begin{array}{r}613 \\
41 \\
522 \\
853 \\
907\end{array}$ & $\begin{array}{r}1654 \\
354 \\
1624 \\
1357 \\
1758\end{array}$ & $\begin{array}{l}2032 \\
1197 \\
2200 \\
1033 \\
2379\end{array}$ & $\begin{array}{l}366 \\
1777 \\
21394 \\
2730 \\
2623\end{array}$ & $\begin{array}{r}72 \\
1983 \\
3233 \\
3249 \\
3012\end{array}$ & $\begin{array}{r}163 \\
224 \\
3330 \\
3447 \\
3268\end{array}$ & $\begin{array}{r}141 \\
941 \\
3195 \\
2105 \\
2944\end{array}$ & $\begin{array}{r}591 \\
1922 \\
1998 \\
270 \\
2490\end{array}$ & $\begin{array}{r}1086 \\
1067 \\
-99 \\
-99 \\
1171\end{array}$ & $\begin{array}{r}915 \\
1191 \\
-97 \\
-49 \\
1093\end{array}$ & $\begin{array}{r}127 \\
1102 \\
232 \\
-99 \\
617\end{array}$ & $\begin{array}{l}118 \\
286 \\
133 \\
137 \\
175\end{array}$ & $\begin{array}{l}0 \\
0 \\
0 \\
0 \\
0\end{array}$ & $\begin{array}{l}0 \\
0 \\
0 \\
0 \\
0\end{array}$ & $\begin{array}{r}8439 \\
12338 \\
-999 \\
-997 \\
22703\end{array}$ \\
\hline $\begin{array}{l}10 \\
17 \\
18 \\
19 \\
2.10\end{array}$ & $\begin{array}{l}0 \\
0 \\
0 \\
0 \\
0\end{array}$ & $\begin{array}{l}0 \\
0 \\
0 \\
0 \\
0\end{array}$ & $\begin{array}{l}0 \\
0 \\
15 \\
15 \\
15\end{array}$ & $\begin{array}{l}308 \\
279 \\
247 \\
250 \\
301\end{array}$ & $\begin{array}{l}907 \\
938 \\
831 \\
919 \\
907\end{array}$ & $\begin{array}{r}1073 \\
1674 \\
91.1 \\
1016 \\
1579\end{array}$ & $\begin{array}{l}2307 \\
2319 \\
1773 \\
2196 \\
2211\end{array}$ & $\begin{array}{l}2922 \\
21102 \\
2188 \\
2513 \\
2799\end{array}$ & $\begin{array}{l}2825 \\
3184 \\
2947 \\
3161 \\
2970\end{array}$ & $\begin{array}{l}2852 \\
-883 \\
3123 \\
3115 \\
2802\end{array}$ & $\begin{array}{l}2188 \\
3058 \\
2802 \\
26118 \\
3054\end{array}$ & $\begin{array}{l}2551 \\
2345 \\
1666 \\
2009 \\
2560\end{array}$ & $\begin{array}{l}2141 \\
1513 \\
-8188 \\
1395 \\
1946\end{array}$ & $\begin{array}{r}1468 \\
812 \\
1052 \\
1029 \\
1240\end{array}$ & $\begin{array}{l}732 \\
2110 \\
587 \\
600 \\
602\end{array}$ & $\begin{array}{r}171 \\
45 \\
87 \\
130 \\
118\end{array}$ & $\begin{array}{l}0 \\
0 \\
0 \\
0 \\
0\end{array}$ & $\begin{array}{l}0 \\
0 \\
0 \\
0 \\
0\end{array}$ & $\begin{array}{r}22921 \\
-999 \\
=999 \\
21601 \\
23152\end{array}$ \\
\hline $\begin{array}{l}21 \\
22 \\
23 \\
24 \\
25\end{array}$ & $\begin{array}{l}0 \\
0 \\
0 \\
1) \\
0\end{array}$ & $\begin{array}{l}0 \\
0 \\
0 \\
0 \\
0\end{array}$ & $\begin{array}{l}19 \\
15 \\
19 \\
19 \\
11\end{array}$ & $\begin{array}{l}331 \\
331 \\
457 \\
366 \\
347\end{array}$ & $\begin{array}{r}991 \\
50100 \\
1204 \\
838 \\
949\end{array}$ & $\begin{array}{l}1792 \\
18.24 \\
1822 \\
2017 \\
1624\end{array}$ & $\begin{array}{l}2491 \\
24911 \\
2513 \\
2.490 \\
22116\end{array}$ & $\begin{array}{l}2970 \\
2970 \\
2994 \\
2978 \\
2753\end{array}$ & $\begin{array}{l}3241 \\
3245 \\
2917 \\
3272 \\
2974\end{array}$ & $\begin{array}{l}3325 \\
3321 \\
3249 \\
3325 \\
3043\end{array}$ & $\begin{array}{l}3127 \\
2791 \\
3222 \\
3127 \\
2312\end{array}$ & $\begin{array}{l}2802 \\
1437 \\
2760 \\
2749 \\
1780\end{array}$ & $\begin{array}{l}2215 \\
1126 \\
2162 \\
2120 \\
1133\end{array}$ & $\begin{array}{l}1502 \\
1390 \\
1372 \\
1380 \\
907\end{array}$ & $\begin{array}{l}678 \\
545 \\
644 \\
629 \\
278\end{array}$ & $\begin{array}{l}87 \\
61 \\
80 \\
83 \\
26\end{array}$ & $\begin{array}{l}0 \\
0 \\
0 \\
0 \\
0\end{array}$ & $\begin{array}{l}0 \\
0 \\
0 \\
0 \\
0\end{array}$ & $\begin{array}{l}25574 \\
23310 \\
25327 \\
25415 \\
21361\end{array}$ \\
\hline $\begin{array}{l}26 \\
27 \\
28 \\
29 \\
30\end{array}$ & $\begin{array}{l}0 \\
0 \\
0 \\
0 \\
0\end{array}$ & $\begin{array}{l}0 \\
u \\
0 \\
0 \\
0\end{array}$ & $\begin{array}{l}0 \\
0 \\
0 \\
0 \\
0\end{array}$ & $\begin{array}{l}232 \\
136 \\
122 \\
1136 \\
171\end{array}$ & $\begin{array}{l}854 \\
171 \\
674 \\
419 \\
827\end{array}$ & $\begin{array}{r}14.3 \\
266 \\
1502 \\
1559 \\
1591\end{array}$ & $\begin{array}{l}17911 \\
508 \\
21011 \\
21155 \\
23011\end{array}$ & $\begin{array}{l}2108 \\
1147 \\
2650 \\
2246 \\
21352\end{array}$ & $\begin{array}{l}1952 \\
2064 \\
2651 \\
2734 \\
3131\end{array}$ & $\begin{array}{l}2696 \\
2825 \\
3146 \\
31031 \\
3325\end{array}$ & $\begin{array}{l}2608 \\
2539 \\
2879 \\
21549 \\
2675\end{array}$ & $\begin{array}{r}-99 \\
2494 \\
2494 \\
2272 \\
2238\end{array}$ & $\begin{array}{l}-99 \\
1799 \\
1777 \\
1803 \\
1788\end{array}$ & $\begin{array}{l}-90 \\
1124 \\
1224 \\
1220 \\
1430\end{array}$ & $\begin{array}{l}-40 \\
305 \\
579 \\
57 ? \\
66 ?\end{array}$ & $\begin{array}{r}-99 \\
41 \\
64 \\
83 \\
95\end{array}$ & $\begin{array}{l}0 \\
0 \\
0 \\
0 \\
0\end{array}$ & $\begin{array}{l}0 \\
0 \\
0 \\
0 \\
0\end{array}$ & $\begin{array}{r}-999 \\
15527 \\
21877 \\
21424 \\
23360\end{array}$ \\
\hline 31 & 0 & ) & $n$ & 2711 & 873 & 10117 & 2108 & 26,73 & 31121 & 3115 & 2921 & 2608 & 2013 & 1238 & -99 & -99 & 0 & 0 & -999 \\
\hline $\begin{array}{r}\text { HEAHI } \\
\text { SD } \\
\text { HUN? }\end{array}$ & $\begin{array}{r}0 \\
0 \\
31\end{array}$ & $\begin{array}{r}11 . \\
0 \\
31\end{array}$ & $\begin{array}{l}17 \\
20 \\
31\end{array}$ & $\begin{array}{r}316 \\
123 \\
31\end{array}$ & $\begin{array}{r}891 \\
293 \\
31\end{array}$ & $\begin{array}{r}1521 \\
4516 \\
31\end{array}$ & $\begin{array}{r}2076 \\
611 \\
30\end{array}$ & $\begin{array}{r}2402 \\
704 \\
30\end{array}$ & $\begin{array}{r}27174 \\
762 \\
31\end{array}$ & $\begin{array}{r}2121 \\
844 \\
30\end{array}$ & $\begin{array}{r}2667 \\
710 \\
31\end{array}$ & $\begin{array}{r}220.3 \\
679 \\
30\end{array}$ & $\begin{array}{r}1710 \\
4830 \\
27\end{array}$ & $\begin{array}{r}1217 \\
565 \\
28\end{array}$ & $\begin{array}{r}569 \\
233 \\
28\end{array}$ & $\begin{array}{r}114 \\
69 \\
29\end{array}$ & $\begin{array}{r}0 \\
0 \\
31\end{array}$ & $\begin{array}{r}0 \\
0 \\
31\end{array}$ & $\begin{array}{r}21739 \\
5148 \\
24\end{array}$ \\
\hline $\begin{array}{l}\operatorname{Mnx} \\
\text { MIN }\end{array}$ & $\begin{array}{l}1) \\
0\end{array}$ & $\begin{array}{l}0 \\
0\end{array}$ & $\begin{array}{r}87 \\
0\end{array}$ & $\begin{array}{r}53 i \\
53\end{array}$ & $\begin{array}{r}1452 \\
11\end{array}$ & $\begin{array}{r}2011] \\
? 0.6)\end{array}$ & $\begin{array}{r}2673 \\
119\end{array}$ & $\begin{array}{r}3140 \\
312\end{array}$ & $\begin{array}{r}3401 \\
72\end{array}$ & $\begin{array}{r}3718 \\
163\end{array}$ & $\begin{array}{r}3558 \\
144\end{array}$ & $\begin{array}{r}3073 \\
270\end{array}$ & $\begin{array}{r}231 \% \\
3115\end{array}$ & $\begin{array}{r}1758 \\
30\end{array}$ & $\begin{array}{r}1102 \\
75\end{array}$ & $\begin{array}{r}297 \\
15\end{array}$ & $\begin{array}{l}0 \\
0\end{array}$ & $\begin{array}{l}0 \\
0\end{array}$ & $\begin{array}{r}27210 \\
5000\end{array}$ \\
\hline
\end{tabular}


CUASTAL - INLAIID 3ILLAI? RADIATIIIN DIFFERENCE STUNY MUHTHLY DATA SUMMARY

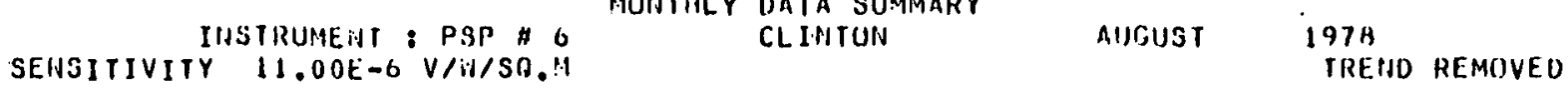

EHERTY KILUJOULES PER SOUARE. METER

\begin{tabular}{|c|c|c|c|c|c|c|c|c|c|c|c|c|c|c|c|c|c|c|c|}
\hline & & & & & & & ENE & & $\begin{array}{l}\text { KILOJ } \\
\text { HIOUR }\end{array}$ & $\begin{array}{l}\text { ULES } \\
\text { ND) I HG }\end{array}$ & ER SO & ARt. MA & TER & & & & & & \\
\hline DATE & 4 & 5 & 6 & 7 & B & 9 & 10 & 11 & 12 & 13 & 14 & 15 & 16 & 17 & IB & 19 & 20 & 21 & TOTAL \\
\hline $\begin{array}{l}1 \\
2 \\
3 \\
4 \\
5\end{array}$ & $\begin{array}{l}0 \\
0 \\
0 \\
0 \\
0\end{array}$ & $\begin{array}{l}0 \\
0 \\
0 \\
0 \\
0\end{array}$ & $\begin{array}{l}47 \\
52 \\
115 \\
58 \\
62\end{array}$ & $\begin{array}{l}379 \\
464 \\
435 \\
435 \\
520\end{array}$ & $\begin{array}{r}1086 \\
549 \\
1129 \\
1196 \\
1151\end{array}$ & $\begin{array}{l}1786 \\
1647 \\
1521 \\
1149 \\
1672\end{array}$ & $\begin{array}{l}2101 \\
2490 \\
1513 \\
26191 \\
1711\end{array}$ & $\begin{array}{r}2526 \\
295 A \\
3174 \\
2037 \\
448\end{array}$ & $\begin{array}{r}2372 \\
1273 \\
2362 \\
3452 \\
935\end{array}$ & $\begin{array}{r}370 \\
1957 \\
3125 \\
3164 \\
824\end{array}$ & $\begin{array}{r}294 \\
1927 \\
3245 \\
2140 \\
464\end{array}$ & $\begin{array}{r}297 \\
2018 \\
2686 \\
2277 \\
1613\end{array}$ & $\begin{array}{r}634 \\
1358 \\
2359 \\
595 \\
1606\end{array}$ & $\begin{array}{r}726 \\
373 \\
1142 \\
392 \\
1014\end{array}$ & $\begin{array}{r}467 \\
98 \\
592 \\
317 \\
258\end{array}$ & $\begin{array}{r}157 \\
58 \\
163 \\
124 \\
68\end{array}$ & $\begin{array}{l}0 \\
0 \\
0 \\
0 \\
0\end{array}$ & $\begin{array}{l}0 \\
0 \\
0 \\
0 \\
0\end{array}$ & $\begin{array}{l}13250 \\
17024 \\
23611 \\
20934 \\
12346\end{array}$ \\
\hline $\begin{array}{r}6 \\
7 \\
8 \\
9 \\
10\end{array}$ & $\begin{array}{l}0 \\
0 \\
0 \\
0 \\
0\end{array}$ & $\begin{array}{l}0 \\
0 \\
0 \\
0 \\
0\end{array}$ & $\begin{array}{l}31 \\
17 \\
32 \\
32 \\
42\end{array}$ & $\begin{array}{l}283 \\
359 \\
474 \\
389 \\
359\end{array}$ & $\begin{array}{r}725 \\
1201 \\
1197 \\
1043 \\
755\end{array}$ & $\begin{array}{l}1713 \\
1767 \\
1901 \\
1626 \\
762\end{array}$ & $\begin{array}{r}2293 \\
1930 \\
2660 \\
2097 \\
850\end{array}$ & $\begin{array}{l}1795 \\
3125 \\
3779 \\
2922 \\
1259\end{array}$ & $\begin{array}{l}241 \\
2346 \\
3131 \\
3501 \\
1606\end{array}$ & $\begin{array}{r}-99 \\
36,35 \\
3413 \\
2732 \\
1865\end{array}$ & $\begin{array}{r}-99 \\
1099 \\
3269 \\
2438 \\
2847\end{array}$ & $\begin{array}{r}-99 \\
2392 \\
2.499 \\
2978 \\
242\end{array}$ & $\begin{array}{r}-99 \\
2421 \\
2317 \\
16138 \\
29\end{array}$ & $\begin{array}{r}-99 \\
726 \\
1613 \\
1502 \\
467\end{array}$ & $\begin{array}{l}-79 \\
323 \\
427 \\
713 \\
297\end{array}$ & $\begin{array}{l}-99 \\
117 \\
199 \\
222 \\
101\end{array}$ & $\begin{array}{l}0 \\
0 \\
0 \\
0 \\
0\end{array}$ & $\begin{array}{l}0 \\
0 \\
0 \\
0 \\
0\end{array}$ & $\begin{array}{r}-999 \\
21460 \\
27211 \\
23920 \\
11471\end{array}$ \\
\hline $\begin{array}{l}11 \\
12 \\
13 \\
14 \\
15\end{array}$ & $\begin{array}{l}0 \\
0 \\
0 \\
0 \\
0\end{array}$ & $\begin{array}{l}0 \\
0 \\
0 \\
0 \\
0\end{array}$ & $\begin{array}{r}0 \\
16 \\
32 \\
16 \\
8\end{array}$ & $\begin{array}{l}153 \\
147 \\
304 \\
304 \\
192\end{array}$ & $\begin{array}{l}654 \\
420 \\
1664 \\
641 \\
656\end{array}$ & $\begin{array}{r}1217 \\
399 \\
922 \\
1001 \\
1101\end{array}$ & $\begin{array}{l}1322 \\
1181 \\
1315 \\
2254 \\
2257\end{array}$ & $\begin{array}{l}2173 \\
1809 \\
2572 \\
2857 \\
2640\end{array}$ & $\begin{array}{l}2830 \\
2997 \\
3030 \\
3066 \\
3117\end{array}$ & $\begin{array}{l}2078 \\
2444 \\
3050 \\
3001 \\
2741\end{array}$ & $\begin{array}{r}9138 \\
2621 \\
2261 \\
3158 \\
21169\end{array}$ & $\begin{array}{l}2382 \\
2440 \\
2470 \\
1201 \\
18711\end{array}$ & $\begin{array}{r}19177 \\
1531 \\
1158 \\
281 \\
1890\end{array}$ & $\begin{array}{r}932 \\
1439 \\
618 \\
225 \\
1489\end{array}$ & $\begin{array}{l}307 \\
503 \\
334 \\
284 \\
745\end{array}$ & $\begin{array}{r}45 \\
127 \\
199 \\
81 \\
1.59\end{array}$ & $\begin{array}{l}0 \\
0 \\
0 \\
0 \\
0\end{array}$ & $\begin{array}{l}0 \\
0 \\
0 \\
0 \\
0\end{array}$ & $\begin{array}{l}17044 \\
18485 \\
19629 \\
19376 \\
21400\end{array}$ \\
\hline $\begin{array}{l}16 \\
17 \\
18 \\
19 \\
20\end{array}$ & $\begin{array}{l}0 \\
0 \\
0 \\
0 \\
0\end{array}$ & $\begin{array}{l}0 \\
0 \\
0 \\
0 \\
0\end{array}$ & $\begin{array}{l}16 \\
19 \\
19 \\
12 \\
13\end{array}$ & $\begin{array}{l}278 \\
346 \\
261 \\
175 \\
320\end{array}$ & $\begin{array}{l}945 \\
955 \\
870 \\
510 \\
3 ? 1\end{array}$ & $\begin{array}{l}1695 \\
1672 \\
1620 \\
1116 \\
1662\end{array}$ & $\begin{array}{l}2382 \\
2281 \\
2267 \\
2178 \\
1960\end{array}$ & $\begin{array}{l}28189 \\
2307 \\
2755 \\
2157 \\
2389\end{array}$ & $\begin{array}{l}2937 \\
3184 \\
2994 \\
2823 \\
2981\end{array}$ & $\begin{array}{l}3122 \\
3053 \\
2690 \\
2914 \\
3259\end{array}$ & $\begin{array}{l}3046 \\
2935 \\
2713 \\
2692 \\
1917\end{array}$ & $\begin{array}{l}2431 \\
1055 \\
2497 \\
18114 \\
2536\end{array}$ & $\begin{array}{r}1832 \\
497 \\
1950 \\
1530 \\
1060\end{array}$ & $\begin{array}{r}1318 \\
39 \\
1217 \\
862 \\
1165\end{array}$ & $\begin{array}{r}785 \\
98 \\
657 \\
254 \\
287\end{array}$ & $\begin{array}{r}170 \\
222 \\
111 \\
80 \\
-99\end{array}$ & $\begin{array}{l}0 \\
0 \\
0 \\
0 \\
0\end{array}$ & $\begin{array}{l}0 \\
0 \\
n \\
0 \\
0\end{array}$ & $\begin{array}{r}23786 \\
19963 \\
22627 \\
191148 \\
-999\end{array}$ \\
\hline $\begin{array}{l}21 \\
22 \\
23 \\
24 \\
25\end{array}$ & $\begin{array}{l}0 \\
0 \\
0 \\
0 \\
0\end{array}$ & $\begin{array}{l}1) \\
0 \\
0 \\
0 \\
0\end{array}$ & $\begin{array}{l}0 \\
16 \\
16 \\
16 \\
13\end{array}$ & $\begin{array}{l}368 \\
359 \\
297 \\
312 \\
255\end{array}$ & $\begin{array}{l}106 ? \\
1053 \\
1050 \\
939 \\
863\end{array}$ & $\begin{array}{l}1795 \\
1799 \\
18.34 \\
1100 \\
159.1\end{array}$ & $\begin{array}{l}2476 \\
2408 \\
2.611 \\
2451 \\
22211\end{array}$ & $\begin{array}{l}2796 \\
2984 \\
2824 \\
2935 \\
2699\end{array}$ & $\begin{array}{l}3340 \\
3209 \\
2961 \\
3273 \\
29118\end{array}$ & $\begin{array}{l}3428 \\
3331 \\
3253 \\
3472 \\
2987\end{array}$ & $\begin{array}{l}3291 \\
3161 \\
3102 \\
3092 \\
2663\end{array}$ & $\begin{array}{l}2872 \\
2735 \\
2758 \\
2287 \\
2454\end{array}$ & $\begin{array}{l}2224 \\
2065 \\
2159 \\
2.1968 \\
1719\end{array}$ & $\begin{array}{l}1278 \\
1426 \\
1423 \\
1374 \\
1037\end{array}$ & $\begin{array}{l}67 h \\
651 \\
651 \\
628 \\
431\end{array}$ & $\begin{array}{r}110 \\
85 \\
85 \\
81 \\
65\end{array}$ & $\begin{array}{l}0 \\
0 \\
0 \\
0 \\
0\end{array}$ & $\begin{array}{l}0 \\
0 \\
0 \\
0 \\
0\end{array}$ & $\begin{array}{l}25919 \\
25345 \\
25032 \\
24328 \\
21915\end{array}$ \\
\hline $\begin{array}{l}26 \\
27 \\
28 \\
29 \\
30\end{array}$ & $\begin{array}{l}0 \\
0 \\
0 \\
0 \\
0\end{array}$ & $\begin{array}{l}0 \\
0 \\
0 \\
0 \\
0\end{array}$ & $\begin{array}{l}0 \\
? \\
8 \\
0 \\
0\end{array}$ & $\begin{array}{l}206 \\
1133 \\
136 \\
2115 \\
278\end{array}$ & $\begin{array}{l}306 \\
451 \\
421 \\
854 \\
B 13 i)\end{array}$ & $\begin{array}{r}782 \\
890 \\
1301 \\
1593 \\
1525\end{array}$ & $\begin{array}{l}1313 \\
1073 \\
1936 \\
2284 \\
22213\end{array}$ & $\begin{array}{l}2647 \\
1875 \\
2545 \\
2794 \\
2241\end{array}$ & $\begin{array}{l}2945 \\
1645 \\
2947 \\
3089 \\
2621\end{array}$ & $\begin{array}{l}1623 \\
2719 \\
2083 \\
3145 \\
3253\end{array}$ & $\begin{array}{l}1407 \\
2533 \\
1723 \\
28199 \\
21363\end{array}$ & $\begin{array}{l}2032 \\
1734 \\
1880 \\
2310 \\
2.461\end{array}$ & $\begin{array}{r}1446 \\
690 \\
1556 \\
1907 \\
2025\end{array}$ & $\begin{array}{r}148 \\
926 \\
663 \\
1117 \\
1161\end{array}$ & $\begin{array}{l}170 \\
373 \\
185 \\
330 \\
404\end{array}$ & $\begin{array}{l}0 \\
49 \\
67 \\
26 \\
52\end{array}$ & $\begin{array}{l}0 \\
0 \\
0 \\
0 \\
0\end{array}$ & $\begin{array}{l}0 \\
0 \\
0 \\
0 \\
0\end{array}$ & $\begin{array}{l}15805 \\
15200 \\
17451 \\
22653 \\
22049\end{array}$ \\
\hline 31 & 0 & 0 & 9 & 202 & 618 & 1011 & 1423 & 2254 & 2719 & 1155 & 2709 & 22131 & 1322 & 1155 & 618 & 52 & 0 & 0 & 17528 \\
\hline $\begin{array}{r}\text { HEAH } \\
\text { SD } \\
\text { HIIM }\end{array}$ & $\begin{array}{r}0 \\
0 \\
31\end{array}$ & $\begin{array}{r}0 \\
0 \\
31\end{array}$ & $\begin{array}{l}21 \\
17 \\
31\end{array}$ & $\begin{array}{r}306 \\
101 \\
31\end{array}$ & $\begin{array}{r}\text { A2. } \\
255 \\
31\end{array}$ & $\begin{array}{r}1123 \\
376 \\
31\end{array}$ & $\begin{array}{r}2020 \\
4830 \\
31\end{array}$ & $\begin{array}{r}2521 \\
5 \% 0 \\
31\end{array}$ & $\begin{array}{r}2673 \\
751 \\
31\end{array}$ & $\begin{array}{r}2603 \\
807 \\
30\end{array}$ & $\begin{array}{r}2401 \\
319 \\
30\end{array}$ & $\begin{array}{r}221 j 4 \\
6 \rightarrow H \\
30\end{array}$ & $\begin{array}{r}1527 \\
630 \\
30\end{array}$ & $\begin{array}{r}977 \\
417 \\
311\end{array}$ & $\begin{array}{r}160 \\
219 \\
30\end{array}$ & $\begin{array}{r}106 \\
58 \\
29\end{array}$ & $\begin{array}{r}0 \\
30\end{array}$ & $\begin{array}{r}0 \\
0 \\
31\end{array}$ & $\begin{array}{r}20210 \\
4052 \\
29\end{array}$ \\
\hline $\begin{array}{l}\text { MAX } \\
\text { UIN }\end{array}$ & $\begin{array}{l}0 \\
0\end{array}$ & $\begin{array}{l}0 \\
0\end{array}$ & $\begin{array}{r}62 \\
1)\end{array}$ & $\begin{array}{l}.320 \\
136\end{array}$ & $\begin{array}{r}1301 \\
36,6\end{array}$ & $\begin{array}{r}1901 \\
390\end{array}$ & $\begin{array}{r}\text { ?inkl } \\
\text { U'jo) }\end{array}$ & $\begin{array}{r}3.79 \\
1148\end{array}$ & $\begin{array}{r}35013 \\
2111\end{array}$ & $\begin{array}{r}3635 \\
376\end{array}$ & $\begin{array}{r}3291 \\
294\end{array}$ & $\begin{array}{r}29713 \\
2.4 ?\end{array}$ & $\begin{array}{r}2421 \\
3 ? 9\end{array}$ & $\begin{array}{r}1613 \\
39\end{array}$ & $\begin{array}{r}9.34 \\
98\end{array}$ & $\begin{array}{r}222 \\
0\end{array}$ & $\begin{array}{l}0 \\
0\end{array}$ & $\begin{array}{l}0 \\
0\end{array}$ & $\begin{array}{r}27211 \\
5000\end{array}$ \\
\hline
\end{tabular}


CIJASIAL - INLAID SIJLAR RADIATION DIFFERENCE STUOY IISSTKUMEIT : PSP 47 MJNTHLY DATA SUMNAKY

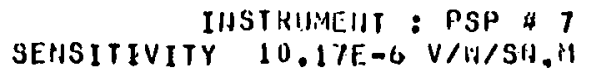
AUGUST

1978

EMERGY KILUJOULES PER SOJUARE METER

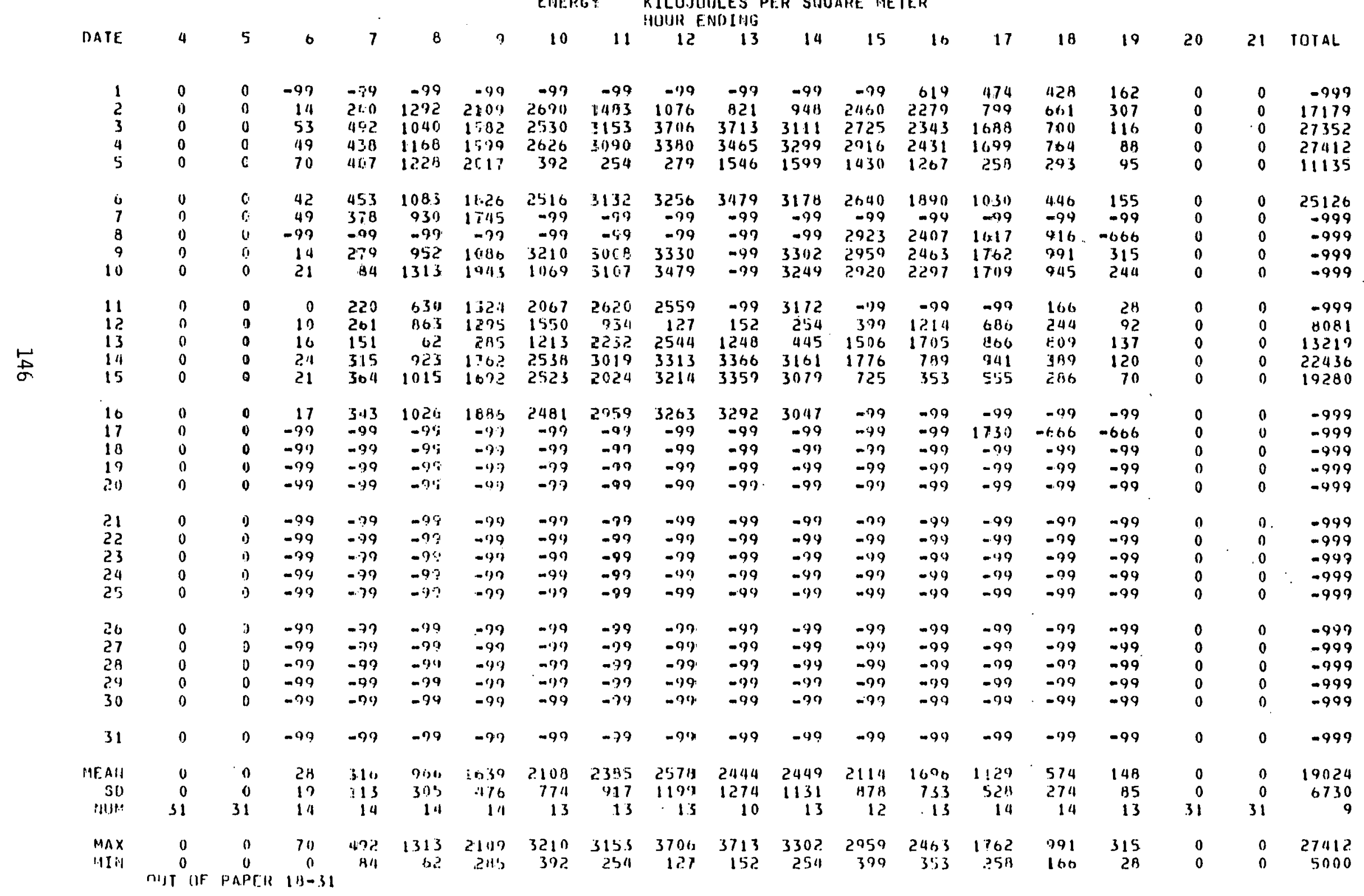


COASTAL - INLAND SILLAR RAUIATIUN OIFFERENCE STUDY MONTILLY DATA SIJMMARY

INISTRUIIENT: HIP A 8
SEIISITIVITY $\$, 70 E-6$ V/HISO.

F.HERGY KILUJOULES PER SIJUARE RETER

E.NERGY KILUJOULES PER SIJUARE HETER
HOUR ENOIHG

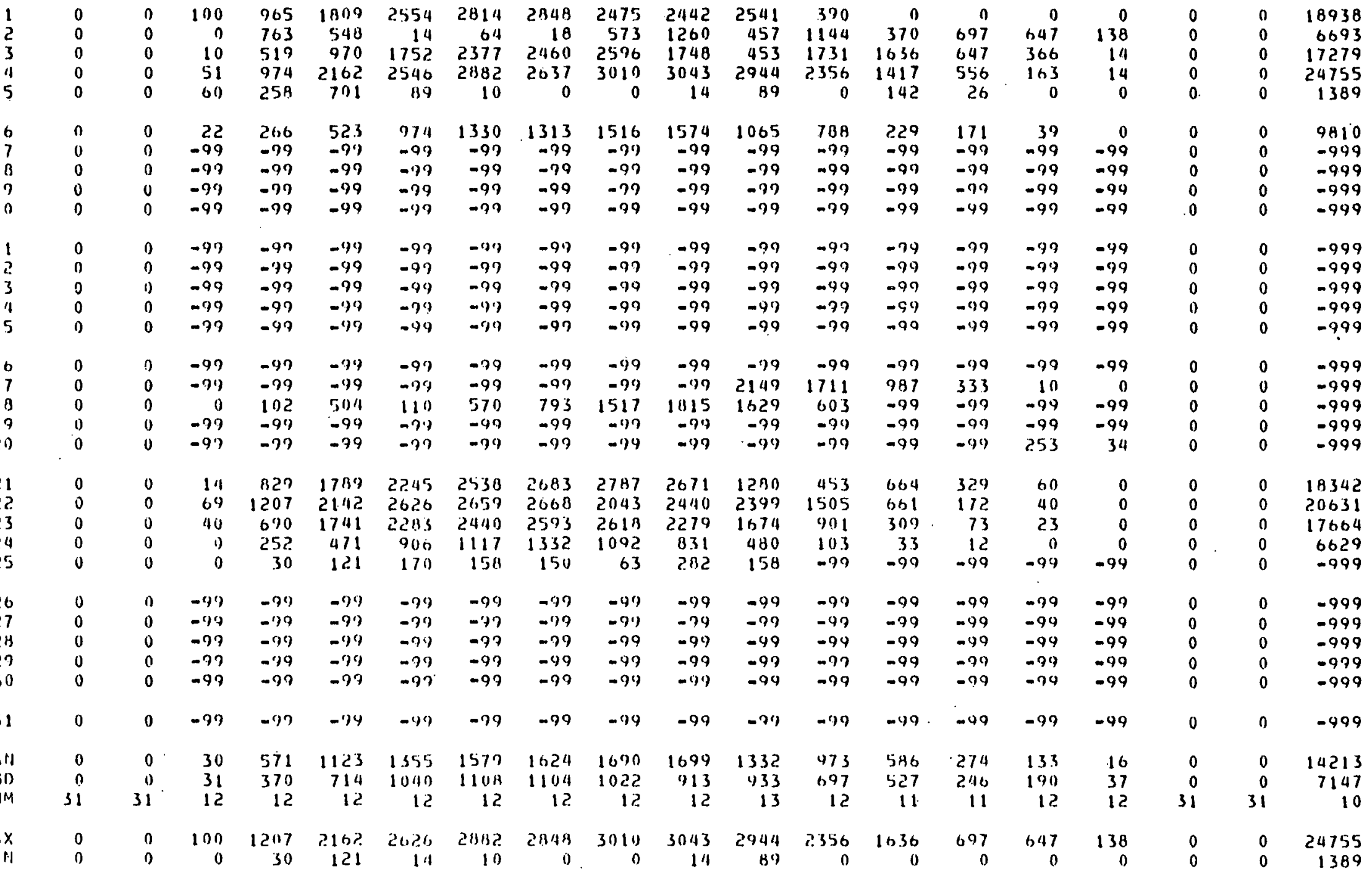

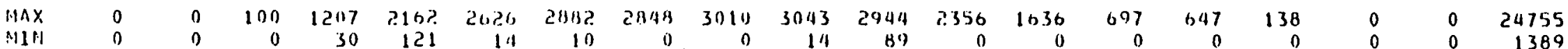


COASTAL - INI. AIJD SOLAR RAOIATIINN DIFFERENCE STULY LHSIRUHEMT: HIP \#10 MONTHLY DATA SUMMARY
SEHSITIVITY H.29E-6 V/H/SO.H

ENFRGY KILUJOULES PER SHUARE METER

\begin{tabular}{|c|c|c|c|c|c|c|c|c|c|c|c|c|c|c|c|c|c|c|c|}
\hline \multicolumn{20}{|c|}{ HOUR ENDIIJG } \\
\hline DATE & 4 & 5 & 6 & 7 & B & 9 & 10 & 11 & 12 & 13 & 14 & 15 & 16 & 17 & 18 & 19 & 20 & 21 & TUTAL \\
\hline $\begin{array}{l}1 \\
2 \\
3 \\
4 \\
5\end{array}$ & $\begin{array}{l}0 \\
0 \\
0 \\
0 \\
0\end{array}$ & $\begin{array}{l}0 \\
0 \\
0 \\
0 \\
0\end{array}$ & $\begin{array}{r}26 \\
0 \\
26 \\
0 \\
69\end{array}$ & $\begin{array}{r}356 \\
616 \\
603 \\
56 \\
955\end{array}$ & $\begin{array}{r}1198 \\
334 \\
1693 \\
1411 \\
1715\end{array}$ & $\begin{array}{r}1590 \\
790 \\
1498 \\
1654 \\
1619\end{array}$ & $\begin{array}{r}933 \\
1836 \\
555 \\
2583 \\
1103\end{array}$ & $\begin{array}{r}1124 \\
2023 \\
2366 \\
24: 23 \\
0\end{array}$ & $\begin{array}{r}790 \\
43 \\
1011 \\
2844 \\
0\end{array}$ & $\begin{array}{r}43 \\
321 \\
1524 \\
2590 \\
0\end{array}$ & $\begin{array}{r}0 \\
308 \\
2114 \\
1311 \\
0\end{array}$ & $\begin{array}{r}0 \\
1363 \\
1910 \\
1758 \\
208\end{array}$ & $\begin{array}{r}0 \\
594 \\
2058 \\
208 \\
182\end{array}$ & $\begin{array}{r}0 \\
0 \\
877 \\
0 \\
8 ?\end{array}$ & $\begin{array}{r}0 \\
0 \\
178 \\
0 \\
0\end{array}$ & $\begin{array}{r}0 \\
0 \\
26 \\
0 \\
0\end{array}$ & $\begin{array}{l}0 \\
0 \\
0 \\
0 \\
0\end{array}$ & $\begin{array}{l}0 \\
0 \\
0 \\
0 \\
0\end{array}$ & $\begin{array}{r}6046 \\
8228 \\
16439 \\
16844 \\
5933\end{array}$ \\
\hline $\begin{array}{r}6 \\
7 \\
8 \\
9 \\
10\end{array}$ & $\begin{array}{l}0 \\
0 \\
0 \\
0 \\
0\end{array}$ & $\begin{array}{l}0 \\
n \\
0 \\
0 \\
0\end{array}$ & $\begin{array}{l}0 \\
21 \\
13 \\
34 \\
13\end{array}$ & $\begin{array}{r}13 \\
238 \\
946 \\
425 \\
260\end{array}$ & $\begin{array}{r}521 \\
1815 \\
1919 \\
1828 \\
277\end{array}$ & $\begin{array}{r}1928 \\
1558 \\
2400 \\
1703 \\
0\end{array}$ & $\begin{array}{r}1545 \\
1278 \\
2688 \\
1741 \\
0\end{array}$ & $\begin{array}{r}937 \\
2523 \\
2740 \\
2219 \\
13\end{array}$ & $\begin{array}{r}-99 \\
1354 \\
2479 \\
2405 \\
21\end{array}$ & $\begin{array}{r}-99 \\
3087 \\
2818 \\
1862 \\
217\end{array}$ & $\begin{array}{r}-99 \\
247 \\
2879 \\
1702 \\
1281\end{array}$ & $\begin{array}{r}-99 \\
1632 \\
21000 \\
2 H ? 2 \\
0\end{array}$ & $\begin{array}{r}-99 \\
1919 \\
2601 \\
1459 \\
0\end{array}$ & $\begin{array}{r}-59 \\
347 \\
2253 \\
1706 \\
0\end{array}$ & $\begin{array}{r}-99 \\
134 \\
1050 \\
961 \\
0\end{array}$ & $\begin{array}{r}-99 \\
43 \\
95 \\
447 \\
0\end{array}$ & $\begin{array}{l}0 \\
0 \\
0 \\
0 \\
0\end{array}$ & $\begin{array}{l}0 \\
0 \\
0 \\
0 \\
0\end{array}$ & $\begin{array}{r}-999 \\
16216 \\
27721 \\
21977 \\
2082\end{array}$ \\
\hline $\begin{array}{l}11 \\
12 \\
13 \\
14 \\
15\end{array}$ & $\begin{array}{l}0 \\
0 \\
0 \\
0 \\
0\end{array}$ & $\begin{array}{l}0 \\
0 \\
0 \\
0 \\
0\end{array}$ & $\begin{array}{r}0 \\
0 \\
13 \\
0 \\
0\end{array}$ & $\begin{array}{r}0 \\
0 \\
65 \\
351 \\
0\end{array}$ & $\begin{array}{r}21 \\
0 \\
10 \\
4+12 \\
17\end{array}$ & $\begin{array}{r}2 \pi 2 \\
0 \\
39 \\
104 \\
321\end{array}$ & $\begin{array}{r}125 \\
490 \\
43 \\
1558 \\
1285\end{array}$ & $\begin{array}{r}7911 \\
63.9 \\
1007 \\
2.240 \\
1849\end{array}$ & $\begin{array}{l}1094 \\
1515 \\
1454 \\
2019 \\
2314\end{array}$ & $\begin{array}{l}1013 \\
1341 \\
1906 \\
1589 \\
1671\end{array}$ & $\begin{array}{r}60 \\
1745 \\
1372 \\
2197 \\
1307\end{array}$ & $\begin{array}{r}1142 \\
2093 \\
2531 \\
234 \\
977\end{array}$ & $\begin{array}{r}1159 \\
1294 \\
503 \\
0 \\
1320\end{array}$ & $\begin{array}{r}06 \\
1524 \\
t 0 \\
0 \\
1024\end{array}$ & $\begin{array}{r}0 \\
625 \\
1237 \\
0 \\
1103\end{array}$ & $\begin{array}{r}0 \\
186 \\
395 \\
0 \\
125\end{array}$ & $\begin{array}{l}0 \\
0 \\
0 \\
0 \\
0\end{array}$ & $\begin{array}{l}0 \\
0 \\
0 \\
0 \\
0\end{array}$ & $\begin{array}{r}4801 \\
11400 \\
10630 \\
10734 \\
13917\end{array}$ \\
\hline $\begin{array}{l}16 \\
17 \\
18 \\
17 \\
20\end{array}$ & $\begin{array}{l}0 \\
0 \\
0 \\
0 \\
0\end{array}$ & $\begin{array}{l}0 \\
0 \\
0 \\
0 \\
0\end{array}$ & $\begin{array}{r}0 \\
17 \\
0 \\
0 \\
0\end{array}$ & $\begin{array}{r}221 \\
042 \\
112 \\
0 \\
260\end{array}$ & $\begin{array}{r}11131 \\
1333 \\
916 \\
4 \\
945\end{array}$ & $\begin{array}{r}1845 \\
1797 \\
1520 \\
805 \\
1919\end{array}$ & $\begin{array}{l}2281 \\
1910 \\
1771 \\
1689 \\
1558\end{array}$ & $\begin{array}{l}23.92 \\
2106 \\
1706 \\
1298 \\
1100\end{array}$ & $\begin{array}{l}1750 \\
2331 \\
1858 \\
1606 \\
2114\end{array}$ & $\begin{array}{l}2106 \\
2006 \\
1321 \\
1684 \\
2297\end{array}$ & $\begin{array}{l}2314 \\
2093 \\
1632 \\
1485 \\
1003\end{array}$ & $\begin{array}{r}1771 \\
2020 \\
1602 \\
920 \\
1119\end{array}$ & $\begin{array}{r}1550 \\
0 \\
1415 \\
977 \\
512\end{array}$ & $\begin{array}{r}1611 h \\
0 \\
n 46 \\
340 \\
1220\end{array}$ & $\begin{array}{r}1589 \\
0 \\
538 \\
21 \\
143\end{array}$ & $\begin{array}{r}421 \\
238 \\
86 \\
0 \\
0\end{array}$ & $\begin{array}{l}0 \\
0 \\
0 \\
0 \\
0\end{array}$ & $\begin{array}{l}0 \\
0 \\
0 \\
0 \\
0\end{array}$ & $\begin{array}{l}20838 \\
15193 \\
15638 \\
10925 \\
15310\end{array}$ \\
\hline $\begin{array}{l}21 \\
22 \\
23 \\
24 \\
25\end{array}$ & $\begin{array}{l}0 \\
0 \\
0 \\
0 \\
0\end{array}$ & $\begin{array}{l}0 \\
0 \\
0 \\
0 \\
0\end{array}$ & $\begin{array}{r}0 \\
13 \\
0 \\
0 \\
0\end{array}$ & $\begin{array}{l}725 \\
768 \\
121 \\
508 \\
160\end{array}$ & $\begin{array}{l}1732 \\
1703 \\
1871 \\
1715 \\
7112\end{array}$ & $\begin{array}{l}2153 \\
2353 \\
2001 \\
2531 \\
1.103\end{array}$ & $\begin{array}{l}2531 \\
2518 \\
2606 \\
26109 \\
1537\end{array}$ & $\begin{array}{l}2766 \\
2709 \\
22811 \\
2696 \\
16513\end{array}$ & $\begin{array}{l}3065 \\
2932 \\
2336 \\
2580 \\
1589\end{array}$ & $\begin{array}{l}3222 \\
2783 \\
2701 \\
2835 \\
1619\end{array}$ & $\begin{array}{l}3365 \\
2718 \\
2710 \\
2579 \\
1294\end{array}$ & $\begin{array}{l}3243 \\
2073 \\
2061 \\
1917 \\
1050\end{array}$ & $\begin{array}{r}2892 \\
2327 \\
2648 \\
2353 \\
985\end{array}$ & $\begin{array}{r}1637 \\
2077 \\
2232 \\
2146 \\
594\end{array}$ & $\begin{array}{r}1558 \\
1320 \\
1511 \\
1446 \\
277\end{array}$ & $\begin{array}{r}173 \\
138 \\
286 \\
351 \\
26\end{array}$ & $\begin{array}{l}0 \\
0 \\
0 \\
0 \\
0\end{array}$ & $\begin{array}{l}0 \\
0 \\
0 \\
0 \\
0\end{array}$ & $\begin{array}{l}29362 \\
27004 \\
26858 \\
25996 \\
13094\end{array}$ \\
\hline $\begin{array}{l}26 \\
27 \\
29 \\
29 \\
30\end{array}$ & $\begin{array}{l}n \\
0 \\
n \\
0 \\
0\end{array}$ & $\begin{array}{l}0 \\
0 \\
0 \\
0 \\
0\end{array}$ & $\begin{array}{l}0 \\
0 \\
0 \\
0 \\
0\end{array}$ & $\begin{array}{r}0 \\
0 \\
0 \\
160 \\
308\end{array}$ & $\begin{array}{r}41 \\
40 \\
912 \\
910\end{array}$ & $\begin{array}{r}810 \\
21 \\
3 \times 0 \\
1193 \\
1272\end{array}$ & $\begin{array}{r}517 \\
0 \\
1003 \\
2001 \\
1819\end{array}$ & $\begin{array}{r}115.7 \\
191 \\
1406 \\
2305 \\
1307\end{array}$ & $\begin{array}{r}1393 \\
108 \\
1237 \\
2505 \\
1285\end{array}$ & $\begin{array}{r}60 \\
781 \\
573 \\
2523 \\
2153\end{array}$ & $\begin{array}{r}47 \\
807 \\
412 \\
2353 \\
1997\end{array}$ & $\begin{array}{r}599 \\
442 \\
529 \\
1706 \\
1487\end{array}$ & $\begin{array}{r}399 \\
0 \\
677 \\
1706 \\
1884\end{array}$ & $\begin{array}{r}0 \\
245 \\
134 \\
1320 \\
1346\end{array}$ & $\begin{array}{r}0 \\
208 \\
13 \\
316 \\
512\end{array}$ & $\begin{array}{r}0 \\
21 \\
34 \\
0 \\
17\end{array}$ & $\begin{array}{l}0 \\
0 \\
0 \\
0 \\
0\end{array}$ & $\begin{array}{l}0 \\
0 \\
0 \\
0 \\
0\end{array}$ & $\begin{array}{r}4264 \\
2822 \\
6378 \\
19270 \\
10405\end{array}$ \\
\hline 31 & 0 & 0 & ) & $n$ & 50 & 116 & 799 & 1593 & 1967 & 395 & 1367 & 1415 & 8911 & $15 \geq \theta$ & 1745 & 243 & 0 & 0 & 12418 \\
\hline $\begin{array}{r}\text { HEAN } \\
\text { SD } \\
\text { HUMM }\end{array}$ & $\begin{array}{r}0 \\
0 \\
31\end{array}$ & $\begin{array}{r}1) \\
0 \\
3.1\end{array}$ & $\begin{array}{r}7 \\
14 \\
31\end{array}$ & $\begin{array}{r}292 \\
297 \\
31\end{array}$ & $\begin{array}{r}870 \\
710 \\
31\end{array}$ & $\begin{array}{r}1215 \\
115 \\
\vdots 1 \\
\vdots 1\end{array}$ & $\begin{array}{r}1452 \\
1327 \\
31\end{array}$ & $\begin{array}{r}16 A 2 \\
804 \\
31\end{array}$ & $\begin{array}{r}1676 \\
B 68 \\
30\end{array}$ & $\begin{array}{r}1604 \\
1002 \\
30\end{array}$ & $\begin{array}{r}1490 \\
936 \\
310\end{array}$ & $\begin{array}{r}1494 \\
199 ? \\
30\end{array}$ & $\begin{array}{r}1150 \\
341 \\
.31\end{array}$ & $\begin{array}{l}1156 \\
1306 \\
30\end{array}$ & $\begin{array}{r}548 \\
590 \\
30\end{array}$ & $\begin{array}{r}121 \\
155 \\
30\end{array}$ & $\begin{array}{r}0 \\
0 \\
31\end{array}$ & $\begin{array}{r}0 \\
0 \\
31\end{array}$ & $\begin{array}{r}14503 \\
7685 \\
30\end{array}$ \\
\hline $\begin{array}{l}\text { MAX } \\
\text { MINI }\end{array}$ & $\begin{array}{l}0 \\
0\end{array}$ & $\begin{array}{l}0 \\
0\end{array}$ & $\begin{array}{r}67 \\
0\end{array}$ & $\begin{array}{r}95 \% \\
0\end{array}$ & $\begin{array}{r}1917 \\
n\end{array}$ & $\begin{array}{r}26(1) \\
1)\end{array}$ & $\begin{array}{r}268 \mathrm{H} \\
1\end{array}$ & $\begin{array}{r}276 ! \\
11\end{array}$ & $\begin{array}{r}3065 \\
0\end{array}$ & $\begin{array}{r}3222 \\
0\end{array}$ & $\begin{array}{r}336 \% \\
0\end{array}$ & $\begin{array}{r}3243 \\
0\end{array}$ & $\begin{array}{r}289 ? \\
0\end{array}$ & $\begin{array}{r}2273 \\
0\end{array}$ & $\begin{array}{r}1745 \\
0\end{array}$ & $\begin{array}{r}473 \\
0\end{array}$ & $\begin{array}{l}0 \\
0\end{array}$ & $\begin{array}{l}n \\
0\end{array}$ & $\begin{array}{r}29362 \\
2082\end{array}$ \\
\hline
\end{tabular}


CIJASTAL - INLAIID SILLAR RAUIATION DIFFERENCE 3TUDY

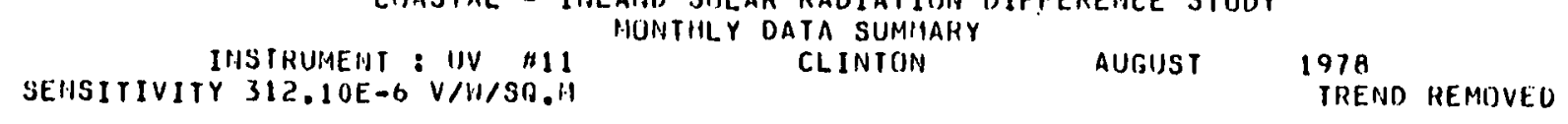

EHFRGY KILIJJOULES PER gIJUARE NETER

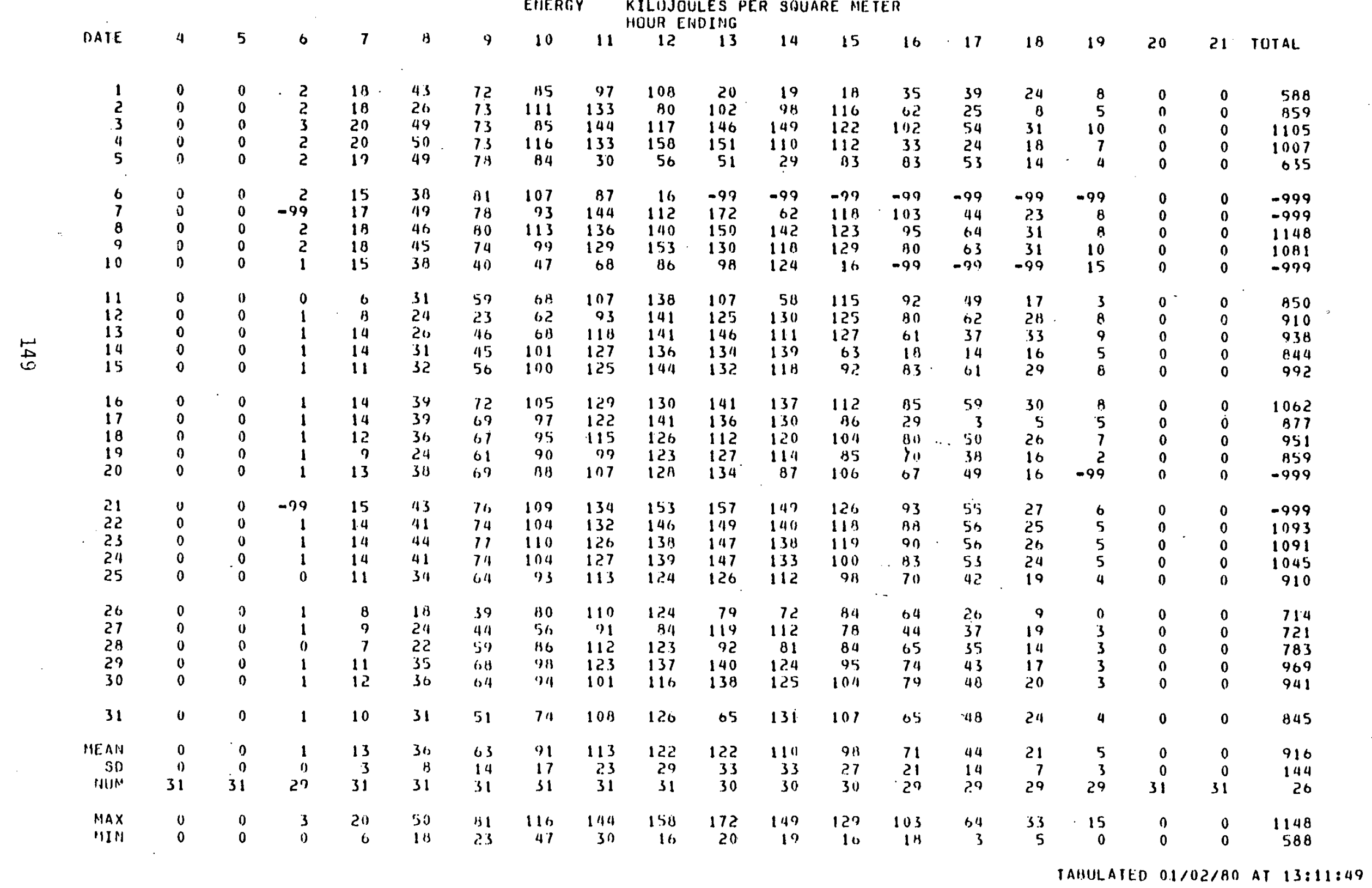


CI.ASTAL - INLANI) SIJLAR RADIATIUN DIFFERENCE STUIOY IHSIRUMEHF : IJV H12 MUNTHLY DATA SUMMARY
SENSITIVITY $154.46 E-6$ VIW/SO.19

ENERGY KILUJOULES PER SIJUARF. HETER

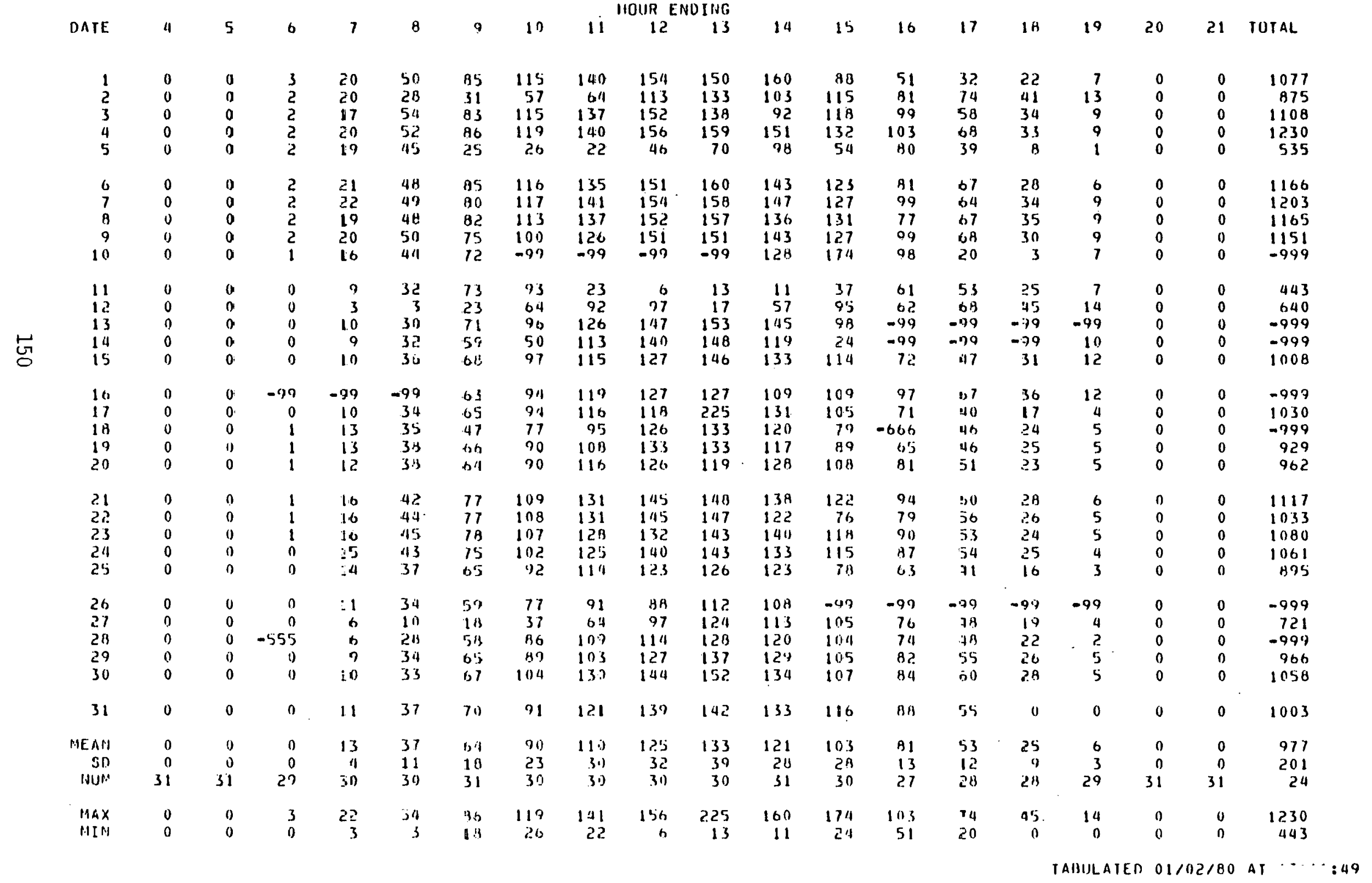


COASTAL - INLAND SOLAR RADIATION DIFFERENCE STUUY IMSTRIJMENT: PSP H ? MONTHLY DATA SIMMMAY
SEHSITIVITY $10.99 E-6$ VIW/SO.

ENERGY KILUJOULES PER SUUARE METER

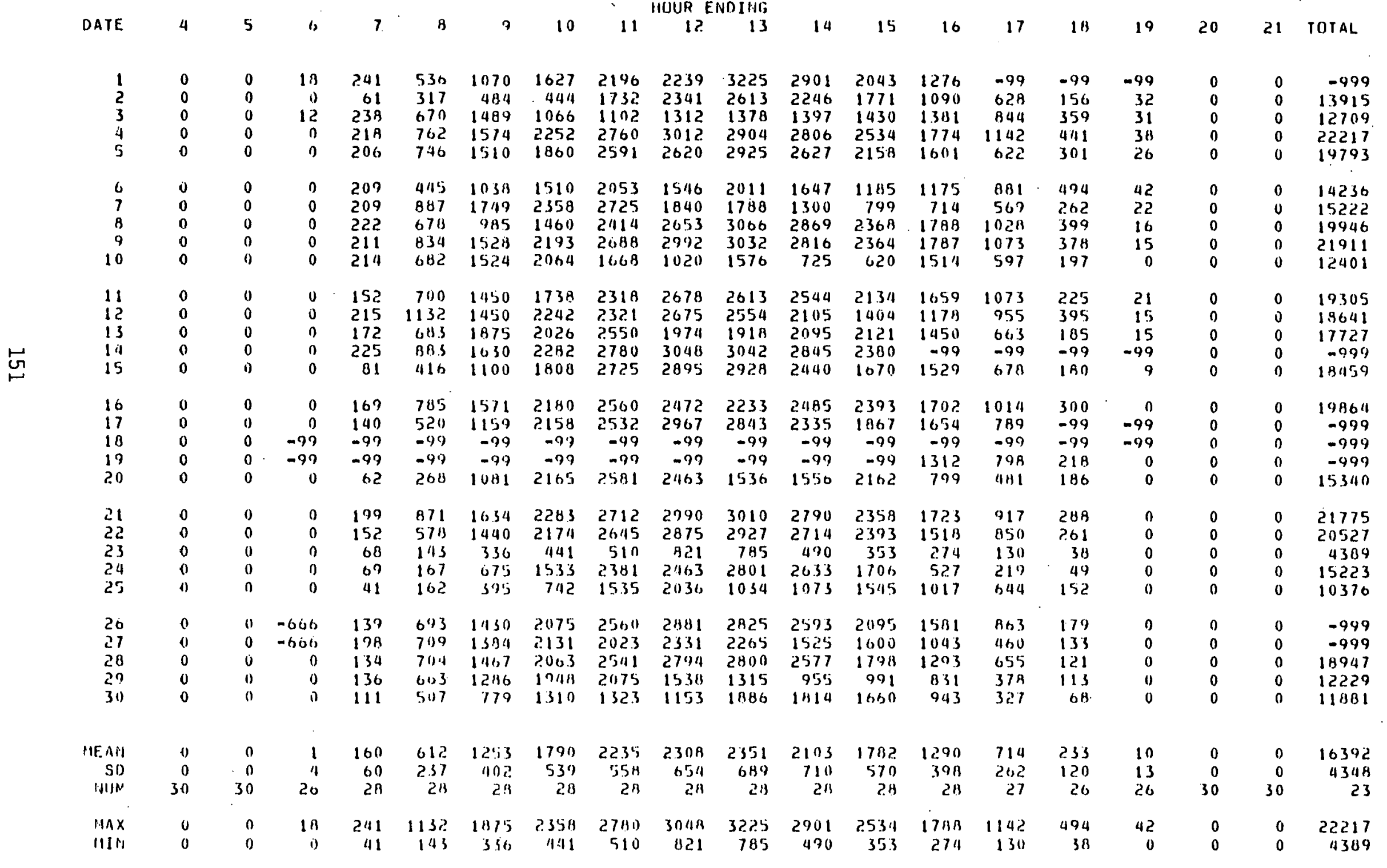


CUASTAL - INLA ID SOLAR RADIATIUN DIFFERENCE STUDY JNSTRIMMEHT : PSP $\$ 3$
SENSITIVITY $11.27 E-6$ V/N/30, MHTHLY DATA SUNMARY

ENERGY KII.UJUULES PER SOUARE METER

Th HIJPR ENDING

HITIS ENDING

\begin{tabular}{|c|c|c|c|c|c|c|c|c|c|c|c|c|c|c|c|c|c|c|c|}
\hline DA TE & 4 & 5 & 6 & $r$ & A & 7 & 10 & 11 & 12 & 13 & 14 & 15 & 16 & 17 & 13 & 19 & 20 & 21 & TUIAL \\
\hline $\begin{array}{l}1 \\
2 \\
3 \\
4 \\
5\end{array}$ & $\begin{array}{l}0 \\
0 \\
0 \\
0 \\
0\end{array}$ & $\begin{array}{l}0 \\
0 \\
0 \\
0 \\
0\end{array}$ & $\begin{array}{r}16 \\
0 \\
10 \\
-79 \\
0\end{array}$ & $\begin{array}{r}303 \\
80 \\
335 \\
-90 \\
150\end{array}$ & $\begin{array}{r}1105 \\
195 \\
959 \\
-97 \\
291\end{array}$ & $\begin{array}{r}1744 \\
502 \\
1384 \\
-49 \\
329\end{array}$ & $\begin{array}{l}2041 \\
1086 \\
1985 \\
2384 \\
840\end{array}$ & $\begin{array}{r}2907 \\
21163 \\
928 \\
2854 \\
1249\end{array}$ & $\begin{array}{r}2095 \\
2789 \\
-99 \\
3107 \\
2976\end{array}$ & $\begin{array}{r}2102 \\
2613 \\
-99 \\
3250 \\
2392\end{array}$ & $\begin{array}{r}3050 \\
1105 \\
-99 \\
3206 \\
2198\end{array}$ & $\begin{array}{r}2198 \\
1361 \\
-99 \\
2806 \\
1833\end{array}$ & $\begin{array}{r}1268 \\
581 \\
-99 \\
1979 \\
1054\end{array}$ & $\begin{array}{r}335 \\
3911 \\
-97 \\
1069 \\
645\end{array}$ & $\begin{array}{l}169 \\
353 \\
-97 \\
399 \\
281\end{array}$ & $\begin{array}{r}0 \\
13 \\
-99 \\
40 \\
19\end{array}$ & $\begin{array}{l}0 \\
0 \\
0 \\
0 \\
0\end{array}$ & $\begin{array}{l}0 \\
0 \\
0 \\
0 \\
0\end{array}$ & $\begin{array}{r}19333 \\
13533 \\
-999 \\
-999 \\
14213\end{array}$ \\
\hline $\begin{array}{r}0 \\
7 \\
9 \\
9 \\
10\end{array}$ & $\begin{array}{l}0 \\
0 \\
0 \\
0 \\
0\end{array}$ & $\begin{array}{l}0 \\
0 \\
0 \\
0 \\
0\end{array}$ & $\begin{array}{r}0 \\
0 \\
0 \\
0 \\
10\end{array}$ & $\begin{array}{l}184 \\
142 \\
222 \\
217 \\
272\end{array}$ & $\begin{array}{l}-79 \\
234 \\
820 \\
920 \\
659\end{array}$ & $\begin{array}{r}-99 \\
487 \\
1501 \\
1543 \\
759\end{array}$ & $\begin{array}{r}-79 \\
397 \\
2196 \\
2229 \\
1304\end{array}$ & $\begin{array}{r}620 \\
710 \\
2602 \\
2737 \\
721\end{array}$ & $\begin{array}{r}676 \\
1272 \\
2490 \\
3044 \\
799\end{array}$ & $\begin{array}{r}1101 \\
1742 \\
2985 \\
3114 \\
90\end{array}$ & $\begin{array}{r}1532 \\
1407 \\
2768 \\
21397 \\
52\end{array}$ & $\begin{array}{r}1401 \\
8313 \\
2311 \\
2417 \\
-99\end{array}$ & $\begin{array}{r}1513 \\
611 \\
1682 \\
1833 \\
-99\end{array}$ & $\begin{array}{r}1097 \\
448 \\
1120 \\
1092 \\
-99\end{array}$ & $\begin{array}{l}417 \\
250 \\
401 \\
427 \\
-97\end{array}$ & $\begin{array}{r}37 \\
20 \\
31 \\
32 \\
-99\end{array}$ & $\begin{array}{l}0 \\
0 \\
0 \\
0 \\
0\end{array}$ & $\begin{array}{l}0 \\
0 \\
0 \\
0 \\
0\end{array}$ & $\begin{array}{r}-999 \\
8558 \\
21189 \\
22530 \\
-999\end{array}$ \\
\hline $\begin{array}{l}11 \\
12 \\
13 \\
14 \\
15\end{array}$ & $\begin{array}{l}0 \\
0 \\
0 \\
0 \\
0\end{array}$ & $\begin{array}{l}n \\
1) \\
0 \\
0 \\
0\end{array}$ & $\begin{array}{l}-97 \\
-99 \\
-99 \\
-99 \\
-99\end{array}$ & $\begin{array}{l}-9.7 \\
-9.7 \\
-97 \\
-9.7 \\
-9.7\end{array}$ & $\begin{array}{l}-99 \\
-99 \\
-99 \\
-99 \\
-99\end{array}$ & $\begin{array}{l}-74 \\
-94 \\
-49 \\
-79 \\
-79\end{array}$ & $\begin{array}{l}-99 \\
-99 \\
-79 \\
-139 \\
-99\end{array}$ & $\begin{array}{l}-99 \\
-99 \\
-99 \\
-99 \\
-99\end{array}$ & $\begin{array}{l}-99 \\
-99 \\
-99 \\
-99 \\
-99\end{array}$ & $\begin{array}{l}-99 \\
-99 \\
-79 \\
-99 \\
-99\end{array}$ & $\begin{array}{l}-79 \\
-99 \\
-99 \\
-99 \\
-79\end{array}$ & $\begin{array}{l}-99 \\
-99 \\
-99 \\
-99 \\
-90\end{array}$ & $\begin{array}{l}-99 \\
-99 \\
-99 \\
-99 \\
-99\end{array}$ & $\begin{array}{l}-99 \\
-99 \\
-99 \\
-97 \\
-99\end{array}$ & $\begin{array}{l}-91 \\
-97 \\
-99 \\
-97 \\
-99\end{array}$ & $\begin{array}{l}-99 \\
-99 \\
-99 \\
-99 \\
-99\end{array}$ & $\begin{array}{l}0 \\
0 \\
0 \\
0 \\
0\end{array}$ & $\begin{array}{l}0 \\
0 \\
0 \\
0 \\
0\end{array}$ & $\begin{array}{l}-999 \\
-999 \\
-999 \\
-999 \\
-999\end{array}$ \\
\hline $\begin{array}{l}16 \\
17 \\
17 \\
17 \\
20\end{array}$ & $\begin{array}{l}0 \\
0 \\
0 \\
0 \\
0\end{array}$ & $\begin{array}{l}0 \\
0 \\
0 \\
0 \\
0\end{array}$ & $\begin{array}{l}-99 \\
-99 \\
-99 \\
-99 \\
-99\end{array}$ & $\begin{array}{l}-97 \\
-97 \\
-97 \\
-97 \\
-97\end{array}$ & $\begin{array}{l}-99 \\
-99 \\
-99 \\
-99 \\
-99\end{array}$ & $\begin{array}{l}-99 \\
-99 \\
-99 \\
-99 \\
-99\end{array}$ & $\begin{array}{r}-99 \\
-91 \\
-99 \\
-99 \\
2251\end{array}$ & $\begin{array}{l}-99 \\
-99 \\
-99 \\
-99 \\
-99\end{array}$ & $\begin{array}{l}-79 \\
-99 \\
-79 \\
-99 \\
-97\end{array}$ & $\begin{array}{l}-99 \\
-99 \\
-99 \\
-99 \\
-99\end{array}$ & $\begin{array}{r}-99 \\
-99 \\
-99 \\
-99 \\
2257\end{array}$ & $\begin{array}{r}-99 \\
-99 \\
-99 \\
-99 \\
21180\end{array}$ & $\begin{array}{r}-99 \\
-99 \\
-91 \\
-99 \\
1813\end{array}$ & $\begin{array}{r}-97 \\
-99 \\
-99 \\
-9.7 \\
1075\end{array}$ & $\begin{array}{l}-99 \\
-99 \\
-99 \\
-99 \\
133\end{array}$ & $\begin{array}{l}-99 \\
-99 \\
-99 \\
-99 \\
158\end{array}$ & $\begin{array}{l}0 \\
0 \\
0 \\
0 \\
0\end{array}$ & $\begin{array}{l}0 \\
0 \\
0 \\
0 \\
0\end{array}$ & $\begin{array}{l}-999 \\
-999 \\
-999 \\
-999 \\
-999\end{array}$ \\
\hline $\begin{array}{l}21 \\
22 \\
23 \\
214 \\
25\end{array}$ & $\begin{array}{l}0 \\
0 \\
0 \\
0 \\
0\end{array}$ & $\begin{array}{l}0 \\
0 \\
0 \\
0 \\
0\end{array}$ & $\begin{array}{l}0 \\
0 \\
0 \\
0 \\
0\end{array}$ & $\begin{array}{r}51 \\
125 \\
13 \\
5 ! \\
107\end{array}$ & $\begin{array}{l}948 \\
654 \\
464 \\
220 \\
280\end{array}$ & $\begin{array}{r}1712 \\
1414 \\
508 \\
658 \\
3 E B\end{array}$ & $\begin{array}{r}2332 \\
2337 \\
605 \\
1741 \\
570\end{array}$ & $\begin{array}{r}2558 \\
2805 \\
347 \\
1818 \\
1497\end{array}$ & $\begin{array}{r}2478 \\
3021 \\
349 \\
2594 \\
1372\end{array}$ & $\begin{array}{r}2645 \\
3078 \\
365 \\
2332 \\
1145\end{array}$ & $\begin{array}{l}2351 \\
2845 \\
1007 \\
2405 \\
1522\end{array}$ & $\begin{array}{r}2127 \\
2286 \\
867 \\
1402 \\
559\end{array}$ & $\begin{array}{r}1096 \\
1657 \\
506 \\
473 \\
538\end{array}$ & $\begin{array}{l}964 \\
231 \\
234 \\
1170 \\
510\end{array}$ & $\begin{array}{r}274 \\
2.38 \\
62 \\
147 \\
104\end{array}$ & $\begin{array}{r}0 \\
0 \\
8 \\
-555 \\
0\end{array}$ & $\begin{array}{l}0 \\
0 \\
0 \\
0 \\
0\end{array}$ & $\begin{array}{l}n \\
0 \\
0 \\
0 \\
0\end{array}$ & $\begin{array}{r}20436 \\
21390 \\
5515 \\
-949 \\
8557\end{array}$ \\
\hline $\begin{array}{l}26 \\
27 \\
28 \\
29 \\
30\end{array}$ & $\begin{array}{l}0 \\
0 \\
0 \\
0 \\
0\end{array}$ & $\begin{array}{l}0 \\
0 \\
0 \\
0 \\
0\end{array}$ & $\begin{array}{l}1 \\
0 \\
0 \\
0 \\
0\end{array}$ & $\begin{array}{l}15 \% \\
170 \\
210 \\
1110 \\
52\end{array}$ & $\begin{array}{l}745 \\
n 012 \\
797 \\
716 \\
324\end{array}$ & $\begin{array}{l}1459 \\
1594 \\
1659 \\
1168 \\
351\end{array}$ & $\begin{array}{r}1930 \\
2189 \\
2155 \\
1769 \\
123\end{array}$ & $\begin{array}{r}2691 \\
2362 \\
21187 \\
2111 \\
809\end{array}$ & $\begin{array}{l}2857 \\
2652 \\
24119 \\
2335 \\
2071\end{array}$ & $\begin{array}{l}2406 \\
1470 \\
2455 \\
1919 \\
1876\end{array}$ & $\begin{array}{l}1250 \\
1554 \\
21126 \\
1293 \\
1578\end{array}$ & $\begin{array}{l}1665 \\
1638 \\
2091 \\
1360 \\
1078\end{array}$ & $\begin{array}{r}927 \\
1164 \\
1369 \\
661 \\
985\end{array}$ & $\begin{array}{l}723 \\
554 \\
4149 \\
561 \\
292\end{array}$ & $\begin{array}{r}138 \\
94 \\
101 \\
107 \\
129\end{array}$ & $\begin{array}{l}0 \\
0 \\
0 \\
0 \\
0\end{array}$ & $\begin{array}{l}0 \\
0 \\
0 \\
0 \\
0\end{array}$ & $\begin{array}{l}0 \\
0 \\
0 \\
0 \\
0\end{array}$ & $\begin{array}{l}16975 \\
16257 \\
18667 \\
14002 \\
10320\end{array}$ \\
\hline $\begin{array}{r}H E A H \\
\text { SO } \\
\text { HIJM }\end{array}$ & $\begin{array}{r}0 \\
0 \\
30\end{array}$ & $\begin{array}{r}0 \\
0 \\
30\end{array}$ & $\begin{array}{r}1 \\
11 \\
17\end{array}$ & $\begin{array}{r}162 \\
81 \\
15\end{array}$ & $\begin{array}{r}618 \\
289 \\
18\end{array}$ & $\begin{array}{r}1018 \\
5: 18 \\
18\end{array}$ & $\begin{array}{r}165 \% \\
67 \\
20\end{array}$ & $\begin{array}{r}1372 \\
1172 \\
20\end{array}$ & $\begin{array}{r}2177 \\
839 \\
19\end{array}$ & $\begin{array}{r}2056 \\
874 \\
19\end{array}$ & $\begin{array}{r}1936 \\
805 \\
20\end{array}$ & $\begin{array}{r}1721 \\
612 \\
17\end{array}$ & $\begin{array}{r}1171 \\
502 \\
19\end{array}$ & $\begin{array}{r}071 \\
311 \\
10\end{array}$ & $\begin{array}{r}2110 \\
126 \\
19\end{array}$ & $\begin{array}{l}19 \\
36 \\
18\end{array}$ & $\begin{array}{r}n \\
0 \\
30\end{array}$ & $\begin{array}{r}0 \\
0 \\
30\end{array}$ & $\begin{array}{r}15432 \\
5156 \\
15\end{array}$ \\
\hline $\begin{array}{l}\text { MAX } \\
\text { MLiv }\end{array}$ & $\begin{array}{l}0 \\
0\end{array}$ & $\begin{array}{l}0 \\
0\end{array}$ & $\begin{array}{r}16 \\
0\end{array}$ & $\begin{array}{r}335 \\
51\end{array}$ & $\begin{array}{r}1105 \\
195\end{array}$ & $\begin{array}{r}1764 \\
3 \geq 16\end{array}$ & $\begin{array}{r}23813 \\
397\end{array}$ & $\begin{array}{r}2907 \\
349\end{array}$ & $\begin{array}{r}31117 \\
3149\end{array}$ & $\begin{array}{r}3250 \\
90\end{array}$ & $\begin{array}{r}3206 \\
52\end{array}$ & $\begin{array}{l}\text { 2HOS } \\
55 \mathrm{~B}\end{array}$ & $\begin{array}{r}1979 \\
473\end{array}$ & $\begin{array}{r}11201 \\
23\end{array}$ & $\begin{array}{r}433 \\
0.2\end{array}$ & $\begin{array}{r}158 \\
0\end{array}$ & $\begin{array}{l}0 \\
0\end{array}$ & $\begin{array}{l}0 \\
0\end{array}$ & $\begin{array}{r}22530 \\
5000\end{array}$ \\
\hline
\end{tabular}


COASTAL. - INLAIID SOLAR RADIATIUN DIFFEREHCE STUDY

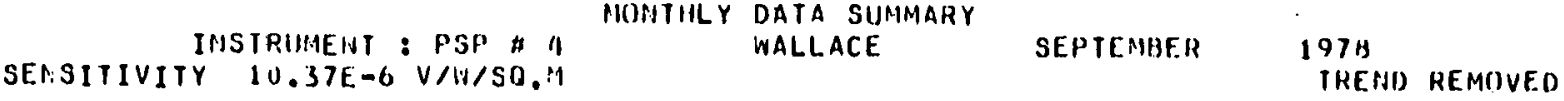

ENERGY KILUJUIILES PER SIJUARE METER

\begin{tabular}{|c|c|c|c|c|c|c|c|c|c|c|c|c|c|c|c|c|c|c|c|}
\hline & & & & & & & EHES & & $\begin{array}{l}\text { KILOJJO } \\
\text { HIJUR E }\end{array}$ & $\begin{array}{l}\text { IJI.ES P } \\
\text { NDING }\end{array}$ & ER 31$\}$ & ARE 11 & TER & & & & & & \\
\hline DAIE & 4 & 5 & 6 & 7 & $B$ & 9 & 10 & 11 & 12 & 13 & 11 & 15 & 16 & 17 & 18 & 19 & 20 & 21 & TOTAL \\
\hline $\begin{array}{l}1 \\
2 \\
3 \\
4 \\
5\end{array}$ & $\begin{array}{l}0 \\
0 \\
0 \\
0 \\
0\end{array}$ & $\begin{array}{l}0 \\
0 \\
0 \\
0 \\
0\end{array}$ & $\begin{array}{r}10 \\
-99 \\
18 \\
11 \\
8\end{array}$ & $\begin{array}{l}340 \\
-99 \\
320 \\
293 \\
261\end{array}$ & $\begin{array}{l}514 \\
233 \\
827 \\
766 \\
775\end{array}$ & $\begin{array}{r}687 \\
385 \\
1674 \\
1671 \\
1563\end{array}$ & $\begin{array}{r}1681 \\
496 \\
626 \\
2299 \\
2077\end{array}$ & $\begin{array}{r}1063 \\
677 \\
897 \\
2792 \\
2768\end{array}$ & $\begin{array}{l}2805 \\
205 ? \\
2014 \\
3018 \\
2931\end{array}$ & $\begin{array}{r}2552 \\
3010 \\
716 \\
2993 \\
3035\end{array}$ & $\begin{array}{l}2864 \\
2531 \\
1560 \\
2844 \\
2848\end{array}$ & $\begin{array}{l}1798 \\
1264 \\
1976 \\
2358 \\
2112\end{array}$ & $\begin{array}{r}1413 \\
816 \\
1528 \\
1754 \\
1244\end{array}$ & $\begin{array}{r}-99 \\
715 \\
650 \\
1035 \\
598\end{array}$ & $\begin{array}{r}-99 \\
45 \\
220 \\
359 \\
244\end{array}$ & $\begin{array}{r}-99 \\
14 \\
22 \\
15 \\
15\end{array}$ & $\begin{array}{l}0 \\
0 \\
0 \\
0 \\
0\end{array}$ & $\begin{array}{l}0 \\
0 \\
0 \\
0 \\
0\end{array}$ & $\begin{array}{r}-999 \\
-999 \\
1.3048 \\
22408 \\
20479\end{array}$ \\
\hline $\begin{array}{r}6 \\
7 \\
9 \\
9 \\
10\end{array}$ & $\begin{array}{l}0 \\
0 \\
0 \\
0 \\
0\end{array}$ & $\begin{array}{l}0 \\
0 \\
0 \\
0 \\
0\end{array}$ & $\begin{array}{r}9 \\
11 \\
7 \\
9 \\
10\end{array}$ & $\begin{array}{l}231 \\
202 \\
210 \\
279 \\
205\end{array}$ & $\begin{array}{l}953 \\
6411 \\
918 \\
749 \\
847\end{array}$ & $\begin{array}{r}1654 \\
1244 \\
1453 \\
1525 \\
1340\end{array}$ & $\begin{array}{l}1897 \\
2386 \\
1118 \\
2254 \\
1396\end{array}$ & $\begin{array}{l}1533 \\
2150 \\
2304 \\
2716 \\
1704\end{array}$ & $\begin{array}{l}1689 \\
2129 \\
3119 \\
2959 \\
1337\end{array}$ & $\begin{array}{r}1713 \\
3175 \\
3029 \\
3014 \\
923\end{array}$ & $\begin{array}{r}1349 \\
1844 \\
2734 \\
2792 \\
371\end{array}$ & $\begin{array}{r}1224 \\
1251 \\
2293 \\
2341 \\
417\end{array}$ & $\begin{array}{r}1078 \\
1299 \\
1696 \\
1765 \\
56 ?\end{array}$ & $\begin{array}{r}929 \\
393 \\
971 \\
1008 \\
323\end{array}$ & $\begin{array}{r}274 \\
178 \\
276 \\
300 \\
97\end{array}$ & $\begin{array}{r}16 \\
0 \\
0 \\
0 \\
0\end{array}$ & $\begin{array}{l}0 \\
0 \\
0 \\
0 \\
0\end{array}$ & $\begin{array}{l}0 \\
0 \\
0 \\
0 \\
0\end{array}$ & $\begin{array}{r}14503 \\
16922 \\
20430 \\
21760 \\
9102\end{array}$ \\
\hline $\begin{array}{l}11 \\
12 \\
13 \\
14 \\
15\end{array}$ & $\begin{array}{l}0 \\
0 \\
0 \\
0 \\
0\end{array}$ & $\begin{array}{l}0 \\
0 \\
0 \\
0 \\
0\end{array}$ & $\begin{array}{l}0 \\
0 \\
0 \\
0 \\
0\end{array}$ & $\begin{array}{l}157 \\
170 \\
229 \\
276 \\
126\end{array}$ & $\begin{array}{l}671 \\
533 \\
705 \\
960 \\
681\end{array}$ & $\begin{array}{r}879 \\
1729 \\
1794 \\
1093 \\
1567\end{array}$ & $\begin{array}{l}2001 \\
2263 \\
2111 \\
2321 \\
1910\end{array}$ & $\begin{array}{l}2021 \\
2378 \\
2354 \\
2779 \\
1667\end{array}$ & $\begin{array}{l}2073 \\
2586 \\
2302 \\
3040 \\
2587\end{array}$ & $\begin{array}{l}2466 \\
2968 \\
1666 \\
3026 \\
2917\end{array}$ & $\begin{array}{l}2459 \\
2795 \\
1788 \\
2727 \\
21428\end{array}$ & $\begin{array}{l}1424 \\
1423 \\
1833 \\
2120 \\
2146\end{array}$ & $\begin{array}{r}1136 \\
851 \\
1486 \\
1641 \\
1497\end{array}$ & $\begin{array}{l}727 \\
719 \\
840 \\
859 \\
431\end{array}$ & $\begin{array}{l}300 \\
233 \\
257 \\
283 \\
293\end{array}$ & $\begin{array}{l}0 \\
0 \\
0 \\
0 \\
0\end{array}$ & $\begin{array}{l}0 \\
0 \\
0 \\
0 \\
0\end{array}$ & $\begin{array}{l}0 \\
0 \\
0 \\
0 \\
0\end{array}$ & $\begin{array}{l}16314 \\
18653 \\
17355 \\
21725 \\
18250\end{array}$ \\
\hline $\begin{array}{l}16 \\
17 \\
18 \\
19 \\
20\end{array}$ & $\begin{array}{l}0 \\
0 \\
0 \\
0 \\
0 .\end{array}$ & $\begin{array}{l}0 \\
0 \\
0 \\
0 \\
0\end{array}$ & $\begin{array}{r}0 \\
11 \\
-79 \\
0 \\
0\end{array}$ & $\begin{array}{l}259 \\
154 \\
197 \\
231 \\
103\end{array}$ & $\begin{array}{l}881 \\
570 \\
920 \\
741 \\
454\end{array}$ & $\begin{array}{l}1589 \\
1348 \\
1144 \\
1488 \\
680\end{array}$ & $\begin{array}{r}22.24 \\
2185 \\
2096 \\
2109 \\
589\end{array}$ & $\begin{array}{l}2614 \\
2560 \\
2523 \\
2529 \\
2131\end{array}$ & $\begin{array}{l}2172 \\
2445 \\
2704 \\
2786 \\
2686\end{array}$ & $\begin{array}{l}2335 \\
2966 \\
2617 \\
2804 \\
3075\end{array}$ & $\begin{array}{l}2703 \\
2594 \\
2471 \\
2526 \\
2162\end{array}$ & $\begin{array}{l}1943 \\
2192 \\
1739 \\
1866 \\
1936\end{array}$ & $\begin{array}{l}1587 \\
1671 \\
1374 \\
1425 \\
1190\end{array}$ & $\begin{array}{l}705 \\
-99 \\
767 \\
8111 \\
175\end{array}$ & $\begin{array}{l}256 \\
-99 \\
194 \\
165 \\
103\end{array}$ & $\begin{array}{r}0 \\
-49 \\
0 \\
0 \\
0\end{array}$ & $\begin{array}{l}0 \\
0 \\
0 \\
0 \\
0\end{array}$ & $\begin{array}{l}0 \\
0 \\
0 \\
0 \\
0\end{array}$ & $\begin{array}{r}19493 \\
-999 \\
-999 \\
19484 \\
13524\end{array}$ \\
\hline $\begin{array}{l}21 \\
22 \\
23 \\
24 \\
25\end{array}$ & $\begin{array}{l}0 \\
0 \\
0 \\
0 \\
0\end{array}$ & $\begin{array}{l}0 \\
0 \\
0 \\
1) \\
0\end{array}$ & $\begin{array}{l}1) \\
0 \\
0 \\
0 \\
0\end{array}$ & $\begin{array}{r}210 \\
174 \\
85 \\
45 \\
36\end{array}$ & $\begin{array}{l}904 \\
708 \\
2.31 \\
257 \\
234\end{array}$ & $\begin{array}{r}1519 \\
1593 \\
335 \\
774 \\
324\end{array}$ & $\begin{array}{r}2272 \\
2145 \\
283 \\
1611 \\
553\end{array}$ & $\begin{array}{r}2744 \\
21193 \\
449 \\
2295 \\
824\end{array}$ & $\begin{array}{r}3001 \\
2822 \\
619 \\
2833 \\
1112\end{array}$ & $\begin{array}{r}3008 \\
2958 \\
845 \\
2680 \\
983\end{array}$ & $\begin{array}{r}2783 \\
2666 \\
685 \\
2076 \\
1233\end{array}$ & $\begin{array}{r}2321 \\
2208 \\
6110 \\
1930 \\
806\end{array}$ & $\begin{array}{r}1693 \\
1527 \\
269 \\
1239 \\
768\end{array}$ & $\begin{array}{l}946 \\
767 \\
130 \\
701 \\
338\end{array}$ & $\begin{array}{r}238 \\
2013 \\
29 \\
97 \\
140\end{array}$ & $\begin{array}{l}0 \\
0 \\
0 \\
0 \\
0\end{array}$ & $\begin{array}{l}0 \\
0 \\
0 \\
0 \\
0\end{array}$ & $\begin{array}{l}0 \\
0 \\
0 \\
0 \\
0\end{array}$ & $\begin{array}{r}21699 \\
20269 \\
4600 \\
16538 \\
7351\end{array}$ \\
\hline $\begin{array}{l}20 \\
27 \\
28 \\
29 \\
30\end{array}$ & $\begin{array}{l}0 \\
0 \\
0 \\
0 \\
0\end{array}$ & $\begin{array}{l}0 \\
0 \\
0 \\
0 \\
0\end{array}$ & $\begin{array}{l}0 \\
0 \\
0 \\
0 \\
0\end{array}$ & $\begin{array}{l}182 \\
173 \\
201 \\
210 \\
78\end{array}$ & $\begin{array}{l}7137 \\
7811 \\
634 \\
516 \\
653\end{array}$ & $\begin{array}{r}1516 \\
1.524 \\
1652 \\
1304 \\
973\end{array}$ & $\begin{array}{l}2115 \\
2089 \\
2128 \\
1786 \\
746\end{array}$ & $\begin{array}{l}2508 \\
2666 \\
2558 \\
1940 \\
1012\end{array}$ & $\begin{array}{l}2473 \\
2509 \\
2791 \\
11125 \\
1193\end{array}$ & $\begin{array}{l}2.903 \\
2339 \\
2747 \\
1002 \\
1649\end{array}$ & $\begin{array}{l}25130 \\
1891 \\
2502 \\
1078 \\
1293\end{array}$ & $\begin{array}{l}1599 \\
1610 \\
1211 \\
1026 \\
1167\end{array}$ & $\begin{array}{r}1101 \\
857 \\
954 \\
590 \\
721\end{array}$ & $\begin{array}{l}717 \\
548 \\
6011 \\
360 \\
179\end{array}$ & $\begin{array}{r}99 \\
121 \\
86 \\
112 \\
117\end{array}$ & $\begin{array}{l}0 \\
0 \\
0 \\
0 \\
0\end{array}$ & $\begin{array}{l}0 \\
0 \\
0 \\
0 \\
0\end{array}$ & $\begin{array}{l}0 \\
0 \\
0 \\
0 \\
0\end{array}$ & $\begin{array}{l}19353 \\
17111 \\
18133 \\
11355 \\
10156\end{array}$ \\
\hline $\begin{array}{r}\text { MEAN } \\
\text { SD } \\
\text { WUI }\end{array}$ & $\begin{array}{r}0 \\
0 \\
30\end{array}$ & $\begin{array}{r}0 \\
0 \\
30\end{array}$ & $\begin{array}{r}3 \\
5 \\
23\end{array}$ & $\begin{array}{r}174 \\
75 \\
29\end{array}$ & $\begin{array}{r}679 \\
2.3 \\
30\end{array}$ & $\begin{array}{r}1215 \\
150 \\
30\end{array}$ & $\begin{array}{r}1714 \\
635 \\
31\end{array}$ & $\begin{array}{r}2070 \\
682 \\
30\end{array}$ & $\begin{array}{r}2356 \\
657 \\
30\end{array}$ & $\begin{array}{r}21107 \\
787 \\
30\end{array}$ & $\begin{array}{r}2172 \\
691 \\
30\end{array}$ & $\begin{array}{r}1602 \\
517 \\
30\end{array}$ & $\begin{array}{r}1234 \\
396 \\
30\end{array}$ & $\begin{array}{r}658 \\
246 \\
28\end{array}$ & $\begin{array}{r}134 \\
4 B \\
285\end{array}$ & $\begin{array}{r}2 \\
6 \\
26\end{array}$ & $\begin{array}{r}0 \\
0 \\
30\end{array}$ & $\begin{array}{r}0 \\
0 \\
30\end{array}$ & $\begin{array}{r}10615 \\
4654 \\
26\end{array}$ \\
\hline $\begin{array}{l}\text { MAx } \\
\text { :III }\end{array}$ & $\begin{array}{l}0 \\
0\end{array}$ & $\begin{array}{l}0 \\
0\end{array}$ & $\begin{array}{r}18 \\
10\end{array}$ & $\begin{array}{r}3110 \\
36\end{array}$ & $\begin{array}{l}96,6 \\
231\end{array}$ & $\begin{array}{r}1781 \\
324\end{array}$ & $\begin{array}{r}2386 \\
283\end{array}$ & $\begin{array}{r}2792 \\
1119\end{array}$ & $\begin{array}{r}3119 \\
619\end{array}$ & $\begin{array}{r}3195 \\
710\end{array}$ & $\begin{array}{r}2864 \\
371\end{array}$ & $\begin{array}{r}2351 \\
417\end{array}$ & $\begin{array}{r}1765 \\
269\end{array}$ & $\begin{array}{r}1035 \\
131)\end{array}$ & $\begin{array}{r}359 \\
27\end{array}$ & $\begin{array}{r}22 \\
0\end{array}$ & $\begin{array}{l}0 \\
0\end{array}$ & $\begin{array}{l}0 \\
0\end{array}$ & $\begin{array}{r}2.2408 \\
4600\end{array}$ \\
\hline
\end{tabular}


CMASTAL - IHLAYD SOLAR RADIATIUN DIFFEREIICE STUJY

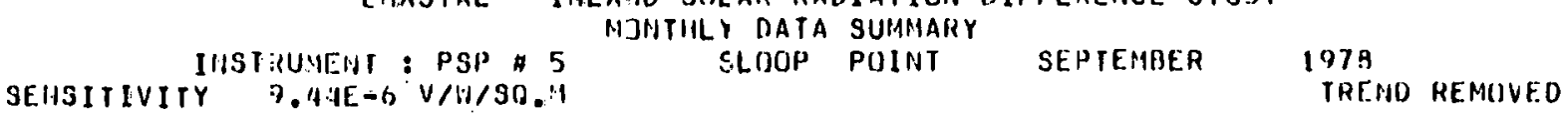

EHERG Y KILUJNULES PER STUARF. HETER

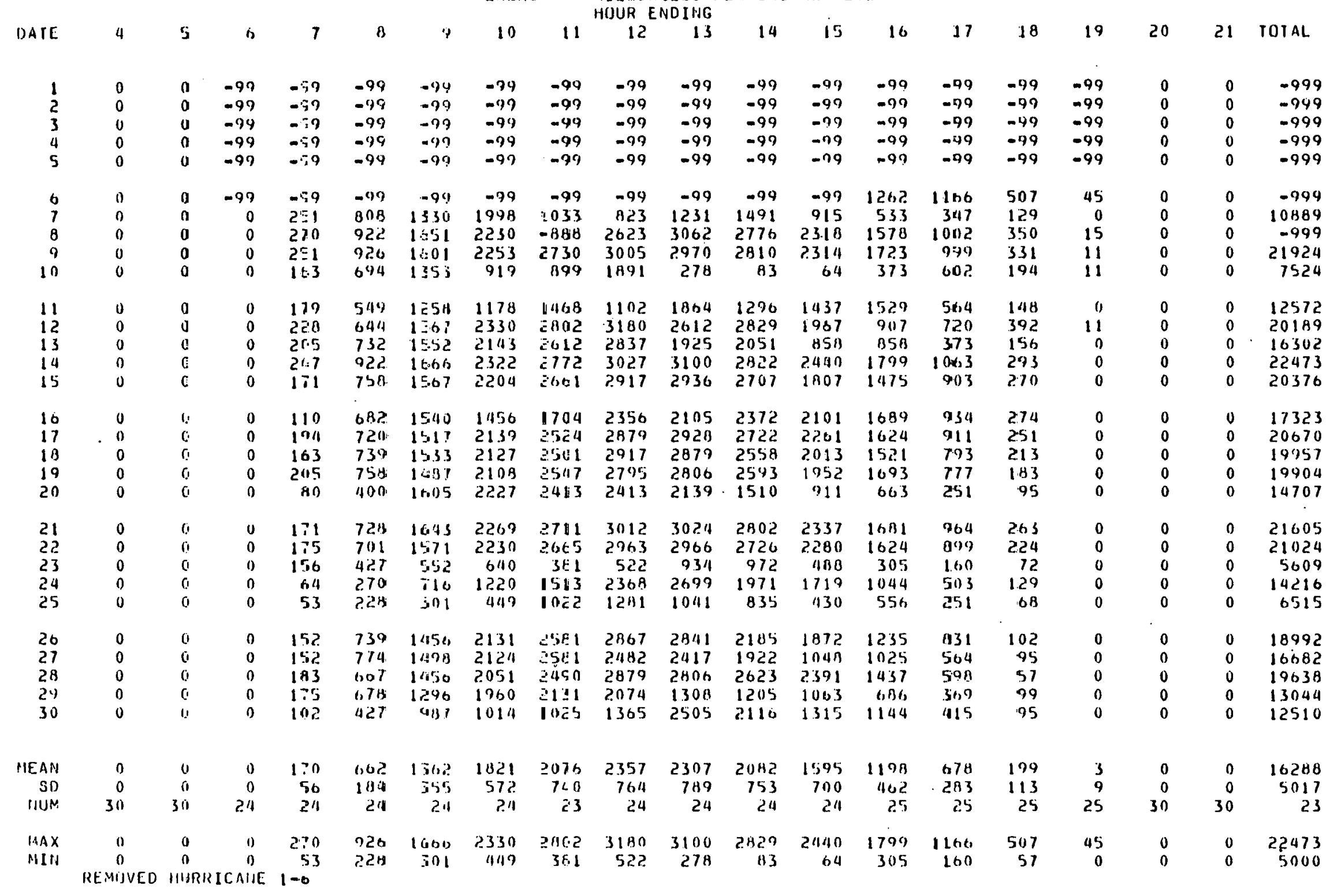


CUASTAL - INLAND SILLAR RADIATIUN DIFFERENCE STUOY MSTIRUMEIILY DATA SUMMARY IISTIRUMENT: PSP H 6
SENSITIVITY $11.00 E-6$ V/HISA.M

FINERTY KILIJJOULES PER SHUARE. METER IIDUR ENDING

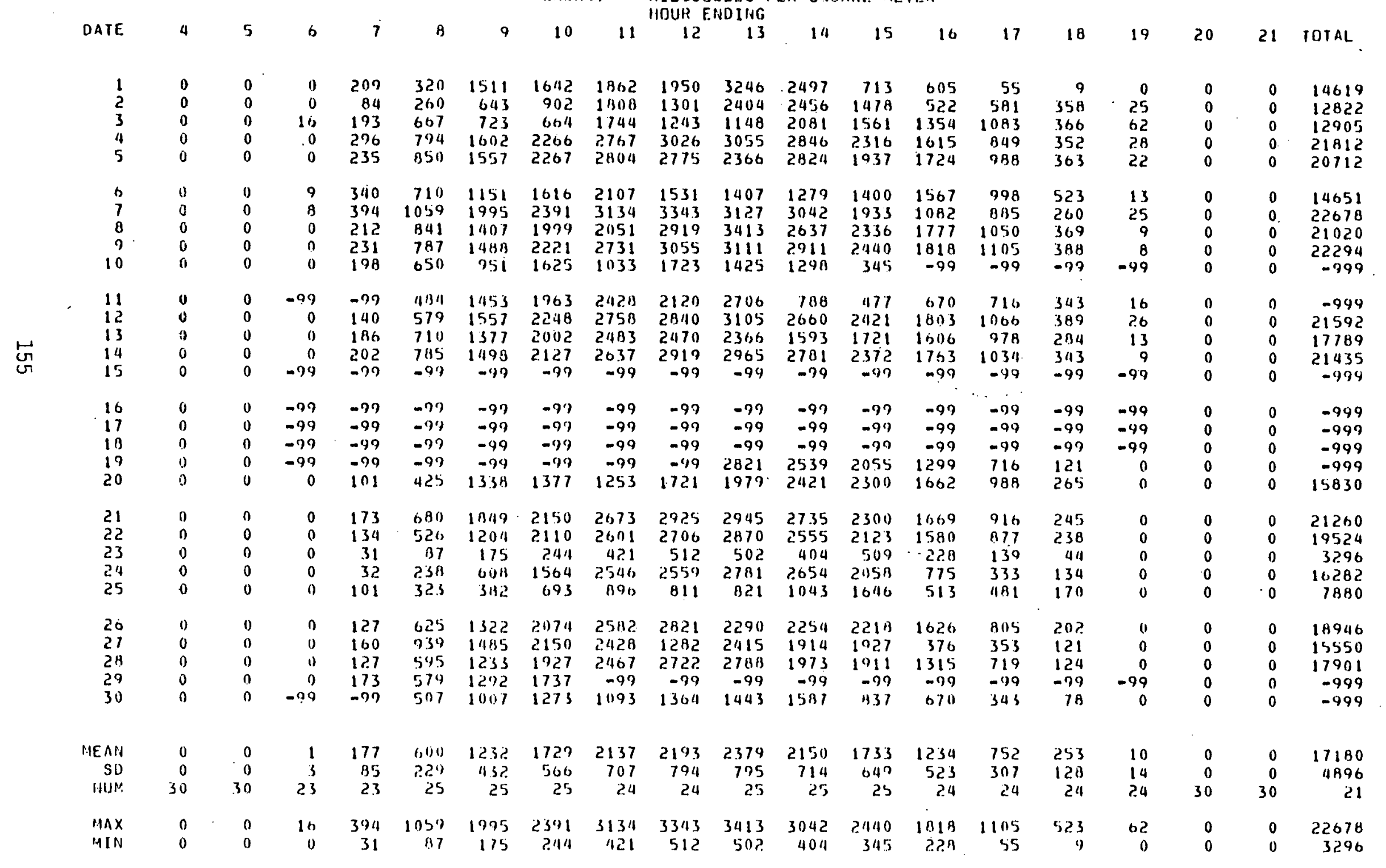


COA3TAL - IILLAHD SULAR RADIATION DIFFEREHCE SIUDY MONTHLY DATA SUMMARY

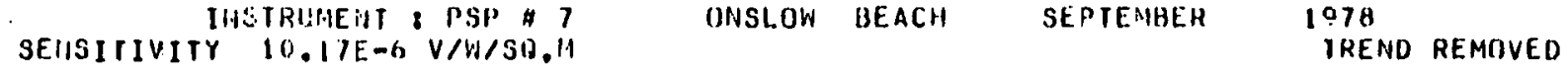

EHERGY KILIJJULLS PER SUUARE HETER HOUR ENDIHG

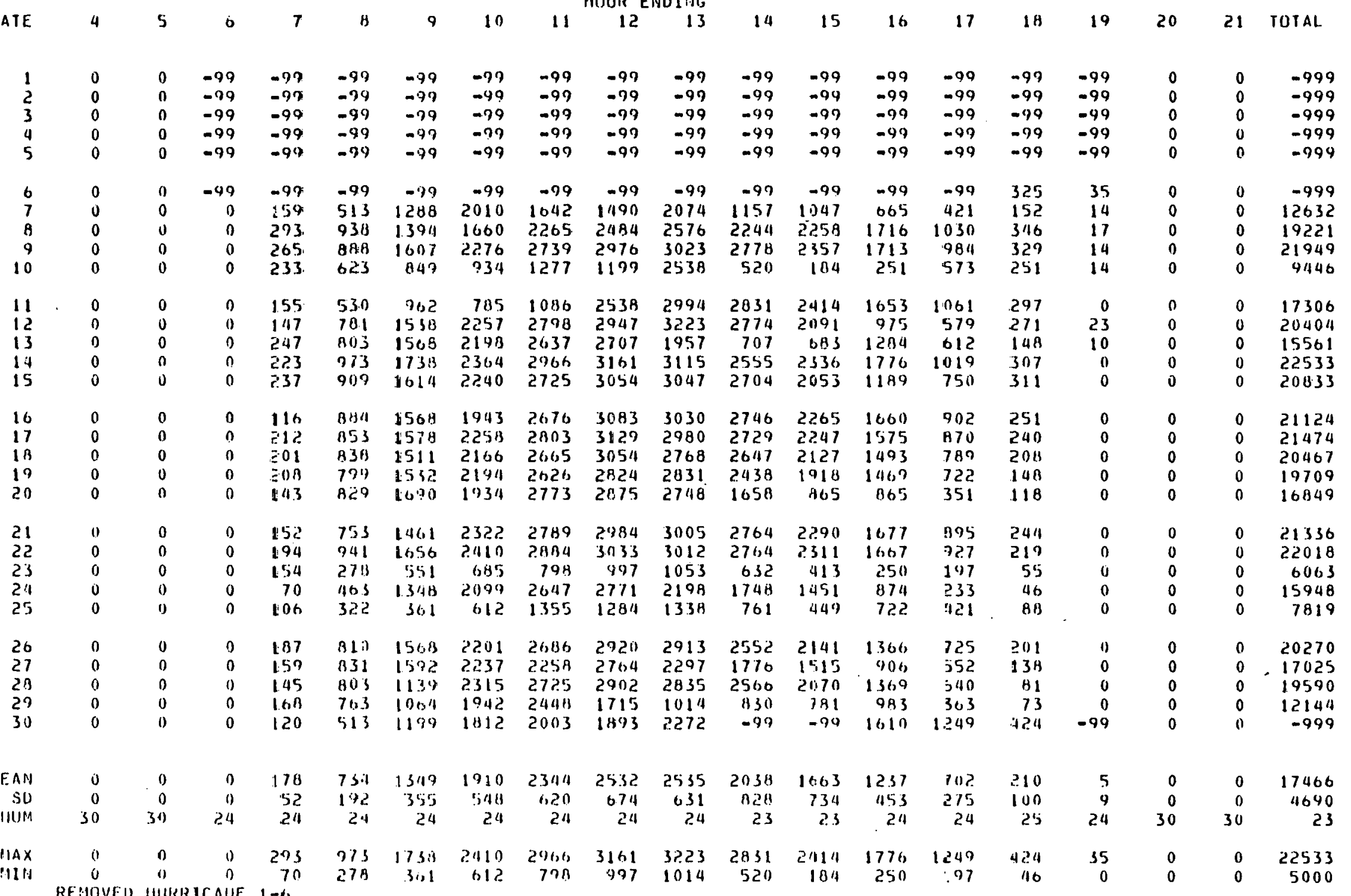
RE:AOVEI IIIKRICAIIE $1-6$ 
CINATTAL - INLAND SULAR RADIATIUN OIFFEREHCE STUOY

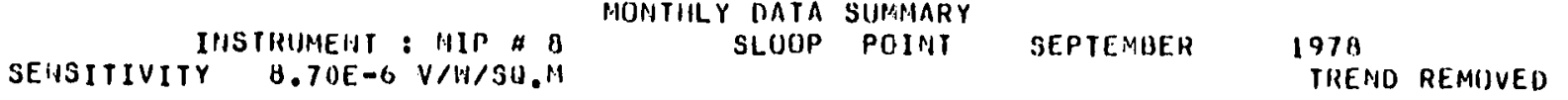

ENERGY KILUJDULE3 PER SGUARE METE.K HOUR ENDIHG

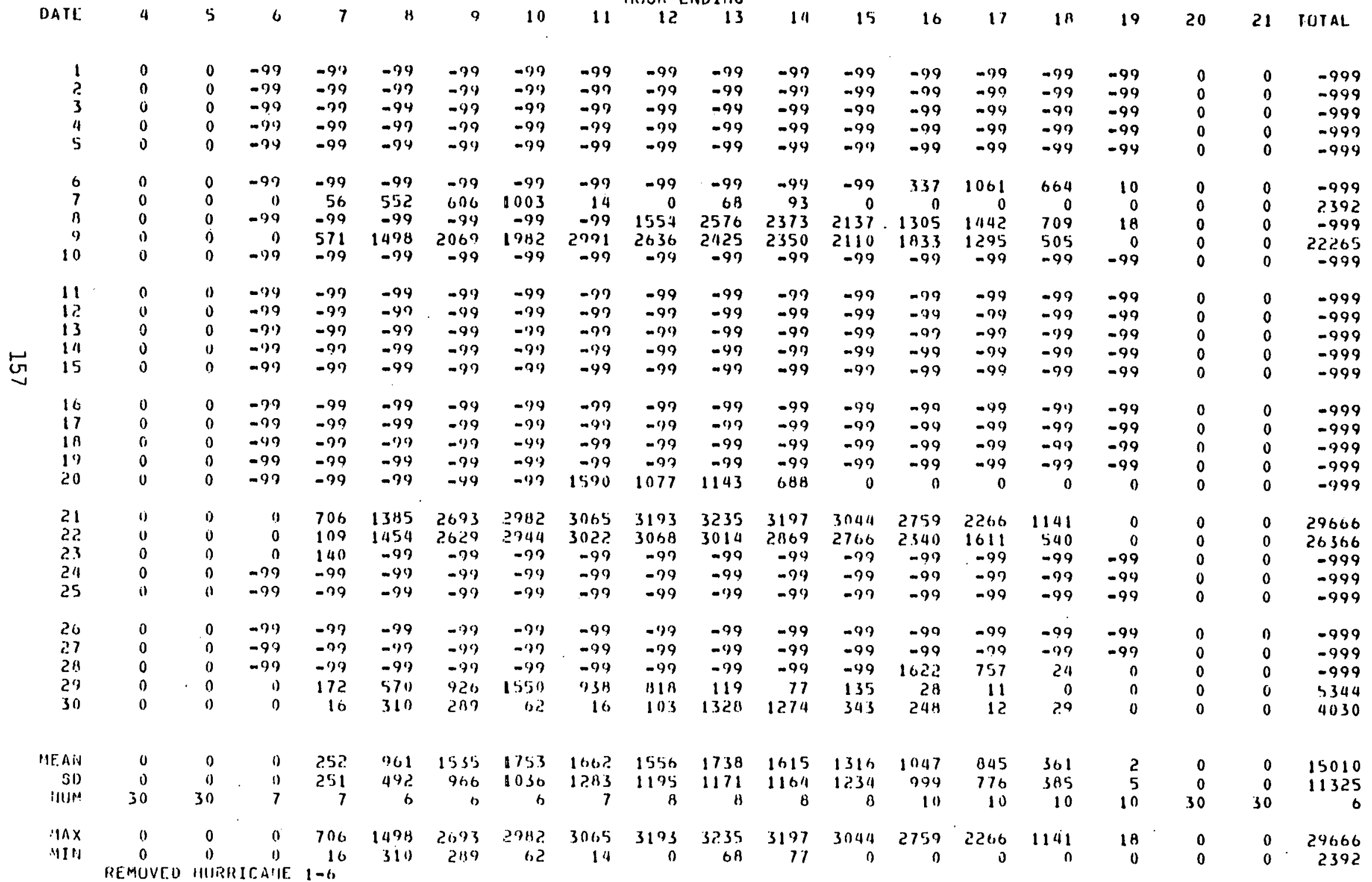


COASTAL - INLAHO SILLAR RAOIATION DIFFEREHCE STUDY

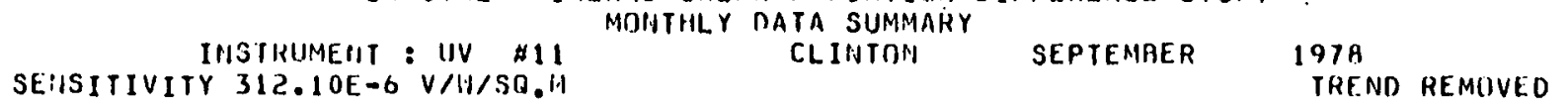

EHERGY KILIJJUULES PER SQUARE METEH.

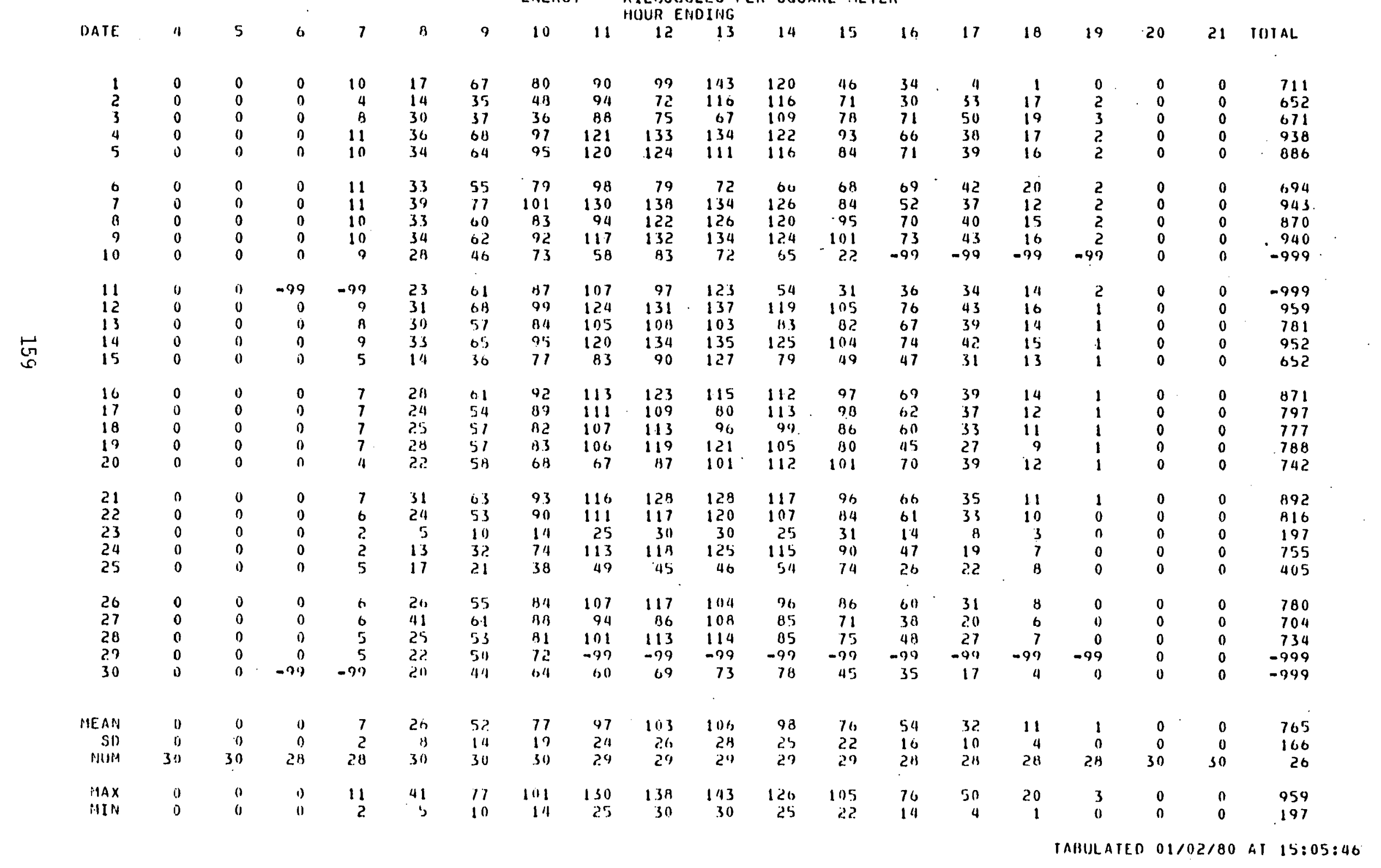


CIIASTAL - INLAHO SOLAR RADIATIOHI DIFFEREHCE STUOY MONTILY YATA SUMLHARY

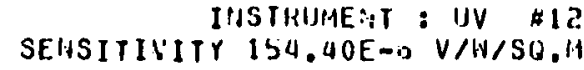
SLOOP SEPTEMRER

1978

ENERG KILUJUHLES PER SOUARE METER HOUR EHDIHA

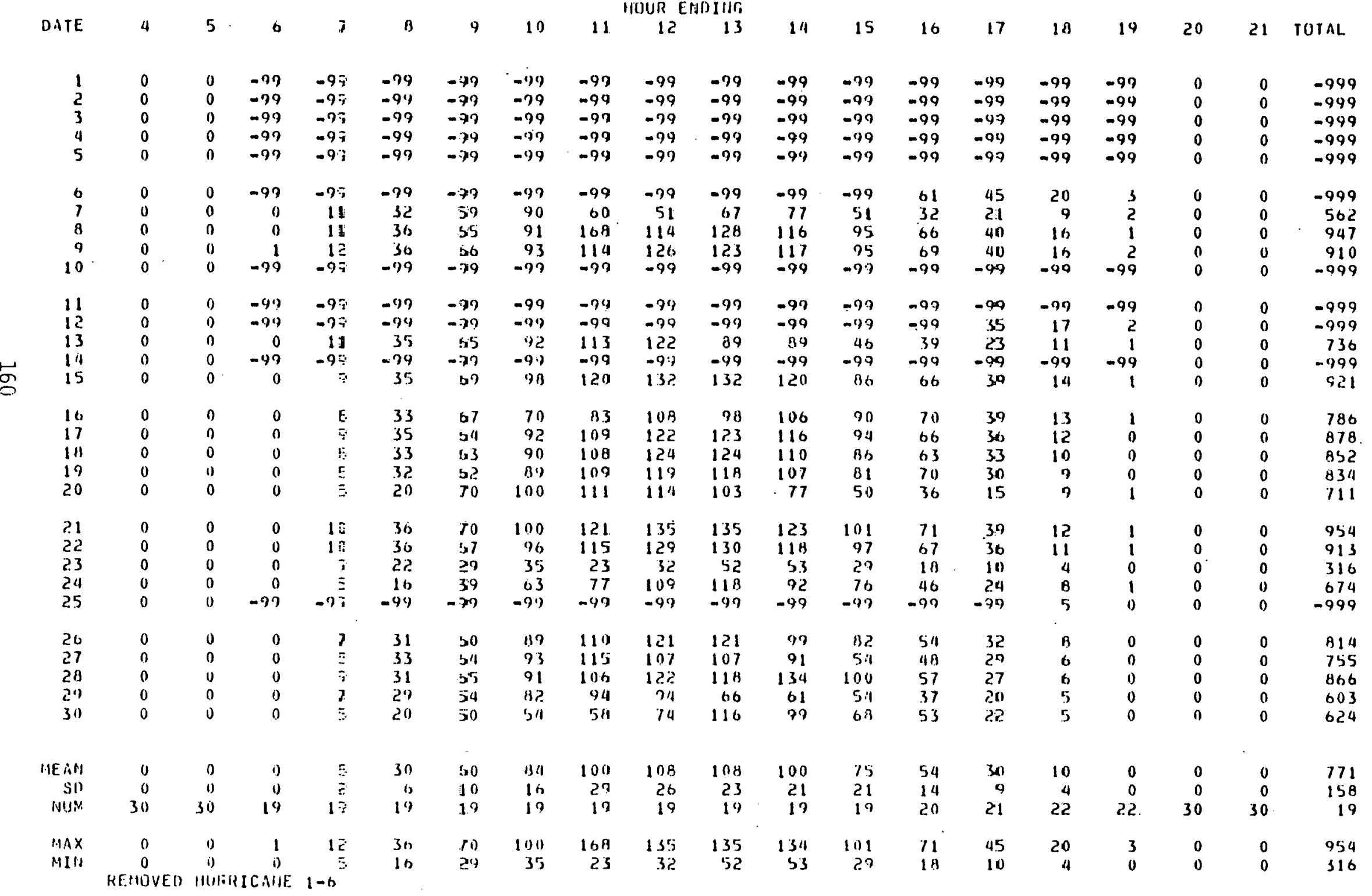




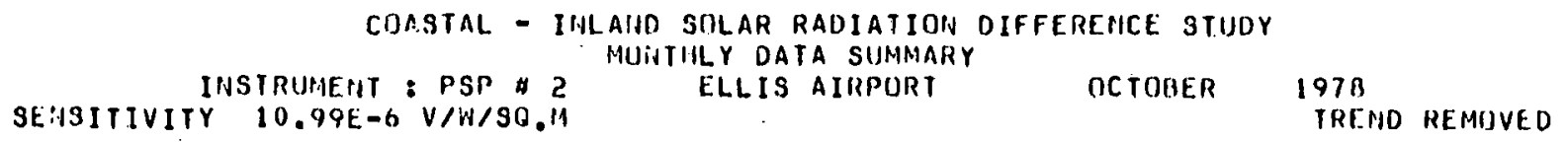

EHERGY KILDJOULES PER SGUARE METER HOUR ENDING

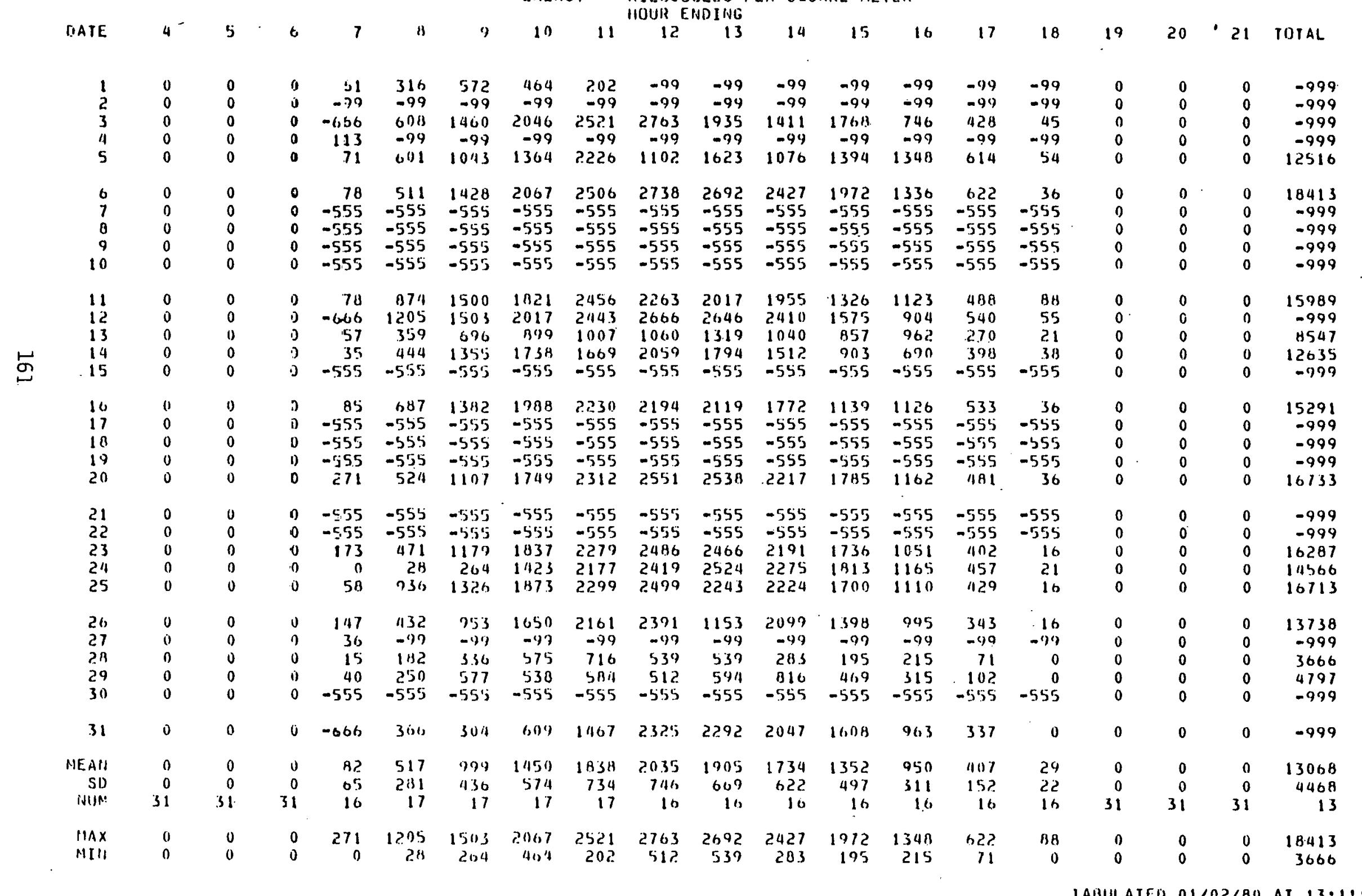


CURSTAL - EMLAHD SOLAR RADIATIUN DIFFEREHCE STULY

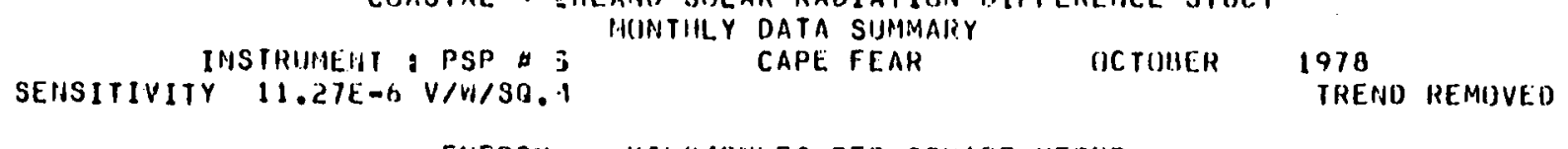

EHERGY KILOJUULES PER SQUIARE METER

\begin{tabular}{|c|c|c|c|c|c|c|c|c|c|c|c|c|c|c|c|c|c|c|c|}
\hline \multicolumn{20}{|c|}{ H(JUUR EHDING } \\
\hline DATE & 4 & 5 & 6 & 7 & $B$ & 9 & 10 & 11 & 12 & 13 & 14 & 15 & 16 & 17 & 18 & 19 & 20 & 21 & TUTAL \\
\hline $\begin{array}{l}1 \\
2 \\
3 \\
4 \\
5\end{array}$ & $\begin{array}{l}0 \\
0 \\
0 \\
0 \\
0\end{array}$ & $\begin{array}{l}0 \\
0 \\
0 \\
0 \\
0\end{array}$ & $\begin{array}{l}0 \\
0 \\
0 \\
0 \\
0\end{array}$ & $\begin{array}{r}61 \\
165 \\
145 \\
61 \\
81\end{array}$ & $\begin{array}{l}1144 \\
781 \\
733 \\
156 \\
707\end{array}$ & $\begin{array}{l}\text { 620 } \\
1431 \\
1441 \\
1050 \\
770\end{array}$ & $\begin{array}{l}1201 \\
2091 \\
2136 \\
2006 \\
1566\end{array}$ & $\begin{array}{r}524 \\
2011 \\
2570 \\
2552 \\
1014\end{array}$ & $\begin{array}{l}1185 \\
2717 \\
2375 \\
2012 \\
1090\end{array}$ & $\begin{array}{l}2220 \\
2433 \\
2570 \\
21140 \\
1764\end{array}$ & $\begin{array}{l}1303 \\
2041 \\
2104 \\
2207 \\
2361\end{array}$ & $\begin{array}{l}1361 \\
1270 \\
2040 \\
2095 \\
2026\end{array}$ & $\begin{array}{r}1220 \\
918 \\
1369 \\
1303 \\
1403\end{array}$ & $\begin{array}{l}7601 \\
550 \\
625 \\
607 \\
669\end{array}$ & $\begin{array}{r}124 \\
123 \\
113 \\
99 \\
54\end{array}$ & $\begin{array}{l}0 \\
0 \\
0 \\
0 \\
0\end{array}$ & $\begin{array}{l}0 \\
0 \\
0 \\
0 \\
0\end{array}$ & $\begin{array}{l}0 \\
0 \\
0 \\
0 \\
0\end{array}$ & $\begin{array}{l}10729 \\
17178 \\
18264 \\
16897 \\
14132\end{array}$ \\
\hline $\begin{array}{r}6 \\
7 \\
8 \\
7 \\
10\end{array}$ & $\begin{array}{l}0 \\
0 \\
0 \\
0 \\
0\end{array}$ & $\begin{array}{l}0 \\
0 \\
0 \\
0 \\
0\end{array}$ & $\begin{array}{l}0 \\
0 \\
0 \\
0 \\
0\end{array}$ & $\begin{array}{r}00 \\
152 \\
150 \\
200 \\
75\end{array}$ & $\begin{array}{l}711 \\
810 \\
511 \\
791 \\
3611\end{array}$ & $\begin{array}{r}1522 \\
1530 \\
1435 \\
1570 \\
752\end{array}$ & $\begin{array}{l}2133 \\
2212 \\
2172 \\
2212 \\
1122\end{array}$ & $\begin{array}{l}2012 \\
2672 \\
2640 \\
2647 \\
2230\end{array}$ & $\begin{array}{l}2842 \\
2 B 48 \\
2900 \\
2899 \\
2379\end{array}$ & $\begin{array}{l}2813 \\
2918 \\
2919 \\
2845 \\
2197\end{array}$ & $\begin{array}{l}2516 \\
2707 \\
2664 \\
2567 \\
1951\end{array}$ & $\begin{array}{l}20013 \\
2183 \\
2130 \\
2078 \\
1692\end{array}$ & $\begin{array}{l}1286 \\
1567 \\
1469 \\
1363 \\
1044\end{array}$ & $\begin{array}{l}527 \\
607 \\
707 \\
633 \\
632\end{array}$ & $\begin{array}{r}95 \\
110 \\
97 \\
53 \\
9 ?\end{array}$ & $\begin{array}{l}0 \\
0 \\
0 \\
0 \\
0\end{array}$ & $\begin{array}{l}0 \\
0 \\
0 \\
0 \\
0\end{array}$ & $\begin{array}{l}0 \\
0 \\
0 \\
0 \\
0\end{array}$ & $\begin{array}{l}19155 \\
20368 \\
19862 \\
19863 \\
14504\end{array}$ \\
\hline $\begin{array}{l}11 \\
12 \\
13 \\
11 \\
15\end{array}$ & $\begin{array}{l}0 \\
0 \\
0 \\
0 \\
0\end{array}$ & $\begin{array}{l}0 \\
0 \\
0 \\
0 \\
0\end{array}$ & $\begin{array}{l}0 \\
0 \\
0 \\
0 \\
0\end{array}$ & $\begin{array}{l}69 \\
39 \\
75 \\
911 \\
37\end{array}$ & $\begin{array}{l}605 \\
720 \\
370 \\
321 \\
701\end{array}$ & $\begin{array}{r}1251 \\
1436 \\
677 \\
1120 \\
1193\end{array}$ & $\begin{array}{r}2049 \\
2049 \\
814 \\
1577 \\
2123\end{array}$ & $\begin{array}{r}2151 \\
2519 \\
792 \\
1864 \\
2580\end{array}$ & $\begin{array}{r}2343 \\
2714 \\
719 \\
1778 \\
2723\end{array}$ & $\begin{array}{r}2797 \\
2599 \\
773 \\
2302 \\
2829\end{array}$ & $\begin{array}{r}2439 \\
2212 \\
680 \\
1041 \\
2519\end{array}$ & $\begin{array}{r}1980 \\
1679 \\
648 \\
1646 \\
1963\end{array}$ & $\begin{array}{r}1343 \\
1155 \\
709 \\
947 \\
1334\end{array}$ & $\begin{array}{l}593 \\
477 \\
113 \\
393 \\
593\end{array}$ & $\begin{array}{r}73 \\
72 \\
5 \\
8 ? \\
53\end{array}$ & $\begin{array}{l}0 \\
0 \\
0 \\
0 \\
0\end{array}$ & $\begin{array}{l}0 \\
0 \\
0 \\
0 \\
0\end{array}$ & $\begin{array}{l}0 \\
0 \\
0 \\
0 \\
0\end{array}$ & $\begin{array}{r}17595 \\
17612 \\
6440 \\
13471 \\
18948\end{array}$ \\
\hline $\begin{array}{l}16 \\
17 \\
18 \\
19 \\
20\end{array}$ & $\begin{array}{l}0 \\
0 \\
0 \\
0 \\
0\end{array}$ & $\begin{array}{l}0 \\
0 \\
0 \\
0 \\
0\end{array}$ & $\begin{array}{l}0 \\
0 \\
0 \\
0 \\
0\end{array}$ & $\begin{array}{l}92 \\
63 \\
89 \\
78 \\
6 ?\end{array}$ & $\begin{array}{l}6513 \\
607 \\
654 \\
549 \\
613\end{array}$ & $\begin{array}{l}130.3 \\
1457 \\
14.1 \\
1308 \\
1351\end{array}$ & $\begin{array}{l}1964 \\
2089 \\
2047 \\
1883 \\
1834\end{array}$ & $\begin{array}{l}21.69 \\
2539 \\
2: 78 \\
2356 \\
2 ? 15\end{array}$ & $\begin{array}{l}2727 \\
2766 \\
2680 \\
2161 \\
2625\end{array}$ & $\begin{array}{l}2708 \\
2724 \\
2635 \\
2273 \\
2638\end{array}$ & $\begin{array}{l}2434 \\
2389 \\
2373 \\
2353 \\
2248\end{array}$ & $\begin{array}{l}2015 \\
1052 \\
1891 \\
1942 \\
1724\end{array}$ & $\begin{array}{r}795 \\
1239 \\
1217 \\
1168 \\
1162\end{array}$ & $\begin{array}{l}341 \\
55 ? \\
507 \\
465 \\
175\end{array}$ & $\begin{array}{l}57 \\
54 \\
41 \\
34 \\
44\end{array}$ & $\begin{array}{l}0 \\
0 \\
0 \\
0 \\
0\end{array}$ & $\begin{array}{l}0 \\
0 \\
0 \\
0 \\
0\end{array}$ & $\begin{array}{l}0 \\
0 \\
0 \\
0 \\
0\end{array}$ & $\begin{array}{l}17623 \\
18371 \\
18029 \\
16523 \\
17101\end{array}$ \\
\hline $\begin{array}{l}21 \\
22 \\
23 \\
24 \\
25\end{array}$ & $\begin{array}{l}0 \\
0 \\
0 \\
0 \\
0\end{array}$ & $\begin{array}{l}0 \\
0 \\
0 \\
0 \\
0\end{array}$ & $\begin{array}{l}0 \\
0 \\
0 \\
0 \\
0\end{array}$ & $\begin{array}{l}76 \\
67 \\
60 \\
29 \\
63\end{array}$ & $\begin{array}{r}629 \\
469 \\
475 \\
38 \\
540\end{array}$ & $\begin{array}{r}1317 \\
148 B \\
1217 \\
1147 \\
1312\end{array}$ & $\begin{array}{l}1948 \\
1683 \\
1891 \\
334 \\
1926\end{array}$ & $\begin{array}{r}2.95 \\
2903 \\
2.331 \\
956 \\
2.535\end{array}$ & $\begin{array}{l}2603 \\
2491 \\
2581 \\
1966 \\
2517\end{array}$ & $\begin{array}{l}2619 \\
.2475 \\
.2389 \\
2292 \\
2481\end{array}$ & $\begin{array}{l}2331 \\
2223 \\
2255 \\
2250 \\
2341\end{array}$ & $\begin{array}{l}1830 \\
1731 \\
1772 \\
1778 \\
1903\end{array}$ & $\begin{array}{l}1204 \\
1098 \\
1118 \\
1148 \\
1143\end{array}$ & $\begin{array}{l}485 \\
42.8 \\
43: \\
452 \\
43:\end{array}$ & $\begin{array}{l}28 \\
25 \\
25 \\
24 \\
19\end{array}$ & $\begin{array}{l}0 \\
0 \\
0 \\
0 \\
0\end{array}$ & $\begin{array}{l}0 \\
0 \\
0 \\
0 \\
0\end{array}$ & $\begin{array}{l}0 \\
0 \\
0 \\
0 \\
0\end{array}$ & $\begin{array}{l}17467 \\
16481 \\
16605 \\
11471 \\
17061\end{array}$ \\
\hline $\begin{array}{l}26 \\
27 \\
28 \\
29 \\
30\end{array}$ & $\begin{array}{l}0 \\
0 \\
0 \\
0 \\
0\end{array}$ & $\begin{array}{l}0 \\
0 \\
0 \\
0 \\
0\end{array}$ & $\begin{array}{l}0 \\
0 \\
0 \\
0 \\
0\end{array}$ & $\begin{array}{r}54 \\
-70 \\
-99 \\
11 \\
51\end{array}$ & $\begin{array}{r}527 \\
-74 \\
-99 \\
82 \\
156\end{array}$ & $\begin{array}{r}1101 \\
-97 \\
-61 \\
171 \\
1079\end{array}$ & $\begin{array}{r}1780 \\
-79 \\
274 \\
321 \\
1728\end{array}$ & $\begin{array}{r}22115 \\
-79 \\
042 \\
679 \\
2133\end{array}$ & $\begin{array}{r}2446 \\
-49 \\
546 \\
650 \\
2351\end{array}$ & $\begin{array}{r}2309 \\
-99 \\
610 \\
753 \\
2322\end{array}$ & $\begin{array}{r}2007 \\
-99 \\
421 \\
576 \\
1766\end{array}$ & $\begin{array}{r}1696 \\
-99 \\
377 \\
685 \\
1517\end{array}$ & $\begin{array}{r}8060 \\
-99 \\
131 \\
366 \\
919\end{array}$ & $\begin{array}{r}380 \\
-99 \\
48 \\
142 \\
306\end{array}$ & $\begin{array}{r}19 \\
-99 \\
0 \\
11 \\
12\end{array}$ & $\begin{array}{l}0 \\
0 \\
0 \\
0 \\
0\end{array}$ & $\begin{array}{l}0 \\
0 \\
0 \\
0 \\
0\end{array}$ & $\begin{array}{l}0 \\
0 \\
0 \\
0 \\
0\end{array}$ & $\begin{array}{r}15724 \\
-999 \\
-999 \\
4467 \\
14640\end{array}$ \\
\hline 31 & 0 & 0 & 1) & 73 & 106 & $30 . y$ & 731 & 977 & 1363 & 2162 & 1013 & 1593 & 955 & 309 & $n$ & 0 & 0 & 0 & 10615 \\
\hline $\begin{array}{r}\text { MEAll } \\
\text { SO } \\
\text { HUM }\end{array}$ & $\begin{array}{r}0 \\
0 \\
31\end{array}$ & $\begin{array}{r}0 \\
0 \\
31\end{array}$ & $\begin{array}{r}n \\
31\end{array}$ & $\begin{array}{l}81 \\
42 \\
25\end{array}$ & $\begin{array}{r}530 \\
2013 \\
29\end{array}$ & $\begin{array}{r}116.7 \\
411 \\
6.7\end{array}$ & $\begin{array}{r}1660 \\
593 \\
311\end{array}$ & $\begin{array}{r}2050 \\
670 \\
30\end{array}$ & $\begin{array}{r}2202 \\
6913 \\
30\end{array}$ & $\begin{array}{r}2326 \\
546 \\
30\end{array}$ & $\begin{array}{r}2060 \\
570 \\
30\end{array}$ & $\begin{array}{r}167 t \\
448 \\
30\end{array}$ & $\begin{array}{r}1105 \\
299 \\
30\end{array}$ & $\begin{array}{r}473 \\
108 \\
30\end{array}$ & $\begin{array}{l}55 \\
37 \\
30\end{array}$ & $\begin{array}{r}0 \\
0 \\
31\end{array}$ & $\begin{array}{r}0 \\
0 \\
31\end{array}$ & $\begin{array}{r}0 \\
0 \\
31\end{array}$ & $\begin{array}{r}15765 \\
3168 \\
29\end{array}$ \\
\hline $\begin{array}{l}\text { MAXX } \\
\text { MIII }\end{array}$ & $\begin{array}{l}0 \\
0\end{array}$ & $\begin{array}{l}0 \\
\text { I) }\end{array}$ & $\begin{array}{l}0 \\
0\end{array}$ & $\begin{array}{r}20 \mathrm{C} \\
11\end{array}$ & $\begin{array}{r}8111 \\
\text { H2 }\end{array}$ & $\begin{array}{r}1 S F 9 \\
1199\end{array}$ & $\begin{array}{r}2212 \\
274\end{array}$ & $\begin{array}{r}2672 \\
53.4\end{array}$ & $\begin{array}{r}29110 \\
546\end{array}$ & $\begin{array}{r}2919 \\
610\end{array}$ & $\begin{array}{r}2707 \\
421\end{array}$ & $\begin{array}{r}2183 \\
377\end{array}$ & $\begin{array}{r}1567 \\
131\end{array}$ & $\begin{array}{l}76 \% \\
4 \%\end{array}$ & $\begin{array}{r}124 \\
0\end{array}$ & $\begin{array}{l}0 \\
0\end{array}$ & $\begin{array}{l}0 \\
0\end{array}$ & $\begin{array}{l}0 \\
0\end{array}$ & $\begin{array}{r}20368 \\
4467\end{array}$ \\
\hline & & & & & & , & 1 & & & & & & & & & IJIJL & & $1 \mathrm{HO}$ & AI 13 \\
\hline
\end{tabular}


CUIASTAL - INLAHD SILLAR RADIATIUN DIFFERENCE STUUY

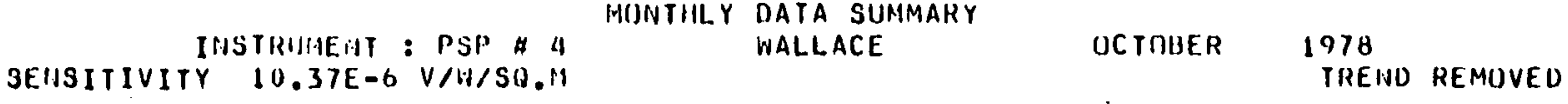

ENERGY KILIIJOULES PER SIJUARE METER IIOUR ENDIHG

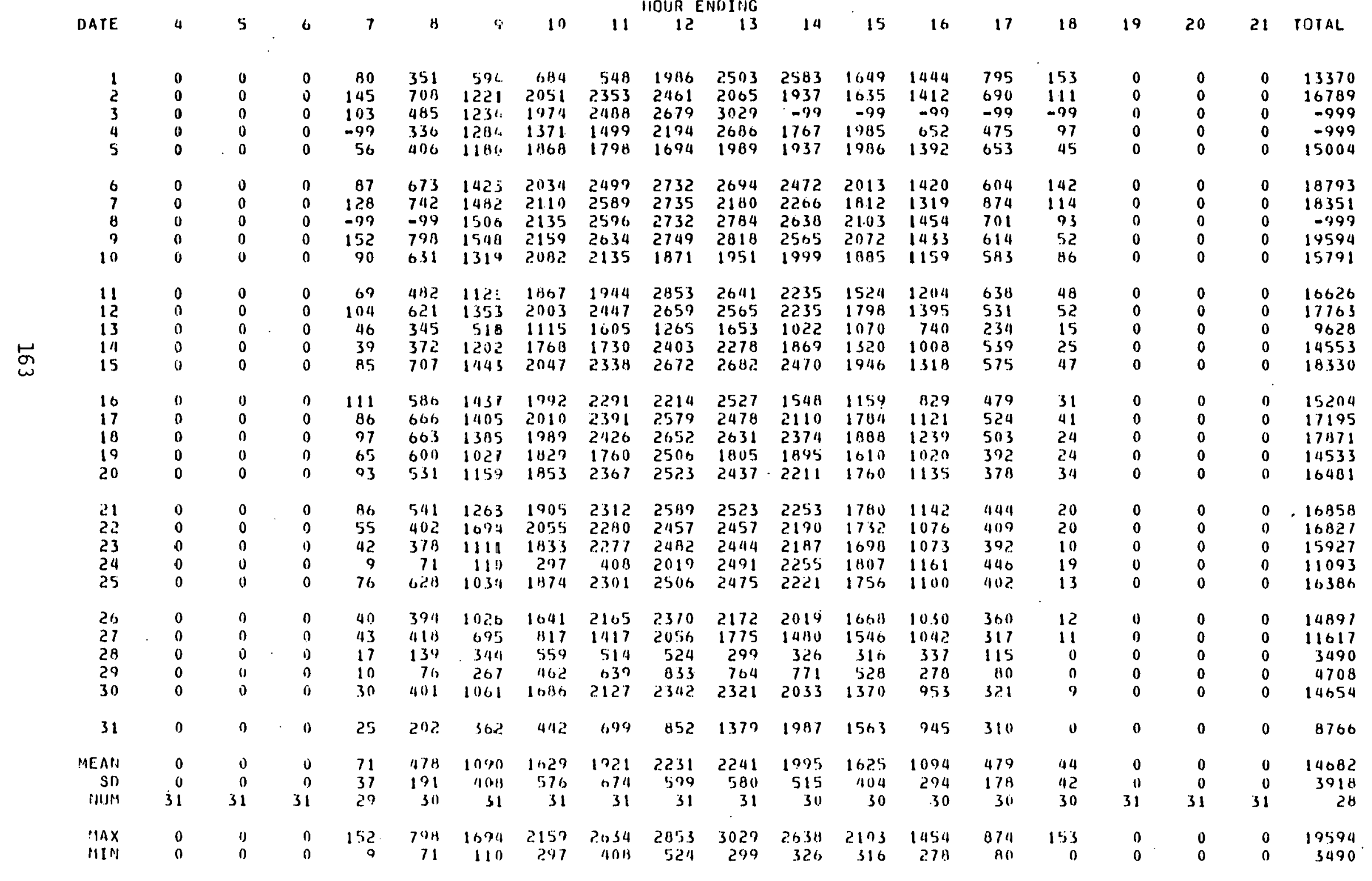


CIJASTAL - IIILAHD STLAR RADIATIUN DIFFEHEHCE STIJUY HONITILLY DATA SIJMMARY

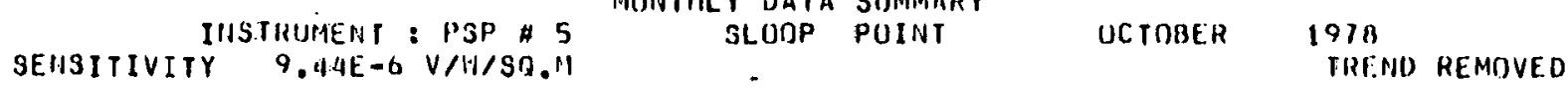

EHERGY KILOJJULES PER STUARF. METER

$10 \quad 111^{110 U R \text { ENOING }}$

STUARF. METER

$\begin{array}{ll}18 & 37 \\ 37 & 72 \\ 29 & 72 \\ 61 & 5 \\ 80 & 2\end{array}$

$\begin{array}{lr}377 & 186 \\ 729 & 1273 \\ 720 & 1372 \\ 583 & 534\end{array}$

$\begin{array}{rr}674 & 659 \\ 1830 & 2337\end{array}$

$\begin{array}{lllll}785 & 1384 & 2104 & 1620 & 1491\end{array}$

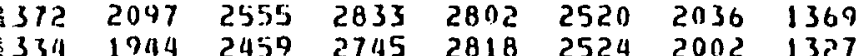

$\begin{array}{llllllllll}91 & 715 & 1399 & 2131 & 2551 & 2791 & 2764 & 2467 & 1960 & 1304\end{array}$

141

$\begin{array}{llll}715 & 1399 & 2131 & 2551 \\ 751 & 1533 & 2177 & 2631\end{array}$

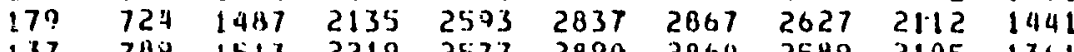

$\begin{array}{lllll}2791 & 2764 & 2467 & 1960 & 1304 \\ 2375 & 2924 & 2558 & 2002 & 1506\end{array}$

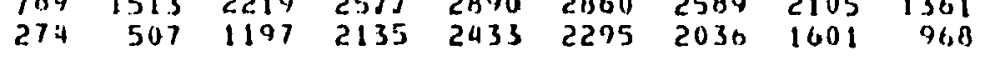

$\begin{array}{lllllllll}443 & 1029 & 1748 & 2533 & 2616 & 2177 & 2600 & 1983 & 1346\end{array}$

$\begin{array}{rrrrrrrrr}563 & 1315 & 1956 & 2479 & 2734 & 1319 & 2242 & 2658 & 1132 \\ 427 & 111 & 705 & 732 & 575 & 655 & 5613 & 562 & 335\end{array}$

$\begin{array}{llllllllll}377 & 1212 & 16113 & 1891 & 1841 & 2177 & 1868 & 1033 & 808\end{array}$

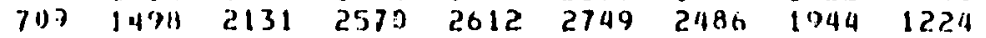

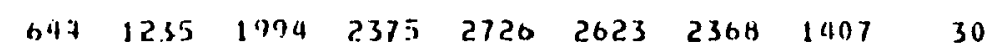

6B; 1449 2.276 $25 ? 4$

697 $1.1872021 \quad 2453 \quad 263626612364$ 1872 1200

$148 \quad 945 \quad 397 \quad 1464 \quad 2073 \quad 1826 \quad 2665 \quad 2368 \quad 1902 \quad 1250$

$\begin{array}{llllllllll}4139 & 1075 & 1.521 & 2322 & 2406 & 2593 & 2341 & 1853 & 1281\end{array}$

1133 1132. 1868 2345 2616 $2631 \quad 2398.1941 \quad 1311$

313 1327 1780 22402516 2631 2398 1941 1311

47: B2s 1563 22)3 2463 2535 2295 1818 1174

3) 100 50s $957 \quad 1544 \quad 2291 \quad 2257 \quad 1864 \quad 1269$

$\begin{array}{lllllllllllll}20 & 44 ? & 1136 & 1761 & 2245 & 2470 & 2524 & 2303 & 1868 & 1239\end{array}$

$\begin{array}{lllllllll}371 & 981 & 1675 & 2173 & 2366 & 2472 & 2183 & 1459 & 1054\end{array}$

4A3 1114 1716 943 1456.2196 1911 $495 \quad 449$

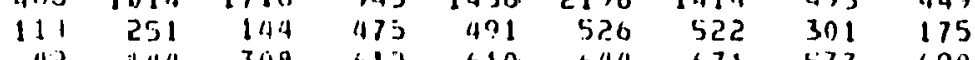

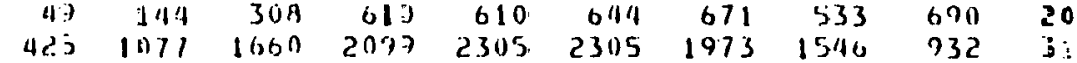

32

233 B?

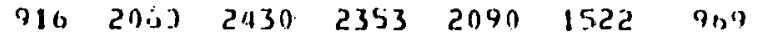

$3: 1$

HEA

SIU

$\begin{array}{rrrrrrrrrr}77 & 199 & 103 i & 1603 & 2017 & 2205 & 2245 & 2035 & 1621 & 1073 \\ 46 & 221 & 416 & 577 & 003 & 727 & 077 & 564 & 547 & 371 \\ 31 & 31 & 31 & 31 & 31 & 31 & 31 & 51 & 31 & 31\end{array}$

10381

16072

19130

18501

13106

18612

19847

19768

19782
14204

17416

16938

5634

13390

18661

15 AS5

17624

18074

16216

17419

16357

15988

11496

16625

15495

10412

3087

4468
14677

13539

15011

4297
31

19847

3087 
CIJASTAL - INLAHID SOLAR RADIATIINM IIFFEREHCE STUDY MUNTILY DATA SUMAMARY

CLINTIJI OCTIMER 1978

SEHSITIVITY $11.0 C E-0 \mathrm{~V} / \mathrm{A} / \mathrm{SH}, \mathrm{i}$ TREND REMOVED

EiJERGY KILOJJUULES PER SQUARE HETER

DATE HOUR ENDING

1
2
3
4
5
0
7
8
7
10
11
12
13
14
15

34

B

14

16

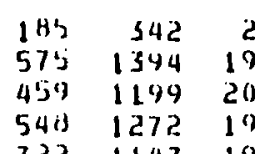

$\begin{array}{rr}213 & 67 \\ 7351\end{array}$

8822640

$252 B \quad 2060 \quad 1272$

54012721052

2462

2627

2590

209
239
207

$\begin{array}{rrr}1633 & 1112 \\ 3 & 1480 & 789\end{array}$

133

$606 \quad 1303 \quad 2073 \quad 2328$

10
10
11

$7113 \quad 1453 \quad 2094 \quad 249$

643 1133 2133 2559

$2666 \quad 2437$

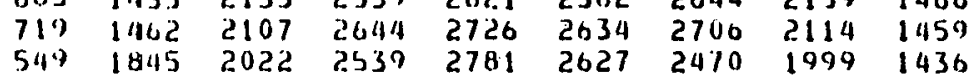

68
281
90
55

$\begin{array}{lllllllll}3144 & 628 & 2143 & 2006 & 1973 & 2588 & 2245 & 1799 & 1345\end{array}$

$\begin{array}{lllllllll}542 & 1158 \quad 1930 & 2362 & 2546 & 2542 & 2271 & 1891 & 1233\end{array}$

$\begin{array}{llllllllll}530 & 725 & 1023 & 938 & 1294 & 1520 & 1327 & 1114 & 804 & 224\end{array}$

$\begin{array}{rrrrrrrrr}530 & 916 & 1313 & 2117 & 2156 & 2025 & 1508 & 998 & 831 \\ 617 & 1353 & 1971 & 2495 & 2361 & 2531 & 2528 & 2027 & 1343\end{array}$

$65 \quad 451 \quad 1741 \quad 1174 \quad 179 b \quad 2228 \quad 1888 \quad 2428 \quad 1750 \quad 1279$

$\begin{array}{llllllllll}57 & 512 & 1265 & 1028 & 1936 & 2250 & 2538 & 2155 & 2001 & 1213\end{array}$

$497 \quad 1263 \quad 1004200526412637290819341239$

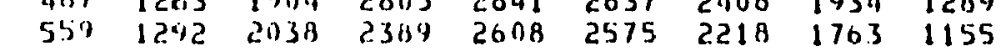

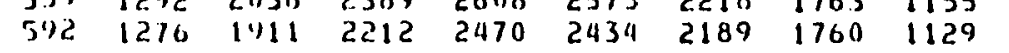

17

18

19

.20

21 TUTAL

189

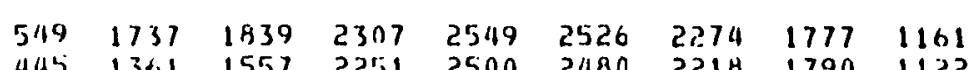

$\begin{array}{lllllllll}445 & 1361 & 1557 & 2251 & 2500 & 2480 & 2218 & 1790 & 1122\end{array}$

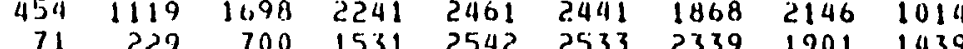

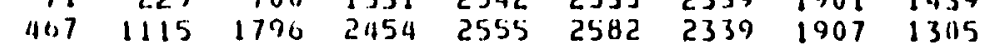

$\begin{array}{llll}209 & 390 & 1629 & 2071 \\ 111 & 360 & 1021 & 1721\end{array}$

$\begin{array}{lllllll}71 & 2339 & 2382 & 2123 & 1606 & 821 & 435 \\ 21 & 2225 & 2281 & 1855 & 1279 & 490 & 349\end{array}$

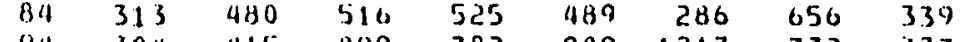

$\begin{array}{llllllll}304 & 415 & 899 & 732 & 909 & 1217 & 772 & 337\end{array}$

$\begin{array}{ll}529 & 172 \\ 713 & 134\end{array}$

524

60

106

129

$542 \quad 77$

454

77
117

121

150

$654 \quad 31$

$B 1$
86

IB

526
607

57

399

42

581

510

39

99

$477 \quad 26$

$510 \quad 22$

-99
65
45

45

35

27

31)

31

112523

$2097 \quad 2379 \quad 2398$

$691711 \quad 1093 \quad 45$

NOS

IHIM 3

$\max$

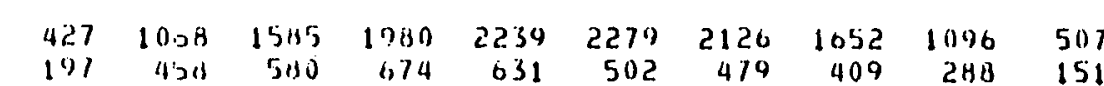

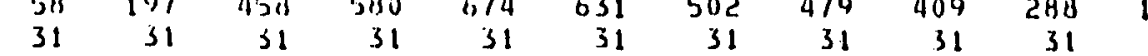

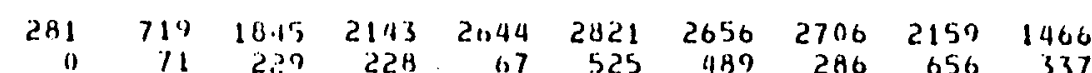

337

87

TABULATED $01 / 02 / 80$ AT $13: 11: 32$ 
CIIASTAL - INLAHD SULAR RADIATION DIFFERENCE STUDY

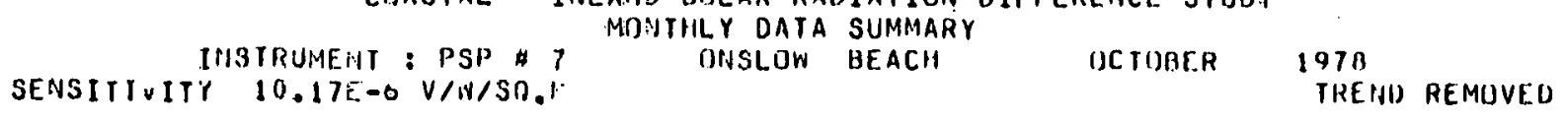

EIIEPGY KILOJOULES PER SOUARE METER

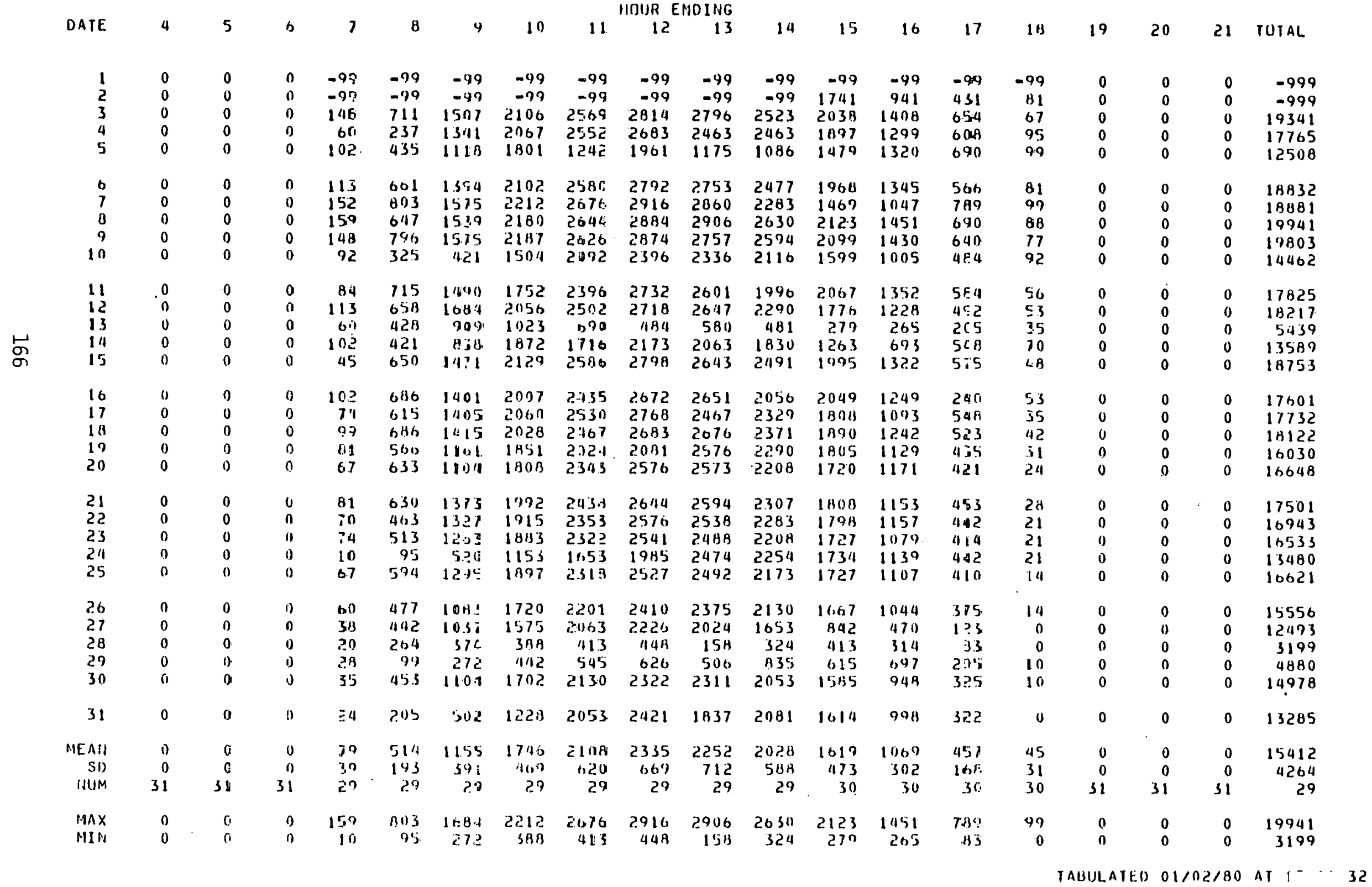


CIIASTAL. - INLAND SILAR RALIATIOH DIFFERENCE BTUDY IHSIRUHEHT: IIIP A 8
SEHSITIVITY MOHTHLY DATA SUMHARY OCTOHER 1978

TREHD REMUVED

EHERGY KILUJOULES FER SUUARE HFTER

HOURR ENDING

DATE

$10 \quad 11^{12} 12^{13}$

$14 \quad 15 \cdot 16 \quad 17$

18

21 TOTAL

\begin{tabular}{|c|c|c|c|c|c|c|c|c|c|c|c|}
\hline $\begin{array}{r}0 \\
164 \\
263 \\
0 \\
0\end{array}$ & $\begin{array}{r}66 \\
1145 \\
1420 \\
224 \\
0\end{array}$ & $\begin{array}{r}0 \\
1025 \\
2004 \\
1035 \\
504\end{array}$ & $\begin{array}{r}53 \\
1728 \\
21115 \\
1627 \\
521\end{array}$ & $\begin{array}{r}16 \\
1687 \\
2610 \\
2103 \\
517\end{array}$ & $\begin{array}{r}19 \\
1861 \\
2788 \\
1846 \\
124\end{array}$ & $\begin{array}{r}165 \\
1372 \\
2630 \\
2169 \\
99\end{array}$ & $\begin{array}{r}587 \\
1017 \\
2370 \\
20128 \\
289\end{array}$ & $\begin{array}{r}1055 \\
710 \\
2130 \\
1594 \\
298\end{array}$ & $\begin{array}{r}2180 \\
652 \\
1575 \\
1019 \\
786\end{array}$ & $\begin{array}{r}2412 \\
623 \\
764 \\
431 \\
397\end{array}$ & $\begin{array}{r}910 \\
152 \\
52 \\
03 \\
45\end{array}$ \\
\hline $\begin{array}{r}16 \\
36 \\
-99 \\
-79 \\
-97\end{array}$ & $\begin{array}{l}269 \\
119 \\
-99 \\
-99 \\
-99\end{array}$ & $\begin{array}{l}649 \\
206 \\
-97 \\
-99 \\
-99\end{array}$ & $\begin{array}{l}885 \\
317 \\
-99 \\
-99 \\
-94\end{array}$ & $\begin{array}{r}1109 \\
379 \\
-99 \\
-99 \\
-99\end{array}$ & $\begin{array}{r}1270 \\
350 \\
-99 \\
-99 \\
-99\end{array}$ & $\begin{array}{r}1216 \\
268 \\
-99 \\
-99 \\
-99\end{array}$ & $\begin{array}{l}980 \\
131 \\
-99 \\
-99 \\
-99\end{array}$ & $\begin{array}{r}658 \\
23 \\
-99 \\
-99 \\
-99\end{array}$ & $\begin{array}{r}343 \\
15 \\
-99 \\
-99 \\
-99\end{array}$ & $\begin{array}{r}49 \\
0 \\
-99 \\
-99 \\
-99\end{array}$ & $\begin{array}{r}0 \\
0 \\
-99 \\
-99 \\
-99\end{array}$ \\
\hline $\begin{array}{l}-97 \\
-99 \\
-99 \\
-99 \\
-99\end{array}$ & $\begin{array}{l}-99 \\
-99 \\
-99 \\
-99 \\
-99\end{array}$ & $\begin{array}{l}-97 \\
-99 \\
-99 \\
-97 \\
-99\end{array}$ & $\begin{array}{l}-79 \\
-99 \\
-99 \\
-99 \\
-99\end{array}$ & $\begin{array}{l}-99 \\
-99 \\
-99 \\
-99 \\
-97\end{array}$ & $\begin{array}{l}-99 \\
-99 \\
-99 \\
-99 \\
-99\end{array}$ & $\begin{array}{l}-99 \\
-99 \\
-99 \\
-99 \\
-99\end{array}$ & $\begin{array}{l}-99 \\
-99 \\
-99 \\
-99 \\
-97\end{array}$ & $\begin{array}{l}-99 \\
-99 \\
-99 \\
-99 \\
-99\end{array}$ & $\begin{array}{l}-99 \\
-99 \\
-99 \\
-99 \\
-99\end{array}$ & $\begin{array}{l}-99 \\
-99 \\
-99 \\
-99 \\
-99\end{array}$ & $\begin{array}{l}-99 \\
-99 \\
-99 \\
-99 \\
-99\end{array}$ \\
\hline $\begin{array}{l}-99 \\
-79 \\
-99 \\
-99\end{array}$ & $\begin{array}{l}-99 \\
-99 \\
-99 \\
-99\end{array}$ & $\begin{array}{l}-139 \\
-99 \\
-99 \\
-99\end{array}$ & $\begin{array}{l}-49 \\
-99 \\
-99 \\
-99\end{array}$ & $\begin{array}{l}-99 \\
-99 \\
-97 \\
-99\end{array}$ & $\begin{array}{l}-99 \\
-99 \\
-99 \\
-49\end{array}$ & $\begin{array}{l}-99 \\
-99 \\
-99 \\
-99\end{array}$ & $\begin{array}{l}-99 \\
-99 \\
-99 \\
-99\end{array}$ & $\begin{array}{l}-99 \\
-97 \\
-99 \\
-97\end{array}$ & $\begin{array}{l}-99 \\
-99 \\
-99 \\
-99\end{array}$ & $\begin{array}{l}-09 \\
-97 \\
-99 \\
-99\end{array}$ & $\begin{array}{l}-99 \\
-99 \\
-99 \\
-99\end{array}$ \\
\hline
\end{tabular}

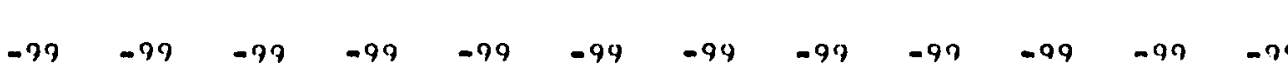

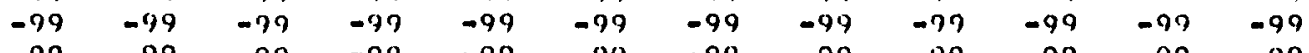

$\begin{array}{lllllll}-99 & -79 & -79 & -99 & -99 & -99 & -99\end{array}$

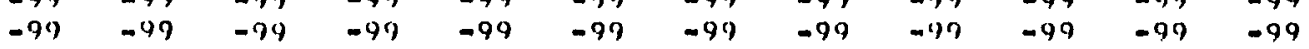

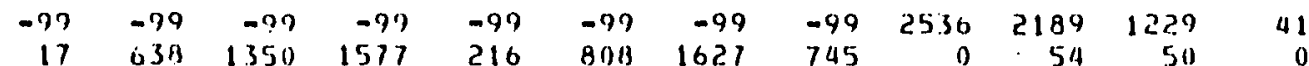

$\begin{array}{rrrrrrrrrrr}0 & 1 & 0 & 0 & 0 & 0 & 0 & 0 & 0 & 0 & 0 \\ 0 & 0 & 0 & 0 & 0 & 0 & 0 & 0 & 0 & 74 & 0\end{array}$

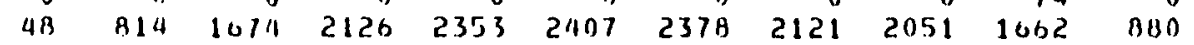

0
0

0
15

$n$
0
0

0
0
0

0

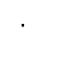

$\begin{array}{rrr}\text { MENII } & 0 & 0 \\ \text { SII } & 0 & 0 \\ \text { MIM } & 31 & 31\end{array}$

MAX
AT N

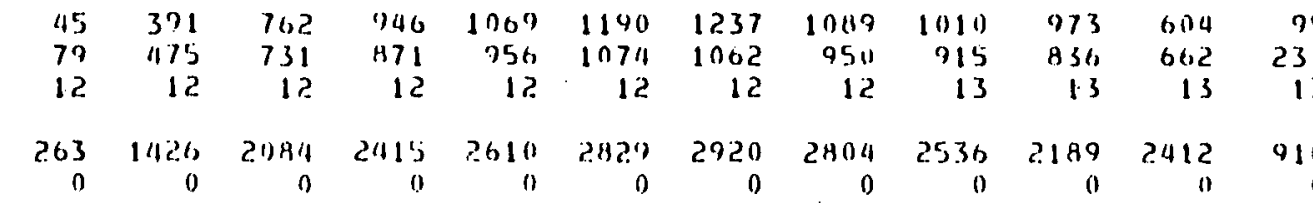


CIIASTAL - IHLANIS SOLAR RADIATION OIFFEREHCE STUDY MONTHLY DATA SUMHARY

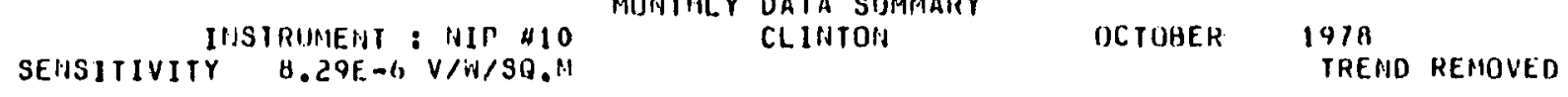

EHERGY KILUJUULES PEH SOUUARE METER HOUR EHDIHG

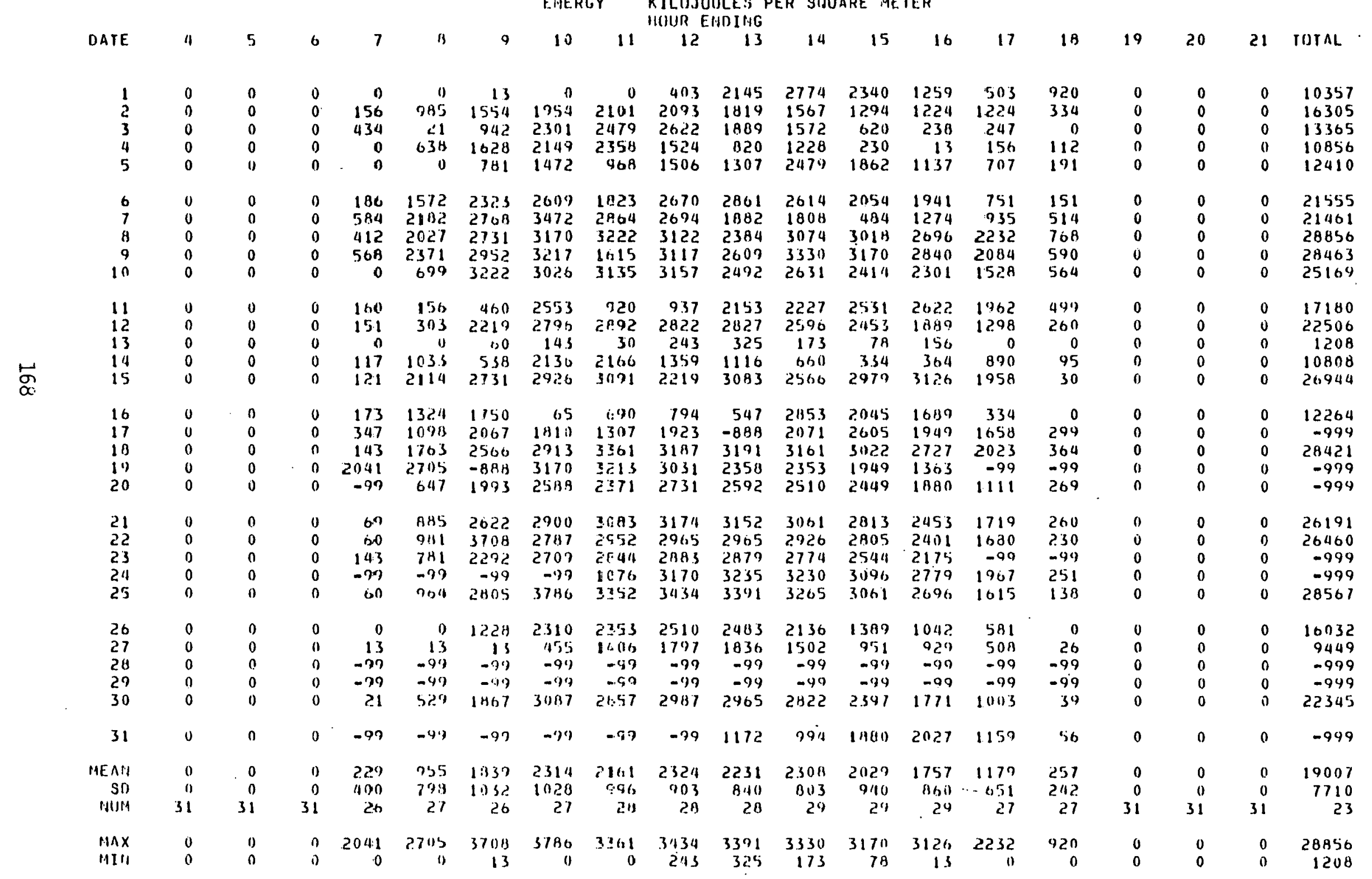


COASTAL - INLAND 3ULAR RADIATIUIN DIFFFRENICE STUOY

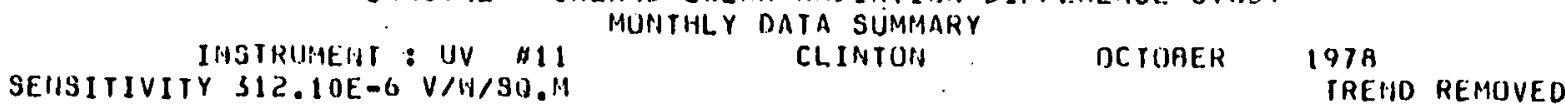

EINERG KILOJOULES PER 3NUARE METER

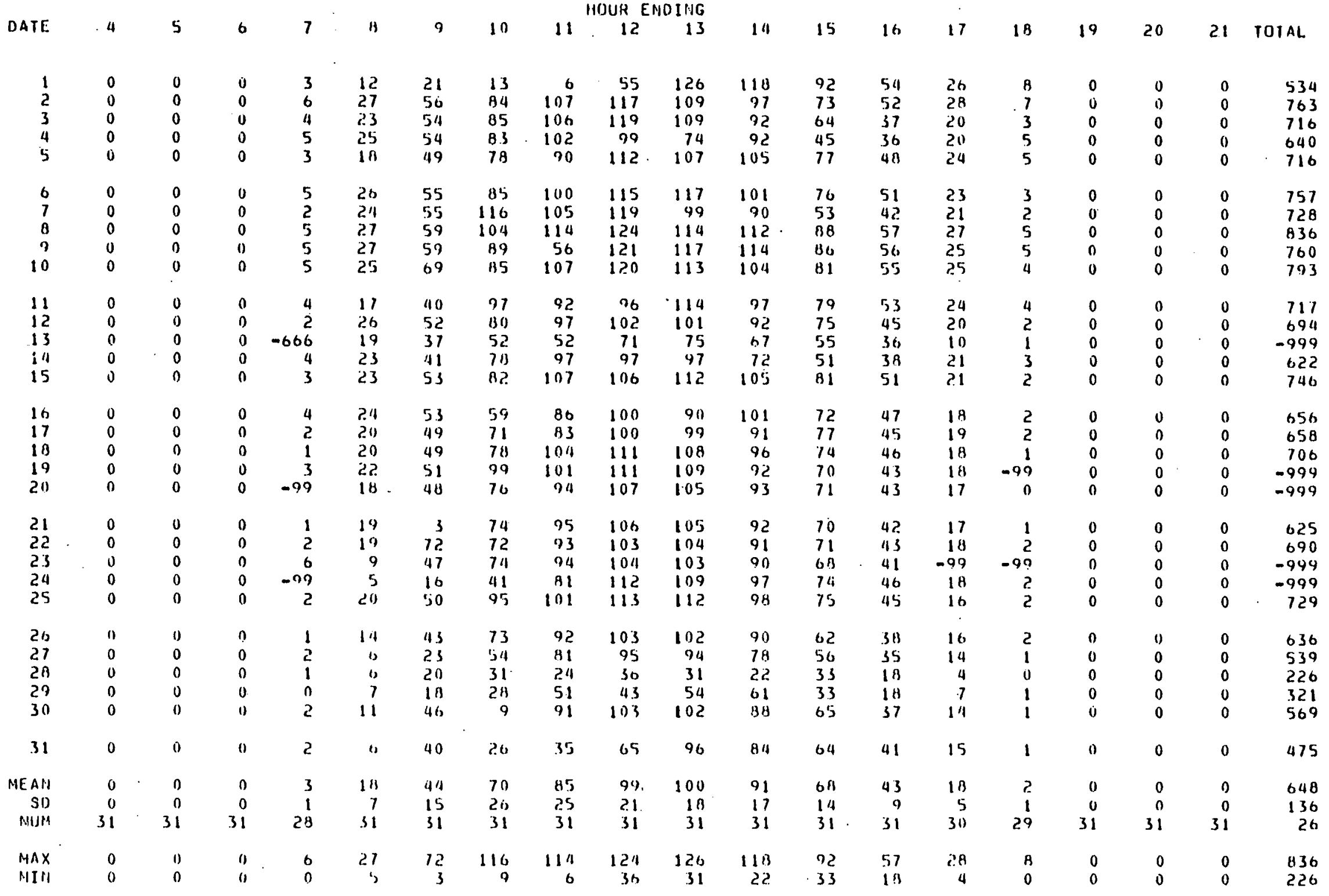


CUNSTAL - INLAND SOLAR RADIATIUIN DIFFEREHCE S:UDY INSTRUMENT: UV \#12 MUHTHLY DATA SUMAARY
SENGTIIITY $154.40 E-6 V / W / S O . M$

EINERGY KILIJJUUL.F. PER SIJUARE ME.TF.R

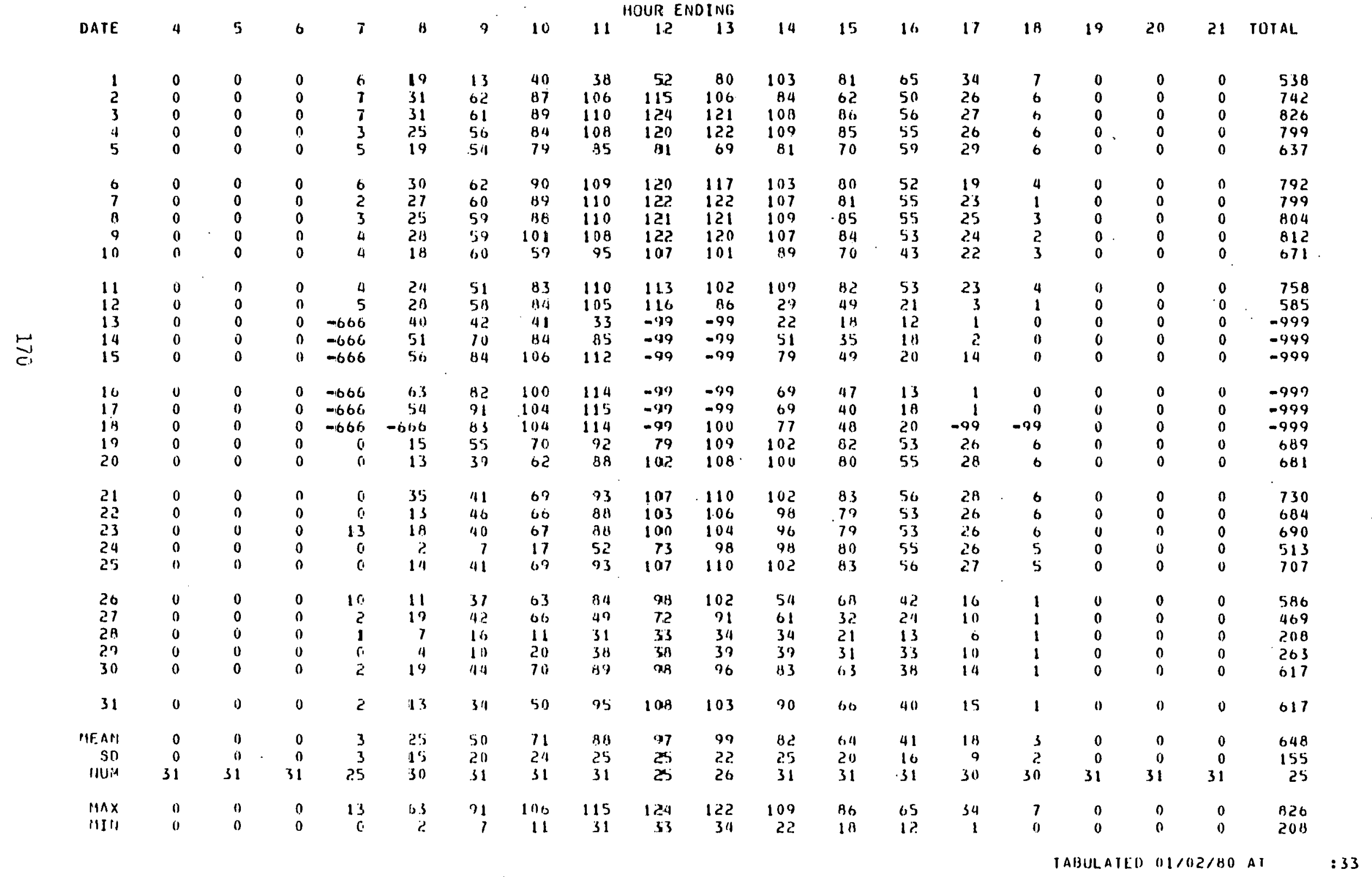


CIJASTAL - IMLAND SIJLAR RAUIATIOH DIFFERENCE STUIOY MOHTHILY DATA SUMMARY

IHSTRUIEUT: PSP 2
SENSITIVITY $10.99 t-6$ V/H/SO.N

ENERGY KILIJJOULES PER SOUARE. METER

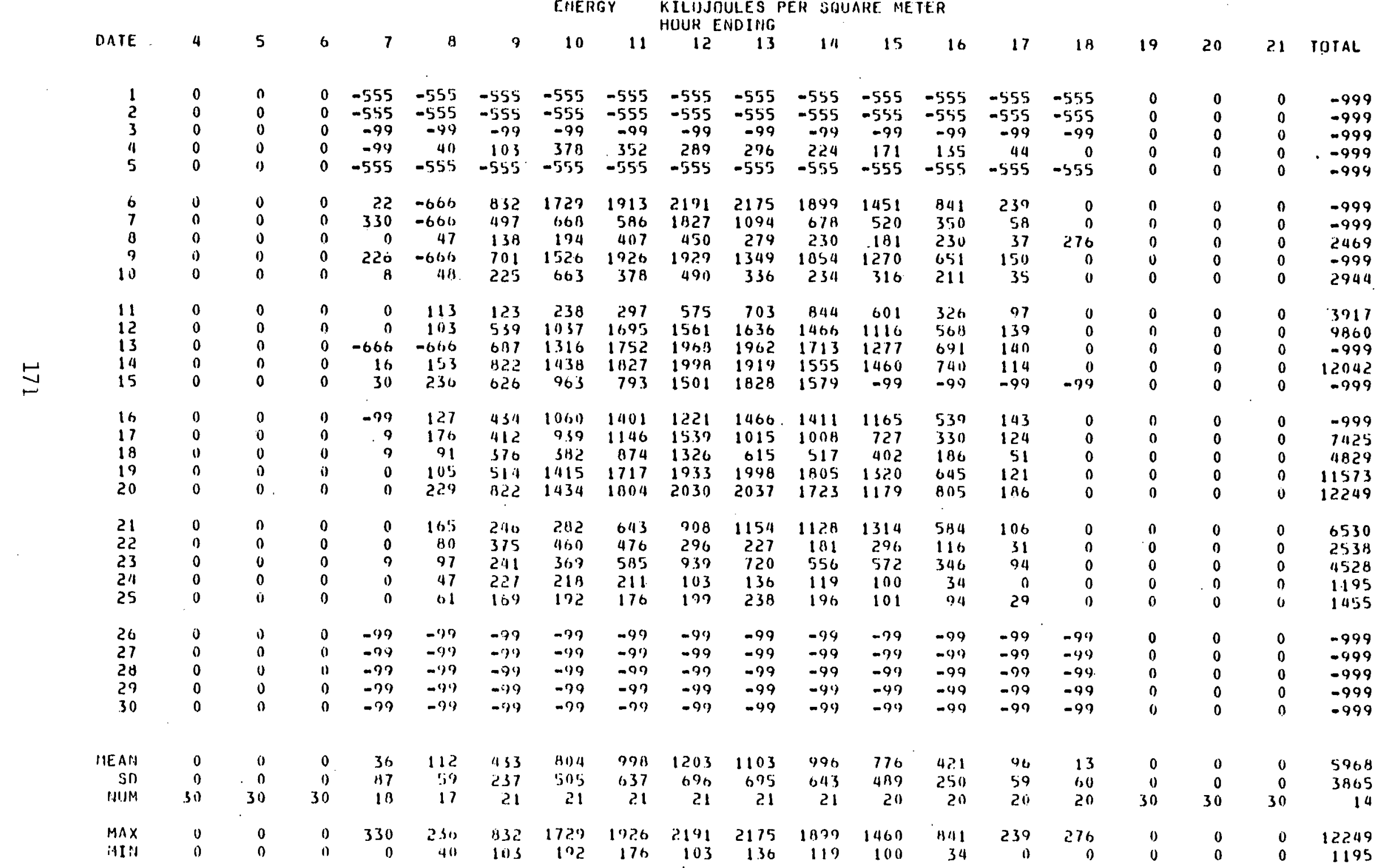


CIASTAL - IULAHO SILAR RAOIATIUN OIFFERENCE STUDY

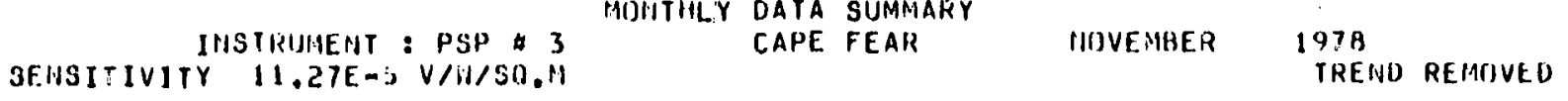

EHERGY KILOJUULLS PER STUARE. MFTER HOUR ENOING

DATE

41
38
21
9

B 9

101

$12 \quad 13$

14

15

16

17

18
0
0
8
0
-99

19

20

21 TIJTLL

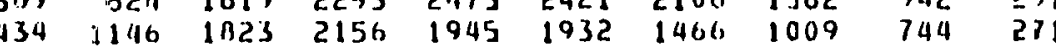

$\begin{array}{llllllllll}423 & 695 & 535 & 954 & 929 & 1002 & 609 & 315 & 171 & 59\end{array}$

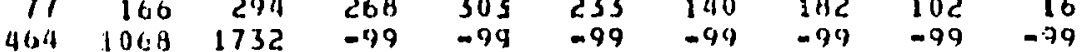

$\begin{array}{llllllllll}-99 & -79 & -97 & 2044 & 2207 & 2184 & 1820 & 1137 & 731 & 178\end{array}$

$\begin{array}{lllllllllll}18 & 340 & 500 & 1017 & 1685 & 1461 & 522 & 385 & 197 & 241 & 104\end{array}$

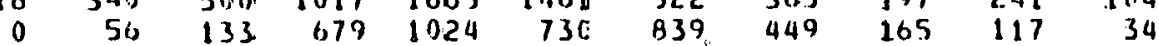

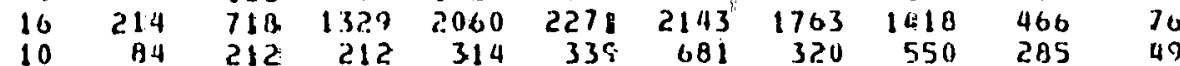

$-9$

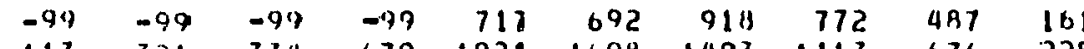

$\begin{array}{llll}113 & 32.1 & 334 & 679\end{array}$

$\begin{array}{llllllllll}191 & 725 & 1195 & 1702 & 2026 & 2053 & 1817 & 1383 & 785 & 239 \\ 290 & 739 & 1427 & 1852 & 2082 & 2045 & 1884 & 1431 & 741 & 194\end{array}$

11 241 6t.5 1065 835 960 2056 1906 1551 768 1月14

19

$\begin{array}{lllllllllll}193 & 650 & 1014 & 1663 & 1947 & 1500 & 1605 & 1631 & 938 & 190\end{array}$

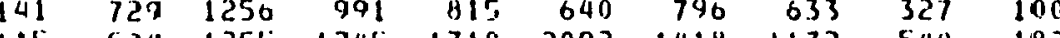

19

$20 \quad 0$

$\begin{array}{llll}21 & 0 & 0 & 0 \\ 22 & 0 & 0 & 0 \\ 23 & 0 & 0 & 0 \\ 24 & 0 & 0 & 0\end{array}$

$15256412541545 \quad 1260 \quad 807.925$ b02 $561 \quad 17$

$\begin{array}{llllllllll}1112 & 109 & 9118 & 1249 & 1226 & 1204 & 1137 & 9118 & 460 & 169\end{array}$

$35 \quad 361 \quad 1041$ 974 $840 \quad 1357 \quad 1019 \quad 565 \quad 268$ 8

147 619. $130801715017002059 \quad 1775 \quad 1487 \quad 925$

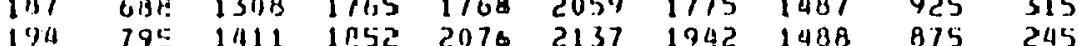

$\begin{array}{lll}26 & 0 & 0 \\ 27 & 0 & 0 \\ 26 & 0 & 0 \\ 29 & 0 & 0\end{array}$

$-9$

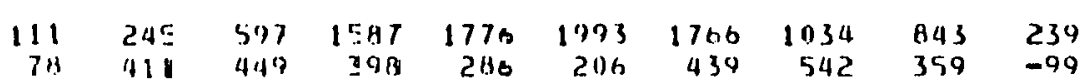

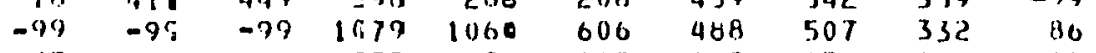

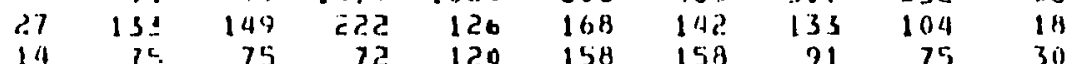

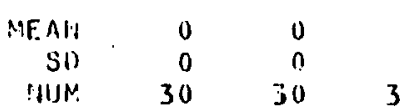

$\begin{array}{rrrrrrrrrrr}7 & 179 & 528 & 950 & 1237 & 1297 & 1301 & 1144 & 997 & 525 & 154 \\ 11 & 1211 & 276 & 491 & 632 & 693 & 730 & 646 & 497 & 277 & 96 \\ 27 & 27 & 28 & 27 & 29 & 29 & 29 & 27 & 29 & 29 & 28 \\ 11 & 1144 & 1146 & 1823 & 2292 & 2475 & 2421 & 2104 & 1631 & 947 & 315 \\ 0 & 14 & 15 & 75 & 76 & 129 & 158 & 100 & 91 & 75 & 16\end{array}$

HAX
HIPI

14

$$
15 \quad 75 \quad 7 \bar{c}
$$


CONSTAL - INLAND SOLAR RADIAIIUH OIFFERENCE STUDY IHSTRUPEHT: PSH 4 MUNTHLY DATA SUMMARY
SEHSITIVITY 10.37 VIALLACE

EHERG KILIJJUULES PER SIJUARE METER

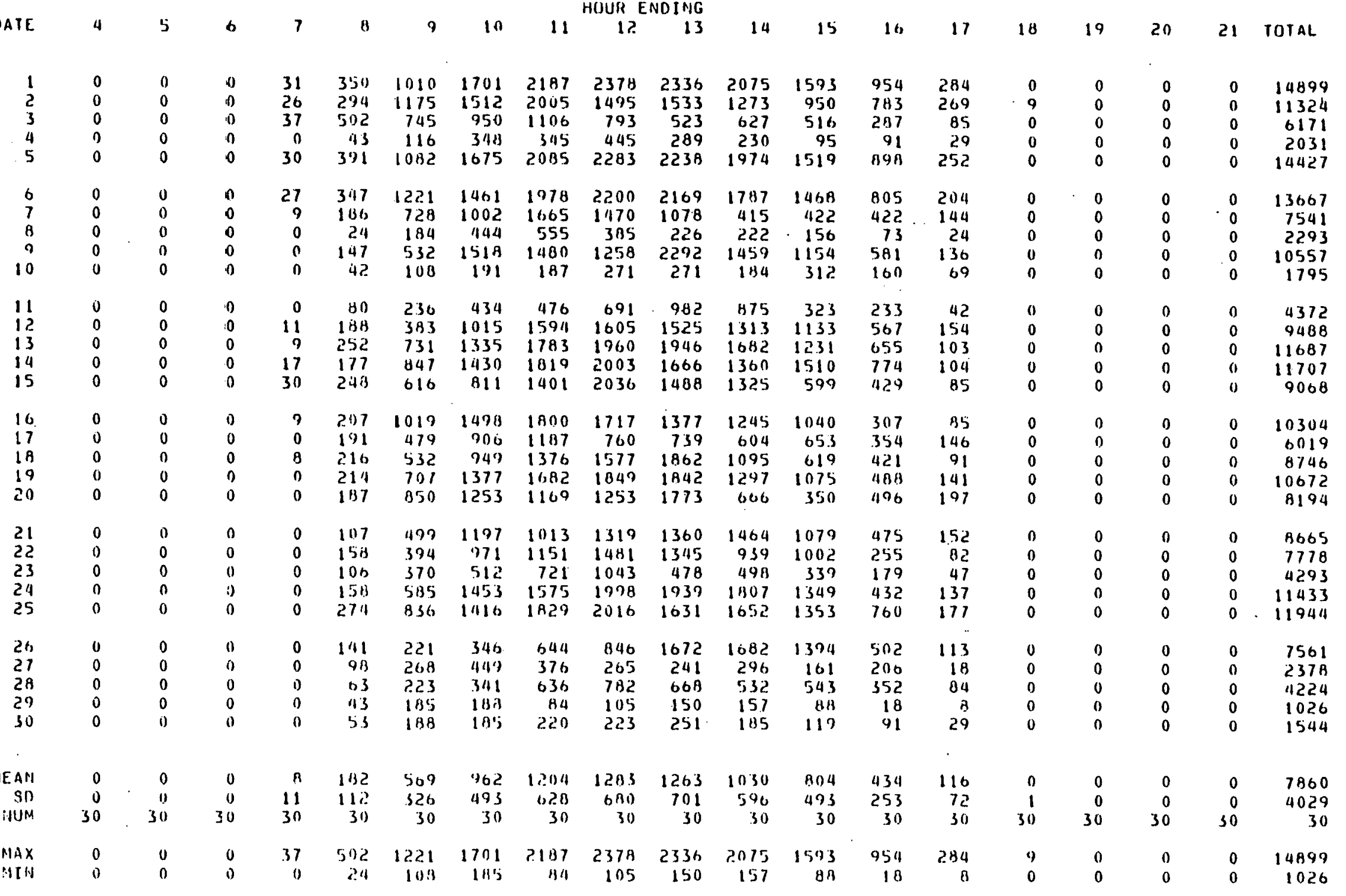


COASTAL. - IHLAHL SILLAR RADIATIUN DIFFERENCE STUUY

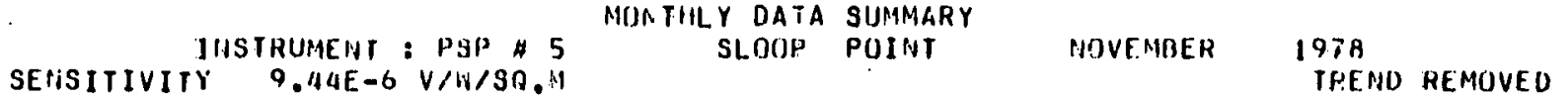

ENERGY KILOJOULES PER SOUARE METER

\begin{tabular}{|c|c|c|c|c|c|c|c|c|c|c|c|c|c|c|c|c|c|c|c|}
\hline \multicolumn{20}{|c|}{ HDUR ENDIFi; } \\
\hline DATE & 4 & 5 & 6 & 7 & H & 9 & 10 & 11 & 12 & 13 & 14. & 15 & 16 & 17 & 18 & 19 & 20 & 21 & IOTAL \\
\hline $\begin{array}{l}1 \\
2 \\
3 \\
4 \\
5\end{array}$ & $\begin{array}{l}0 \\
0 \\
0 \\
0 \\
0\end{array}$ & $\begin{array}{l}0 \\
0 \\
0 \\
0 \\
0\end{array}$ & $\begin{array}{l}0 \\
0 \\
0 \\
0 \\
0\end{array}$ & $\begin{array}{r}26 \\
33 \\
29 \\
0 \\
15\end{array}$ & $\begin{array}{r}339 \\
399 \\
281 \\
62 \\
415\end{array}$ & $\begin{array}{r}930 \\
103 \\
559 \\
184 \\
1109\end{array}$ & $\begin{array}{r}1555 \\
1570 \\
452 \\
329 \\
1689\end{array}$ & $\begin{array}{r}2078 \\
1604 \\
1003 \\
646 \\
2131\end{array}$ & $\begin{array}{r}21155 \\
2405 \\
898 \\
82 . \\
2333\end{array}$ & $\begin{array}{r}2410 \\
1360 \\
933 \\
358 \\
2291\end{array}$ & $\begin{array}{r}2124 \\
1303 \\
597 \\
341 \\
2036\end{array}$ & $\begin{array}{r}1609 \\
1657 \\
288 \\
230 \\
1590\end{array}$ & $\begin{array}{l}961 \\
647 \\
132 \\
135 \\
957\end{array}$ & $\begin{array}{r}312 \\
174 \\
25 \\
20 \\
296\end{array}$ & $\begin{array}{l}0 \\
0 \\
0 \\
0 \\
0\end{array}$ & $\begin{array}{l}0 \\
0 \\
0 \\
0 \\
0\end{array}$ & $\begin{array}{l}0 \\
0 \\
0 \\
0 \\
0\end{array}$ & $\begin{array}{l}0 \\
0 \\
0 \\
0 \\
0\end{array}$ & $\begin{array}{r}14807 \\
12615 \\
4997 \\
3174 \\
14852\end{array}$ \\
\hline $\begin{array}{r}6 \\
7 \\
8 \\
9 \\
10\end{array}$ & $\begin{array}{l}0 \\
0 \\
0 \\
0 \\
0\end{array}$ & $\begin{array}{l}0 \\
0 \\
0 \\
0 \\
0\end{array}$ & $\begin{array}{l}0 \\
0 \\
0 \\
0 \\
0\end{array}$ & $\begin{array}{r}21 \\
13 \\
0 \\
0 \\
0\end{array}$ & $\begin{array}{r}372 \\
287 \\
72 \\
159 \\
57\end{array}$ & $\begin{array}{l}944 \\
531 \\
236 \\
910 \\
350\end{array}$ & $\begin{array}{r}1547 \\
665 \\
278 \\
1436 \\
301\end{array}$ & $\begin{array}{r}2020 \\
1511 \\
865 \\
1760 \\
255\end{array}$ & $\begin{array}{l}2241 \\
1328 \\
1533 \\
1333 \\
377\end{array}$ & $\begin{array}{r}2203 \\
787 \\
884 \\
2077 \\
408\end{array}$ & $\begin{array}{r}700 \\
428 \\
777 \\
1764 \\
141\end{array}$ & $\begin{array}{r}1467 \\
257 \\
236 \\
1051 \\
278\end{array}$ & $\begin{array}{l}834 \\
127 \\
110 \\
544 \\
148\end{array}$ & $\begin{array}{r}246 \\
66 \\
41 \\
94 \\
22\end{array}$ & $\begin{array}{l}0 \\
0 \\
0 \\
0 \\
0\end{array}$ & $\begin{array}{l}0 \\
0 \\
0 \\
0 \\
0\end{array}$ & $\begin{array}{l}0 \\
0 \\
0 \\
0 \\
0\end{array}$ & $\begin{array}{l}0 \\
0 \\
0 \\
0 \\
0\end{array}$ & $\begin{array}{r}12595 \\
6000 \\
5032 \\
11628 \\
2337\end{array}$ \\
\hline $\begin{array}{r}11 \\
12 \\
13 \\
114 \\
\hdashline 15\end{array}$ & $\begin{array}{l}0 \\
0 \\
0 \\
0 \\
0\end{array}$ & $\begin{array}{l}0 \\
0 \\
0 \\
0 \\
0\end{array}$ & $\begin{array}{l}0 \\
0 \\
0 \\
0 \\
0\end{array}$ & $\begin{array}{r}0 \\
0 \\
11 \\
11 \\
-97\end{array}$ & $\begin{array}{l}120 \\
102 \\
251 \\
334 \\
-99\end{array}$ & $\begin{array}{l}411 \\
2 \geq 1 \\
771 \\
-79 \\
-79\end{array}$ & $\begin{array}{r}6410 \\
670 \\
1311 \\
-97 \\
-917\end{array}$ & $\begin{array}{r}1022 \\
1830 \\
1826 \\
-99 \\
-99\end{array}$ & $\begin{array}{r}1 \geq 47 \\
1351 \\
2903 \\
-99 \\
-99\end{array}$ & $\begin{array}{r}1426 \\
1685 \\
2017 \\
-99 \\
-99\end{array}$ & $\begin{array}{r}1357 \\
1399 \\
1742 \\
-99 \\
-99\end{array}$ & $\begin{array}{r}13011 \\
1197 \\
1292 \\
-99 \\
-99\end{array}$ & $\begin{array}{l}564 \\
591 \\
697 \\
-99 \\
-99\end{array}$ & $\begin{array}{l}175 \\
171 \\
175 \\
-99 \\
-99\end{array}$ & $\begin{array}{r}0 \\
0 \\
0 \\
-99 \\
-99\end{array}$ & $\begin{array}{l}0 \\
0 \\
0 \\
0 \\
0\end{array}$ & $\begin{array}{l}0 \\
0 \\
0 \\
0 \\
0\end{array}$ & $\begin{array}{l}0 \\
0 \\
0 \\
0 \\
0\end{array}$ & $\begin{array}{r}8275 \\
9747 \\
12086 \\
-999 \\
-999\end{array}$ \\
\hline $\begin{array}{l}16 \\
17 \\
18 \\
19 \\
20\end{array}$ & $\begin{array}{l}0 \\
0 \\
0 \\
0 \\
0\end{array}$ & $\begin{array}{l}0 \\
0 \\
0 \\
0 \\
0\end{array}$ & $\begin{array}{l}0 \\
0 \\
0 \\
0 \\
0\end{array}$ & $\begin{array}{l}-99 \\
-99 \\
-97 \\
-99 \\
-99\end{array}$ & $\begin{array}{l}-99 \\
-99 \\
-99 \\
-94 \\
-99\end{array}$ & $\begin{array}{l}-99 \\
-99 \\
-99 \\
-99 \\
-99\end{array}$ & $\begin{array}{l}-99 \\
-99 \\
-79 \\
-89 \\
-79\end{array}$ & $\begin{array}{l}-99 \\
-99 \\
-99 \\
-99 \\
-99\end{array}$ & $\begin{array}{l}-99 \\
-89 \\
-99 \\
-99 \\
-99\end{array}$ & $\begin{array}{l}-99 \\
-99 \\
-99 \\
-99 \\
-97\end{array}$ & $\begin{array}{l}-99 \\
-99 \\
-99 \\
-99 \\
-99\end{array}$ & $\begin{array}{l}-99 \\
-99 \\
-99 \\
-79 \\
-99\end{array}$ & $\begin{array}{l}-99 \\
-99 \\
-99 \\
-99 \\
-99\end{array}$ & $\begin{array}{l}-99 \\
-99 \\
-99 \\
-99 \\
-99\end{array}$ & $\begin{array}{l}-79 \\
-99 \\
-99 \\
-99 \\
-99\end{array}$ & $\begin{array}{l}0 \\
0 \\
0 \\
0 \\
0\end{array}$ & $\begin{array}{l}0 \\
0 \\
0 \\
0 \\
0\end{array}$ & $\begin{array}{l}0 \\
0 \\
0 \\
0 \\
0\end{array}$ & $\begin{array}{l}-999 \\
-999 \\
-999 \\
-999 \\
-999\end{array}$ \\
\hline $\begin{array}{l}21 \\
22 \\
23 \\
24 \\
25\end{array}$ & $\begin{array}{l}0 \\
0 \\
0 \\
0 \\
0\end{array}$ & $\begin{array}{l}0 \\
0 \\
0 \\
0 \\
0\end{array}$ & $\begin{array}{l}0 \\
0 \\
0 \\
0 \\
0\end{array}$ & $\begin{array}{l}-99 \\
-99 \\
-79 \\
-99 \\
-99\end{array}$ & $\begin{array}{l}-99 \\
-99 \\
-99 \\
-99 \\
-99\end{array}$ & $\begin{array}{l}-99 \\
-99 \\
-99 \\
-99 \\
-99\end{array}$ & $\begin{array}{l}-99 \\
-79 \\
-79 \\
-99 \\
-79\end{array}$ & $\begin{array}{l}-99 \\
-99 \\
-99 \\
-99 \\
-99\end{array}$ & $\begin{array}{l}-97 \\
-99 \\
-09 \\
-49 \\
-99\end{array}$ & $\begin{array}{l}-97 \\
-97 \\
-97 \\
-97 \\
-97\end{array}$ & $\begin{array}{l}-99 \\
-99 \\
-99 \\
-99 \\
-99\end{array}$ & $\begin{array}{l}-99 \\
-99 \\
-99 \\
-99 \\
-99\end{array}$ & $\begin{array}{l}-99 \\
-99 \\
-99 \\
-99 \\
-99\end{array}$ & $\begin{array}{l}-99 \\
-99 \\
-99 \\
-99 \\
-99\end{array}$ & $\begin{array}{l}-199 \\
-99 \\
-.19 \\
-99 \\
-99\end{array}$ & $\begin{array}{l}0 \\
0 \\
0 \\
0 \\
0\end{array}$ & $\begin{array}{l}0 \\
0 \\
0 \\
0 \\
0\end{array}$ & $\begin{array}{l}0 \\
0 \\
0 \\
0 \\
0\end{array}$ & $\begin{array}{l}-999 \\
-999 \\
-999 \\
-999 \\
-999\end{array}$ \\
\hline $\begin{array}{l}20 \\
27 \\
28 \\
29 \\
30\end{array}$ & $\begin{array}{l}0 \\
0 \\
0 \\
0 \\
0\end{array}$ & $\begin{array}{l}0 \\
0 \\
0 \\
0 \\
0\end{array}$ & $\begin{array}{l}0 \\
0 \\
0 \\
0 \\
0\end{array}$ & $\begin{array}{l}-99 \\
-99 \\
-99 \\
-99 \\
-99\end{array}$ & $\begin{array}{l}-99 \\
-99 \\
-97 \\
-91 \\
-99\end{array}$ & $\begin{array}{l}-49 \\
-99 \\
-99 \\
-99 \\
-99\end{array}$ & $\begin{array}{l}-79 \\
-97 \\
-79 \\
-79 \\
-97\end{array}$ & $\begin{array}{l}-99 \\
-99 \\
-49 \\
-99 \\
-99\end{array}$ & $\begin{array}{r}-59 \\
-59 \\
-59 \\
-59 \\
-59\end{array}$ & $\begin{array}{l}-99 \\
-99 \\
-49 \\
-99 \\
-99\end{array}$ & $\begin{array}{l}-99 \\
-99 \\
-99 \\
-99 \\
-90\end{array}$ & $\begin{array}{l}-99 \\
-99 \\
-99 \\
-99 \\
-99\end{array}$ & $\begin{array}{l}-99 \\
-99 \\
-99 \\
-99 \\
-49\end{array}$ & $\begin{array}{l}-99 \\
-99 \\
-99 \\
-99 \\
-99\end{array}$ & $\begin{array}{l}-99 \\
-99 \\
-99 \\
-99 \\
-99\end{array}$ & $\begin{array}{l}0 \\
0 \\
0 \\
0 \\
0\end{array}$ & $\begin{array}{l}0 \\
0 \\
0 \\
0 \\
0\end{array}$ & $\begin{array}{l}0 \\
0 \\
0 \\
0 \\
0\end{array}$ & $\begin{array}{l}-999 \\
-999 \\
-999 \\
-999 \\
-999\end{array}$ \\
\hline $\begin{array}{r}\text { HEAH } \\
\text { SD } \\
\text { HHMM }\end{array}$ & $\begin{array}{r}0 \\
0 \\
30\end{array}$ & $\begin{array}{r}0 \\
0 \\
30\end{array}$ & $\begin{array}{r}0 \\
0 \\
30\end{array}$ & $\begin{array}{l}11 \\
11 \\
14\end{array}$ & $\begin{array}{r}236 \\
130 \\
14\end{array}$ & $\begin{array}{r}658 \\
377 \\
13\end{array}$ & $\begin{array}{l}1513 \\
5.31 \\
.13\end{array}$ & $\begin{array}{r}1411 \\
594 \\
13\end{array}$ & $\begin{array}{r}1646 \\
6.15 \\
13\end{array}$ & $\begin{array}{r}145 ? \\
690 \\
13\end{array}$ & $\begin{array}{r}1131 \\
646 \\
13\end{array}$ & $\begin{array}{r}958 \\
570 \\
13\end{array}$ & $\begin{array}{r}405 \\
315 \\
13\end{array}$ & $\begin{array}{r}139 \\
98 \\
13\end{array}$ & $\begin{array}{r}0 \\
0 \\
13\end{array}$ & $\begin{array}{r}0 \\
0 \\
30\end{array}$ & $\begin{array}{r}0 \\
0 \\
30\end{array}$ & $\begin{array}{r}0 \\
0 \\
30\end{array}$ & $\begin{array}{r}9080 \\
4210 \\
.13\end{array}$ \\
\hline $\begin{array}{l}1.1 A x \\
11111\end{array}$ & $\begin{array}{l}0 \\
0\end{array}$ & $\begin{array}{l}0 \\
0\end{array}$ & $\begin{array}{l}0 \\
0\end{array}$ & $\begin{array}{r}3.3 \\
0\end{array}$ & $\begin{array}{r}715 \\
157\end{array}$ & $\begin{array}{r}146.3 \\
1334\end{array}$ & $\begin{array}{r}1649 \\
278\end{array}$ & $\begin{array}{r}3131 \\
255\end{array}$ & $\begin{array}{r}245.5 \\
377\end{array}$ & $\begin{array}{r}2410 \\
396\end{array}$ & $\begin{array}{r}2124 \\
141\end{array}$ & $\begin{array}{r}1657 \\
230\end{array}$ & $\begin{array}{l}96,1 \\
110\end{array}$ & $\begin{array}{r}312 \\
20\end{array}$ & $\begin{array}{l}0 \\
0\end{array}$ & $\begin{array}{l}0 \\
0\end{array}$ & $\begin{array}{l}0 \\
0\end{array}$ & $\begin{array}{l}0 \\
0\end{array}$ & $\begin{array}{r}14852 \\
2337\end{array}$ \\
\hline
\end{tabular}


CIIASTAL - IINLAHD SIJLAR RADIATIUN DIFFEREMCE STUDY

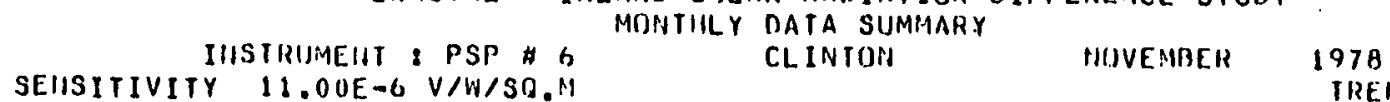

1978
IREND REMOVED

EHERGY KILOJIUULES PER SIJUARE METER

DATE $4 \quad 5 \quad 6 \quad 4 \quad H \quad 4 \quad 10 \quad 11$ HOUR ENIDING

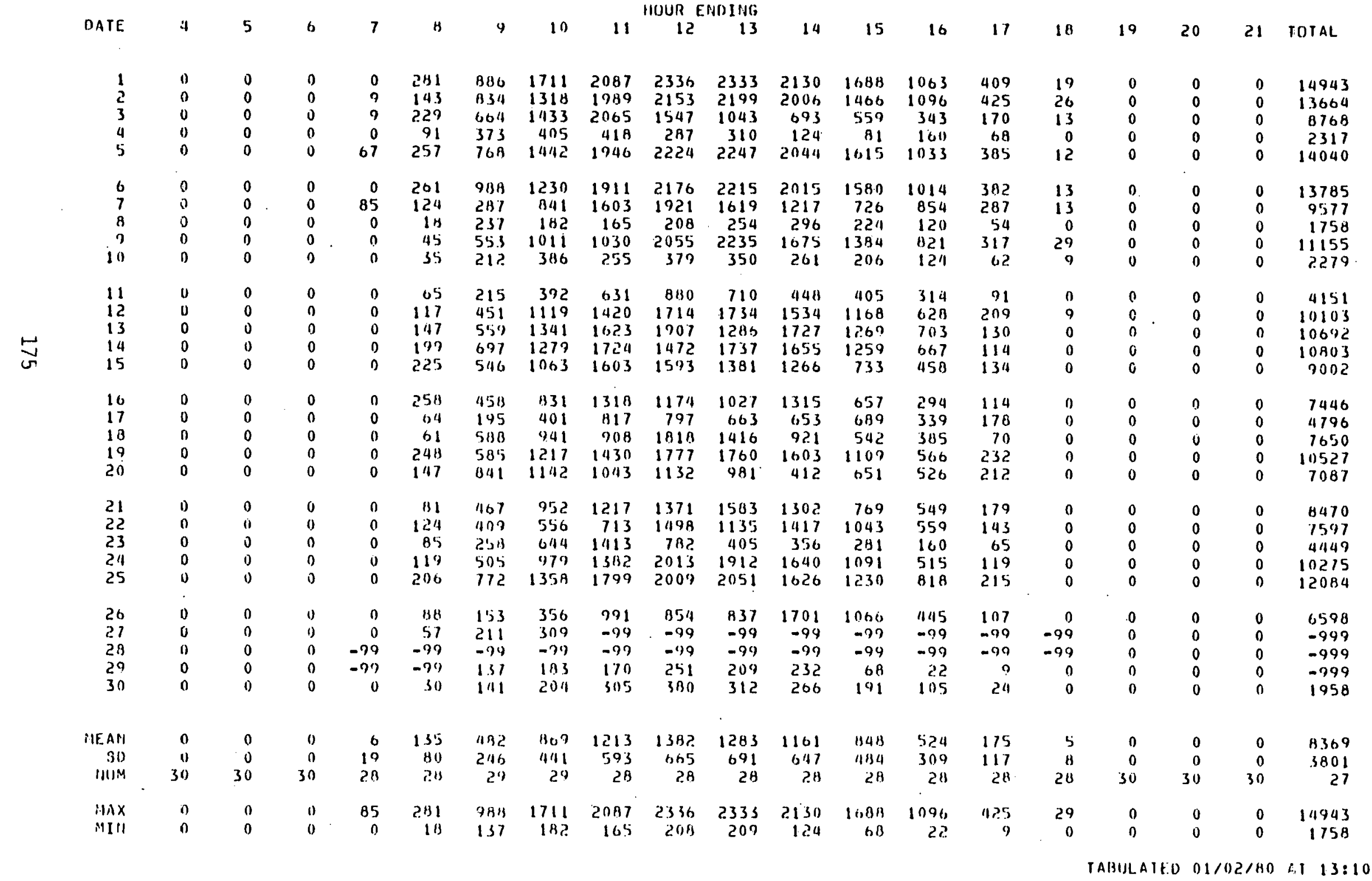

IABULATEO 01/02/BO :I I $13: 10: 39$ 
COASTAL - IILLAHD SILAR RADIATIUN DIFFERENCE. STUQY

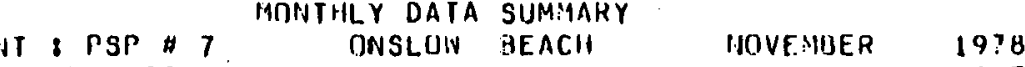

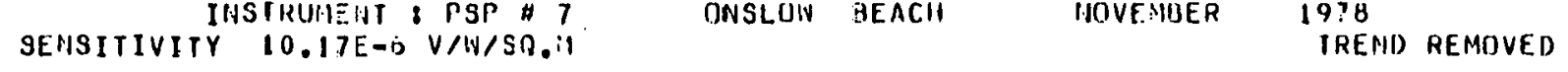

ENERG KILOJOULES PER SUUARE HETER. HOUR ENDING

\begin{tabular}{|c|c|c|c|c|c|c|c|c|c|c|c|c|c|c|c|c|c|c|c|}
\hline ATE. & 4 & 5 & 6 & 7 & 3 & 9 & 10 & 11 & 12 & 13 & 14 & 15 & 16 & $: 7$ & 18 & 19 & 20 & 21 & TUTAL \\
\hline $\begin{array}{l}1 \\
2 \\
3 \\
4 \\
5\end{array}$ & $\begin{array}{l}0 \\
0 \\
0 \\
0 \\
0\end{array}$ & $\begin{array}{l}0 \\
0 \\
0 \\
0 \\
0\end{array}$ & $\begin{array}{l}0 \\
0 \\
0 \\
0 \\
0\end{array}$ & $\begin{array}{r}28 \\
24 \\
35 \\
0 \\
20\end{array}$ & $\begin{array}{r}207 \\
121 \\
1103 \\
0: 3 \\
253\end{array}$ & $\begin{array}{r}523 \\
1069 \\
750 \\
204 \\
1014\end{array}$ & $\begin{array}{r}1461 \\
1751 \\
704 \\
735 \\
1712\end{array}$ & $\begin{array}{r}2502 \\
1430 \\
730 \\
871 \\
2138\end{array}$ & $\begin{array}{r}2456 \\
2392 \\
860 \\
990 \\
2321\end{array}$ & $\begin{array}{r}2407 \\
1720 \\
796 \\
1110 \\
2289\end{array}$ & $\begin{array}{r}2109 \\
651 \\
453 \\
1761 \\
2030\end{array}$ & $\begin{array}{r}1599 \\
506 \\
315 \\
583 \\
1598\end{array}$ & $\begin{array}{l}952 \\
140 \\
134 \\
246 \\
986\end{array}$ & $\begin{array}{r}307 \\
0 \\
24 \\
37 \\
324\end{array}$ & $\begin{array}{l}0 \\
0 \\
0 \\
0 \\
0\end{array}$ & $\begin{array}{l}0 \\
0 \\
0 \\
0 \\
0\end{array}$ & $\begin{array}{l}0 \\
0 \\
0 \\
0 \\
0\end{array}$ & $\begin{array}{l}0 \\
0 \\
0 \\
0 \\
0\end{array}$ & $\begin{array}{r}14773 \\
10116 \\
5273 \\
6626 \\
14655\end{array}$ \\
\hline $\begin{array}{r}6 \\
7 \\
8 \\
3 \\
10\end{array}$ & $\begin{array}{l}0 \\
0 \\
0 \\
0 \\
0\end{array}$ & $\begin{array}{l}0 \\
0 \\
0 \\
0 \\
0\end{array}$ & $\begin{array}{l}0 \\
0 \\
0 \\
0 \\
0\end{array}$ & $\begin{array}{r}24 \\
10 \\
0 \\
9 \\
0\end{array}$ & $\begin{array}{r}403 \\
293 \\
43 \\
137 \\
87\end{array}$ & $\begin{array}{r}1047 \\
771 \\
197 \\
551 \\
423\end{array}$ & $\begin{array}{r}1614 \\
945 \\
172 \\
1422 \\
198\end{array}$ & $\begin{array}{r}2028 \\
811 \\
555 \\
1373 \\
738\end{array}$ & $\begin{array}{r}2247 \\
750 \\
1131 \\
2168 \\
462\end{array}$ & $\begin{array}{l}2247 \\
1277 \\
1022 \\
1931 \\
303\end{array}$ & $\begin{array}{r}1978 \\
414 \\
9 \\
1591 \\
668\end{array}$ & $\begin{array}{r}1511 \\
155 \\
190 \\
1358 \\
4148\end{array}$ & $\begin{array}{r}870 \\
134 \\
101 \\
437 \\
151\end{array}$ & $\begin{array}{r}251 \\
49 \\
55 \\
719 \\
41\end{array}$ & $\begin{array}{l}0 \\
0 \\
0 \\
0 \\
0\end{array}$ & $\begin{array}{l}0 \\
0 \\
0 \\
0 \\
0\end{array}$ & $\begin{array}{l}0 \\
0 \\
0 \\
0 \\
0\end{array}$ & $\begin{array}{l}0 \\
0 \\
0 \\
0 \\
0\end{array}$ & $\begin{array}{r}14220 \\
5559 \\
3490 \\
11116 \\
3819\end{array}$ \\
\hline $\begin{array}{l}11 \\
12 \\
13 \\
14 \\
15\end{array}$ & $\begin{array}{l}0 \\
0 \\
0 \\
0 \\
0\end{array}$ & $\begin{array}{l}0 \\
0 \\
0 \\
0 \\
0\end{array}$ & $\begin{array}{l}0 \\
0 \\
0 \\
0 \\
0\end{array}$ & $\begin{array}{r}0 \\
0 \\
10 \\
-99 \\
42\end{array}$ & $\begin{array}{r}153 \\
91 \\
251 \\
-97 \\
293\end{array}$ & $\begin{array}{l}552 \\
452 \\
753 \\
-39 \\
736\end{array}$ & $\begin{array}{l}1012 \\
1248 \\
1405 \\
-119 \\
1204\end{array}$ & $\begin{array}{l}12 B 8 \\
1584 \\
1847 \\
1929 \\
1054\end{array}$ & $\begin{array}{l}1507 \\
1701 \\
2031 \\
2102 \\
1978\end{array}$ & $\begin{array}{l}1568 \\
1429 \\
2021 \\
2056 \\
1709\end{array}$ & $\begin{array}{l}1136 \\
1191 \\
1780 \\
1780 \\
1745\end{array}$ & $\begin{array}{l}679 \\
1174 \\
1314 \\
1313 \\
1330\end{array}$ & $\begin{array}{l}630 \\
675 \\
753 \\
715 \\
435\end{array}$ & $\begin{array}{l}237 \\
100 \\
109 \\
110 \\
176\end{array}$ & $\begin{array}{l}0 \\
0 \\
0 \\
0 \\
0\end{array}$ & $\begin{array}{l}0 \\
0 \\
0 \\
0 \\
0\end{array}$ & $\begin{array}{l}0 \\
0 \\
0 \\
0 \\
0\end{array}$ & $\begin{array}{l}0 \\
0 \\
0 \\
0 \\
0\end{array}$ & $\begin{array}{r}8764 \\
9735 \\
12375 \\
-999 \\
10772\end{array}$ \\
\hline $\begin{array}{l}16 \\
17 \\
18 \\
19 \\
20\end{array}$ & $\begin{array}{l}0 \\
0 \\
0 \\
0 \\
0\end{array}$ & $\begin{array}{l}0 \\
0 \\
0 \\
0 \\
0\end{array}$ & $\begin{array}{l}0 \\
0 \\
0 \\
0 \\
0\end{array}$ & $\begin{array}{r}24 \\
13 \\
0 \\
14 \\
10\end{array}$ & $\begin{array}{l}315 \\
314 \\
151 \\
2410 \\
279\end{array}$ & $\begin{array}{l}181 \\
725 \\
356 \\
669 \\
500\end{array}$ & $\begin{array}{r}1412 \\
915 \\
1032 \\
1415 \\
063\end{array}$ & $\begin{array}{l}1922 \\
1740 \\
1747 \\
1061 \\
1646\end{array}$ & $\begin{array}{l}2031 \\
1811 \\
1351 \\
1865 \\
1415\end{array}$ & $\begin{array}{r}1617 \\
1011 \\
1570 \\
1922 \\
930\end{array}$ & $\begin{array}{r}1454 \\
413 \\
1584 \\
1656 \\
1479\end{array}$ & $\begin{array}{r}722 \\
721 \\
1145 \\
1214 \\
520\end{array}$ & $\begin{array}{l}470 \\
558 \\
377 \\
615 \\
371\end{array}$ & $\begin{array}{r}130 \\
1 ? 2 \\
37 \\
1: 16 \\
1.2\end{array}$ & $\begin{array}{l}0 \\
0 \\
0 \\
0 \\
0\end{array}$ & $\begin{array}{l}0 \\
0 \\
0 \\
0 \\
0\end{array}$ & $\begin{array}{l}0 \\
0 \\
0 \\
0 \\
0\end{array}$ & $\begin{array}{l}0 \\
0 \\
0 \\
0 \\
0\end{array}$ & $\begin{array}{r}10578 \\
8416 \\
4400 \\
11577 \\
8195\end{array}$ \\
\hline $\begin{array}{l}21 \\
22 \\
23 \\
211 \\
25\end{array}$ & $\begin{array}{l}0 \\
0 \\
0 \\
0 \\
0\end{array}$ & $\begin{array}{l}0 \\
0 \\
0 \\
0 \\
0\end{array}$ & $\begin{array}{l}0 \\
0 \\
0 \\
0 \\
0\end{array}$ & $\begin{array}{r}10 \\
0 \\
0 \\
13 \\
0\end{array}$ & $\begin{array}{l}205 \\
17.3 \\
1201 \\
2140 \\
322\end{array}$ & $\begin{array}{l}690 \\
311 \\
315 \\
724 \\
962\end{array}$ & $\begin{array}{r}1044 \\
909 \\
732 \\
1468 \\
1536\end{array}$ & $\begin{array}{r}1100 \\
906 \\
1079 \\
1361 \\
1329\end{array}$ & $\begin{array}{l}107 \% \\
1670 \\
2024 \\
196 . \\
2123\end{array}$ & $\begin{array}{l}1069 \\
1058 \\
1649 \\
2023 \\
1961\end{array}$ & $\begin{array}{r}1069 \\
934 \\
849 \\
1754 \\
1550\end{array}$ & $\begin{array}{r}679 \\
559 \\
626 \\
1259 \\
1345\end{array}$ & $\begin{array}{l}322 \\
293 \\
315 \\
515 \\
707\end{array}$ & $\begin{array}{r}53 \\
38 \\
49 \\
101 \\
134\end{array}$ & $\begin{array}{l}0 \\
0 \\
0 \\
0 \\
0\end{array}$ & $\begin{array}{l}0 \\
0 \\
0 \\
0 \\
0\end{array}$ & $\begin{array}{l}0 \\
0 \\
0 \\
0 \\
0\end{array}$ & $\begin{array}{l}0 \\
0 \\
0 \\
0 \\
0\end{array}$ & $\begin{array}{r}7323 \\
6908 \\
7758 \\
11427 \\
12569\end{array}$ \\
\hline $\begin{array}{l}20 \\
27 \\
28 \\
29 \\
30\end{array}$ & $\begin{array}{l}0 \\
0 \\
0 \\
0 \\
0\end{array}$ & $\begin{array}{l}n \\
0 \\
0 \\
0 \\
0\end{array}$ & $\begin{array}{l}0 \\
0 \\
0 \\
0 \\
0\end{array}$ & $\begin{array}{c}10 \\
0 \\
0 \\
0 \\
0\end{array}$ & $\begin{array}{r}233 \\
13.3 \\
1983 \\
74 \\
35.5\end{array}$ & $\begin{array}{l}378 \\
498 \\
3143 \\
297 \\
85\end{array}$ & $\begin{array}{l}337 \\
692 \\
460 \\
307 \\
124\end{array}$ & $\begin{array}{r}523 \\
1096 \\
9.84 \\
247 \\
96\end{array}$ & $\begin{array}{r}916 \\
496 \\
1426 \\
180 \\
12 i\end{array}$ & $\begin{array}{r}1359 \\
377 \\
633 \\
279 \\
160\end{array}$ & $\begin{array}{r}1663 \\
278 \\
424 \\
148 \\
181\end{array}$ & $\begin{array}{l}1185 \\
197 \\
286 \\
116 \\
106\end{array}$ & $\begin{array}{r}707 \\
94 \\
258 \\
63 \\
70\end{array}$ & $\begin{array}{r}134 \\
16 \\
63 \\
10 \\
1.8\end{array}$ & $\begin{array}{l}0 \\
0 \\
0 \\
0 \\
0\end{array}$ & $\begin{array}{l}0 \\
0 \\
0 \\
0 \\
0\end{array}$ & $\begin{array}{l}0 \\
0 \\
0 \\
0 \\
0\end{array}$ & $\begin{array}{l}0 \\
0 \\
0 \\
0 \\
0\end{array}$ & $\begin{array}{l}7447 \\
3879 \\
5075 \\
1746 \\
1016\end{array}$ \\
\hline $\begin{array}{r}\text { IEAN } \\
\text { SO } \\
\text { MUUM }\end{array}$ & $\begin{array}{r}1) \\
0 \\
30\end{array}$ & $\begin{array}{r}0 \\
0 \\
30\end{array}$ & $\begin{array}{r}0 \\
0 \\
30\end{array}$ & $\begin{array}{l}10 \\
11 \\
99\end{array}$ & $\begin{array}{r}216 \\
109 \\
29\end{array}$ & $\begin{array}{r}575 \\
259 \\
79\end{array}$ & $\begin{array}{r}1007 \\
465 \\
20\end{array}$ & $\begin{array}{r}1506 \\
567 \\
30\end{array}$ & $\begin{array}{r}1510 \\
667 \\
30\end{array}$ & $\begin{array}{r}1383 \\
626 \\
30\end{array}$ & $\begin{array}{r}1157 \\
639 \\
30\end{array}$ & $\begin{array}{r}826 \\
4135 \\
30\end{array}$ & $\begin{array}{r}435 \\
272 \\
30\end{array}$ & $\begin{array}{r}110 \\
13 \\
30\end{array}$ & $\begin{array}{r}0 \\
0 \\
30\end{array}$ & $\begin{array}{r}0 \\
0 \\
30\end{array}$ & $\begin{array}{r}0 \\
0 \\
30\end{array}$ & $\begin{array}{r}0 \\
0 \\
30\end{array}$ & $\begin{array}{r}8434 \\
3670 \\
29\end{array}$ \\
\hline $\begin{array}{l}\text { MAX } \\
\text { MINI }\end{array}$ & $\begin{array}{l}0 \\
0\end{array}$ & $\begin{array}{l}1) \\
0\end{array}$ & $\begin{array}{l}0 \\
0\end{array}$ & $\begin{array}{r}42 \\
0\end{array}$ & $\begin{array}{r}1463 \\
515\end{array}$ & $\begin{array}{r}106 ? \\
81\end{array}$ & $\begin{array}{r}1755 \\
121\end{array}$ & $\begin{array}{r}2562 \\
76\end{array}$ & $\begin{array}{r}2456 \\
127\end{array}$ & $\begin{array}{r}21107 \\
166\end{array}$ & $\begin{array}{r}2.109 \\
9\end{array}$ & $\begin{array}{r}1599 \\
106\end{array}$ & $\begin{array}{r}946 \\
63\end{array}$ & $\begin{array}{r}3 ? .4 \\
0\end{array}$ & $\begin{array}{l}0 \\
0\end{array}$ & $\begin{array}{l}0 \\
0\end{array}$ & $\begin{array}{l}0 \\
0\end{array}$ & $\begin{array}{l}0 \\
0\end{array}$ & $\begin{array}{r}14773 \\
1016\end{array}$ \\
\hline
\end{tabular}


COJASTAL - INLAND SULAR RADIATIUN DIFFEREHCE STUDY

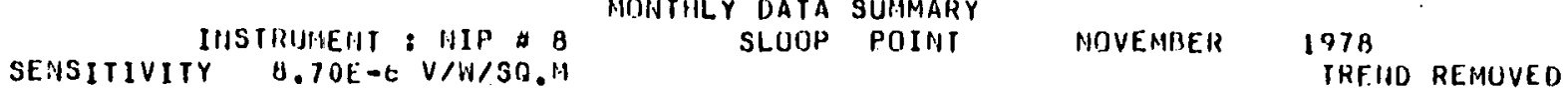

EHERIGY KILOJOULES PER SHUARE METER HOUR ENDING

\begin{abstract}
0
\end{abstract}
$0 \quad 254 \quad 626$

0
22
27
523

0
20
18
242

12
23
22
$2567 \quad 2567$

$20 \quad 20$

44 135

593

242
279

482.2567

$\begin{array}{ll}9 & 20 \\ 19 & 18\end{array}$

$33 \quad 1$

12
15
14

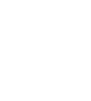

0
11

23
0
30

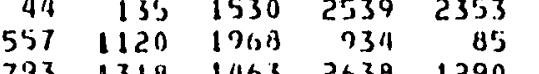

$\begin{array}{lllll}793 & 318 & 1163 & 2638 & 1290\end{array}$

$10732108 \quad 1454$

1702
$4 \quad 391$

180

i

0
0
0
0
0

0
0
0
0
0

19979

8260

62
0

2005

532

73
43

213

$\begin{array}{rrrrrrrrrrrr}211 & 62 & 37 & 54 & 120 & 173 & 0 & 0 & 0 & 0 & 0 & 0 \\ 0 & 533 & 513 & 147 & 199 & 37 & 306 & 310 & 0 & 0 & 0 & 0 \\ 0 & 71 & 12 & 53 & 153 & 0 & 0 & 0 & 0 & 0 & 0 & 0 \\ 31 & 79 & 154 & 352 & 572 & 758 & 725 & 320 & 55 & 0 & 0 & 0 \\ -79 & -99 & -99 & -37 & -97 & -97 & -99 & -99 & -99 & -99 & -99 & -29\end{array}$

$\begin{array}{rrr}0 & 0 & 0 \\ 83 & 197 & 0\end{array}$

0
0
0

0

$\begin{array}{ll}0 & 0 \\ 0 & 0\end{array}$

ก

9596

4675

7705

7506
-999

587 1897

$-99$

$-94$

$-99$

$-99$

-99
-99

-94
-99

-99
-99

$-997$.

$-999$

$-999$

$-999$

$-999$

\begin{tabular}{|c|c|c|c|c|c|c|c|c|c|c|c|c|c|c|}
\hline MEAN & 0 & 0 & 0 & 14 & 161 & Si) $\mathrm{H}$ & 5' & $59 B$ & 626 & 510 & 4513 & 353 & 178 & 61 \\
\hline 30 & 0 & 0 & $n$ & 12 & 241 & 116 & 733 & ถค7 & 933 & 806 & 815 & 701 & 486 & 184 \\
\hline NuIH & $3 n$ & 30 & 30 & 24 & 24 & 24 & 21 & 24 & 24 & 24 & 24 & 24 & 24 & 2.4 \\
\hline HAX & $n$ & 0 & 0 & 211 & 7113 & 13111 & 22713 & 2799 & $302 ?$ & 3056 & 2911 & 2447 & 1831 & 1329 \\
\hline "MI IV & 0 & 0 & 0 & 0 & 0 & 0 & 1) & 0 & (i) & () & 0 & 0 & () & 1 \\
\hline
\end{tabular}


CDASTAL - INLAHD SULAR HADIATION DIFFERENCE STUIJY

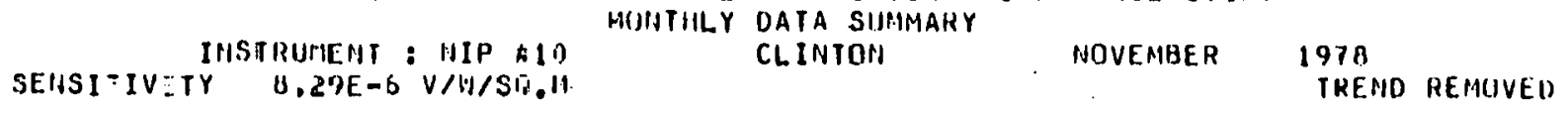

F.HERGY KILINOULES PEH SHUARE METER HDUR ENOING

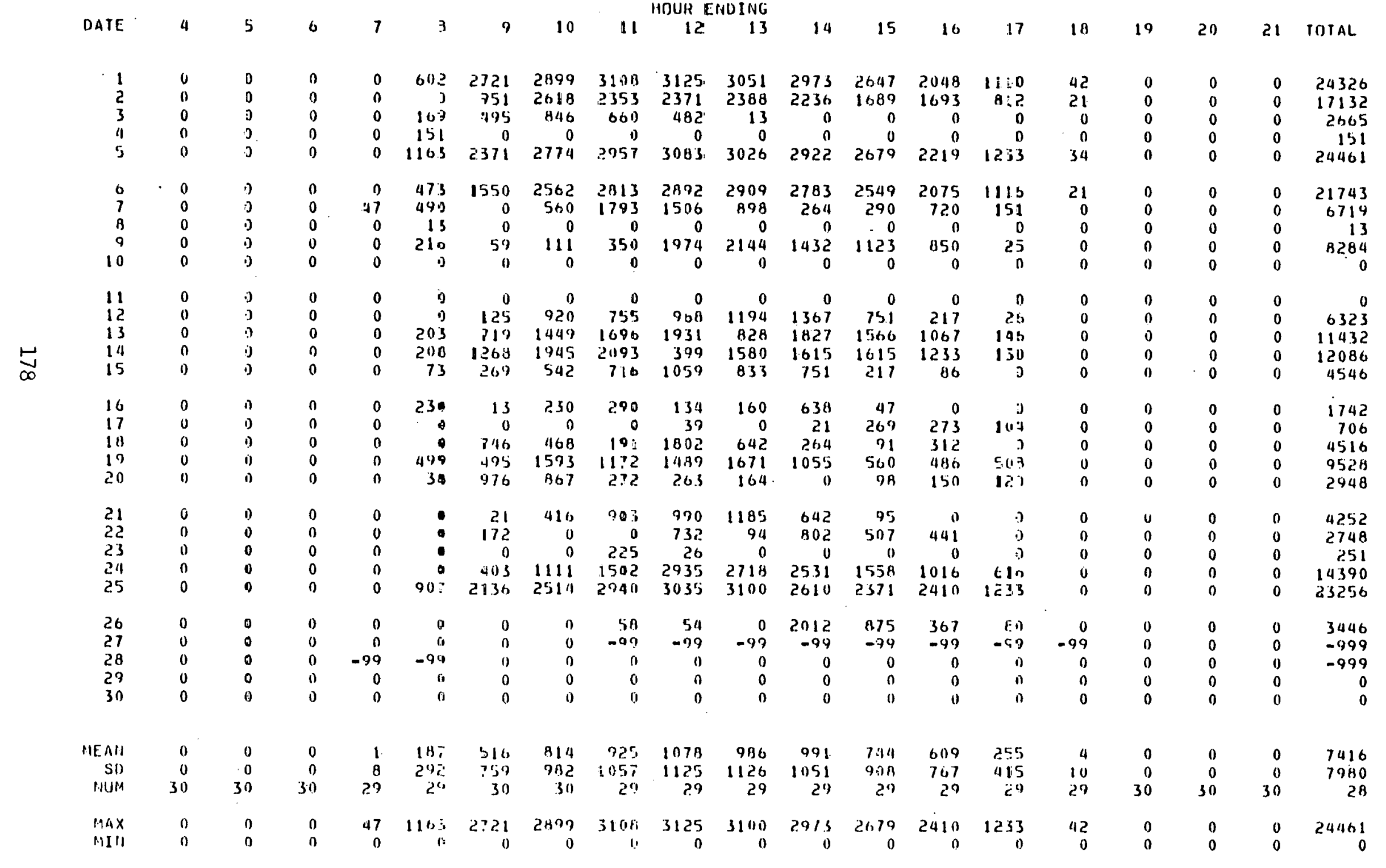


CUASTAL - INLAHID SOLAR RAUIATIOIN DIFFEREHICE STUOY

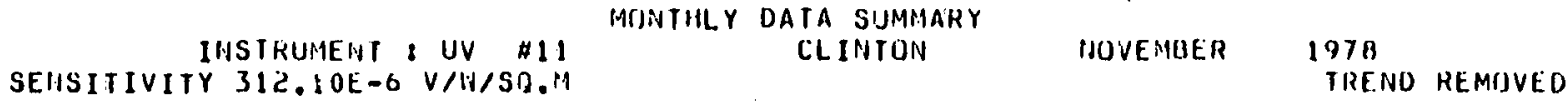

ENERIYY KILOJOULES PER SOUARE METER

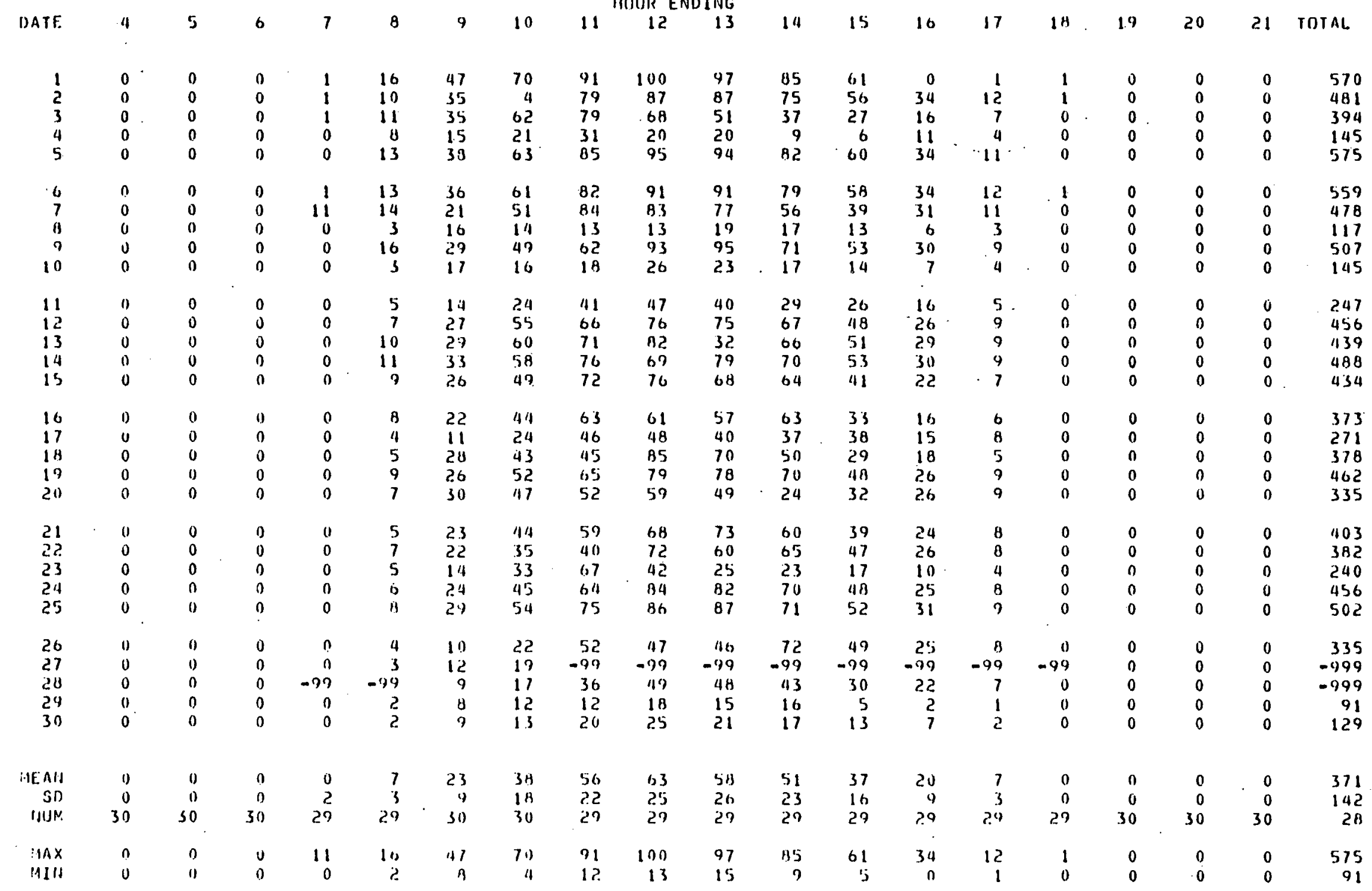


CON3TAL - IHLAHD SILAK RADIATIOT DIFFEREHCE STUDY MNTHLY DATA SUIMAFY

$$
\begin{array}{r}
\text { INSTRUHEII : UV \#12 } \\
\text { SEHSITIVITY } 154.40 E-D V / W / 39 . M
\end{array}
$$

\begin{tabular}{|c|c|c|c|c|c|c|c|c|c|c|c|c|c|c|c|c|c|c|c|}
\hline \\
\hline DATE & 4 & 5 & 6 & $?$ & 8 & 9 & 10 & 11 & 12 & 13 & 14 & 15 & 16 & 17 & 18 & 19 & 20 & 21 & TUIAL \\
\hline 1 & 0 & 0 & 0 & 2 & 18 & 97 & 76 & 95 & 104 & 101 & 87 & 63 & 38 & 14 & 1 & 0 & 0 & 0 & 646 \\
\hline 2 & 0 & 0 & 0 & 2 & 19 & 15 & 70 & 73 & 99 & 67 & 60 & 64 & 31 & 10 & $i$ & 0 & 0 & 0 & 541 \\
\hline 3 & 0 & 0 & 0 & $\ddot{i}$ & 11 & ;0 & 27 & 45 & 48 & 50 & 34 & 18 & 9 & 2 & 0 & 0 & 0 & 0 & 218 \\
\hline 4 & 0 & 0 & 0 & 0 & $b$ & $: 2$ & 23 & 42 & 49 & 26 & 22 & 15 & 10 & 3 & 0 & 0 & 0 & 0 & 208 \\
\hline 5 & 0 & 0 & 0 & 2 & 18 & 16 & 71 & 92 & 101 & 100 & 87 & 65 & $3 B$ & 13 & 1 & 0 & 0 & 0 & 634 \\
\hline 6 & 0 & 0 & 0 & $\vdots$ & 16 & ;9 & 62 & 80 & 91 & 90 & 28 & $5 S$ & 32 & 12 & 1 & 0 & 0 & 0 & 507 \\
\hline 7 & 0 & 0 & 0 & $\vdots$ & 14 & 27 & 38 & 71 & 68 & 45 & 28 & 18 & 10 & 5 & 1 & 0 & 0 & 0 & 326 \\
\hline$\dot{\theta}$ & 0 & 0 & 0 & 0 & 5 & -3 & 18 & 50 & 75 & 44 & 41 & 11 & 1 & ? & 0 & 0 & 0 & 0 & 269 \\
\hline 9 & 0 & 0 & 0 & 0 & ) & 66 & 63 & $\dot{\theta u}$ & 86 & B8 & 81 & 50 & 26 & 7 & 0 & 0 & 0 & 0 & 526 \\
\hline in & 0 & 0 & 0 & $?$ & 4 & 21 & 19 & 10 & 25 & 27 & 12 & 19 & 11 & 2 & 0 & 0 & 0 & 0 & 160 \\
\hline 11 & 0 & 0 & 0 & $j$ & 9 & 2.5 & 36 & 55 & 67 & 74 & 68 & 58 & 30 & 11 & 0 & 0 & 0 & 0 & 432 \\
\hline 12 & 0 & 0 & 0 & 0 & 6 & 15 & 31 & 80 & $B 2$ & 76 & 63 & 51 & 27 & 9 & 1 & 0 & 0 & 0 & 447 \\
\hline 13 & 0 & 0 & 0 & 1 & 13 & 34 & $5 B$ & 76 & 85 & 83 & 72 & 53 & 30 & 10 & 0 & 0 & 0 & 0 & 315 \\
\hline 11 & 0 & 0 & 0 & $i$ & 13 & -89 & -99 & -99 & -99 & -99 & -99 & -99 & -99 & -89 & -99 & 0 & 0 & 0 & -999 \\
\hline 15 & 0 & 0 & 0 & -99 & -99 & -99 & -39 & -99 & -90 & -99 & -99 & -99 & -99 & -59 & -5.9 & 0 & 0 & 0 & -1999 \\
\hline 16 & () & 0 & ) & -97 & -94 & -99 & -29 & -99 & -79 & -99 & -99 & -97 & -99 & $-\leq 9$ & $-9: 9$ & 0 & 0 & 0 & -999 \\
\hline 17 & 0 & 0 & 0 & -97 & -99 & -99 & -79 & -99 & -99 & -99 & -99 & -97 & $\therefore 99$ & $-c .0$ & -99 & 0 & 0 & 0 & -999 \\
\hline 18 & 0 & 0 & 0 & -97 & -97 & $-3 \%$ & -97 & -99 & -97 & -99 & -99 & -911 & -99 & -60 & -9.7 & 0 & 0 & 0 & -999 \\
\hline 17 & 0 & 0 & 0 & -99 & -97 & -99 & -79 & .99 & -99 & -99 & -99 & -97 & -99 & $-(x)$ & -99 & 0 & 0 & 0 & -999 \\
\hline 20 & 0 & n & 0 & -97 & $-17 ?$ & .99 & -99 & -99 & -99 & -99 & -99 & -99 & -99 & -69 & -619 & 0 & 0 & 0 & -999 \\
\hline 21 & 0 & 0 & 0 & -9.7 & -99 & .99 & -97 & -99 & -99 & -99 & -97 & -99 & -99 & -69 & -49 & 0 & 0 & 0 & -999 \\
\hline 22 & 0 & 0 & 0 & -97 & -49 & -99 & -49 & -99 & -99 & -99 & -97 & -29 & -99 & -99 & -99 & 0 & 0 & 0 & -999 \\
\hline 2.3 & 0 & 0 & 0 & -97 & -79 & -.09 & -94 & -90 & -99 & -99 & -99 & -99 & -99 & -99 & -99 & 0 & 0 & 0 & -999 \\
\hline 21 & 0 & 0 & 0 & -97 & -99 & -.19 & -99 & $-9 n$ & -99 & -99 & -99 & -99 & .99 & -99 & -99 & 0 & 0 & 0 & -999 \\
\hline 25 & 0 & 0 & 0 & -99 & -97 & -99 & -99 & -99 & -99 & -49 & -99 & -99 & -99 & -99 & -09 & 0 & 0 & 0 & -999 \\
\hline 26 & 0 & 0 & 0 & -97 & -97 & -79 & -99 & -99 & -99 & -99 & -97 & -99 & -99 & -99 & .99 & 0 & 0 & 0 & -999 \\
\hline 27 & 0 & 0 & $n$ & -97 & -99 & -70 & -91 & -99 & -29 & -99 & -99 & -30 & -99 & $-\infty 9$ & -60 & 0 & 0 & 0 & -999 \\
\hline 28 & 0 & 1) & ) & -97 & -97 & -34 & -97 & -99 & -97 & .99 & -91 & -99 & -99 & .99 & -99 & 0 & 0 & 0 & -997 \\
\hline 29 & 0 & 0 & 0 & -97 & -49 & .79 & -79 & -99 & -171 & -99 & -99 & -79 & .99 & -99 & -99 & 0 & 0 & 0 & -999 \\
\hline 30 & ) & 0) & 0 & -9.7 & -97 & -37 & -37 & -99 & -99 & -99 & -94 & -90 & -90 & -90 & -44 & 0 & 0 & 0 & -999 \\
\hline
\end{tabular}

ENERGY KILOJOHLES PEH SHUARE HEIEIT

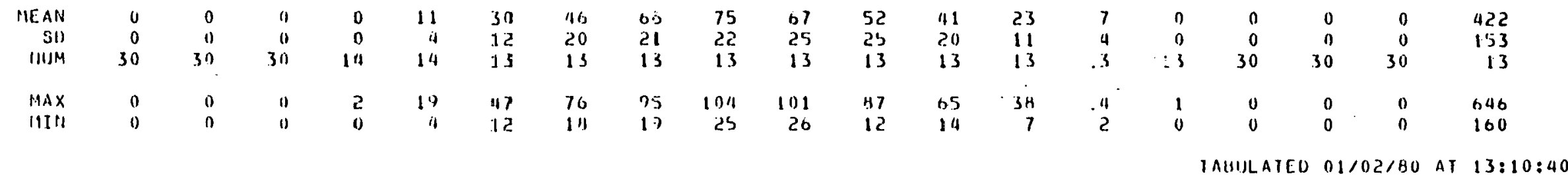


COASTAL - INLAND SULAR RADIATION DIFFERENCE BTUDY MONTHLY DATA SUMMAKY ELLIS AIRPORT DECEMBER $197 \mathrm{H}$

IHSTRUMENT: PSP 2
TY $10.99 E-6$ VIW/SU.

EMERgy KILUJOULES PER siJUARE METEh HOUR ENDING,

\begin{tabular}{|c|c|c|c|c|c|c|c|c|c|c|c|c|c|c|c|}
\hline & \multirow[b]{2}{*}{ DATE } & \multirow[b]{2}{*}{4} & \multirow[b]{2}{*}{5} & \multirow[b]{2}{*}{6} & \multicolumn{11}{|c|}{ HOUR ENDING } \\
\hline & & & & & 7 & 8 & 9 & 10 & 11 & 12 & 13 & 14 & 15 & 16 & 17 \\
\hline : & $\begin{array}{l}1 \\
2 \\
3 \\
4 \\
5\end{array}$ & $\begin{array}{l}0 \\
0 \\
0 \\
0 \\
0\end{array}$ & $\begin{array}{l}0 \\
0 \\
0 \\
0 \\
0\end{array}$ & $\begin{array}{l}0 \\
0 \\
0 \\
0 \\
0\end{array}$ & $\begin{array}{l}0 \\
0 \\
0 \\
0 \\
0\end{array}$ & $\begin{array}{r}76 \\
202 \\
195 \\
70 \\
15\end{array}$ & $\begin{array}{r}89 \\
840 \\
781 \\
388 \\
100\end{array}$ & $\begin{array}{r}427 \\
1332 \\
1057 \\
610 \\
156\end{array}$ & $\begin{array}{r}411 \\
1758 \\
1450 \\
1288 \\
133\end{array}$ & $\begin{array}{r}702 \\
1974 \\
1545 \\
1701 \\
234\end{array}$ & $\begin{array}{r}640 \\
1964 \\
1499 \\
1609 \\
356\end{array}$ & $\begin{array}{r}672 \\
1758 \\
831 \\
1609 \\
182\end{array}$ & $\begin{array}{r}1101 \\
1273 \\
654 \\
1174 \\
310\end{array}$ & $\begin{array}{l}699 \\
706 \\
624 \\
633 \\
342\end{array}$ & $\begin{array}{r}171 \\
172 \\
107 \\
155 \\
25\end{array}$ \\
\hline & $\begin{array}{r}6 \\
7 \\
8 \\
9 \\
10\end{array}$ & $\begin{array}{l}0 \\
0 \\
0 \\
0 \\
0\end{array}$ & $\begin{array}{l}0 \\
0 \\
0 \\
0 \\
0\end{array}$ & $\begin{array}{l}0 \\
0 \\
0 \\
0 \\
0\end{array}$ & $\begin{array}{l}0 \\
0 \\
0 \\
0 \\
0\end{array}$ & $\begin{array}{l}157 \\
162 \\
135 \\
194 \\
124\end{array}$ & $\begin{array}{l}714 \\
621 \\
230 \\
803 \\
723\end{array}$ & $\begin{array}{r}690 \\
1220 \\
649 \\
1295 \\
1329\end{array}$ & $\begin{array}{r}1608 \\
1207 \\
964 \\
1612 \\
1798\end{array}$ & $\begin{array}{l}1962 \\
1384 \\
1219 \\
1734 \\
2034\end{array}$ & $\begin{array}{l}1985 \\
1754 \\
1462 \\
1300 \\
2047\end{array}$ & $\begin{array}{r}1780 \\
1286 \\
1006 \\
810 \\
1831\end{array}$ & $\begin{array}{r}1352 \\
1109 \\
843 \\
885 \\
1392\end{array}$ & $\begin{array}{l}786 \\
356 \\
630 \\
633 \\
799\end{array}$ & $\begin{array}{r}103 \\
97 \\
181 \\
96 \\
196\end{array}$ \\
\hline & $\begin{array}{l}11 \\
12 \\
13 \\
14 \\
15\end{array}$ & $\begin{array}{l}0 \\
0 \\
0 \\
0 \\
0\end{array}$ & $\begin{array}{l}0 \\
0 \\
0 \\
0 \\
0\end{array}$ & $\begin{array}{l}0 \\
0 \\
0 \\
0 \\
0\end{array}$ & $\begin{array}{l}0 \\
0 \\
0 \\
0 \\
0\end{array}$ & $\begin{array}{r}68 \\
24 \\
251 \\
111 \\
250\end{array}$ & $\begin{array}{r}275 \\
328 \\
1021 \\
045 \\
1000\end{array}$ & $\begin{array}{r}497 \\
679 \\
1263 \\
1020 \\
1431\end{array}$ & $\begin{array}{r}917 \\
1396 \\
1728 \\
1170 \\
1749\end{array}$ & $\begin{array}{l}1297 \\
1969 \\
1967 \\
1391 \\
2001\end{array}$ & $\begin{array}{l}1339 \\
1992 \\
1990 \\
1516 \\
2027\end{array}$ & $\begin{array}{l}1100 \\
1793 \\
1787 \\
1303 \\
1827\end{array}$ & $\begin{array}{r}940 \\
1363 \\
1368 \\
1280 \\
1320\end{array}$ & $\begin{array}{l}366 \\
787 \\
794 \\
795 \\
841\end{array}$ & $\begin{array}{r}68 \\
197 \\
188 \\
196 \\
180\end{array}$ \\
\hline$\stackrel{\mapsto \infty}{\bullet}$ & $\begin{array}{l}16 \\
11 \\
19 \\
17 \\
20\end{array}$ & $\begin{array}{l}0 \\
0 \\
0 \\
0 \\
0\end{array}$ & $\begin{array}{l}0 \\
0 \\
0 \\
0 \\
0\end{array}$ & $\begin{array}{l}0 \\
0 \\
0 \\
0 \\
0\end{array}$ & $\begin{array}{l}0 \\
0 \\
0 \\
0 \\
0\end{array}$ & $\begin{array}{r}152 \\
96 \\
54 \\
23 \\
11\end{array}$ & $\begin{array}{r}333 \\
656 \\
375 \\
78 \\
70\end{array}$ & $\begin{array}{r}523 \\
1295 \\
654 \\
239 \\
253\end{array}$ & $\begin{array}{r}1096 \\
1750 \\
9811 \\
373 \\
371\end{array}$ & $\begin{array}{r}1803 \\
2002 \\
778 \\
275 \\
361\end{array}$ & $\begin{array}{r}1790 \\
2028 \\
696 \\
173 \\
407\end{array}$ & $\begin{array}{r}1617 \\
1835 \\
1050 \\
259 \\
453\end{array}$ & $\begin{array}{r}939 \\
1406 \\
722 \\
363 \\
322\end{array}$ & $\begin{array}{l}523 \\
820 \\
342 \\
252 \\
358\end{array}$ & $\begin{array}{r}113 \\
217 \\
136 \\
29 \\
155\end{array}$ \\
\hline & $\begin{array}{l}21 \\
22 \\
23 \\
24 \\
25\end{array}$ & $\begin{array}{l}0 \\
0 \\
0 \\
0 \\
0\end{array}$ & $\begin{array}{l}0 \\
0 \\
0 \\
0 \\
0\end{array}$ & $\begin{array}{l}0 \\
0 \\
0 \\
0 \\
0\end{array}$ & $\begin{array}{l}0 \\
0 \\
0 \\
0 \\
0\end{array}$ & $\begin{array}{r}15 \\
1189 \\
121 \\
18 \\
75\end{array}$ & $\begin{array}{r}150 \\
985 \\
805 \\
28 \\
547\end{array}$ & $\begin{array}{r}101 \\
1218 \\
1172 \\
48 \\
1120\end{array}$ & $\begin{array}{r}235 \\
1686 \\
1362 \\
38 \\
1621\end{array}$ & $\begin{array}{r}353 \\
1945 \\
1546 \\
35 \\
1876\end{array}$ & $\begin{array}{r}491 \\
1981 \\
1700 \\
54 \\
1952\end{array}$ & $\begin{array}{r}584 \\
1795 \\
1339 \\
353 \\
1798\end{array}$ & $\begin{array}{r}1077 \\
1369 \\
923 \\
172 \\
1395\end{array}$ & $\begin{array}{l}157 \\
789 \\
416 \\
100 \\
828\end{array}$ & $\begin{array}{r}203 \\
193 \\
127 \\
64 \\
235\end{array}$ \\
\hline & $\begin{array}{l}20 \\
27 \\
28 \\
27 \\
30\end{array}$ & $\begin{array}{l}0 \\
0 \\
0 \\
0 \\
0\end{array}$ & $\begin{array}{l}0 \\
0 \\
0 \\
0 \\
0\end{array}$ & $\begin{array}{l}0 \\
0 \\
0 \\
0 \\
0\end{array}$ & $\begin{array}{l}0 \\
0 \\
0 \\
0 \\
0\end{array}$ & $\begin{array}{r}173 \\
52 \\
36 \\
75 \\
45\end{array}$ & $\begin{array}{l}746 \\
5133 \\
265 \\
583 \\
2913\end{array}$ & $\begin{array}{r}1159 \\
1238 \\
638 \\
1195 \\
455\end{array}$ & $\begin{array}{r}1628 \\
1703 \\
923 \\
1680 \\
1048\end{array}$ & $\begin{array}{r}1903 \\
1981 \\
979 \\
1952 \\
1942\end{array}$ & $\begin{array}{l}1939 \\
2027 \\
1061 \\
2011 \\
1418\end{array}$ & $\begin{array}{r}1708 \\
1844 \\
825 \\
1837 \\
1723\end{array}$ & $\begin{array}{r}1359 \\
1270 \\
625 \\
1431 \\
1365\end{array}$ & $\begin{array}{l}805 \\
632 \\
494 \\
845 \\
61 H\end{array}$ & $\begin{array}{l}232 \\
160 \\
124 \\
232 \\
248\end{array}$ \\
\hline & 31 & 0 & 0 & D & 0 & 71 & 346 & 739 & 1630 & 1515 & 1361 & 1500 & 1233 & 690 & 113 \\
\hline & $\begin{array}{r}\text { HEAH } \\
\text { SD } \\
\text { HUH }\end{array}$ & $\begin{array}{r}0 \\
0 \\
31\end{array}$ & $\begin{array}{r}0 \\
0 \\
31\end{array}$ & $\begin{array}{r}0 \\
0 \\
31\end{array}$ & $\begin{array}{r}0 \\
0 \\
31\end{array}$ & $\begin{array}{r}100 \\
69 \\
31\end{array}$ & $\begin{array}{r}50.3 \\
302 \\
31\end{array}$ & $\begin{array}{r}837 \\
120 \\
31\end{array}$ & $\begin{array}{r}1213 \\
540 \\
31\end{array}$ & $\begin{array}{r}1431 \\
628 \\
31\end{array}$ & $\begin{array}{r}1440 \\
623 \\
31\end{array}$ & $\begin{array}{r}1301 \\
542 \\
31\end{array}$ & $\begin{array}{r}1043 \\
370 \\
31\end{array}$ & $\begin{array}{r}601 \\
218 \\
31\end{array}$ & $\begin{array}{r}154 \\
58 \\
31\end{array}$ \\
\hline & $\begin{array}{l}\text { MNX } \\
M I N\end{array}$ & $\begin{array}{l}0 \\
0\end{array}$ & $\begin{array}{l}0 \\
11\end{array}$ & $\begin{array}{l}0 \\
0\end{array}$ & $\begin{array}{l}0 \\
0\end{array}$ & $\begin{array}{r}258 \\
11\end{array}$ & $\begin{array}{r}1021 \\
20\end{array}$ & $\begin{array}{r}1431 \\
413\end{array}$ & $\begin{array}{r}1798 \\
38\end{array}$ & $\begin{array}{r}20311 \\
35\end{array}$ & $\begin{array}{r}2047 \\
54\end{array}$ & $\begin{array}{r}1844 \\
182\end{array}$ & $\begin{array}{r}1431 \\
112\end{array}$ & $\begin{array}{l}1845 \\
100\end{array}$ & $\begin{array}{r}244 \\
25\end{array}$ \\
\hline
\end{tabular}


COASTIAL - INLAND SULAR RADIATION DIFFEREHCE STUDY

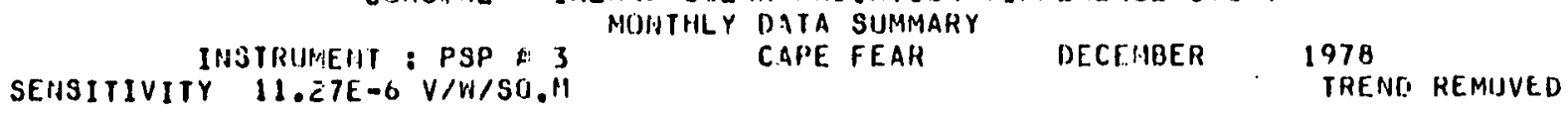

EMERGY KILOJOULES PER SOUARE METER

\begin{tabular}{|c|c|c|c|c|c|c|c|c|c|c|c|c|c|c|c|c|c|c|c|}
\hline \\
\hline RATE. & 4 & 5 & 6 & 7 & 8 & 9 & 10 & 11 & 12 & 13 & 14 & 15 & 16 & 17 & 18 & 19 & 20 & 21 & TOTAL \\
\hline $\begin{array}{l}1 \\
2 \\
3 \\
11 \\
5\end{array}$ & $\begin{array}{l}0 \\
0 \\
0 \\
0 \\
0\end{array}$ & $\begin{array}{l}0 \\
0 \\
0 \\
0 \\
0\end{array}$ & $\begin{array}{l}0 \\
0 \\
0 \\
0 \\
0\end{array}$ & $\begin{array}{l}0 \\
0 \\
0 \\
0 \\
0\end{array}$ & $\begin{array}{r}40 \\
193 \\
190 \\
117 \\
40\end{array}$ & $\begin{array}{r}193 \\
669 \\
692 \\
366 \\
53\end{array}$ & $\begin{array}{r}171 \\
1113 \\
909 \\
586 \\
91\end{array}$ & $\begin{array}{r}312 \\
1567 \\
1273 \\
950 \\
347\end{array}$ & $\begin{array}{r}385 \\
1979 \\
1545 \\
1886 \\
465\end{array}$ & $\begin{array}{r}395 \\
2014 \\
1615 \\
1896 \\
353\end{array}$ & $\begin{array}{r}462 \\
1803 \\
1046 \\
1723 \\
168\end{array}$ & $\begin{array}{r}328 \\
1362 \\
847 \\
1069 \\
628\end{array}$ & $\begin{array}{l}232 \\
787 \\
641 \\
605 \\
117\end{array}$ & $\begin{array}{r}62 \\
187 \\
145 \\
145 \\
24\end{array}$ & $\begin{array}{l}0 \\
0 \\
0 \\
0 \\
0\end{array}$ & $\begin{array}{l}0 \\
0 \\
0 \\
0 \\
0\end{array}$ & $\begin{array}{l}0 \\
0 \\
0 \\
0 \\
0\end{array}$ & $\begin{array}{l}0 \\
0 \\
0 \\
0 \\
0\end{array}$ & $\begin{array}{r}2860 \\
11674 \\
8946 \\
9343 \\
2286\end{array}$ \\
\hline $\begin{array}{r}6 \\
7 \\
8 \\
7 \\
10\end{array}$ & $\begin{array}{l}0 \\
0 \\
0 \\
0 \\
0\end{array}$ & $\begin{array}{l}0 \\
0 \\
0 \\
0 \\
0\end{array}$ & $\begin{array}{l}0 \\
0 \\
0 \\
0 \\
0\end{array}$ & $\begin{array}{l}0 \\
0 \\
0 \\
0 \\
0\end{array}$ & $\begin{array}{r}99 \\
155 \\
144 \\
-99 \\
86\end{array}$ & $\begin{array}{l}150 \\
666 \\
336 \\
-99 \\
705\end{array}$ & $\begin{array}{r}945 \\
1225 \\
576 \\
-99 \\
1335\end{array}$ & $\begin{array}{r}1753 \\
1315 \\
9 E 8 \\
-60 \\
1830\end{array}$ & $\begin{array}{l}2009 \\
1292 \\
1211 \\
1449 \\
2085\end{array}$ & $\begin{array}{l}2012 \\
1909 \\
1361 \\
1772 \\
2117\end{array}$ & $\begin{array}{r}1804 \\
1423 \\
-99 \\
1375 \\
1926\end{array}$ & $\begin{array}{r}1379 \\
1027 \\
-99 \\
701 \\
1510\end{array}$ & $\begin{array}{l}788 \\
494 \\
-99 \\
427 \\
907\end{array}$ & $\begin{array}{r}191 \\
85 \\
-99 \\
85 \\
268\end{array}$ & $\begin{array}{l}0 \\
0 \\
0 \\
0 \\
0\end{array}$ & $\begin{array}{l}0 \\
n \\
0 \\
0 \\
0\end{array}$ & $\begin{array}{l}0 \\
0 \\
0 \\
0 \\
0\end{array}$ & $\begin{array}{l}0 \\
0 \\
0 \\
0 \\
0\end{array}$ & $\begin{array}{r}11430 \\
.9591 \\
-999 \\
-999 \\
12769\end{array}$ \\
\hline $\begin{array}{l}11 \\
12 \\
13 \\
14 \\
15\end{array}$ & $\begin{array}{l}0 \\
0 \\
0 \\
0 \\
0\end{array}$ & $\begin{array}{l}0 \\
0 \\
0 \\
0 \\
0\end{array}$ & $\begin{array}{l}0 \\
0 \\
0 \\
0 \\
0\end{array}$ & $\begin{array}{l}0 \\
0 \\
0 \\
0 \\
0\end{array}$ & $\begin{array}{r}75 \\
25 \\
-11 \\
95 \\
: 91\end{array}$ & $\begin{array}{l}359 \\
172 \\
632 \\
520 \\
913\end{array}$ & $\begin{array}{r}727 \\
712 \\
1239 \\
1175 \\
1229\end{array}$ & $\begin{array}{l}110.7 \\
11618 \\
1737 \\
162.5 \\
1721\end{array}$ & $\begin{array}{r}887 \\
1996 \\
1999 \\
1983 \\
2006\end{array}$ & $\begin{array}{r}978 \\
2054 \\
2044 \\
2073 \\
2044\end{array}$ & $\begin{array}{r}925 \\
1868 \\
1868 \\
1843 \\
1875\end{array}$ & $\begin{array}{l}631 \\
1960 \\
1472 \\
1373 \\
1488\end{array}$ & $\begin{array}{l}372 \\
8 B A \\
884 \\
910 \\
910\end{array}$ & $\begin{array}{l}110 \\
278 \\
281 \\
290 \\
293\end{array}$ & $\begin{array}{l}0 \\
0 \\
0 \\
0 \\
0\end{array}$ & $\begin{array}{l}0 \\
0 \\
0 \\
0 \\
0\end{array}$ & $\begin{array}{l}0 \\
0 \\
0 \\
0 \\
0\end{array}$ & $\begin{array}{l}0 \\
0 \\
0 \\
0 \\
0\end{array}$ & $\begin{array}{l}6191 \\
10567 \\
12207 \\
111887 \\
12670\end{array}$ \\
\hline $\begin{array}{l}16 \\
17 \\
18 \\
17 \\
20\end{array}$ & $\begin{array}{l}0 \\
0 \\
0 \\
0 \\
0\end{array}$ & $\begin{array}{l}0 \\
0 \\
0 \\
0 \\
0\end{array}$ & $\begin{array}{l}0 \\
0 \\
0 \\
0 \\
0\end{array}$ & $\begin{array}{l}0 \\
0 \\
0 \\
0 \\
0\end{array}$ & $\begin{array}{l}101 \\
42 \\
56 \\
18 \\
211\end{array}$ & $\begin{array}{l}318 \\
017 \\
423 \\
137 \\
248\end{array}$ & $\begin{array}{r}605 \\
1262 \\
682 \\
318 \\
602\end{array}$ & $\begin{array}{r}954 \\
1757 \\
915 \\
552 \\
807\end{array}$ & $\begin{array}{r}1439 \\
2055 \\
637 \\
670 \\
848\end{array}$ & $\begin{array}{r}9771 \\
6115 \\
855 \\
714 \\
1136\end{array}$ & $\begin{array}{r}1858 \\
1911 \\
1247 \\
599 \\
1644\end{array}$ & $\begin{array}{r}1104 \\
1521 \\
781 \\
535 \\
922\end{array}$ & $\begin{array}{l}542 \\
927 \\
433 \\
344 \\
051\end{array}$ & $\begin{array}{r}216 \\
301 \\
152 \\
91 \\
181\end{array}$ & $\begin{array}{l}0 \\
0 \\
0 \\
0 \\
0\end{array}$ & $\begin{array}{l}0 \\
0 \\
0 \\
0 \\
0\end{array}$ & $\begin{array}{l}0 \\
0 \\
0 \\
0 \\
0\end{array}$ & $\begin{array}{l}0 \\
0 \\
0 \\
0 \\
0\end{array}$ & $\begin{array}{r}9178 \\
12520 \\
6181 \\
3960 \\
7146\end{array}$ \\
\hline $\begin{array}{l}21 \\
22 \\
23 \\
24 \\
25\end{array}$ & $\begin{array}{l}0 \\
0 \\
0 \\
0 \\
0\end{array}$ & $\begin{array}{l}0 \\
0 \\
0 \\
0 \\
0\end{array}$ & $\begin{array}{l}0 \\
0 \\
0 \\
0 \\
0\end{array}$ & $\begin{array}{l}0 \\
0 \\
0 \\
0 \\
0\end{array}$ & $\begin{array}{r}43 \\
41 \\
105 \\
0 \\
37\end{array}$ & $\begin{array}{r}347 \\
578 \\
536 \\
15 \\
519\end{array}$ & $\begin{array}{r}213 \\
1223 \\
1050 \\
11 \\
1126\end{array}$ & $\begin{array}{r}120 \\
1728 \\
1616 \\
118 \\
1644\end{array}$ & $\begin{array}{r}152 \\
1990 \\
1769 \\
24 \\
1960\end{array}$ & $\begin{array}{r}321 \\
2044 \\
1747 \\
101 \\
2011\end{array}$ & $\begin{array}{r}535 \\
1868 \\
1456 \\
299 \\
1867\end{array}$ & $\begin{array}{r}676 \\
1482 \\
10119 \\
75 \\
1493\end{array}$ & $\begin{array}{r}762 \\
881 \\
453 \\
50 \\
725\end{array}$ & $\begin{array}{l}328 \\
260 \\
178 \\
98 \\
318\end{array}$ & $\begin{array}{l}0 \\
0 \\
0 \\
0 \\
0\end{array}$ & $\begin{array}{l}0 \\
0 \\
0 \\
0 \\
0\end{array}$ & $\begin{array}{l}0 \\
0 \\
0 \\
0 \\
0\end{array}$ & $\begin{array}{l}0 \\
0 \\
0 \\
0 \\
0\end{array}$ & $\begin{array}{r}3497 \\
12043 \\
9919 \\
691 \\
11900\end{array}$ \\
\hline $\begin{array}{l}26 \\
27 \\
28 \\
29 \\
30\end{array}$ & $\begin{array}{l}0 \\
0 \\
0 \\
0 \\
0\end{array}$ & $\begin{array}{l}0 \\
0 \\
0 \\
0 \\
0\end{array}$ & $\begin{array}{l}0 \\
0 \\
0 \\
0 \\
0\end{array}$ & $\begin{array}{l}0 \\
0 \\
0 \\
0 \\
0\end{array}$ & $\begin{array}{l}70 \\
54 \\
25 \\
67 \\
34\end{array}$ & $\begin{array}{l}552 \\
346 \\
172 \\
530 \\
353\end{array}$ & $\begin{array}{r}1149 \\
1133 \\
507 \\
1162 \\
676\end{array}$ & $\begin{array}{r}16 \leq 1 \\
17 \div 7 \\
6=6 \\
16=3 \\
10=5\end{array}$ & $\begin{array}{r}1734 \\
2037 \\
852 \\
1974 \\
1813\end{array}$ & $\begin{array}{l}2025 \\
2095 \\
2038 \\
2053 \\
2624\end{array}$ & $\begin{array}{r}1868 \\
1881 \\
776 \\
1922 \\
1592\end{array}$ & $\begin{array}{r}1504 \\
1379 \\
568 \\
1555 \\
1408\end{array}$ & $\begin{array}{l}926 \\
737 \\
396 \\
967 \\
387\end{array}$ & $\begin{array}{l}319 \\
198 \\
172 \\
268 \\
324\end{array}$ & $\begin{array}{l}0 \\
0 \\
0 \\
0 \\
0\end{array}$ & $\begin{array}{l}0 \\
0 \\
0 \\
0 \\
0\end{array}$ & $\begin{array}{l}0 \\
0 \\
0 \\
0 \\
0\end{array}$ & $\begin{array}{l}0 \\
0 \\
0 \\
0 \\
0\end{array}$ & $\begin{array}{r}12002 \\
11657 \\
5132 \\
12181 \\
9776\end{array}$ \\
\hline 31 & 0 & 0 & $n$ & 0 & 68 & 2511 & 870 & 1036 & 1767 & : 969 & 1655 & 1269 & 796 & 2.24 & 0 & 0 & 0 & 0 & $9 A 04$ \\
\hline $\begin{array}{r}\text { MEAH } \\
\text { SO } \\
\text { HIJPH }\end{array}$ & $\begin{array}{r}0 \\
0 \\
31\end{array}$ & $\begin{array}{l}0 \\
0 \\
31\end{array}$ & $\begin{array}{r}0 \\
0 \\
31\end{array}$ & $\begin{array}{r}0 \\
0 \\
31\end{array}$ & $\begin{array}{l}78 \\
52 \\
30\end{array}$ & $\begin{array}{r}123 \\
211 \\
31\end{array}$ & $\begin{array}{r}433 \\
369 \\
30\end{array}$ & $\begin{array}{r}1152 \\
530 \\
50\end{array}$ & $\begin{array}{r}1454 \\
640 \\
31\end{array}$ & $\begin{array}{r}1551 \\
625 \\
31\end{array}$ & $\begin{array}{r}1436 \\
550 \\
30\end{array}$ & $\begin{array}{r}11198 \\
417 \\
30\end{array}$ & $\begin{array}{r}654 \\
261 \\
30\end{array}$ & $\begin{array}{r}202 \\
87 \\
30\end{array}$ & $\begin{array}{r}0 \\
31 \\
31\end{array}$ & $\begin{array}{r}0 \\
0 \\
31\end{array}$ & $\begin{array}{r}0 \\
0 \\
31\end{array}$ & $\begin{array}{r}0 \\
0 \\
31\end{array}$ & $\begin{array}{r}8970 \\
3537 \\
29\end{array}$ \\
\hline $\begin{array}{l}\text { MAX } \\
\text { MIN }\end{array}$ & $\begin{array}{l}0 \\
0\end{array}$ & $\begin{array}{l}0 \\
0\end{array}$ & $\begin{array}{l}0 \\
0\end{array}$ & $\begin{array}{l}0 \\
0\end{array}$ & $\begin{array}{r}193 \\
0\end{array}$ & $\begin{array}{r}413 \\
15\end{array}$ & $\begin{array}{r}1335 \\
11\end{array}$ & $\begin{array}{r}1850 \\
19\end{array}$ & $\begin{array}{r}2085 \\
24\end{array}$ & $\begin{array}{r}2117 \\
101\end{array}$ & $\begin{array}{r}1926 \\
168\end{array}$ & $\begin{array}{r}1555 \\
75\end{array}$ & $\begin{array}{r}967 \\
50\end{array}$ & $\begin{array}{r}328 \\
24\end{array}$ & $\begin{array}{l}0 \\
0\end{array}$ & $\begin{array}{l}0 \\
0\end{array}$ & $\begin{array}{l}0 \\
0\end{array}$ & $\begin{array}{l}0 \\
0\end{array}$ & $\begin{array}{r}12769 \\
691\end{array}$ \\
\hline
\end{tabular}


CUASTIL. - IILLAIJ SOLAR RADIATION DIFFEREHCE 9TUDY IMSIRUMENT: PSP A 4 MONTILY DATA SUMMARY
SEHSITIVITY $10.37 E-6$ VIW/SO. HALLACE

EHERGY KILOJOJULES PER SIJUARE. METER

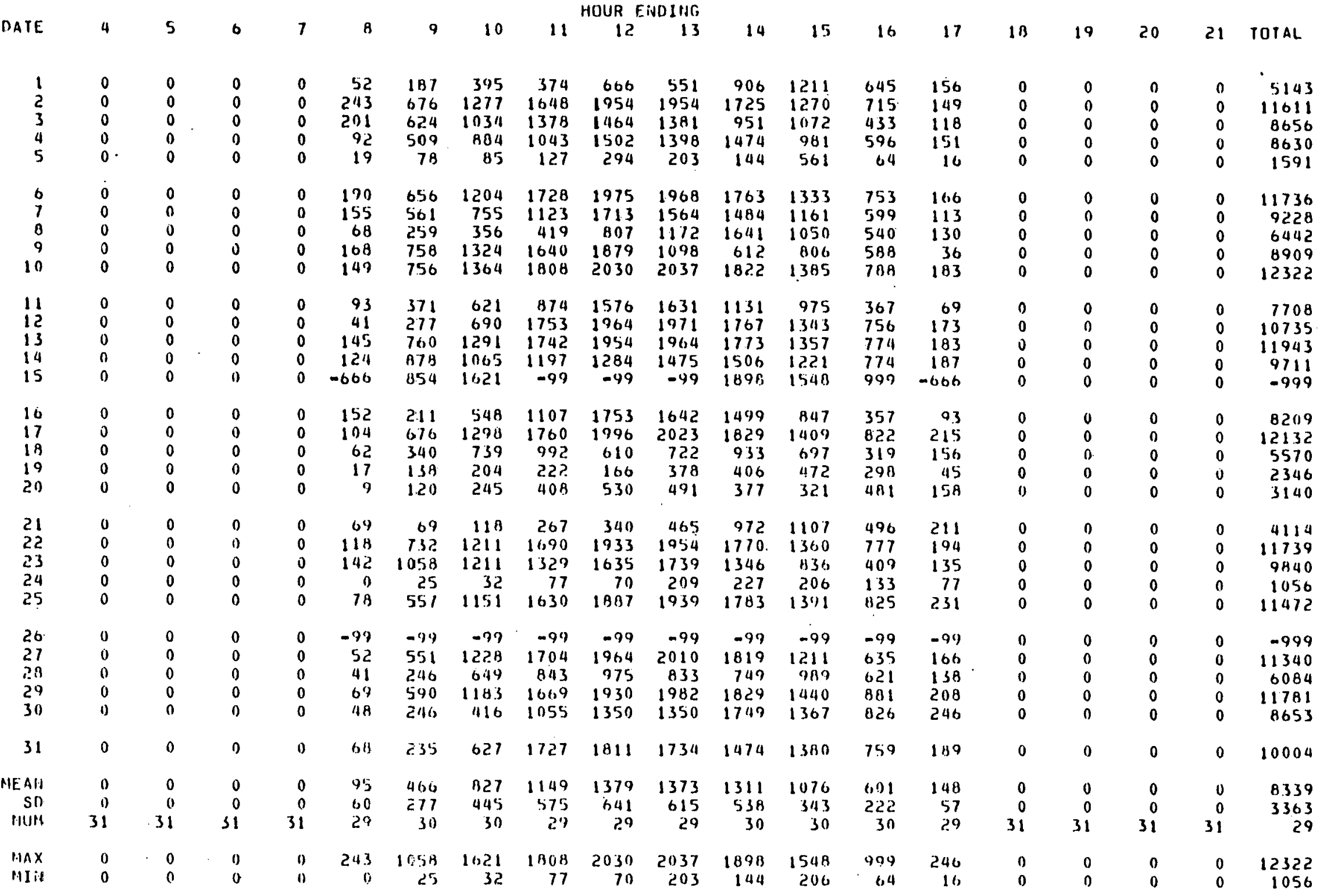


CIASTAL - II.LAIID SOLAR RADIATIOH DIFFEREIICE STUDY MONTHLY DATA SUMMARY

INSTRUHENT: PSP $\$ 5$
SENSITIVITY $9.4 A E-6 . V / W / S O .11$

EHERGY KILOJOULES PER SIJJARE METER

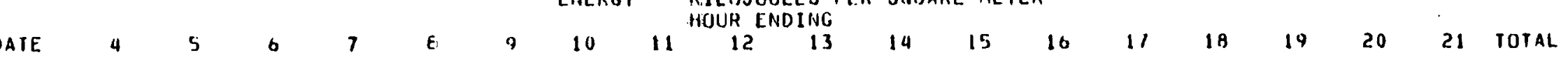

$\begin{array}{ll}1 & 0 \\ 2 & 0 \\ 3 & 0 \\ 4 & 0 \\ 5 & 0 \\ & 0 \\ 6 & 0 \\ 7 & 0 \\ 8 & 0 \\ 9 & 0 \\ 0 & 0 \\ 1 & \\ 1 & 0 \\ 2 & 0 \\ 3 & 0 \\ 4 & 0 \\ 5 & 0\end{array}$

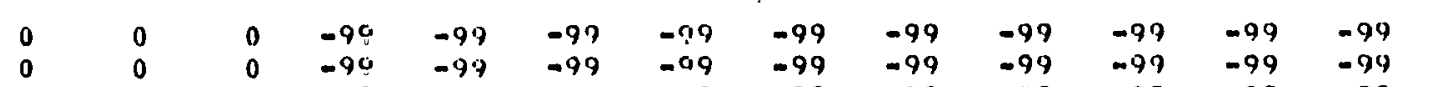

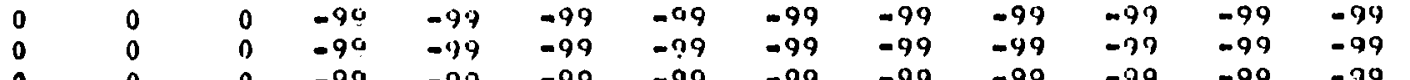

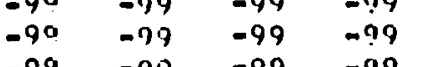

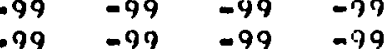

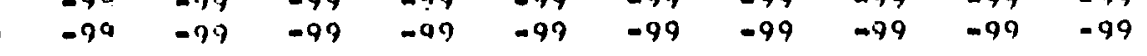

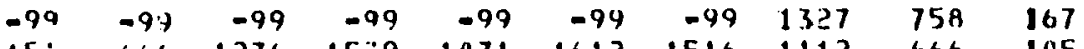

15: $6650.51276 \quad 1539 \quad 1971 \quad 1612 \quad 1516 \quad 1112 \quad 666 \quad 115$

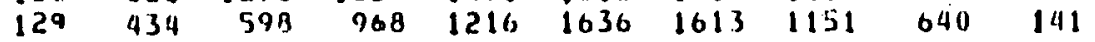

$\begin{array}{rrrrrrrrrr}145 & 655 & 1193 & 732 & 1361 & 1861 & 1521 & 659 & 369 & 99 \\ 106 & 755 & 1372 & 1826 & 2059 & 2074 & 1857 & 1414 & 816 & 190\end{array}$

8. 366 804 1132 968 980 899 633 343 613

$\begin{array}{rrrrrrrrrr}19 & 198 & 750 & 11: 7 & 2013 & 2013 & 1811 & 1372 & 797 & 186 \\ 137 & 690 & 1277 & 1727 & 1941 & 1983 & 1796 & 1365 & 793 & 194\end{array}$

1395331346 18: 2013200515591426

$286-060 \quad 1319 \quad 1735 \quad 1794 \quad 2024 \quad 1790 \quad 1384 \quad 800 \quad 190$

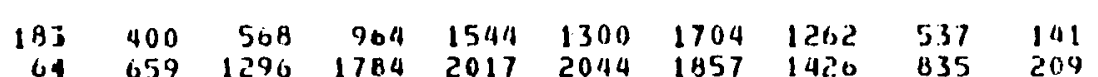

$\begin{array}{rrrrrrrrrr}64 & 659 & 1296 & 1784 & 2017 & 20144 & 1857 & 1420 & 835 & 209 \\ 68 & 1142 & 732 & 1044 & 594 & 736 & 1128 & 709 & 327 & 100\end{array}$

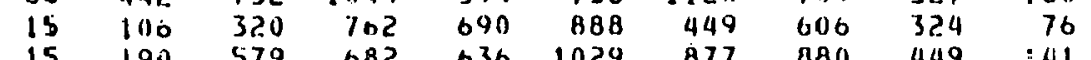

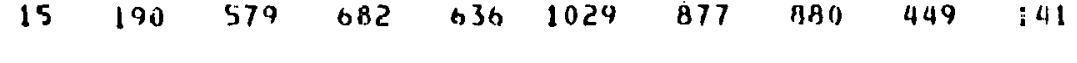

$\begin{array}{rrrrrrrrrr}49 & 316 & 118 & -99 & -79 & -99 & -99 & -99 & -99 & 293 \\ 61 & 579 & 1178 & 1677 & 1906 & 2009 & 1845 & 1445 & 873 & 255\end{array}$

$\begin{array}{lllllllllll}167 & 732 & 1163 & 1571 & 1323 & 1609 & 1475 & 1193 & 461 & 214\end{array}$

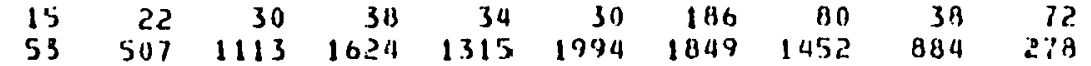

$72 \quad 376 \quad 1330 \quad 1624 \quad 1540 \quad 1990 \quad 182 ? \quad 1437 \quad 8880270$

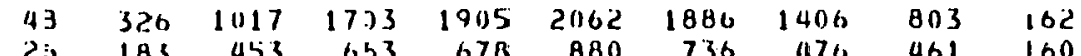

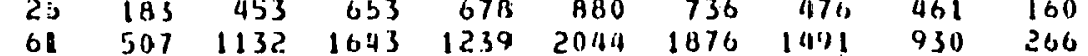

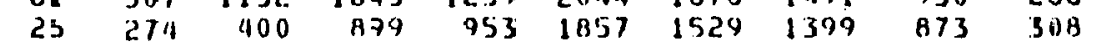

30

$$
53
$$

$\begin{array}{rr}\text { MENH } & 0 \\ \text { NUN: } & 0 \\ \text { NUN: } & 31\end{array}$

$\operatorname{lnx}$

\begin{tabular}{|c|c|c|c|}
\hline 0 & 0 & 0 & -999 \\
\hline 0 & 0 & 0 & -999 \\
\hline 0 & 0 & 0 & -999 \\
\hline 0 & 0 & 0 & -999 \\
\hline 0 & 0 & 0 & .999 \\
\hline 0 & o & 0 & -999 \\
\hline 0 & 0 & 0 & 10114 \\
\hline 0 & 0 & 0 & 8526 \\
\hline 0 & 0 & 0 & 月541 \\
\hline 0 & 0 & 0 & 12469 \\
\hline 0 & 0 & 0 & 6280 \\
\hline 0 & 0 & 0 & 10284 \\
\hline ) & 0 & 0 & 11903 \\
\hline 0 & 0 & 0 & 11870 \\
\hline 0 & 0 & 0 & -999 \\
\hline 0 & 0 & 0 & $A 603$ \\
\hline 0 & 0 & 0 & 12191 \\
\hline 0 & 0 & 0 & 5940 \\
\hline 0 & 0 & 0 & 4236 \\
\hline 0 & 0 & 0 & 5478 \\
\hline 0 & 0 & 0 & -999 \\
\hline 0 & 0 & 0 & 11828 \\
\hline 0 & 0 & 0 & 9838 \\
\hline 0 & 0 & 0 & 545 \\
\hline 0 & 0 & 0 & 11069 \\
\hline 0 & 0 & 0 & 11377 \\
\hline 0 & 0 & 0 & 11318 \\
\hline 0 & 0 & 0 & 4716 \\
\hline 0 & o & 0 & 11189 \\
\hline 0 & 0 & 0 & 8518 \\
\hline 0 & 0 & 0 & 8831 \\
\hline 0 & 0 & 0 & 8941 \\
\hline 0 & 0 & 0 & 3043 \\
\hline 31 & 31 & 3.1 & 23 \\
\hline 0 & 0 & 0 & 12469 \\
\hline 0 & 0 & 0 & 545 \\
\hline
\end{tabular}


CIJA3TAL - IMLAHU SULAR RADIATION DIFFERENCE STUUY MUNTHLY DATA SUMHARY

IIISTRUMENT: PSP \# 6
SENSITIVITY $11.00 E-6$ VIW/SH.

EHERgY KILOJUULES PER SQUARE METER

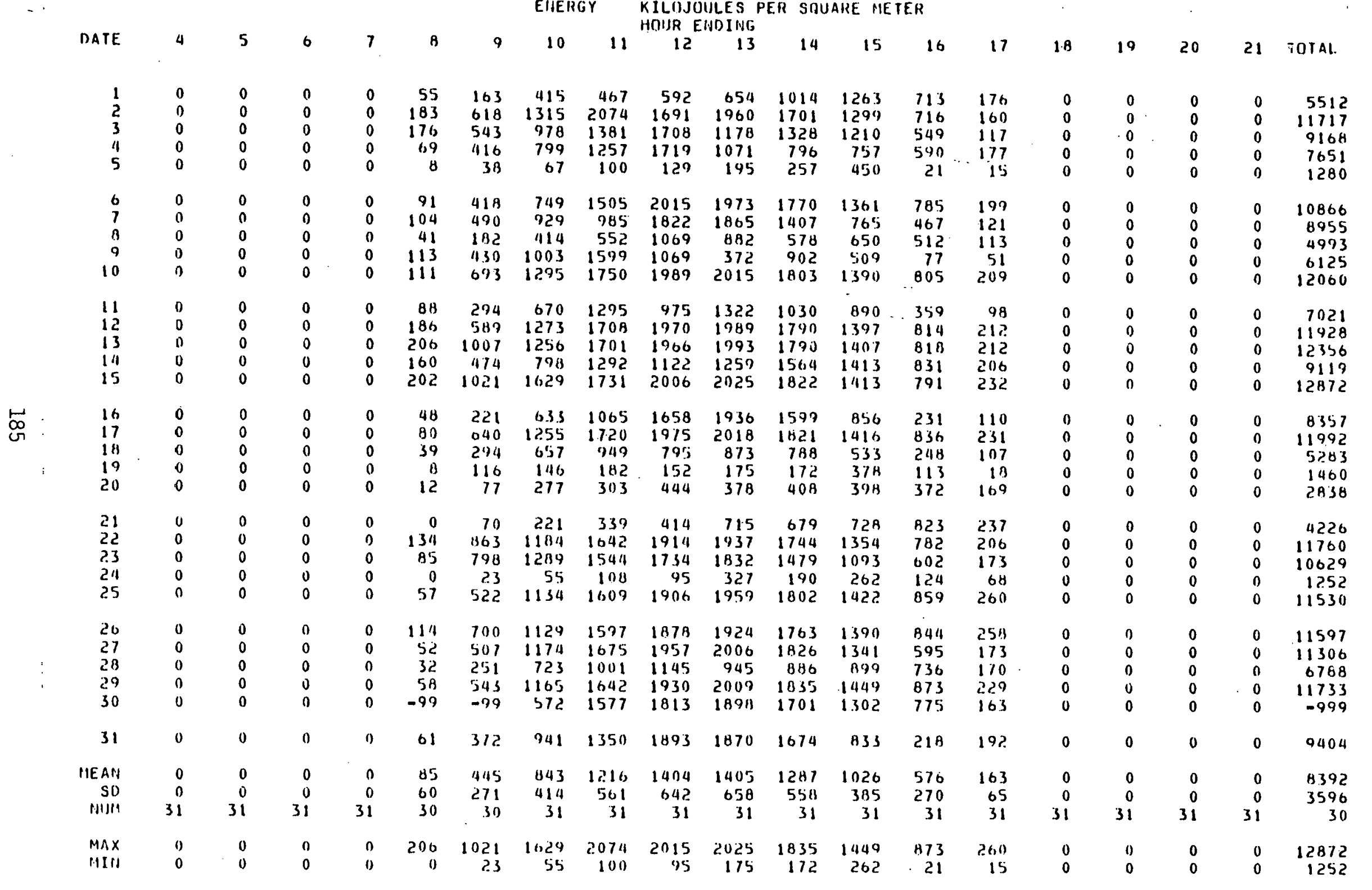


CTASTAL - INLANID SOLAR RADIATIOH DIFFEREHCE STIJEY MOIITHLY DATA SUMMARY

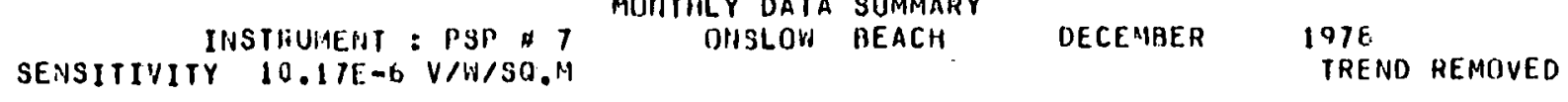

ENERGY KILOJJOJLES PER SIJUARE METER

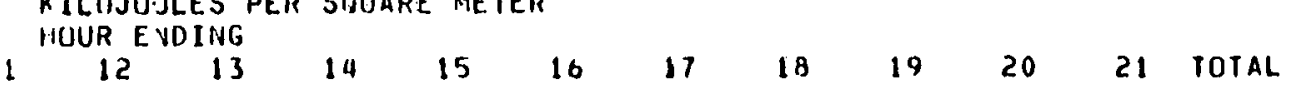

\begin{tabular}{|c|c|c|c|c|c|c|c|c|c|c|c|c|c|c|c|}
\hline $\begin{array}{l}0 \\
0\end{array}$ & $\begin{array}{r}45 \\
243 \\
229 \\
105 \\
20\end{array}$ & $\begin{array}{l}232 \\
767 \\
6155 \\
200 \\
175\end{array}$ & $\begin{array}{l}310 \\
1531 \\
1131 \\
1160 \\
154\end{array}$ & $\begin{array}{r}268 \\
1829 \\
1538 \\
1496 \\
245\end{array}$ & $\begin{array}{r}430 \\
2020 \\
1577 \\
1843 \\
222\end{array}$ & $\begin{array}{r}423 \\
1991 \\
1358 \\
1846 \\
144\end{array}$ & $\begin{array}{r}406 \\
1740 \\
1262 \\
1701 \\
398\end{array}$ & $\begin{array}{r}572 \\
1262 \\
466 \\
1053 \\
190\end{array}$ & $\begin{array}{l}268 \\
639 \\
352 \\
565 \\
487\end{array}$ & $\begin{array}{r}74 \\
109 \\
84 \\
54 \\
24\end{array}$ & $\begin{array}{l}n \\
0 \\
0 \\
0 \\
0\end{array}$ & $\begin{array}{l}0 \\
0 \\
0 \\
0 \\
0\end{array}$ & $\begin{array}{l}0 \\
0 \\
0 \\
0 \\
0\end{array}$ & $\begin{array}{l}0 \\
0 \\
0 \\
0 \\
0\end{array}$ & $\begin{array}{r}2988 \\
12151 \\
8692 \\
10123 \\
2067\end{array}$ \\
\hline $\begin{array}{l}0 \\
0 \\
0 \\
0\end{array}$ & $\begin{array}{l}186 \\
204 \\
147 \\
-99 \\
-99\end{array}$ & $\begin{array}{r}-600 \\
770 \\
501 \\
-79 \\
-97\end{array}$ & $\begin{array}{r}1478 \\
1425 \\
579 \\
-99 \\
-99\end{array}$ & $\begin{array}{r}1819 \\
1669 \\
777 \\
-99 \\
-99\end{array}$ & $\begin{array}{r}2020 \\
1878 \\
961 \\
-99 \\
-99\end{array}$ & $\begin{array}{r}1991 \\
1213 \\
1468 \\
-99 \\
-99\end{array}$ & $\begin{array}{r}1737 \\
1425 \\
1627 \\
-99 \\
-97\end{array}$ & $\begin{array}{r}1266 \\
1156 \\
-79 \\
-99 \\
-99\end{array}$ & $\begin{array}{l}682 \\
519 \\
-99 \\
-99 \\
-99\end{array}$ & $\begin{array}{l}1 \geq 7 \\
112 \\
-49 \\
-49 \\
-99\end{array}$ & $\begin{array}{l}0 \\
0 \\
0 \\
0 \\
0\end{array}$ & $\begin{array}{l}0 \\
0 \\
0 \\
0 \\
0\end{array}$ & $\begin{array}{l}0 \\
0 \\
0 \\
0 \\
0\end{array}$ & $\begin{array}{l}0 \\
0 \\
0 \\
0 \\
0\end{array}$ & $\begin{array}{r}-999 \\
10371 \\
-999 \\
-999 \\
-999\end{array}$ \\
\hline $\begin{array}{l}0 \\
0 \\
0 \\
0\end{array}$ & $\begin{array}{r}-99 \\
-99 \\
-99 \\
161 \\
-660\end{array}$ & $\begin{array}{r}-99 \\
-97 \\
-97 \\
781 \\
1015\end{array}$ & $\begin{array}{r}-27 \\
-29 \\
-99 \\
1531 \\
1387\end{array}$ & $\begin{array}{r}-90 \\
-49 \\
-98 \\
1828 \\
1436\end{array}$ & $\begin{array}{r}-79 \\
-99 \\
-99 \\
2091 \\
2053\end{array}$ & $\begin{array}{r}-99 \\
=99 \\
-99 \\
1758 \\
2074\end{array}$ & $\begin{array}{r}-99 \\
-99 \\
-99 \\
1478 \\
1865\end{array}$ & $\begin{array}{r}-79 \\
-99 \\
1355 \\
1128 \\
11115\end{array}$ & $\begin{array}{l}-99 \\
-99 \\
775 \\
643 \\
792\end{array}$ & $\begin{array}{l}-99 \\
-99 \\
197 \\
183 \\
187\end{array}$ & $\begin{array}{l}0 \\
0 \\
0 \\
0 \\
0\end{array}$ & $\begin{array}{l}n \\
0 \\
0 \\
n \\
0\end{array}$ & $\begin{array}{l}0 \\
0 \\
0 \\
0 \\
0\end{array}$ & $\begin{array}{l}0 \\
0 \\
0 \\
0 \\
0\end{array}$ & $\begin{array}{r}-999 \\
-999 \\
-999 \\
11583 \\
-999\end{array}$ \\
\hline $\begin{array}{l}0 \\
0\end{array}$ & $\begin{array}{r}184 \\
84 \\
81 \\
27 \\
20\end{array}$ & $\begin{array}{r}470 \\
764 \\
495 \\
98 \\
69\end{array}$ & $\begin{array}{r}605 \\
1112 \\
768 \\
282 \\
299\end{array}$ & $\begin{array}{r}1054 \\
1876 \\
998 \\
548 \\
36 ?\end{array}$ & $\begin{array}{r}1706 \\
2109 \\
785 \\
498 \\
515\end{array}$ & $\begin{array}{r}1299 \\
2092 \\
718 \\
257 \\
731\end{array}$ & $\begin{array}{r}1150 \\
1876 \\
828 \\
317 \\
728\end{array}$ & $\begin{array}{r}1281 \\
1426 \\
722 \\
314 \\
B 80\end{array}$ & $\begin{array}{l}5911 \\
814 \\
389 \\
222 \\
554\end{array}$ & $\begin{array}{r}120 \\
208 \\
102 \\
27 \\
140\end{array}$ & $\begin{array}{l}0 \\
0 \\
0 \\
0 \\
0\end{array}$ & $\begin{array}{l}0 \\
0 \\
0 \\
0 \\
0\end{array}$ & $\begin{array}{l}0 \\
0 \\
0 \\
0 \\
0\end{array}$ & $\begin{array}{l}0 \\
0 \\
0 \\
0 \\
0\end{array}$ & $\begin{array}{r}8463 \\
12061 \\
5886 \\
2582 \\
4299\end{array}$ \\
\hline $\begin{array}{l}0 \\
0 \\
0 \\
0 \\
0 \\
1\end{array}$ & $\begin{array}{r}91 \\
109 \\
180 \\
43 \\
102\end{array}$ & $\begin{array}{r}519 \\
6.10 \\
615 \\
43 \\
615\end{array}$ & $\begin{array}{r}1613 \\
1306 \\
1352 \\
18 \\
1217\end{array}$ & $\begin{array}{r}144 \\
1706 \\
1684 \\
28 \\
1684\end{array}$ & $\begin{array}{r}175 \\
1789 \\
1051 \\
46 \\
1753\end{array}$ & $\begin{array}{r}2683 \\
2007 \\
1430 \\
50 \\
1971\end{array}$ & $\begin{array}{r}515 \\
1798 \\
1539 \\
198 \\
1773\end{array}$ & $\begin{array}{r}678 \\
1394 \\
1192 \\
74 \\
1373\end{array}$ & $\begin{array}{r}717 \\
745 \\
502 \\
32 \\
007\end{array}$ & $\begin{array}{r}225 \\
194 \\
116 \\
71 \\
22.6\end{array}$ & $\begin{array}{l}0 \\
0 \\
0 \\
0 \\
0\end{array}$ & $\begin{array}{l}0 \\
0 \\
0 \\
0 \\
0\end{array}$ & $\begin{array}{l}0 \\
0 \\
0 \\
0 \\
0\end{array}$ & $\begin{array}{l}0 \\
0 \\
0 \\
0 \\
0\end{array}$ & $\begin{array}{r}3500 \\
11988 \\
10461 \\
603 \\
11721\end{array}$ \\
\hline $\begin{array}{l}0 \\
0 \\
0 \\
0 \\
0\end{array}$ & $\begin{array}{r}120 \\
-99 \\
52 \\
116 \\
56\end{array}$ & $\begin{array}{l}658 \\
-119 \\
260 \\
665 \\
213\end{array}$ & $\begin{array}{r}1231 \\
-99 \\
533 \\
1288 \\
513\end{array}$ & $\begin{array}{r}1706 \\
-99 \\
820 \\
1740 \\
053\end{array}$ & $\begin{array}{r}1971 \\
-99 \\
784 \\
2.035 \\
1405\end{array}$ & $\begin{array}{r}1990 \\
-99 \\
912 \\
2063 \\
1203\end{array}$ & $\begin{array}{r}1752 \\
-99 \\
809 \\
1861 \\
1578\end{array}$ & $\begin{array}{r}-79 \\
1387 \\
540 \\
1447 \\
1391\end{array}$ & $\begin{array}{l}-99 \\
633 \\
646 \\
877 \\
824\end{array}$ & $\begin{array}{l}-99 \\
134 \\
1.59 \\
215 \\
3.23\end{array}$ & $\begin{array}{l}0 \\
0 \\
0 \\
0 \\
0\end{array}$ & $\begin{array}{l}0 \\
0 \\
0 \\
0 \\
0\end{array}$ & $\begin{array}{l}0 \\
0 \\
0 \\
0 \\
0\end{array}$ & $\begin{array}{l}0 \\
0 \\
0 \\
0 \\
0\end{array}$ & $\begin{array}{r}-999 \\
-999 \\
5514 \\
12347 \\
8329\end{array}$ \\
\hline ( & 105 & 645 & 675 & 1443 & 1673 & 1790 & 1578 & 696 & 540 & 1608 & 0 & 0 & 0 & 0 & 9373 \\
\hline 0 & $\begin{array}{r}112 \\
64 \\
24\end{array}$ & $\begin{array}{r}50 ? \\
250 \\
3.4\end{array}$ & $\begin{array}{r}874 \\
500 \\
25\end{array}$ & $\begin{array}{r}1201 \\
626 \\
25\end{array}$ & $\begin{array}{r}138.3 \\
709 \\
25\end{array}$ & $\begin{array}{r}1322 \\
682 \\
25\end{array}$ & $\begin{array}{r}1278 \\
557 \\
25\end{array}$ & $\begin{array}{r}985 \\
425 \\
25\end{array}$ & $\begin{array}{r}586 \\
205 \\
25\end{array}$ & $\begin{array}{r}140 \\
54 \\
255\end{array}$ & $\begin{array}{r}0 \\
0 \\
3.1\end{array}$ & $\begin{array}{r}0 \\
31\end{array}$ & $\begin{array}{r}0 \\
0 \\
31\end{array}$ & $\begin{array}{r}0 \\
0 \\
31\end{array}$ & $\begin{array}{r}7890 \\
3829 \\
21\end{array}$ \\
\hline ri & $\begin{array}{r}243 \\
20\end{array}$ & $\begin{array}{r}1015 \\
43\end{array}$ & $\begin{array}{r}1531 \\
18\end{array}$ & $\begin{array}{r}1876 \\
21]\end{array}$ & $\begin{array}{r}2109 \\
46\end{array}$ & $\begin{array}{r}2092 \\
50\end{array}$ & $\begin{array}{r}1876 \\
198\end{array}$ & $\begin{array}{r}1447 \\
74\end{array}$ & $\begin{array}{r}877 \\
3 ?\end{array}$ & $\begin{array}{r}226 \\
27\end{array}$ & $\begin{array}{l}0 \\
0\end{array}$ & $\begin{array}{l}0 \\
0\end{array}$ & $\begin{array}{l}0 \\
0\end{array}$ & $\begin{array}{l}0 \\
0\end{array}$ & $\begin{array}{r}12661 \\
603\end{array}$ \\
\hline
\end{tabular}


COASTAL - INLAIID SOLAR RADIATION DIFFEREHCE STUDY

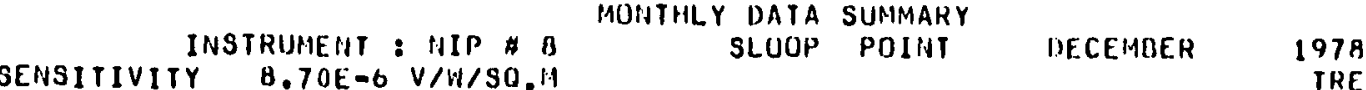

EIIERGY KILIJJUULES PER SQUARE METER

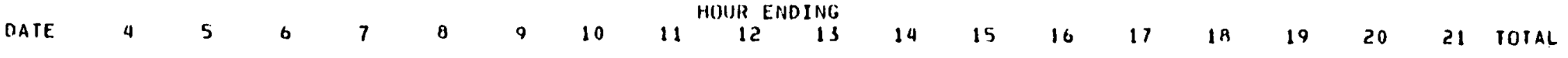

$\begin{array}{rrrrrrrrrr}-99 & -99 & -99 & -99 & -99 & -99 & -99 & -99 & -99 & -99 \\ -99 & -99 & -79 & -99 & -99 & -99 & -99 & -97 & -99 & -99 \\ -99 & -99 & -99 & -99 & -99 & -99 & -99 & -99 & -99 & -99 \\ -99 & -99 & -99 & -99 & -99 & -99 & -99 & -99 & -99 & -99 \\ -99 & -97 & -99 & -99 & -99 & -99 & -99 & -99 & -99 & -99 \\ & & & & & & & & & \\ -99 & -99 & -99 & -99 & -99 & -97 & -99 & -99 & 2511 & 1071 \\ 308 & 1367 & 2489 & 1777 & 1326 & 1082 & 1901 & 1346 & 1450 & 0 \\ 173 & 206 & 94 & 82 & 222 & 2097 & 2465 & 1890 & 1373 & 330 \\ 610 & 1934 & 2265 & 130 & 1305 & 2472 & 2158 & 366 & 101 & 0\end{array}$

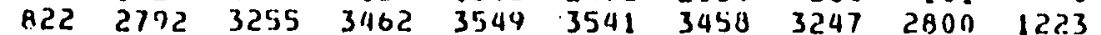

978

$\begin{array}{rrrrrrrrrr}178 & 0 & 33 & 157 & 45 & 111 & 124 & 24 & 0 & 0 \\ 0 & 0 & 273 & 546 & 3355 & 3393 & 3331 & 3115 & 2681 & 1162 \\ 1133 & 2598 & 3074 & 3293 & 3335 & 3351 & 3293 & 3107 & 2664 & 1167 \\ 602 & 1698 & 3072 & 2177 & 2522 & 1913 & 1524 & 2662 & 2720 & 1169 \\ 1189 & 2104 & 3184 & 3370 & 3494 & 3490 & 3295 & 3175 & 2712 & 1207\end{array}$

$\begin{array}{llllllllll}327 & 115 & 0 & 149 & 1978 & 1167 & 2904 & 2623 & 1092 & 140\end{array}$ $\begin{array}{llllllllll}297 & 2481 & 3164 & 3421 & 3451 & 3425 & 3363 & 3185 & 2734 & 1252\end{array}$

$\begin{array}{rrrrrrrrrr}0 & 140 & 115 & 306 & 0 & 24 & 190 & 62 & 0 & 62 \\ 0 & 0 & 0 & 30 & 0 & 42 & 0 & 13 & 0 & 0 \\ 0 & 13 & 63 & 0 & 0 & 50 & 67 & 659 & 15 & 19\end{array}$

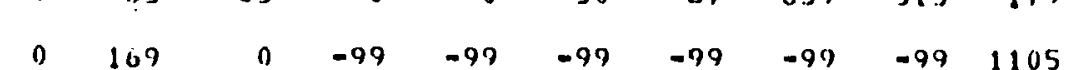

$\begin{array}{llllllllll}384 & 2118 & 3074 & 3101 & 3389 & 3471 & 3376 & 3165 & 2677 & 1253\end{array}$

$53244 \% 2962 \quad 2578$ 1882 $12451109 \quad 1435$ 62 12

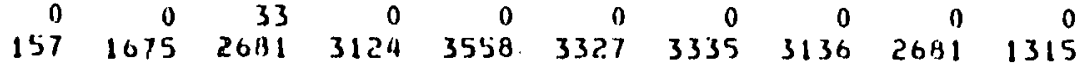

$\begin{array}{llllllllll}627 & 2133 & 2956 & 327 ! & 2638 & 3428 & 3320 & 3151 & 2716 & 1330\end{array}$

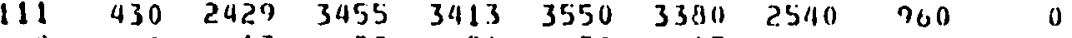

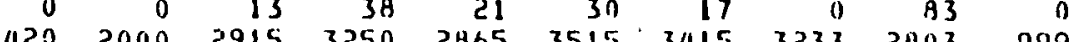

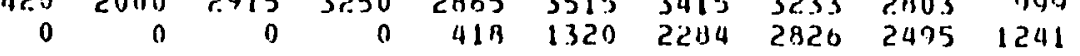

$\begin{array}{lllllllllll}419 & 795 \quad 0 & 816 & 1660 & 3084 & 2794 & 2666 & 100 ? & 232\end{array}$

$\begin{array}{llllllllll}312 & 1114 & 1525 & 1047 & 1851 & 2072 & 2129 & 1984 & 1515 & 633\end{array}$

$\begin{array}{lllllllll}25 & 25 & 25 & 24 & 24 & 24 & 24 & 24 & 25\end{array}$

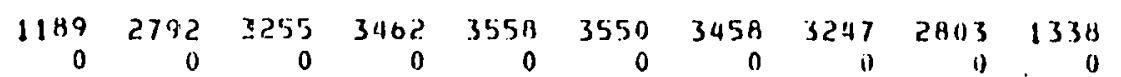

$\begin{array}{rrrrr}0 & 0 & 0 & 0 & -999 \\ 0 & 0 & 0 & 0 & -999 \\ 0 & 0 & 0 & 0 & -999 \\ 0 & 0 & 0 & 0 & -999 \\ 0 & 0 & 0 & 0 & -999 \\ 0 & 0 & 0 & 0 & -999 \\ 0 & 0 & 0 & 0 & 13646 \\ 0 & 0 & 0 & 0 & 8940 \\ 0 & 0 & 0 & 0 & 11341 \\ 0 & 0 & 0 & 0 & 28149 \\ 0 & 0 & 0 & 0 & 672 \\ 0 & 0 & 0 & 0 & 17856 \\ 0 & 0 & 0 & 0 & 27019 \\ 0 & 0 & 0 & 0 & 20759 \\ 0 & 0 & 0 & 0 & 27815 \\ 0 & 0 & 0 & 0 & 10495 \\ 0 & 0 & 0 & 0 & 26776 \\ 0 & 0 & 0 & 0 & 899 \\ 0 & 0 & 0 & 0 & 85 \\ 0 & 0 & 0 & 0 & 1396 \\ 0 & 0 & 0 & 0 & -999 \\ 0 & 0 & 0 & 0 & 26308 \\ 0 & 0 & 0 & 0 & 13783 \\ 0 & 0 & 0 & 0 & 33 \\ 0 & 0 & 0 & 0 & 24989 \\ 0 & 0 & 0 & 0 & 25578 \\ 0 & 0 & 0 & 0 & 20268 \\ 0 . & 0 & 0 & 0 & 202 \\ 0 & 0 & 0 & 0 & 25415 \\ 0 & 0 & 0 & 0 & 10584 \\ 0 & 0 & 0 & 0 & 13468 \\ 0 & 0 & 0 & 0 & 14853 \\ 0 & 0 & 0 & 0 & 10179 \\ 0 & 31 & 31 & 31 & 24 \\ 0 & 0 & 0 & 0 & 28149 \\ 0 & 0 & 0 & 0 & 33\end{array}$


COASTAL - IMLAHD SILLAR RADIATIOH DIFFERENCE STUUY MUNTIILY DATA SUMMARY

INSTRUMENT: MIP $\# ! 0$
SENSITIVITY $0,29 E-6$ V/W/SO.M

EMERG KILUJOULES PER SGUARE. METER HDISR ENDING

DA

0
80
16
8
0

$\begin{array}{rrrrrrrrrr}0 & 20 & 91 & 2348 & 3374 & 3369 & 3322 & 3126 & 2696 & 1480 \\ 121 & 633 & 606 & 685 & 2144 & 2921 & 1714 & 637 & 307 & 98\end{array}$

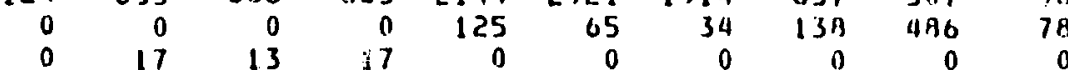

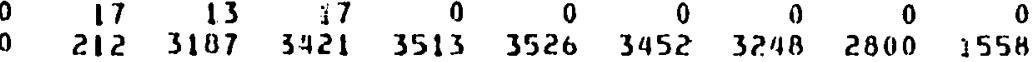

$\begin{array}{rrrrrrrrrr}143 & 247 & 82 & 924 & 121 & 577 & 26 & 243 & 0 & 0 \\ 81 & 1927 & 3021 & 3251 & 3347 & 3364 & 3303 & 3133 & 2721 & 1475\end{array}$

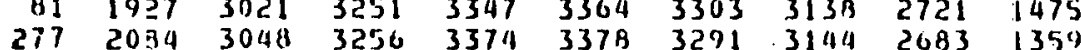

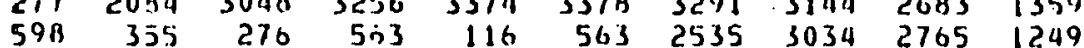
650 2552 $31473336 \quad 3499 \quad 3468 \quad 33993156 \quad 2435 \quad 1392$ $\begin{array}{rrrrrrrrrr}0 & 0 & 24 & 976 & 1826 & 2329 & 2177 & 458 & 0 & 0 \\ 560 & 2579 & 3152 & 33.32 & 3465 & 3482 & 3417 & 3217 & 2809 & 1585\end{array}$

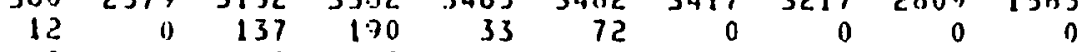
$\begin{array}{rrrrrrrrrr}0 & 0 & 0 & 0 & 0 & 0 & 0 & 0 & 0 & 0 \\ 0 & 0 & 0 & 0 & 0 & 0 & 0 & 0 & 347 & 421\end{array}$

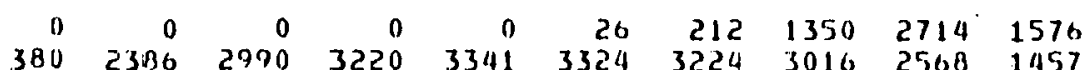
$\begin{array}{llllllllll}69 & 868 & 2353 & 2601 & 2440 & 2527 & 1658 & 1174 & 634 & 117\end{array}$

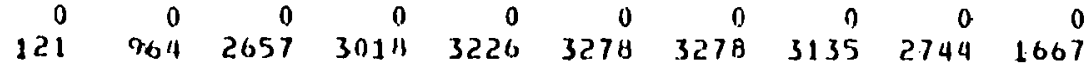

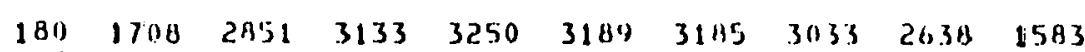

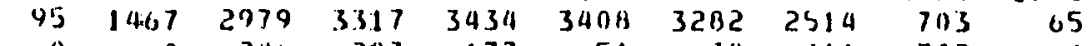
$\begin{array}{rrrrrrrrrr}0 & 0 & 246 & 203 & 177 & 51 & 38 & 311 & 732 & 0 \\ 390 & 2236 & 2957 & 3226 & 3361 & 3121 & 3352 & 3122 & 2679 & 1029\end{array}$ $\begin{array}{lllllllll}-99 & -99 & -99 & 1684 & 1975 & 3087 & 3078 & 28110 & 2397\end{array}$ 
CUASTAL - IHLAND SOLAR RADIATIUN DIFFERENCE STUDY MIINTHLY DATA SUMMARY

INSTRUMENT: UV \#11
SEMSITIVITY $312.10 E-6$ CLINTON DE.

ENERGY KILOJOULES PER SQUARE METER

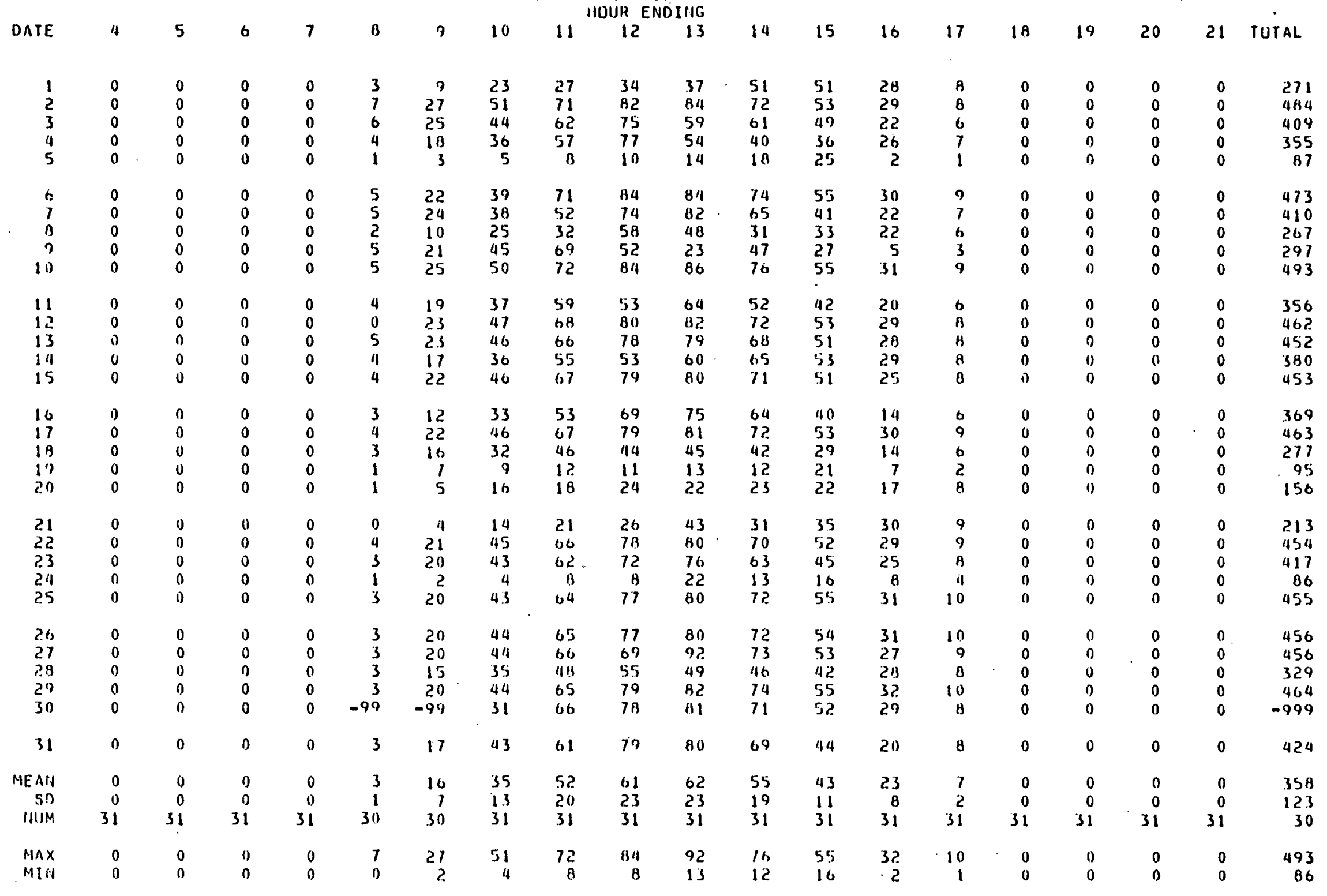


CUASTAL - IHLAHD SOLAR RADIATION DIFFERE.HCE STUDY INBTRUMENT: UV \#IZ MONTILY DATA SUMMARY

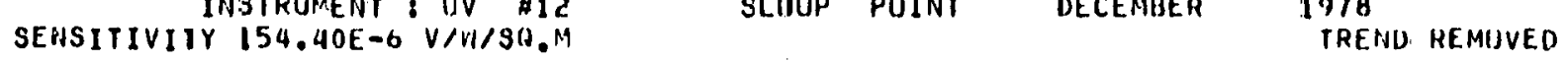

EHERGY KILOJDULES PER SHUARE METER HOUR ENDING

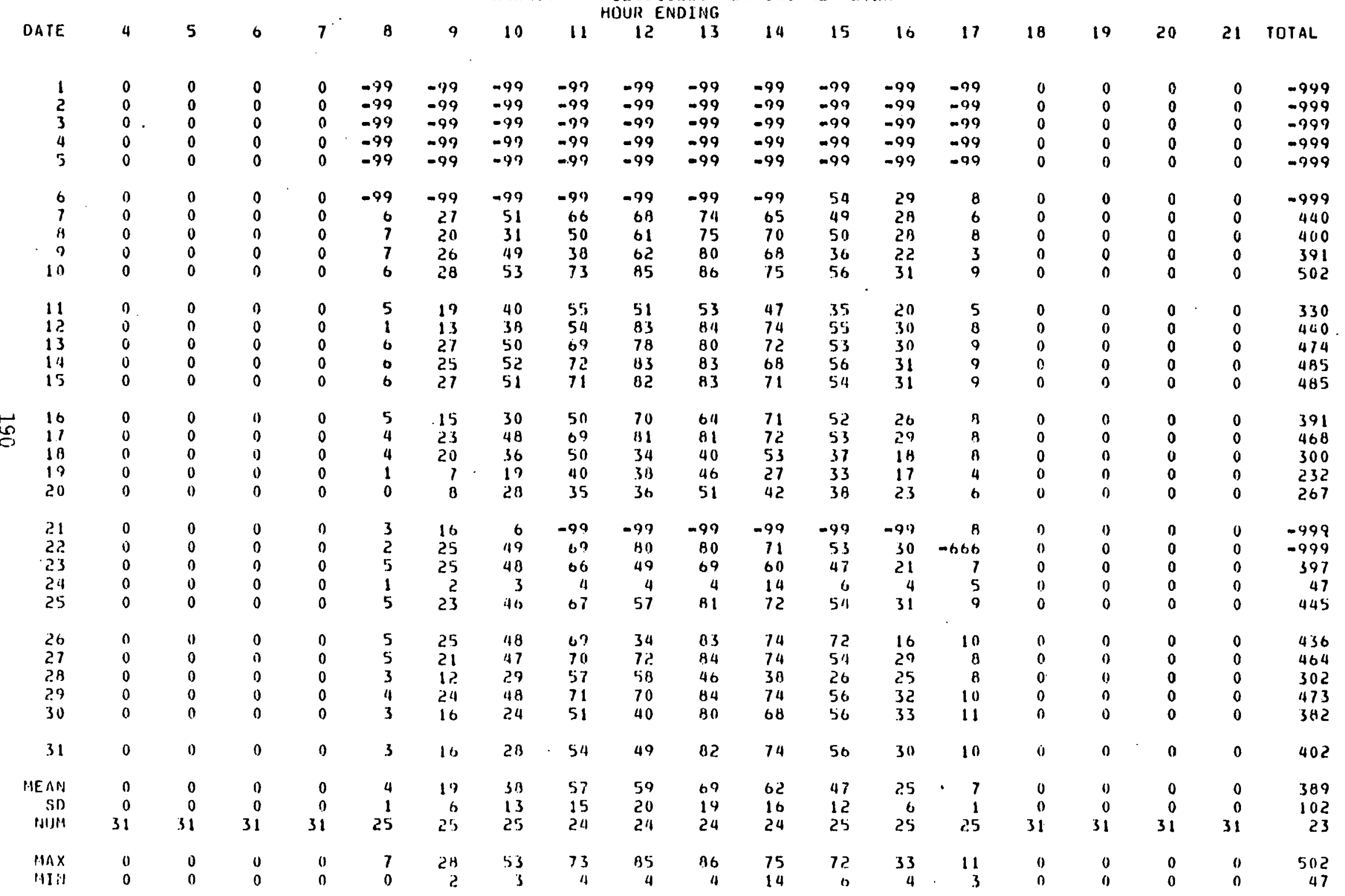


APPENDIX 3

EFFECTS OF THE SEA BREEZE FRONTAL PASSAGE AND ASSOCIATED SEA BREEZE INDUCED CLOUD COVER ON THE GLOBAL INSOLATION OF THE ONSLOW BAY REGION OF NORTH CAROLINA

by

Paul J. Gunthorpe

School of Forestry and Environmental Studies

Duke University

\begin{abstract}
A thesis submitted in partial fulfillment of the requirements for the degree of Master of Forestry in the School of Foresty and Environmental Studies of Duke University
\end{abstract}

1979 
INTRODUCTION

Practical utilization of solar radiation as an energy source in the United States requires detailed, area-specific solar insolation data. Development of data on the effects of mesoscale climate upon the solar radiation received along the coastal zone of the continental area is important for two main reasons. Sixty percent of the nation's population live within $110 \mathrm{~km}$ of the Atlantic, Gulf, Great Lakes, and Pacific coasts. Thus, these coastal regions, with higher population densities, have greater energy requirements than do regions of equal area further inland. Also, sea breeze circulations which frequently develop along this coastal zone influence the spatial and temporal variations in available solar energy.

A major part of a solar resource assessment program is the development of a solar radiation data base over various geographic regions of the country. The State of North Carolina, in particular, has a strong interest in the use of solar radiation as a source of energy. North Carolina has no significant hydro or fossil fuel reserves of its own and imports a major portion of its energy requirements. In response to the national search for alternative energy sources, the Research Triangle Institute of Research Triangle Park, N. C. is conducting a program to analyze the solar energy resources of coastal North Carolina. This program under the sponsorship of the U. S. Department of Energy utilizes a one-year solar data sample to define the coastal-inland solar radiation difference within the state.

The southeastern coastal plain of North Carolina is an ideal area for the investigation of sea breeze circulation effects on global insolation. Sea breezes frequently occur along the Onslow Bay coast beginning in late May and continuing through October. Coastal areas immediately north of Onslow Beach, near Morehead City and New Bern, are less suitable as study locations because of the complexity of their land-water interfaces. In these regions the sea breeze solenoid circulation is frequently poorly defined, resulting in sporadic areas of enhanced convergence or areas of divergence. 
The sea breeze convergence zone, commonly referred to as the sea breeze front, associated with these solenoids forms at the boundary between the cool air from the ocean and the air warmed over the land. Strong development of the heating differential during the day initiates the movement inland of the sea breeze front. Fair weather cumulus clouds, frequently accompanied by sea breeze cumulus clouds arranged in a cloud line positioned over the sea breeze frontal zone, often appear over land on sea breeze days. In general, a ground location experiencing a sea breeze frontal passage is initially covered by air mass cumulus, followed by sea breeze cumulus cloud cover, and eventually clearing behind the front.

This paper attempts to describe and quantify the effects of sea breeze frontal passages and their associated cumulus cloud cover on the global insolation received along the Onslow Bay region of North Carolina. Satellite photographs obtained from the National Weather Service office in Raleigh, N. C. and global insolation measured by Research Triangle Institute (RTI) at six study location sites of varying distance from the Onslow Bay coast were used to determine relationships of global insolation versus cloud cover for both air mass and sea breeze cumulus. Similar data were also used to determine the spatial and temporal effects of sea breeze frontal passages on the global insolation measured at study sites in the Onslow Bay region during selected case study days. 


\section{BACKGROUND INFORMATION}

\section{Sea Breeze Circulations}

Sea breeze circulations are most prevalent in late spring and early summer when land and water temperature differences are maximum; their frequency decreases towards the end of the warm season as temperature differences decrease. The sea breeze operates within an area of convergence. This sea breeze convergence zone modeled by Pielke (1974) and Hsu (1967) moves inland during the day as the cooler air from the ocean is advected over the warmer land air (Fig. 1). Sea breeze circulations along Onslow Bay are often dominated by changes in the general wind pattern. Banerjee (1975) observed sea breezes as much as $50 \mathrm{~km}$ to $100 \mathrm{~km}$ inland from the coast during synoptically undisturbed days. Vertical extention of the sea breeze may vary from as little as $15 \mathrm{~m}$ to as much as $1400 \mathrm{~m}$ depending on the strength of the onshore flow. Maximum sea breeze flow is normally located between $30 \mathrm{~m}$ and $70 \mathrm{~m}$ above the ground surface (Anderson, 1974). The rate of advance of the sea breeze front over most coastal regions normally decreases with greater distance inland due to increased friction (hills and forests) and decreased velocity. Oscillatory passages of sea breeze fronts are believed to be due in part to the slow speeds that the fronts advance.

The most extensive research done in the field of sea breeze prediction and air property changes with sea breeze frontal passages for the southeastern United States has been conducted by Williams (1969, 1972, 1974).

Williams developed a prediction technique for sea breeze frontal passages based on data collccted from portions of coactal Georgia. He determined that temperatures are higher, relative humidity is lower, and wind speeds are less during the early morning hours on days dominated by sea breeze circulations. A sea breeze frontal passage usually results in a drop in the dry bulb temperature, a rise in the dewpoint temperature, an increase in relative humidity, and a significant shift in wind direction. Due to the variability in the development of a sea breeze circulation, sea breeze fronts will not advance to inland stations on some days, while on other days a sea breeze 


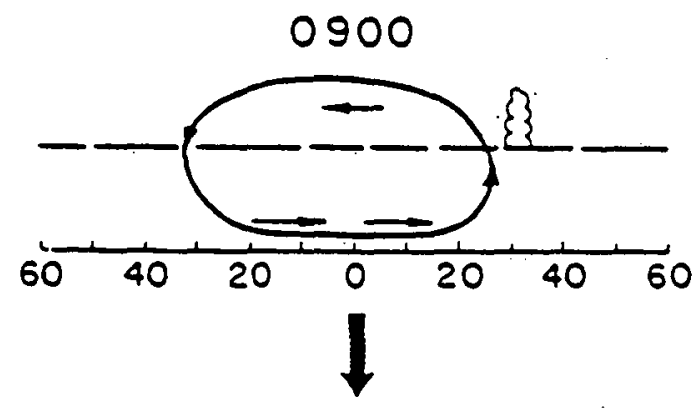

1200
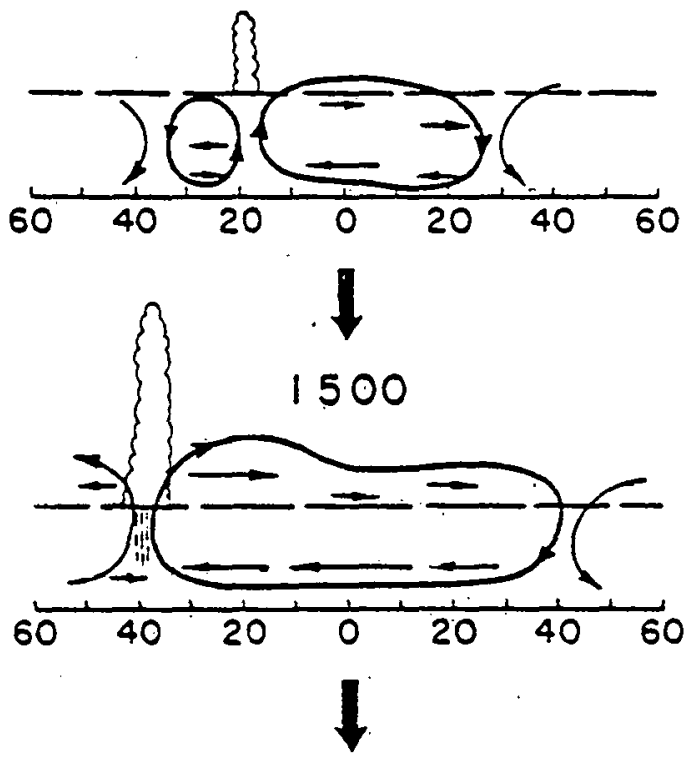

1800

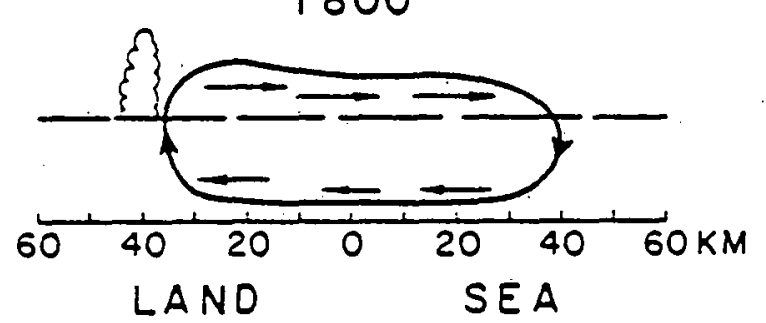

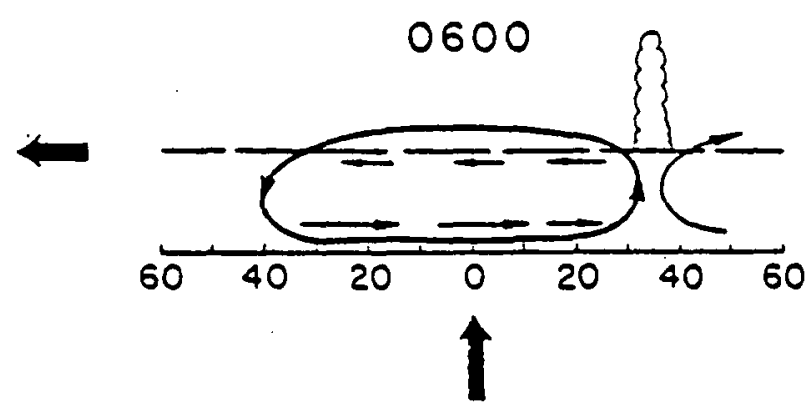

0300

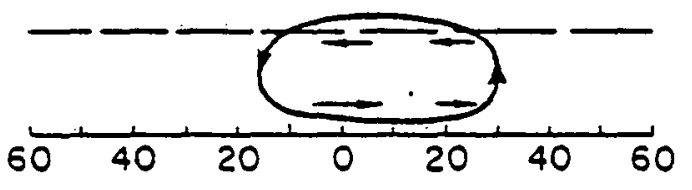

$\hat{1}$

0000

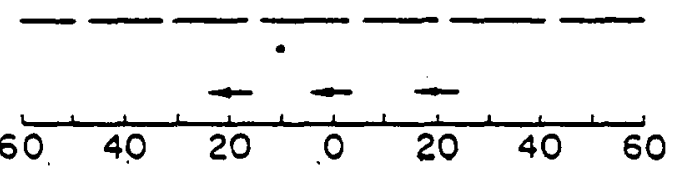

2100

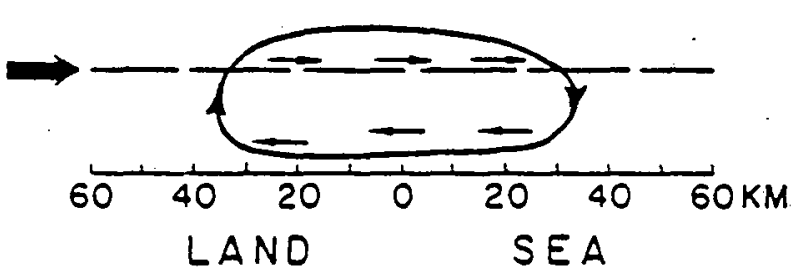

SEA

WINO SPEED SCALE

Figure 1. Synthesized empirical model of the land-sea breeze system along the Texas Gulf Coast. 
front will form short distances onshore. Passage times of the sea breeze front tend to be later as distance from the coast increases.

\section{Cloud Cover Effect on Insolation}

Several variables influence the quantity and quality of shortwave radiation reaching the earth's surface. Absorption and scattering of the solar beam through the gaseous composition of the atmosphere is a function of both height and time (Robinson, 1966). Depletion of the solar beam is relatively small through pure air but increases with the level of contamination, polluLivi, or turbidity, aceociated with varlable sumpuients such as water vapor, dust, and haze. Maritime air over the coastal stations of Onslow Bay frequently has different absorption and scattering properties due to salt spray or maritime aerosols and multiple scattering from the sea surface. Solar radiation received at sites further inland undergoes scattering from smaller particles. Insolation is also a function of the path length of the solar beam through the atmosphere, increasing with greater solar elevation.

On the average, approximately 24 percent of the incident radiant energy is reflerted and scattered back into space by clouds, and another 3 percent is lost through absorption by clouds (Sellers, 1965). Several meteorological. factors are responsible fur lle specific cloud cover development nver the Onslow Bay coastal zone. Cumulus clouds of variable depths almost always fnrm alnng the sea breeze frontal convergence zone during the onshore flow of warm season days. Fog conditions are much more common over coastal stations than over sites further inland. Cyclogenesis over the Gulf Stream often influences the develupment of increased cloudiness over the coastal zone llut experienced by inland locations. The effects of the cloud cover over the coastal zone un the reduction of global insolation may he minimal for scattered cumulus clouds but can be significant for thick, unbroken blankcta of fog.

The radiation regime of the atmosphere is determined not only by the quantity of cloud cover, but also by the thickness of the clouds and their distribution throughout the sky. All radiation fluxes exhibit a non-linear dependence on cloud cover amount, since individual clouds differ with respect to their thickness and height (Kasten, 1977). However, in specific quantita- 
tive intervals of cloud cover for similar cloud types, linear relationships have been found between total hemispheric radiation and the measured sky cover (Pochop, 1968), such that decreasing levels of insolation result from increasing amounts of cloud cover. Other independent studies have revealed that reductions in insolation due to scattered cumulus clouds cannot be represented adequately by the traditional linear relationships developed for other cloud types (Kaiser, 1976 and Pyldmaa, 1964).

The albedo of cumulus clouds depends on the thickness and form of the clouds and also on the solar altitude. Observations by Kondratyev (1969) confirmed an increase of albedo for increasing cloud thickness. This trend is more rapid at smaller cloud thicknesses (up to $300 \mathrm{~m}$ ) and slows considerably with increasing thickness. Albedo of high density clouds also changes as the cloud form fluctuates (Mironova, 1973).

Cumulus Cloud Characteristics and Effects on Insolation

Cumulus clouds are formed by the condensation of water vapor as heated air from the surface rises and cools by expansion; they are clouds with vertical development. Base heights of cumulus clouds range anywhere from 0 to $3 \mathrm{~km}$. The tops of these clouds rise to heights less than $10 \mathrm{~km}$ for cumulus, 10 to $14 \mathrm{~km}$ for cumulus congestus (growing stage), and greater than 14 $\mathrm{km}$ for cumulonimbus. Cumulonimbus clouds predominate in substantial convective cloud cover, while smaller cumulus clouds predominate in lower fractions of sky cover (Avaste, 1964).

Table 1. Mean albedo for various cumulus cloud types as determined from the brightness of cloud-cover TV pictures Cloud cover more than $80 \%$ of the area (from Kondratyev, 1972).

\begin{tabular}{lc}
\hline \multicolumn{1}{c}{ Cloud Type } & Albedo (\%) \\
\hline Cumulus humilis clouds--over land & 29 \\
Cumulonimbus clouds, small, cloud tops up to $6 \mathrm{~km}$ & 86 \\
Cumulonimbus clouds, large and thick & 92 \\
\hline
\end{tabular}


Kondratyev (1972) measured the mean albedo for different types of cumulus using the brightness characteristic of cloud covers displayed on television pictures (Table 1). Albedo increases as cumulus clouds increase in vertical development. Albedo values for the air mass and sea breeze cumulus clouds developing during sea breeze circulation occurrences are similar to those observed for cumulus humilis and for clouds of vertical development midway between cumulus humilis and small cumulonimbus, respectively.

Solar zenith angles greater than $50^{\circ}$ enhance the coverage of the direction of sighting for cumulus clouds having considerable vertical dimensions (Avaste, 1964). Consequently, the duration of sunshine decreases for an unchanged state of cumulus cloud cover and a diminishing solar altitude. Analyses conducted by Galperin (1964) showed that for the sdllte allilule of the sun, shape and quantity of cumulus clouds, and intensity of solar radiation, there is no significant difference in the insolation received at various stations. Considerable research into cloud cover effects on insolation has been pursued by Pyldmaa. The quantity $Q *$, a dimensionless value representing the ratio of the total radiation received on a plane surface to the average diurnal fluctuation of the possible total radiation of a cloudless sky, was subjected to an intensive analysis. Pyldmaa determined that the average flux ratio $\bar{Q} *$ increases from clouds of the lower levels to clouds of the middle and upper levels. For cumulus cloud cover greater than two-tenths sky cover, the magnitude $\bar{Q}^{*}=1.20-0.08 \mathrm{n}$, where $\mathrm{n}$ is Lhe cumulus cloud cover in tenths. This linear dependence calculated by Pyldmaa is not adequate for cumulus clouds that change in vertical extent as a function of sky cover, a characteristic typically observed in sea breeze cumulus. There is no evidence in the published scientific journals of any research describing the relationship between sea breeze cumulus cloud cover and the quantity $\bar{Q} *$.

During sea breeze circulation occurrences, cumulus clouds with extensive vertical development frequently form in the sea breeze frontal zone of the Onslow Bay region of coastal North Carolina. Air mass cumulus differ from the above in appearance, being smaller in size and vertical extent. In comparison of the two types of cumulus, sea breeze cumulus cloud cover appears much brighter on a visual spectrum satellite photograph (reflects a greater percentage of incoming solar radiation) than the less vertically developed air mass cumulus (Fig. 2). With the aid of maximum vertical 


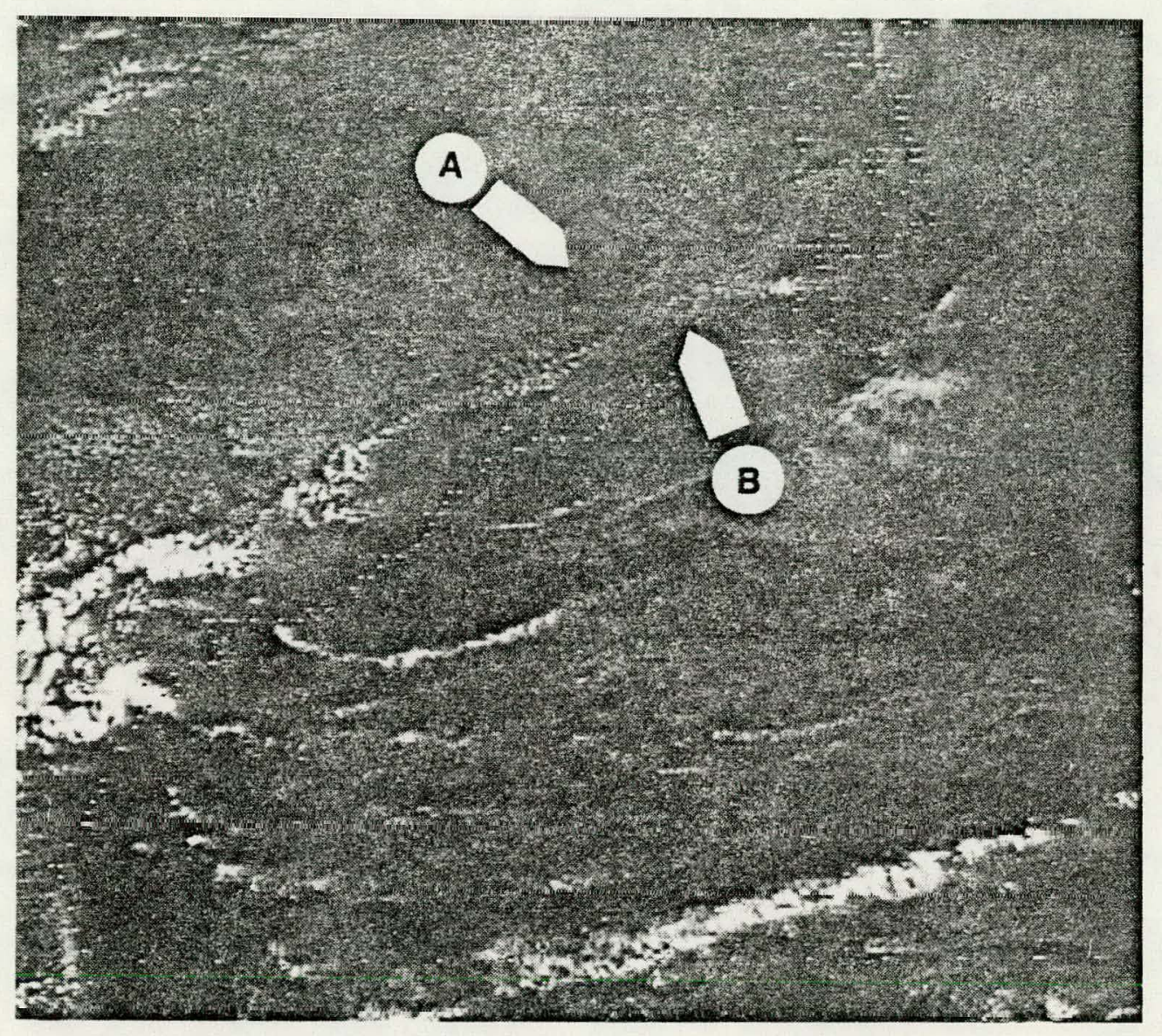

Figure 2 . Visual spectrum satellite photograph showing the difference in relative brightness between the sea breeze cumulus along the sea breeze convergence zone and the air mass cumulus ahead of the sea breeze front in southeastern North Carolina. 
development, sea breeze cumulus can transform into large and thick

cumulonimbus with albedo values in excess of 90 percent (Fig. 3). Thus, the type and amount of cumulus cloud cover could be variables primarily responsible for the differences observed in the global insolation received amongst coastal and inland locations during the sea breeze days.

The foregoing review of the literature has summarized the effects of air mass cumulus cloud cover on global insolation. However, at the present time, no studies were found that determined the effects of sea breeze cumulus cloud cover on global insolation. Since coastal areas are frequently influenced by the sea breeze cumulus associated with sea breeze circulations, research was necessary to describe this mesoscale effect on the incident solar radiation of these areas. This paper evaluates the effects of sea breeze frontal passages on global insolation, the effects of sea breeze cumulus cloud cover on global insolation, and the difference in effects of air mass and sea breeze cumulus on the global insolation measured during sea breeze days in the Onslow Bay region. 


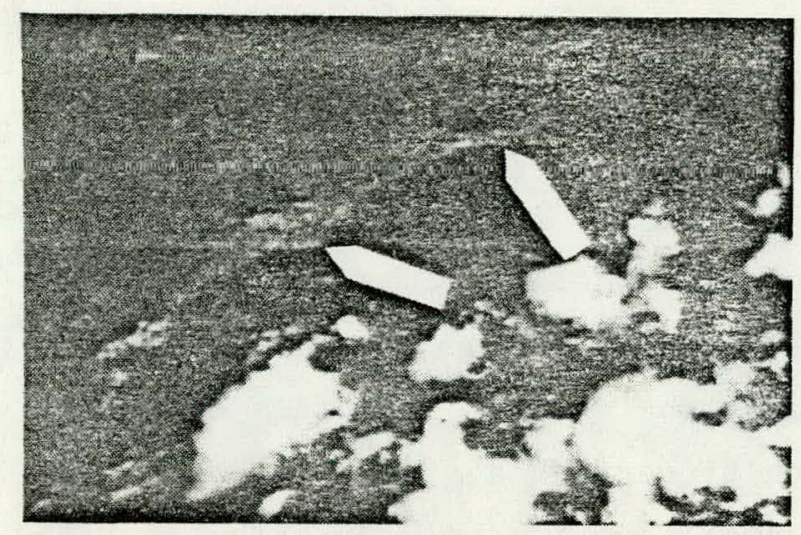

1101 EST

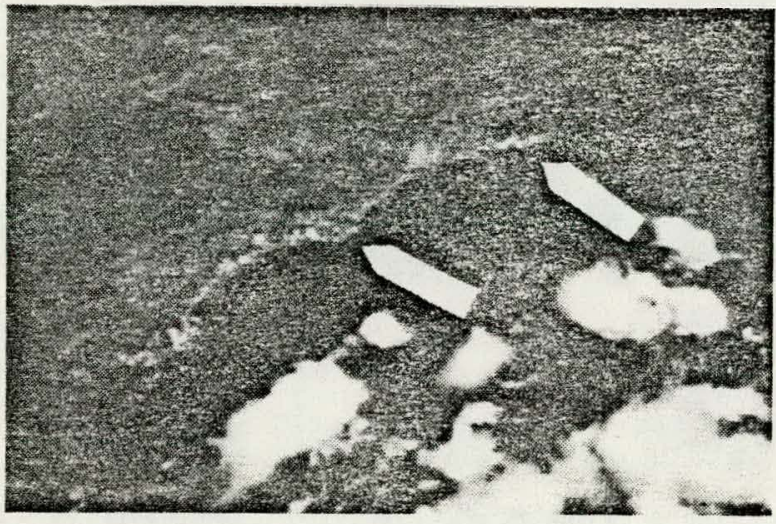

1131 EST

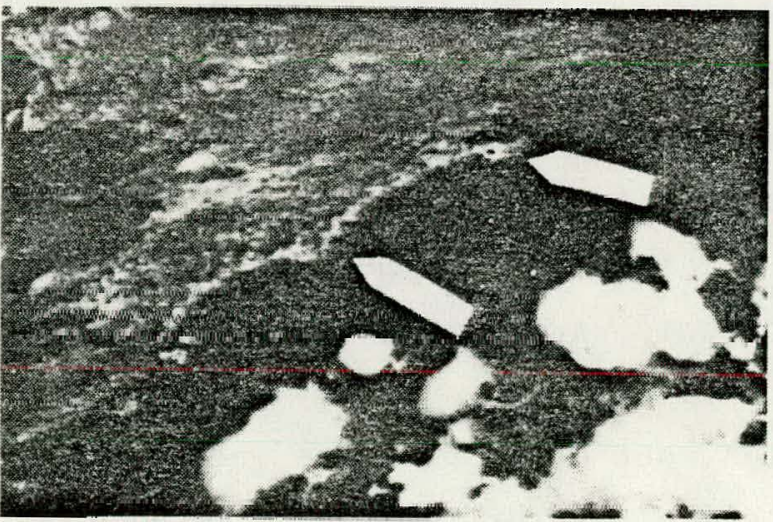

1201 EST

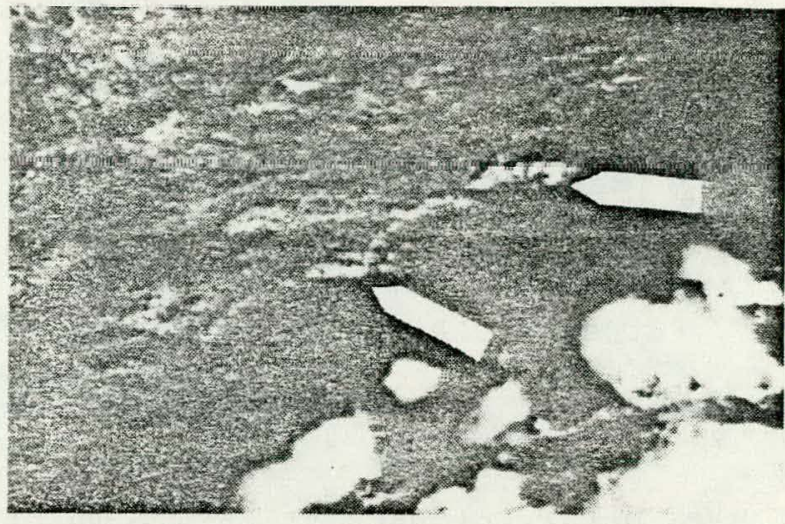

1231 EST

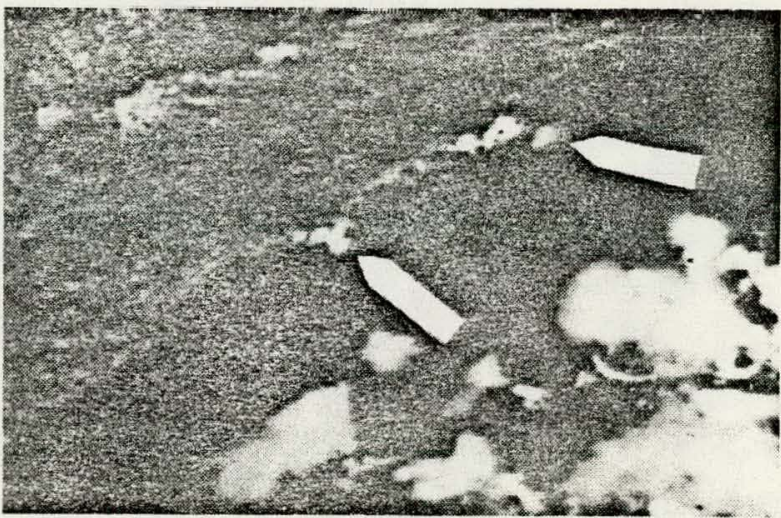

1301 EST

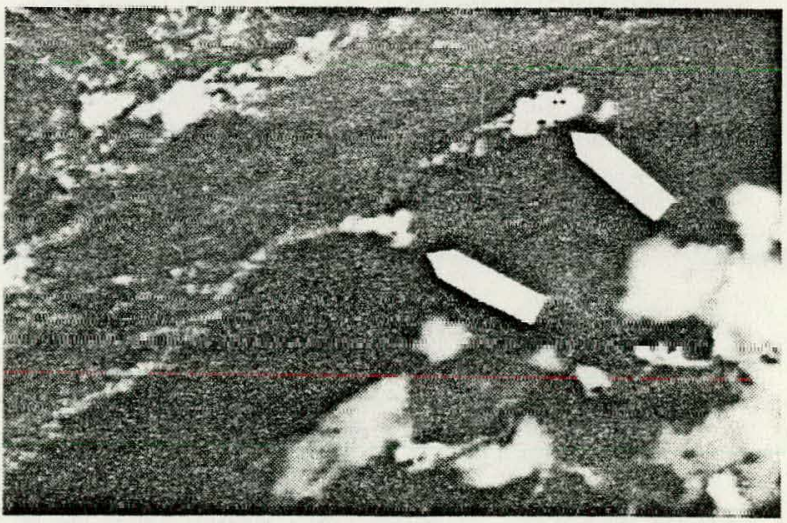

1331 EST

Figure 3. Time series of visual spectrum satellite photographs showing the development of thunderstorms (cumulonimbus) at the confluence of the sea breeze frontal zone along the Onslow Bay coast. 


\section{METHODS AND MATERIALS}

\section{The Onslow Bay Region}

Land adjacent to Onslow Bay is similar in topography and climate to other locations along North Carolina's coastal plain. The area's elevation is quite low, averaging $30 \mathrm{~m}$ of rise for the first $100 \mathrm{~km}$ from the coast. Major land use is primarily focused on timber and agricultural production. Centers of population are relatively small and sparsely distributed. The southeast facing coastline of the study location is very uniform, broken infrequently by small inlets separating the coastal islands.

In the summer months, the entire coastal plains region of North Carolina is warm, with little variation of average temperature between locations. An average July temperature of $27^{\circ} \mathrm{C}$ is typical of the Onslow Bay area. The cooling effect of the summer sea breezes is well illustrated by the differences in temperature between Wilmington and Raleigh, N. C. (Carney, 1955). At Wilmington the temperature reaches $32^{\circ} \mathrm{C}$ on an average of only 24 days a year, while inland at Raleigh $32^{\circ} \mathrm{C}$ temperatures occur 41 days a year on the average.

Plevailing winds in the study location are trom the northeast from November through February, while they come from the southwest from March through August. The greatest frequency of high winds occur during midwinter. Lighter winds are more common during the summer months when sea breeze circulations frequently develop. Sea breeze solenoid circulations are nearly an every day occurrence along the Onslow Bay coast during the warm season. The inland boundary of this circulation frequently extends greater than $50 \mathrm{~km}$ from the shore, dependent upon the 1and-sea temperature difference and the magnitude and direction of the synoptic winds.

The greatest amount of rainfall occurs in the summer months, averaging $73 \mathrm{~cm}$ during the warm season. Most of this warm season rainfall romes principally from thundershowers. Rainfall from this highly developed cumulus convection is usually short in duration, but often heavy and unevenly distributed. Thundershowers occur about 1 out of 3 days from June through August during the late afternoon hours. Thus the effect of clouds associated with 
warm season rainfall on the solar radiation received in this area is intermittent and of short duration.

Winter rainfall is ordinarily associated with large, slow-moving, low pressure systems. This precipitation, averaging $58 \mathrm{~cm}$ annually, is of the slow, steady type, generally lasting 1 or 2 days. Unlike the summer thundershowers, winter showers often occur in the early morning hours. The effect of cloud cover associated with winter precipitation on the solar radiation received in the Onslow Bay region is more frequent and of longer duration.

\section{Description of Study Sites}

Selection of the study site locations in the Onslow Bay region was made by $\mathrm{Dr}$. Walter D. Bach of RTI after a detailed inspection of the area. Sites were distributed so that an analysis of the coastal-inland solar radiation gradient would be possible (Fig. 4). Three stations along the coast were under the influence of sea breeze circulations on nearly all synoptically undisturbed days during the summer months. Two other stations were located a sufficient distance from the coast so that sea breeze circulation occurrences were less frequent than along the immediate coastal region. A sixth site was located approximately $100 \mathrm{~km}$ from the Atlantic Ocean, where the effects of sea breeze circulations were nonexistent. Among these six sites two were chosen as primary measurement stations and the others as secondary measurement stations. A summary of Bach's description of each of these study sites follows.

At the two primary sites, one on the Onslow Bay coast and another, approximately $100 \mathrm{~km}$ from the ocean, measurements of global, direct, and ultraviolet radiation were recorded. Both installations also had a mechanical weather station to continuously monitor the daily wind, temperature, and rainfall. The primary inland station was located on the Horticultural Crops Research Station of the North Carolina Department of Agriculture near Clinton, N. C. This site had excellent exposure in all directions. The tallest trees are approximately $15 \mathrm{~m}$ high and $150 \mathrm{~m}$ to the south-southeast of the site. The coastal primary site was located approximately $5 \mathrm{~km}$ from the ocean at Sloop Point, beside an unpaved road separating adjacent cornfields. This station had an excellent view of the horizon in all directions. The 


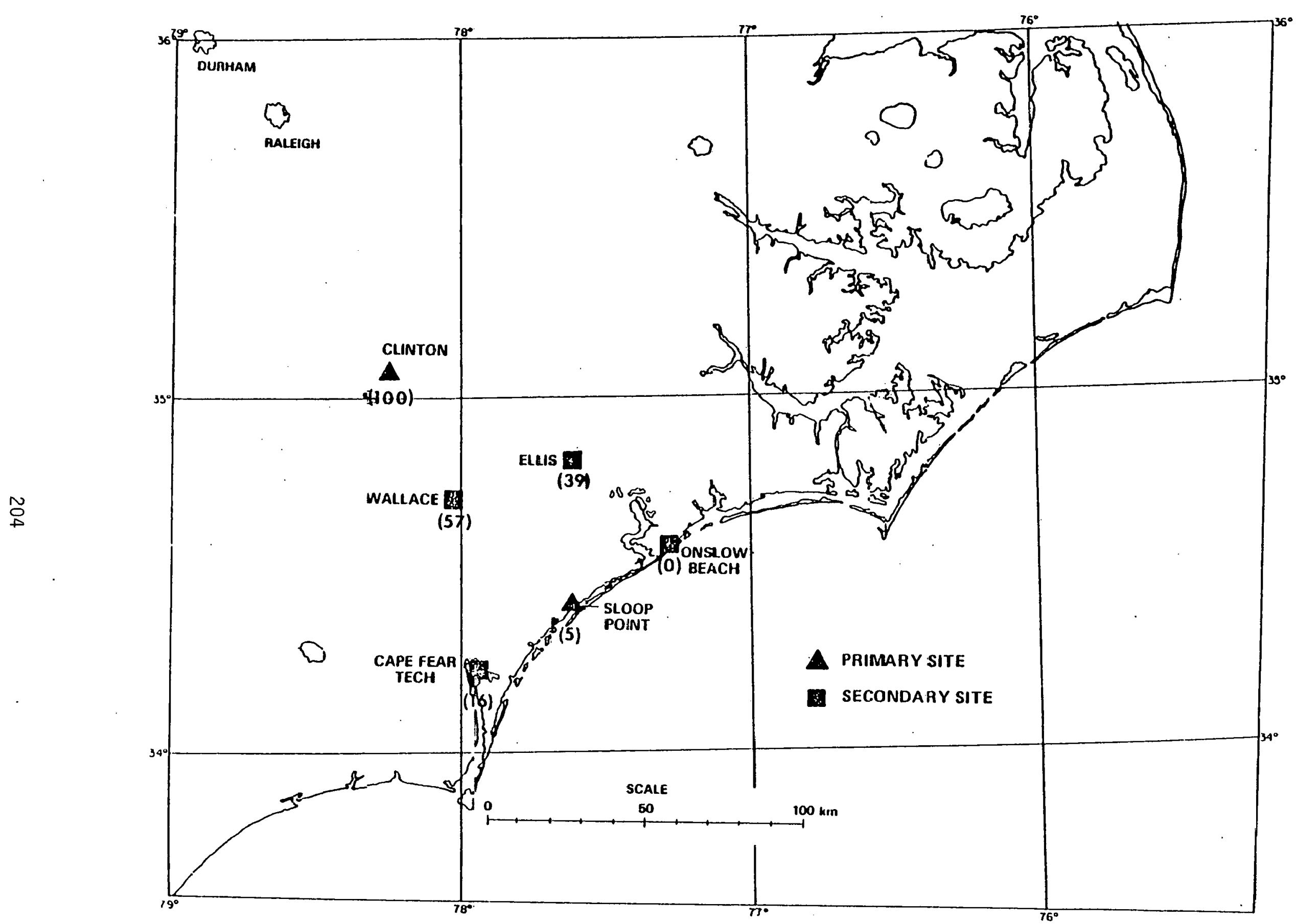

Figure 4. Map of the Onslow Bay study area in southeastern North Carolina and the six study site locations. 
corn crop, which never grew more than $1 \mathrm{~m}$ high, did not interfere with the instrument exposure. The coastal station was positioned inland from the shore to minimize the accumulation of salt spray on the solar radiation equipment.

Four secondary sites were selected to assess the spatial variability of the solar radiation in the Onslow Bay area. Only the global insolation was monitored at these sites. The Onslow Beach site was located at the sewage treatment plant as part of the U. S. Marine Corps Camp LeJeune base. This site, located only $100 \mathrm{~m}$ northwest of the Atlantic Ocean, had excellent exposure to the horizon in all directions. Another secondary coastal site was located on the deck of a moored barge of a marine laboratory at the Cape Fear Technical Institute, adjacent to downtown Wilmington, N. C. To the east and southeast two buildings extend approximately $10^{\circ}$ above the horizon. There were no obstructions to the horizon in the remaining quadrants of the Cape Fear station. Natural oscillations of the barge have small periods and do not affect hourly average values. The two remaining secondary sites were located midway between the coastal and inland stations. One of these stations was located atop the one story terminal building of the Henderson Airport, Wallace, N. C. The site, located approximately $57 \mathrm{~km}$ from the ocean, had an unobstructed view of the sky in all but the northern quadrant, where a utility pole was located. The other secondary site was located $20 \mathrm{~m}$ from the end of the Ellis Airport runway, approximately $40 \mathrm{~km}$ from the Onslow Bay coastline. The horizon was obscured in all directions by a pine stand. However, the stand height was not more than $10 \mathrm{~m}$ and the trees were no closer than $800 \mathrm{~m}$ in any direction.

\section{Global Insolation Measurements}

Global insolation was measured at each of the six study location sites using Eppley precision spectral pyranometers and Eppley Model 411-6140 integrator/printers. The radiation detectors operate as differential thermopiles with blackened hot-junction receivers and wire-wound reference coils kept at approximate ambient air temperature. Each instrument was calibrated against a standard and all individual sensitivities were determined before the field study. A prefield check of all the instruments and recorders was conducted 
at RTI. Electronic signals from the detecting units were passed through the integrators which provided hourly values printed out on paper tape.

Study site attendants performed daily inspections of all the instruments, and recorded weather information and any instrument problems. The outer glass hemisphere of each radiation instrument was cleaned daily. Printouts of the hourly integrated values were collected daily and mailed to RTI. Qualified personnel made quality assurance field checks on each instrument similar to the intercomparisons made in the prefield checkout at RTI. When pyranometers became unreliable or inoperative, they.were replaced by working spare units.

Hourly integrated values of global insolation were entered on disk at RTI's computing facilities. These energy counts were converted to units of $\mathrm{KJ} / \mathrm{M}^{2}$ using the instrument's sensitivity value. After the storage of the global insolation values onto disk, they were easily accessible for data analyses. Missing global insolation data were given specific identification on the disks. Some of the causes of missing data at particular sites were: (1) power failures, (2) instrument failures, (3) quality assurance field checks underway, (4) exhaustion of paper tape in printers, or 5) data was lost by site attendants. Global insolation values were also validated before using them in study analyses. Anomalies in the data were identified by visually examining the outputs of the time series of insolation data. Date and time of unusual patterns of insolation were identified and checked against the original energy counts on paper tape. Spurious signals producing nighttime energy counts were also identified, and such trends were removed from the data.

\section{Determination of Cloud Cover Amount}

At the present time there are two basic methods of determining cloud cover--ground or satellite observations. Ground based observations provide measurements of relative cloud amount determined by means of projerting the clouds onto an imaginary hemisphere, the center of which is an observer on the earth's surface. Satellite photographs can be used to measure near absolute cloud cover amounts determined by means of projecting the clouds onto a horizontal surface. The differences between these two methods are 
attributed to projection problems by ground base observers, where the sides of clouds are viewed and included in the estimates of total cloud cover.

Satellite photographs were chosen as the sole basis in determining cloud cover amount over the study location sites in order to avoid the effect of cumulus cloud vertical extent present in ground base measurements. Visual spectrum satellite photographs of the study location are produced every 30 minutes by the Geostationary Operational Environmental Satellite (GOES). Timanovskya (1964) analyzed the steady state nature and optimum period of observation for cumulus clouds. Results from this analysis verify that a point of cumulus cloud cover in summer is usually preserved at a constant value for one to two hours. Thus, it was assumed that cloud cover observations taken at 30 minute intervals along with corresponding hourly integrated global insolation measurements would represent an adequate sampling frequency for the development of relationships between cumulus cloud cover and the associated global radiation received at each of the six study location sites.

Cloud amount measurements, using three different sizes of cloud field areas, were evaluated on four sea breeze case study days. Two of the cloud field areas were circular, having radii of 10 and $20 \mathrm{~km}$ respectively. The third area was a $40 \mathrm{~km}$ wide quadrilateral. All three areas were centered over each of the study site locations. Correlation coefficients for each group of measured cloud amounts and corresponding $Q *$ 's were calculated for both sea breeze and air mass cumulus type cloudiness. As indicated in the Results section, correlation coefficients for sea breeze cumulus decreased significantly from the smaller $10 \mathrm{~km}$ radius areas to the quadrilateral sampling areas. Just the opposite trend occurred for the air mass cumulus cloud measurements. To provide adequate cloud measurements for both the air mass and sea breeze cumulus types, intermediate size circular cloud fields with 20 $\mathrm{km}$ radii above the observation points were used to determine cloud amount, as previously recommended by Avaste (1964). Measurements of the percentage cloud cover over each of the study sites were made using a transparency placed over the visual spectrum satellite photographs. This transparency includes reference points for positioning, observation points marking locations for each of the study sites, and $20 \mathrm{~km}$ radius circles centered on each of the observation points. Within these circles, visual estimates of cloud amount in tenths of sky cover for both sea breeze and air mass cumulus were 
made from each satellite photograph. Hourly cloud cover data were calculated by averaging cloud amounts measured from two 30 minute satellite photographs taken during a particular hour.

\section{Determination of Cloud Type}

Visible spectrum statellite photographs with slightly less than $1 \mathrm{~km}$ resolution were used to measure both cloud amount and cloud type over each of the study location sites. Usually cumulus convection over land appears as a cellular pattern consisting of cumulus humilis and more vertically developed cumulus congestus. Air mass and sea breeze cumulus were classified on the basis of their vertical extent as determined by their relative brightness and, in some cases, the size of their shadows. Sea breeze cumulus cloud cover appears on satellite photographs as cloud lines positioned along the sea breeze front some distance from the coast and accompanied hy a typically cloud-free area along the coastline and extending offshore. The air mass cumulus clouds develop inland of the sea breeze front and have lower relative brightness.

\section{Sed Breeze Case Study Daỹs}

Daily weather maps published by NOAA were used in the first step of determination of sea breeze circulation days. Surface weather maps and station weather for the East Coast at 0700 EST were analyzed for all days bcginning with April 1, 1978. These wealler maps were used solely as indirators of synoptically disturbed days. Synoptic disturbances over the study location tend to mask or impede the development of a sea breeze circulation. All days in which cold or warm fronta paracd the atudy lacation weie eliminated from possible sea breeze case study days.

The second step in the sea breeze case study day selection process required the use of temperature and wind data. Surface weather observations recorded along the coast of North Carolina and at inland airfields within the study location were compiled and checked for evidence of two sea breeze frontal passage prediction factors. Possible sea breeze solenoids require that: (1) The dry bulb temperature over the land rises at least $4^{\circ} \mathrm{C}$ above 
the average sea surface temperature (Table 2); and (2) the prevailing wind at mid-morning across the study locations blows in an offshore direction with a magnitude sufficiently light to allow a sea breeze front to move against it (Table 3).

The third criterion used in the sea breeze case study day selection process was evidence of sea breeze cumulus on the visual spectrum satellite photographs. Days in which synoptic cloudiness dominated the sky above the study location were immediately eliminated from consideration. Case study days were then selected from the remaining days which showed evidence of sea breeze cumulus and sea breeze frontal cloud lines. These cloud lines positioned along the sea breeze front will frequently parallel the coastline. A total of 32 case study days from May through September were selected using the previously described methods. The following analyses on these 32 case study days focus on the effects that sea breeze frontal passages and associated sea breeze cumulus have on the global insolation of the Onslow Bay region. 
Table 2. Land temperatures required for a sea breeze front along the North Carolina coast south of Hatteras (Williams, 1974)

\begin{tabular}{|c|c|c|}
\hline Month & Dates & Temperature $\left({ }^{\circ} \mathrm{C}\right)$ \\
\hline January & $\begin{array}{r}1-10 \\
11-20 \\
21-31\end{array}$ & $\begin{array}{l}14 \\
12 \\
11\end{array}$ \\
\hline February & $\begin{array}{r}1-10 \\
11-20 \\
21-29\end{array}$ & $\begin{array}{l}11 \\
11 \\
12\end{array}$ \\
\hline March & $\begin{array}{r}1-10 \\
11-20 \\
21-29\end{array}$ & $\begin{array}{l}13 \\
15 \\
17\end{array}$ \\
\hline April & $\begin{array}{r}1-10 \\
11-20 \\
21-30\end{array}$ & $\begin{array}{l}18 \\
19 \\
21\end{array}$ \\
\hline May & $\begin{array}{r}1-10 \\
11-20 \\
21-31\end{array}$ & $\begin{array}{l}22 \\
23 \\
24\end{array}$ \\
\hline June & $\begin{array}{r}1-10 \\
11-20 \\
21-30\end{array}$ & $\begin{array}{l}26 \\
27 \\
28\end{array}$ \\
\hline July & $\begin{array}{r}1-10 \\
11-20 \\
21-31\end{array}$ & $\begin{array}{l}28 \\
29 \\
29\end{array}$ \\
\hline August & $\begin{array}{r}1-10 \\
11-20 \\
21-31\end{array}$ & $\begin{array}{l}29 \\
29 \\
29\end{array}$ \\
\hline September & $\begin{array}{r}1-10 \\
11-20 \\
21-30\end{array}$ & $\begin{array}{l}29 \\
28 \\
27\end{array}$ \\
\hline October & $\begin{array}{r}1-10 \\
11-20 \\
21-31\end{array}$ & $\begin{array}{l}26 \\
25 \\
24\end{array}$ \\
\hline November & $\begin{array}{r}1-10 \\
11-20 \\
21-30\end{array}$ & $\begin{array}{l}22 \\
21 \\
19\end{array}$ \\
\hline December & $\begin{array}{r}1-10 \\
11-20 \\
21-31\end{array}$ & $\begin{array}{l}18 \\
17 \\
16\end{array}$ \\
\hline
\end{tabular}


Table 3. Land to sea and sea to land wind directions along the Atlantic Coast (Williams, 1974)

\begin{tabular}{lll}
\hline \hline \multicolumn{1}{c}{ Portion of coast } & Land to sea & Sea to land \\
\hline North Carolina north of Hatteras & $\mathrm{NW}, \mathrm{W}, \mathrm{SW}, \mathrm{S}$ & $\mathrm{N}, \mathrm{NE}, \mathrm{E}, \mathrm{SE}$ \\
$\begin{array}{l}\text { North Carolina south of Hatteras } \\
\text { and South Carolina }\end{array}$ & $\mathrm{NNE}, \mathrm{N}, \mathrm{NW}, \mathrm{W}, \mathrm{WSW}$ & $\mathrm{ENE}, \mathrm{E}, \mathrm{SE}, \mathrm{S}, \mathrm{SSW}$ \\
Georgia & $\mathrm{N}, \mathrm{NW}, \mathrm{W}, \mathrm{SW}$ & $\mathrm{NE}, \mathrm{E}, \mathrm{SE}, \mathrm{S}$ \\
Florida southward to Palm Beach & $\mathrm{NW}, \mathrm{W}, \mathrm{SW}, \mathrm{S}$ & $\mathrm{N}, \mathrm{NE}, \mathrm{E}, \mathrm{SE}$ \\
\hline
\end{tabular}




\section{RESULTS AND DISCUSSION}

\section{Sea Breeze Frontal Passages}

Visual spectrum satellite photographs of the study location for all case study days were uṣed to determine the time of passage of a sea breeze front over each of the six sites. Time of passage is defined as that time when the seaward edge of the sea breeze cumulus cloud line passes over the site and a clearing trend develops. In later analyses, sites located seaward with respect to the position of sea breeze frontal zone development were classified as coastal sites. Sites experiencing a sea breeze frontal passage at any time during a case study day were classified as frontal. Sites not affected by a sea breeze frontal passage for a particular case study day due to their distance inland from the coast were classified as inland.

Table 4 summarizes the sea breeze frontal passage times and number of occurrences for all six study location sites and all sea breeze case study days. Sea breeze fronts formed inland of the three coastal sites (Onslow Beach, Sloop Foint, and Cape Fear) on one-quarter to one-third of the sea breeze days examined. These three coastal sites experienced the most sea breeze frontal passages during the period of 1100 to 1400 EST. Sites located further inland (Ellis and Wallace) experienced fewer passages, most occurring between the hours of 1400 to 1800 EST. The Clinton station never experienced a sea breeze frontal passage during the 32 days studied because of its greater distance from the coast.

The mean Lines of sea breeze frontal passage in the Onslow Bay region are shown on figure 5 . 'The average time of a sea breeze frontal passage increases with distance from the Atlantic Ocean. The horizontal distance separating the isochrones also increases with distance inland from the coast. Mean time of sea breeze frontal passage increases from 1200 EST at Sloop Point and Onslow Beach, 1300 EST at Cape Fear Tech, 1500 EST at Ellis, to 1530 EST at the Wallace station.

The maximum number of sea breeze frontal passages occurred within a 10 $\mathrm{km}$ wide area approximately $20 \mathrm{~km}$ from the coast (Fig. 6). Fewer passages 


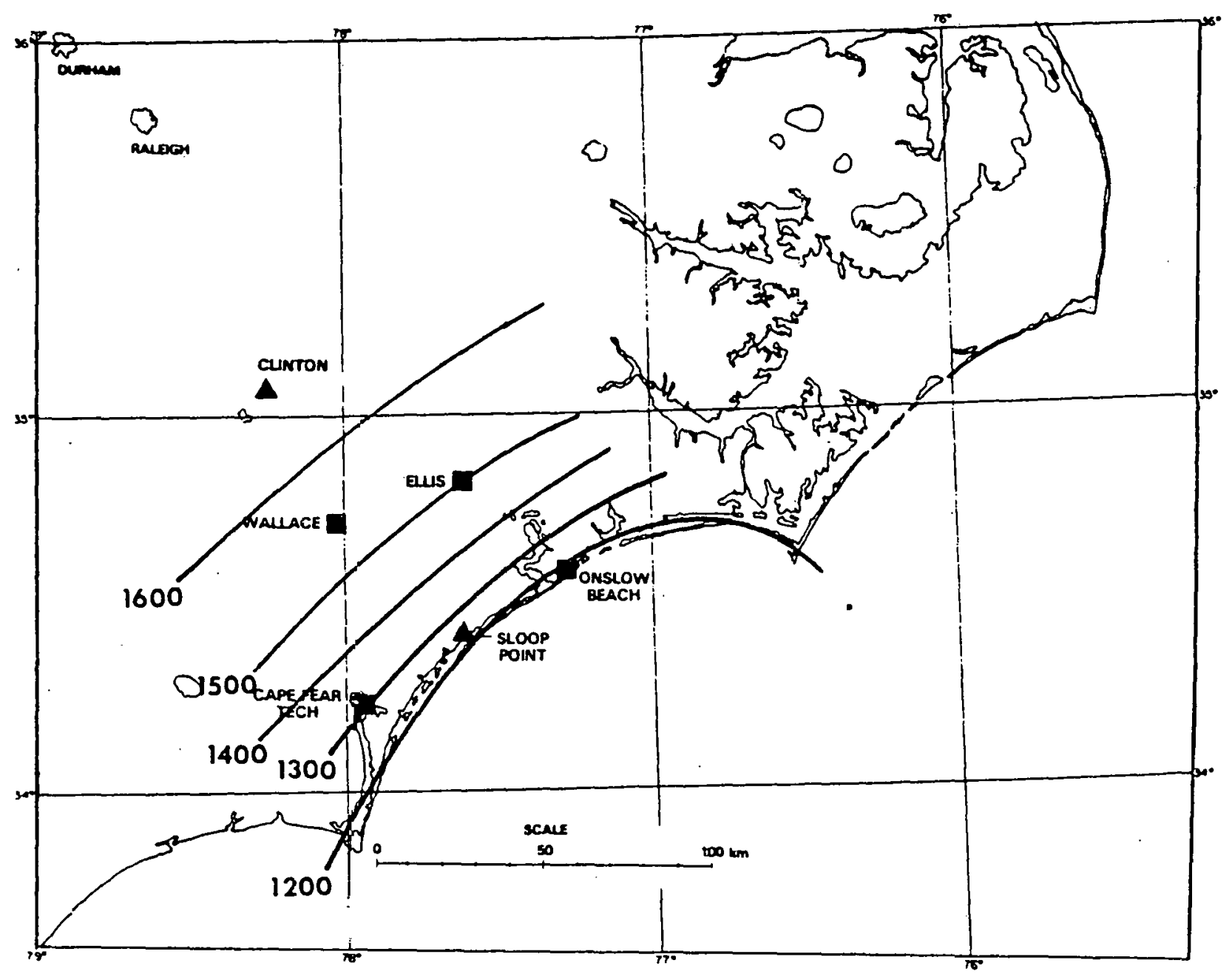

Figure 5. Mean isochrones (EST) of sea breeze frontal passages in the Ons low Bay region determined from 32 sea breeze case study days during the months of May through September 1978. 


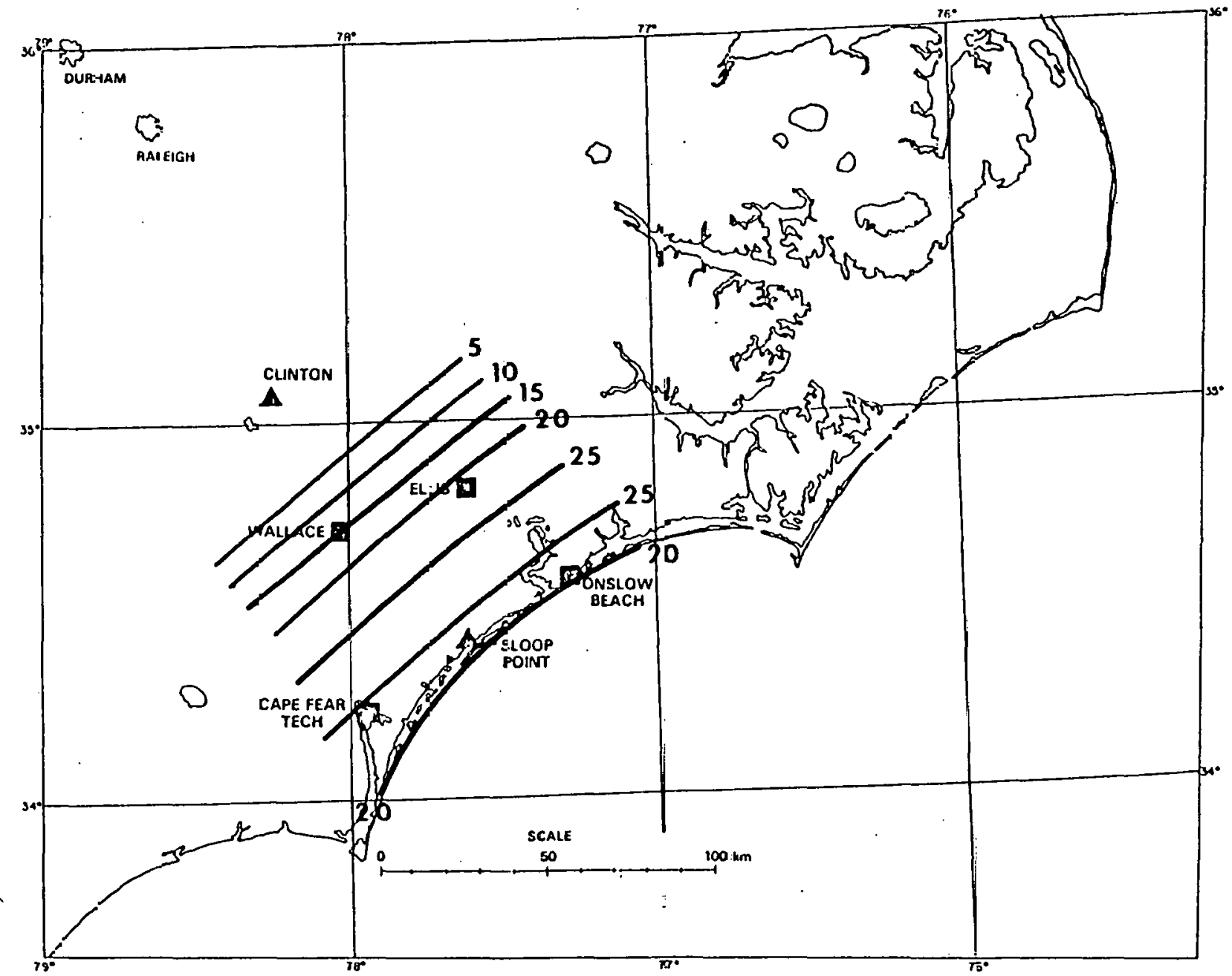

Figure 6. Isopleths of sea breeze frontal passages in the onslow Bay study location determined from 32 sea breeze case study days during the months of May through September 1978. 
Table 4. Sea breeze frontal passage times and number of occurrences for six study location sites ordered in increasing distance from the Onslow Bay coast

\begin{tabular}{lrrrrrr}
\hline \hline & \multicolumn{5}{c}{ Site Location } \\
\cline { 2 - 6 } Passage Time & $(1)$ & $(2)$ & $(3)$ & $(4)$ & $(5)$ & $(6)$ \\
\hline Coastal & 12 & 11 & 8 & 0 & 0 & 0 \\
$0600-1100$ EST & 7 & 7 & 3 & 0 & 0 & 0 \\
$1100-1400$ & 11 & 13 & 16 & 6 & 3 & 0 \\
$1400-1800$ & 2 & 1 & 5 & 15 & 12 & 0 \\
Inland & 0 & 0 & 0 & 11 & 17 & 32 \\
\hline \hline
\end{tabular}

Study location sites are: (1) Onslow Beach, (2) Sloop Point, (3) Cape Fear, (4) Ellis, (5) Wallace, and (6) Clinton

occurred closer to the coast due to occasional sea breeze fronts forming short distances inland from the coastline. Sea breeze fronts passed over the Cape Fear Tech station on 24 of the 32 days studied. Passages occurred on 21 days at Sloop Point and Ellis, 20 days at Onslow Beach, and 15 days at Wallace, N. C.

Earliest, mean, and latest times of sea breeze frontal passage all increased with distance inland (Fig. 7). Greatest separation between earliest and latest times of sea breeze frontal passage occurred at the Cape Fear Tech. The Ellis station experienced the most consistent times of sea breeze frontal passage of all the study location sites. The earliest passage time over Ellis was $1200 \mathrm{EST}$ and the latest occurred at 1630 EST.

\section{Sampling Area Size}

The sampling area surrounding each of the study location sites for all cloud cover observations is a critical factor that determines the accuracy of any derived relationship between cloud cover and $Q *$. Data of cloud cover and global insolation for four case study days during the month of June were initially analyzed using two different sampling area sizes (Fig. 8 and 9). Linear regressions of $Q *$ versus percent of clear sky were developed for air mass and sea breeze cumulus cloud cover and for both sampling area sizes (Fig. 10 through 13). 


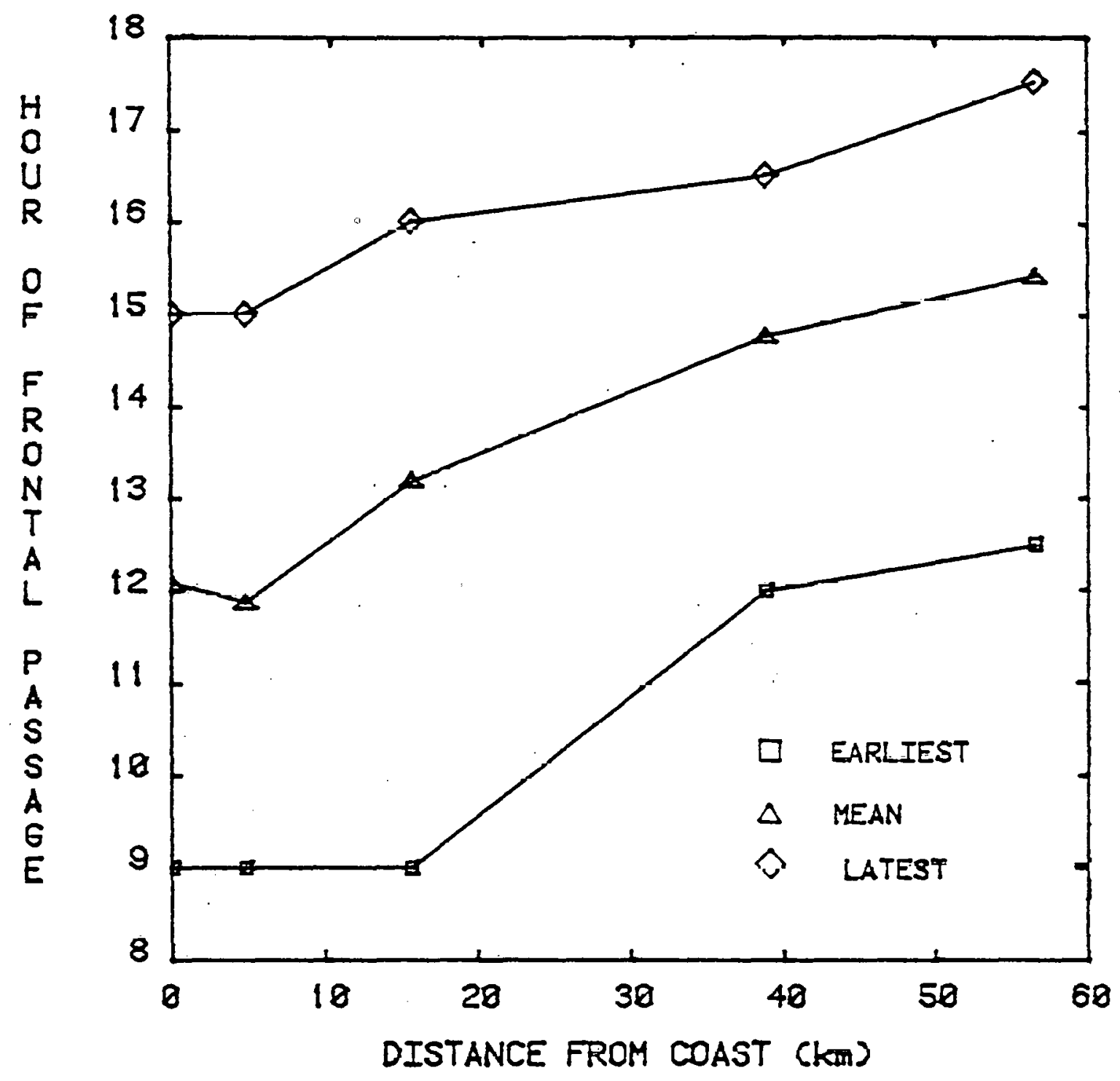

Figure 7. Relationship between the time of sea breeze frontal passage (EST) and the distance from the coast $(\mathrm{km})$. 


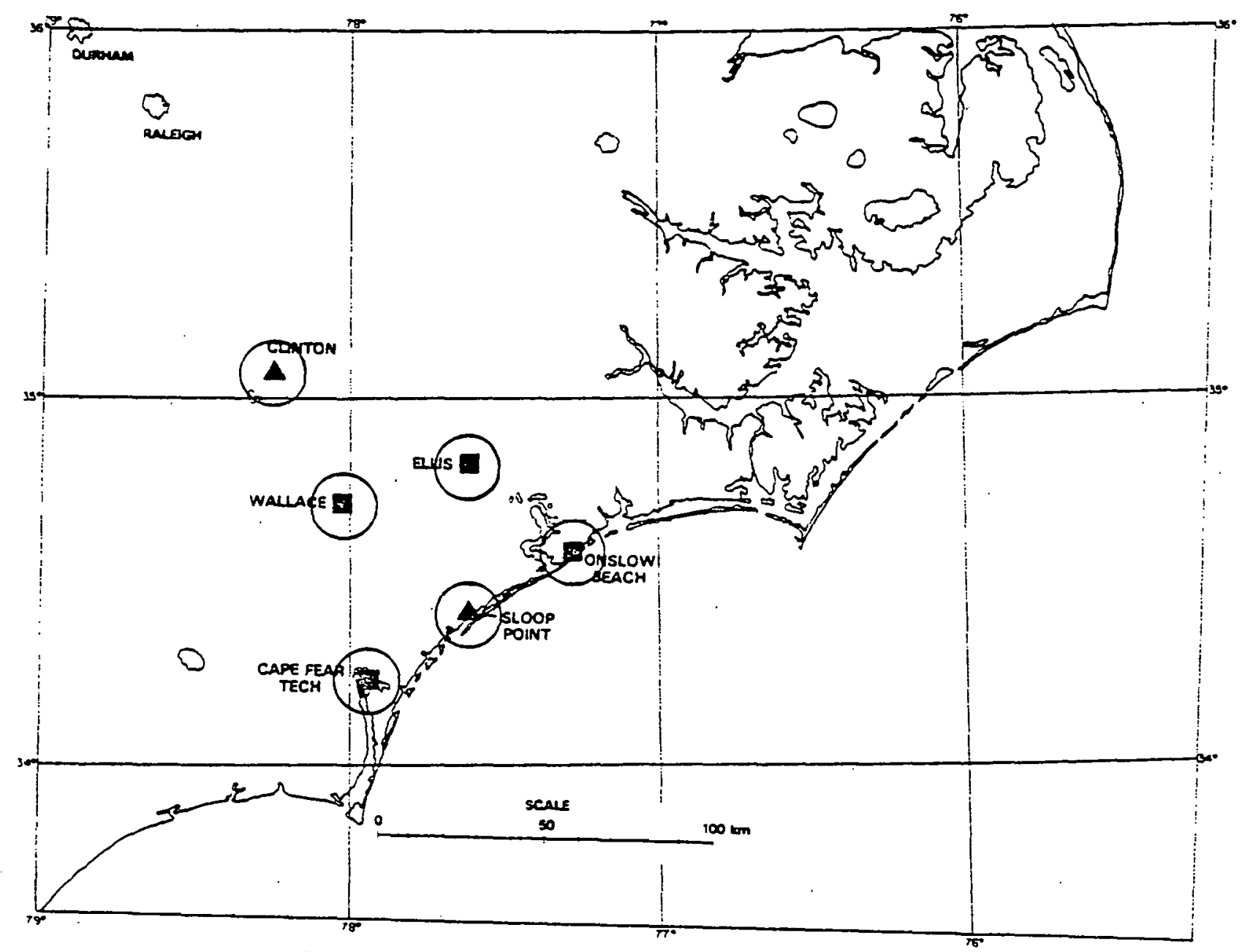

Figure 8. Circular cloud sampling areas with $10 \mathrm{~km}$ radij and centered over each of the study sites. 


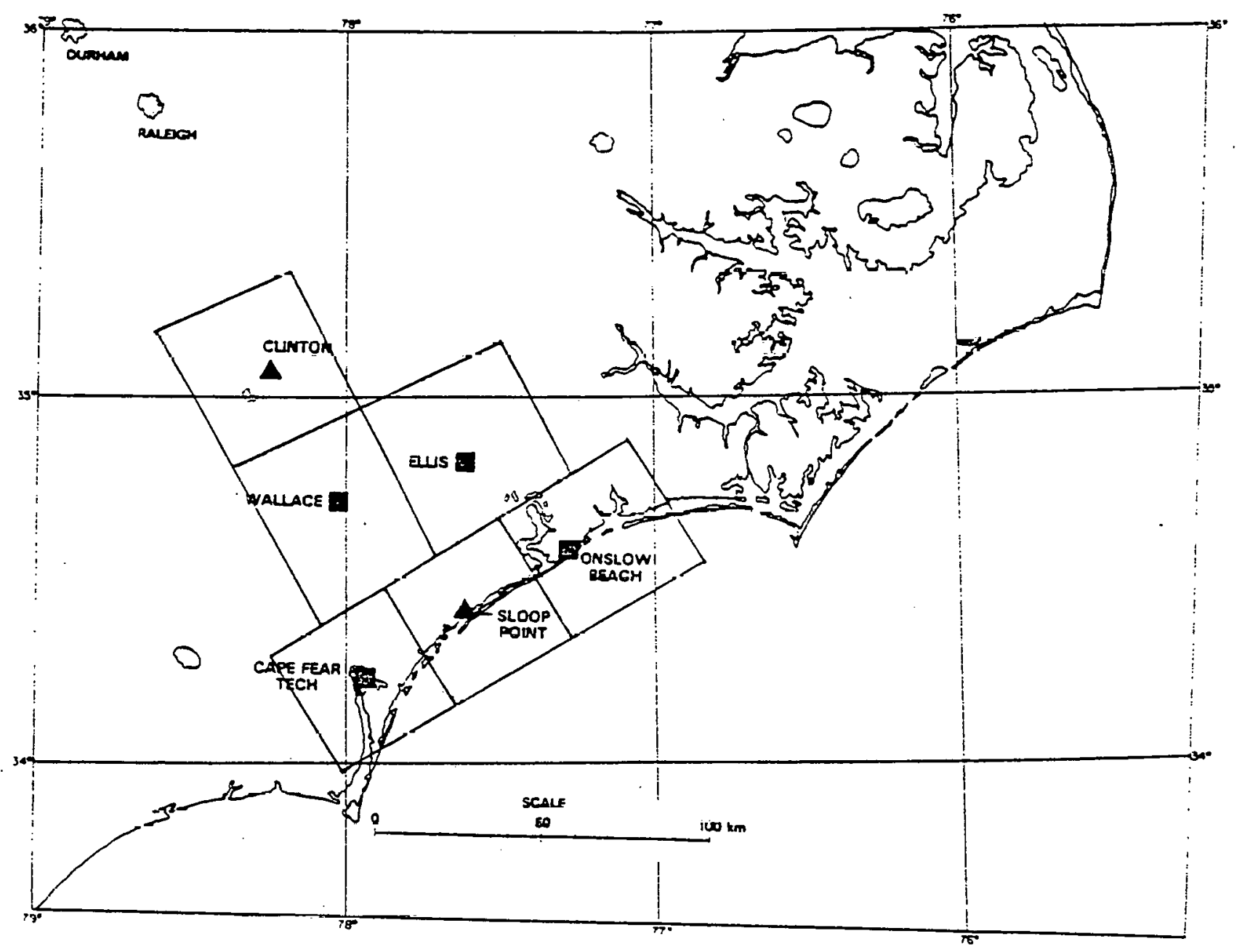

Figure 9. Contiguous $40 \mathrm{~km}$ wide quadrilateral cloud sampling areas approximately centered nuer each of the six study sites. 


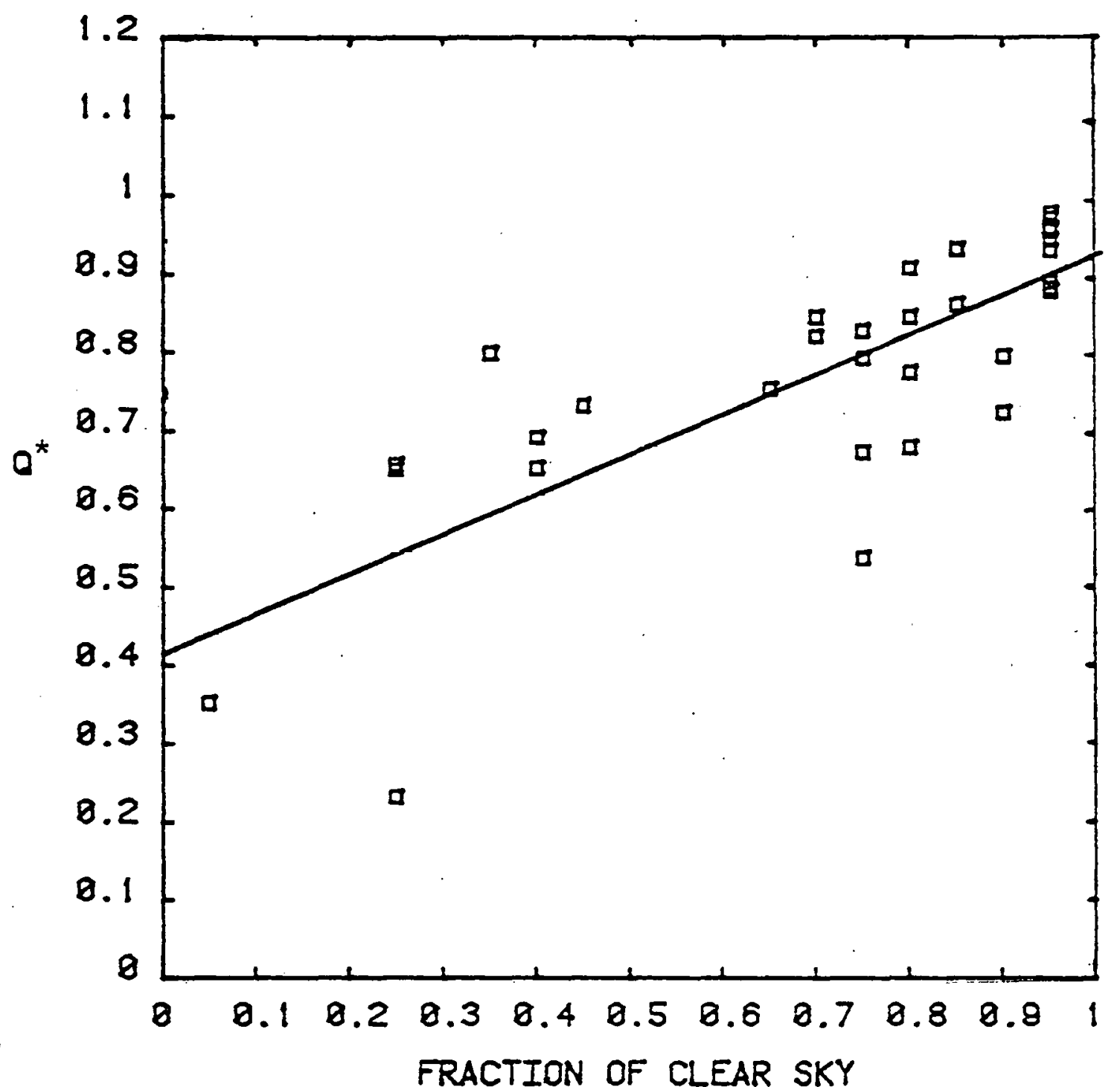

Figure 10. Scatter diagram and linear regression line for $Q^{*}$ versus percent of clear sky under sea breeze cumulus cloud cover measured with $10 \mathrm{~km}$ radius circular areas over the Onslow Bay study location. 


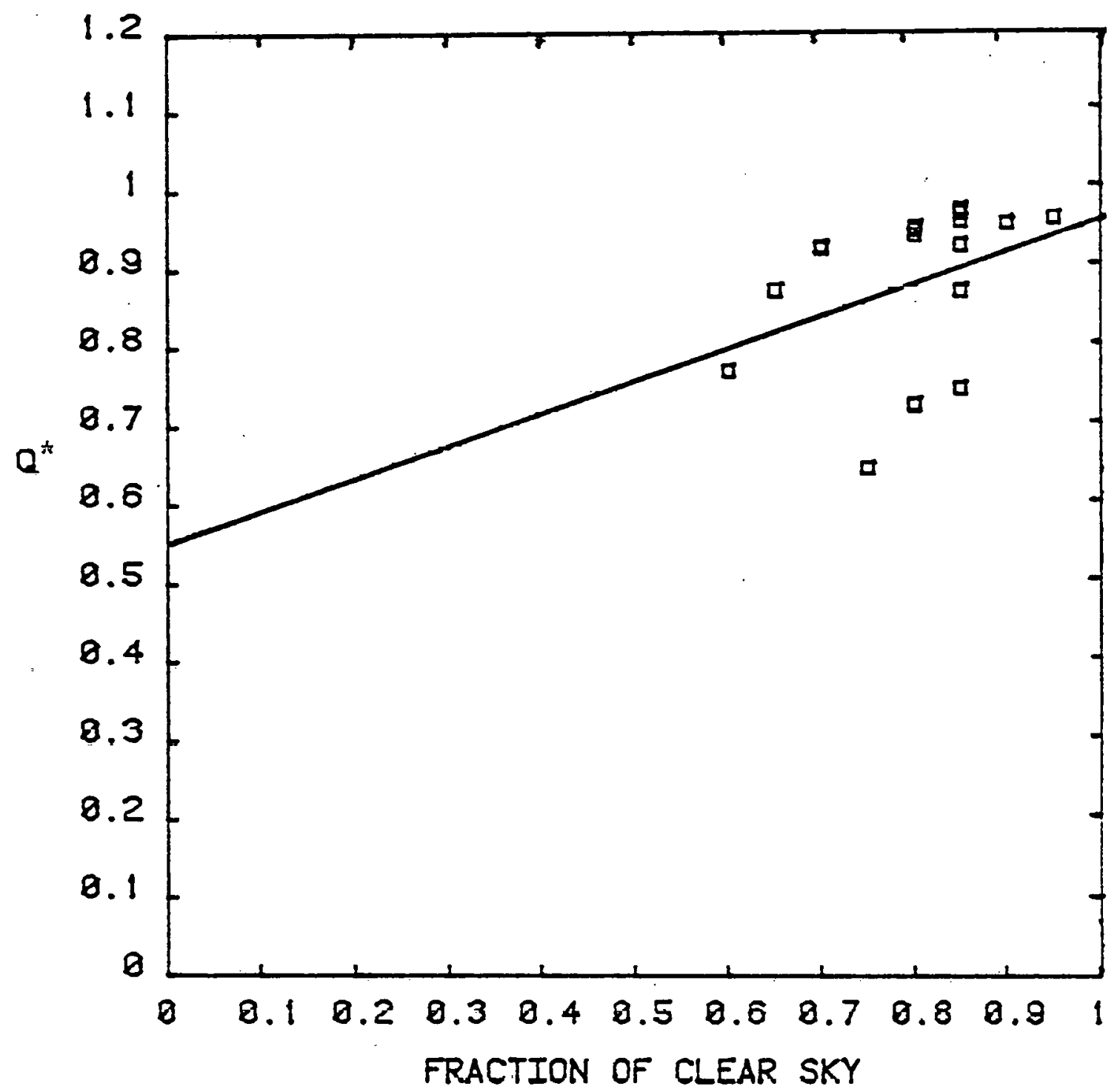

Figure 11. Scatter diagram and linear regression line for $Q^{\star}$ versus percent of clear sky under sea breeze cumulus cloud cover measured with $40 \mathrm{~km}$ wide quadrilateral areas over the Onslow Bay study location. 


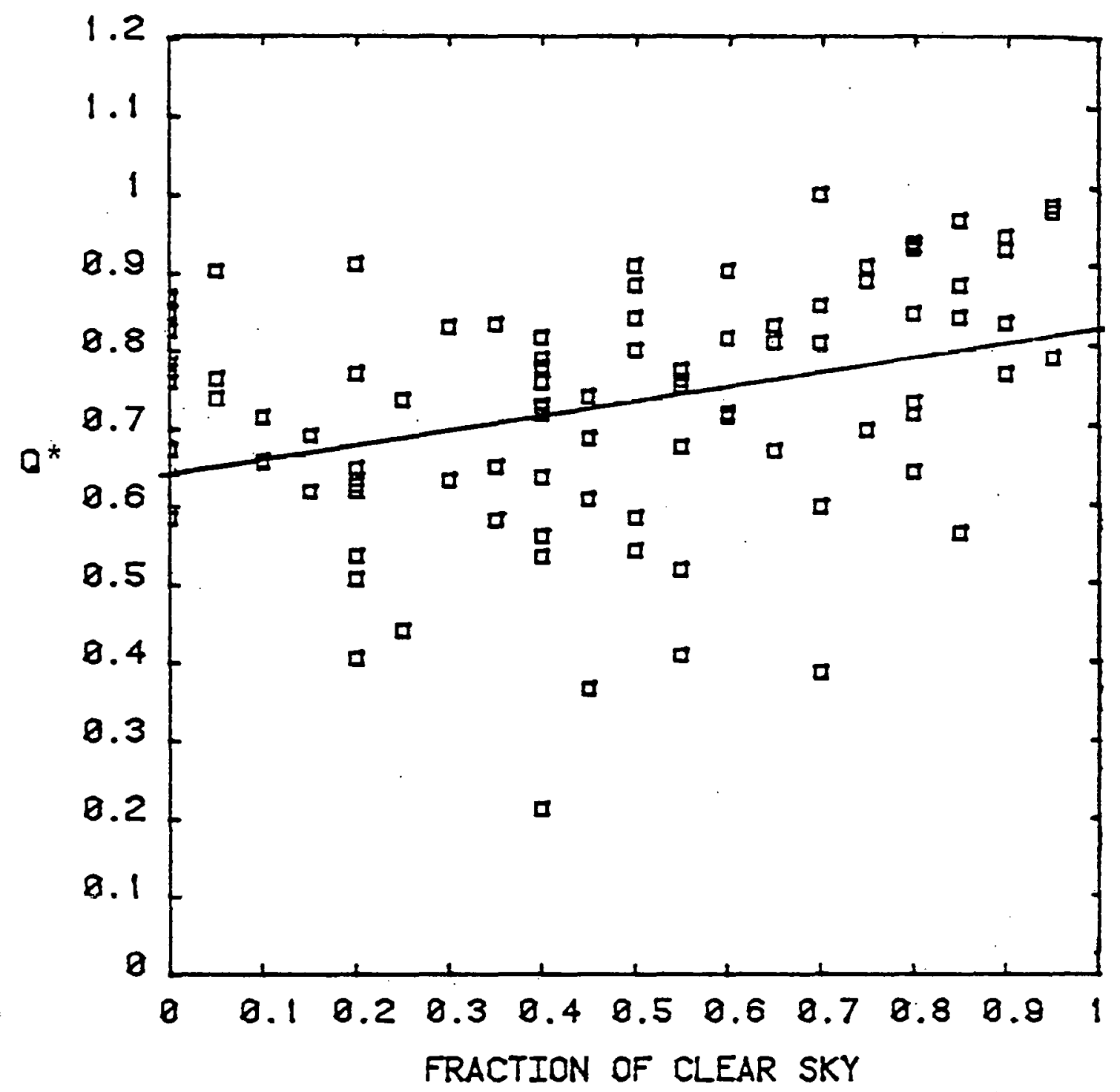

Figure 12. Scatter diagram and linear regression line for $Q^{*}$ versus percent of clear sky under air mass cumulus cloud cover measured with $10 \mathrm{~km}$ radius circular areas over the Onslow Bay study location. 


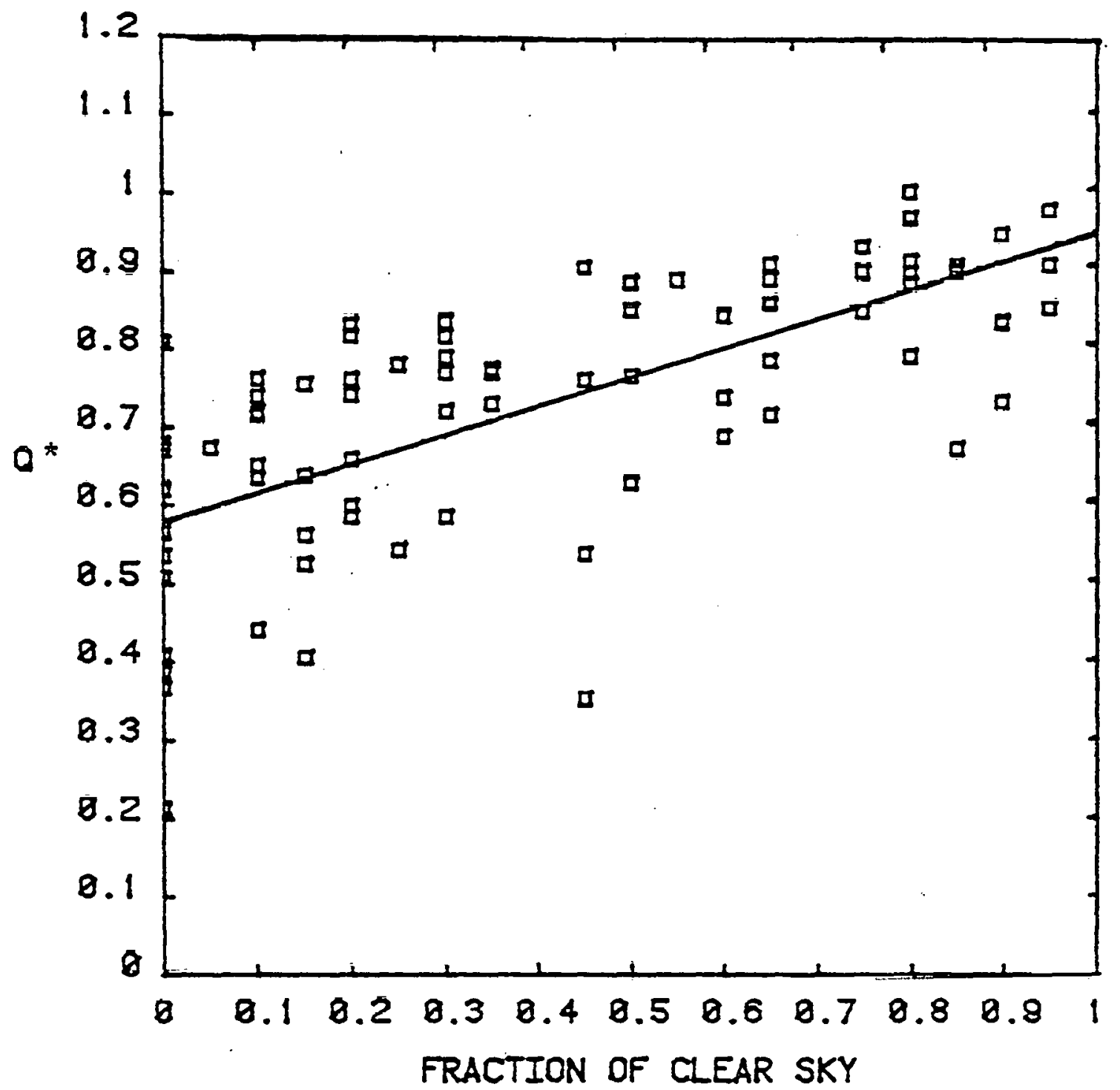

Figure 13. Scatter diagram and linear regression line for $Q^{*}$ versus percent of clear sky under air mass cumulus cloud cover measured wi th $40 \mathrm{~km}$ wide quadrilateral areas over the Orislow Bay sludy location. 
Measured sea breeze cumulus cloud covers using a $10 \mathrm{~km}$ radius sampling area fit a linear regression line with a correlation coefficient of 0.7762 . For the same cloud type, but using a $40 \mathrm{~km}$ wide quadrilateral area, the linear regression line has a correlation coefficient of 0.3775 . The use of a smaller sampling area gives better estimates of the effects of sea breeze cumulus cloud cover on the value of $Q *$. In contrast, air mass cumulus cloud covers measured with a $10 \mathrm{~km}$ radius sampling area are fit poorly by a linear regression line having a correlation coefficient of 0.3173 . However, the correlation coefficient of the linear regression fit using the quadrilateral sampling area increased to 0.6772 . Based on the magnitude of correlation coefficients, the use of a larger sampling area gives better estimates of the effect of air mass cumulus cloud cover on the global insolation received in the Onslow Bay region.

Air mass cumulus convection represents a larger scale (synoptic) than the smaller (mesoscale) sea breeze cumulus convection zone. Since neither small sampling areas for air mass cumulus nor larger sampling areas for sea breeze cumulus are adequate for measuring cumulus cloud amounts, a sampling area size in the midrange between the two areas previously discussed was determined. A $20 \mathrm{~km}$ radius circle was finally chosen as an adequate sampling area size for measurement of both types of cumulus cloud cover in the Onslow Bay Region (Fig. 14). This sampling area size was specifically recommended by Avaste (1964). Linear regressions performed on the cumulus cloud cover measured with $20 \mathrm{~km}$ radius circles versus $Q *$ produced correlation coefficients of 0.542 for sea breeze cumulus and 0.636 for air mass cumulus (Fig. 15 and 16). Correlation coefficients for air mass cumulus cloud cover versus $Q *$ increase in magnitude with increasing sampling area size. Magnitudes of correlation coefficients for the cloud cover versus $Q^{*}$ relationship decrease as the sampling area size for sea breeze cumulus is increased.

\section{Solar Elevation Effect}

To analyze the solar elevation effect on global insolation under cumulus cloud cover, all measured global insolation values were converted to a dimensionless quantity $Q *$. The quantity $Q *$ is the ratio of the measured global insolation $Q$ for a particular day and hour to the monthly maximum global insolation $Q_{0}$ for the study location. Qo was calculated by averaging the 


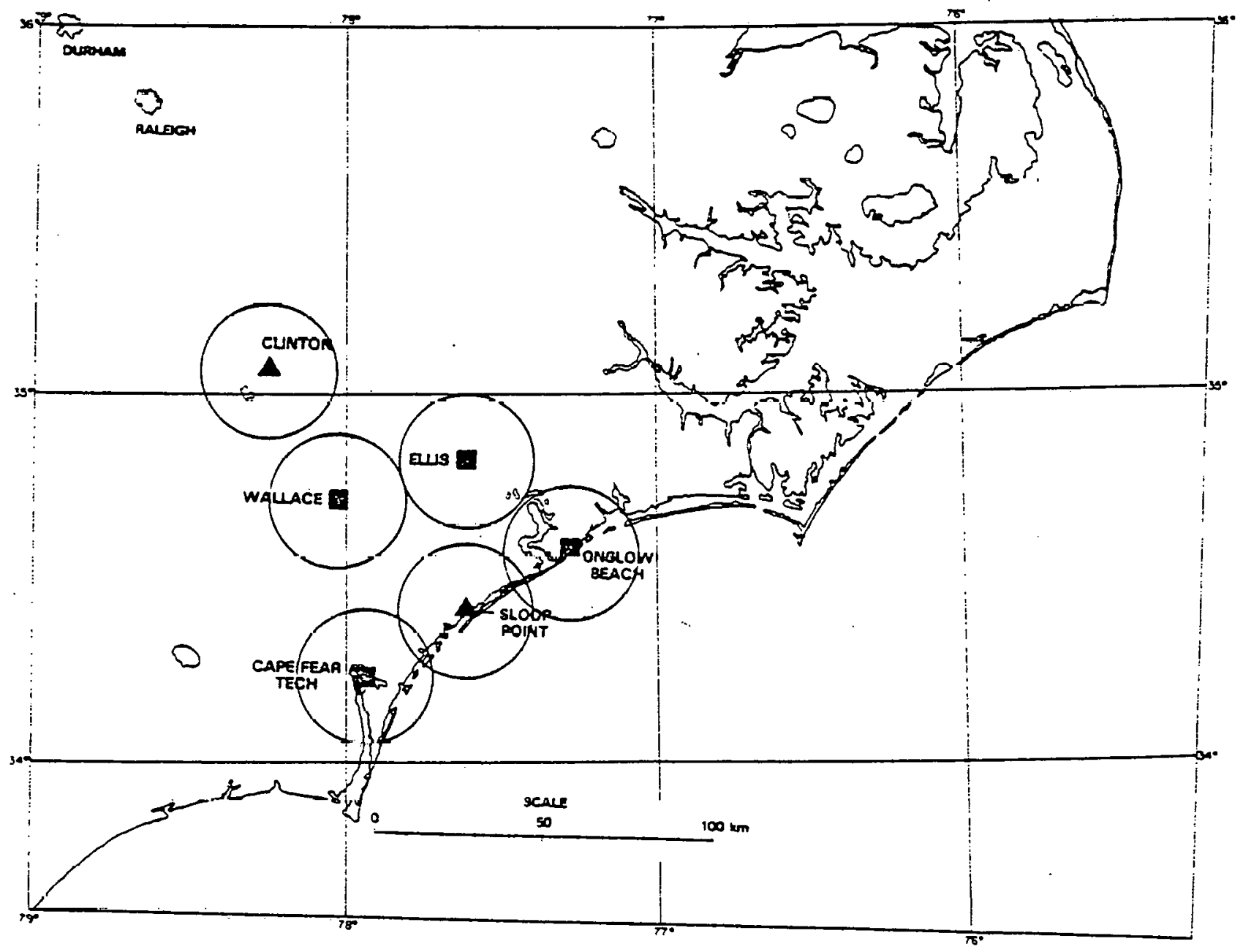

Figure 14. Circular areas with $20 \mathrm{~km}$ radii used to determine both air mass and sea breeze cumulus cloud cover over the six study sites in the Onslow Bay region. 


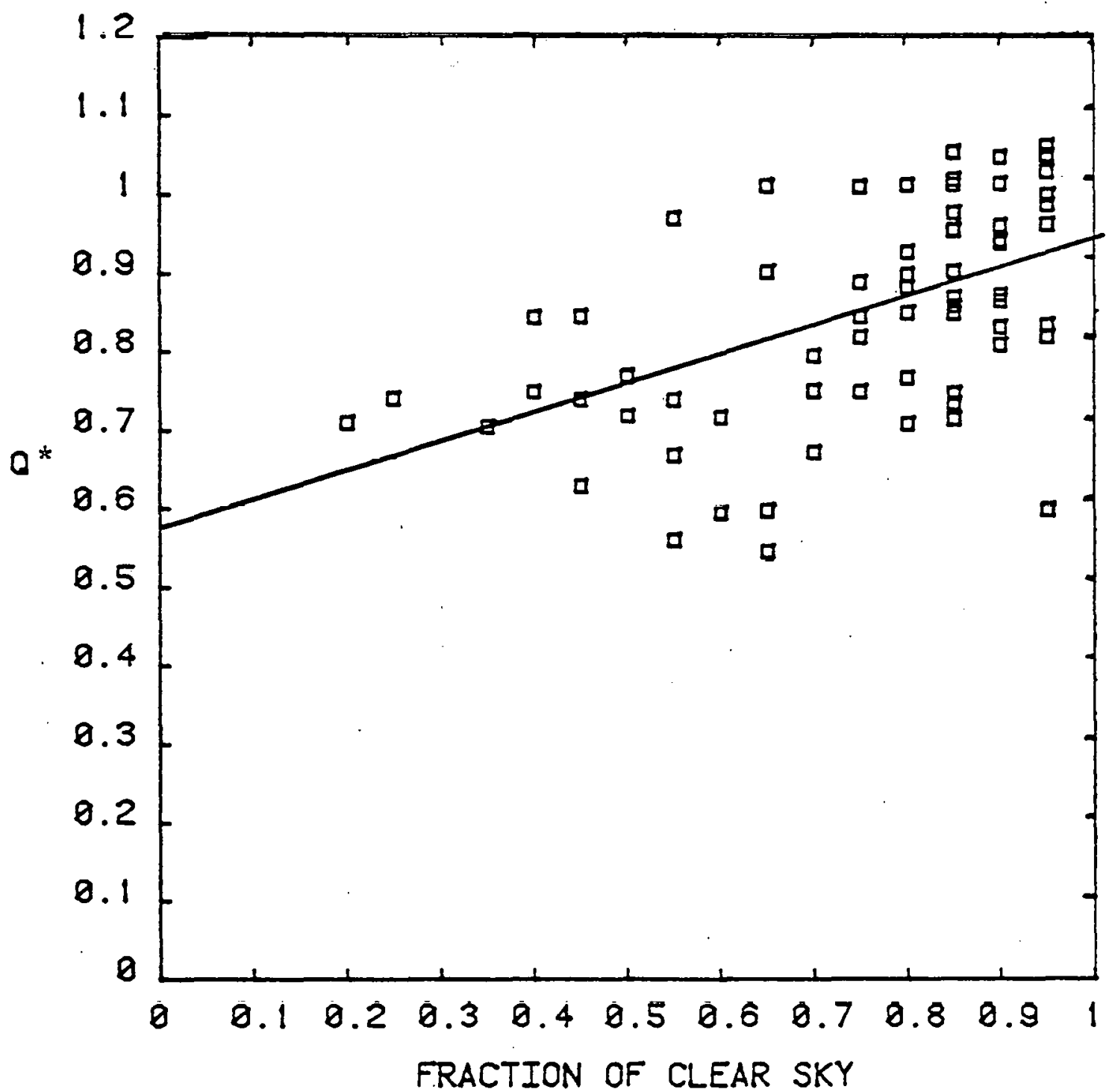

Figure 15. Scatter diagram and linear regression line for $Q^{*}$ versus percent of clear sky under sea breeze cumulus cloud cover measured with $20 \mathrm{~km}$ radius circular areas over the Onslow Bay study location. 


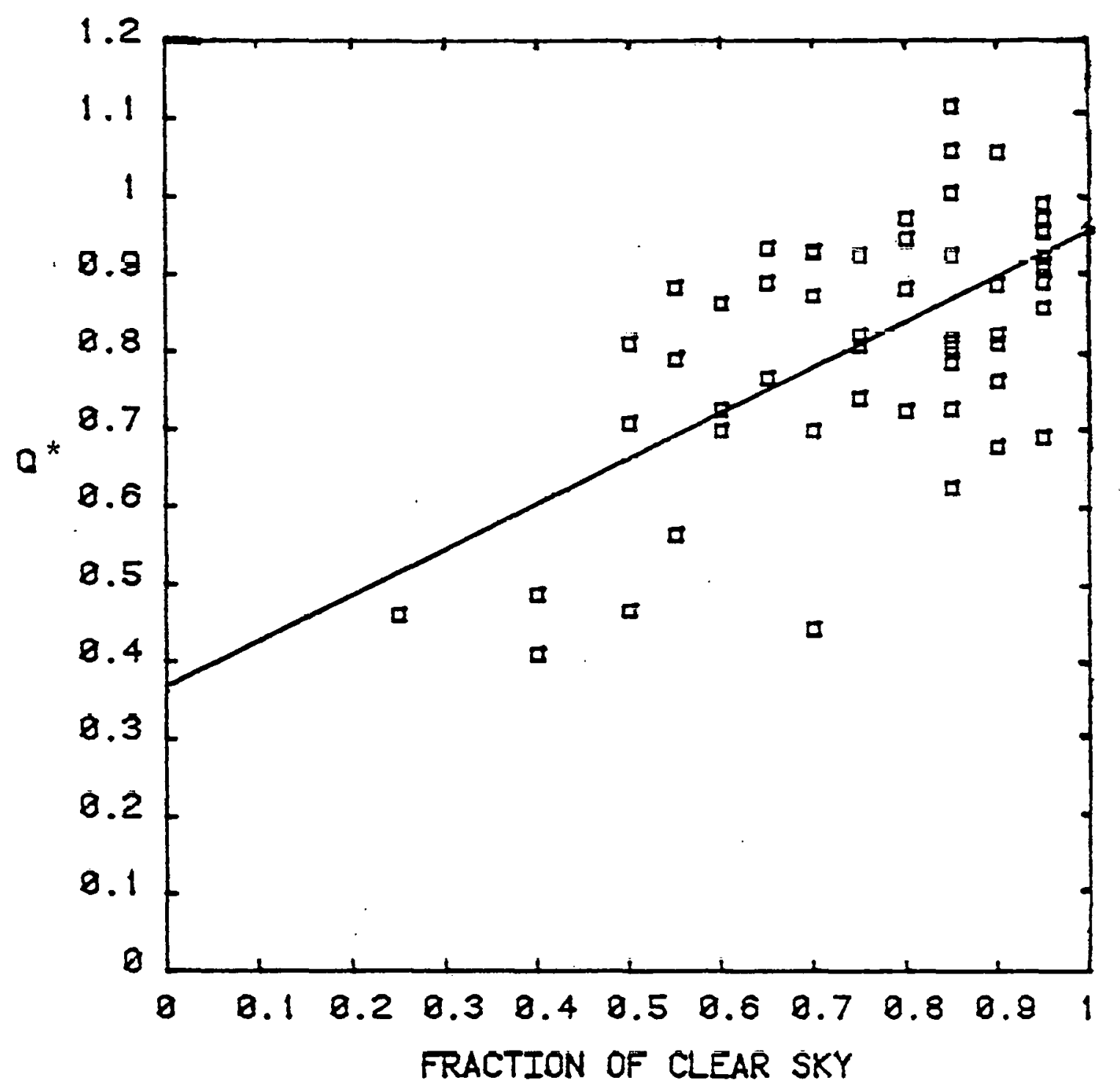

Figure 16. Scatter diagram and linear regression $l$ ine for $Q^{*}$ versus percent of clear sky under air mass cumulus cloud cover measured with $20 \mathrm{~km}$ radius circular areas over the Onslow Bay study location. 
maximum measured global insolation of all six study location sites for a particular month and hour.

Scatter diagrams of solar elevation versus $Q *$ for the Onslow Bay study location show that the solar elevation effect on relative amount of shortwave radiation received at the ground surface through cumulus cloud cover is almost negligible for both sea breeze and air mass cumulus (Fig. 17 and 18). These plots reveal no evidence of solar elevation effect for angles greater than $40^{\circ}$. On the average, for any given amount of sea breeze or air mass cumulus cloud cover, the decrease in $Q *$ values for solar elevaton angles less than $40^{\circ}$ is less than 10 percent. Therefore, solar elevation is not a major influence on differences in $Q *$ for the same cloud cover type and amount. Further analyses on the global insolation received under similar cloud types and amounts will not be significantly influenced by solar elevation since only a small number of the data used fall into the lower solar elevation range.

Cumulus Cloud Cover and Global Insolation

The dimensionless ratio $Q *$ of measured to maximum global insolation is nearly a linear function of cloud cover for air mass cumulus cloud amounts up to 50 percent of total sky covered. For air mass cumulus cloud cover greater than 50 percent, the rate of decrease in the magnitude of $Q *$ increases slowly with rising cloud cover amount. The vertical extent of air mass cumulus clouds observed over the Onslow Bay region did not change significantly for cloud amounts up to 50 percent. As air mass cumulus cloud amounts increased from 50 percent, the clouds also increased in thickness and corresponding values of $Q *$ decreased at an increasing rate. Values of $Q *$ range from 0.93 for scattered air mass cumulus, to approximately 0.62 for up to 90 percent sky cover (Fig. 19).

The trendline for sea breeze cumulus cloud cover versus $Q *$ reveals changes in the effect of cloud amount on $Q *$ as cloud amount increases (Fig. 20). Small variations in values of $Q *$ result from increases in sea breeze cumulus up to 20 percent sky cover. A considerable fraction of the global radiation received at the Onslow Bay study sites and influenced by scattered 


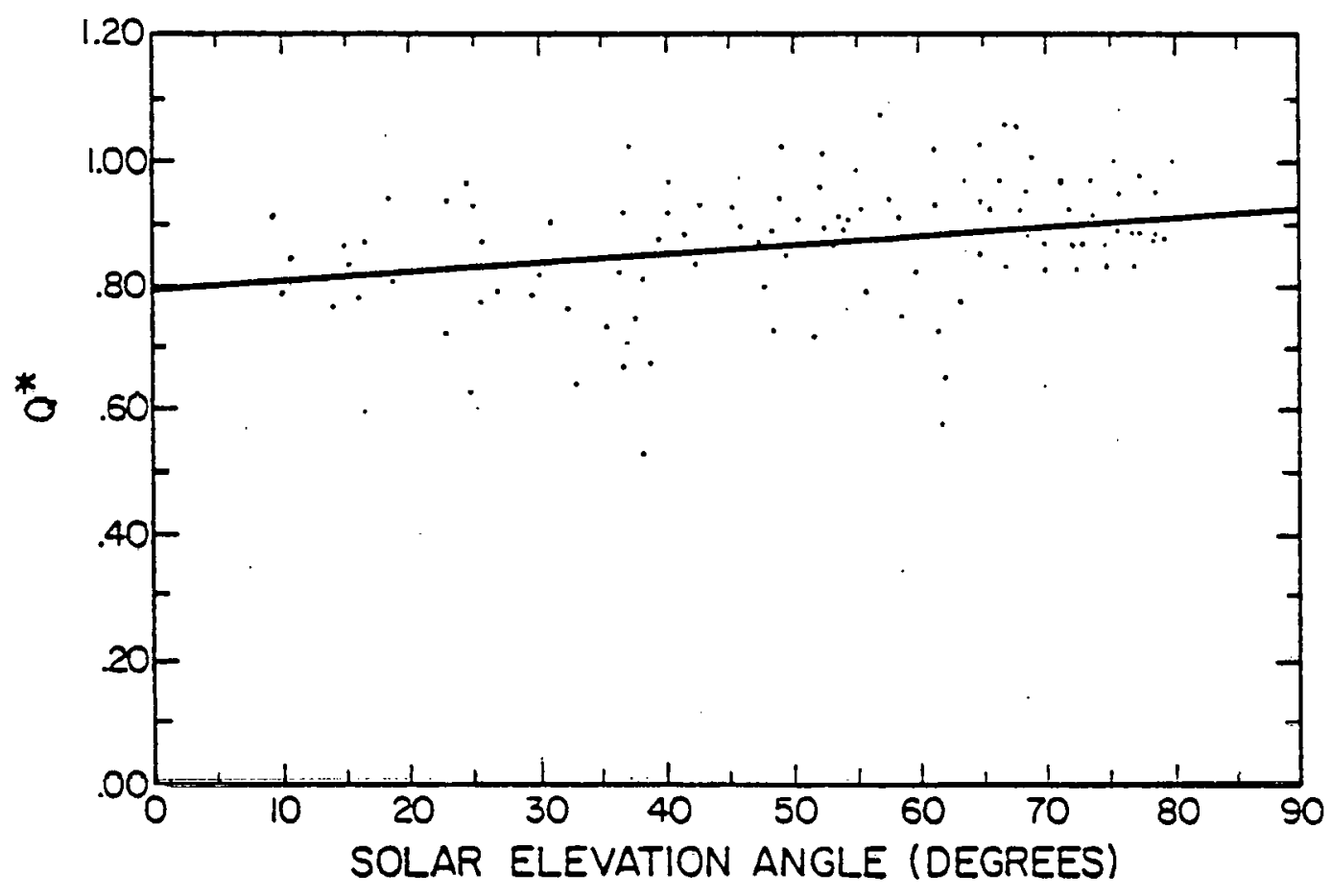

Figure 17. Scatter diagram of $Q^{\star}$ versus solar elevation angle for two-tenths sea breeze cumulus cloud cover over the Onslow Bay study location. 


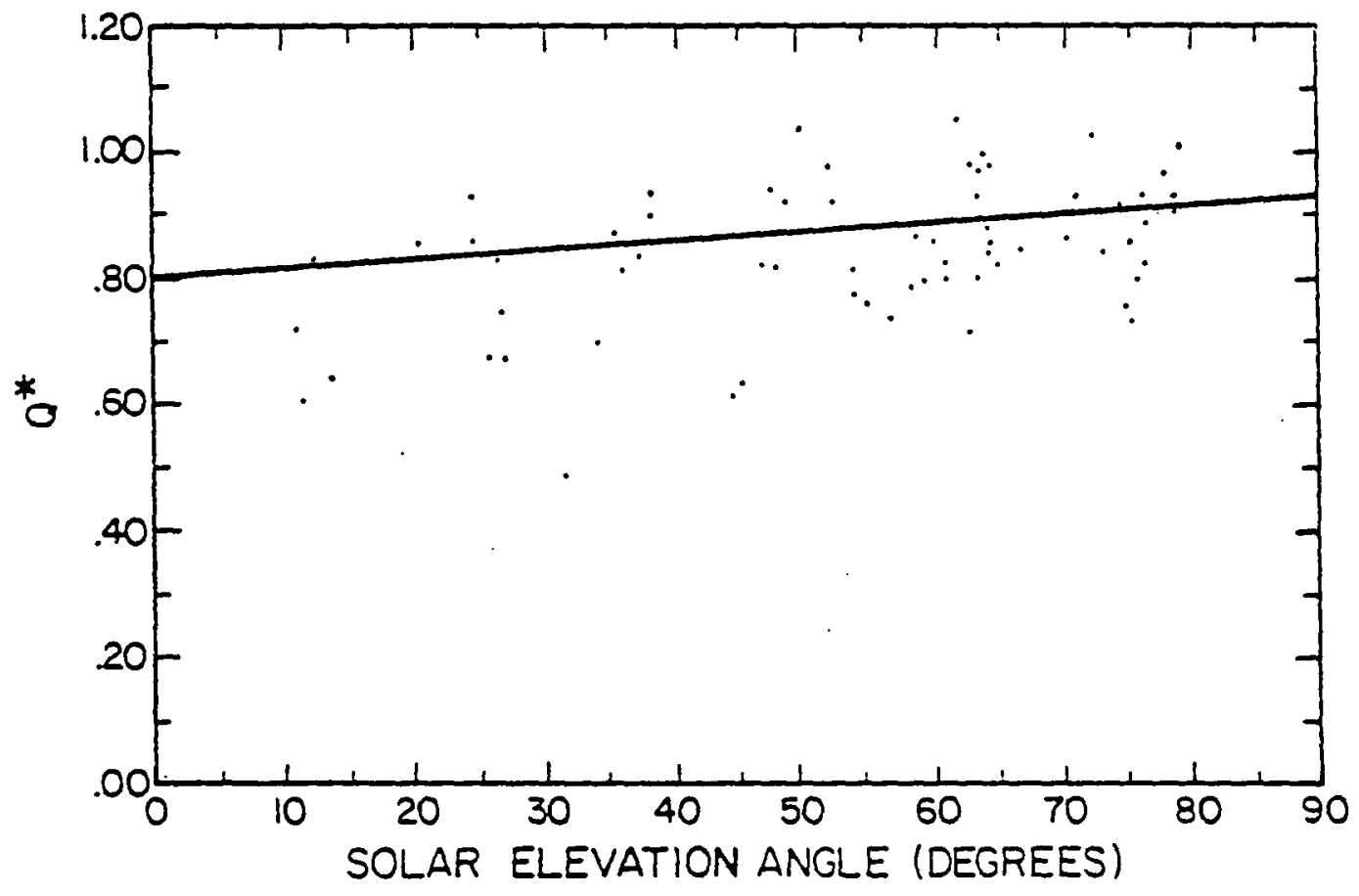

Figure 18. Scatter diagram of $Q^{*}$ versus solar elevation angle for two-tenths air mass cumulus cloud cover over the
Ons low Bay study location. 


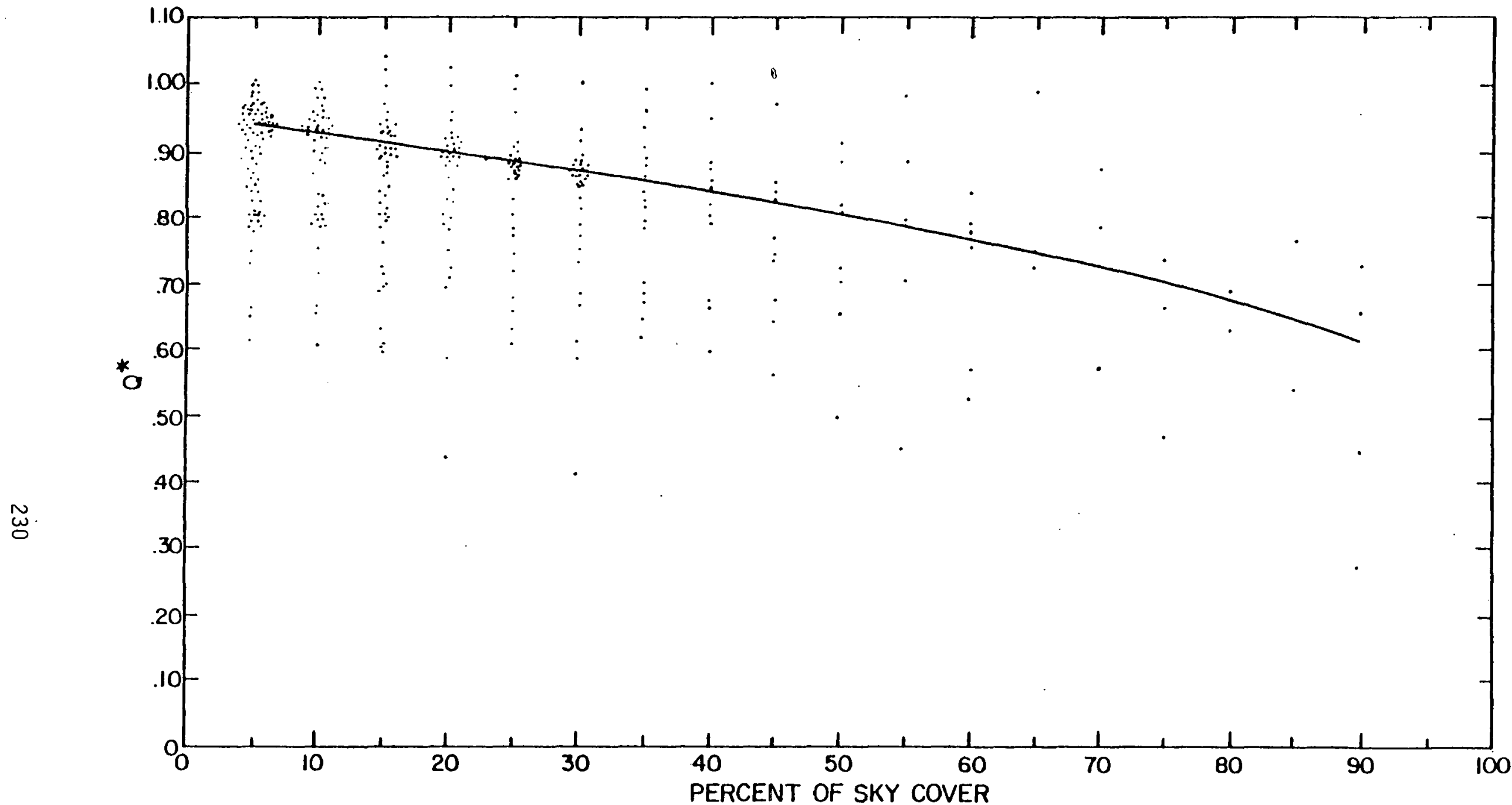

Figure 19. Scatter diagram and a yisually approximated trend lire showing the relationship between $Q^{*}$ and amount of air mass cumulus cloud cover. 


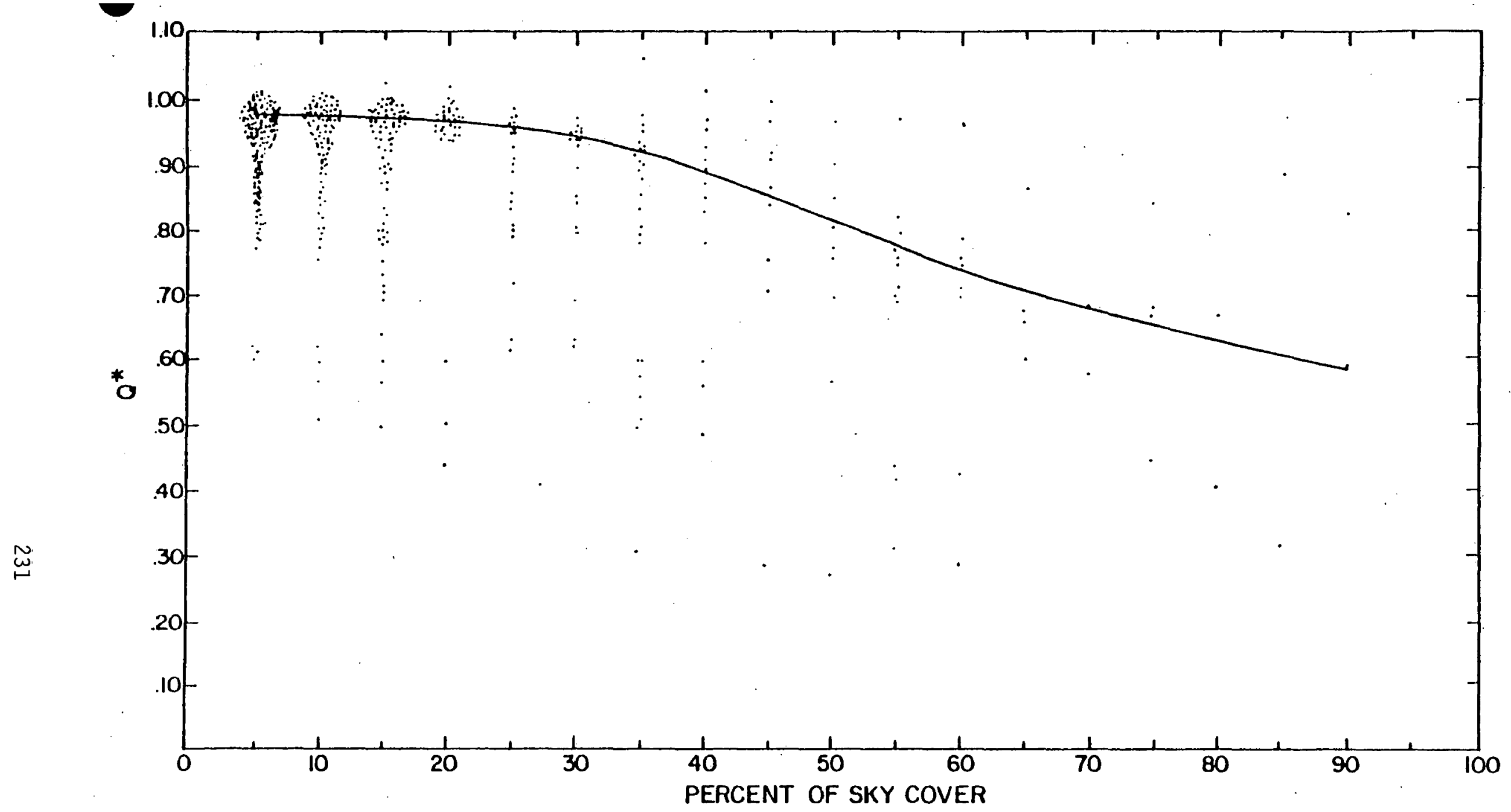

Figure 20. Scatter diagram and a visually approximated trend line showing the relationship between $Q^{*}$ and amount of sea breeze cumulus cloud cover. 
sea breeze cumulus cloudiness consisted of reflected radiation from the sides of these relatively thick clouds. Values of $Q *$ greater than 0.95 were received at sites influenced by scattered sea breeze cumulus cloud. cover. The magnitude of $Q *$ decreased significantly for fractions of sky cover greater than 20 percent as the vertical extent of the sea breeze cumulus increased. Values of $Q *$ dropped below 0.60 for locations beneath a 90 percent sea breeze cumulus cloud cover.

The difference between the effects of air mass and sea breeze cumulus on the global radiation received at the Onslow Bay region is directly related to the vertical extent of each of these cloud types. Sea breeze cumulus clouds reduce a greater amount of global radiation than do air mass cumulus clouds due to their larger relative vertical extent.

Mean Effect of Cloud Cover and Time of Day

The quantity $Q *$ is a function of the cloud amount present but is also dependent on the diurnal variations in cloud thickness and solar elevation angles. As a consequence of the complex diurnal effects of cloud cover on the magnitude of $Q *$, no attempt was made to relate quantity of cumulus cloud cover to corresponding values of $Q *$ using linear or nonlinear models. Instead, values of the mean $Q *$ for both sea breeze and air mass cumulus clouds and for specific intervals of cloud amount were calculated from observations made during sea breeze days in the Onslow Bay region.

The value of $\bar{Q} *$ associated with a constant amount of sea breeze cumulus cloud cover decreases with time of day for three-tenths or less sky covered (Fig. 21). Increasing thickness of individual clouds in the sea breeze convergence zone with time of day resulted in a reduction of $\bar{Q} *$ over the Onslow Bay region trom 0.896 in the morning hours to 0.763 during the late afternoon hours for a constant 1 to 2 tenths sky cover. Air mass cumulus clouds exhibit a much smaller diurnal increase in vertical extent for small, constant cloud amounte. The diurnal change in solar elevation angle alnng with a small increase in cloud thickness for constant amounts of air mass cumulus cloud cover influence the corresponding diurnal change in the magnitude of $\bar{Q} *$ (Fig. 22). Maximum values of $\bar{Q} *$ for given air mass cumulus cloud cover amounts were obtained during the midday hours of the sea breeze case 
CLOUD COVER (tenths)

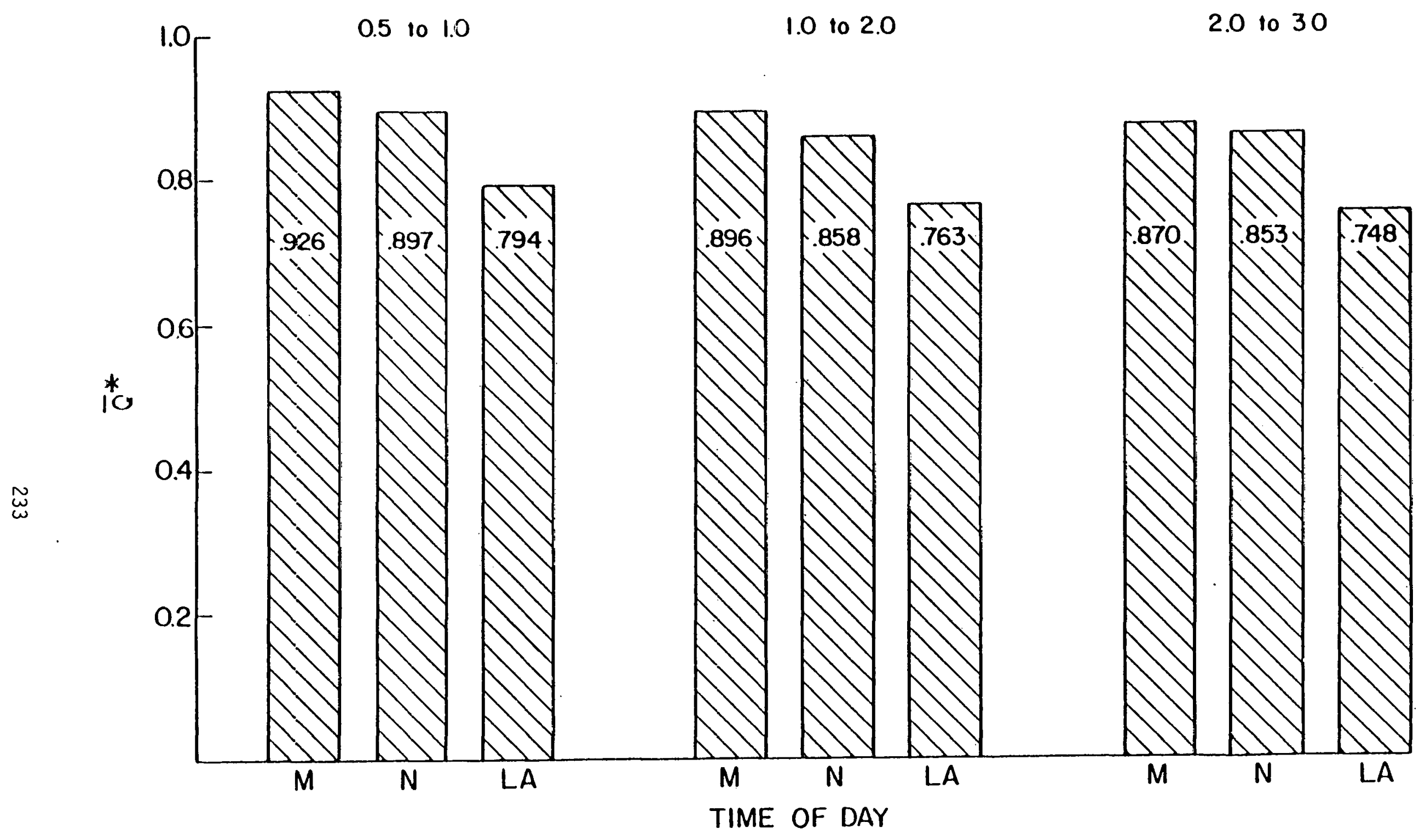

Figure 21. Histogram of the mean global insolation ratio, $\bar{Q}^{\star}$, under specific amounts of sea breeze cumulus cloud cover. 


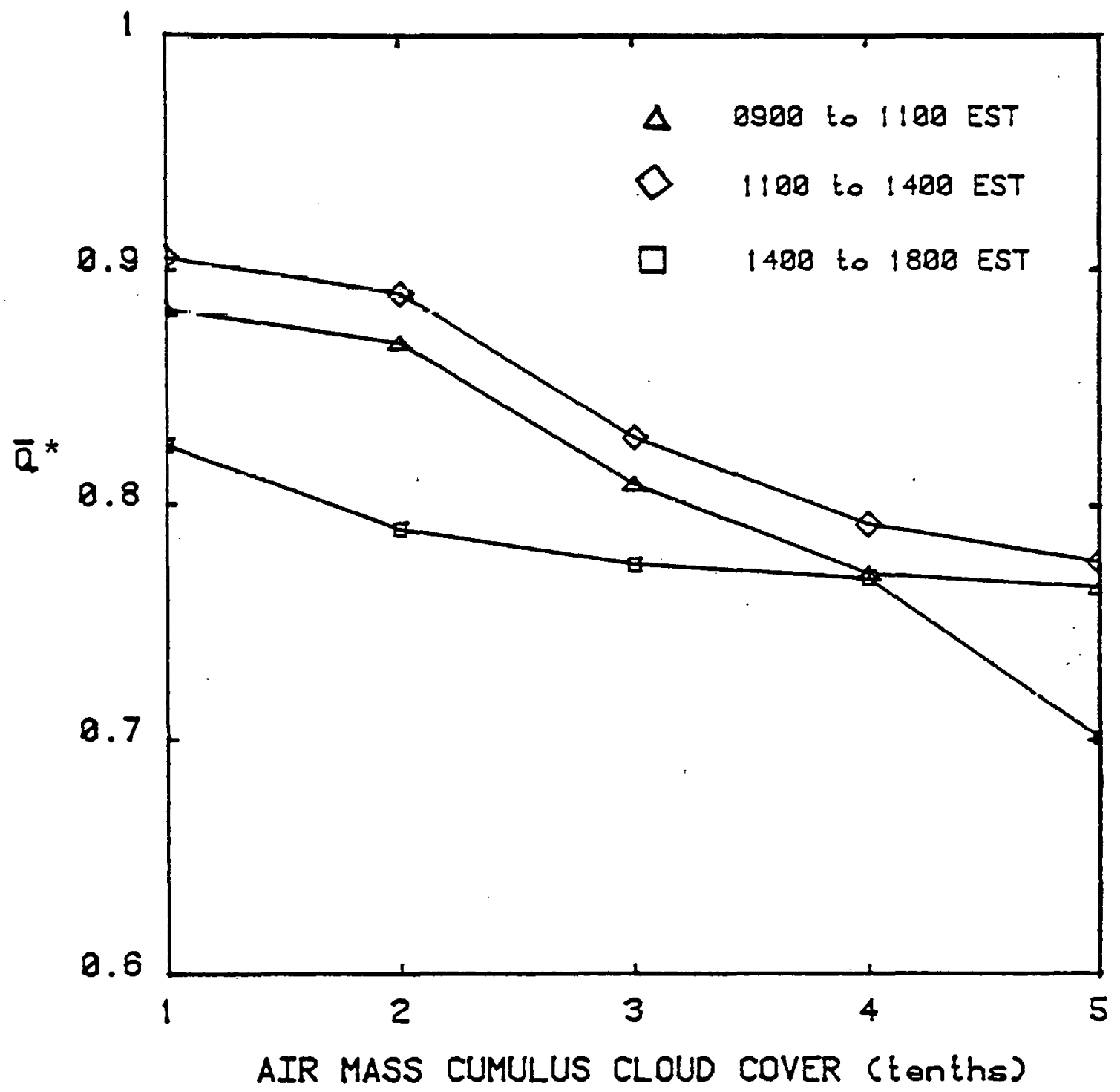

Figure 22. Relationship between the mean global insolation ratio and the amount of air mass cumulus cloud cover observed on sea breeze case study days, Sunmer 1978. 
study days. For a constant cloud amount ranging from 1 to 5 tenths sky cover, magnitudes of $\bar{Q} *$ consistently decreased from the midday, to morning, and finally to late afternoon hours. Midday values of $Q^{*}$ were greater than at other times of the day because the reduction of global radiation by the sides of clouds is minimal with large solar elevation angles. $\bar{Q} *$ is consistently less under similar air mass cumulus cloud amounts during the late afternoon hours than during the morning hours as a consequence of the minor influence of diurnal increases in cloud thickness.

A significant number of cloud cover observations taken during the late afternoon period made it possible to analyze and compare the quantity $\bar{Q} *$ for both sea breeze and air mass cumulus. Values of $\bar{Q}^{*}$ corresponding to specific amounts of sea breeze cumulus were consistently smaller than values of $\bar{Q} *$ existing under the same amount of air mass cumulus (Fig. 23). The decrease in $\bar{Q} *$ with increasing amounts of either air mass or sea breeze cumulus cloud cover observed in the Onslow Bay region is noticeably less than that observed by Pyldmaa. Pyldmaa suggests that $\bar{Q} *$ is linearly dependent upon the quantity of cumulus cloud cover. He obtained $Q^{*}$ values of 0.98 and 0.64 for corresponding cumulus cloud amounts of 3 tenths and 7 tenths, respectively. For equal cloud cover amounts, air mass cumulus reduced $\bar{Q} *$ from 0.774 to 0.638 and sea breeze cumulus reduced $\bar{Q} *$ from 0.7 .48 to 0.618 . Pyldma's estimate of the $\bar{Q} *$ versus cumulus cloud amount relationship probably differs from the one found in the Onslow Bay region because of dissimilar times of observations and differences in the specific types of cumulus clouds under analysis. The difference in $\bar{Q} *$ for the two types of cumulus clouds observed in the Onslow Bay region during the late afternoon hours corresponds directly to the difference in cloud thickness between the shallow air mass cumulus and the more vertically developed sea breeze cumulus.

\section{Temporal Effect of Sea Breeze Frontal Passage}

Passage of a sea breeze front is usually accompanied by a marked decrease in cloud cover and an increase in $Q *$ over study location sites behind the passing front. This change in $Q^{*}$ is dependent upon the amount of cloud cover ahead of the front, the time of passage, and the orientation of the sea breeze convergence zone paralleling the coast. Cloud amount and 


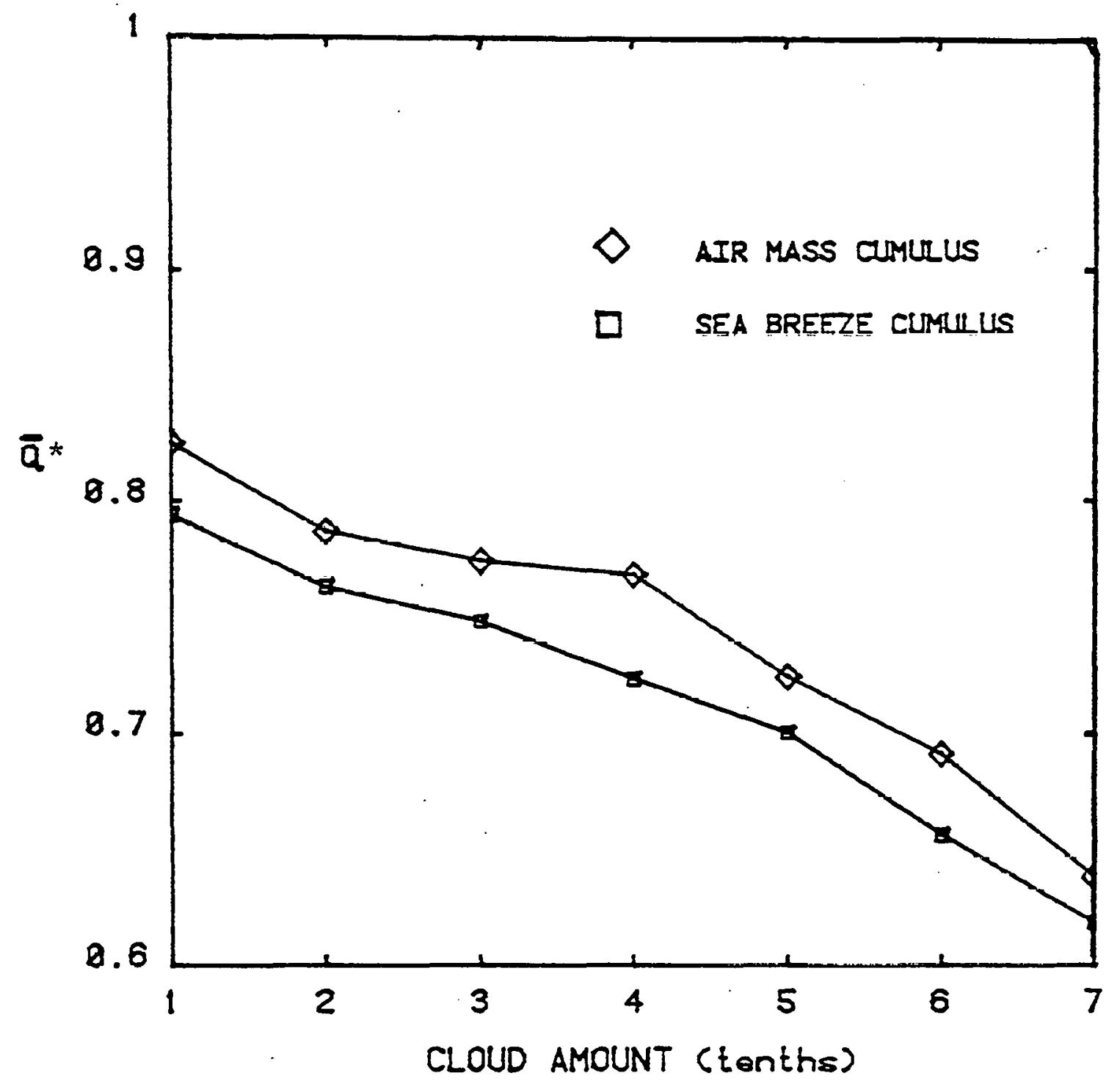

Figure 23. Dependence of mean global insolation ratio on the amount of air mass and sea breeze cumulus eloud cover observed from 1400 to 1800 EST on sea breeze case study days, Summer 1978. 
global insolation measurements taken at all the study location sites in the Onslow Bay region were grouped together according to whether or not a sea breeze front had passed a particular location and also according to the hour which the observation was made. Hourly mean values of $Q *$ were calculated for sites ahead and behind the sea breeze front between the hours of 0900 and 1800 EST (Fig. 24). Study location sites behind the sea breeze front consistently exhibit larger $\bar{Q} *$ values than for sites ahead of the front observed during the same hour of the day. $\bar{Q} *$ values observed ahead of the front range from 0.883 in the morning to 0.622 in the late afternoon. Values of $\bar{Q} *$ behind the sea breeze front range from 0.923 at 1000 EST to 0.699 at 1800 EST.

The difference between $\bar{Q} *$ values ahead and behind the sea breeze front increases with time of day after the midday period. This spatial difference in $\bar{Q} *$ increases from 0.043 at 1400 EST to 0.105 at 1700 EST. A major influence on this noticeable temporal increase in the difference of $\bar{Q} *$ values ahead and behind the sea breeze front is the significant increase of cumulus cloud cover amount and cloud thickness of individual sea breeze cumulus. Values of $\bar{Q} *$ received at stations ahead of the sea breeze front diminish with time after solar noon as a consequence of this strong cumulus convection. Values of $\bar{Q} *$ for locations behind the front also experience a decrease in magnitude from solar noon on, but this trend is likely a function of the position of the individual sites with respect to the sea breeze front and also a function of the solar elevation. Given these assumptions, $\bar{Q} \div$ will decrease for sites along east coasts close to and behind the coastal edge of a sea breeze frontal zone as the solar elevation of the afternoon sun diminishes. For sites located along west coasts under similar sea breeze conditions, a decrease in $\bar{Q} *$ for locations close to the coastal edge of the frontal zone is unlikely since cumulus clouds ahead of the front would not obstruct the direct radiation from an afternoon sun. Cumulus clouds in the sea breeze frontal zone could in fact increase $\bar{Q} *$ values of sites for the latter situation during late afternoon hours resulting from their high albedo.

Total daily global radiation received at any study location site is also a function of the sea breeze frontal passage time. Observations of frontal passages for all sites and case study days were grouped into three major 


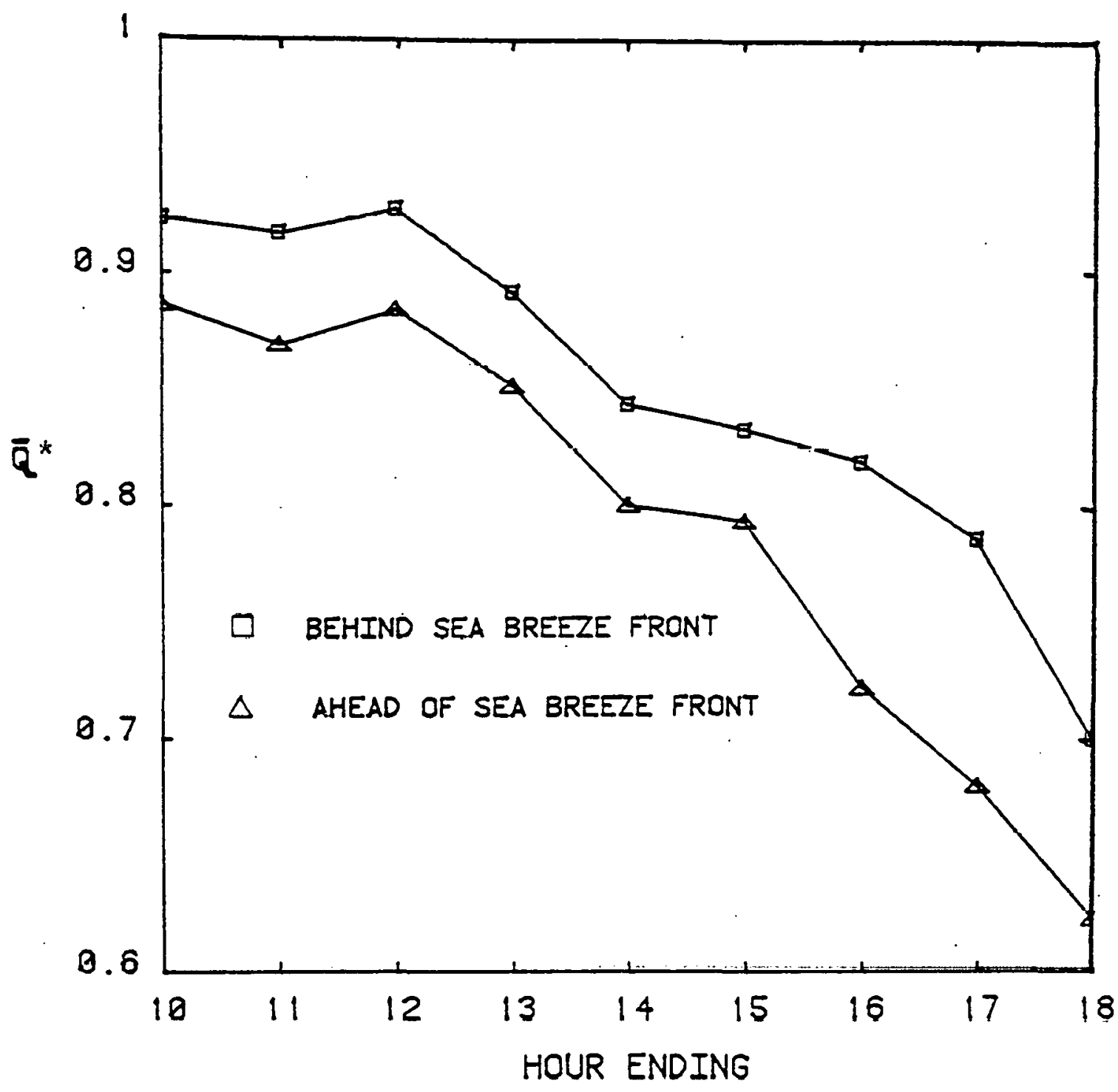

Figure 24. Mean global insolation ratio for locations ahead and behind sea breeze fronts in the Onslow Bay region during hours ending 1000 through 1800 EST, Summer 1978. 
categories: (1) The front forms inland with respect to the site (coastal), (2) the frontal passage over the site occurs during daylight hours (frontal), and (3) the front's location is coastal with respect to the site for the duration of daylight hours (inland). The mean of the daily global insolation measured at all sites for these three classifications was calculated for the months of June and July (Table 5).

Sites not experiencing a sea breeze frontal passage because of their coastal location had the highest mean daily global insolation values, followed in decreasing magnitude by sites experiencing a frontal passage and, finally, inland sites not experiencing a sea breeze frontal passage. This gradient of mean daily global insolation occurs for both the months analyzed. Total global insolation received at coastal locations in the Onslow Bay region exceeded values measured at inland sites an average of 10.3 percent during the month of June and 9.8 percent in July, 1978. The results of this analysis suggest that the daily global radiation received at any study location site will, on the average, decrease with increasing delay of the sea breeze frontal passage. Since sea breeze frontal passages usually occur later in the day for locations further inland from the coast, daily global insolation will decrease with increasing distance from the Onslow Bay coast on synoptically undisturbed days dominated by sea breeze solenoids and associated cloudiness.

Table 5. Mean daily global insolation for coastal, frontal, and inland sile classifications.

\begin{tabular}{|c|c|c|c|}
\hline Month & Site Classification & Mean Global Insolation & $\left(\mathrm{KJ} / \mathrm{M}^{2} / \mathrm{day}\right)$ \\
\hline June & $\begin{array}{l}\text { Coastal } \\
\text { Frontal } \\
\text { Inland }\end{array}$ & $\begin{array}{l}28264 \\
26056 \\
25632\end{array}$ & \\
\hline July & $\begin{array}{l}\text { Coastal } \\
\text { Frontal } \\
\text { Inland }\end{array}$ & $\begin{array}{l}27240 \\
25940 \\
24802\end{array}$ & \\
\hline
\end{tabular}

Trends for Horizontal Gradients of Global Insolation

Sea breeze study location sites were separated according to the time of the sea breeze frontal passage. The classifications used are similar to Lhuse described in the proviouc analysis. Coastal sites are defined as 
locations which experienced a frontal passage before 1100 EST. This classification would also include sites not experiencing a sea breeze frontal passage because of their close proximity to the coast. Frontal sites experienced a sea breeze frontal passage after 1100 EST. Sea breeze fronts did not pass over locations classified as inland sites.

A subset of the sea breeze case study days was chosen to include all days for which coastal, frontal, and inland sites exist. For each of these select days the mean global insolation of three time periods was calculated for each of the threp site classificatione, dorived from measured values obtained from all six study location sites. Values if glnhal insolation for the three site classifications were then summarized in the form of trend lines. The inland site classification was used as a reference for the global insolation values. The mean global insolation value for frontal sites was considered significantly higher or lower in magnitude than the reference value using a threshold of \pm 5 percent. The mean global insolation for coastal sites was defined as significantly greater in magnitude than for frontal sites if their difference exceeded a threshold of 5 percent. Given the defined threshold values, there are nine possible trend representations (Fig. 25).

Fourteen days were selected for this trend analysis. A summary of the trend analysis results is shown in Table 6 . The major trend occurring during the period from 0600-1100 EST was I, indicating an insignificant difference in mean global insolation amongst the three site classifications for sea breeze days. Trend classification I's dominance decays during the midday period as trend VI increases in frequency. Betwepn the hours of 1100 and

Table 6. Number of days represented by particular horizontal gradients of global insolation for morning, midday, and late afternoon periods

\begin{tabular}{lccccccccccc}
\hline & \multicolumn{10}{c}{ Trend Representations } & \\
\cline { 2 - 11 } HOURS (EST) & I & II & III & IV & V & VI & VII & VIII & IX & Total \\
\hline $0600-1100$ & 8 & 2 & 0 & 1 & 0 & 3 & 0 & 0 & 0 & 14 \\
$1100-1400$ & 4 & 1 & 1 & 1 & 0 & 6 & 0 & 0 & 0 & 13 \\
$1400-1800$ & 1 & 4 & 1 & 3 & 0 & 4 & 0 & 1 & 0 & 14 \\
\hline Total & 13 & 7 & 2 & 5 & 0 & 13 & 0 & 1 & 0 & 41 \\
\hline \hline
\end{tabular}



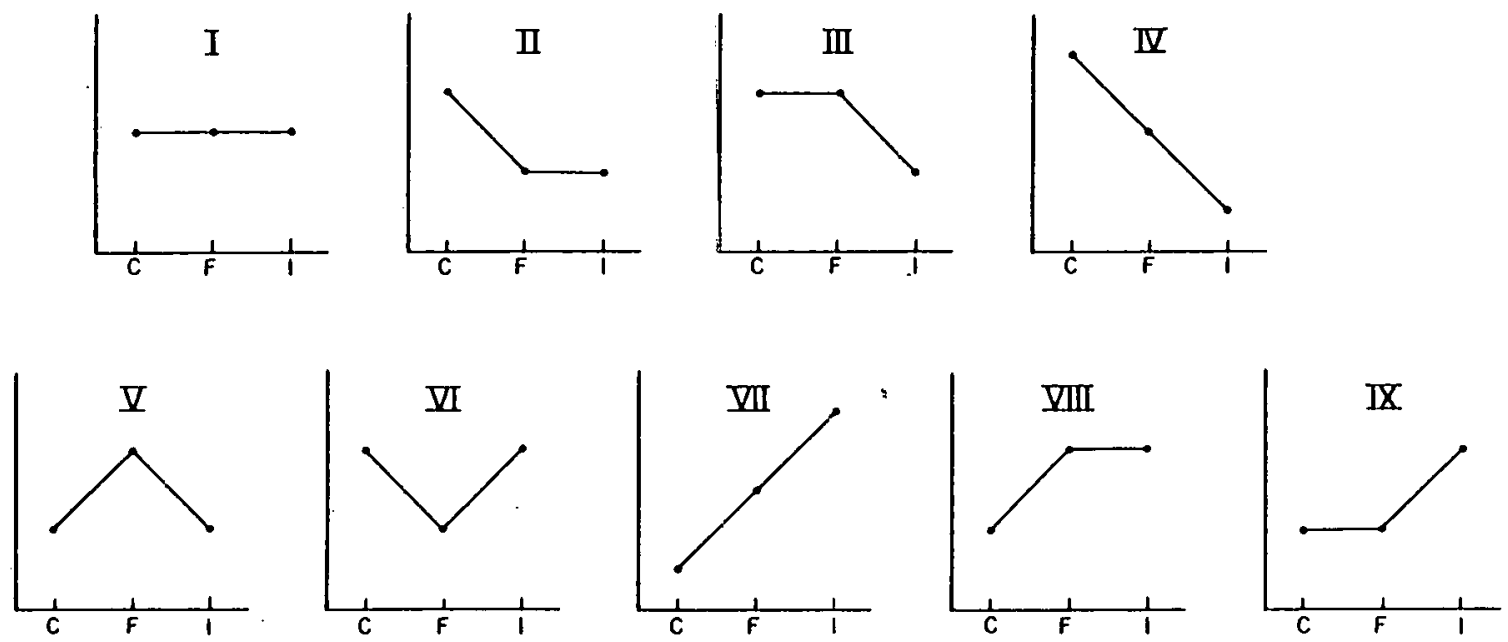

Figure 25. Nine possible global insolation gradients for the Onslow Bay region. 
1400 EST frontal sites received significantly lower levels of global insolation than either coastal or inland sites on 6 of the 14 days studied resulting from increased cloud cover with greater vertical extent along the sea breeze convergence zone. Trend classifications II, IV, and VI dominate during the late afternoon hours. Trends II and VI occurring in the late afternoon represent cases when the sea breeze front passes frontal sites relatively late in the day. Trend II will most likely occur when the sea breeze cumulus convection along the front is not maximally developed. Trend VI will occur when sea breeze cumulus along the front are more vertically developed than the air mass cumulus above inland sites. A positive global inoolation horizental gradient from the inland reference is represented by trend IV. This trend develops between the hours of 1400 and 1800 EST on those days when a sea breeze front has passed a majority of the frontal sites. The frontal cloudiness, however, remains close enough to these locations to reduce their global insolation values under lower solar elevation angles.

\section{Effect of Cloud Cover Ahead of Sea Breeze Front}

The variation in amount of cumulus cloud cover ahead of the sea breeze front is a major factor contributing to the differences in global 1nsulaliun ahead of and behind the front. A relationship was determined between the amount of cumulus cloud cover ahead of the sea breeze front and the calculated value of $\bar{Q} *$. No discrimination was made between the air mass and sea breeze cumulus cloud types for this particular analȳis. $\bar{Q}^{\lambda}$ delermined fium all clear sky observations behind the front was used as a reference value for analyzing the effect of increased cloud cover ahead of the sea breeze front on $\bar{Q} *$.

The effect of a sea breeze frontal passage on the change in $\bar{Q} *$ received at sites in the Onslow Bay region increases as the amount of cumulus cloud cover increases (Fig. 26). On days when little or no cloudiness forms ahead of the sea breeze front, the change in $\bar{Q} *$ at locations after a sea breeze frontal passages is small. A change in $\bar{Q} *$ from 0.859 to 0.924 was observed for sites experiencing a sea breeze frontal passage with two-tenths cumulus cloud cover ahead of the front. When the cumulus cloud cover ahead of the 


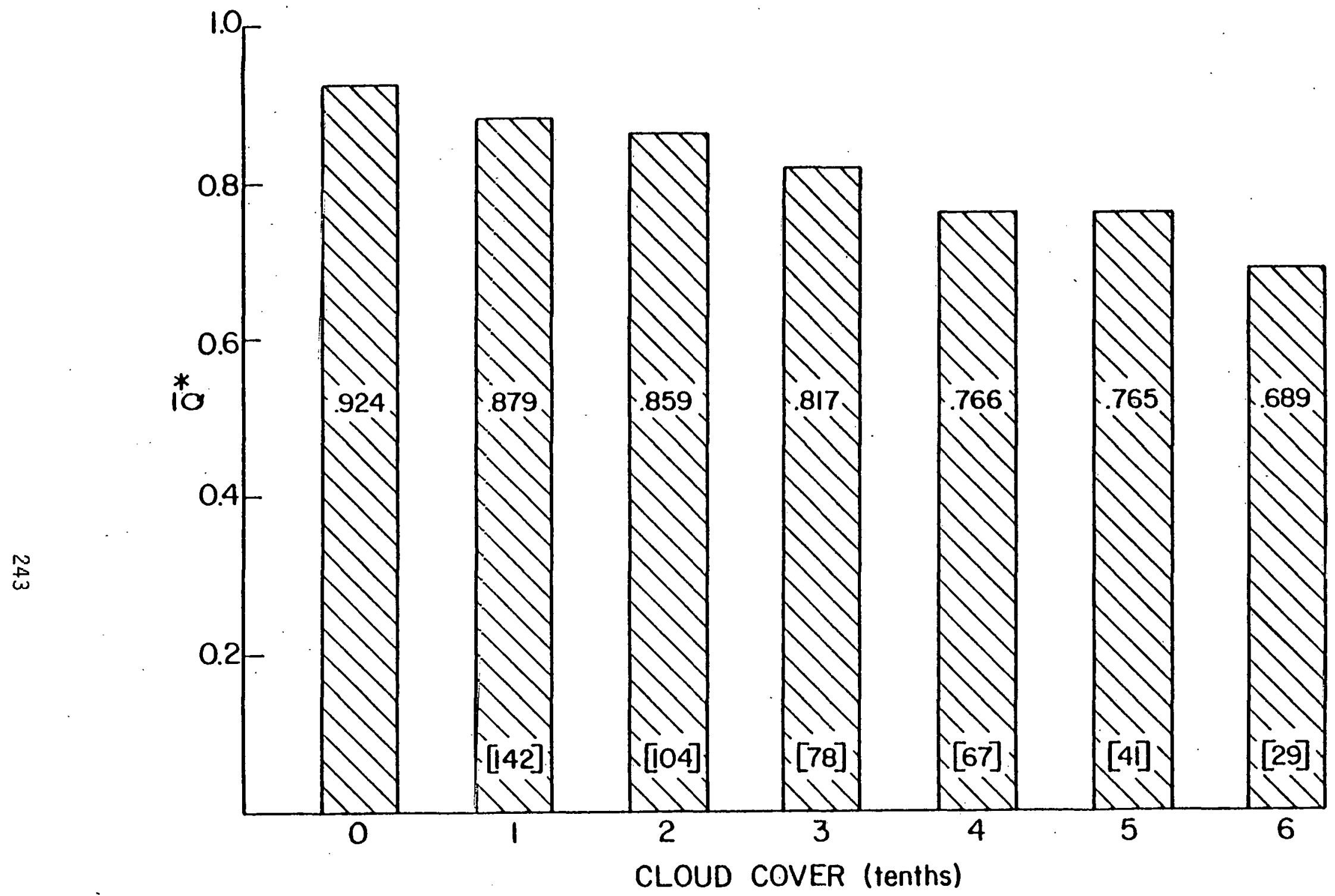

Figure 26. Relationship between the mean global insolation ratio and the cloud amount ahead of sea breeze fronts over the Onslow Bay region, Summer 1978. 
sea breeze front increased to six-tenths, an increase in $\bar{Q} *$ of 34.1 percent was observed at locations in the Onslow Bay area experiencing a sea breeze frontal passage. In general, the ratio of $\bar{Q}^{*}$ at locations ahead of and behind a sea breeze front rose significantly as the amount of cumulus cloud cover ahead of the front decreased. 
SUMMARY AND CONCLUSIONS

Distinct differences in the physical characteristics of sea breeze and air mass cumulus clouds influence the spatial variation in global insolation values measured within the Onslow Bay region. Sea breeze cumulus clouds located along the sea breeze convergence zone exhibit greater horizontal and vertical development than do the air mass cumulus clouds that form beyond this zone. Global insolation, or more specifically, the normalized insolation $\bar{Q}:$ is more significantly reduced at locations under sea breeze cumulus than air mass cumulus for any specific cloud amount. The reduction of global insolation by sea breeze cumulus cloud cover exceeds the reduction attributed to air mass cumulus by as much as 5 percent in the Onslow Bay region. $\bar{Q}^{*}$ is a nonlinear function of cumulus cloud amount; its value is also a function of the changing cloud vertical extent with increasing cloud amounts. The diurnal changes in calculated $\bar{Q} *$ values for specific amounts of sea breeze cumulus cloud cover is also related to the increase of the cloud's vertical extent with time of day. Increasing thickness of sea breeze cumulus for a constant cloud amount of 1 to 2 tenths decreased the quantity of $\bar{Q} *$ by 14.8 percent between the hours of 0900 and 1800 EST.

The clearing effect produced after the passage of a sea breeze front in the Onslow Bay area is unquestionably reflected in the increase of $\bar{Q} *$ values behind the front. The absolute change in $\bar{Q} \div$ following a frontal passage is determined by the amount and type of cumulus cloud cover that develops ahead of the sea breeze front. $\bar{Q} *$ values increased by 34.1 percent after a sea breeze frontal passage at locations under the influence of 6 tenths cumulus cloud cover before the passage. Average summer values of daily global insolation were reduced significantly (up to 10.3 percent) with an increasing delay of the sea breeze frontal passage over the Onslow Bay region. For most of the sea breeze case study days there were only minor differences (less than 5 percent) in the global insolation values amongst sites of unequal distance from the sea breeze front before the hour of 1100 EST. Later in the day, as the sea breeze front moves inland and convective type cloudiness 
increases, coastal-inland global insolation differences exceeding 10 percent frequently develop during sea breeze circulation days. Frontal stations received in excess of 5 percent less global radiation than was received at either coastal or inland sites between the hours of 1100 and 1400 EST on over a third of the sea breeze days studied. After 1400 EST values of global insolation measured at coastal and frontal stations in the Onslow Bay region were significantly higher (in excess of 5 percent) than those measured at inland sites on sea breeze case study days.

More detailed investigations of the sed breeze fronta $\perp$ passagc and sea breczc cumulus cloud effects on global insolation will require several years of data aquisitiun and a significant increase in the number of measuring sites to insure a sufficient number of observations required for many of the desired analyses. Increased resolution and magnification of the satellite photographs in both the visible and infrared spectrum will improve the accuracy and precision of any analysis incorporating variables such as cumulus cloud cover amount or cumulus cloud vertical extent. The relationships developed between sea breeze frontal passages and the values of global insolation or $Q *$ for the Onslow Bay region can be applied equally well to other regions along the southeastern coast of the United States having similar mesoscale climates. Further research investigating the effects of sea breeze circulations upon global insolation in coastal regions, where orographic features have major influences on the mesoclimate, will become increasingly urgent as this country depends more heavily upon solar radiation as a source of energy. 


\section{IITERATURE CITED}

Anderson, Ralph K., et al., 1974: Application of meteorological satellite data in analysis and forecasting. ESSA Technical Report NESC 51, (with Supplement 1 and 2), Environmental Science Services Administration, Washington, D. C., $350 \mathrm{pp}$.

Avaste, 0. A., 1964: Transfer of solar radiation in the atmosphere. NASA Technical Translation F-323, National Aeronautics and Space Administration, Washington, D. C., pp. 54-66.

Avaste, 0. A., et al., 1964: On the coverage of the sky by clouds. NASA Technical. Translation F-323, National Aeronautics and Space Administration, Washington, D.C., pp. 173-181.

Banerjee, A. K., A. Chowdhury, and T. H. Bhattucharjee, 1975: On deep inland penetration of sea breeze. Indian J. Met. Hydrol. Geophys., 26, pp. 501-505.

Carney, Charles B., 1955: Weather and climate in North Carolina. Agricultural Experiment Station Bulletin No. 396, North Carolina State University, Raleigh, N. C., 47 pp.

Galperin, B. M., and L. P. Seryakova, 1964: Scattered and total solar radiation under various conditions. NASA Technical Translation F-323, National Aeronautics and Space Administration, Washington, D. C., pp. $1-17$.

Hsu, S.-A., 1967: Mecoscale surface temperature characteristics of the Texas coast sea breeze. Atmospheric Science Group Technical Report, No. $\delta$, The Univ. of Texas at Austin, $74 \mathrm{pp}$.

Kaiser, Jack A. C., and Robert H. Hill, 1976: On skies with scattered cumulus cloud cover. J. Geophys. Res., 81, pp. 395-398.

Kasten, F., 1977: Ground radiation as affected by clouds. Radiation in the Atmosphere, Science Press, Princeton, N. J., pp. 193-195.

Kondratyev, K. Ya., 1969: Radiation in the Atmosphere, Academic Press, New York, $912 \mathrm{pp}$.

Kondratyev, K. Ya., 1972: Radiation Processes in the Atmosphere. World Meteorological Organization, No. 309, 214 pp. 
Mironova, Z. F., 1973: Albedo of Earth's surface and clouds. NASA Technical Translation F-678, National Aeronautics and Space Administration, Washington, D. C., pp. 192-247.

Pielke, Roger A., 1974: A three dimensional numerical model of the sea breezes over South Florida. Mon. Weater Rev., 102, pp. 115-139.

Pochop, Larry O., Milton D. Shanklin, and David A. Horner, 1968: Sky cover influence on total hemispheric radiation during daylight hours. J. Applied Met., 7, pp. 484-489.

Pyldmaa, V. K., and R. G. Timanovskaya, 1964: Total radiation at the surface of the Earth in various conditions of cloud cuver. NASA lechnical Translation FNational Aeronautics and Space Administration, Washingtuu, D. C., pp. $131-137$.

Robinson, N., 1966: Solar Radiation. Elsevier Publ. Co., New York, 347 pp.

Sellers, William D., 1965: Physical Climatology, Univ. of Chicago Press, Chicago, $272 \mathrm{pp}$.

Timanovskaya, R. G., and Ye. M. Feygelson, 1964: On the methodology of the study of the statistical structure of ground fluxes of solar radiation in cloudy conditions. NASA Technical Translation F-323, National Aeronautics and Space Administration, Washington, D. C., pp. 125-130.

Williams, Dansy T., 1969: Unusual wind shifts of two wildfires. USDA, Forest Service Researrh Paper SE50; Southeasteru Furest Experiment Station, Asheville, N. C., 8 pp.

Williams, Dansy T., 1972: Spring season sea breeze fronts in coastal Georgia. Project THEO Report, Southeastern Forest Experiment Station, Asheville, N.C., 21 pp.

Williams, Dansy T., 1974: Predicting the Atlantic sea breeze in the Southeastern states. Weatherwise, 27, pp. 106-109. 